Running head: fruiting body genomics

\title{
Lessons on fruiting body morphogenesis from genomes and transcriptomes of Agaricomycetes
}

\author{
László G. Nagy ${ }^{1 *}$, Peter Jan Vonk ${ }^{2}$, Markus Künzler ${ }^{3}$, Csenge Földi ${ }^{1}$, Máté Virágh ${ }^{1}$, Robin A. \\ $\mathrm{Ohm}^{2}$, Florian Hennicke ${ }^{4}$, Balázs Bálint ${ }^{1}$, Árpád Csernetics ${ }^{1}$, Botond Hegedüs ${ }^{1}$, Zhihao Hou ${ }^{1}$, \\ Xiao-Bin Liu ${ }^{1}$, Shen Nan ${ }^{5}$, Manish Pareek ${ }^{1}$, Neha Sahu ${ }^{1}$, Benedek Szathmári ${ }^{1}$, Torda Varga ${ }^{1}$, \\ Hongli Wu ${ }^{1}$, Xiao Yang ${ }^{5}$, Zsolt Merényi ${ }^{1}$ \\ 1 Synthetic and Systems Biology Unit, Biological Research Center, Szeged, 6726, Hungary \\ 2 Microbiology, Department of Biology, Faculty of Science, Utrecht University, Padualaan 8, \\ $3584 \mathrm{CH}$, Utrecht, The Netherlands \\ 3 Eidgenössische Technische Hochschule Zürich, Department of Biology, Institute of \\ Microbiology, Zürich, Switzerland \\ 4 Project Group Genetics and Genomics of Fungi, Chair Evolution of Plants and Fungi, \\ Ruhr-University Bochum, 44780, Bochum, North Rhine-Westphalia, Germany \\ 5 Institute of Applied Mycology, Huazhong Agricultural University, 430070 Hubei Province, \\ PR China \\ *To whom correspondence should be addressed: Inagy@fungenomelab.com
}

\section{Abstract}

Fruiting bodies of mushroom-forming fungi (Agaricomycetes) are among the most complex structures produced by fungi. Unlike vegetative hyphae, fruiting bodies grow determinately and follow a genetically encoded developmental program that orchestrates tissue differentiation, growth and sexual sporulation. In spite of more than a century of research, our understanding of the molecular details of fruiting body morphogenesis is limited and a general synthesis on the genetics of this complex process is lacking. In this paper, we aim to comprehensively identify conserved genes related to fruiting body morphogenesis and distill novel functional hypotheses for functionally poorly characterized genes. As a result of this analysis, we report 921 conserved developmentally expressed gene families, only a few dozens of which have previously been reported in fruiting body development. Based on literature data, conserved expression patterns and functional annotations, we provide informed hypotheses on the potential role of these gene families in fruiting body development, yielding the most complete description of molecular processes in fruiting body morphogenesis to date. We discuss genes related to the initiation of fruiting, differentiation, growth, cell surface and cell wall, defense, transcriptional regulation as well as signal transduction. Based on these data we derive a general model of fruiting body development, which includes an early, proliferative phase that is mostly concerned with laying out the mushroom body plan (via cell division and differentiation), and a second phase of growth via cell expansion as well as meiotic events and sporulation. Altogether, our discussions cover 1480 genes of Coprinopsis cinerea, and their orthologs in Agaricus bisporus, Cyclocybe aegerita, Armillaria ostoyae, Auriculariopsis ampla, Laccaria bicolor, Lentinula edodes, Lentinus tigrinus, Mycena kentingensis, Phanerochaete chrysosporium, Pleurotus ostreatus, 
Running head: fruiting body genomics

and Schizophyllum commune, providing functional hypotheses for $\sim 10 \%$ of genes in the genomes of these species. Although experimental evidence for the role of these genes will need to be established in the future, our data provide a roadmap for guiding functional analyses of fruiting related genes in the Agaricomycetes. We anticipate that the gene compendium presented here, combined with developments in functional genomics approaches will contribute to uncovering the genetic bases of one of the most spectacular multicellular developmental processes in fungi.

Key words: functional annotation; comparative genomics; cell wall remodeling; development; fruiting body morphogenesis; mushroom; transcriptome

\section{Abstract}

1.1. What are fruiting bodies and how do they develop

1.2. The evolution of Agaricomycete fruiting bodies: from simple to complex morphogenesis

1.3. Cellular and regulatory processes involved in fruiting body development

1.4. Genomic and transcriptomic resources

4. Results: developmentally expressed gene classes in the Agaricomycetes 13

4.1. Cell division, proliferation and growth

4.1.1. Meiotic and mitotic gene expression show two distinct expression peaks

4.1.3. Growth by cell expansion and turgor manipulation - a biphasic model of fruiting body development 
Running head: fruiting body genomics

4.5.4.1. Histone gene expression correlates with fruiting body initiation and

4.5.4.2. Other chromatin related genes

4.8.2.1. Chitin-active enzyme families (GH18, GH20, X325, AA9, AA14, CBM5/12,

4.8.2.2. Glucan-linked families (GH3, GH5, GH16, GH17, GH30, GH55, GH71,

4.8.2.3. Miscellaneous cell wall remodeling (GH27, GH72, GH76, GH79, GH88, GT18, pectinesterases)

4.8.3. Miscellaneous cell wall related families (HTP, DyP, AA3, AA5, GH76, GT8, SIt2, Cfs1, Eln3, peptidases)

4.9.3. Other cell surface proteins (HsbA, Con6, PriA, AA7, CFEM, wax synthases, fasciclins, peptidases, intradiol ring cleavage dioxygenase) 
Running head: fruiting body genomics

4.13. Ubiquitin-proteasome system 90

4.13.1. Cop9 signalosome 90

4.13.2. Protein ubiquitination $\quad 90$

4.14. Other gene groups $\quad 94$

4.14.1. Transporters 94

4.14.2. Stress response genes 95

4.14.3. Unannotated genes 98

4.14.4. Functionally poorly known genes 98

4.14.5. Ferric reductase superfamily proteins and iron metabolism 100

5. A synoptic model of fruiting body development 101

5.1. Early events: primordium formation and cell proliferation 101

5.2. Transition to growth by expansion 103

$\begin{array}{ll}\text { 5.3. Late events: meiosis and spore production } & 104\end{array}$

6. Outstanding questions related to fruiting body morphogenesis 105

$\begin{array}{ll}\text { 7. Conclusions } & 107\end{array}$

$\begin{array}{ll}\text { Acknowledgements } & 107\end{array}$

$\begin{array}{ll}\text { References } & 108\end{array}$

$\begin{array}{ll}\text { Figures } & 146\end{array}$

$\begin{array}{ll}\text { Table Legends } & 158\end{array}$

\section{Introduction}

Fungi are one of the five main extant lineages in which complex macroscopic structures emerged over the course of evolution. The best known macroscopic structures of fungi are sexual fruiting bodies, which enclose spore-producing cells into a 3-dimensional, protective environment and facilitate spore dispersal(Nagy et al. 2018). Sexual fruiting bodies are complex multicellular structures based on the definition of Knoll(Knoll 2011) and show evidence for repeated evolution across the fungal tree of life(Stajich et al. 2009; Merényi et al. 2020a). They are found in at least 8 lineages, with best known representatives belonging to the Pezizomycotina (e.g. morels, truffles), Agaricomycotina (e.g. agarics, boletes), however, spectacular examples evolved in several other lineages, such as Endogone spp. in the Mucoromycota(Chang et al. 2019b) or the enigmatic Neolecta genus in the Taphrinomycotina(Nagy 2017; Nguyen et al. 2017). Fruiting bodies reached highest complexity in the Agaricomycetes, which include developmentally integrated morphologies that follow a spatially and temporally tightly regulated developmental program(Hibbett 2004; Virágh et al. 2021). In terms of complexity level, certain Agaricomycete fruiting bodies approach the complexity of simple animals and plants(Taylor and Ellison 2010; Nagy et al. 2018). 
Running head: fruiting body genomics

160

161

162

163

164

165

166

167

168

169

170

171

172

173

174

175

176

177

178

179

180

181

182

183

184

185

186

187

188

189

190

191

192

193

194

195

196

197

198

199

200

201

202

203

204

205
Fruiting body morphogenesis has been a subject of intense research, however, despite tremendous efforts in this field, morphogenesis of Basidiomycota fruiting bodies is quite poorly known. In comparison, fruiting body development and its underlying genetics are considerably better explored in the Ascomycota (see e.g.(Pöggeler et al. 2006a)). This may be due to a mixture of factors, including the complexity of Basidiomycota developmental programs, the lack of easy-to-use model systems or the relative difficulty of hypothesis testing via genetic manipulation (e.g. due to low frequency of homologous recombination(De Jong et al. 2010)). Research on Basidiomycete fruiting body morphogenesis has recently been relying largely on comparative -omics techniques, which provided insights into the gene repertoires and the regulation of gene expression during fruiting body development. This complements the rich body of literature on functionally characterized genes in Coprinopsis cinerea (summarized in Kües(Kües 2000)), Schizophyllum commune and other species. There are several recent milestones in fruiting body research such as the publication of key genome sequences(Ohm et al. 2010; Stajich et al. 2010; Morin et al. 2012) or comparative transcriptomic datasets (e.g. (Morin et al. 2012; Plaza et al. 2014a; Kües and Navarro-González 2015; Almási et al. 2019; Krizsán et al. 2019; Merényi et al. 2021)). An emerging driving force of research on fruiting body morphogenesis is the mushroom industry, which produces sexual fruiting bodies for food or as sources of bioactive compounds(Royse et al. 2017; Ma et al. 2018). Fruiting body development has been a subject of several excellent reviews, surprisingly, however, the last gene-centric review of fruiting body development in mushroom-forming fungi was published in 2000 (Whiteford and Thurston 2000). This paper aims to complement recent reviews on the morphogenesis of vegetative hyphae(Riquelme et al. 2018), and on the genome of Neurospora crassa(Borkovich et al. 2004) with knowledge on fruiting body morphogenesis in the Agaricomycetes.

The aim of this paper is to systematically catalog genes and genetic processes related to fruiting body morphogenesis in the Agaricomycetes, based on both literature reviews and a meta-analysis of published developmental transcriptomes. We provide a handbook-like inventory of gene families and their putative functions based on expression data and functional annotations borrowed from well-researched model systems. Because a negligible proportion of morphogenesis genes has so far been functionally characterized in the Agaricomycetes, it is currently impossible to build a comprehensive picture based on mechanistic studies alone, as has recently been done for hypha morphogenesis(Riquelme et al. 2018). Instead, we derive functional hypotheses for genes based on comparative expression data across multiple species. Our starting point is that genes which are developmentally regulated in the majority of species for which transcriptome data are available, should belong to the core pathways of fruiting body morphogenesis, whereas genes that show species-specific expression patterns should be responsible for sculpting species-specific morphologies or represent transcriptional or technical noise. Finally, we incorporate novel and previous observations into a new synthesis on processes active during fruiting body morphogenesis.

\subsection{What are fruiting bodies and how do they develop}

Fruiting bodies, also called basidiomes, basidiocarps or simply 'mushrooms' in lay language, may be some of the best known structures of fungi. They are sexual reproductive organs that facilitate spore dispersal. While in simple Basidiomycota spores are born on naked 
Running head: fruiting body genomics

206 basidia, mushroom-forming fungi evolved complex 3-dimensional structures that offer 207 tremendous advantages in spore dispersal efficiency.

208 Developmental events that take place in fruiting bodies have been investigated

209 previously in several species, of which Coprinopsis/Coprinellus spp. and S. commune are

210 the two most widely used groups of models. The development of fruiting bodies in these

211 species has been described in previous reviews(Kües 2000; Kües and Liu 2000; Palmer and

212 Horton 2006; Kües and Navarro-González 2015), therefore, we here only provide a short

213 introduction, and describe the process in more detail (combining new results) in Chapter 5.

214 Briefly, fruiting body development is initiated on sexually competent mycelia as the

215 reprogramming of hyphal branching patterns, which results in the interlacing of hyphae to

216 form a 3-dimensional fruiting body initial(Kües and Liu 2000; Kües and Navarro-González

217 2015). Rather than relying on zygotic (e.g. maternal) mRNA cargo as animals and plants do,

218 fungal hyphae undergo transcriptional reprogramming during fruiting body initiation. This

219 reprogramming is influenced and triggered by a mixture of - often species-specific - factors,

220 such as starvation, light or temperature changes (reviewed by(Sakamoto 2018)). Once

221

222

223

224

225

226 appropriate internal and external signals converge on the initiation of fruiting, morphogenesis starts with an undifferentiated initial (hyphal knot, aggregate), which undergoes tissue differentiation to form primordia. In subsequent late primordium and young fruiting body stages rapid growth (stipe elongation and cap expansion) and meiosis happen in a coordinated manner, which ultimately results in species-specific mature morphologies and culminates in spore formation and release.

Development of the fruiting bodies is a tightly regulated process that has a high degree of autonomy. That is, once started, the developmental program is not interrupted by even large perturbations, such as injuries, or removal of the fruiting body from the supporting colony at or after certain stages(Moore 2013). In fruiting bodies not only morphogenetic events, but also several internal processes (e.g. nutritional and sexual) are developmentally regulated. For example, in $C$. cinerea, stipe elongation starts immediately after meiosis takes place, after which spore production, maturation and cap expansion follow in a tightly choreographed chronology(Kües 2000; Moore 2013). For detailed description of the process and molecular summaries of current knowledge on fruiting body morphogenesis the reader is referred to recent reviews(Kües 2000; Kües and Navarro-González 2015; Virágh et al. 2021) and the following detailed chapters of this paper.

\subsection{The evolution of Agaricomycete fruiting bodies: from simple to complex}

\section{9 morphogenesis}

240 How fruiting body complexity evolved has interested mycologists for decades and has received considerable attention recently. Therefore we provide only a brief introduction here and refer the reader to recent reviews on the topic (e.g.(Kües and Navarro-González 2015; in uncovering broad patterns of morphological transformation and trends in fruiting body evolution (e.g. (Hibbett 2004; Varga et al. 2019; Sánchez-García et al. 2020)). Fruiting body morphologies show a great diversity in the Basidiomycota, from naked lawns of basidia, to resupinate, coralloid or highly complex, or the so called pileate-stipitate fruiting bodies of well-known agarics (e.g. C. cinerea) (Fig. 1).

Agaricomycete fruiting bodies might have emerged in or around the last common 
Running head: fruiting body genomics

252 This is partly due to the presence of jelly-like telia reminiscent of fruiting bodies in the 253 Pucciniomycotina, uncertainties in the branching order of Basidiomycota subphyla and 254 reductive evolution in early-diverging clades (Bartheletiomycetes, Wallemiomycetes) of the 255 Agaricomycotina subphylum (reviewed in (Prasanna et al. 2020)). Nevertheless, all 256 Agaricomycetes share a single, fruiting body-forming ancestor, which suggests there should 257 be a core, deeply conserved genetic toolkit of fruiting body development. Phylogenetic 258 comparative studies have provided sound evidence that this ancestor had 259 corticioid/resupinate/jelly like fruiting bodies (e.g. Phanerochaete chrysosporium) and that 260 more complex forms derived from such morphologies repeatedly(Hibbett 2004; Varga et al. 261 2019). Within the Agaricomycetes, tissues that protect and enclose basidia became thicker 262 and more complex, eventually leading to the mushroom fruiting body structures we know 263 today. From the perspective of this paper, it is important to mention that phylogenetic studies 264 have also established that fruiting body evolution is highly convergent, that is, many of the 265 characteristic traits (e.g. cap, gills) evolved independently several times. Therefore, an 266 evolutionary framework is important for understanding how fruiting bodies evolved and for 267 establishing homologies among tissues. Gills, caps and stipes, for example, although 268 superficially similar, are not homologous across Polyporales and Agaricales. This implies 269 that such, convergent forms (e.g. gills of Lentinus tigrinus in the Polyporales and that of $C$. cinerea in the Agaricales) are not homologous across lineages and their development should not be expected to follow the same genetic principles. On the other hand, basidia, the sexual processes that happen therein and the development of 3-dimensional fruiting body tissue, are homologous across all mushroom-forming fungi (i.e. they have a single origin in the Basidiomycota), providing an reliable framework for analysis.

These examples illustrate how the diversity of fruiting bodies can complicate the identification of core components of their morphogenesis. Ideally, we are interested in developmental processes and underlying genetic components that uniformly characterize fruiting bodies of all mushroom-forming fungi, i.e. those that can be traced back to the first fruiting body-forming Agaricomycete ancestor.

Therefore, building 'minimal models', which encompass the minimum set of genes necessary to build a fruiting body are highly relevant. Such minimal models could be, among others, species that produce resupinate fruiting bodies (e.g. Ph. chrysosporium)(Krizsán et al. 2019). Recently, Cryptococcus neoformans (Tremellomycetes, Basidiomycota) proved to be a useful model organism, because it produces simple lawns of basidia, sometimes referred to as fruiting bodies, in which basidium differentiation, meiosis and spore formation take place(Liu et al. 2018). Therefore, C. neoformans 'fruiting bodies' encompass all processes needed for Basidiomycota sexual reproduction but lack features essential to the definition of fruiting bodies such as three-dimensional organization, tissue differentiation and others. Merenyi et al recently sorted developmental genes of Pleurotus ostreatus and seven other complex species into groups that were developmentally regulated both in $C$. neoformans and mushroom-forming fungi and those in which developmental expression was observed only in mushroom-forming fungi(Merényi et al. 2021). 'Shared' orthogroups comprised most mitosis/meiosis related genes, but also some previously thought to be specific for complex morphogenesis (e.g. fasciclins). This indicates that sexual morphogenesis, even at the very simple forms that exist in $C$. neoformans, involves a morphogenetic program. Comparing genes that are developmentally regulated in fruiting bodies to those regulated in $C$. neoformans, thus, can help constructing more precise genetic models of complex fruiting body morphogenesis. 
bioRxiv preprint doi: https://doi.org/10.1101/2021.12.09.471732; this version posted December 10,2021 . The copyright holder for this preprint (which was not certified by peer review) is the author/funder, who has granted bioRxiv a license to display the preprint in perpetuity. It is made available under aCC-BY-NC-ND 4.0 International license.

Running head: fruiting body genomics

299

300

301

302

303

304

305

306

307

308

309

310

311

312

313

314

315

316

317

318

319

320

321

322

323

324

325

326

327

328

329

330

331

332

333

334

335

336

337

338

339

340

341

342

343

344

345

\subsection{Cellular and regulatory processes involved in fruiting body development}

Fruiting body development is an incredibly complex process and this complexity is reflected in the underlying cellular and genetic processes. Several cellular processes are emerging as important regulatory mechanisms underlying fruiting body morphogenesis, whereas some others that proved developmentally relevant in animals and plants seem to be of limited importance in fungi (e.g. gene body methylation(A et al. 2018; Wen et al. 2019)). This chapter summarizes the most important - mostly transcription related - processes described in fruiting bodies recently. Splicing and RNA interference are discussed below under chapters 4.5 .3 and 4.6 , respectively.

Probably the most influential and most commonly assayed mechanisms of fruiting body development are transcriptional reprogramming and gene expression changes. Gene expression changes naturally associate with developmental processes and are informative regarding the cellular processes that take place during fruiting. In silico functional analyses of differentially or developmentally expressed genes have been a primary tool for mycologists to understand fruiting body functions. Developmentally dynamic gene expression patterns form the primary data source for this paper and the main part of the paper (Chapter 4 ) is devoted to discussing genes with conserved expression patterns. A significant unresolved question is how gene expression changes are regulated in various stages of fruiting body development. It is not known currently if transcriptional reprogramming events (e.g. during fruiting body initiation) are mediated by broad regulation of chromatin accessibility, by regulating the expression and activity of regulatory genes (e.g. transcription factors), or a mixture of these. A potentially significant regulatory mechanism is post-translational modification of transcription factors or molecular switches in intracellular signaling pathways, as demonstrated for the Hom2 or the Ras 1 gene of $S$. commune(Knabe et al. 2013; Pelkmans et al. 2017b). Another exciting avenue of future research is the regulatory potential of selective post-translational protein modification by the ubiquitin/proteasome system. Although direct evidence for its role in fruiting body development has not yet been published, indirect evidence was provided by the remarkable expansion of genes encoding F-box, BTB/POZ and RING-type zinc finger proteins in the genomes of mushroom-forming fungi(Krizsán et al. 2019). These gene families provide the substrate-specificity of ubiquitin ligases and have been shown, in other lineages, to be involved in transcriptional regulation(Kipreos and Pagano 2000).

Natural antisense transcripts (NATs) are RNA transcripts that show complementarity to transcripts of protein coding genes. NATs occur ubiquitously across eukaryotes and have been detected in mushroom-forming fungi too(Ohm et al. 2010; Muraguchi et al. 2015; Merényi et al. 2021). Because NATs do not encode proteins, they often evolve very fast and are hard to link to specific functions. Nevertheless, their expression is developmentally dynamic in all species examined to date, suggesting that they may be involved in regulating development. Muraguchi et al reported that an antisense transcript of the stipe elongation related AspE gene is transcribed just before stipe elongation(Muraguchi et al. 2015). However, it should also be kept in mind that many NATs may result from random transcription initiation and may therefore be transcriptional noise in fruiting body transcriptomes(Merényi et al. 2021).

Allele-specific expression (ASE) refers to the imbalance of gene expression from two divergent alleles in diploid or dikaryotic organisms. Fungal dikaryons almost always harbor two allelically different monokaryons (heterokaryons), which can lead to intermediate, A- or B-dominated expression. ASE has so far been detected in fruiting body transcriptomes of $A$. 
Running head: fruiting body genomics

346 bisporus(Gehrmann et al. 2018) and P. ostreatus(Merényi et al. 2021), but may be

347 widespread in the Agaricomycetes. In A. bisporus, Gehrmann et al suggested that ASE may

348 facilitate the division of labor between nuclei in a dikaryon(Gehrmann et al. 2018). Another

349 study, based on $P$. ostreatus, suggested that, at macroevolutionary timescales, ASE may be

350 a neutrally arising phenomenon that can nevertheless generate adaptive gene expression

351 variation(Merényi et al. 2021). It was found to be mostly characteristic of evolutionarily young

352 genes, consistent with neutral evolution. Allele-specific expression has agriculturally broad

353 consequences in hybrid genetics of plants(Bartoš et al. 2019) and could become a similarly

354 central question in the mushroom industry.

355 RNA editing has recently been detected in fruiting body transcriptomes of several

356 Ascomycota species(H et al. 2016; Liu et al. 2017; Teichert et al. 2017) and subsequently

357 reported in Basidiomycota also(Zhu et al. 2014; Wu et al. 2019). Like alternative splicing,

358 RNA editing provides a (post-)transcriptional mechanism for diversifying transcripts encoded

359 by a single gene, by enzymatically modifying certain bases of the primary RNA transcript. A

360 critical analysis recently found no evidence for RNA editing in fruiting body transcriptomes of

$361 P$. ostreatus(Merényi et al. 2021). This study raised the possibility that, because allele-

362 specific expression and RNA editing can generate similar signatures in RNA-Seq data, ASE

363 may have been mistaken for RNA editing in some prior Basidiomycota analyses and that

364

365

366

367

368

369

370

371

372

373

more research is needed to prove a role of RNA editing in mushroom development.

Several other exciting phenomena may be involved in the morphogenesis of

Basidiomycota fruiting bodies, including regulatory processes that may yet to be discovered.

For example, epigenetic regulation represents a potentially widespread but largely

underexplored regulatory mechanism for fruiting body morphogenesis(Vonk and Ohm 2021).

Similarly, upstream open reading frames (uORFs), which are open reading frames located in the 5' untranslated region of an mRNA, can regulate (e.g. via encoded peptides) or interfere with the transcription of the gene(HM et al. 2009). uORFs are known to be important in the asexual development of Ascomycota(Han and Adams 2001) [and have recently been detected in developmental genes of $C$. cinerea (Hegedus et al in prep)].

\subsection{Genomic and transcriptomic resources}

375 Genomic resources are also available for most model species of fruiting body development

376 (e.g. C. cinerea (Schaeff.) Redhead, Vilgalys \& Moncalvo, S. commune Fr., Cyclocybe

377 aegerita (V. Brig.) Vizzini)(Ohm et al. 2010; Stajich et al. 2010; Muraguchi et al. 2015; Gupta

378 et al. 2018; Almási et al. 2019) and those used by the mushroom industry, including

379 Hericium erinaceus (Bull.) Pers.(Gong et al. 2020; W et al. 2020), Ganoderma spp.(Chen et

380 al. 2012), Flammulina spp.(Park et al. 2014, 2019; Chen et al. 2020), L. edodes (Berk.)

381 Pegler(Chen et al. 2016; Shim et al. 2016; Sakamoto et al. 2017a), Pleurotus spp(A et al.

382 2018; Wang et al. 2018a; Dai et al. 2019; Lee et al. 2021), Agaricus spp(Morin et al. 2012;

383 O'Connor et al. 2019; Sonnenberg et al. 2020), Tricholoma matsutake (S. Ito \& S. Imai)

384 Singer(Min et al. 2020), Sparassis crispa (Wulfen) Fr.(Kiyama et al. 2018), Hypsizygus

385 marmoreus (Peck) H.E. Bigelow(Min et al. 2018), among others. More broadly, in the

386 Agaricomycetes, draft genome sequences have been proliferating at a steady pace, due to

387 interest in biodiversity, lignocellulose decomposition(Martinez et al. 2004, 2009a; Floudas et

388 al. 2012; Riley et al. 2014) and mycorrhiza-formation(Martin et al. 2008; Kohler et al. 2015;

389 Miyauchi et al. 2020). This trend has virtually eliminated genome data being the bottleneck in

390 biological discovery in fungi. This is further supported by the spread of third generation

391 sequencing techniques, long-read sequencing data, which allow more and more complete 
bioRxiv preprint doi: https://doi.org/10.1101/2021.12.09.471732; this version posted December 10,2021 . The copyright holder for this preprint (which was not certified by peer review) is the author/funder, who has granted bioRxiv a license to display the preprint in perpetuity. It is made available under aCC-BY-NC-ND 4.0 International license.

Running head: fruiting body genomics

392 (telomere-to-telomere) assemblies and a range of analyses that draft genomes did not 393 permit.

394

395

396

397

398

399

400

401

402

403

404

405

406

407

408

409

410

411

412

413

414

415

416

417

418

419

420

421

422

423

424

425

426

427

428

The continuous development of technologies has also influenced the identification of genes related to fruiting body morphogenesis. While early studies isolated fruiting bodyspecific proteins/genes using mutant analyses, hybridization techniques (among others), these were later substituted by RT-PCR and microarray studies, then by high throughput sequencing analyses, such as 5'-serial analysis of gene expression (SAGE(WW et al. 2008; Cheng et al. 2013a)) and most recently RNA-Seq. As of today, RNA-Seq datasets are available for a wide range of species and conditions. These include $C$. cinerea(Plaza et al. 2014a; Muraguchi et al. 2015; Krizsán et al. 2019; Xie et al. 2020), Flammulina spp(Yan et al. 2019; Liu et al. 2020b), L. edodes(Park et al. 2017; Sakamoto et al. 2017b; Song et al. 2018b; Y et al. 2018; Yoo et al. 2019; Kim et al. 2020), Agaricus spp(Gehrmann et al. 2018; Lu et al. 2020; O'Connor et al. 2021), Pleurotus ostreatus (Jacq.) P. Kumm.(Wen et al. 2019; Merényi et al. 2021), H. marmoreus(Zhang et al. 2015b), C. aegerita(Orban et al. 2021), Phanerochaete chrysosporium Burds.(Krizsán et al. 2019), Lentinus tigrinus (Bull.) Fr.(Krizsán et al. 2019), Mycena kentingensis Y.S. Shih, Chi Y. Chen, W.W. Lin \& H.W. Kao(Ke et al. 2020a), Rickenella mellea (Singer \& Clémençon) Lamoure(Krizsán et al. 2019), Armillaria ostoyae (Romagn.) Herink(Sipos et al. 2017a), Pisolithus microcarpus (Cooke \& Massee) G. Cunn (Pereira et al. 2017), Laccaria bicolor (Maire) P.D. Orton (Ruytinx et al in prep), S. commune(Ohm et al. 2010) and Auriculariopsis ampla (Lév.) Maire(Almási et al. 2019). Many of these datasets build the foundation of this study, by providing windows into developmentally expressed genes in different fungal species.

A general observation we made during the analyses we present hereafter is that the resolution of the transcriptomic data is key to identifying expression dynamics and patterns. Both tissue-wise and temporal resolution of the data allowed the discovery of more developmentally relevant expression patterns. We found several cases where tissueresolved transcriptomes provided clear signal for conserved, tissue-specific expression of a gene, whereas in non-resolved transcriptomes the same gene showed a more or less flat expression curve. This is an inherent nature of bulk RNA-Seq data: spatial expression patterns average out in samples that contain large populations of multiple cell types.

Beyond gene expression, a broad array of other types of information could be leveraged for understanding fruiting body development. Notable recent examples include assays of the overrepresentation of certain gene families in the genomes of focal species(Krizsán et al. 2019), a population genomics study combined with selection analyses and gene expression profiling in $L$. edodes(Zhang et al. 2021c) to identify genes involved in fruiting body development(Zhang et al. 2021c), or ChIP-Seq analyses for assaying promoter occupancy and thus transcriptional activity(Vonk and Ohm 2021).

\section{Description of the approach used in this paper}

430 In this study, we address the puzzle of how a fruiting body develops and what novel insight 431 can be gleaned from systematic comparisons of developmental transcriptomes. We aim at 432 providing a comprehensive listing of putatively development-related gene families in the 433 Agaricomycetes. We provide, for each discussed functional group or gene family, 434 information on their expression, orthology to well-established model organisms 435 (Saccharomyces cerevisiae, Schizosaccharomyces pombe, Aspergillus nidulans, 436 Neurospora crassa) and to key mushroom-forming fungi as tables. We also annotate several 
bioRxiv preprint doi: https://doi.org/10.1101/2021.12.09.471732; this version posted December 10,2021 . The copyright holder for this preprint (which was not certified by peer review) is the author/funder, who has granted bioRxiv a license to display the preprint in perpetuity. It is made available under aCC-BY-NC-ND 4.0 International license.

Running head: fruiting body genomics

437 conserved pathways in C. cinerea, based on strict 1-to-1 orthology to known pathway 438 members in well-researched model systems (see Methods for details).

439 Each chapter is structured so that it starts with a literature review of the given gene

440 family followed by a discussion of novel findings (if any) based on our meta-analysis of 441 transcriptomes. Our main units of investigation are conserved developmentally expressed

442 (CDE) orthogroups, which are groups of single-copy genes in which the majority $\left(>65 \%{ }^{1}\right)$ of

443 the genes were developmentally expressed (see Methods and Merenyi et al 2021(Merényi et

444 al. 2021)). For simplicity CDE orthogroups are considered to be groups of genes sharing

445 similar functions (see limitations below) in fruiting body development.

446

447

448

449

450

451

452

453

We performed a meta-analysis of 12 developmental transcriptomes (Agaricus bisporus(Gehrmann et al. 2018), C. aegerita(Orban et al. 2021), A. ostoyae(Sipos et al. 2017a), A. ampla(Almási et al. 2019), C. cinerea(Krizsán et al. 2019), L. bicolor (Ruytinx et al in prep), L. edodes(Zhang et al. 2021d), L. tigrinus(Krizsán et al. 2019), M. kentingensis(Ke et al. 2020a), Ph. chrysosporium(Krizsán et al. 2019), P. ostreatus(Krizsán et al. 2019), S. commune(Almási et al. 2019)) (Fig. 1). These species belong to two orders (Agaricales, Polyporales), which span an estimated 200 million years of evolution(Varga et al. 2019), and represent diverse ecologies and morphological adaptations, such as simple resupinate $(P h$. chrysosporium), coralloid (Pterula gracilis), pileate-stipitate (e.g. C. cinerea) and cyphelloid (A. ampla, S. commune) forms. Each of these species and their ecological adaptations show several unique aspects (e.g. fruiting body ecotypes) which could be related to gene expression data. However, we are here interested in only the shared aspects of fruiting body development, and therefore use the diversity of the compared species in focusing our attention to widely conserved fruiting body genes which should help define the core building blocks required for fruiting body morphogenesis.

The comparative approach we follow is paramount to separate 'wheat from chaff', i.e. developmentally regulated genes that are relevant for the development of fruiting bodies in general from genes with taxonomically restricted or species-specific developmental roles. This builds on the assumption that a gene involved in core fruiting body functions should be conserved across Agaricomycetes and should show similar expression dynamics in all species. Therefore, in our approach we focus on conservation of both sequence and expression. A comparative approach is also desirable because it is now well-known that gene expression is associated with 'biological noise', which may influence the detection of differentially expressed or developmentally regulated genes(Merényi et al. 2021) in any single species, whereas across-species comparisons effectively cancel the effects of gene expression noise.

This study, like all others, has limitations. First, it should be noted that evidence for gene function remains, in an overwhelming majority of cases, circumstantial, inferred from expression patterns or extrapolated from the functions of orthologous genes in model fungi. At the moment we rely on these types of evidence due to the scarcity of developmental genetic and in-depth functional studies in most Agaricomycetes. Despite the inferential nature of the study, many orthologs display very conserved expression patterns across Agaricomycetes species (e.g. aquaporins in stipe tissues, see below), allowing us to make confident predictions on the function of such genes. Second, a perfect comparative transcriptomic study would compare corresponding developmental stages across species. In the Agaricomycetes establishing homology relationships among individual species is

\footnotetext{
${ }^{1}$ We deviaed from this rule where justified.
} 
bioRxiv preprint doi: https://doi.org/10.1101/2021.12.09.471732; this version posted December 10,2021 . The copyright holder for this preprint (which was not certified by peer review) is the author/funder, who has granted bioRxiv a license to display the preprint in perpetuity. It is made available under aCC-BY-NC-ND 4.0 International license.

Running head: fruiting body genomics

482 complicated (see above). Furthermore, the developmental transcriptomes we used differ in 483 the sampled time points and resolution (e.g. tissue-wise or bulk) and do not follow a unified 484 nomenclature for developmental stages (e.g. aggregate in $S$. commune vs. hyphal knot in $C$. 485 cinerea). Therefore, we here focused broadly on comparing expression dynamics and the 486 shape of the expression curve, rather than attempting to find 1-to-1 correspondence 487 between stages of different species. Finally, in the present study, we focus on genes with 488 developmentally dynamic expression. However, not all morphogenetically relevant genes 489 show expression dynamics; constantly expressed genes can often be important for 490 development, for example, if regulated post-transcriptionally (e.g. alternative splicing 491 isoforms(Gehrmann et al. 2016; Krizsán et al. 2019)) or post-translationally (e.g. 492 phosphorylation, ubiquitylation etc.(Knabe et al. 2013; Pelkmans et al. 2017a)).

493

\section{Methods}

495 Bioinformatic analyses of RNA-Seq data

496 The raw RNA-Seq data of 12 previously published Basidiomycota species were reanalysed

497 following Merenyi et al(Merényi et al. 2021). The following reference genomes were used: $C$. 498 cinerea (AmutBmut pab1-1 v1.0(Muraguchi et al. 2015)), A. ostoyae (C18/9(Sipos et al. 499 2017b)), A. ampla (NL-1724 v1.0(Almási et al. 2019)), S. commune (H4-8a and H4-8b(Ohm 500 et al. 2010)), L. bicolor (v2.0(Martin et al. 2008)), L. edodes (Le(Bin) 0899 ss11 v1.0(Zhang 501 et al. 2021c)), L. tigrinus (RLP-9953-sp(Wu et al. 2018)), Ph. chrysosporium (RP-78 502 v2.2(Ohm et al. 2014)), P. ostreatus (PC15 v2.0(Riley et al. 2014)), M. kentingensis(Ke et al. 503 2020a), A. bisporus (var. bisporus H97(Morin et al. 2012)), C. aegerita (AAE-3(Gupta et al. 504 2018)). To remove adaptors, ambiguous nucleotides and any low quality read ends, reads 505 were trimmed using bbduk.sh and overlapping read pairs were merged with bbmerge.sh 506 (part of BBMap/BBTools; http://sourceforge.net/projects/bbmap/) with the following 507 parameters: qtrim $=r l$ trimq $=25$ minlen=40. A two-pass STAR alignment(Veeneman et al. 508 2016) was performed against reference genomes with the same parameters as in our 509 previous study(Krizsán et al. 2019) except that the maximal intron length was reduced to 5103000 nt. Reads were counted and summarized for each exon with FeatureCounts(Liao et al. 511 2014) taking into account the mode of sequencing (paired-end, single-end). Since the 512 Cyclocybe transcriptome was obtained with the Quantseq method, reads were counted only 513 in the $-100-+400$ region of the 3' ends of the genes. Read count data were normalized 514 using EdgeR(Robinson et al. 2010). Expression levels were calculated as fragments per 515 kilobase of transcript per million mapped reads (FPKM).

$516 \quad$ Developmentally regulated genes were identified as before(Krizsán et al. 2019; 517 Merényi et al. 2021), based on a stricter four-fold or a more permissive two-fold change 518 cutoff between adjacent developmental stages or tissue types. Genes showing a 4-fold 519 upregulation in the first primordium stage relative to vegetative mycelium were termed ' $F B$ 520 init', and considered regulated during fruiting body initiation. It should be noted that the data 521 we analyze is noisy, based on different chemistries, different labs and protocols, etc., which 522 introduces a certain amount of data loss in our analyses. Nevertheless, the transition to 523 fruiting body development is such a dramatic change that we expect similar gene expression 524 changes to be discoverable across species, despite the heterogeneity of the data. This is 525 supported by previous comparative transcriptomic studies(Plaza et al. 2014b; Krizsán et al. 
bioRxiv preprint doi: https://doi.org/10.1101/2021.12.09.471732; this version posted December 10,2021 . The copyright holder for this preprint (which was not certified by peer review) is the author/funder, who has granted bioRxiv a license to display the preprint in perpetuity. It is made available under aCC-BY-NC-ND 4.0 International license.

Running head: fruiting body genomics

526 2019; Merényi et al. 2020b, 2021) as well as the biologically meaningful conclusions 527 obtained here.

528

529

530

531

532

533

534

535

536

537

538

539

540

541

542

543

544

545

546

547

548

549

550

551

552

553

554

555

556

557

558

559

560

\section{Orthology based on reciprocal best hits}

Proteins of each species were searched against the proteomes of other species using the reciprocal best hit (RBH) module of MMSeqs2(Steinegger and Söding 2017). To remove spurious RBHs, a bidirectional $50 \%$ coverage and at least $1 \mathrm{e}-6$ e-value were required. Proteins were clustered into single copy clusters using a connected component clustering algorithm in the igraph package([CSL STYLE ERROR: reference with no printed form.]), based on reciprocal best hits. This step clustered $74.3 \%$ of the proteins in the examined species. The other $25.7 \%$ of proteins form clusters with duplication in any or more species. For these 1:1 orthogroups were circumscribed using the 'ortholog coding' algorithm of the COMPARE pipeline(Nagy et al. 2014). In the case of terminal duplications, orthogroup membership was decided based on connectivity; the protein which showed more hits to other members of the orthogroup was selected while the other(s) moved to separate orthogroup(s). Complete orthology relationships of genes across all 12 species are given in this paper are presented in Supplementary Table 1.

This approach is suitable for identifying moderately to very conserved gene groups, whereas for highly volatile genes (i.e those that show frequent duplication/loss), a more liberal approach building on orthologous groups (like(Krizsán et al. 2019)) is more appropriate. For fast-evolving genes, approaches looking at sequence conservation might not work. Therefore, in the case of certain gene families, such as defense genes or cell wall remodeling CAZymes, we relied on primary gene family classification instead of reciprocal best hit based orthogroups. In the case of CAZymes, we followed the cazy.org classification(Levasseur et al. 2013).

\section{Identification of CDE orthogroups}

The central unit of discussion in this paper is referred to as 'conserved developmentally expressed (CDE) orthogroup', which refers to groups of single-copy genes (one gene per species) in which the majority of genes is developmentally regulated either at fold change $>2$ or $>4$. As a principal rule, we considered an orthogroup to be a CDE orthogroup if $>65 \%$ of the genes in it were developmentally regulated, and at least 8 species were represented. Deviations from this rule were allowed in unique cases (e.g. taxonomically restricted but important gene family) and are transparently presented in tables and supplementary tables under each of the following chapters.

561 4. Results: developmentally expressed gene classes in the 562 Agaricomycetes

563 In this paper we synthesized literature reviews with a meta-analysis of developmental 564 transcriptomes to identify conserved gene families involved in fruiting body development. For 565 the latter we reanalysed data for 12 species and identified developmentally regulated genes, 566 i.e. those that showed $>2$ or $>4$-fold changes between successive developmental stages or 567 adjacent tissue types (Supplementary Table 2). Statistics on developmentally expressed 568 genes are provided in Supplementary Table 2/b. Numbers of developmentally regulated 569 genes were similar to those reported by previous studies,(Almási et al. 2019; Krizsán et al. 
bioRxiv preprint doi: https://doi.org/10.1101/2021.12.09.471732; this version posted December 10,2021 . The copyright holder for this preprint (which was not certified by peer review) is the author/funder, who has granted bioRxiv a license to display the preprint in perpetuity. It is made available under aCC-BY-NC-ND 4.0 International license.

Running head: fruiting body genomics

570 2019; Merényi et al. 2020b, 2021) therefore, we here do not discuss these in detail, rather 571 focus on those that are conserved across species.

$572 \quad$ We organized developmentally regulated genes into strict 1-to-1 orthogroups

573 following the logic of Merenyi et al(Merényi et al. 2021). Orthogroups in which $>65 \%$ of the

574 genes were developmentally regulated were designated as conserved developmentally

575 expressed (CDE) orthogroups at fold change values of $>2$ or $>4$. We identified $921 \mathrm{CDE}$

576 orthogroups across the twelve Agaricomycetes species; these form the basis of the

577 discussion presented in the rest of this paper. Statistics on the number of species which had

578 developmentally regulated genes in these orthogroups are shown on Fig. 2/A. The

579 transcriptome data of $A$. bisporus was an outlier in several aspects. We currently do not

580 know if this is due to biological differences of this species or technical reasons, nevertheless

581 this transcriptome was assigned lower weight and discussed rarely in the following chapters.

582 By relying on conserved developmentally expressed orthogroups, we here take shared

583 expression dynamics as a proxy for conservation of function. It should be mentioned that this

584 approach may be, in several cases, only a crude approximation of real conservation of

585 function, but facilitates comparative discussion of gene families and their speculated

586 functions in fruiting body morphogenesis.

$587 \quad$ We functionally characterized CDE orthogroups and arranged them into broader

588 groups based on function inferred by orthology to genes in well-known model systems ( $S$.

589 cerevisiae, Schizosaccharomyces pombe, A. nidulans, N. crassa) and/or their Gene

590 ontology and InterPro annotations. We defined 17 broader functional groups, cell

591 division/proliferation, defense, transcriptional regulators, signal transduction, cell wall

592 biosynthesis and remodeling, cell surface proteins, secondary metabolism, cytoskeleton,

593 basic metabolism and acetyl-CoA production, lipid metabolism, storage carbohydrate

594 metabolism, ubiquitin/proteasome system, transporters, functionally poorly known genes,

595 unannotated genes, stress response related genes and ferric reductases (Fig. 2/B). The

596 main categories were further subdivided into finer groups to facilitate discussions of putative

597 functions. We categorized developmental genes manually to the best of our knowledge

598 based on inferred gene function, though the subdivision is probably not impeccable. For

599 example, overlaps exist between chromatin remodeling and DNA replication related

600 regulatory factors or between the latter and cytoskeleton related genes (e.g. kinesins

601 involved in moving chromatids during mitosis).

602 The two largest categories were functionally poorly characterized and unannotated

603 genes, these contained 182 and 176 CDE orthogroups, respectively (Fig. 2/B). The first,

604 'functionally poorly characterized genes' contains orthogroups which we could not

605 confidently link to any fruiting body functions. It is possible that this category could be

606 subdivided and with further manual annotation more orthogroups could be explained in the

607 context of fruiting body development. On the other hand, unannotated genes are those that

608 contain no existing Pfam or InterPro domain signatures and lack all kinds of functional

609 annotation. The largest functional group for which a function could be determined was

610 related to cell proliferation (DNA replication, DNA repair, mitosis, meiosis) followed by cell

611 wall biosynthesis and remodeling. Transcriptional regulators (including transcription factors,

612 chromatin-related and RNA-binding regulatory proteins), transporters and Acetyl-CoA

613 production related genes were also represented by a considerable number of genes. Certain

614 functional categories, such as 'defense' and 'secondary metabolism' contained only a small

615 number of CDE orthogroups. In these cases, these low numbers reflect the lack of

616 conservation of gene sequences, rather than the lack of a role of these gene families in

617 fruiting bodies. 
bioRxiv preprint doi: https://doi.org/10.1101/2021.12.09.471732; this version posted December 10,2021 . The copyright holder for this preprint (which was not certified by peer review) is the author/funder, who has granted bioRxiv a license to display the preprint in perpetuity. It is made available under aCC-BY-NC-ND 4.0 International license.

Running head: fruiting body genomics

618 Our discussion is guided here by conservation of developmentally regulated families.

619 However, there are gene families that do not form conserved orthogroups, but are

620 nevertheless very important for fruiting body development. These can be either multigene

621 families that undergo frequent duplication and thus orthology relationships are intricate (e.g.

622 hydrophobins), or fast-evolving families in which divergence quickly erases orthology

623 relationships (e.g. F-Box proteins, defense-related proteins). Therefore, in addition to gene

624 families/pathways identified through CDE orthogroups, we discuss broader, fast-evolving

625 gene families based on annotations at the gene family level only (i.e. without orthogroups)

626 and performed manual annotations to identify broader containing gene families or cellular

627 pathways. Accordingly, in addition to the 921 CDE orthogroups, we discuss further 558 gene

628 groups which belong to broad functionalities involved in fruiting body development. For some

629 processes we were able to reconstruct (nearly) complete pathways, such as in the case of

630 mitosis/meiosis related genes or fatty acid biosynthesis, whereas in other cases we only

631 document the widespread developmental regulation and expression profile conservation of a

632 gene family, but without information on the containing pathway of broader cellular function.

633 For some gene groups, such as cytoskeletal genes, GPCRs or MAP kinase pathways, we

634 provide annotations despite their limited expression dynamics during fruiting body

635 development. Such gene families may be important in fruiting body development despite

636 their low expression dynamics, therefore, we decided to annotate them in order to facilitate

637 comparative discussion across mushroom-forming fungi and more thoroughly investigated

638 Ascomycota model systems.

639 In the following chapters we discuss each of the more important gene groups in

640 detail, with emphasis on expression profiles, putative function and role in fruiting body

641 development. Besides well-known ones, we intentionally focus on lesser-known gene

642 families with widespread developmental expression, to potentially distill new insights into

643 fruiting body development. We provide catalogues and references to recent reviews for well-

644 researched gene families as well (e.g. hydrophobins or laccases).

645

646 4.1. Cell division, proliferation and growth

647 4.1.1. Meiotic and mitotic gene expression show two distinct expression peaks

648 Mitosis and meiosis are key to the development and growth of multicellular organisms.

649 Mushroom fruiting bodies are no exception: mitosis leads to cell proliferation early in fruiting

650 body development, whereas meiosis happens in basidia, which are localized to gills, to

651 produce spores.

652

653

Transcriptomic data provided clear evidence for expression peaks of genes related to

654

655

656

657

658

659

660

661

662 mitotic or meiotic cell division, as well as associated processes in DNA replication, repair, chromosome dynamics/movement etc., during fruiting body development. The enrichment of meiotic/mitotic genes during fruiting body development and sporulation was noted in $C$. cinerea(Burns et al. 2010; Muraguchi et al. 2015; X et al. 2015; Krizsán et al. 2019), Pisolithus microcarpus(Pereira et al. 2017), Tricholoma matsutake(Tang et al. 2020) and L. edodes(Song et al. 2018b), Agaricus blazei(Lu et al. 2020) as well as in a six-species comparison published by Krizsan et al(Krizsán et al. 2019), among others. Similar signal for meiotic gene upregulation has been detected in Ascomycota fruiting bodies as well(Rodenburg et al. 2018). C. cinerea has been used as a model system of Basidiomycota meiosis, in particular in so called white cap mutants that have meiosis-associated defects(Lu 
bioRxiv preprint doi: https://doi.org/10.1101/2021.12.09.471732; this version posted December 10,2021 . The copyright holder for this preprint (which was not certified by peer review) is the author/funder, who has granted bioRxiv a license to display the preprint in perpetuity. It is made available under aCC-BY-NC-ND 4.0 International license.

Running head: fruiting body genomics

663 et al. 2003). These works revealed many conserved and some unique aspects of the meiotic 664 process as well as resulted in meiosis being one of the best understood processes in this 665 species(Seitz et al. 1996; NY et al. 1997; L et al. 1999; Gerecke and Zolan 2000; Nara et al. 666 2001; Cummings et al. 2002; Lu et al. 2003; SH et al. 2004; Muraguchi et al. 2008a; AM et 667 al. 2009).

668 Probably the most detailed such study was performed by Burns et al(Burns et al. 669 2010), who investigated by microarray the transcriptional events that happen in C. cinerea 670 gills during a time course that encompasses the meiotic events. They found that 2,721 671 genes exhibited changing probe intensity during the six time points around meiosis. Using 672 dikaryotic mycelium as reference, they identified 886 genes that were expressed in gill tissue 673 only; these genes included core meiotic components. Like in other species, genes were 674 induced in successive waves during the meiotic process; these waves were arranged into 9 675 clusters based on expression trajectories. The clusters separated genes into functional 676 groups, e.g. those required for prophase I or sporulation in early and late in the time series, 677 respectively. Early genes further included ones related to DNA replication, cytoskeleton 678 organization and regulation. Late induced genes in their study are mostly related to spore 679 formation and show overlaps with genes related to ascospore formation in S. cerevisiae(S et 680 al. 1998a; Primig et al. 2000) and Sch. pombe(Mata et al. 2002). Ribosomal protein coding 681 gene expression was found to be high up to karyogamy, at which point it starts to decrease 682 and does not lift up again. This might reflect the shutting down of protein synthesis as the 683 fruiting body enters the sporulating phase.

$684 \quad$ Freitas Pereira et al examined sporogenesis in Pisolithus, an ectomycorrhizal fungus 685 in the Boletales, and found that meiotic genes (annotated by KEGG) showed dynamics 686 throughout the development of basidiospore-containing peridioles(Pereira et al. 2017). In this 687 species spores are produced in peridioles, lentil-shaped to globular compartments within the 688 fruiting body. Several genes that were differentially expressed in peridioles of different 689 maturation stages were annotated as meiotic/cell cycle or cell division related, most of which 690 showed an expression peak in unconsolidated, young, and mature peridioles. These 691 observations are consistent with microscopic observations on the sporogenesis of $P$. 692 microsporus(AN and MD 2010).

693 We annotated 180 conserved orthogroups as involved in cell proliferation, such as 694 mitosis, meiosis, DNA replication, DNA repair or the cell cycle, based on expression 695 conservation 141 of these qualified as CDE orthogroup (Table 1). These included previously 696 functionally characterized genes such as ku70, rad50 and Mre11 of $C$. cinerea(AM et al. 697 2009; Nakazawa et al. 2011), DMC1 (=Lenedo1_1211634) of L. edodes(Sakamoto et al. 698 2009), ku80 of S. commune(De Jong et al. 2010) and Msh4 of $P$. ostreatus(Lavrijssen et al. 699 2020). Most of these genes showed developmental regulation and similar expression profiles 700 in most of the species. This is not surprising, given that both mitosis and meiosis have well701 documented roles in sculpting fruiting bodies. Nevertheless, to our knowledge, these genes 702 represent the most comprehensive, although surely not complete, list of cell proliferation 703 related genes to date. We tentatively subdivided CDE orthogroups into ones related to DNA 704 replication, DNA repair, meiosis as well as both mitosis and meiosis; these contained 87, 26, 705 13, 54 genes, respectively (Table 1). These include members of several well-characterized 706 protein complexes described from S. cerevisiae, such as Ku, SMC, MCM, Ndc80, Gins and 707 cohesin complexes (Table 1). Orthologs of $C$. cinerea arp9, which was described as a 708 putative actin-related protein involved in chromatin remodeling(Nakazawa et al. 2016), were 709 also found to follow similar expression profiles, although at moderate dynamics (Table 1). 
Running head: fruiting body genomics

$710 \quad$ These genes showed characteristic expression profiles in most species. The 711 expression of meiotic genes shows a distinct peak in gill tissues (Fig. 3, Supplementary Fig.

712 1) or in stages that contain meiotic tissue. Meiotic gene expression precedes early phases of

713 sporulation and was clearly discernible in $C$. neoformans or $P$. ostreatus in previous

714 transcriptomic studies(Liu et al. 2018; Merényi et al. 2021). Because the onset of meiosis is

715 tightly regulated in most species (among mushrooms best known in C. cinerea(Kües 2000))

716 meiotic gene expression also provides a landmark to calibrate developmental chronologies

717 among species where this may be hard to do because of morphological divergence. It

718 should be noted that some species develop in ways so that we do not expect a clear peak.

719 For example, cell proliferation and spore production is not synchronized in S. commune,

720 where it appears that sporulation progresses from the basal, older parts and progresses

721 towards the edges of the fruiting body(Kües and Liu 2000). In S. commune, this may be

722 explained by the highly derived morphology of the fruiting bodies, consisting of an

723 assemblage of cyphelloid fruiting bodies. Therefore, we would not expect one clear peak of

724 meiotic gene expression, unless we sample different zones of the fruiting body separately.

725

On the other hand, mitotic, DNA replication and repair genes showed a clear peak associated with primordium stages, and their expression leveled off in mature fruiting bodies (Fig. 3, Supplementary Fig. 1). This probably reflects intense cell proliferation in primordia, which establishes the main tissues and developmental modules of the mushroom. The expression of these genes later decreases to low levels in young fruiting body stipes and caps (also high in meiotic tissues where one round of mitosis happens) and in mature fruiting bodies. This peak was also detected in previous studies on Agaricus blazei(Lu et al. 2020) and $P$. ostreatus(Merényi et al. 2021) and probably relates to the biphasic development of fruiting bodies, where the first phase is concerned with cell proliferation and tissue formation whereas the second with growth by cell expansion without much change in cell numbers. Our data suggest that this pattern should be conserved across agarics, but potentially missing or less clear in species that follow different developmental patterns (e.g. polypores).

Cryptococcus neoformans recently proved to be a particularly useful model for teasing apart morphogenetic processes(Merényi et al. 2021). This species forms simple fruiting bodies composed of lawns of aerial hyphae bearing basidia. Liu et al pointed out in Cryptococcus, that genes co-induced with meiotic genes might be related to basidium morphogenesis, a process that greatly overlaps with meiosis(Liu et al. 2018). Based on transcriptome data it may be hard to separate such genes from meiotic genes, because of the temporal and spatial overlap of their expected expression. Nevertheless, one of the genes reported by Liu et al, C. neoformans Csa1 (C. cinerea protein ID: 471238 , see Table TRA below), which has been implicated in basidium development (and to a lesser extent meiosis(Liu et al. 2018)), is developmentally expressed in 10/10 species in our data and shows expression peaks that overlap with that of meiotic genes.

An orthogroup that includes the serine-threonine kinases Sch. pombe Ran1 and S. cerevisiae Sks1 (represented by $C$. cinerea 456276, Table 1) showed marked peaks in gills of mature fruiting bodies in all agaricoid species (except $C$. cinerea) and in mature fruiting bodies in species with no separable gills or caps (S. commune, A. ampla). Ran1 is a negative regulator of Sch. pombe meiosis (with Mei2 as target(Caligiuri et al. 1997)), whereas Sks1 is involved in the adaptation to low glucose concentrations and pseudohyphal growth in yeast. Despite this apparent strong contradiction, the characteristic expression peaks in meiotic tissues and developmental stages in mushroom-forming fungi suggest roles in sporulation-related processes, possibly the repression of meiotic processes during spore morphogenesis. 
Running head: fruiting body genomics

758

759

760

761

762

763

764

765

766

767

768

769

770

771

772

773

774

775

776

777

778

779

780

781

782

783

784

785

786

787

788

789

790

791

792

793

794

795

796

797

798

799

800

801

802

803
In summary, mitotic and meiotic (including associated processes such as DNA replication and repair) gene expression showed conserved patterns across the examined mushroom-forming fungi. This probably reflects, on one hand, intense cell proliferation during primordium development and, on the other hand, meiosis that precedes sporulation in basidia. These gene expression patterns are easily recognizable in transcriptomic data and provide landmarks for calibrating developmental series. The downregulation of mitosis related genes in the second half of fruiting body development coincides with ceasing cell proliferation and the transition of the mushroom to growth by cell expansion.

\subsubsection{Ribosomal genes}

Ribosomes produce proteins, the necessary building blocks for living cells. They are composed of four RNA subunits and up to 80 different proteins. The amount of ribosomes, and thus ribosomal protein expression, in a cell is proportional to its protein synthesis demand(Kraakman et al. 1993; Jorgensen et al. 2002). Therefore, ribosomal protein expression has been used as a proxy for the proliferative activity of a cell or a tissue and we expect it can be similarly informative in mushrooms too.

We observed a preponderance of ribosomal protein genes among CDE orthogroups, especially at FC>2 and to a lesser extent at FC>4. To look globally at structural proteins of ribosomes, we identified ribosomal protein genes based on previously reported ribosomal compositions, excluding mitochondrial ribosomal proteins and ribosome biogenesis-related genes. The 74 orthogroups identified this way (Table RIB) showed very characteristic expression dynamics, albeit at low fold change values, in most species. Ribosomal genes were highly expressed in the early developmental stages of most species followed by gradual shutting down in young and mature fruiting bodies. A second, sharp expression peak was observed in gill tissues of $P$. ostreatus, A. ostoyae, A. bisporus and $C$. cinerea (Fig. 4, Supplementary Fig. 2). Like in the case of mitotic and meiotic genes, we hypothesize that these two peaks correspond to an early, proliferative stage of development and the protein synthesis demand of spore production/meiosis. Ribosomal genes seem to be co-expressed with genes involved in cell division and proliferation (meiotic, DNA replication and repair, see above) in both primordia and in the gills. This could signify the need for increased protein production for cell proliferation in primordia and for meiosis and spore production, although other processes happening in gills requiring a large amount of protein cannot be ruled out. The characteristic expression of ribosomal genes is a conspicuous pattern and was detected in partial or complete form in several transcriptomic papers in both Basidiomycota(Cheng et al. 2013b; Zhou et al. 2014; Zhang et al. 2015b; Song et al. 2018b; Krizsán et al. 2019; Liu et al. 2020a; Lu et al. 2020) and Ascomycota(Rodenburg et al. 2018; Tong et al. 2020).

The vegetative mycelium generally showed high ribosomal protein-coding gene expression, though not in all species, which we hypothesize depended on whether samples for RNA-Seq were harvested from older, inactive or actively growing (e.g. colony edge) parts of the mycelium.

A ribosome-bound Hsp70 chaperone protein formed a CDE orthogroup developmentally regulated in 9 species (FC>2) (C. cinerea protein ID: 438977) (Table 2). This gene was also detected previously as upregulated in primordia of $C$. cinerea(Cheng et al. 2013b). It is orthologous to S. cerevisiae Ssb2, a Hsp70-family chaperone that is bound by the ribosome and escorts the folding of nascent polypeptides(Peisker et al. 2010).

In summary, a characteristic expression trajectory of ribosomal genes is characteristic of most mushroom-forming fungi, which likely reflects high protein demands of 
bioRxiv preprint doi: https://doi.org/10.1101/2021.12.09.471732; this version posted December 10,2021 . The copyright holder for this preprint (which was not certified by peer review) is the author/funder, who has granted bioRxiv a license to display the preprint in perpetuity. It is made available under aCC-BY-NC-ND 4.0 International license.

Running head: fruiting body genomics

804 intensely growing tissue in primordial stages and gills and supports a bi-phasic model of 805 fruiting body development (see below).

806 4.1.3. Growth by cell expansion and turgor manipulation - a biphasic model of fruiting 807 body development

808 A key difference in fruiting bodies and hyphae is that in the latter growth and expansion 809 takes place at the tip, whereas in the former, cell expansion can vary between apical and 810 isotropic. Already de Bary established, by studying Mycena vulgaris, that mushroom fruiting 811 bodies grow mostly by cell expansion not by cell proliferation(Bary et al. 1887). This appears 812 to be a rather widespread feature of fruiting bodies and shows broad similarity to growth by 813 cell expansion in plant fruits(Krizsán et al. 2019). More precisely, fruiting body development 814 seems to be divided into a first proliferative stage, during which cell division, differentiation 815 as well as tissue formation takes place, and a growth phase, which is primarily concerned 816 with cell expansion to achieve the mature shapes and sizes of the fruiting bodies(MOORE et 817 al. 1979; Kües 2000; G et al. 2013; Kües and Navarro-González 2015; Krizsán et al. 2019; 818 Liu et al. 2021). Of the better investigated species, this two-phased development is probably 819 most spectacular in $C$. cinerea, which is adapted to very rapidly lifting cap above ground and 820 expanding it for spore dispersal(Kües 2000).

It should be noted that the two-phase development is not fully universal among fungi and does not always mean a complete separation of proliferative and expansive phases. For example, Craig et al(Craig et al. 1977) showed that the top regions of $A$. bisporus stipes elongated up to 9-fold, whereas cell lengths increased up to 2.2-fold, and from these data derived that cell division also occurs to achieve this elongation rate. In S. commune, it appears that outer parts of the fruiting body are still growing (by cell proliferation) while older parts are already sporulating(Kues and Liu 2000).

Whether for the entire fruiting body or only parts of it, growth by cell expansion seems to be a widespread trait during the second half of development of mushrooms. How cell expansion is achieved, in general, is poorly known, but it probably requires water supply, turgor manipulation (by osmolytes), cell wall elasticity and new cell wall material deposition. Water supply may be realized by the regulated expression of aquaporin genes (see below). On the other hand, the identity of the osmolytes has not been resolved yet. In the Ascomycota ions, ion channels, glycerol and sugars were reported as main players of turgor manipulation(Fischer et al. 2004). In Basidiomycota fruiting bodies, sugars (mannitol, trehalose) offer themselves as straightforward candidates, but Moore([CSL STYLE ERROR: reference with no printed form.]) speculated that high molecular weight sugars are unlikely to be the osmolytes that drive stipe expansion. Trehalose and polyols have been excluded as osmolytes(Kües 2000; F et al. 2005), whereas potassium oxaloacetate has been hypothesized, which would be consistent with the upregulation of oxalate metabolism (see below), but not confirmed(Knoll; Kües 2000). On the other hand, ions would either need to be transported to the fruiting body from the mycelium, or released locally.

Factors modulating cell wall elasticity and remodeling are better known. During fruiting body growth, cell wall deposition occurs throughout the cell surface, and not only in the tip region as in hyphae, which was termed diffuse extension(Kües 2000). In Agaricus bisporus larger proportion of $\beta$-1,6-linked glucose side branches in a $\beta$-1,3-glucan backbone was observed in the rapidly elongating top segment of the stipe(Mol and Wessels 1990). This causes hydrogen-bonds among glucan chains to be weaker in rapidly elongating stipe compared with mature stipe and vegetative hyphae. It has been hypothesized that these 
bioRxiv preprint doi: https://doi.org/10.1101/2021.12.09.471732; this version posted December 10,2021 . The copyright holder for this preprint (which was not certified by peer review) is the author/funder, who has granted bioRxiv a license to display the preprint in perpetuity. It is made available under aCC-BY-NC-ND 4.0 International license.

Running head: fruiting body genomics

850 weak bonds are disrupted and reformed by turgor pressure rather than hydrolysing 851 enzymes(Mol et al. 1990). The architecture of cell wall also regulates stipe elongation; in 852 primordial stages chitin microfibrils are arranged isotropically which is remodeled to helical 853 arrangement during development(Kamada et al. 1991). The turgor pressure acting on the 854 cell wall and insertion of new chitin microfibrils allow elongation and in that dynamic 855 rearranges of microfibrils chitin synthases, chitinases and glucanases play the major role in 856 C. cinerea(Kamada et al. 1991). Niu et al observed differences in structure of chitin 857 microfibrils in elongating and mature stipe regions(Niu et al. 2015). Extension of the cell wall 858 happens by insertion of newly synthesized microfibrils. In contrast, studies in Flammulina 859 and Coprinopsis suggested that stipe cell wall extension is mediated by endogenous 860 expansin-like proteins, not turgor pressure or cell wall glycoside hydrolases(Fang et al. 2014; 861 W et al. 2014). Plant expansins mediate Turgor-mediated cell expansion(Baccelli 2015) and 862 several other morphogenetic processes (e.g. organ abscission, fruit softening(Sampedro and 863 Cosgrove 2005)). The auxin-driven expansion of plant cells without changes in cell number 864 is known as acid growth(Rayle and Cleland 1992; Arsuffi and Braybrook 2018), because low $865 \mathrm{pH}$ is required by cell wall modifying proteins (e.g. expansins) to loosen the cell wall. In fungi, 866 it is not known if an analogous process exists, but the acidifying effect of external $\mathrm{CO}_{2}$ has 867 been hypothesized as a factor in stipe cell elongation(Liu et al. 2021) and there is evidence 868 for expansins participating in the process (see below). Accordingly, high external $\mathrm{CO}_{2}$ during 869 mushroom cultivation was speculated to cause malformed, long-stipe fruiting bodies by 870 acidifying the extracellular space and activating cell wall modifying proteins(Liu et al. 2021).

\subsection{Acetyl-CoA production by central carbon metabolism}

872 We found evidence for widespread developmental expression of multiple acetyl-coenzyme $A$

873 (Ac-CoA) producing enzyme families. Ac-CoA is a key intermediate in central carbon 874 metabolism as well as other processes (biosynthesis of lipids and certain amino acids(Chen 875 et al. 2015)). Under normal carbon metabolic conditions, the generated Ac-CoA, which 876 comes from either glycolysis or fatty acid beta-oxidation enters the TCA cycle, where it is 877 oxidized to $\mathrm{CO}_{2}$ for energy (ATP) production. However, Ac-CoA is required also for the 878 production of biological membranes(Strijbis and Distel 2010; Chen et al. 2015), and our 879 results point in the direction of multiple basic metabolic pathways converging on excess Ac880 CoA production for membrane biosynthesis. Combined with gene expression profiles of 881 these genes, we hypothesize that excess Ac-CoA is being channeled into ergosterol and 882 fatty acid production during fruiting body development, as detailed below.

883 For this work we started from Ac-CoA related CDE orthogroups detected in the 884 transcriptomes of the examined species and developmental stages, which belonged to 885 several pathways. We reasoned that if there is an increased demand for Ac-CoA at certain 886 developmental stages of fruiting bodies, then this should be reflected in the regulation of Ac887 CoA-producing pathways. Thus, we annotated the TCA cycle, the glyoxal cycle, the fatty 888 acid beta-oxidation, ethanol utilization, glycolytic as well as pyruvate decarboxylation 889 pathways of $C$. cinerea, based on protein orthology to corresponding pathways of $S$. 890 cerevisiae (Fig. 5, Supplementary Fig. 3, Table 3). These led to a model we put forth on Fig. 8915 , with the additional note that it reflects one possible synthesis of the observations into a 892 mechanistic model compatible with what we know about fruiting body development, but that 893 other interpretation may also be viable.

$894 \quad$ First of all, we found that oxaloacetase (oxaloacetate lyase) genes were 895 developmentally expressed in 11 species (FC>4, C. cinerea 499734). Oxaloacetases 
Running head: fruiting body genomics

896 produce oxalate and acetate from oxaloacetate, which is an intermediate of both the TCA 897 and the Glyoxylate cycles. In S. cerevisiae acetate is converted to Ac-CoA by acetyl-CoA 898 synthases (Acs1), orthologs of which were developmentally regulated in 11 and 4 species at 899 fold change of 2 and 4, respectively (represented by C. cinerea 500719). We also found a 900 CDE orthogroup comprising putative orthologs of the S. cerevisiae Ac-CoA hydrolase Ach1 901 (represented by $C$. cinerea 442787, $\mathrm{FC}>2 / 4: 7 / 5$ ). Ach1 is a relatively poorly known gene, 902 which was recently shown to be responsible for the conversion of Ac-CoA to acetate, which 903 can then be easily transferred from the mitochondrion to the cytoplasm(Chen et al. 2015). 904 Cytoplasmic acetate is then converted to cytoplasmic Ac-CoA, a starting material for lipid 905 synthesis, suggesting that Ach1 mediated Ac-CoA supply for lipid synthesis. Remarkably, 906 Ach1 orthologs are present in only 8 species and completely missing in A. bisporus (missing 907 even in telomere-to-telomere assemblies(ASM et al. 2020)), M. kentingensis, Ph.

908 chrysosporium, L. tigrinus, Pt. gracilis. This patchiness might indicate that the role of these 909 genes is dispensable and probably others can take over their roles.

Acetate is also an intermediate of the ethanol utilization pathway, in which two enzymes form a linear pathway that terminates in the production of Acetyl-CoA(M et al. 2003). Aldehyde dehydrogenases orthologous to AldA of $A$. nidulans and Ald4 of $S$. cerevisiae formed a CDE orthogroup (C. cinerea protein ID: 500663, 9/7); the encoded enzymes also contribute to acetate production and, in $C$. cinerea, showed peak expression in young fruiting body caps. Ald4/AldA converts the toxic compound acetaldehyde to acetate(B et al. 2001). Although the ethanol utilization pathway is mostly discussed in the context of extracellular ethanol (a nonfermentable carbon source) uptake and degradation, as well as anaerobic fermentation, acetaldehyde can also be converted to ethanol (Fig. 5), if it is present in excess amounts in the cells. This may explain the developmental regulation of orthologs of $S$. cerevisiae Adh3 and A. nidulans AlcA/AlcC (represented by $C$. cinerea 500663), which were developmentally expressed in 11/7 species (FC>2/4, Table 3). Enzymes of the alcohol utilization pathway were recently detected in proteomics data of fruiting bodies of Guyanagaster necrorhiza, where their presence was interpreted as evidence for anaerobic fermentation within the fruiting body, as part of an intriguing mutualism with $\mathrm{N}_{2}$ fixing bacteria(Koch et al. 2021).

The last step of glycolysis is catalyzed by the pyruvate decarboxylase Pdc1 in $S$. cerevisiae, which converts pyruvate to acetaldehyde. Pdc1 generates cytoplasmic Ac-CoA, which is essential for the biosynthesis of lipids(Chen et al. 2015). Pdc1 orthologs (represented by $C$. cinerea 422402 ) were developmentally expressed in $11 / 8$ species (FC>2/4), whereas genes encoding other enzymes in glycolysis showed much smaller dynamics (Supplementary Fig. 3). Together, these observations suggest a widespread upregulation of acetaldehyde and from that either acetate or ethanol producing enzyme genes in fruiting bodies.

Upstream of oxaloacetases, enzymes responsible for oxaloacetate production in the TCA (yeast Mdh1, C. cinerea 500663, 11/3) and glyoxylate cycles (yeast Mdh2, C. cinerea $449845,9 / 3$ ) were also quite widely developmentally regulated. In the glyoxylate cycle we found orthologs of S. cerevisiae Mls1 (malate synthase, C. cinerea 530292) and Icl1 (isocitrate lyase, $C$. cinerea 495730 ) to form CDE orthogroups developmentally regulated in $12 / 11$ and $12 / 7$ species (FC>2/4), respectively. This suggests that the glyoxylate cycle is strongly induced in fruiting bodies. On the other hand, members of the TCA cycle showed lower developmental dynamics (Fig. 5).

What happens to the oxalate that is produced by oxaloacetases? It is possible that the resulting oxalate is a waste product, but it may also be used somehow by the fungus, 
Running head: fruiting body genomics

944 e.g. as a developmental signal molecule(Palmieri et al. 2019) or as an electron donor, as 945 has been observed in wood-decay(Hastrup et al. 2012; Zhu et al. 2016; Graz et al. 2017), 946 where it acts as an electron donor for oxidative enzymes. One hypothesis for the role of 947 oxalate is that oxidative enzymes, which might participate in the modification of the fungal 948 cell wall during development (see above), utilize it as an electron donor, as do enzymes 949 involved in wood-decay. On the other hand, if oxalate is a by-product, excess amounts may 950 need to be broken down actively. We found evidence for the upregulation of oxalate 951 catabolism in the compared species. The two oxalate degradation pathway in fungi 952 comprises oxalate decarboxylase, which converts oxalate to formate and $\mathrm{CO}_{2}$ and formate 953 dehydrogenase, which breaks formate down to $\mathrm{CO}_{2}$ (Mäkelä et al. 2014). We detected two 954 CDE orthogroups of oxalate decarboxylases (C. cinerea protein ID: $361522,8 / 6$,

955 Schco3_2751877, 7/6) and one of formate dehydrogenases (C. cinerea protein ID: 452378,

$9567 / 6$, orthologous to Fdh1 of $S$. cerevisiae)(Table 3), which were upregulated in fruiting bodies 957 widely, suggesting that fruiting bodies utilize enzymatic mechanisms for the neutralization of 958 oxalate. An alternative route of oxalate degradation in S. cerevisiae is catalyzed by PCS60, 959 an oxalyl-CoA synthetase(Foster and Nakata 2014). Orthologs of this gene were found in 960 only a subset of species (represented by C. cinerea 474928), but were developmentally 961 regulated in most of them. In addition, a CDE orthogroup of formate transporters, 962 orthologous to the Postia placenta gene reported in Martinez et al(Martinez et al. 2009b), 963 was developmentally expressed in 8/5 species (FC>2/4, represented by Schco3_2738153, 964 missing in C. cinerea).

$965 \quad$ Building on the evidence for intense Ac-CoA production, we annotated and analyzed 966 the Coenzyme-A producing pathway as well as two other sources of Ac-CoA, the fatty acid 967 beta-oxidation pathway and the cellular cycle for pyruvate decarboxylation to Ac-CoA. These 968 pathways were reconstructed and annotated based on corresponding pathways in $S$. 969 cerevisiae(JM et al. 2012). Members of these pathways did not show remarkable 970 developmental dynamics (Table 3).

971 Several genes connected to acetate metabolism and transport also formed CDE 972 orthogroups. Orthologs of S. cerevisiae Ady2, an acetate transporter(S et al. 2004), are 973 widely developmentally regulated (represented by $C$. cinerea $441597,7 / 6$ ), and were 974 strongly induced in young fruiting body caps of $C$. cinerea. A CDE orthogroup of putative 975 monocarboxylate (lactate, acetate, succinate, etc.) transporters (C. cinerea protein ID: 976375404 [7/5])(Table 3) orthologous to S. cerevisiae Jen1(M et al. 1999) were also highly 977 induced in YFB-caps in C. cinerea. We also identified putative orthologs of $S$. cerevisiae 978 Yat1 (represented by $C$. cinerea 443703, 10/5), a carnitine acetyltransferase that shuttles 979 Ac-CoA across the mitochondrial membrane. A CDE orthogroup of aquaglyceroporins 980 orthologous to $S$. cerevisiae Fps 1, which is involved in the uptake of acetate (C. cinerea 981 protein ID: $3731417 / 6$ ), were developmentally regulated in $7 / 6$ species. This gene encodes 982 a protein that has been associated with either glycerol influx/efflux(Luyten et al. 1995) or 983 acetate uptake(Mollapour and Piper 2007; Watcharawipas et al. 2018), which leaves some 984 uncertainty on which role it fulfills in fruiting bodies. Orthologues of $S$. cerevisiae Sym 1 also 985 formed a CDE orthogroup, although only at a fold change cutoff of 2 (C. cinerea protein ID: $986147126,9 / 3$ ) (Table 3). This gene is said to be involved in ethanol utilization and stress 987 response, but mechanistic details are not known(Reinhold et al. 2012; Gilberti et al. 2018). 988 Similarly, orthologs of yeast Sfc1, a succinate/fumarate antiporter that transports C4 989 succinate (which is produced from acetate(Bojunga et al. 1998)), to the mitochondria, are 990 developmentally regulated in several species (represented by $C$. cinerea $374148,12 / 7$ ). The 991 Sfc1 ortholog is also strongly induced late in the development of $C$. neoformans(Liu et al. 
bioRxiv preprint doi: https://doi.org/10.1101/2021.12.09.471732; this version posted December 10,2021 . The copyright holder for this preprint (which was not certified by peer review) is the author/funder, who has granted bioRxiv a license to display the preprint in perpetuity. It is made available under aCC-BY-NC-ND 4.0 International license.

Running head: fruiting body genomics

992 2018). Finally, a CDE orthogroup of putative malate symporters orthologous to Sch. pombe 993 Mae1, which is involved in the uptake of extracellular malic acid was detected as 994 developmentally regulated in $9 / 5$ species ( $F C>2 / 4$, represented by $C$. cinerea 534888 , Table 995 3). Whether these genes are related to the above discussed observation of acetate/Ac-CoA 996 related gene upregulation, or how cell surface transporters may be related remains to be 997 determined. The induction of these genes does not happen in the same or similar 998 developmental stage in all species (although there is consistency within species, see the 999 upregulation of several genes in YFB-caps of $C$. cinerea), nevertheless, their induction 1000 suggests differential acetate concentrations and an important role for acetate during in 1001 fruiting body development.

$1002 \quad \mathrm{CO}_{2}$ produced by the TCA cycle may be fixed into oxaloacetate by the conversion of $1003 \mathrm{CO}_{2}$ to hydrogencarbonate by carbonic anhydrases, then conversion of $\mathrm{HCO}_{3}{ }^{-}$and pyruvate 1004 into oxaloacetate by pyruvate carboxylases(Elleuche and Pöggeler 2010) (Pyc1 in $S$. 1005 cerevisiae). We found that several carbonic anhydrases (2-9 genes in Agaricomycetes 1006 genomes) are developmentally regulated in mushroom forming fungi and they form at least 1007 1008 one CDE orthogroup (represented by C. cinerea 450047)(Table 3). C. cinerea 450047 shows an upregulation in young fruiting bodies, coincident with the induction of Ac-CoA producing enzymes. This observation raises the possibility that $\mathrm{CO}_{2}$ produced by the TCA cycle in fruiting bodies may be fixed into oxaloacetate, which can further be converted to AcCoA (Fig. 5). Of note, carbonic anhydrases are involved in the regulation of sexual development in the Ascomycota(Elleuche and Pöggeler 2010).

Taken together, we observed developmental regulation of key enzymes in several pathways that seem to converge on increased acetyl-CoA production, either directly, or indirectly (through acetate or oxaloacetate). Several connected pathways, including oxalate degradation, the ethanol degradation pathway, the terminal reaction of glycolysis as well as evidence from the TCA and glyoxylate cycles were pointing in this direction. The expression of these genes tended to be upregulated late during development and showed good consistency within, but not necessarily across, species. A possible interpretation of these data is that increased Ac-CoA production is needed for growth by cell expansion late in development, as a starting material for lipid and sterol biosynthesis. This is underpinned by the fact that biosynthetic pathways of ergosterol and other membrane components are also upregulated during late developmental stages (e.g. the young fruiting body stage in $C$. cinerea), probably for membrane assembly needed for rapid cell growth. This interpretation would be consistent with the two-phased development of several mushroom-forming species, which consists of a first stage of cell division and differentiation and a second, during which cell numbers hardly change but their volumes increase considerably via Turgor-mediated expansion. Such a reprogramming of the fungus's basic metabolism from using Ac-CoA for energy production (when citrate is in the mitochondrion) to using it for lipid metabolism (when citrate is in the cytoplasm) seems particularly well-suited for fruiting body formation, which requires tight regulation of lipid metabolism. It should be mentioned that AcCoA is also required for melanin production(Lee et al. 2019), although it is unlikely to be the driving force of our observation, because pigment production is not conserved across the examined species. A previously reported reprogramming of the glyoxylate cycle in Fomitopsis palustris for the production of oxalate(Munir et al. 2001) seems related, however, in that case, the main product in demand was hypothesized to be oxalate that facilitates wood-decay, whereas in our case we hypothesize the product in demand is acetate and finally Ac-CoA. Both variants of the metabolic pathways nevertheless pass through 1039 oxaloacetate(Munir et al. 2001). The excess Ac-CoA production may rely on, or be 
bioRxiv preprint doi: https://doi.org/10.1101/2021.12.09.471732; this version posted December 10,2021 . The copyright holder for this preprint (which was not certified by peer review) is the author/funder, who has granted bioRxiv a license to display the preprint in perpetuity. It is made available under aCC-BY-NC-ND 4.0 International license.

Running head: fruiting body genomics

1040 connected to carbohydrate reserves that are deposited in the subhymenial part of the cap of 1041 the mushroom(Ji and Moore 1993; Muraguchi et al. 2015) during primordium development.

1042 However, there may be alternative interpretations and the topic is certainly of interest for 1043 detailed, further research, that integrates more functional data beyond gene expression 1044 observations.

1045

1046 4.3. Lipid metabolism

1047 Lipid metabolism is pivotal to very basic organismal processes such as growth,

1048 differentiation, cell division, energy storage and cell membrane composition, to name a few.

1049 Therefore, in depth understanding of gene expression changes associated with lipid

1050 metabolism, catabolism and transport can be expected to provide novel insights into fruiting

1051 body development. Indeed, our meta-analysis of Agaricomycete transcriptomes revealed

1052 several CDE orthogroups related to lipid metabolism. We discuss these below,

1053 complemented with annotations of major related pathways (but no attempt is made to

1054 comprehensively annotate all pathways) and processes. We base our annotations on data

1055 from well-studied model organisms, such as S. cerevisiae, A. nidulans and Sch. pombe, with

1056 orthology relationships to Agaricomycetes genes established on the basis of 1-to-1

1057 reciprocal best Blast hits.

\section{4.3.1. Yeast beta oxidation}

1059 We reconstructed the beta-oxidation pathway (Table 4) in C. cinerea based on the yeast 1060 beta-oxidation pathway(van Roermund et al. 2003) (Fig. 5). The expression of genes 1061 comprising the beta-oxidation pathway in general does not appear to be widely dynamic in 1062 fruiting bodies. We found orthologs of yeast CTA1, a catalase A, to form a CDE orthogroup. 1063 However, this catalase in yeast is only a marginal component of the beta-oxidation pathway, 1064 and may be involved in a wide range of other functions (e.g. signaling). The more 1065 widespread developmental expression of ACS1 orthologs is remarkable (11/4 species at $1066 \mathrm{FC}=2 / 4$ ), for a possible connection with sterol biosynthesis (see above). ACS1 produces 1067 Acetyl-CoA, which is the starting point for ergosterol and membrane lipid biosynthesis.

\section{4.3.2. Ergosterol biosynthesis}

1069 We reconstructed the ergosterol biosynthesis pathway based on orthology to known 1070 pathway member genes in S. cerevisiae(JM et al. 2012) (Table 5). ERG pathway genes of

1071 Agaricomycetes are generally higher expressed in fruiting bodies than in vegetative mycelia 1072 (VM/P1>1 in 20 out of 21 genes in C. cinerea), with expression peaking in young fruiting 1073 bodies of $C$. cinerea (Fig. 6, Table 5). Expression peaks at similar stages in other species 1074 too, in primordium 3/young fruiting body in $P$. ostreatus and young fruiting bodies/mature 1075 fruiting bodies stages in A. ostoyae (Supplementary Fig. 4). Similar pictures were obtained 1076 for other species. It should be noted that although the ergosterol biosynthesis pathway 1077 shows clear expression trends, the dynamics of expression is relatively low, mostly between

$10782<\mathrm{FC}<4$. Therefore, ergosterol biosynthesis genes mostly do not form CDE orthogroups, nevertheless, the expression patterns are distinctive so they warrant discussion here.

In these species expression peaks seem to coincide with intense growth by cell

1082 expansion in fruiting bodies. Above we showed that mitotic, ribosomal and histone gene expression level off in late primordium stages (P2 in C. cinerea, see Fig. 3), after which 
bioRxiv preprint doi: https://doi.org/10.1101/2021.12.09.471732; this version posted December 10,2021 . The copyright holder for this preprint (which was not certified by peer review) is the author/funder, who has granted bioRxiv a license to display the preprint in perpetuity. It is made available under aCC-BY-NC-ND 4.0 International license.

Running head: fruiting body genomics

1083 growth by cell expansion takes place. This might create an increased demand for membrane 1084 constituents during rapid cell expansion/growth by turgor, which is what we might see in the 1085 expression patterns of genes in the ergosterol biosynthesis pathway (Fig. 6, Supplementary 1086 Fig. 4). This hypothesis would also explain why there is no clear peak in C. neoformans and 1087 $P t$. gracilis, where sudden growth by cell expansion is lacking.

Similarly, a homolog of the Sterol-Regulatory Element Binding Proteins (SREBP(CM and PJ 2010)), similar to the $S$. cerevisiae transcription factor HMS1 shows a very similar expression pattern: in $C$. cinerea (C. cinerea protein ID: 488426) expression peak in YFB-S and YFB-C (Fig. 6), whereas in $P$. ostreatus it peaks in mature fruiting body tissues (PleosPC15_2_174910). This suggests, based on expression data, that these putative SREBPs might be regulating ergosterol biosynthesis genes also in the Agaricomycetes.

A connection with the above-mentioned Ac-CoA producing basic metabolic pathways, where certain, but not all, components were found widely upregulated, is possible here. These pathways produce Acetyl-CoA, which is the starting point of ergosterol biosynthesis. This raises the possibility that the upregulation of the ethanol utilization pathway components (see above, Fig. 5) and certain components of the glyoxylate cycle reflect increased Ac-CoA production which is required as input to ergosterol biosynthesis and eventually membrane assembly. A competing hypothesis was put forth in $P$. microcarpus, where upregulation of glyoxylate shunt genes was observed and suggested that it underlies the production of storage materials for spores(Pereira et al. 2017). Using our more resolved transcriptome data and based on the observation of ergosterol pathway expression dynamics, we propose that the connection to the glyoxylate shunt might represent a link to membrane constituent synthesis, although more data would be needed to verify this hypothesis.

The upregulation of the ergosterol synthesis pathway was observed in F. velutipes, where most ergosterol biosynthesis genes were upregulated in the young fruiting body stage(Wang et al. 2019) (approximately in agreement with our definition of young fruiting bodies) and showing intense growth. Sterol metabolism genes were also found to be more abundant in fruiting bodies of $H$. erinaceus compared to vegetative mycelia(Zeng et al. 2018). It was reported that orthologs of the lanosterol-alpha-demethylase Erg11 of $S$. cerevisiae, a component of the ergosterol biosynthesis pathway (corresponding $C$. cinerea ortholog: 397292), was upregulated in fruiting bodies of Antrodia cinnamomea(Lu et al. 2014) and $P$. ostreatus(Sunagawa and Magae 2005). This is consistent with the observations made in this study.

We also checked the sphingolipid biosynthesis pathway based on orthology to pathway members in S. cerevisiae. Sphingolipids, along with sterols are important in membrane rafts and microdomains and form key components of fungal cell membranes. Although some yeast genes did not have clear orthologs in Agaricomycetes, genes involved in sphingolipid biosynthesis showed a similar expression profile in C. cinerea (Fig. 6, Table 5). This may underscore our hypothesis on the link between growth by cell expansion and membrane assembly.

A characteristic CDE orthogroup of putative C14-sterol reductase genes was developmentally expressed in 12/7 species (represented by C. cinerea 539004)(Table 5, Fig. 6). These proteins are not part of the ergosterol biosynthesis pathway, but show a high similarity to $A$. nidulans and $C$. albicans membrane phospholipid metabolism proteins AN2040 and Erg24, respectively, which are involved in ergosterol metabolism. Genes in this orthogroup show peak expression in late development, coincident with rapid expansion of fruiting bodies. In several species they were also upregulated in primordia relative to 
bioRxiv preprint doi: https://doi.org/10.1101/2021.12.09.471732; this version posted December 10,2021 . The copyright holder for this preprint (which was not certified by peer review) is the author/funder, who has granted bioRxiv a license to display the preprint in perpetuity. It is made available under aCC-BY-NC-ND 4.0 International license.

Running head: fruiting body genomics

1131 vegetative mycelium samples. These expression patterns resemble that of ergosterol

1132 biosynthesis genes, and suggest that this orthogroup may be linked to ergosterol

1133 metabolism.

\section{4.3.3. Sterol transport}

1135 We detected several CDE orthogroups that were annotated as related to ergosterol transport 1136 and showed similar upregulation in late development as ergosterol biosynthesis genes did. 1137 We therefore inventoried sterol transport related genes of the families Niemann-Pick type C 1138 protein family (S. cerevisiae Npc2), the yeast Ncr1 family, the nonspecific sterol transport 1139 protein (Scp2) family, StAR-related lipid-transfer (START) proteins as well as members of the Oxysterol Binding Protein Homologue (OSH) protein family(Schulz and Prinz 2007).

Of these, the Ncr1, Npc2, Scp2 and START gene families are single-copy families in mushroom-forming fungi, whereas the $\mathrm{OSH}$ family is represented by 5 members in all mushroom-forming fungi (except $S$. commune which has 6, Table 6). Npc2 (ortholog $C$. cinerea 475807), Scp2 (ortholog C. cinerea 478741) and START proteins as well as one member of the OSH family were (C. cinerea protein ID: 427232)(Table 6) upregulated during the second half of development, at or after the young fruiting body stage. This is similar to the expression patterns seen in ergosterol biosynthesis genes. One member of the OSH family orthogroup (C. cinerea protein ID: 378276 ) showed a clearly stipe-specific expression, with peaks in stipes of young fruiting bodies or fruiting bodies of $C$. cinerea, $A$. ostoyae, $M$. kentingensis, $A$. bisporus and $P$. ostreatus, albeit mostly with low fold change values $(2<\mathrm{FC}<4)$. Interestingly, the corresponding gene was cap-upregulated in L. bicolor (Lacbi2_456536), whereas in simpler species (S. commune, A. ampla), it showed diverse expression peaks. Ncr1 orthologs (represented by $C$. cinerea 494709 ) showed consistently higher expression in fruiting bodies than in vegetative mycelia. Fold changes were generally low in these gene families, but nevertheless the patterns are phylogenetically very consistent.

These expression patterns resemble those of ergosterol biosynthesis genes, which also showed upregulation in mid- to late development, coincident with cell expansion and growth. This suggests that ergosterol transport, in addition to biosynthesis, is an important process in the second half of the development of mushroom fruiting bodies. Like synthesis, transport of sterols might be required to fulfill the needs of rapid cell expansion, which obviously coincides with cell- and membrane surface expansion too.

Although marginally related, we mention here the CAP domain/Pry1 protein family, which includes known sterol-binding proteins. The family has been associated with diverse functionalities, including cell adhesion, pathogenicity (pathogenicity-related PR proteins belong here), morphogenesis, endocrine and paracrine functions, among others (GM et al. 2008; Schneiter and Pietro 2013). This protein family includes S. cerevisiae Pry1, a lipid and sterol binding protein involved in sterol export(Darwiche et al. 2017). To our best knowledge, there are no previous reports of this family in fruiting body development, whereas, their role in morphogenesis in mammals is well-established(GM et al. 2008). We detected, on average 2-5 copies of CAP proteins in the Agaricomycetes, with expansions up to 9 in Pt. gracilis, $L$. bicolor and $A$. ostoyae as well as a CDE orthogroup of Pry1-related CAP-domain proteins (represented by $C$. cinerea 470387, FC2/4: 7/6, Table 6, Fig. 6). Members of this orthogroup showed peak expression late in development, at or after the young fruiting body stage in most species. The sterol transporting ability and expression patterns suggest that this family may have a role in sterol transport during the second half of development in Agaricomycetes 
bioRxiv preprint doi: https://doi.org/10.1101/2021.12.09.471732; this version posted December 10,2021 . The copyright holder for this preprint (which was not certified by peer review) is the author/funder, who has granted bioRxiv a license to display the preprint in perpetuity. It is made available under aCC-BY-NC-ND 4.0 International license.

Running head: fruiting body genomics

1177 fruiting bodies. It should be noted that neither Polyporales species (Ph. chrysosporium and 1178 L. tigrinus) had developmentally regulated genes in this family, indicating that their role, if 1179 any, may not be universal.

\section{4.3.4. Phospholipid and fatty acid biosynthesis}

1181 The phospholipid and fatty acid biosynthesis pathways are conserved in Agaricomycetes, 1182 with most genes showing clear 1-to-1 orthologs to described pathway members of $S$. 1183 cerevisiae (Table 7). Because active cell growth and expansion requires an excess amount 1184 of membrane components (ergosterol, phospholipids), we examined pathways involved in 1185 membrane phospholipid and triacylglycerol synthesis. We reconstructed these pathways 1186 based on well-known reaction steps in S. cerevisiae and Yarrowia lipolytica(Carman and 1187 Han 2011; Fakas 2017). The first half of the pathway, which starts from glycerol-3-phosphate 1188 and leads to the production of phosphatidic acid, shows upregulation in young and mature 1189 fruiting bodies of $C$. cinerea and $A$. ostoyae (Fig. 7). Phosphatidic acid sits in a branching 1190 point of the pathway, with one branch leading to membrane phospholipids (CDP-DAG 1191 pathway), whereas the other leads to triacylglycerol (TAG), a major storage material. The 1192 CDP-DAG pathway shows a general upregulation in the second half of development (young 1193 and mature fruiting bodies) in C. cinerea, A. ostoyae, M. kentingensis, A. bisporus

1194 (Supplementary Fig. 5). These patterns are consistent with increased membrane lipid 1195 biosynthesis in these stages, possibly serving the needs of increasing cell volumes during 1196 rapid growth. On the other hand, TAG pathway members appear upregulated somewhat 1197 later, mostly in mature fruiting bodies in most species (Fig. 7, Supplementary Fig. 5), which 1198 coincides with spore formation and could be related to storage material synthesis in spores. 1199 In $P$. ostreatus, both the CDP-DAG and TAG pathways show remarkably uniform upregulation in primordium stages (Fig. 7), which is quite different from the pattern seen in other species. This pattern in $P$. ostreatus is also observable in fatty acid biosynthesis pathways (see below).

Similar to the CDP-DAG and TAG pathways, fatty acid biosynthesis (Table 7) shows a signal for upregulation in young fruiting body tissues, with $C$. cinerea orthologs of $S$. cerevisiae Acc1, Fas2, Elo2 and Faa1 showing peak expression in the YFB stage. Similar patterns exist in A. ostoyae, M. kentingensis and to some extent in A. bisporus (Fig. 7). In $P$. ostreatus, fatty acid biosynthesis genes are co-expressed with the other phospholipid biosynthesis genes, showing an upregulation in primordia.

Both phospholipid synthesis and fatty acid biosynthesis require Acetyl-CoA as a starting point. For the former, it is needed for acylation reactions in the conversion of lysophosphatidic acid to phosphatidic acid (last element of the shared part of the pathway) whereas in the latter, Ac-CoA is the starting point of fatty acid synthesis(Fakas 2017). This provides another link with the dynamics of Ac-CoA biosynthesis genes noted above. As in the case of ergosterol biosynthesis pathway members, phospholipid and fatty acid metabolism genes showed low expression dynamics (and hence did not form CDE orthogroups), but their expression patterns were consistent across stages and species.

The expression of mitochondrial membrane components (cardiolipin and phosphatidylglycerol) is also peaking in young fruiting bodies, mostly in stipe, but not very characteristic (Fig. 7). 
bioRxiv preprint doi: https://doi.org/10.1101/2021.12.09.471732; this version posted December 10,2021 . The copyright holder for this preprint (which was not certified by peer review) is the author/funder, who has granted bioRxiv a license to display the preprint in perpetuity. It is made available under aCC-BY-NC-ND 4.0 International license.

Running head: fruiting body genomics

1220

1221

1222

1223

1224

1225

1226

1227

1228

1229

1230

1231

1232

1233

1234

1235

1236

1237

1238

1239

1240

1241

1242

1243

1244

1245

1246

1247

1248

1249

1250

1251

1252

1253

1254

1255

1256

1257

1258

1259

1260

1261

1262

1263

1264

1265

1266

\subsubsection{Linoleic acid producing fatty acid desaturases}

Several CDE orthogroups of fatty acid desaturases (FAD) were detected among conserved developmentally expressed genes. Members of these orthogroups show high similarity to $C$. albicans Fad2 and Fad3 and $A$. nidulans OdeA, which are involved in the synthesis of alphalinoleic acid. Linoleic acid is a major membrane component in fungi, which, as an unsaturated fatty acid, regulates membrane fluidity. It has also been speculated to be the starting point for the biosynthesis of volatiles, such as 1-octen-3-ol(A et al. 2020). Linoleic acid showed increased proportions in fruiting bodies and reproductive structures in general in both the Asco- and Basidiomycota(SHAW 1967; Goodrich-Tanrikulu et al. 1998; H and S 2003; Gessler et al. 2017; Song et al. 2018a). Linoleic acid is also a precursor of oxylipins, key developmental signaling molecules that are actively produced by reproductive cells(Combet et al. 2006; Gessler et al. 2017; Orban et al. 2021). Accordingly, in the Ascomycota, several fatty acid desaturase gene mutations were reported to asexual/sexual defects(Pöggeler et al. 2006b). The higher proportion of linoleic acid in fruiting bodies of Agaricomycetes was already noted in 1967(SHAW 1967) with several similar reports(H and S 2003; Song et al. 2018a) later. The proportion of unsaturated fatty acid (which include linoleic acid) increases during fruiting body formation(H and S 2003) in L. edodes. Three fatty acid desaturases, two in L. edodes (Le.Fad1 and Le.Fad2(H and S 2005)) and one in C. cinerea (Cc.odeA(H and S 2003, 2005; S et al. 2007b)) have been shown to be capable of producing linoleic acid. Fad genes were found to be differentially expressed in fruiting bodies of $A$. bisporus previously(Morin et al. 2012).

We found 7-16 fatty acid desaturase genes in the examined Agaricomycetes, many of which were developmentally regulated, although clear orthology of these genes to experimentally characterized Ascomycota genes is missing. Each species had at least 2-3 FAD encoding genes upregulated in primordia relative to vegetative mycelium, suggesting that their functions are key to the initiation of fruiting body development. FAD genes formed five CDE orthogroups (Table 8). One of these (C. cinerea protein ID: 363743 ) was FB-init in most species (C. cinerea, $A$. ostoyae, $A$. ampla, $M$. kentingensis, $P$. ostreatus, $S$. commune, $C$. aegerita, $L$. bicolor), showed high expression in primordium stages, then expression levels dropped significantly in later developmental stages. The CDE orthogroup containing Le.FAD1 (Lenedo1_1050661) was upregulated in primordia of most species relative to mycelium samples (C. aegerita, A. ampla, S. commune, L. edodes. L. bicolor, L. tigrinus, $M$. kentingensis, but not $A$. ostoyae, $P$. ostreatus and $A$. bisporus, ortholog missing in $C$. cinerea), although only slightly (fold change $=1.5-2 x$, except in $L$. edodes and $P t$. gracilis which showed $>4$-fold upregulation). The orthogroup encompassing $C$ c.ode $A$ (C. cinerea protein ID: 422393) and Le.Fad2 (orthogroup represented by S. commune 2511002) was upregulated in primordia relative to vegetative mycelia of several species ( $A$. ampla, $C$. aegerita, S. commune, $C$. cinerea, $L$. edodes, L. tigrinus and $P$. ostreatus, but not in $A$. ostoyae, M. kentingensis, Pt. gracilis and A. bisporus). Our expression data are consistent with previous reports of transcripts/protein abundance in case of both Le.FAD1 and Le.FAD2 in L. edodes(H and S 2003, 2005). The other two FAD orthogroups showed less conservation in expression (C. cinerea protein ID: 471478, 422976, Fig. 8), including peaks in later developmental stages, such as young fruiting body caps.

The linoleic acid-producing ability of the proteins encoded by these genes suggests that the detected CDE orthogroups and expression dynamics relate to linoleic acid production. It follows from our observations of expression dynamics that linoleic acid production is upregulated in the primordia of several species. This is consistent with previous 
bioRxiv preprint doi: https://doi.org/10.1101/2021.12.09.471732; this version posted December 10,2021 . The copyright holder for this preprint (which was not certified by peer review) is the author/funder, who has granted bioRxiv a license to display the preprint in perpetuity. It is made available under aCC-BY-NC-ND 4.0 International license.

Running head: fruiting body genomics

1267 reports of higher linoleic and unsaturated fatty acid proportions in fruiting bodies of

1268 Agaricomycetes (see above). There may be at least three explanations for this observation, 1269 including (i) regulation of membrane fluidity in response to temperature changes, (ii) the 1270 same being genetically encoded in fruiting body development, or (iii) fulfilling the 1271 requirements of oxylipin production. We argue that hypothesis (i) is unlikely, however, we 1272 can't speculate on hypotheses (ii) and (iii).

1273 Regulation of membrane fluidity by increasing the proportion of unsaturated fatty 1274 acids is a common mechanism across organisms( $H$ and $S$ 2005). Therefore, a question 1275 arises whether the higher inferred proportion of linoleic acid and corresponding changes in gene expression are genetically coded traits of fruiting bodies or simply a consequence of lower temperature required by several species for fruiting. At least for the transcriptomes we produced(Sipos et al. 2017a; Almási et al. 2019; Krizsán et al. 2019) we know that vegetative mycelium samples were harvested at the same temperature as fruiting body samples for all species, which rules out the possibility that higher linoleic acid proportion is simply a response to lower fruiting temperatures. Rather, this suggests that higher linoleic acid concentration is a genetically encoded trait in fruiting bodies. Similar conclusions were reached in the context of unsaturated fatty acids in $L$. edodes(H and S 2003). From an evolutionary point of view, such a genetic adaptation could go back to the exposed nature of fruiting bodies; in the natural environments (soil, wood) the vegetative mycelium is in more stable conditions than fruiting bodies are, which might therefore need to be adapted to colder temperatures by increasing the proportion of unsaturated fatty acids in fruiting body tissues. At the same time, due to their large surfaces and continuous evaporation, fruiting bodies cool themselves (on average $\sim 2.5 \mathrm{C}$ colder than their environments(Dressaire et al. 2016)), which may explain why genetic encoding of higher linoleic acid proportions is beneficial for their development. It should be noted that linoleic acid production is also required for the synthesis of several oxylipin species(Combet et al. 2006), which might be involved in cell-tocell communication.

\subsubsection{Other potentially membrane lipid related genes}

We detected several CDE orthogroups which are not parts of the above-mentioned pathways, yet might be related to lipid metabolism, membrane organization or assembly. In many cases the functions of these orthogroups is not clear, but their widespread developmental expression suggests they might be interesting players in fruiting body development. Therefore, we discuss them below, with details on potential functions where available. developmentally expressed in 11 species (FC>4), which makes it one of the most widely developmentally expressed families. However, it is hard to make functional inferences as these proteins show only limited similarity to experimentally characterized fungal proteins (highest to Pgc1 of S. cerevisiae). S. cerevisiae Pgc1 (Phosphatidylglycerol phospholipase C) negatively regulates phosphatidylglycerol degradation, leading to increased concentrations in deletion mutants(Šimočková et al. 2008). The glycerophosphodiester phosphodiesterase family (IPR030395), is represented by three copies in most examined Agaricomycetes (some species have 4), of which only one is developmentally expressed in all species. These form a CDE orthogroup in which most genes are FB-init ( $C$. aegerita, $A$. ostoyae, A. ampla L. bicolor, L. tigrinus, P. ostreatus, S. commune), whereas expression had 
bioRxiv preprint doi: https://doi.org/10.1101/2021.12.09.471732; this version posted December 10,2021 . The copyright holder for this preprint (which was not certified by peer review) is the author/funder, who has granted bioRxiv a license to display the preprint in perpetuity. It is made available under aCC-BY-NC-ND 4.0 International license.

Running head: fruiting body genomics

1313 different dynamics in C. cinerea, L. edodes, M. kentingensis, Ph. chrysosporium). These 1314 expression patterns suggest that the role of this orthogroup, although unknown at the moment, may be key to the initiation of fruiting body formation.

We noticed that the detected glycerophosphodiester phosphodiesterases showed strongly correlated expression with members of another CDE orthogroup (represented by $C$. cinerea 380149, Fig. 7) in six species (data not shown). This contains putative orthologs of S. cerevisiae Cpt1, a choline/ethanolamine phosphotransferase that catalyzes the last step (formation of phosphatidylcholine) of the CPD-choline (Kennedy) pathway in S. cerevisiae. The strongly correlated expression in the case of six species (Pearson $r>0.8$ ) suggests that the two genes are linked and possibly form a linear pathway. However, their role in the context of fruiting body formation requires further research.

Marvel protein family - We also detected two CDE orthogroups (represented by $C$. cinerea 444998, 462849) (Table 9) of Marvel proteins showing low similarity to $A$. nidulans mrvA, C. albicans Mrv2 and S. cerevisiae Fhn1. The gene family seems to be generally poorly characterized. In $S$. cerevisiae, it may be involved in nonclassical protein secretion, might act as a sensor of sphingolipids or be related to membrane organization( $\mathrm{M}$ et al. 2010). In metazoans, marvel proteins are related to sterol-rich membrane region positioning(Sánchez-Pulido et al. 2002). Members of the first orthogroup (C. cinerea protein ID: 444998) were FB-init in several species (A. ostoyae, $M$. kentingensis, $P$. ostreatus, $S$. commune, $L$. edodes), suggesting that they fulfill key functions in fruiting body initiation. The second orthogroup (C. cinerea protein ID: 462849) showed less characteristic expression patterns, with upregulation mostly late in development in most species.

Sphingolipid C9-methyltransferases - We found two CDE orthogroups of sphingolipid C9-methyltransferases (C. cinerea protein ID: 395241, 365330)(Table 9) that were FB-init in nearly all species. One of the orthogroups (C. cinerea protein ID: 395241) contains orthologs of Mts 1 of $C$. albicans, which is involved in glucosylceramide biosynthesis. Glucosylceramides are glycosphingolipids that form structural components of the fungal cell membrane, with diverse roles (cell division, spore germination, pathogenicity, lipid rafts(Poeta et al. 2014)), although little mechanistic detail to date.

PAP2 enzyme family - A CDE orthogroup of diacylglycerol pyrophosphate phosphatases (C. cinerea protein ID: 380219)(Table 9) was FB-init in most species in which the gene was present. The encoded proteins show high similarity (but not 1-to-1 orthology) to $A$. nidulans and $C$. albicans membrane phospholipid metabolism proteins AN1671 and Dpp3, respectively. The latter is required also for farnesol biosynthesis(Nickerson et al. 2006). These genes belong to the broader PAP2 family, which produce diacylglycerol and are key in the synthesis of phospholipids, triacylglycerol and the generation of signaling molecules with a lipid nature. The upregulation of these genes in fruiting bodies might be related to increased membrane material production associated with cell proliferation during early development.

Caleosins - Caleosins are well-characterized in plants and indirect data indicate they may be involved in oxylipin signaling, lipid metabolism, reproduction(Rahman et al. 2018), though exact functional knowledge seems to be missing. In fungi, caleosins are involved in sporulation, pathogenicity and lipid storage(Y et al. 2015). A previous study found that the caleosin family is present in $\sim 30 \%$ of fungal genomes(Rahman et al. 2018), whereas we found that it is present in most of the examined Agaricomycetes, mostly as a single-copy gene. Members of a detected caleosin CDE orthogroup (C. cinerea protein ID: 376602)(Table 9) were FB-init in most species and consistently had peak expression late in 
bioRxiv preprint doi: https://doi.org/10.1101/2021.12.09.471732; this version posted December 10,2021 . The copyright holder for this preprint (which was not certified by peer review) is the author/funder, who has granted bioRxiv a license to display the preprint in perpetuity. It is made available under aCC-BY-NC-ND 4.0 International license.

Running head: fruiting body genomics

1360 development, which is compatible with presumed roles in fruiting body initiation and 1361 sporulation, respectively.

\section{4.4. Storage carbohydrate metabolism}

1363 Fungal colonies accumulate and mobilize various carbohydrates as reserve material and for 1364 energy storage. Previous research has revealed that there are very specific and tightly 1365 regulated accumulation and mobilization patterns of glycogen, trehalose and mannitol within fungal fruiting bodies( $U$ et al. 2000). Storage carbohydrate metabolism and distributions in the mycelium and fruiting bodies have been studied in a number of economically important mushroom-forming species, including F. velutipes(Kitamoto et al. 2001), Pleurotus spp.(Han et al. 2003; Zhou et al. 2016a; Zhu et al. 2019), Pisolithus microcarpus(AN and MD 2010), A. bisporus(WELLS et al. 1987; TM et al. 2008; Patyshakuliyeva et al. 2013), L. bicolor(Deveau et al. 2008) and described to a high precision/resolution in $C$. cinerea in a series of papers by Moore et al(Kuhad et al. 1987; Ji and Moore 1993; Moore 2013), including links with cAMP levels (summarized by ( $U$ et al. 2000)). Briefly, these studies provided evidence on tightly regulated glycogen, trehalose and mannitol levels in mycelia and various tissues and developmental stages of fruiting bodies. They also revealed that the identity and quantities of major sugars in fruiting bodies differ from species to species; trehalose dominates in $L$. bicolor(Deveau et al. 2008) and C. cinerea(Kues 2000), mannitol in A. bisporus(Morton et al. 1985 ) and $L$. edodes(Tan and Moore), and both present in similar quantities in $P$. ostreatus(Hong Jai-Sik;Kim 1988; Zhou et al. 2016a).

More recently, genome sequences allowed the reconstruction of carbohydrate metabolism pathways based on gene orthology information to better studied model systems such as $S$. cerevisiae. Deveau et al found that most of the genes in known fungal pathways related to glycogen, trehalose and mannitol metabolism were found in the genome of the ectomycorrhizal L. bicolor(Deveau et al. 2008) and similar patterns can be expected in these very conserved pathways upon examination of other Agaricomycetes genomes.

Transcriptomic studies further allowed previous biochemical observations to be interpreted in the light of gene expression patterns, although this has not yet been systematically performed in any species. In the following sections we review information on the metabolism of glycogen, trehalose and mannitol gleaned from transcriptomic studies of mushroom development. Many of these studies highlighted that shared patterns of gene expression related to storage carbohydrate metabolism exist among mushroom-forming species. For example, Merenyi et al showed that dynamic glycogen accumulation/breakdown is shared between $C$. neoformans basidium development and complex fruiting body morphogenesis, two extremes of Basidiomycota development along the complexity gradient(Merényi et al. 2021). On the other hand, trehalose concentrations were found to react to heat shock in fruiting bodies of Flammulina filiformis(Liu et al. 2016). Our discussion hereafter is based on well-described pathways of storage carbohydrate metabolism in yeast(François and Parrou 2001; JM et al. 2012).

1399 Glycogen metabolism - Glycogen production and mobilization takes place in various tissues and developmental stages and follows tight regulation that may differ from species to species (reviewed in (Ji and Moore 1993; Kues 2000; Xie et al. 2020), see also (Ceccaroli et al. 2011) for Ascomycota). In the Agaricomycetes, this is best known in C. cinerea and $A$. bisporus, whereas mechanistically glycogen metabolism is best known in $S$. 
bioRxiv preprint doi: https://doi.org/10.1101/2021.12.09.471732; this version posted December $10,2021$. The copyright holder for this preprint (which was not certified by peer review) is the author/funder, who has granted bioRxiv a license to display the preprint in perpetuity. It is made available under aCC-BY-NC-ND 4.0 International license.

Running head: fruiting body genomics

1406 glycogenins and elongation and branching by glycogen synthases. Glycogenins (orthologs of 1407 S. cerevisiae Glg1) are upregulated in young fruiting body gills and/or mature fruiting body 1408 gills of most compared species (Table 10), respectively. This likely corresponds to intense 1409 glycogen synthesis during basidiospore maturation(Kües 2000). Consistent with this, 1410 orthologs of yeast Gac1, a phosphatase that positively regulates the activity of glycogen 1411 synthases, is upregulated in gills and mature fruiting bodies of multiple species. Gac1 in $S$. 1412 cerevisiae regulates glycogen synthases together with Glc7. The latter has orthologs in 1413 mushroom-forming fungi, but these are not developmentally regulated. In S. cerevisiae, a 1414 number of other CBM21 domain proteins (Pig1, Pig2) also participate in the regulation of 1415 glycogen synthase activity, however, these have no clear orthologs in mushroom-forming 1416 fungi. On the other hand, the glycogen branching enzyme Glc3 orthologs (represented by $C$. 1417 cinerea 365699) show more diverse expression peaks, and orthologs are upregulated mostly 1418 in stipe tissues (in C. cinerea, L. bicolor, P. ostreatus and A. ostoyae) (Fig. 9, Supplementary 1419 Fig. 6), indicating that linear glycogen chain production is transcriptionally decoupled from 1420 the addition of branched side chains to the glycogen backbone.

Genes that encode proteins upstream of glycogen synthesis, for example orthologs of the uridinephosphoglucose pyrophosphorylase Ugp1 (C. cinerea protein ID: 359312) and the phosphoglucomutase PGM1 (C. cinerea protein ID: 497782) of S. cerevisiae, are developmentally regulated in multiple species (Table 10). They show some overlap in expression patterns, in particular, a tendency for upregulation late in development, but their pattern of expression is quite unique and show lower expression fold changes.

It should be noted that the glycogen synthase kinase 3 (GSK-3)(Chang et al. 2019a) gene in $C$. cinerea (C. cinerea protein ID: 362899), despite its name, it functionally not relevant to glycogen metabolism, it is an orthologue of $S$. cerevisiae Rim11, a pleiotropic kinase involved in cell cycle regulation.

Glycogen mobilization happens through three primary genes in $S$. cerevisiae(François and Parrou 2001) - all these have orthologs in mushroom-forming fungi (Table 10). Orthologs of the yeast glycogen phosphorylase Gph1 (represented by $C$. cinerea 495987) mostly show high expression in vegetative mycelia or early development, whereas those of the glycogen debranching enzyme Gdb1 are upregulated also in mature fruiting bodies in all species (Fig. 9). This probably indicates differential usage of phosphorolysis and debranching activities in different stages of development. Sga1 is a sporulation specific glucoamylase in S. cerevisiae. In our species orthologs (represented by $C$. cinerea 473268) of this gene do not show expression peaks in gills, the tissues in which spore development takes place. Rather, in $C$. cinerea, this gene is upregulated in young fruiting body cap tissues. How this upregulation corresponds to patterns of glycogen metabolism remains to be understood. Large-scale postmeiotic utilization of glycogen accumulated in subhymenial tissues was reported in C. cinerea(Kües 2000), however, subhymenial tissues were part of gill samples in previous RNA-Seq experiments, which excludes the possibility that Sga1 orthologs were involved in that process. In other species, expression patterns were different, ranging from upregulation in mature fruiting bodies in $A$. ampla, to peak expression in vegetative mycelium ( $P$. ostreatus and $M$. kentingensis). We also detected a CDE orthogroup of alpha-amylases (represented by $C$. cinerea 495249), which find $A$. nidulans

1449 AmyA as best hit and are probably involved in glycogen catabolism. In $C$. cinerea, this gene showed upregulation in primordia and peak expression in young fruiting body caps, similar to other genes related to glycogen mobilization.

In addition to known members of the glycogen catabolism pathways, we identified two CBM21 domain containing CDE orthogroups that were developmentally regulated in 
bioRxiv preprint doi: https://doi.org/10.1101/2021.12.09.471732; this version posted December $10,2021$. The copyright holder for this preprint (which was not certified by peer review) is the author/funder, who has granted bioRxiv a license to display the preprint in perpetuity. It is made available under aCC-BY-NC-ND 4.0 International license.

Running head: fruiting body genomics

$145411 / 7$ and $11 / 6$ species (C. cinerea protein ID: 544130 and 455149) (Table 10). In most

1455 species, this gene had the highest expression in vegetative mycelia, but showed significant

1456 expression dynamics also during fruiting body morphogenesis. This seems to be a

1457 Basidiomycota-specific orthogroup, with no significantly similar proteins in model

1458 Ascomycota. The CBM21 domain mostly occurs in starch-binding proteins (glucoamylase

1459 and a-amylase(Ashikari et al. 1986)) whereas in this orthogroup, the CBM21 domain occurs

1460 alone, providing no specific clues to function. Therefore, our placement of this CDE

1461 orthogroup under this chapter should be viewed tentatively until functional studies prove or

1462 disprove the role of the family in glycogen metabolism.

1463

1464

1465

1466

1467

1468

1469

1470

1471

1472

1473

1474

1475

1476

1477

1478

1479

1480

1481

1482

1483

1484

1485

1486

1487

1488

1489

1490

1491

1492

1493

1494

1495

1496

1497

1498

1499

1500

Beyond these gene families, glycogen utilization patterns in fruiting bodies raise a number of exciting research avenues, such as the mode of transport within the fruiting body and the identity of transporters involved in that process.

Trehalose metabolism - Out of the at least five pathways known to be involved in trehalose synthesis, two have been found in Agaricomycetes(Deveau et al. 2008). The first, canonical pathway comprises the action of a protein complex formed by four proteins in $S$. cerevisiae (Tps1 - C. cinerea 478544, Tps2 - 473004, Tsl1 and Tps3 - no ortholog(François and Parrou 2001)). Of these, Tsl1 (C. cinerea protein ID: 503099) and Tps3 are paralogs that arose from a recent whole genome duplication and thus there is only one corresponding gene in filamentous fungi. The three genes showed widespread developmentally regulated expression in examined Agaricomycetes (Table 10), with upregulation mostly late in development with notable co-regulation between the three genes, as expected for members of a protein complex (Fig. 9).

A second, noncanonical pathway involving trehalose phosphorylase (TP) exists in fungi and was detected in multiple mushroom-forming species( $\mathrm{K}$ et al. 1998; Eis and Nidetzky 1999; Wannet et al. 1999; Deveau et al. 2008; Thammahong et al. 2017). Trehalose phosphorylase gene formed a CDE orthogroup (C. cinerea protein ID: 540817 ) in the Agaricomycetes we examined(Merényi et al. 2021) and show upregulation in stipes and in late stages of development in most species (Table 10), although deviations from this pattern exist (e.g in C. cinerea). For example, the gene showed peak expression in primordium stages of $A$. ampla, S. commune and M. kentingensis(Almási et al. 2019; Krizsán et al. 2019; Ke et al. 2020b).

For catabolizing trehalose, fungi exhibit two types of trehalose hydrolyzing activities, one termed acid trehalase and the other called neutral trehalase(François and Parrou 2001). Agaricomycetes species possess clear orthologs of neutral trehalases orthologous to Nth1 of $S$. cerevisiae. On the other hand, and in contrast to Deveau et al (Deveau et al. 2008), we did not find proteins in the genomes of Agaricomycetes showing significant sequence similarity (based on BLAST) to acid trehalase. A single neutral trehalase gene was found in Agaricomycetes, this formed a CDE orthogroup at FC>2 ( $C$. cinerea protein ID: 437212, Table 10) and showed varied expression patterns in the species examined.

Orthologs of previously described fungal trehalose transporters were not detectable in Agaricomycetes genomes, based on sequence similarity, in agreement with a previous study on L. bicolor(Deveau et al. 2008). However, it should be noted that fungal trehalose transporters are poorly known and current knowledge likely misses a large proportion of genes with this activity(Thammahong et al. 2017).

Mannitol metabolism - Mannitol accumulates in high concentrations in fruiting bodies of certain species, possibly as an osmolyte assisting fruiting body growth(Chakraborty et al. 2004; Zhou et al. 2016b) (see also opposing evidence above). A 
bioRxiv preprint doi: https://doi.org/10.1101/2021.12.09.471732; this version posted December 10,2021 . The copyright holder for this preprint (which was not certified by peer review) is the author/funder, who has granted bioRxiv a license to display the preprint in perpetuity. It is made available under aCC-BY-NC-ND 4.0 International license.

Running head: fruiting body genomics

1502 NADP-dependent mannitol 2-dehydrogenase was reported from A. bisporus(JM and H 1998;

1503 S et al. 2001). In agreement, in L. bicolor Deveau et al reported a single mannitol

1504 2-dehydrogenase-dependent pathway of mannitol biosynthesis(Deveau et al. 2008), which

1505 works via the direct reduction of fructose. Using the A. bisporus NADP-dependent mannitol

1506 2-dehydrogenase gene(JM and $\mathrm{H}$ 1998) as a query, we identified an orthogroup

1507 (represented by $C$. cinerea 429272) of short-chain dehydrogenase/reductase genes that

1508 contained only A. bisporus, L. bicolor, C. cinerea, and $P$. ostreatus, but was missing from

1509 other species (formed multiple orthogroups probably due to duplications in A. bisporus).

1510 However, members of this orthogroup were developmentally regulated in all species (Table

1511 10); they were upregulated at the initiation of fruiting and showed expression peaks in gills of

1512 C. cinerea and $P$. ostreatus, young and mature caps of $L$. bicolor, as well as a marked

1513 upregulation in the stipe tissues of $A$. bisporus. These observation agree with mannitol

1514 metabolism being enhanced in fruiting bodies of $L$. bicolor (Deveau et al. 2008).

1515 In summary, the genomes of Agaricomycetes contain clear orthologs of

1516

1517 experimentally characterized members of glycogen, trehalose and mannitol metabolism

1518

1519

1520

1521

1522 pathways. Many of the genes in these pathways are developmentally regulated in fruiting bodies, often showing conserved expression peaks in multiple species, reflecting a conservation of storage carbohydrate mobilization strategies within fruiting bodies. Interestingly, in L. bicolor trehalose and glycogen metabolism was upregulated in both fruiting bodies and mycorrhizae, providing links between fruiting bodies and ECM(Deveau et al. 2008; Martin et al. 2008).

1523

1524 4.5. Transcriptional regulators

\subsubsection{Transcription factors}

Transcription factors are proteins that modulate gene expression by binding to specific DNA sequences and subsequently activating or inhibiting transcription. Transcription factors play a major role in the regulation of development across the tree of life, and mushroom-forming fungi are no exception. They are involved in many stages of development, ranging from the establishment of a dikaryon to various aspects of mushroom development.

The best studied are the homeodomain transcription factors of the MatA mating-type locus that are essential for the establishment of a fertile dikaryon, which is required for fruiting body formation in heterothallic basidiomycetes(Raudaskoski and Kothe 2010; Vonk and Ohm 2018). During mating the homeodomain transcription factors expressed from compatible MatA loci dimerize and activate dikaryon-specific transcriptional pathways, including the formation of clamp connections. The mechanism of mate recognition is very similar to that found in the yeast S. cerevisiae(Haber 2012) and is present across the fungal kingdom, although many ascomycetes do not use homeodomain transcription factors for mating(Houbraken and Dyer 2015). In some Agaricales, like C. cinerea and S. commune, the MatA locus has undergone expansion, improving the chances of finding a compatible mate(Freihorst et al. 2016).

Several transcription factors have been identified that act downstream of the MatA homeodomain transcription factors. Deletion of the transcription factors hom2, tea1 and wc2 in S. commune resulted in strains that did not show dikaryotic development beyond clamp formation(Ohm et al. 2011, 2013; Pelkmans et al. 2017a). Generally, the resulting colonies remained symmetrical and showed no sign of mushroom development. Hom2 is highly 
bioRxiv preprint doi: https://doi.org/10.1101/2021.12.09.471732; this version posted December 10,2021 . The copyright holder for this preprint (which was not certified by peer review) is the author/funder, who has granted bioRxiv a license to display the preprint in perpetuity. It is made available under aCC-BY-NC-ND 4.0 International license.

Running head: fruiting body genomics

1547 conserved in mushroom-forming fungi and features four putative phosphorylation sites.

1548 Disruption of these phosphorylation sites leads to a constitutively active Hom2 and the

1549 inhibition of vegetative growth and monokaryotic fruiting, indicating that Hom2 is post-

1550 translationally regulated(Pelkmans et al. 2017a). WC2 is part of the blue light sensing white

1551 collar complex first identified in N. crassa. In N. crassa, WC1 and WC2 interact through PAS

1552 domains and WC1 contains a LOV domain that binds a FAD chromophore when exposed to

1553 blue light. This results in conformation changes in the white collar complex and activates

1554 transcription factor domains on both WC1 and WC2(Corrochano 2019). Orthologs of WC1

1555 (dst1 in C. cinerea) and WC2 (Cc.wc2 in C. cinerea) in S. commune and C. cinerea fulfil a

1556 similar role and deletion ( $S$. commune wc1 and wc2, C. cinerea wc2) or mutation (C. cinerea

1557 wc1) of either wc1 or wc2 prevents the formation of mature fruiting bodies and is called a

1558 blind phenotype(Terashima et al. 2005; Kuratani et al. 2010; Nakazawa et al. 2011; Ohm et

1559 al. 2013). In S. commune this results in a phenotype where no dikaryotic development is

1560 observed and in increased sensitivity to intense light. However, in C. cinerea a blind

1561 phenotype leads to the so called 'dark stipe' phenotype(Kamada et al. 2010). In this

1562 phenotype, the primordial shaft is elongated, but pileus and stipe development cannot be

1563 completed. In S. commune, WC1 does not contain a DNA-binding domain, unlike WC1 in N.

1564 crassa, making transcriptional activity dependent on WC2(Ohm et al. 2010). Orthologs of

1565 white collar complex members with the same function have been reported in $L$. edodes(Sano

1566 et al. 2009) and P. ostreatus(Qi et al. 2020). Furthermore, developmental expression of wc

1567 members was reported for $F$. filiformis(Liu et al. 2020b). Mutants of the gene tea1, which is

1568 downregulated in $\Delta w c 1, \Delta w c 2, \Delta h o m 2, \Delta b r i 1$ and $\Delta f s t 4$ dikaryons, also did not form

1569 mushrooms(Pelkmans et al. 2017a). It should be noted that in tea1 deletion mutants,

1570

1571

1572 dikaryotic colonies sporadically produced clusters of mushrooms, indicating that Tea1 is not essential for fruiting body formation and may act as a "switch" that can be bypassed. The

1573 ARID/BRIGHT DNA binding domain transcription factor Bri1 was initially also thought to play a major role in fruiting development(Ohm et al. 2011). However, later characterization of bri1 deletion strains revealed that inactivation leads to significant growth defects that result in a delay in fruiting(Pelkmans et al. 2017a). The fungal specific transcription factor fst4 has a very similar phenotype where no mushrooms are formed, but deletion strains of this transcription factor do form asymmetrical colonies after mating, which is the first step in mushroom development(Kües and Liu 2000; Ohm et al. 2010). In P. ostreatus, mutations in gat1 resulted in a strain that did not fruit(Nakazawa et al. 2019). The missense mutation inside the zinc-finger binding domain resulted in a dominant gain of function mutation, which suggests that the mutated transcription factor has an altered DNA-binding specificity. This is in line with disruption of the ortholog gat1 in S. commune, where the phenotype was not dominant and resulted in the formation of more, but smaller mushrooms(Ohm et al. 2011). A general overview of Zn(II)2Cys6 zinc cluster transcription factors, including expression patterns in $P$. ostreatus fruiting bodies, was published by Hou et al(Hou et al. 2020). In Ganoderma lucidum, silencing of the $\mathrm{c}_{2} \mathrm{~h}_{2}$-type transcription factor pacC also resulted in the absence of fruiting bodies, as well as altered mycelial growth(Wu et al. 2016). Interestingly, $\mathrm{PacC}$ is conserved across the fungal kingdom and activates genes under alkaline conditions, and inhibits genes under acidic conditions(Peñalva et al. 2008). Therefore, it is unclear if the absence of fruiting can be attributed to fruiting-specific transcription or general homeostasis. In L. edodes, PriB was described as a primordium-upregulated transcription factor(Endo et al. 1994). Finally, silencing of hada-1 resulted in delayed fruiting, as well as multiple growth defects, in Hypsizygus marmoreus(Zhang et al. 2021b). 
bioRxiv preprint doi: https://doi.org/10.1101/2021.12.09.471732; this version posted December 10,2021 . The copyright holder for this preprint (which was not certified by peer review) is the author/funder, who has granted bioRxiv a license to display the preprint in perpetuity. It is made available under aCC-BY-NC-ND 4.0 International license.

Running head: fruiting body genomics

1594

1595

1596

1597

1598

1599

1600

1601

1602

1603

1604

1605

1606

1607

1608

1609

1610

1611

1612

1613

1614

1615

1616

1617

1618

1619

1620

1621

1622

1623

1624

1625

1626

1627

1628

1629

1630

1631

1632

1633

1634

1635

1636

1637

1638

1639

1640

1641
Downstream in development, S. commune c2h2, fst1 and zfc7 gene disruption resulted in arrested fruiting body formation, where primordia were formed that did not develop further into mature fruiting bodies(Ohm et al. 2011; Vonk and Ohm 2021). Interestingly, overexpression of $c 2 h 2$ could increase the rate of fruiting in $A$. bisporus, indicating that $c 2 h 2$ expression is a limiting step in fruiting body development(Pelkmans et al. 2016). The transcription factors Fst1 and Zfc7 were identified due to enrichment of histone 3 lysine 4 (H3K4) dimethylation in their genes during fruiting body development after 4 days, which indicates increased genetic accessibility and transcription during this developmental stage(Vonk and Ohm 2021). Further downstream, the $C$. cinerea highmobility group transcription factor Exp1 regulates pileus expansion and autolysis to complete fruiting(Muraguchi et al. 2008b). Notably, this transcription factor is conserved and developmentally regulated in all studied mushroom-forming fungi, except for $A$. ampla, Pt. gracilis and $S$. commune, whose fruiting bodies do not feature a pileus.

Instead of promoting fruiting body development, several transcription factors are involved in promoting vegetative growth or repressing fruiting. These include $F$. velutipes Ifc1 and $h m g 1$, where silencing increased the rate of fruiting(Wu et al. 2020a; Meng et al. 2021). Additional transcription factors are P. ostreatus Pofst 3 and $S$. commune fst 3 , gat 1 and hom1(Ohm et al. 2011; Qi et al. 2019). These genes are differentially regulated in the majority of mushroom forming species, and conserved in all of them. When the genes are deleted ( $S$. commune) or silenced ( $P$. ostreatus) it results in the formation of more, smaller mushrooms. Finally, premature stop codons in pcc1 in $C$. cinerea (natural variation, truncated from 561 to 210 amino acids, HMG-box not disrupted) and P. ostreatus (CRISPR/Cas9-assisted gene mutagenesis, truncated from 471 to 58 amino acids, HMGbox disrupted) resulted in monokaryotic fruiting and the formation of pseudoclamps(Murata et al. 1998; Boontawon et al. 2021). In C. cinerea these pseudoclamps were formed during monokaryotic growth, indicating a relation between the MatA homeodomain transcription factors and Pcc1. In P. ostreatus however, dikaryotic strains formed pseudoclamps, suggesting a role in clamp formation.

Many transcription factors are conserved across fungi or specifically in mushroomforming fungi. Both wc2 and pacC are conserved in both ascomycetes and basidiomycetes and except for $h m g 1$ ( $F$. velutipes), Ifc1 (F. velutipes), pdd1 (F. velutipes), pcc1 (C. cinerea and $P$. ostreatus) and $z f c 7$ (S. commune) all characterized transcription factors are at least conserved in mushroom-forming fungi. Cag1 (C. cinerea protein ID: 466216) and Cc.Tup1 (C. cinerea protein ID: 369655 ) of $C$. cinerea are further examples of highly conserved transcription factors(Masuda et al. 2016). They are homologs of the highly conserved general transcriptional co-repressor Tup1 of $S$. cerevisiae, which can form repressive complexes with a wide range of sequence-specific transcription factors. Deletion of Cag1 resulted in a cap-growthless phenotype, which, upon closer inspection turned out to be caused by developmental defects of gills(Masuda et al. 2016). However, the expression patterns are generally not conserved during fruiting body development(Almási et al. 2019; Krizsán et al. 2019). For example, the developmental stage with the highest expression level of the ortholog can vary greatly between species. This can indicate either that transcriptional regulation of fruiting body development is poorly conserved between species, or that transcription factors are regulated post-translationally. The MatA mating type genes are an example of post-translational regulation, where the transcription factors are constitutively expressed, but only become activated when heterodimers are formed between compatible homeodomain transcription factors. Similarly, in N. crassa WC2 is activated by structural changes in its interaction partner WC1 when blue light is detected. Furthermore, Hom2 in $S$. 
bioRxiv preprint doi: https://doi.org/10.1101/2021.12.09.471732; this version posted December 10,2021 . The copyright holder for this preprint (which was not certified by peer review) is the author/funder, who has granted bioRxiv a license to display the preprint in perpetuity. It is made available under aCC-BY-NC-ND 4.0 International license.

Running head: fruiting body genomics

1642 commune is constitutively expressed, but post-translationally phosphorylated to become 1643 inactive.

1644 While many expression patterns of transcription factors are not conserved in 1645 mushroom-forming fungi, there are several interesting and conserved transcription factors.

1646 We identified 51 orthogroups of TFs that were developmentally regulated in a significant 1647 number of species (Table 11). Among the functionally characterized transcription factors, 1648 orthologs of dst1 (=WC1, C. cinerea protein ID: 369621) and Cc.wc2 (C. cinerea protein ID: 1649 8610) of $C$. cinerea formed CDE orthogroups developmentally regulated in 10/4 and 10/6 1650 (FC>2/4) species, respectively. Orthologs of dst1 and Cc.wc2 showed varied expression 1651 profiles in the examined species. An orthogroup of homeodomain transcription factors ( $C$. 1652 cinerea protein ID: 497620) is developmentally regulated during fruiting body formation in all 1653 mushroom forming fungi, except for Pt. gracilis, which is known for its simplified fruiting body 1654 structure. The detected CDE orthogroups also included exp1 of $C$. cinerea (represented by 1655 C. cinerea 421729), which were developmentally regulated in 9/5 species (FC>2/4). In 1656 accordance with its reported function in $C$. cinerea(Muraguchi et al. 2008b), it showed peak 1657 expression in young fruiting body caps. However, the A. ostoyae, L. bicolor and $M$. 1658 kentingensis orthologs were upregulated in stipe tissues. In $P$. ostreatus we found the 1659 highest expression in cap cuticle. Furthermore, a $\mathrm{C}_{2} \mathrm{H}_{2}$-type transcription factor (C. cinerea 1660 protein ID: 494418) is conserved and expressed in all mushrooms except for A. ampla, Pt. 1661 gracilis and $S$. commune. It may therefore play a similar role to Exp1 in $C$.

1662 cinerea(Muraguchi et al. 2008b), which is also conserved and developmentally regulated in 1663 most fungi except for A. ampla, Pt. gracilis and S. commune. While many other transcription 1664 factor orthogroups are conserved in all studied mushroom-forming fungi, their expression 1665 patterns are not strongly conserved. Therefore, proteomics or functional analyses are 1666 required to further elucidate the role of transcription factors in fruiting body formation.

\section{4.5.2. RNA binding proteins}

Pumilio family - This family comprises evolutionarily conserved sequence-specific RNA-binding proteins that bind 3' UTR regions of specific mRNAs. This family has diverse roles in fungi, including the regulation of meiotic and sexual processes (e.g. Mcp2 of $S$. pombe)(Wilinski et al. 2017; Liu et al. 2018). Pumilio proteins were found to be active during fruiting body formation in both the Asco- and Basidiomycota(Krizsán et al. 2019; Merényi et al. 2020b). With a few exceptions, all Agaricomycetes contain six Pumilio protein encoding genes. These formed two CDE orthogroups (Table 12), one of which (C. cinerea protein ID: 378537) contains orthologs of the Sch. pombe meiotic coiled-coil protein Mcp2 and shows expression dynamics reminiscent of mitotic and meiotic genes (see Chapter 4.1.1), and another, comprising orthologs of Sch. pombe Puf3 and C. neoformans Pum1 (C. cinerea protein ID: 354109$)$. Both orthogroups are developmentally regulated in a large number of species (8/7 and 7/6 at FC2/4, respectively). In C. neoformans, pum1 has been associated with basidium development and morphogenesis, suggesting that its orthologs may have similar roles in Agaricomycetes too.

RRM family - We identified five very conserved CDE orthogroups of RNA recognition motif (RRM) family proteins. One of these (C. cinerea protein ID: 471238) were annotated as related to mitosis and meiosis and is discussed under that chapter. The RRM family comprises proteins that bind diverse RNA types and shows considerable diversity in fungi, with 68-88 genes in the Agaricomycetes. Overall, relatively few members of the family were developmentally expressed, however, the orthogroups we detected showed a conserved 
bioRxiv preprint doi: https://doi.org/10.1101/2021.12.09.471732; this version posted December 10,2021 . The copyright holder for this preprint (which was not certified by peer review) is the author/funder, who has granted bioRxiv a license to display the preprint in perpetuity. It is made available under aCC-BY-NC-ND 4.0 International license.

Running head: fruiting body genomics

1688 expression pattern. One of them (C. cinerea protein ID: 357717) was developmentally 1689 regulated in all 12 species (Table 12) and was upregulated in the second half of fruiting body 1690 development (young fruiting body and mature fruiting body in $C$. cinerea), in particular in gills 1691 (A. ostoyae, $P$. ostreatus, $M$. kentingensis, $C$. cinerea) where gill tissue was sampled 1692 separately, or caps (L. bicolor, L. edodes). It was also upregulated considerably in primordia 1693 relative to vegetative mycelia. A less characteristic, but similar and very conserved late 1694 upregulation was observed also in the second CDE orthogroup (C. cinerea protein ID: 1695 356106), which contains reciprocal best hits of Sch. pombe Vip1.

1696

1697

1698

The third CDE orthogroup (C. cinerea protein ID: 193545, 11/11 species at FC=2/4)

1699

1700

1701

1702

1703

1704

1705

1706

1707

1708

1709 contains $C$. neoformans Csa2. Members of this orthogroup showed strong induction in the first primordium stage in $C$. cinerea, $A$. ostoyae, $C$. aegerita, $L$. bicolor, $L$. edodes, $P$. ostreatus, S. commune, A. ampla, M. kentingensis. In $C$. neoformans it shows a strong peak $24 \mathrm{~h}$ after the induction of sexual development, which, combined with evidence for regulation by pum1, led Liu et al to conclude it is involved in basidium development and sporulation(Liu et al. 2018). It's an orthologue of Sch. pombe Mei2, which is related to meiosis. The other three basidium-related genes reported by Liu et al (Fad1, Fas1, CNA00260)(Liu et al. 2018) do not have clear orthologs in Coprinopsis.

The CDE orthogroup represented by $C$. cinerea 424800 (Table 12) was consistently upregulated in gills and sporulating stages (in A. ampla, S. commune) in all species, suggesting roles in hymenium and/or spore formation. In model Ascomycota, proteins in this orthogroup find Pab1 of both Sch. pombe and S. cerevisiae as their best Blast hits, which are related to mRNA export and the control of poly(A) tail length.

Finally, the CDE orthogroup comprising $C$. cinerea 368527 includes best reciprocal hosts of S. cerevisiae Nam8, a meiosis-specific splicing factor. Our expression data are consistent with a meiosis-specific role: the gene is developmentally regulated in 6/4 species (FC2/4, see above, Table 1) and shows a peak expression in meiotic tissues in all species (gills, cap+gills or mature fruiting bodies). Based on these data, we hypothesize that this gene also participates in meiosis-specific splicing events in the basidia during fruiting body formation.

\subsubsection{RNA interference, Small RNA}

1718 RNA interference is a conserved eukaryotic gene-silencing mechanism that acts via base pairing of small noncoding RNAs of $\sim 20-30$ nucleotides to DNA/RNA targets. RNAi is key to developmental and differentiation processes, genome defense against viruses or transposons. There is very limited knowledge on the role of RNAi in mushroom-forming fungi (but see(Lau et al. 2018)), whereas the processes are extensively studied in the Ascomycota (in particular N. crassa and Sch. pombe(Dang et al. 2011)), where RNAi has welldocumented roles in defense against viruses, transposons, meiotic silencing of unpaired DNA, translational repression and even inter-organismal pathogenic interactions(Weiberg et al. 2013).

A fully functional RNAi machinery exists in the Basidiomycota. Based on orthology to 1730 438955)(Lau et al. 2018), whereas Stajich et al annotated the argonaute family proteins 1731 AGO (C. cinerea protein ID: 355234), an AGO-like protein (C. cinerea protein ID: 473372) 1732 and Qde-2 (C. cinerea protein ID: 363313)(Stajich et al. 2010) (Table 13). We identified 3-16 1733 genes encoding argonaute and argonaute-like proteins in the examined Agaricomycetes. 
bioRxiv preprint doi: https://doi.org/10.1101/2021.12.09.471732; this version posted December 10,2021 . The copyright holder for this preprint (which was not certified by peer review) is the author/funder, who has granted bioRxiv a license to display the preprint in perpetuity. It is made available under aCC-BY-NC-ND 4.0 International license.

Running head: fruiting body genomics

1734 Furthermore, fungal RNA interference involves RNA-dependent RNA polymerases, which 1735 are required for generating double-stranded RNA or for amplifying sRNA signals(Dang et al. 1736 2011). The genomes of the examined Agaricomycetes contain 5-13 RNA-dependent RNA 1737 polymerases; of these, the orthogroup represented by $C$. cinerea 498671 is most similar to 1738 the RDP1 protein of $S$. pombe, which was shown to be integral to RNA 1739 interference(Carmichael et al. 2004). Other RNA-dependent RNA polymerases (Table 11) 1740 might participate in alternative RNAi pathways or signal amplification(S et al. 2012b).

1741 Interestingly, we found no clear orthologs of SAD-1, a meiotic silencing protein(Borkovich et 1742 al. 2004), in the examined genomes.

1743

1744

1745

1746

1747

1748

1749

1750

1751

1752

1753

1754

1755

1756

1757

1758

1759

1760

1761

1762

1763

1764

1765

1766

1767

1768

1769

RNAi components show some expression dynamics during fruiting body development. Genes encoding Dcl-2, Dcl-3 and AGO-like proteins were upregulated in $C$. cinerea primordia relative to vegetative mycelium(Lau et al. 2018). Similarly, AGO-like and Qde-2 were found developmentally regulated in C. cinerea by Krizsan et al(Krizsán et al. 2019), although Dcl-1-3 genes were not. All of the seven RNA-dependent RNA polymerases were developmentally regulated in the data of Krizsan et al. In our meta-analysis, the abovelisted components did not show significant developmental expression dynamics (Table 13). The only noteworthy orthogroup (represented by $C$. cinerea 502116), was developmentally regulated in 9/6 species ( $\mathrm{FC}>2 / 4)$ and contains Argonaute-like proteins that share highest similarity with $A$ nidulans RsdA and Sch. pombe Ago1, the first of which is involved in RNAsilencing(TM et al. 2008) whereas the second has a large suite of described functions, from RNA silencing to chromosome segregation and cell cycle arrest(Carmichael et al. 2004). We found no conserved expression pattern in this orthogroup across the examined species.

The most direct evidence for the role of microRNA-like RNAs (milRNA) in mushroom development was reported by Lau et al(Lau et al. 2018), who compared small RNA transcriptome profiles of vegetative mycelium and primordium stages and detected 22 milRNA in C. cinerea, several of which were differentially expressed between vegetative mycelia and primordia. These results point to the potential roles of milRNA in mushroom development, although more studies are needed to understand its roles.

RNA interference has been exploited as a tool of genetic manipulation in mushroomforming fungi, often to compensate for the lack of reliable reverse genetics tools in these organisms. Gene knockdown has been demonstrated in S. commune(de Jong et al. 2006), C. cinerea(Namekawa et al. 2005; MA et al. 2006; Heneghan et al. 2007), L. bicolor(M et al. 2009a), Ph. chrysosporium(Matityahu et al. 2008), P. ostreatus, A. bisporus(Costa Ana S.M.B.; Thomas 2009), Grifola frondosa(Sato et al. 2015) and L. edodes(Nakade et al. 2011), among others, demonstrating the utility of knockdown techniques in cases/species when gene knockout was not feasible (particularly in the pre-CRISPR times).

\subsubsection{Chromatin regulators}

1771

Chromatin remodeling is a fundamental and general mode of epigenetic gene expression

1772 regulation. Dynamic chromatin states are generated primarily via chromatin remodeling

1773

1774

1775

1776

1777 complexes and histone modifications in fungi, in response to environmental or internal signals. Importantly, chromatin remodeling often provides the basis for broad changes in gene expression, by modulating the accessibility of DNA for transcription, as opposed to sequence-specific transcription factors, which regulate only specific genes. Epigenetic changes often associate with developmental processes; these receive increasing attention in multiple lineages, including fungi. Accordingly, studies addressing the role of chromatin 1779 remodeling in mushroom development, although quite few at the moment, revealed 
bioRxiv preprint doi: https://doi.org/10.1101/2021.12.09.471732; this version posted December 10,2021 . The copyright holder for this preprint (which was not certified by peer review) is the author/funder, who has granted bioRxiv a license to display the preprint in perpetuity. It is made available under aCC-BY-NC-ND 4.0 International license.

Running head: fruiting body genomics

1780 fundamental roles of chromatin states in fruiting-related gene transcription. For example, 1781 deleting CC.snf5 (C. cinerea protein ID: 365798, see Table 14), a member of the SWI/SNF 1782 chromatin remodeling complex, leads to the failure to initiate fruiting body development in $C$. 1783 cinerea (in addition to defects in dikaryon formation)(Ando et al. 2013). In C. cinerea two 1784 further genes identified in mutant analyses, ich1(Muraguchi and Kamada 1998) and Cc. 1785 rmt1(Nakazawa et al. 2010), may be related to chromatin regulation. Recent ChIP-Seq 1786 analyses of epigenetic states revealed substantial changes in transcriptional activity (with H3K4me2 as a proxy) during the transition to fruiting(Vonk and Ohm 2021), whereas histone acetyltransferase complex members were found in quantitative trait loci in $L$. edodes(Zhang et al. 2021d). The presence of SET domain methyltransferases in the Basidiomycota is also worth mentioning(Freitag 2017). Although evidence at the moment is scanty, the few available studies and extrapolation from other organisms suggests that chromatin remodeling may be a significant factor in fruiting body formation. Therefore, in the following chapters we annotate and discuss expression patterns of genes related to chromatin maintenance and remodeling based on homology to known chromatin remodeling components in S. cerevisiae and N. crassa.

4.5.4.1. Histone gene expression correlates with fruiting body initiation and sporulation

Histones - Histones are highly conserved proteins that assemble into octameric nucleosomes and condense DNA into chromatin in a tightly regulated manner. Shuffling of histone variants can generate nucleosome variants, which, combined with diverse posttranslational modifications of histones (acetylation, methylation, phosphorylation) can generate a finely diversified histone code contributing to fine-tuned regulation of chromatin states, which, in turn, impact transcriptional patterns of genes(Borkovich et al. 2004). Histone modifications are often used to make readouts of transcriptional states associated with development(Vonk and Ohm 2021). Agaricomycetes have 14-40 histone and histonevariant encoding genes. They show a very similar overall expression pattern in all species that resembles the expression of meiotic/mitotic and ribosomal genes: upregulation in primordium stages as well as meiotic tissues (gills or caps+gills) coincident with sporulation (Fig. 10, Supplementary Fig. 7). Though overall very conserved, there are slight variations to this general pattern: $A$. ampla shows a peak only in primordia, whereas in $C$. cinerea and $L$. tigrinus a meiosis/sporulation associated peak is better visible, while the primordium-peak is modest. We detected four CDE orthogroups of histones and histone variants (Table 14). These include a basidiomycetes-specific histone H2A component (C. cinerea protein ID: $363625)$, which is developmentally expressed in $8 / 6$ species (FC2/4) and show considerable upregulation in primordia relative to vegetative mycelium in most species. Other CDE orthogroups also showed primordium- and gill- upregulation. A straightforward interpretation of the similar expression patterns of histones and cell proliferation related genes is a mutual link to DNA repair. H2A histone phosphorylation is associated with DNA repair(JA et al. 2000; Borkovich et al. 2004), providing a potential mechanistic explanation for the observed expression patterns. Somewhat contradicting this is the fact that relatives of the Mec1 kinase (C. cinerea protein ID: 19106), which phosphorylates H2A.Z in S. cerevisiae(JA et al. 2000), are not developmentally regulated in Agaricomycetes. On the other hand, histone upregulation in primordia and meiotic tissues may also be interpreted as evidence for intense transcriptional reprogramming, which has been hypothesized for the myceliumfruiting body transition(Krizsán et al. 2019; Vonk and Ohm 2021). Alternatively, intense DNA replication may require increased supply of histones in proliferating tissues. 
bioRxiv preprint doi: https://doi.org/10.1101/2021.12.09.471732; this version posted December 10,2021 . The copyright holder for this preprint (which was not certified by peer review) is the author/funder, who has granted bioRxiv a license to display the preprint in perpetuity. It is made available under aCC-BY-NC-ND 4.0 International license.

Running head: fruiting body genomics

1826

1827

1828

1829

1830

1831

1832

1833

1834

1835

1836

1837

1838

1839

1840

1841

1842

1843

1844

1845

1846

1847

1848

1849

1850

1851

1852

1853

1854

1855

1856

1857

1858

1859

1860

1861

1862

1863

1864

1865

1866

1867

1868

1869

1870

1871

Histone chaperone - We detected a histone chaperone CDE orthogroup containing homologs of $S$. cerevisiae Chz1 (represented by $C$. cinerea 498885)(Table 14), which forms a single-copy gene family in the Agaricomycetes. Members of this orthogroup are involved in histone replacement during transcription and showed the same expression pattern as histones (peaking in primordia and meiotic tissues), so we speculate they are involved in the same cellular processes during fruiting body initiation and sporulation.

We next inventoried histone acetyltransferases, histone deacetylases, histone methyltransferases, histone ubiquitylases, histone kinases and histone ADP-ribosylases based on homology to corresponding $N$. crassa genes described by Borkovich et al(Borkovich et al. 2004) as well as diagnostic conserved domains.

Histone acetyltransferase (HAT) - We found one HAT gene per species in the examined Agaricomycetes (represented by C. cinerea 420113), in contrast to Ascomycota, that have multiple histone acetyltransferase genes(Borkovich et al. 2004). This family showed no developmental expression dynamics in our species. However, we detected several protein $\mathrm{N}$-acetyltransferases, which resemble HATs, but lack orthology to known HATs and do not contain diagnostic conserved domains of HATs. They share an identical domain composition with Sch. pombe Ard1, a Gcn5-related N-acetyltransferase (GNAT), which acetylates diverse proteins, including transcriptional regulators. A CDE orthogroup of GNATs (C. cinerea protein ID: 457726) was developmentally expressed in 8/4 species (FC2/4, Table 14), however, given the lack of clear histone link we only tentatively discuss this family here, noting that functional analysis is needed to confirm their functional annotation.

Histone deacetylases (HDACs) - we distinguished two groups of HDACs, one related to $S$. cerevisiae Hda1 and the Sirtuin family, which were detected in 6-7 (11 in $A$. ostoyae) and 4-9 copies in the examined Agaricomycetes, respectively. Neither groups showed considerable developmental expression dynamics, except for one CDE orthogroup comprising sirtuins (represented by $C$. cinerea 455597), which was developmentally expressed in 9/9 species (FC2/4, Table 14). This family is orthologous to Hst4 of Sch. pombe and Hst3 of $S$. cerevisiae, which regulate DNA damage response and repair by regulating histone $\mathrm{H} 3 \mathrm{~K} 56$ acetylation(Konada et al. 2018). Consistent with their presumed role, the expression of this gene family followed closely that of mitosis/meiosis and DNA repair related genes in all species: they showed a small expression peak in primordia and a larger one in meiotic tissues coinciding with sporulation (Fig. 10).

Histone ADP-ribosylases are poly(ADP-ribose) polymerases (PARPs) and are among the least understood factors in epigenetic regulation; they attach (poly-)ADP-ribose moieties to proteins, including histones and are thought to regulate histone turnover and DNA damage response. In $N$. crassa the family is represented by a single gene, parp(Borkovich et al. 2004). The examined Agaricomycetes' genomes harbor 3-9 copies of PARPs, of which two formed CDE orthogroups (C. cinerea protein ID: 459336, 384835), which were developmentally expressed in $8 / 4$ and $6 / 5$ species (FC2/4, Table 14), respectively. Their expression patterns did not follow that of histones, suggesting that their actions are independent of those.

Histone methyltransferases and histone kinases showed no noticeable expression dynamics in fruiting bodies (Table 14), suggesting that they are equally important in fruiting bodies and vegetative mycelia. 
bioRxiv preprint doi: https://doi.org/10.1101/2021.12.09.471732; this version posted December 10,2021 . The copyright holder for this preprint (which was not certified by peer review) is the author/funder, who has granted bioRxiv a license to display the preprint in perpetuity. It is made available under aCC-BY-NC-ND 4.0 International license.

Running head: fruiting body genomics

1872

1873

1874

1875

1876

1877

1878

1879

1880

1881

1882

1883

1884

1885

1886

1887

1888

1889

1890

1891

1892

1893

1894

1895

1896

1897

1898

1899

1900

1901

1902

1903

1904

1905

1906

1907

1908

1909

1910

1911

1912

1913

1914

1915

1916

1917

\subsubsection{Other chromatin related genes}

Ich1 gene family - The only methyltransferase that might be connected to chromatin regulation was the gene underlying the 'Ichijiku' mutant, which was first identified by Muraguchi and Kamada(Muraguchi and Kamada 1998), as a mutant that produced malformed, barrel-shaped primordia, in which cap development fails. The corresponding ich1 gene encodes a nuclear methyltransferase that contains a winged-helix DNA binding domain and was reported to be upregulated in primordia(Muraguchi et al. 2015). Our results agree and extend these observations: Ich1 orthologs form a CDE orthogroup (represented by $C$. cinerea 545797) that is developmentally expressed in 8/6 species (FC2/4)(Table 14) and shows a marked upregulation in primordia relative to vegetative mycelium in $C$. cinerea, $A$. ostoyae (weakly), A. ampla, S. commune, A. bisporus, M. kentingensis and $P$. ostreatus. The exact mechanism of how lch1 regulates tissue formation is not known, however, this gene represents a very interesting target for detailed studies, especially given its putative role in chromatin regulation and/or methylation. On a related note, Cc.rmt1, an arginine methyltransferase which was suggested to participate in histone methylation and to be required for hyphal knot and fruiting body formation(Nakazawa et al. 2010), forms an orthogroup which is developmentally regulated in nine species (at FC>2) (Table 14). The exact role of Cc.rmt1 is not known so its role in histone methylation remains preliminary.

Histone isomerases - We found a CDE orthogroup that was widely developmentally expressed (represented by C. cinerea 493226)(Table 14). Ascomycota orthologs of these genes are histone proline isomerases in the peptidyl-prolyl cis-trans isomerase family, which contribute to nucleosome assembly and thus regulate gene expression. This orthogroup shows upregulation in gills of $C$. cinerea, $A$. ostoyae and $P$. ostreatus (all species in which gills were sampled separate) and in cap tissues of $L$. edodes, M. kentingensis, L. tigrinus. These expression patterns are consistent with a role in sporulation. Genes in this orthogroup also show upregulation in primordia relative to vegetative mycelia, although this is usually modest (2-3x, 10x in $P$. ostreatus).

HMGA and AT-hook protein - We detected a CDE orthogroup (C. cinerea protein ID: 473747)(Table 14) that includes primarily stipe-upregulated genes (in $C$. cinerea, $A$. ostoyae, $L$. tigrinus, $P$. ostreatus, $M$. kentingensis, but not $L$. bicolor) that shows expression peaks also in YFB cap of $C$. cinerea and FB cap of $P$. ostreatus. We found no expression dynamics in Schizophyllaceae species, which lack stipes. The orthogroup belongs to the High mobility group A family, which comprises AT-rich DNA binding proteins with an important role in chromatin organization. This family appears to be patchily distributed in the examined species, but nevertheless seems to be important for fruiting body development.

SRA-YDG domain proteins - We detected a CDE orthogroup ( $C$. cinerea protein ID: 511209)(Table 14) of proteins containing an SRA-YDG (IPR003105) domain, which occurs in histone and chromatin binding proteins and may be associated with the cell cycle. Proteins with this domain seem to be missing in Ascomycota model species (results not shown), but show expression patterns similar to those of histones. They are upregulated in primordia and in meiotic stages (gills, caps with gills) in C. cinerea, A. ostoyae, P. ostreatus and $L$. edodes. In the Schizophyllaceae as well as $P$. ostreatus, the gene family is upregulated in primordia relative to vegetative mycelium samples. Based on their expression patterns, we hypothesize they are related to cell proliferation, the cell cycle or chromatin modification, but available data do not allow us to further narrow down their putative function among these categories. 
bioRxiv preprint doi: https://doi.org/10.1101/2021.12.09.471732; this version posted December 10,2021 . The copyright holder for this preprint (which was not certified by peer review) is the author/funder, who has granted bioRxiv a license to display the preprint in perpetuity. It is made available under aCC-BY-NC-ND 4.0 International license.

Running head: fruiting body genomics

1918

1919

1920

1921

1922

1923

1924

1925

1926

1927

1928

1929

1930

1931

1932

1933

1934

1935

1936

1937

1938

1939

1940

1941

1942

1943

1944

1945

1946

1947

1948

1949

1950

1951

1952

1953

1954

1955

1956

1957

1958

1959

1960

1961

1962

1963

\subsection{Alternative splicing and spliceosome components}

Alternative splicing is a post-transcriptional mechanism for regulating transcript identity and abundances. mRNA variants generated by splicing can increase the diversity of proteins transcribed from a single gene, but can also destine transcripts to the nonsense-mediated decay pathway and thus reduce transcript abundance posttranscriptionally(Krizsán et al. 2019). Transcriptomes allow us to describe alternative splicing complexity of organisms at an unprecedented depth. Several studies looked at alternative splicing in fungi(Zhao et al. 2013; Grützmann et al. 2014; Jin et al. 2017), including Agaricomycetes model systems(Lugones et al. 1999a; Gehrmann et al. 2016; Krizsán et al. 2019). The Basidiomycota and, in particular, the Agaricomycetes displayed a higher percentage of spliced genes than did other groups of fungi(Grützmann et al. 2014; Krizsán et al. 2019). Results of these studies highlighted that, of the several possible splicing events, intron retention $(\mathrm{IR})$ is the most common in fungi while exon-skipping, which most amply contributes to generating protein isoforms, accounts for only $0.5-2.5 \%$ of fungal splicing events. Most research on splicing focuses on the latter, however, it appears that exon skipping-rich transcriptomes represent an animal innovation(Grau-Bové et al. 2018). IR events have been considered either as transcriptional noise or as a regulatory mechanism(Middleton et al. 2017). IR can introduce premature stop codons in transcripts or new functional elements for RNA binding proteins. Premature stop codons can activate nonsense-mediated decay, a surveillance mechanism that results in the degradation of the mRNA transcript. Recent results suggest that IR may not only regulate transcript levels, but can be a key driver of posttrancriptional processes(Middleton et al. 2017). There is evidence that in the Basidiomycota IR exerts its effect on transcripts independent of the nonsensemediated decay pathway(Gonzalez-Hilarion et al. 2016).

Functionally characterized examples of developmentally relevant splicing events are known in the Ascomycota. For example, alternative splicing of the blue light receptor wc-1 gene was observed in Phaeosphaeria spp. and it was hypothesized that AS may control various light-dependent reactions(Chiu et al. 2010). In Neurospora wc-1 transcription is controlled by multiple distinct cis-regulatory regions (termed different promoters), which responded differently (not, weakly or strongly) to light and resulted in alternative protein isoforms(Káldi et al. 2006). These observations, although probably just scratching the surface, implicate AS as an important contributor to the development of fruiting bodies.

Alternative transcript isoforms affect a considerable portion of genes during fruiting body development. IR-generated transcript isoforms with a dynamic expression are abundant in fruiting bodies(Gehrmann et al. 2016; Krizsán et al. 2019), affecting 20-46\% of expressed genes, pointing towards potential important regulatory roles. In S. commune, 69\% of the alternative spliced transcripts had altered predicted functional properties or subcellular localization(Gehrmann et al. 2016). Gehrmann et al reported the largest number of splicing events at the fruiting body initiation stage in S. commune, whereas Krizsan et al did not find substantial differences in the number of splicing events across developmental stages.

Splicing can influence transcript levels significantly during fruiting body development. A recent study found 425 genes in $S$. commune for which an alternative transcript replaced the highest expressed transcript in some stage of development. Many such events happened at fruiting body initiation (referred to as the aggregate stage), pinpointing the magnitude of transcriptome reprogramming at this developmental transition. A conceptually similar scenario, genes whose expression do not vary considerably during fruiting body 
bioRxiv preprint doi: https://doi.org/10.1101/2021.12.09.471732; this version posted December 10,2021 . The copyright holder for this preprint (which was not certified by peer review) is the author/funder, who has granted bioRxiv a license to display the preprint in perpetuity. It is made available under aCC-BY-NC-ND 4.0 International license.

Running head: fruiting body genomics

1964 development, but have splicing isoforms that vary in their expression significantly, was 1965 detected for $\sim 160-1300$ genes in six Agaricomycetes (Krizsán et al. 2019).

1966

1967

1968

1969

1970

1971

1972

1973

1974

1975

1976

1977

1978

1979

Taken together, recent studies on Agaricomycetes suggest that splicing, in particular intron retention, is an important process in fruiting body development, contributing to posttranscriptional regulation of transcript levels or generates functional diversity in proteins translated from alternative transcripts. The prevalence of IR in fungal transcriptomes suggests that the main role of splicing in fungi might be posttranscriptional transcript level regulation rather than the expansion of the proteome space, although mechanistic or proteome-level studies are missing at the moment.

We here annotated spliceosome components and splicing-associated proteins in $C$. cinerea based on knowledge in S. cerevisiae and Sch. pombe(Käufer and Potashkin 2000; JM et al. 2012). We annotated 50 orthogroups associated with splicing, however, none of them showed remarkable expression dynamics in fruiting bodies (Supplementary Table 3). These included the gene described as Le.cdc5 and Cc.cdc5 in L. edodes and C. cinerea, respectively(Miyazaki et al. 2004), a pre-mRNA splicing factor.

\subsection{Signal transduction systems and communication}

1981 One of the most important multicellular processes.

\subsubsection{Oxylipins}

1983 In fungi, oxylipins are responsible for a range of signaling functionalities and act as paracrine hormone-like substances or as so-called 'infochemicals'(Combet et al. 2006; Holighaus and Rohlfs 2019). Ascomycota species produce diverse oxylipin compounds (reviewed in(Gessler et al. 2017)) and changes in their levels influence development. For example, oxylipins can regulate the yeast-to-hypha transition in dimorphic fungi, as well as immune evasion(Rennemeier et al. 2011) and communication with the host in pathogens, while a well-known group of oxylipins called psi (precocious sexual inducer) factors regulates sexual and asexual development in Ascomycota(Tsitsigiannis and Keller 2007). Basidiomycota oxylipins are less known. The best-known oxylipin of mushroom-forming fungi is 1-octen-3ol, an enantiomeric compound that is mainly produced as $(\mathrm{R})-(-)-1$-octen-3-ol by widelyknown species(Zawirska-Wojtasiak 2004). On the one hand, it acts as an interkingdomsignaling molecule. It may attract or repel fungivorous arthropods(Fäldt et al. 1999; Holighaus and Rohlfs 2019) or derogate insect larval development. In combination with the equally prominent octan-3-one that may attract specialist fungivores, it has also been shown to attract predators of such fungivores(Fäldt et al. 1999). Furthermore, 1-octen-3-ol has been shown to retard plant seed germination and vegetative growth(Hung et al. 2014). On the other hand, 1-octen-3-ol shows a quorum-sensing like action as it inhibits colony growth(Yin et al. 2015; Kües et al. 2018), conidiation(Fäldt et al. 1999; Yin et al. 2015), fruiting initiation(Noble et al. 2009; Eastwood et al. 2013) and germination where spores are crowded(Chitarra et al. 2004; Combet et al. 2006).

The composition of the oxylipin cocktail emitted by intact mushroom tissue changes in the course of mushroom development while measurement of the most abundant oxylipins during that process crucially depends on the sampling method: disruption of fungal tissue has been shown to trigger the release of oxylipins that are not emitted when fungal material is intact(Fäldt et al. 1999). The changing specific oxylipin bouquet emitted by a developing mushroom may not solely reflect adaptation to insect attraction for spore dispersal and 
bioRxiv preprint doi: https://doi.org/10.1101/2021.12.09.471732; this version posted December $10,2021$. The copyright holder for this preprint (which was not certified by peer review) is the author/funder, who has granted bioRxiv a license to display the preprint in perpetuity. It is made available under aCC-BY-NC-ND 4.0 International license.

Running head: fruiting body genomics

2009 repellence of fungivores. It is also possible that, e.g. octan-3-one and 1-octen-3-ol are 2010 produced by the fruiting body to inhibit on-colony spore germination(Combet et al. 2006) or 2011 colonization by mycoparasites(Berendsen et al. 2013). During fruiting body development, 2012 octan-3-one emission either positively(Combet et al. 2009) or negatively(Zhang et al. 2008; 2013 A et al. 2020; Orban et al. 2021) correlates with the amount of 1-octen-3-ol produced by (not 2014 yet) sporulating tissue samples of certain species.

2015 The biosynthesis pathway of 1-octen-3-ol has interested mycologists for a long 2016 time(Combet et al. 2006; Kües et al. 2018; Orban et al. 2021), but remained unresolved to 2017 date. The process starts by oxygenation of polyunsaturated fatty acids (PUFAs) with linoleic 2018 acid as the predominant PUFA in Asco- and Basidiomycota(Combet et al. 2006; Brodhun 2019 and Feussner 2011). It was suggested that lipoxygenases (LOXs), linoleate diol synthases 2020 (LDSs that combine a dioxygenase - DOX - and an isomerase activity), hydroperoxide 2021 lyases (HPLs) and alcohol dehydrogenases (ADHs) participate in 1-octen-3-ol 2022 biosynthesis(Combet et al. 2006). Still, it is possible that 1-octen-3-ol can be produced by 2023 incomplete beta-oxidation or by multiple pathways(Combet et al. 2006; Brodhun and 2024 Feussner 2011). Orban et al. recently attempted to identify genes involved in oxylipin 2025 production based on correlations between 1-octen-3-ol concentrations and gene expression 2026 patterns(Orban et al. 2021) including a gene of an ene-reductase for octan-3-one formation 2027 from ADH-mediated conversion of 1-octen-3-ol.

2028

2029

2030

2031

2032

2033

2034

2035

2036

2037

2038

2039

2040

2041

2042

2043

2044

2045

2046

2047

2048

2049

2050

2051

2052

2053

2054

2055

2056

Two main enzyme classes have been implicated in fungal oxylipin production, LOXs and LDSs(Combet et al. 2006; Orban et al. 2021). Both act on linoleic acid as the starting point, from which various compounds are produced by first an oxidation reaction, then by further modification(Combet et al. 2006). We found LOXs to be highly patchy in Agaricomycetes, whereas LDSs (basically the Ppo family of proteins) are widely conserved. Incomplete beta-oxidation has also been suggested as a possible source of oxylipins(Venter et al. 1997; Brodhun and Feussner 2011), this hypothesis is compatible with the lack of certain enzymes in many species.

Lipoxygenases (LOXs) - Out of the examined species, we only found lipoxygenases in $C$. aegerita, $P$. ostreatus, $L$. bicolor and $L$. edodes, which have 1-5 copies. These did not form conserved orthogroups and their expression patterns mostly did not vary substantially during development. Exceptions include the gene previously described as PoLOX1(Plagemann et al. 2013; Y et al. 2013) (PleosPC15_2_1068582). PoLOX1 displays a remarkable upregulation (1880 fold, FPKM 94.000) in fruiting bodies relative to vegetative mycelium. The gene showed consistently higher expression in stipes compared to caps, which confirms the previous report on the gene(Y et al. 2013). A lipoxygenase gene (CaeLOX4, gene ID AAE3_04864/Genbank accession no. CAA7262624) of C. aegerita also showed a strong upregulation ( 150-fold) during late development (post sporulation stage) in this species, as noted by Orban et al (Orban et al. 2021). Notably, two other LOXs show strong phase-specific expression increase in fruiting stage tissue: on the one hand, CaeLOX5 (AAE3_07753) shows an expression pattern quite congruent with the maturationassociated increase of the 1-octen-3-ol levels in the headspace of the $C$. aegerita culture. On the other hand, CaeLOX1 (AAE3_00896) shows transcriptional upregulation before fruiting body maturation sets in. Such patterns may imply stage-specific action or (partial) functional redundancy among these genes that must, prospectively, be tested by functional characterization studies.

Linoleate diol synthases (LDSs) - The other well-known gene family involved in the production of oxylipins is the one of linoleate diol synthases, which makes up to PpoA-C family in A. nidulans. The main products of the Ppo family are the so-called psi factors, 
bioRxiv preprint doi: https://doi.org/10.1101/2021.12.09.471732; this version posted December 10,2021 . The copyright holder for this preprint (which was not certified by peer review) is the author/funder, who has granted bioRxiv a license to display the preprint in perpetuity. It is made available under aCC-BY-NC-ND 4.0 International license.

Running head: fruiting body genomics

2057 which regulate diverse developmental processes from yeast-to-hypha transition and cell 2058 aggregation (reviewed in ref(Tsitsigiannis and Keller 2007)). We identified 1-4 LDS genes in 2059 Agaricomycetes with most species having two copies. Although several genes have 2060 reciprocal best hits among Ascomycota (e.g. C. cinerea 423716 to PpoC of $A$. nidulans), a 2061 gene tree analysis showed that these are not true orthologs (results not shown) but likely 2062 resulted from a duplication event that predated the split of Asco- and Basidiomycota.

2063 Therefore, it is likely that Basidiomycota linoleate diol synthases do not have the psi factor 2064 producing activity that $A$. nidulans PpoA-C genes and their homologs have(Tsitsigiannis and 2065 Keller 2007).

2066

2067

Linoleate diol synthases grouped into three conserved orthogroups (Table 15), of which two (C. cinerea protein ID: 398037,423716$)$ were developmentally expressed in a considerable number of species. One of these (C. cinerea protein ID: 423716 ) includes $U$. maydis Ssp1 (Ustma2_2_11710), which was found to be specifically expressed in teliospores(Huber et al. 2002). Genes within these orthogroups seem to show diverse expression patterns, without noticeable conservation of expression across species. In the absence of functional information of these genes in Basidiomycota, it is impossible to speculate on their mechanistic role in fruiting body formation. They are likely to be involved in oxylipin production(Orban et al. 2021), but further studies are necessary to elucidate their functions.

Alcohol dehydrogenases - the alcohol dehydrogenase family participates in a broad range of cellular processes, including oxylipin biosynthesis(Orban et al. 2021). We identified a CDE orthogroup containing $C$. aegerita Adh1-1 (represented by $C$. cinerea 363127), which showed partial conservation of expression: in most species it was upregulated during late development (C. aegerita, $A$. ostoyae, L. edodes, L. tigrinus, $C$. cinerea). In addition, in $S$. commune, Ph. chrysosporium, $A$. ostoyae and $L$. edodes it was also upregulated $>4 \mathrm{x}$ in primordia relative to vegetative mycelium. In $M$. kentingensis and $A$. ampla the gene was not developmentally regulated, whereas $L$. bicolor and $P$. ostreatus did not have orthologs. Based on a previous report of $C$. aegerita Adh1-1(Orban et al. 2021), it is possible that this orthogroup of alcohol dehydrogenases participate in oxylipin production.

\subsubsection{Pheromone and pheromone receptor pathways}

2088 The structure and evolution of mating systems, including HD transcription factors (A locus), 2089 pheromone receptors and pheromone precursor genes (B locus) is among the best known 2090 aspects of Agaricomycete biology (e.g.(Kües 2000, 2015; James 2015) refs). The primary 2091 role of pheromone/receptor systems is regulating dikaryotization, i.e. the compatibility of 2092 monokaryons, nuclear movement and other downstream events. Hyphal fusion is believed to occur ubiquitously in the Basidiomycota, therefore, pheromone/receptor systems may regulate post-fusion behavior(Raudaskoski and Kothe 2010; Jones and Bennett 2011). However, pheromone/receptor systems are involved in the regulation of sexual events too. For example, mating type loci are involved in fruiting body formation in $C$. cinerea(Kues et al. 1998, 2002). Basidiomycete mating systems, pheromone and pheromone receptor genes have been reviewed recently in detail (e.g.(Raudaskoski and Kothe 2010; Jones and Bennett 2011; James 2015; Kuees 2015)). We here annotate components of the Agaricomycete 2100 mating systems, with special emphasis on copy number variation across the twelve species 2101 examined in this paper and expression dynamics during fruiting body development. 
bioRxiv preprint doi: https://doi.org/10.1101/2021.12.09.471732; this version posted December 10,2021 . The copyright holder for this preprint (which was not certified by peer review) is the author/funder, who has granted bioRxiv a license to display the preprint in perpetuity. It is made available under aCC-BY-NC-ND 4.0 International license.

Running head: fruiting body genomics

2102 Pheromone precursor genes - Basidiomycetes produce mating pheromones that 2103 are structurally analogous to S. cerevisiae a-factor(Jones and Bennett 2011). Mating 2104 pheromones are short peptides that undergo a multi-step maturation process and regulate 2105 intercellular communication and sexual development in a wide range of fungi(Jones and 2106 Bennett 2011). Copy numbers of pheromone precursor genes vary across the

2107 Agaricomycetes and are often found in the vicinity of corresponding pheromone

2108 GPCRs(Raudaskoski and Kothe 2010). Four pheromone precursor genes were found in $L$. 2109 edodes(Wu et al. 2013), P. eryngii(Kim et al. 2014), A. bisporus(Foulongne-Oriol et al. 2021), Moniliopthora roreri(Díaz-Valderrama and Aime 2016), five in Ph. chrysosporium(James et al. 2011), seven in Pycnoporus cinnabarinus(Levasseur et al. 2014), two in P. touliensis(Gao et al. 2018) and several genes (up to 20) in $C$.

2114 cinerea(Riquelme et al. 2005) and S. commune(Specht 1995). Synthetic pheromone administration affected PriA (a primordium-expressed gene) expression in L. edodes, indicating the involvement of mating pheromones in fruiting body formation(Ha et al. 2018). One of the pheromone precursor genes was expressed in glucose-limited conditions following blue light induction of fruiting in $C$. cinerea(Sakamoto et al. 2018).

In our dataset, we found up to nine pheromone-precursor encoding genes, however, we did not find any in A. ostoyae, A. bisporus, M. kentingensis, Ph. chrysosporium and $L$. tigrinus. The explanation for this may be that they are simply missing from the genome annotations. Pheromone precursors are short $\sim 50$ amino acid peptides and several genome annotation protocols omit predicted proteins that are $<50$ or $<100$ amino acids long. For this same reason, it is likely that our inventories of pheromone-precursor genes in other examined species is incomplete. Nevertheless, we found pheromone precursors in $C$. cinerea, A. ampla, L. bicolor, L. edodes, S. commune and P. ostreatus; these genes were often developmentally regulated, implying that they may participate in developmental processes, possibly as signal molecules. While knowledge on the role of pheromones in mating and dikaryon formation is abundant, whether and how they participate in fruiting body development requires further research.

Pheromone modification - pheromone precursor proteins are post-translationally modified during their maturation process. This involves prenylated, carboxymethylation as well as proteolytic cleavage to remove $\mathrm{N}$-terminal sequence fragments(Jones and Bennett 2011).

Post-translational prenylation (mostly farnesylation) on CAAX motifs is performed by RAM proteins in S. cerevisiae(Caldwell et al. 1995). Based on orthology with S. cerevisiae pheromone prenylating enzymes, we identified two genes as putatively involved in pheromone prenylation. The first is orthologous to $S$. cerevisiae RAM1 (C. cinerea protein ID: 491483) and shows very modest expression dynamics (Table 16), whereas the second is an ortholog of RAM2 (C. cinerea protein ID: 473005), encoding a general prenylation enzyme that also modifies other peptides, including proteins related to cell wall glucan biosynthesis. Following prenylation, the S. cerevisiae a-factor is cleaved by Rce1 (ortholog: C. cinerea 377901) and Ste24 (ortholog: C. cinerea 419650). Orthologs of these genes showed constant expression during fruiting body development (Table 16).

On the other hand, we found evidence for the developmental expression of carboxymethylating enzymes. Two CDE orthogroups of protein-S-isoprenylcysteine Omethyltransferases (homologous to S. cerevisiae Ste14) were detected among conserved developmentally expressed genes. Ste14 mediates methylation of the isoprenylated Cterminal CAAX motif in the mating pheromone a-factor(Romano and Michaelis 2001). Whether these homologs are related to mating pheromone maturation in mushroom-forming 
bioRxiv preprint doi: https://doi.org/10.1101/2021.12.09.471732; this version posted December 10,2021 . The copyright holder for this preprint (which was not certified by peer review) is the author/funder, who has granted bioRxiv a license to display the preprint in perpetuity. It is made available under aCC-BY-NC-ND 4.0 International license.

Running head: fruiting body genomics

2150 fungi remains unclear, therefore, we only tentatively classified these as signal transduction2151 related genes. Two Ste14 homologs of $C$. cinerea were previously found to be expressed 2152 after blue light exposure(Sakamoto et al. 2018). We detected two CDE orthogroups of Ste14 2153 homologs (Table 16). One of the orthogroups (represented by C. cinerea 152667) is present 2154 only in five species and upregulated in primordia of $C$. cinerea, $A$. ostoyae, A. bisporus and

$2155 P$. ostreatus but interestingly shows the reverse pattern, high in vegetative mycelium and no 2156 expression in any fruiting body stage in $M$. kentingensis. The other orthogroup (represented 2157 by $C$. cinerea 354499) was upregulated in primordia of $C$. cinerea, $A$. ostoyae, Pt. gracilis

2158 and $S$. commune (but not in $A$. ampla and $P$. ostreatus). More broadly, members of this gene 2159 family were developmentally regulated in several species, although they were not conserved 2160 across species. On the other hand, 1-to-1 orthologs of S. cerevisiae Ste14 (represented by 2161 C. cinerea 153902), show constant expression in all species.

2162 As a final step of a-factor maturation, Ste24 and Axl1/Ste23 proteolytically cleave the 2163 N-terminus in two steps(Jones and Bennett 2011). The corresponding Agaricomycete 2164 orthologs did not show developmentally dynamics expression (C. cinerea protein ID: 419650 2165 and 358206, respectively)(Table 16).

2166 Pheromone sensing GPCRs - Seven-transmembrane domain G-protein coupled 2167 receptors have been studied in multiple Agaricomycetes(Kües 2000; Raudaskoski and Kothe 2168 2010; Jones and Bennett 2011; Wirth et al. 2021). Pheromone-sensing GPCRs are located 2169 in the B mating type locus in Basidiomycota, together with pheromone precursor genes. 2170 Pheromone sensing GPCRs transduce signals intracellularly to a mitogen-activated protein 2171 kinase (MAPK) cascade (see next chapter). Homology relationships and copy numbers of 2172 Basidiomycete pheromone-sensing GPCRs vary significantly across species, but all 2173 Basidiomycete pheromone GPCRs are homologous to yeast Ste3, which is involved in 2174 mating (reviewed recently(Raudaskoski and Kothe 2010; Fraser et al. 2014)). For example, 2175 four pheromone-like GPCR genes have recently been annotated in S. commune, of which 2176 one was suggested to participate in mating, whereas the others were proposed to have a 2177 role in directing growth in vegetative mycelia(Wirth et al. 2021).

2178 We identified 4-19 pheromone GPCR-encoding genes in the examined species. 2179 These genes in general showed low expression dynamics in fruiting bodies (Table 16), 2180 although several genes were upregulated slightly $(F C<4)$ in stipes in certain species.

A-factor export proteins - We identified a CDE orthogroup (represented by $C$. cinerea 540181)(Table 16) of plasma membrane ATPases which are othologous to $S$. cerevisiae Ste6, the protein that exports farnesylated a-factor from the cell. This orthogroup showed partial conservation of expression across the examined species. In A. ostoyae, $L$. bicolor, L. edodes and $M$. kentingensis it was upregulated in stipe tissues relative to caps, whereas in $C$. cinerea, $S$. commune, $A$. ampla (FC>4) and $C$. aegerita (though only at $\mathrm{FC}>2$ ) the gene was upregulated in primordia relative to vegetative mycelium.

2190 MAP kinase pathways - The mitogen-activated protein kinase (MAPK) cascades are evolutionary conserved intracellular signal transduction pathways, which regulate important

\subsubsection{MAPK, TOR and cAMP pathways} cellular processes(Román et al. 2007). The extracellular signal is sensed by membrane proteins or receptors, which is relayed by sequential phosphorylation and activation of three kinases (MAPKKK, MAPKK, MAPK) to transcription factors that induce or repress specific target genes. MAPK cascades usually consist of further components, like various adaptors, 
bioRxiv preprint doi: https://doi.org/10.1101/2021.12.09.471732; this version posted December $10,2021$. The copyright holder for this preprint (which was not certified by peer review) is the author/funder, who has granted bioRxiv a license to display the preprint in perpetuity. It is made available under aCC-BY-NC-ND 4.0 International license.

Running head: fruiting body genomics

2196 docking and scaffold proteins which are implicated in the spatial and temporal regulation of 2197 MAP kinase signaling(Frawley and Bayram 2020).

$2198 \quad$ While in Ascomycota MAPK members have been extensively examined

2199 (e.g.(Teichert et al. 2014)), relatively little information is available on the expression and role 2200 of MAPK pathways (except the pheromone, see above) in Agaricomycetes. In Pleurotus

2201 tuoliensis pheromone-dependent and starvation-dependent MAPK signaling pathway

2202 members were reported to be up-regulated during fruiting(Fu et al. 2017). Le.MAPK, a MAP

2203 kinase of $L$. edodes (Lenedo1_1058304) was reported to be developmentally regulated and

2204 one of its interacting partners was characterized(Szeto et al. 2007). In this paper, we

2205 annotated Agaricomycetes MAPK signaling pathways based on the four main MAPK

2206 pathways known in S. cerevisiae: the peptide pheromone pathway (Fus3), the starvation

2207 responding pathway (Kss1), the high osmolarity glycerol stress pathway (Hog1) and the cell

2208 wall integrity (Slt2/Mpk1) pathway (Table 17). We found relatively few clear orthologs in $C$.

2209 cinerea (Table 17). We performed transitive ortholog and domain-based search to find

2210 further putative S. cerevisiae orthologs in C. cinerea. MAPK pathway components do not

2211 show dynamic expression patterns during fruiting body development.

2212 TOR pathway - The target of rapamycin (TOR) is an evolutionary conserved

2213 serine/threonine kinase that is a key regulator in multiple cellular events(Tatebe and

2214 Shiozaki 2017). In fungi, the TOR pathway plays role in the response to nutrients (especially

2215 sugar and nitrogen) but also involved in stress responses, such as osmotic and oxidative

2216 stresses. TOR signaling pathways are well-studied in yeasts, but data about their functional

2217 analysis in multicellular agaricomycetes is rare. In G. lucidum TOR signaling was reported to

2218 play a role in the regulation of chitin and $\beta-1,3$-glucan synthesis and hence of cell wall

2219 thickness in an SLT2-MAPK dependent manner(Chen et al. 2019). Orthologs of $P h$.

2220 chrysosporium were previously annotated(Nguyen et al. 2020). According to a 5'-SAGE

2221 study of $C$. cinerea, TOR pathway members are constantly expressed during the fruiting

2222 body formation, except Rheb (C. cinerea protein ID: 493094) a Ras-like small GTPase,

2223 which is able to prevent FKBP12 inhibition of TOR(Cheng et al. 2013a).

2224

2225

2226

2227

2228

2229

2230

2231

2232

2233

2234

2235

2236

2237

2238

2239

2240

2241

2242

2243

Here we annotated TOR pathway components in mushroom-forming fungi and could find orthologs for all but three yeast genes reported to participate in the pathway (Table 17). Consistent with previous reports, TOR pathway components showed modest expression dynamics during fruiting body development. Nevertheless, based on the broad role of the pathway in fungal physiology it will be interesting to investigate its connection with fruiting body morphogenesis in the future.

cAMP pathway - Adenylyl cyclase dependent pathway (cAMP-pathway) has diverse roles in fungi, including cell growth, metabolism, stress resistance and nutrient-sensing (especially glucose) or starvation in fungi(D'Souza and Heitman 2001). Accordingly, the cAMP pathway has been intensely researched in mushroom-forming fungi(D'Souza and Heitman 2001; Tamaki 2007). Genetic manipulation of several cAMP and connected pathway components in $S$. commune resulted in altered fruiting characteristics, either repression or complete lack of fruiting body formation(Yamagishi et al. 2002, 2004; Knabe et al. 2013; Pelkmans 2016). The cAMP pathway has also been linked to morphogenesis in F. velutipes: the deletion of a Gß-like protein CPC-2 completely impaired fruiting body formation, but the addition of cAMP or it analog 8-Bromo-cAMP (also a PKA-activator) into the medium restored fruiting in Fv.cpc2 knockdown mutants(Wu et al. 2020b). Ras and G-protein $\square$ subunits and their regulators (e.g. Thn1) might also be connected to the cAMP pathway(Yamagishi et al. 2002; Palmer and Horton 2006; Knabe et al. 2013) and were repeatedly reported to affect morphogenesis of fruiting bodies (e.g.(Schubert et al. 2006)). 
bioRxiv preprint doi: https://doi.org/10.1101/2021.12.09.471732; this version posted December 10,2021 . The copyright holder for this preprint (which was not certified by peer review) is the author/funder, who has granted bioRxiv a license to display the preprint in perpetuity. It is made available under aCC-BY-NC-ND 4.0 International license.

Running head: fruiting body genomics

2244

2245

2246

2247

2248

2249

2250

2251

2252

2253

2254

2255

2256

2257

2258

2259

2260

2261

2262

2263

2264

2265

2266

2267

2268

2269

2270

2271

2272

2273

2274

2275

2276

2277

2278

2279

2280

2281

2282

2283

2284

2285

2286

2287

2288

2289

In general, cAMP is a known inducer of fruiting in several basidiomycetes, such as $C$. macrorhizus(Uno and Ishikawa 1973), C. cinerea(Swamy et al. 1985), S. commune(Schwalb 1974) and Ph. chrysosporium(Gold and Cheng 1979). In the latter, cAMP was speculated to induce fruiting by overriding the repressive effect of glucose (i.e. mimicked starvation in the fungus(Gold and Cheng 1979)). More recent studies in S. commune reported a repressing effect of cAMP on fruiting, and suggested this was because cAMP is able to mimic high $\mathrm{CO}_{2}$ levels(Pelkmans 2016). Genes of the cAMP pathway were annotated in S. commune and the pde2 phosphodiesterase (Schco3_2636760), which can degrade cAMP was functionally analyzed(Pelkmans 2016). The overexpression of pde2 results in fruiting body development even at elevated $\mathrm{CO}_{2}$ levels. These results were interpreted as evidence for $\mathrm{CO}_{2}$ being sensed via the cAMP pathway(Pelkmans 2016).

We annotated members of the cAMP pathway with $C$. cinerea as reference (Table 17). Consistent with data in S. commune(Pelkmans 2016), we could identify most members of the cAMP pathway in the examined species. Our expression data revealed that CAMP pathway members show relatively little expression dynamics in mushroom-forming fungi (Table 17). Nevertheless, this does not preclude an important role of cAMP signaling in fruiting body formation.

\subsubsection{G-protein coupled receptors}

G-protein coupled receptors (GPCRs) comprise the largest receptor family in fungi. GPCRs are seven transmembrane cell surface receptors, which transduce signals from a wide range of external stimuli by a G-protein heterotrimer(Xue et al. 2008)._Fungal GPCRs originally divided into six classes based on their structural similarity and homology(Xue et al. 2008), however, this classification was later expanded with additional classes (reviewed in (Brown et al. 2018)).

Pheromone sensing GPCRs are among the most widely studied in connection with fruiting body development. In Flammulina filiformis six pheromone receptor-like genes were characterized whereby four were highly expressed in the fruiting body, while two exhibited the maximal expression level in the mycelia(Meng et al. 2020)._Six pheromone-like GPCRs were characterized also in S. commune (bar3, bbr2, brl1, brl2, brl3 and brl4); it was reported that the overexpression of $b r l 2, b r l 3$ and $b r l 4$ enhanced mating or fruiting body formation in this species(Wirth et al. 2021). We annotated 7 mating-type pheromone receptors in $C$. cinerea (Table 16). Most of the pheromone receptors are highly expressed during the fruiting, but do not have orthologs in other species. In addition, five further GPCR genes were detected, which, similarly to pheromone-sensing GPCRs mostly did not have orthologs in other species (Table 18). This may suggest that fungal species have a specialised GPCR repertoire, that is hard to examine using orthology-based approaches.

While GPCRs are not conserved, we detected three CDE orthogroups that may be related to GPCR signaling and were developmentally regulated across several species. An RGS (regulator of G-protein signaling) domain containing protein family (C. cinerea protein ID: 479562) was developmentally regulated in $11 / 7$ species (FC>2/4)(Table 18). RGS proteins are involved in signal transduction by regulating G-protein activity and rapidly switching on or off G-protein coupled receptors pathways. We also found a CDE orthogroup containing putative Ran-binding proteins (C. cinerea protein ID: 472370), which are members of the Ras superfamily that regulates all receptor-mediated transport between the nucleus and the cytoplasm. Finally, we detected two arrestin superfamily orthogroups $(C$. 
bioRxiv preprint doi: https://doi.org/10.1101/2021.12.09.471732; this version posted December 10,2021 . The copyright holder for this preprint (which was not certified by peer review) is the author/funder, who has granted bioRxiv a license to display the preprint in perpetuity. It is made available under aCC-BY-NC-ND 4.0 International license.

Running head: fruiting body genomics

2290 cinerea protein ID: 473465, S. commune:Schco3_2490312) (Table 18). Arrestins are 2291 involved in signal transduction through the inactivation of $\mathrm{G}$ protein-coupled receptors, but 2292 some members of the superfamily have been reported in receptor endocytosis, cell cycle 2293 progression and pH sensing as well also(Herranz et al. 2005; Telzrow et al. 2019).

\section{4.7.5. Other signal transduction related families}

2295 Kinases - The majority of CDE orthogroups classified as signal transduction were kinases 2296 (Table 15). We found 13 conserved and developmentally expressed kinase orthogroups, 2297 however, most of these proved difficult to functionally characterize, likely due to the high rate 2298 of evolution in kinases and the lack of orthology to well-known genes in model species. One 2299 exception is the CDE orthogroup (C. cinerea protein ID: 451611) that contains orthologs of 2300 S. cerevisiae Rck2, which is a member of the high osmolarity glycerol (HOG) MAPK 2301 pathway. The HOG pathway is involved in responding to oxidative and osmotic stress. In 2302 most species, Rck2 orthologs showed early upregulation, with highest expression values in 2303 primordia, though its functional significance is not known. Rck2 orthologs were differentially 2304 expressed between dikaryons and A and B type monokaryons of $C$. cinerea as well(Stajich 2305 et al. 2010), and it was concluded that RCK2 genes in C. cinerea must serve different 2306 functions from that of $S$. cerevisiae orthologs. The remaining kinase orthogroups are 2307 functionally uncharacterized and may be interesting targets for functional studies, their 2308 dynamic expression patterns indicate potentially conserved and important functions in 2309 fruiting body development.

2310 Velvet complex - The velvet complex has been identified as a central regulatory hub in 2311 fungal development and secondary metabolism(Bayram et al. 2008). The complex was 2312 characterized in the Ascomycota, where it consists of VeA, LaeA, VelB as well as VelC and 2313 VosA, which assemble into condition-specific (including light-responsive) complexes. It has 2314 been shown that the velvet domain, an 200 amino acid fold, binds DNA and resembles the 2315 structure of the metazoan transcription factors NF-kB(Ahmed et al. 2013). Proteins containing a velvet domain exist outside the Pezizomycotina as well, however, these proteins did not appear to be orthologous to members of the Velvet complex in the aspergilli(Ojeda-López et al. 2018); accordingly, how they function and whether they form complexes also in the Basidiomycota and early-diverging fungi is not known.

The first evidence on a potential role of Velvet proteins in Basidiomycota fruiting body development was provided by Plaza et al(Plaza et al. 2014b). They detected the upregulation of a velvet domain containing protein in primordia of $C$. cinerea relative to vegetative mycelia. Following these trails and combining detailed mechanistic knowledge from the velvet regulon of $A$. nidulans(Krijgsheld et al. 2013), they could show that some, albeit a minor proportion, of orthologs of the $A$. nidulans velvet-regulated genes are upregulated in fruiting bodies in C. cinerea, L. bicolor and S. commune(Plaza et al. 2014b) also. This suggested some extent of conservation of function in the Dikarya, although the lack of orthology between Asco- and Basidiomycota proteins casts some uncertainty on how these data should be interpreted. Velvet protein-encoding genes were reported to be developmentally regulated in multiple Agaricomycetes species in the comparative analyses of Krizsan et al(Krizsán et al. 2019).

Our current analysis indicated that the velvet domain occurs in 5-6 proteins (only 4 in $P$. ostreatus) encoded in the genomes of mushroom-forming fungi. Surprisingly, the yeastlike $C$. neoformans also possess 6 genes with a velvet domain, in contrast to Ascomycota yeasts, which have lost velvet genes(Nagy et al. 2014). Interestingly, Ascomycota on 
bioRxiv preprint doi: https://doi.org/10.1101/2021.12.09.471732; this version posted December 10,2021 . The copyright holder for this preprint (which was not certified by peer review) is the author/funder, who has granted bioRxiv a license to display the preprint in perpetuity. It is made available under aCC-BY-NC-ND 4.0 International license.

Running head: fruiting body genomics

2336 average have 4 genes that encode velvet-domain containing proteins, indicating an 2337 expansion in the Basidiomycota. Consistent with results of Ojeda-Lopez et al(Ojeda-López 2338 et al. 2018), we found that Basidiomycota velvet proteins lack clear orthology to Ascomycota 2339 genes. Only VelB and VosA of $A$. nidulans have reciprocal best hits in $C$. cinerea ( $C$. cinerea 2340 protein ID: 365674 and 374867 , respectively).

In terms of expression, observed patterns vary widely among species. For example, all six genes are developmentally regulated in $C$. cinerea, but none in $A$. ostoyae (both $F C>4$ ), indicating considerable expression plasticity through evolution. We identified three orthogroups whose members were developmentally regulated in a notable number of species (Table 19). Of these, genes in one orthogroup (represented by $C$. cinerea 385561) were mostly upregulated in stipe tissues in the pileate-stipitate species $C$. cinerea, $A$. ostoyae, M. kentingensis, L. edodes (but only in mature FBs of L. bicolor). The gene was not developmentally regulated in $L$. tigrinus and $P$. ostreatus, possibly indicating the distant relationship of these species from ones in which the gene is stipe-upregulated. The second most interesting CDE orthogroup ( $C$. cinerea protein ID: 496341) was developmentally regulated in 10 species (FC>2), but expression peaks varied from primordia ( $A$. ampla, $S$. commune, $A$. ostoyae), to young fruiting body caps ( $C$. cinerea) and stipes ( $M$. kentingensis).

Based on the above data, velvet protein-encoding genes represent an interesting target for phylogenetic and functional studies of sexual fruiting bodies in the Agaricomycetes; we expect their analyses will reveal interesting insights into fruiting body development.

STRIPAK complex - The striatin-interacting phosphatase and kinase, STRIPAK (known as Far complex in(Kück et al. 2019)), is a widely conserved multi-protein complex that can be found from yeast to metazoans(Reschka et al. 2018). Its function in metazoans is unknown, whereas in Ascomycota model systems (e.g. Sordaria macrospora) it is involved in hyphal fusion and fruiting body development. It was first discovered in 2004 in fungi(S and U 2004) and its function has been increasingly clarified in a series of papers since then (see in(Reschka et al. 2018; Riquelme et al. 2018)). Mechanistically, the STRIPAK complex provides links between multiple signaling pathways in eukaryotic cells (reviewed in(Kück et al. 2019)), although many of the details of its interactions with other proteins are not known. Its role in fruiting body formation in the Basidiomycota has not yet been investigated.

We queried the $C$. cinerea genome with members of the STRIPAK complex described from Sordaria and S. cerevisiae (listed in(Reschka et al. 2018)). C. cinerea seems to have a fully functional STRIPAK complex, with all genes having clear orthologs, except for Far3/7 which only had weak hits. Further, the $C$. cinerea proteins were members of conserved single-copy Agaricomycetes orthogroups, which indicated that the complex is conserved in all the examined Agaricomycetes species. Members of this complex did not show significant expression dynamics (Table 20). The conservation of the complex in the Agaricomycetes suggests it might have important functions, however, its role in fruiting body development remains unclear at the moment and requires further research.

\subsubsection{Light sensing systems and light receptors}

2377 Fungi adapt developmental processes to light signals in time and space to ensure that 2378 sporulating cells can maximize spore dispersal efficiency(Yu and Fischer 2018). The 2379 photobiology and its connection to developmental events are quite well-known in both the 2380 Asco- and Basidiomycota. In the last decade, the molecular architectures and regulatory 2381 networks of sensing and responding to blue, red and green light have been uncovered in 
bioRxiv preprint doi: https://doi.org/10.1101/2021.12.09.471732; this version posted December $10,2021$. The copyright holder for this preprint (which was not certified by peer review) is the author/funder, who has granted bioRxiv a license to display the preprint in perpetuity. It is made available under aCC-BY-NC-ND 4.0 International license.

Running head: fruiting body genomics

2382 multiple species(Corrochano 2011; Fuller et al. 2015). In this chapter we discuss light 2383 sensing systems in Agaricomycetes and analyze their expression patterns in fruiting bodies. 2384 We note that the best known such system, the white collar complex has transcription factor 2385 activity and is discussed under chapter 4.5.1.

2386 genes that function as blue light photoreceptors and are involved in DNA repair(Fuller et al. 2015). Cryptochromes are regulated by the white collar complex in N. crassa(Froehlich et al. 2010) and regulate fruiting body development in certain Ascomycota (e.g. Cordyceps militaris(F et al. 2017)), but to our best knowledge, no such information is available in the Basidiomycota. Our analysis suggests that cryptochromes are single copy genes in the Agaricomycetes, except in L. edodes, which has two genes. The CDE orthogroup containing Agaricomycetes cryptochromes (represented by C. cinerea 492962)(Table 21) was developmentally regulated in $9 / 7$ species ( $F C>2 / 4)$, and showed strong induction in primordia relative to vegetative mycelium in $A$. ostoyae, $M$. kentingensis, L. edodes, $P$. ostreatus, Ph. chrysosporium and at FC>2 in $C$. aegerita and $C$. cinerea. The gene was constantly expressed in $A$. ampla and $S$. commune and was missing in L. bicolor. Based on these data, cryptochromes may be relevant to fruiting body formation in the Agaricomycetes.

Phytochromes are red- and far-red receptors that influence sexual and asexual sporulation in fungi. The first circumscribed fungal phytochrome was the fphA gene in $A$. nidulans, which represses sexual development under red light(A et al. 2005). Since then, the repression of sexual development has been proven for $N$. crassa too and several details of the process (e.g. interaction with WCC and velvet factors) have been clarified(A and $\mathrm{J} 2005$; Wang et al. 2016; Yu et al. 2016). In the Basidiomycota, the few reports of phytochromes include a systematic mutant library in $C$. neoformans, which circumscribed the gene encoding TCO3 (Lee et al. 2016), as well as observations of phytochrome upregulation in browning mycelium of $L$. edodes(Tang et al. 2013) and in rhizomorphs of $A$. ostoyae(Sipos et al. 2017b). In U. maydis red and blue light receptors have been shown to be required for the development of fruiting body-like structures(Sánchez-Arreguin et al. 2020).

Phytochromes seem to be single-copy genes in the Agaricomycetes examined here. They formed a conserved orthogroup (represented by C. cinerea 470504, Table 21), however, genes in this orthogroup did not show significant expression dynamics during fruiting body formation, except in $A$. ostoyae and $C$. cinerea. In $A$. ostoyae the expression of the phytochrome gene was $\sim 10 x$ higher in stipes than caps in stage 2 primordia and young fruiting bodies. Similarly, in $C$. cinerea peak expression was observed in elongating stipes in the young fruiting body stage. However, other species did not display this expression profile, indicating that phytochrome expression is not conserved across mushroom-forming fungi. Although expression patterns did not seem to be conserved in fruiting bodies, it is possible that red light sensing phytochromes are part of a photoreceptor system that signals position in open air, which is required for sporulation and fruiting body formation for the majority of species(J et al. 2010; Fuller et al. 2015).

Fungal light sensing systems also include rhodopsins, seven-transmembrane domain proteins involved in sensing green light(Bieszke et al. 1999). We detected rhodopsins only in L. tigrinus and Ph. chrysosporium, but none of the Agaricales in our dataset.

Genes related to blue light receptors - Blue light is one of the most important signals for fungi and responses as well as molecular aspects of the fungal blue light response are among the best known aspects of fungal photobiology in both the Asco- and Basidiomycota. The most important receptors of blue light are the white collar complex (WCC), which comprises the photoreceptors/transcription factors WC2 and WC1. These 
bioRxiv preprint doi: https://doi.org/10.1101/2021.12.09.471732; this version posted December 10,2021 . The copyright holder for this preprint (which was not certified by peer review) is the author/funder, who has granted bioRxiv a license to display the preprint in perpetuity. It is made available under aCC-BY-NC-ND 4.0 International license.

Running head: fruiting body genomics

2430 have been discussed under the chapter on transcription factors (see above). We here only 2431 discuss putative accessory proteins similar to VIVID of $N$. crassa. These proteins harbor a 2432 single PAS domain and act as repressors of the WCC in N. crassa and were suggested to 2433 provide a mechanism for adapting to changing light intensities during the day(Fuller et al. 2434 2015). Although evidence for that is missing in the Agaricomycetes, a CDE orthogroup of

2435 PAS domain proteins (represented by $C$. cinerea 500409) was developmentally regulated in $24369 / 7$ species ( $F C>2 / 4)$ and showed strong induction in primordia relative to vegetative mycelia 2437 in A. ostoyae, C. aegerita, C. cinerea, L. bicolor, L. tigrinus, M, kentingensis, $P h$.

2438 chrysosporium but not in $A$. ampla and $P$. ostreatus (and missing from $L$. edodes and $S$. 2439 commune). It will be interesting to see if this or similar genes are involved in light responses 2440 in the Agaricomycetes.

2441 Dst2 family - We found that orthologs of the dst2 gene of $C$. cinerea formed a CDE 2442 orthogroup (represented by C. cinerea 361584). Dst2 has been described as a FAD-binding 2443 and Berberine-like domain containing protein that, if mutated, results in a blind dark stipe 2444 phenotype similar to what is observed in mutations on the white collar complex(Kamada et 2445 al. 2010; Kuratani et al. 2010). Based on these observations, it has been hypothesized that 2446 dst2 represents a novel photoreceptor(Kuratani et al. 2010). The typical expression profile of 2447 these genes (seen in A. ostoyae, C. aegerita, C. cinerea, L. edodes and P. ostreatus) 2448 involved upregulation in primordia relative to mycelium and, in later stages, higher 2449 expression in stipes compared to caps. Variations to this pattern exist, such as only being 2450 FB-init in L. bicolor and S. commune, or being only stipe upregulated but not FB-init in $M$. 2451 kentingensis and L. tigrinus. We hypothesize that dst2 genes might be related to stipe 2452 development and, potentially, photomorphogenesis.

2453

\subsection{Cell wall biosynthesis and modification}

2455

2456

2457

2458

2459

2460

2461

2462

2463

2464

2465

2466

2467

2468

2469

2470

2471

2472

2473

2474

The cell wall is an organelle that functions as an extracellular matrix in fungi. It provides integrity, rigidity to cells, defines cell shape, harbors extracellular proteins, represents a channel of communication with the environment, and provides the first line of physical defense, to name just a few of its major roles in fungal physiology. Structural roles are particularly important for the Agaricomycetes that form above ground fruiting bodies that should be rigid yet plastic enough to withstand environmental forces and support spore dispersal. In fruiting bodies, the cell wall must serve structural and defense roles but must also be sufficiently plastic to allow changes in shape and size during development. Although mechanisms of cell shape determination are not known in mushroom-forming fungi, regulated cell wall deposition and remodeling by hydrolytic enzymes residing in the wall itself(Verdín et al. 2019), are undoubtedly of key importance.

There is plenty of evidence for cell wall remodeling and differential cell wall compositions in fruiting bodies of various species (e.g. (Buser et al. 2010a; Almási et al. 2019; Krizsán et al. 2019; Zhou et al. 2019)), although most of that is based on transcriptome rather than functional studies. For example, stipe growth may be realized by the breaking of chitin-glucan cross-links by hydrolytic enzymes(Liu et al. 2021). Similarly, there are cross-linking (transglycosidases) and lytic (e.g. glycoside hydrolases) enzymes that rigidify and make the cell wall more plastic, respectively(Verdín et al. 2019). Mannosylation might also rigidify the cell wall and mannoproteins are often N-glycosylated, which contributes to water retention and adhesion(Lesage and Bussey 2006). In the 2475 Agaricomycetes, Buser et al reported a fruiting body-specific N-glycan from C. cinerea(Buser 
bioRxiv preprint doi: https://doi.org/10.1101/2021.12.09.471732; this version posted December 10,2021 . The copyright holder for this preprint (which was not certified by peer review) is the author/funder, who has granted bioRxiv a license to display the preprint in perpetuity. It is made available under aCC-BY-NC-ND 4.0 International license.

Running head: fruiting body genomics

2476 et al. 2010b). The regulated interplay of these enzymes might result in the formation of 2477 stage-, tissue-, and species-specific cell wall architectures in fruiting bodies.

In this chapter we review current knowledge on cell wall related genes in mushroomforming fungi and analyze CDE orthogroups that are likely linked to cell wall synthesis or modification. We will not review cell wall composition in fruiting bodies, but refer to earlier reviews(Kües 2000). Cell wall functioning is quite well-researched in the Ascomycota (e.g. in $S$. cerevisiae and $N$. crassa), and to a much smaller extent in the Basidiomycota. We therefore base much of our functional hypotheses on knowledge in the Ascomycota, noting that the process may be different and/or significantly more complex in the Basidiomycota. For example, while some chitin-glucan cross linking enzyme families appear similarly important both in Asco- and Basidiomycota (e.g. GH16/GH17), there are some (e.g. GH72 and GH76) which are involved in cell wall remodeling in the Ascomycota, but apparently not in the Basidiomycota. Hereafter we discuss cell wall related CDE orthogroups broken down into synthetic and remodeling enzymes as well as by substrate (chitin/glucan).

From these analyses a few broad observations emerge, which we discuss here. Most importantly, the diversity of enzymes that show developmental regulation in fruiting bodies suggests that the fruiting body cell wall may be significantly more complex than we think, and we still know quite little about its exact structure and composition. A general pattern that also arises is that more tissue- and stage-resolved transcriptomes reveal proportionately more developmentally expressed genes, which indicates that cell wall remodeling and, consequently, cell wall architectures, likely show high tissue-specificity in fruiting bodies. Based on our results, it appears that fungal cell wall (FCW) remodeling and crosslinking genes show significantly more expression dynamics than genes encoding chitin and glucan synthases. In line with this observation, we found that intracellular components of cell wall biosynthesis (chaperones, glucan synthase complex, pathways involved in glucan monomer synthesis) also show little expression dynamics.

Comparisons across species revealed limited conservation in FCW related gene expression patterns across species, which suggests it diverges rapidly during evolution. This lends support to the quick evolution of cell wall architectures and corresponding enzyme profiles in fruiting bodies. Another observation related to this is that FCW-related genes show complex gene duplication/loss patterns, which complicates the inference of wellconserved groups of 1-to-1 orthologous genes. Thus, these genes might be more appropriately analyzed in the context of broader orthogroups (e.g. based on Orthofinder(Emms and Kelly 2015)) than based on the strict 1-to-1 orthogroups we used. However, for the sake of uniformity, we stick to the latter, with notes on overall prevalence of developmental expression in a given family.

An interesting observation is that several FCW active genes upregulated in fruiting bodies are also expressed during mycorrhiza establishment in ECM fungi such as $L$. bicolor(Martin et al. 2008; C et al. 2014). Such signals provide clues to shared morphogenetic mechanisms between ectomycorrhizal development and fruiting bodies.

Finally, an important consideration for cell wall biosynthesis/remodeling genes is whether they are upregulated in fruiting bodies is the vegetative mycelium sample that was used as control in the RNA-Seq. Non-growing inactive hyphae (where cell wall synthesis rate is low) as control of actively growing fruiting bodies can yield more gene expression differences than an actively growing mycelium sample. Although we do not think this has significantly influenced our comparisons, because we do not know the sampling conditions for all species, this possibility should be kept in mind in the interpretation of results. 
bioRxiv preprint doi: https://doi.org/10.1101/2021.12.09.471732; this version posted December 10,2021 . The copyright holder for this preprint (which was not certified by peer review) is the author/funder, who has granted bioRxiv a license to display the preprint in perpetuity. It is made available under aCC-BY-NC-ND 4.0 International license.

Running head: fruiting body genomics

2523

2524

2525

2526

2527

2528

2529

2530

2531

2532

2533

2534

2535

2536

2537

2538

2539

2540

2541

2542

2543

2544

2545

2546

2547

2548

2549

2550

2551

2552

2553

2554

2555

2556

2557

2558

2559

2560

2561

2562

2563

2564

2565

2566

2567

We attempted to as comprehensively analyze cell wall related gene expression as possible, at least with consideration of genes that show some expression conservation at the gene family level. Nevertheless, it is certain that several important, especially speciesspecific genes are left out from our inventory. For example, mannosylation, a potentially interesting process contributing to cell wall functioning, is not discussed here due to the lack of orthology to experimentally characterized mannosylation components in the Ascomycota. These aspects will need to be covered in future studies.

\subsubsection{Cell wall biosynthesis}

\subsubsection{Chitin biosynthesis (GT2)}

Chitin is one of the most important, although usually not the most abundant structural polymer of the fungal cell wall. This is true for fruiting bodies as well, in which, although cell wall composition is altered relative to vegetative mycelia(M and C 1981; Mol and Wessels 1990; Kües 2000), chitin is an important component that confers rigidity. Chitin is also a scaffold for the modification of cell wall structure either by cross-linking, incorporation of glycoproteins or chemical modifications to the chitin polymers, among others(Verdín et al. 2019). Cell wall chitin assembly involves the action of the UDP-N-acetylglucosamine biosynthesis pathway (in S. cerevisiae Gfa1p, Gna1p, Pcm1p, Qri1p), chitin synthases (Chs1-3) which elongate the growing chitin polymer as well as chitin deacetylases, which modify the chitin on the cell surface(Shahinian and Bussey 2000; Klis et al. 2006; Verdín et al. 2019) (the latter is discussed in chapter 4.8.2.1). While yeasts have up to three chitin synthases, filamentous fungi have additional chitin synthase genes (on average up to seven(Verdín et al. 2019)), including highly processive ones that harbor a myosin motor domain and that were lost in yeasts( $\mathrm{N}$ et al. 2006).

We examined chitin synthesis and modification related genes based primarily on functional annotations and recent reviews(Shahinian and Bussey 2000; Klis et al. 2006; Verdín et al. 2019). Clear orthologs of the S. cerevisiae UDP-N-acetylglucosamine biosynthesis pathway could be identified in all examined Agaricomycetes species (Table 22). We identified 8-13 (17 in L. bicolor) chitin synthases of the GT2 family, 2-3 chitin synthase export chaperones as well as orthologs of S. cerevisiae SKT5 (activator of chitin synthase 3) in the examined Agaricomycetes. Interestingly, UDP-N-acetylglucosamine biosynthesis pathway genes showed more expression dynamics than most chitin synthase genes (Table 22). Among chitin synthases, although many formed conserved orthogroups, only a single appeared developmentally regulated in a wide array of species. Nevertheless, each species had developmentally regulated and/or FB-init (sensu Krizsan et al 2019) chitin synthases, though these did not always belong in conserved orthogroups. The most interesting orthogroup (represented by C. cinerea 359180 ) was developmentally regulated in $7 / 6$ (at fold change 2/4) species and showed upregulation and high expression in primordia relative to vegetative mycelium in several species (C. cinerea, A. ampla, P. ostreatus, S. commune, $C$. aegerita and $L$. edodes). Comparative genomic data suggests that this orthogroup of chitin synthases is specific to mushroom-forming fungi or the Basidiomycota, suggesting that it may be a fruiting body-specific chitin synthase. A second CDE orthogroup of GT2 chitin synthases (C. cinerea protein ID: 519767 ) was also detected (Table 22).

A chitin synthase regulator described as Csr2 from C. neoformans(Banks et al. 2005) and Skt5 from S. cerevisiae(S et al. 1993), is conserved in the Agaricomycetes (orthogroup 
bioRxiv preprint doi: https://doi.org/10.1101/2021.12.09.471732; this version posted December 10,2021 . The copyright holder for this preprint (which was not certified by peer review) is the author/funder, who has granted bioRxiv a license to display the preprint in perpetuity. It is made available under aCC-BY-NC-ND 4.0 International license.

Running head: fruiting body genomics

2568 represented by $C$. cinerea 538650), but did not show significant expression dynamics (Table 2569 22).

$2570 \quad$ 4.8.1.2. $\beta$-glucan biosynthesis

2571 4.8.1.2.1. $\beta-1,6-$ glucan (Kre9/Knh1, GH16, GH63)

2572 In addition to $\beta-1,3$-glucan, $\beta-1,6$-glucan is also found in fungal cell walls and is

2573 hypothesized to serve gluing purposes by covalently linking $\beta-1,3-$ glucan to chitin and

2574 mannoproteins(Lesage and Bussey 2006). Its synthesis builds on UDP-glucose produced by

2575 the corresponding pathway in S. cerevisiae. We find that most members of the yeast beta-

2576 1,6-glucan biosynthesis pathway have no clear 1-to-1 ortholog in the Agaricomycetes (Kre9,

2577 Knh1, Dfg5, Dcw1, Kre5, Kre1). Only Kre6 (C. cinerea protein ID: 447635), Rot1 (C. cinerea

2578 protein ID: 241938), Rot2 (C. cinerea protein ID: 359282) and CWH41 (C. cinerea protein ID:

2579 486333) had clear orthologs. However, at the family level, all known members of the $\beta-1,6-$

2580 glucan synthesis machinery are represented in the Agaricomycetes (Table 23), except Kre1,

2581 which seems missing.

2582 Kre9/Knh1 - In yeasts and filamentous Ascomycota, the Kre9/Knh1 and GH16 families

2583 include the main glucan synthases(Lesage and Bussey 2006). Several additional well-

2584 characterized proteins in the endoplasmic reticulum are necessary for $\beta$-1,6-glucan

2585 synthesis (e.g. Big1, Rot1, Rot2, Cwh41). It should be noted that there is a strong overlap

2586 between genes participating in $\beta$-1,6-glucan synthesis and remodeling, therefore, separating

2587

2588

2589

2590

2591

2592

2593

2594

2595

2596

2597

2598

2599

2600

2601

2602

2603

2604

2605

2606

2607

2608

2609

2610

2611

2612

2613 these two processes is harder than it is in the case of chitin or $\beta-1,3-$ glucan.

The Kre9/Knh1 family of cell surface proteins are involved in $\beta-1,6$-glucan assembly in the cell wall of fungi and have been characterized in a number of Ascomycota species(Brown and Bussey 1993; Dijkgraaf et al. 1996; S et al. 1998b; Costachel et al. 2005), predominantly yeasts. A member of this family has been independently reported as a developmentally regulated gene, DRMIP, of L. edodes(Szeto et al. 2007), which was reported to be primarily expressed in primordia. Reports of this family in fruiting body transcriptomes just started to emerge recently(Almási et al. 2019; Krizsán et al. 2019; Liu et al. 2020b), and no mechanistic study proved yet a link to cell wall in fruiting bodies. The family is overrepresented in Agaricomycetes(Krizsán et al. 2019), suggesting it might have experienced duplications specific to mushroom-forming fungi.

We identified 5-15 Kre9/Knh1 genes in mushroom-forming fungi. Of these 3-4 are developmentally regulated in each species. Notably, each species has 2-3 genes in this family that show a considerably higher expression in all fruiting body tissues than in vegetative mycelia, suggesting that these genes are involved in generating fruiting-body specific cell walls. For example, in $C$. cinerea we found three FB-init Kre9/Knh1 genes ( $C$. cinerea protein ID: 470185, 359649, 465604), whereas in L. edodes, there was one FB-init gene, in addition to DRMIP, which was highly expressed and two-fold upregulated in all fruiting body tissues compared to vegetative mycelium. The family displayed more expression dynamics in more resolved transcriptomes (e.g. A. ostoyae, C. cinerea), suggesting that it has considerable tissue-specificity. In addition, each species showed for some genes an expression peak in vegetative mycelium, which is consistent with a partitioning of fruiting body and mycelium-specific roles. Taken together, the Kre9/Knh1 family is likely involved in $\beta-1,6$-glucan assembly in the cell wall, with several genes showing fruiting body-specific expression. We detected five CDE orthogroups of Kre9/Knh1 genes (represented by C. cinerea 359649, 465604, 470186, 417746, S. commune 2557059)(Table 23). 
bioRxiv preprint doi: https://doi.org/10.1101/2021.12.09.471732; this version posted December $10,2021$. The copyright holder for this preprint (which was not certified by peer review) is the author/funder, who has granted bioRxiv a license to display the preprint in perpetuity. It is made available under aCC-BY-NC-ND 4.0 International license.

Running head: fruiting body genomics

2614 GH16 - The other major $\beta$-1,6-glucan synthase family is GH16, which includes both glucan 2615 synthases (e.g. Kre6 of $S$. cerevisiae, $=$ C. cinerea 447635) and glucan-chitin cross-linking 2616 enzymes that participate in cell wall remodeling(Patel and Free 2019). We discuss this family 2617 in detail under the 'Cell wall remodeling' chapter.

2618 Several ER-localized $\beta$-1,6-glucan synthesis related proteins have been described in 2619 yeasts and filamentous Ascomycota(Lesage and Bussey 2006; Orlean 2012; Essen et al. 2620 2020), including Kre5, Big1, Rot1, Rot2 and Cwh41. Rot2 and Cwh41 are also involved in 2621 protein N-glycosylation(Lesage and Bussey 2006). These are conserved in the

2622 Agaricomycetes, mostly forming single-copy families with low expression dynamics during 2623 fruiting body development. The GT24 (glycoprotein a-glucosyltransferase) family includes 2624 the Kre5 gene in S. cerevisiae and $C$. albicans. Kre5 has an indirect role in $\beta-1,6$-glucan 2625 synthesis and may be involved in the refolding of misfolded $\mathrm{N}$-glycoproteins in the $\mathrm{ER}$, in 2626 particular $\beta$-1,6-glucan synthases(Lesage and Bussey 2006). GT24 is present as a single2627 copy family in Agaricomycetes (Table 23); their expression is approximately constant in 2628 fruiting bodies. Big1 is an ER membrane protein of unknown function that is required for $\beta$ 2629 1,6-glucan synthesis in S. cerevisiae(Lesage and Bussey 2006). It is a single-copy gene 2630 family (represented by $C$. cinerea 432992) in the Agaricomycetes, without developmentally 2631 relevant expression patterns (Table 23). Rot1 is a chaperone involved in protein folding (in 2632 general) located in the ER. It is required for cell wall synthesis and N-glycosylation and O2633 mannosylation in S. cerevisiae(Lesage and Bussey 2006). Rot1 orthologs are single copy 2634 genes (C. cinerea protein ID: 241938) in the Agaricomycetes that showed no developmental 2635 dynamics in the species we examined (Table 23). Rot2 is an ER-localized alpha-glucosidase 2636 involved in protein $\mathrm{N}$-glycosylation, required for normal levels of $\beta$-1,6-glucan in the cell wall 2637 of $S$. cerevisiae (Lesage and Bussey 2006). It belongs to the GH31 family, which is present in 2638 3-8 copies in Agaricomycetes. They form two CDE orthogroup (FC2/4: 8/6 species, 2639 represented by $C$. cinerea 499280 and FC2/4: $6 / 5$ species, represented by $S$. commune 2640 2543909)(Table 23), which were developmentally regulated in $6 / 5$ and $5 / 4$ species, however, 2641 expression patterns of these orthogroups did not show marked conservation. 1-to-1

2642 orthologs of $S$. cerevisiae Rot2 (represented by $C$. cinerea 359282) showed very small gene 2643 expression dynamics.

2644 The GH63 family includes alpha-glucosidases, such as Cwh41 of S. cerevisiae, 2645 involved in cell wall beta-1,6-glucan assembly in the ER. The family is represented by 2 2646 copies in all but one of the examined species (L. edodes has 5 genes). GH63 genes form 2647 two conserved orthogroups, of which one (C. cinerea protein ID: 486333), containing 2648 orthologs of $S$. cerevisiae Cwh41) did not appear to be interesting, whereas members of the 2649 other (C. cinerea protein ID: 494484) showed marked expression peaks coinciding with 2650 spore formation in all species (Table 23). In C. neoformans, the gene showed upregulation 2651 at $72 \mathrm{~h}$, also coinciding with sporulation. Whether this GH63 orthogroup is needed for spore 2652 wall assembly or other processes that temporally overlap with sporulation cannot be 2653 determined based on these data, nevertheless, the family seems to be interesting in the 2654 context of fruiting body development.

2655 4.8.1.2.2. $\beta$-1,3-glucan (GT48)

2656 In S. cerevisiae $\beta-1,3-$ glucan is synthesized by the FKS (Fks1-3) and the GAS (Gas1-4 2657 families as well as a few accessory proteins, such as Smi1 (C. cinerea protein ID: 181364) 2658 and Rho1 (C. cinerea protein ID: 528086)(Lesage and Bussey 2006; Verdín et al. 2019). Of 2659 the glucan synthases only FKS1 and Gas1 had clear 1-to-1 orthologs is the Agaricomycetes 2660 (C. cinerea protein ID: 446030 and 543646, respectively). The glucan synthase complex, 
bioRxiv preprint doi: https://doi.org/10.1101/2021.12.09.471732; this version posted December 10,2021 . The copyright holder for this preprint (which was not certified by peer review) is the author/funder, who has granted bioRxiv a license to display the preprint in perpetuity. It is made available under aCC-BY-NC-ND 4.0 International license.

Running head: fruiting body genomics

2661 which is formed by FKS1, Rho1, Smi1(Verdín et al. 2019), seems to be conserved in the 2662 Agaricomycetes.

2663 Most Agaricomycetes species have two beta-1,3-glucan synthase genes of the GT48 2664 family (except $M$. kentingensis and L. tigrinus, which have 5 and 7 respectively). Somewhat 2665 surprisingly, GT48 genes showed very modest expression dynamics and were mostly not 2666 developmentally regulated. Similarly, the Gas1 family of glucanosyltransferases is present in 2667 1-2 copies in the examined species (L. bicolor has 7 and $M$. kentingensis has 4 ) and they 2668 were not developmentally regulated. Accessory members of the glucan synthase complex 2669 (Smi1, Rho1) showed constant expression in fruiting bodies. Collectively, these data suggest 2670 that $\beta-1,3$-glucan synthesis is present, but it is not dynamically regulated in fruiting bodies.

2671

2672

\subsubsection{Cell wall remodeling}

2673

4.8.2.1. Chitin-active enzyme families (GH18, GH20, X325, AA9, AA14, CBM5/12, CBM50, 2674 CE4)

2675

2676

2677

2678

2679

GH18 - Glycoside hydrolase family 18 contains chitinases (both endo- and exochitinases). Chitinases remodel the chitin structure by cleaving $\beta-1,4$ linkage of chitin or modify oligosaccharide(Verdín et al. 2019) (e.g. cleaving chitobiose from the cell wall). Chitinases are one of the most straightforward players in fruiting body development and accordingly have been reported in several previous genomic(Chen et al. 2012; Park et al. 2014), transcriptomic(Patyshakuliyeva et al. 2013; Sakamoto et al. 2017b; Xie et al. 2018; Krizsán et al. 2019) and mechanistic(Niu et al. 2016; Zhou et al. 2016c) studies. Despite the clear prediction of their role in morphogenesis, this has not been mechanistically shown in the Basidio- or Ascomycota( $L$ et al. 2011), possibly because multiple paralogous genes in each species' genome complicate functional studies.

In the Basidiomycota, Zhou et al reported chiB1 (C. cinerea protein ID: 368217) (Table 24), a class $V$ exochitinase purified from $C$. cinerea and suggested to be involved in cap autolysis(Zhou et al. 2016c). Expression data agree with this, the corresponding gene shows a very strong induction in mature fruiting body caps. Similarly, Chilll(Niu et al. 2016) of $C$. cinerea (C. cinerea protein ID: 354859 ) was reported to have both endo- and exochitinase activity, and to be expressed mostly in fruiting bodies, with peak expression in mature fruiting bodies (which is confirmed by our expression data). Most recently, Zhou et al assayed the role of chitinases in stipe cell wall extension(Zhou et al. 2019) and named six further chitinases in C. cinerea, ChiE1, (C. cinerea protein ID: 543586), ChiE2 (C. cinerea protein ID: 520359), ChiEn1 (C. cinerea protein ID: 91051$)$, ChiEn2 (C. cinerea protein ID: 90984), ChiEn3 (C. cinerea protein ID: 470416), and ChiEn4 (C. cinerea protein ID: 358869). Chitinases have also been examined in the context of autolysis of the model mushrooms $C$. cinerea and Coprinellus congregatus( $\mathrm{H}$ and $\mathrm{HT} 2009)$.

GH18 genes are found in 8-12 copies in Agaricomycetes (up to 20 in Polyporales, see (Chen et al. 2012)), some of which harbor carbohydrate binding modules (CBMs), but their function has not been examined. Most chitinases are developmentally expressed in fruiting bodies. Mycelium-upregulated chitinases are also found in most species; these, although display peak expression in vegetative mycelia (often $\sim 10 \mathrm{x}$ that of fruiting bodies), often also show $>4$ fold expression dynamics within fruiting bodies. We detected seven CDE orthogroups (Table 24), which included C. cinerea ChiB1, ChiE2, ChiEn1 and ChiEn3. In general, although, the detected chitinase orthogroups were developmentally regulated in many species, they did not show conserved expression patterns across species, which could 
bioRxiv preprint doi: https://doi.org/10.1101/2021.12.09.471732; this version posted December $10,2021$. The copyright holder for this preprint (which was not certified by peer review) is the author/funder, who has granted bioRxiv a license to display the preprint in perpetuity. It is made available under aCC-BY-NC-ND 4.0 International license.

Running head: fruiting body genomics

2707 indicate quick divergence of function or the failure of our reciprocal-best-hit based approach 2708 to detect functionally related genes (in the latter case phylogenetic analyses might help).

2709 One orthogroup (represented by C. cinerea 520359) shows gill-specific expression peaks in 2710 the species in which separate RNA-Seq data are available for gills (A. ostoyae, $P$. ostreatus) 2711 or late upregulation in Pt. gracilis, C. aegerita, L. edodes, L. bicolor, Ph. chrysosporium, A. 2712 ampla and S. commune, suggesting it may have something to do with processes that take 2713 place in the hymenium late in development, possibly sporulation.

2714 GH20 and chitin degradation products - A chitin-connected GH family is GH20, which has $2715 \quad \beta-1,6-\mathrm{N}$-acetylglucosaminidase activity and thus can cleave chitobiose released by chitinases further into N-acetylglucosamine (GlcNac). GlcNac may be taken up by the cell for repurposing of cleaved cell wall components as nutrient source(López-Mondéjar et al. 2009; de Oliveira et al. 2018) or, to trigger upregulation of chitinolytic genes in a transcriptional feedback-loop(Langner and Göhre 2015). The latter hypothesis seems more plausible for cases when the fungus feeds on chitin rather than modifies it for developmental purposes. In previous reports, GH20 genes have been identified in the post-harvest transcriptome of $L$. edodes(Sakamoto et al. 2017b). We identified 2-8 GH20 genes in the examined Agaricomycetes, some of which were developmentally regulated (including the single gene in C. neoformans), but did not form conserved CDE orthogroups. The role of this gene family in fruiting body development may be worth examining further.

If GlcNac is indeed taken up and metabolized in the cell, then two of the detected CDE orthogroups may provide clues to its mechanistic bases. An orthogroup of GlcNac transporters, which contains reciprocal best hits of $C$. albicans NGT1, was developmentally regulated in 5/4 species (C. cinerea protein ID: 464567, Table 24). C. albicans NGT1 encodes a specific transporter of GlcNac(FJ and JB 2007; Q et al. 2020). We speculate that this orthogroup may be involved in the uptake of GlcNac monomers generated by $\mathrm{GH} 20$ enzymes extracellularly.

In fungi, the GlcNac catabolic pathway is best described in C. albicans(Kumar et al. 2000; Yamada-Okabe et al. 2001), however, only a few of the corresponding genes are conserved in Agaricomycetes. Only the GlcN6P deaminase (Nag1, C. cinerea 356467 FC2/4: 7/4 species) and a GlcNac permease Nag4 (C. cinerea protein ID: 544873, FC2/4: 6/4)(Table 24) genes have clear reciprocal best hits in Agaricomycetes, whereas we could not identify clear orthologs of $\mathrm{N}$-acetylglucosamine kinase (Nag5) or $\mathrm{N}$-acetylglucosamine deacetylase (Nag2). Our annotations identified a different gene as $\mathrm{N}$-acetylglucosamine deacetylase, however (C. cinerea protein ID: 446427), which was developmentally regulated in $8 / 6$ species $(F C=2 / 4)$. We also detected a GlcNac deacetylase orthogroup (C. cinerea protein ID: 446427), which may be involved in intracellular GlcNac catabolism.

In summary, the evidence presented here on GlcNac uptake and catabolism in mushroom forming-fungi is circumstantial, at best, however, provides clues for further research on this topic. GlcNac (and any other breakdown intermediate) produced during cell wall modification may be precious compounds in the fruiting body, which is otherwise dependent on distal supply of nutrients from the mycelium. This might have prompted the evolution of efficient repurposing mechanisms in fruiting bodies to mitigate its dependence on mycelial nutrient supply.

2751

2752 CE4 chitin deacethylases - Chitin deacetylases (CDA) are involved in chitosan production, a minor but key constituent of the cell wall. Chitosan, a more soluble polymer than chitin, occurs in both mycelia and fruiting bodies of basidiomycetes, although its role in fruiting body formation is thought to be limited(M and C 1981; Crestini et al. 1996; Baker et al. 2007). It is produced by the synergistic activities of chitin synthases and CDAs, where the former 
bioRxiv preprint doi: https://doi.org/10.1101/2021.12.09.471732; this version posted December $10,2021$. The copyright holder for this preprint (which was not certified by peer review) is the author/funder, who has granted bioRxiv a license to display the preprint in perpetuity. It is made available under aCC-BY-NC-ND 4.0 International license.

Running head: fruiting body genomics

2755 synthesize and the latter deacetylate chitin in various spatial or temporal patterns and to 2756 varying degrees(Sebastian et al. 2020). CDAs belong to the carbohydrate esterase 4 family 2757 (CE4) in the CAZy classification and are homologous to bacterial nodB factors that produce 2758 signal molecules in the rhizobia-legume symbiosis(Buhian and Bensmihen 2018).

2759 Deacetylation of chitin to chitosan makes it resistant to chitinases, which might provide a 2760 mechanism for regulating the extent of chitinase-mediated wall plasticity and thus cell 2761 expansion (e.g. in stipe elongation(Liu et al. 2021)). Fungal CDAs have a potential in 2762 biotechnology, as chitosan is an additive in food, cosmetic and pharmaceutical 2763 products(Morin-Crini et al. 2019). CDAs have poorly known roles in Ascomycota fruiting 2764 body development, with both $N$. crassa CDAs, nevertheless, being highly expressed during 2765 perithecium development, suggesting roles in sexual morphogenesis(Patel and Free 2019). 2766 It should be noted that CE4 also contains acetyl xylan esterases, which are able to break 2767 down the xylan backbone of hemicellulose during wood decay ( $X$ and $S$ 2009). Therefore, 2768 2769 some genes of the family in the Agaricomycetes may be related to wood decay too.

CDA genes have been detected in several RNA-Seq studies focusing on fruiting body development(Morin et al. 2012; Sipos et al. 2017b; Xie et al. 2018; Krizsán et al. 2019). Three chitin deacetylases have been experimentally characterized in $C$. cinerea, CDA1 ( $C$. cinerea protein ID: 42776), CDA2 (C. cinerea protein ID: 502602), and CDA3 (C. cinerea protein ID: 464952)(Table 24), all of which were reported to have high expression in the elongating region of the stipe(Wang et al. 2018b; Bai et al. 2020). Another chitin deacetylase, primarily expressed in the fruiting bodies, was investigated in F. velutipes(M et al. 2008).

Searching the genomes of fruiting body forming fungi reveals 5-18 chitin deacetylase genes, several of which formed conserved single-copy orthogroups. The previously investigated CDA1-3 genes of $C$. cinerea formed species-specific orthogroups. The majority of CDA genes in the examined species were developmentally regulated in fruiting bodies, indicating key functions in fruiting body development. Six out of 17 C. cinerea CDA genes (Table 24) formed CDE orthogroups. These showed diverse expression peaks with no apparent trend in their expression. However, several genes were upregulated in stipes of young or mature fruiting bodies. One of them (C. cinerea protein ID: 355445$)$ comprise proteins representing reciprocal best hits of $S$. cerevisiae CDA1, which is involved in the biosynthesis of the ascospore wall, required for spore wall rigidity. Expression of genes in this orthogroup did not peak during spore development, suggesting that this specific gene orthogroup is not involved in basidiospore wall synthesis. On the other hand, the expression of one and three genes were highest in young fruiting body gills and mature fruiting body gills and caps, respectively, which might be involved in producing chitosan in spore walls.

On a related note, we could not detect chitosanases, which degrade chitosan, in the genomes of mushroom-forming fungi, suggesting that chitosan is a terminal cell wall carbohydrate, not modified or cleaved further.

Carbohydrate binding modules (CBMs) - Carbohydrate binding modules, as their name suggests, bind various carbohydrates, and thus can frequently be attached to cell wall modifying enzymes. Chitin binding ability has been shown for at least 9 CBM families(Sánchez-Vallet et al. 2015). Of the most widely known chitin-binding CBMs (1, 5/12, $14,18,19,50)$, only CBM1, 5/12, 18 and 50 families are conserved in the Agaricomycetes we examined. The CBM1 family, which primarily binds cellulose and according to some reports chitin, does not appear to be involved in fruiting body development: most genes that encode CBM1 containing proteins have virtually no expression in fruiting bodies. Some such proteins show significant expression dynamics (e.g. C. cinerea 363583, 499390), but are not 
bioRxiv preprint doi: https://doi.org/10.1101/2021.12.09.471732; this version posted December $10,2021$. The copyright holder for this preprint (which was not certified by peer review) is the author/funder, who has granted bioRxiv a license to display the preprint in perpetuity. It is made available under aCC-BY-NC-ND 4.0 International license.

Running head: fruiting body genomics

2803 conserved across species. The CBM18 family occurs in GH16 proteins, which have been 2804 described above.

2805 CBM5/12 - The CBM5/12 family comprises chitin-binding modules, which assist the 2806 action of cell wall modifying enzymes in fungal development(Hartl et al. 2011). The CBM5 2807 family is sometimes considered a lectin due to its carbohydrate binding ability(Ismaya et al. 2808 2020). Most CBM5/12 modules occur in combination with GH18 domains, where their chitin 2809 binding ability is believed to increase the efficiency of the chitinase(Hartl et al. 2011). We 2810 identified 3-6 CBM5/12 domains in the examined Agaricomycetes. All but one CBM5/12 2811 modules are attached to $\mathrm{GH} 18$ chitinases and have thus been discussed above. The single 2812 CBM5/12 module that is not attached to a chitinase occurs on proteins that also harbor a 2813 domain of unknown function (DUF3421) and a DM9 repeat as well. Such proteins occur in 2814 single copy in Agaricomycetes genomes and comprise a CDE orthogroup (C. cinerea protein 2815 ID: 413423) that show high expression in vegetative mycelia and mature stipe tissues of 2816 most species (A. ostoyae, P. ostreatus, M. kentingensis, C. cinerea, L. tigrinus, L. bicolor, $L$. 2817 edodes) (Table 24). The protein is functionally uncharacterized. Both the DUF3421 and the 2818 DM9 repeat occur primarily in insects and other animals (but are found also in early2819 diverging fungi), but their activity is not known. Their developmental expression and the 2820 presence of a CBM5/12 domain suggests they may be linked to the chitin content in the cell 2821 wall, however, how exactly they function remains unclear.

2822 CBM50 - also known as LysM domain, this family binds chitin and can occur in a 2823 number of chitin-active proteins or on its own(GB et al. 2015). It has been reported to show developmentally dynamic expression in previous studies of fruiting body development(Sakamoto et al. 2017b; Sipos et al. 2017b; Krizsán et al. 2019; Huang et al. 2020). LysM domain proteins are widely known as effectors of plant pathogenic fungi, in which solitary secreted CBM50 modules can mask chitin residues and thus can dampen the immune response of plants(GB et al. 2015). Solitary CBM50 modules expressed in fruiting bodies could be involved in defense against insects(Kunzler 2015).

The examined Agaricomycetes contain 3-9 CBM50/LysM domain encoding genes (except $A$. ostoyae, which has 18, but its expansion may be related to the pathogenic nature of Armillaria(Sipos et al. 2017b)). The CBM50 module occurs almost exclusively on its own in the examined Agaricomycetes, only $C$. aegerita has proteins that harbored combinations of CBM50 and chitinase domain signatures. Interestingly, CBM50 encoding genes showed relatively little expression dynamics within fruiting bodies with 0-2 developmentally expressed genes per species. This, and reports on the induction of one of the CBM50 genes of $C$. cinerea in response to bacterial and nematode challenge(Kombrink et al. 2018; Tayyrov et al. 2018, 2019) could indirectly support a role in defense. However, some genes had high and constant expression during fruiting body development (e.g. C. cinerea 448942), suggesting chitin-linked functions.

AA9 family (Lytic polysaccharide monooxygenases) - The family has been reported only recently to have fruiting body specific expression patterns(Almási et al. 2019; Krizsán et al. 2019). AA9 proteins were initially considered to be endoglucanases and classified in glycoside hydrolase family 61 , but turned out to be lytic polysaccharide monooxygenases (LPMOs) that cleave polysaccharide chains using a distinct oxidative mechanism(G et al. 2010).

The examined Agaricomycetes possess 7-34 AA9 encoding genes, of which 1-10 were developmentally regulated. A part of this copy number variation can be explained by lifestyle, as wood-decay species are known to have expanded, while biotrophic or mycorrhizal species have contracted AA9 repertoires(Miyauchi et al. 2020). AA9 LPMOs 
bioRxiv preprint doi: https://doi.org/10.1101/2021.12.09.471732; this version posted December 10,2021 . The copyright holder for this preprint (which was not certified by peer review) is the author/funder, who has granted bioRxiv a license to display the preprint in perpetuity. It is made available under aCC-BY-NC-ND 4.0 International license.

Running head: fruiting body genomics

2851 might be active on $\beta$-glucan or on both glucan and cellulose as well, providing them with the 2852 activity required to remodel the fungal cell wall(Krizsán et al. 2019). The identified AA9 2853 genes hardly formed conserved orthogroups, which may be a consequence of high 2854 duplication/loss rates.

2855 Several species showed interesting LPMO expression patterns, which were quite 2856 consistent within, but different between species. For example, in C. cinerea several genes 2857 had a late upregulation, suggesting that LPMOs might be involved in autolysis. On the other 2858 hand, in Ph. chrysosporium many genes showed high expression in vegetative mycelium 2859 and mature fruiting bodies but low in young fruiting bodies, creating U-shaped expression 2860 curves. In L. tigrinus several genes showed a marked peak in cap and gill samples of mature 2861 fruiting bodies. The single AA9 gene (Cryne_JEC21_1_562) of C. neoformans had a strong 2862 (45-fold) induction in $24 \mathrm{~h}$ samples, coincident with basidium formation(Liu et al. 2018). In 2863 general, all species displayed several genes with high expression in vegetative mycelium, 2864 but negligible (<5 FPKM) expression in any of the fruiting body tissues, suggesting the 2865 existence of separate vegetative mycelium-specific and fruiting body-specific AA9 2866 subgroups. Yet other AA9 genes displayed uniformly low ( $<5$ FPKM) expression; these 2867 LPMO-s might be specific for the plant cell wall, and thus not induced during development. 2868 Nevertheless, the developmentally dynamic expression of several AA9 genes suggests that 2869 this family is involved in fungal cell wall remodeling during fruiting body development.

AA14 family - We found a conserved orthogroup (represented by $C$. cinerea 362412 ) that contained members of the recently circumscribed AA14 family of lytic xylan oxidases(Couturier et al. 2018). These proteins require copper as their native cofactor and were speculated to unlock xylan-coated cellulose fibers in the plant cell wall. We identified members of the AA14 family based on BlastP (query Pycnoporus coccineus AUM86167 and AUM86166): we find that blastp with e-value cutoff of $10^{-10}$ yielded copy numbers that match perfectly those reported by Couturier et al (Suppl Data Set 1)(Couturier et al. 2018). Fruiting body forming fungi contained 2-5 copies of the family, whereas yeast-like fungi (U. maydis and $C$. neoformans) contained none. Gene belonging to this family did not display characteristic expression peaks across species. However, as with other GH and AA families, their developmental expression in fruiting bodies suggests they may be active on some component of the fungal cell wall as well or have other extracellular roles.

X325 family - We also detected the recently described X325 family of coppercontaining, LPMO-like proteins(Labourel et al. 2020). As a new gene family, the only functional knowledge available currently is that it displays a fold similar to fungal LPMOs, may be involved in copper homeostasis(Garcia-Santamarina et al. 2020), is upregulated in several mycorrhizal fungi and resides on the surface (via a GPI anchor) of the fungal cell wall(Labourel et al. 2020). A morphogenetic role for the family has been speculated. We here report that members of this family form a CDE orthogroup (represented by $C$. cinerea 454418) and are developmentally regulated in a wide range of species (9/6 at FC4/2), although expression patterns differ between species. Additional developmentally regulated X325 genes from other species were segregated into other orthogroups. The L. bicolor gene is slightly upregulated in primordia relative to free living mycelium $(F C=1,8)$ and has a peak expression in young fruiting body stipes.

AA10 and AA11, two well-known chitinolytic LPMO families(Langner and Göhre 2015; Polonio et al. 2021), are missing in the examined Agaricomycetes, so they are unlikely to be contributors to fruiting body development. 
bioRxiv preprint doi: https://doi.org/10.1101/2021.12.09.471732; this version posted December $10,2021$. The copyright holder for this preprint (which was not certified by peer review) is the author/funder, who has granted bioRxiv a license to display the preprint in perpetuity. It is made available under aCC-BY-NC-ND 4.0 International license.

Running head: fruiting body genomics

2897 4.8.2.2. Glucan-linked families (GH3, GH5, GH16, GH17, GH30, GH55, GH71, GH128, 2898 GH152, Cdc43, WSC)

2899 Cdc43 - We detected orthologs of S. cerevisiae Cdc43 (C. cinerea protein ID: 376392) as a 2900 CDE orthogroup in this study (Table 25). Cdc43, together with Ram2, forms the type I 2901 geranylgeranyl transferase of $S$. cerevisiae, which transfers a geranyl-geranyl moiety to 2902 proteins having a CaaX sequence on their C-terminal(AA et al. 1991). One of the targets in 2903 S. cerevisiae is Rho1p, a rho-type GTPase, which is the regulatory subunit of beta-1,32904 glucan synthase(SB et al. 1999). Cdc43 orthologs were developmentally expressed in 11/6 2905 (FC=2/4) (Table 25) species as well as $C$. neoformans. They showed a marked upregulation 2906 from vegetative mycelium to primordia in A. ampla, A. ostoyae, C. aegerita, L. bicolor, $L$. 2907 edodes, C. cinerea, S. commune, and more or less constant expression in M. kentingensis, 2908 Pt. gracilis and P. ostreatus. On the other hand, orthologs of Ram2 (C. cinerea protein ID: 2909 473005) as well as those of the main substrate, Rho1 (C. cinerea protein ID: 528086) did not 2910 show strong expression dynamics. We hypothesize that the expression dynamics of Cdc43 2911 mirrors the dynamics of cell wall glucan biosynthesis in fruiting bodies.

2912 GH17 - Enzymes belonging to the GH17 family are known to crosslink $\beta$-1,3-glucan in the 2913 cell wall of yeast species and presumably N. crassa too(Patel and Free 2019). The family 2914 has been reported in a transcriptomic study of mushroom development(Krizsán et al. 2019) 2915 (as endo- $\beta$-1,3-glucanosyltransferase), and it was suggested to be involved in $\beta-1,3-g l u c a n$ 2916 crosslinking, although direct evidence for this is lacking at the moment. The examined 2917 Agaricomycetes possess 1-4 GH17 encoding genes. C. cinerea has four GH17 genes, of 2918 which only one appear to be clear orthologs of Ascomycota genes: C. cinerea 389921 is 2919 reciprocal best hit to SCW10 of $S$. cerevisiae a soluble cell wall protein with a poorly known 2920 function. GH17-s formed 2 CDE orthogroups (Table 25). One of the CDE orthogroups 2921 (represented by C. cinerea 476698) showed clear tissue-specific expression (cap- or stipe 2922 upregulation), but interestingly, this was not fully consistent across species.

2923 GH16 - The GH16 family shows glycosylhydrolase and glycosyltransferase activity and 2924 contain glucan synthase and crosslinking enzymes, which are active in cell wall synthesis

2925 (e.g. Kre6 and Skn1 of S. cerevisiae) and remodeling. GH16s were shown to crosslink $\beta-1,3-$ 2926 glucan and $\beta$-1,6-glucan to chitin fibers in Candida spp and $N$. crassa(Patel and Free 2019). 2927 Chitin-glucan crosslinking is key to morphogenesis in fungi(Arroyo et al. 2016). GH16s 2928 comprise the $\mathrm{CRH}$ (Congo Red Hypersensitive) family, three members of which (Crh1p, 2929 Crh2p and Crr1p) were originally circumscribed in S. cerevisiae. Of these, only Crr1 has a 2930 clear 1-to-1 ortholog in Agaricomycetes (C. cinerea protein ID: 449960), the other two do 2931 not. At the transcriptome level, the upregulation of $\mathrm{GH} 16$ genes have been reported before 2932 in a comparative study(Krizsán et al. 2019), in A. bisporus(Morin et al. 2012;

2933 Patyshakuliyeva et al. 2013), Leucocalocybe mongolica(Duan et al. 2021), Pleurotus spp(Xie 2934 et al. 2018; Ye et al. 2021) as well as during post-harvest development on $L$.

2935 edodes(Sakamoto et al. 2009, 2017b). Mlg1 is a functionally characterized member of the 2936 GH16 family in L. edodes assayed for its glucanase activity during postharvest 2937 senescence(Sakamoto et al. 2009).

2938 The species in this study have 21-36 GH16 encoding genes, which is consistent with 2939 a previous report on L. edodes(Sakamoto et al. 2017b). About half of the GH16 genes in 2940 each species also carry a 'Beta-glucan synthesis-associated Skn1' domain, which signifies 2941 relationship with the Kre6/Skn1 family, which is involved in $\beta-1,6$-glucan synthesis(Garcia2942 Rubio et al. 2020). We found five GH16 CDE orthogroups (C. cinerea protein ID: 502321, $2943381870,411452,169822$, S. commune 2686544) (Table 25), that are not orthologous to 
bioRxiv preprint doi: https://doi.org/10.1101/2021.12.09.471732; this version posted December $10,2021$. The copyright holder for this preprint (which was not certified by peer review) is the author/funder, who has granted bioRxiv a license to display the preprint in perpetuity. It is made available under aCC-BY-NC-ND 4.0 International license.

Running head: fruiting body genomics

2944 functionally characterized GH16 genes in the Ascomycota. Although most genes do not form 2945 conserved orthogroups, possibly because of volatility (frequent duplication/loss), it is 2946 noteworthy that each species has 1-3 genes which show a marked upregulation in the 2947 primordium stage and remain highly expressed in the fruiting body relative to vegetative 2948 mycelium. These might be involved in the formation of 3-dimensional fungal tissues. Another 2949 interesting observation is that eight out of $21 \mathrm{GH} 16$ genes in C. neoformans are also 2950 developmentally regulated, with several of them showing peak expression at $24 \mathrm{~h}$, coincident 2951 with basidium development.

2952 GH30 - Glycoside hydrolase family 30 (GH30) includes enzymes with diverse activities, 2953 which have been mostly discussed for their role as endo-xylanases in wood decay in the 2954 fungal literature(Wymelenberg et al. 2009; Katsimpouras et al. 2019). However, GH30 also 2955 includes $\beta$-1,6-glucanases, which were found to be upregulated in L. bicolor 2956 ectomycorrhizae( $C$ et al. 2014), raising the possibility of morphogenetic roles. GH30 2957 members were also upregulated during postharvest development of $L$. edodes(Sakamoto et 2958 al. 2017b). Of the 1-3 (6 in L. bicolor) GH30 genes in the examined Agaricomycetes, at most 2959 1-2 are developmentally expressed in fruiting bodies and only a single orthogroup 2960 (represented by C. cinerea 381637) was so in several species (Table 25). Genes in this 2961 orthogroup were most often upregulated in stipe tissues relative to pileus in fruiting bodies. $2962 \mathrm{GH} 30$ genes of $C$. cinerea have been proposed to facilitate stipe elongation by in the stipe 2963 base may contribute to replacing $\beta$-1,6-glucan to $\beta-1,3$-glucan crosslinking in the stipe base, 2964 which may reduce wall extensibility, leading to the cessation of growth(Liu et al. 2021).

2965 Further, the shared upregulation of GH30 genes in multiple fruiting body transcriptomes and 2966 in ectomycorrhizae provide strong evidence for its role in FCW remodeling during 2967 morphogenesis in the Basidiomycota.

2968 GH3 - The GH3 family comprises enzymes with diverse activities, including exo- $\beta-1,3-$ 2969 glucanase activity, and has been suggested to participate in cell wall remodeling in 2970 Ascomycetes(Patel and Free 2019). The upregulation of GH3 genes in fruiting bodies has 2971 previously been noted in a number of species(Zhou et al. 2018; Krizsán et al. 2019), raising 2972 the possibility that they participate in cell wall remodeling during fruiting body development. 2973 A GH3 family member, that was upregulated in fruiting bodies of Auricularia heimuer, was 2974 recently cloned and characterized(Sun et al. 2020). GH3 encoding genes are present in 7-20 2975 copies in examined Agaricomycetes. Three CDE orthogroups belonging to the GH3 family 2976 (C. cinerea protein ID: 369876, 373418, S. commune Schco3_2629974,) were detected 2977 (Table 25), genes in these orthogroups showed diverse expression patterns in the fruiting 2978 bodies of different species. It should be mentioned that some GH3 enzymes, mostly in 2979 bacteria, possess $\beta$-N-acetylhexosaminidase activity, which allows the encoded enzymes to 2980 be involved in chitin utilization, by cleaving $\mathrm{N}$-acetylglucosamine dimers into monomeric 2981 GlcNac(de Oliveira et al. 2018). Most such enzymes are bacterial, with sporadic reports of $\beta$ $2982 \mathrm{~N}$-acetylhexosaminidase activity in fungi(S et al. 2014). Therefore, whether GH3 2983 upregulation is linked to chitin utilization or glucan remodeling cannot be determined here. 2984 GH5 - The GH5 family is a very diverse group that includes $\beta$-1,4-glucanases, as well as 2985 several other activities. It includes classic cellulases involved in wood decay(Floudas et al. 2986 2012), whereas Ascomycota GH5 genes, such as Exg1, Exg2 and Exg3 of S. pombe, or 2987 Exg1 of $S$. cerevisiae, are well-known components of the cell wall glucan remodeling 2988 machinery(Dueñas-Santero et al. 2010). GH5s were shown to not only hydrolyze $\beta-1,3-$ 2989 glucan, but also to act as transglycosylases, and thus contribute to cell wall 2990 crosslinking(Arroyo et al. 2016). Based on expression patterns, GH5s were speculated to be 2991 active on beta-glucan in fruiting body cell walls also(Y et al. 2005; C et al. 2014; Almási et al. 
bioRxiv preprint doi: https://doi.org/10.1101/2021.12.09.471732; this version posted December $10,2021$. The copyright holder for this preprint (which was not certified by peer review) is the author/funder, who has granted bioRxiv a license to display the preprint in perpetuity. It is made available under aCC-BY-NC-ND 4.0 International license.

Running head: fruiting body genomics

2992 2019; Krizsán et al. 2019). Krizsan et al reported multiple subfamilies (GH5_7, GH5_9, 2993 GH5_15, GH5_49) with endo- $\beta$-1,4-mannanase, endo- $\beta-1,6$ - and exo- $\beta$-1,3-glucanase 2994 activities to be upregulated in fruiting bodies(Krizsán et al. 2019). The family includes the 2995 exo-beta-1,3-glucanase Exg1 from $L$. edodes, which was reported to be fruiting body 2996 specific(Y et al. 2005). Our re-analysis confirms that Exg1 (Lenedo1_1176007) has 2997 consistently higher expression in stipe than in cap tissues.

$2998 \quad$ GH5 is a large family with 16-20(25) copies in the examined Agaricomycetes. 2999 Consistent with their diversity, we detected six CDE orthogroups in our data (C. cinerea 3000 protein ID: $546300,405828,505989,496411,414471,373700)$ (Table 25). One of these ( $C$. 3001 cinerea protein ID: 546300), contains reciprocal best hits of Sch. pombe Exg3, a cytoplasmic $3002 \beta-1,6$-glucanase with unknown cellular function. All species in which this orthogroup is 3003 developmentally regulated showed upregulation in mature fruiting bodies. This could indicate 3004 a link to sporulation, or other late developmental events. Another orthogroup (C. cinerea 3005 protein ID: 414471) contains 1-to-1 orthologs of Sch. pombe and S. cerevisiae Exg1, which 3006 is involved in cell expansion and sporulation, picturing potentially similar roles in

3007 Agaricomycetes too. Members of yet another orthogroup (C. cinerea protein ID: 373700), are 3008 consistently higher expressed in all fruiting stages (primordia to mature FB) than in 3009 vegetative mycelia in A. ostoyae, C. aegerita, A. ampla, C. cinerea, P. ostreatus, $S$. 3010 commune and to some extent in L. bicolor and L. edodes (but not in $A$. bisporus and $P t$. 3011 gracilis).

3012 GH55 - This family contains exo- or endo- $\beta$-1,3-glucanases that have been characterized 3013 functionally in fruiting bodies of two Agaricomycetes. Exg2 was found to be expressed higher 3014 in the stipe than in the cap of L. edodes(Sakamoto et al. 2005). The GH55 family has also 3015 been reported to be upregulated during cap expansion of Volvariella volvacea and the 3016 corresponding gene was named Exg2(Tao et al. 2013). We detected on average two GH55 3017 genes in the examined Agaricomycetes (5 in A. ostoyae). One of these formed a CDE orthogroup (represented by $C$. cinerea 492834), which included L. edodes Exg2 and showed very strong induction in young fruiting body caps and stipes of $C$. cinerea, caps of $A$. ostoyae and stipes of $M$. kentingensis. Overall, these data are consistent with the suggested role of GH55 in cap expansion in V. volvacea. In L. edodes, the expression of Exg2 was higher in stipes than in cap tissues. Interestingly, the second GH55 gene of $C$. cinerea ( $C$. cinerea protein ID: 454337) (Table 25) also shows this characteristic induction and high expression in young fruiting body caps and stipes. In species with simpler morphology (S. commune, $A$. ampla) the family did not show characteristic expression dynamics. A third GH55 gene (Armosto1_267056) in A. ostoyae showed an induction in primordia relative to vegetative mycelium.

GH128 - GH128 has been first circumscribed in the context of fruiting body development, based on a fruiting body expressed $\beta$-1,3-glucanase, Glu1, in L. edodes(Y et al. 2011). Since its discovery, some reports have mentioned upregulation of $\mathrm{GH} 128$ genes in fruiting bodies(Krizsán et al. 2019; Duan et al. 2021; Merényi et al. 2021), with a single report also in connection with wood decay(Mäkinen et al. 2019). On average, the GH128 family has 2-8 members in Agaricomycetes. We detected two CDE orthogroups (represented by C. cinerea 463000,413757 ) which were expressed in the majority of species (Table 25) and several GH128 genes which were developmentally expressed in a species-specific way. In $C$. cinerea, protein 413757 represents the best hit of $L$. edodes GLU1. Available data here and in previous publications suggest that $\mathrm{GH} 128$ might be a primarily fruiting body-related gene family, worthy of further functional examination. 
bioRxiv preprint doi: https://doi.org/10.1101/2021.12.09.471732; this version posted December $10,2021$. The copyright holder for this preprint (which was not certified by peer review) is the author/funder, who has granted bioRxiv a license to display the preprint in perpetuity. It is made available under aCC-BY-NC-ND 4.0 International license.

Running head: fruiting body genomics

3039 GH71 - Four orthogroups containing developmentally regulated GH71 alpha-1,3-glucanases 3040 and beta-glucuronidases were detected (C. cinerea protein ID: 22054, 464399, 456054, 3041 492754)(Table 25). GH71 genes (also called mutanases) are involved in fungal cell wall 3042 remodeling in a wide range of species, both yeasts and filamentous fungi(Dekker et al. 2004; 3043 C et al. 2014; $\mathrm{H}$ et al. 2015). A well-characterized member of this family is the Sch. pombe 3044 endo-1,3-alpha-glucanase Agn1, which facilitates cell fission via the degradation of the 3045 septum material(Dekker et al. 2004). In previous studies of mushroom development, this 3046 family was reported only by Krizsan et al(Krizsán et al. 2019), Park et al(Park et al. 2014), 3047 and Morin et al(Morin et al. 2012). In Pleurotus eryngii, GH71 genes were found 3048 3049 downregulated in primordia after blue light stimuli(Xie et al. 2018). GH71 genes are present in 3-12 copies in the examined Agaricomycetes. One of the CDE orthogroups (represented by $C$. cinerea 456054) is upregulated in primordia relative to vegetative mycelium in 7 of the 10 species (C. cinerea, A. ostoyae, C. aegerita, L. tigrinus, L. bicolor, Ph. chrysosporium, $P$. ostreatus) in which it is present.

GH152 - The GH152 family, also known as thaumatin-like proteins, which include both putatively defense-related antimicrobial peptides and $\beta-1,3-$ glucanases. They show homology to plant thaumatin-like proteins. Because the literature reports both antimicrobial and glucanase activity for this family(Kunzler 2015), we do not attempt to subdivide them and refer to them as GH152/thaumatin proteins. The GH152/thaumatins include an experimentally characterized gene, Tlg1 in L. edodes (Lenedo1_561169), which was detected in a post-harvest screen of cell wall and lentinan degrading genes(Sakamoto et al. 2006). Lentinan is a glucan-like cell wall component with important medicinal properties in $L$. edodes. The expression of the gene was detected only after harvest, raising questions as to what the physiological role of the gene might be under natural circumstances. Our analysis of the data from Zhang et al did not reveal developmentally dynamic expression for Tlg1 using RNA-Seq data(Zhang et al. 2021c). Structure prediction for an A. ostoyae developmentally expressed protein indicated an acidic cleft in the 3D structure, characteristic of antimicrobial members of the family(Krizsán et al. 2019). A new report of antifungal activity of the thaumatin-like protein Le.TLP1 in L. edodes further supports putative defense roles for the GH152 family(Ma et al. 2021).

The family comprises 2-8 (19 in Pt. gracilis) copies in examined Agaricomycetes, many of which are poorly conserved. Therefore, while several genes are developmentally expressed in each species, only a single CDE orthogroup was detected (represented by $C$. cinerea 496344)(Table 25). The role of GH152/thaumatins in fruiting body development is unclear at the moment, it could be involved in defense against microbes, cell wall glucan remodeling, or both(Kunzler 2015).

Additional beta-glucan active families (GH1, GH6, GH12) were detected in the broader orthology-based approach of Krizsan et al(Krizsán et al. 2019), which were not detected in this study, probably because of the strict orthology definition we use. WSC family - this family of lectin-like proteins comprises a widely conserved but poorly known group of fungal proteins. The family was originally described as a putative stress receptor in S. cerevisiae(AL et al. 1999) and as a domain in an extracellular $\beta-1,3-$ exoglucanase in Trichoderma harzianum(Cohen-Kupiec et al. 1999), and later demonstrated to be a carbohydrate binding module, similar to CBMs(S et al. 2019). The best-characterized member in the Basidiomycota seems to be involved in beta-glucan binding/FCW remodeling during plant-fungal interaction in Serendipita indica(Wawra et al. 2019). Whether this is true for other WSC family members too, is not known, but is indicative of a potential role in adhesion/remodeling at the cell surface. 
bioRxiv preprint doi: https://doi.org/10.1101/2021.12.09.471732; this version posted December 10,2021 . The copyright holder for this preprint (which was not certified by peer review) is the author/funder, who has granted bioRxiv a license to display the preprint in perpetuity. It is made available under aCC-BY-NC-ND 4.0 International license.

Running head: fruiting body genomics

3087

3088

3089

3090

3091

3092

3093

3094

3095

3096

3097

3098

3099

3100

3101

3102

3103

3104

3105

3106

3107

3108

3109

3110

3111

3112

3113

3114

3115

3116

3117

3118

3119

3120

3121

3122

3123

3124

3125

3126

3127

3128

3129

3130

3131

3132

The examined Agaricomycetes harbored 2-12 copies of WSC-domain encoding genes. Of these, several showed strong induction in primordia and fruiting bodies in $A$. ostoyae, C. aegerita, C. cinerea, L. bicolor, L. edodes, L. tigrinus, Ph. chrysosporium, $P$. ostreatus and $S$. commune (but not in A. ampla and M. kentingensis). Genes with a predicted WSC domain in their encoded proteins were typically primordium-upregulated in all species. In $C$. cinerea these genes had high expression in early (proliferative) development (hyphal knots to stage 2 primordia), followed by very low expression in most genes in late developmental stages. The WSC domain can occur on its own or in combination with other enzymes (most often GH71 and glyoxal oxidase) and uncharacterized domains, indicating that it often fulfills a binding role in larger proteins. For example, WSC domain is present on GH71 proteins that form a 10/8 orthogroup (represented by C. cinerea 464399), which we discussed above.

\subsubsection{Miscellaneous cell wall remodeling (GH27, GH72, GH76, GH79, GH88, GT18, pectinesterases)}

In addition to chitin- and glucan-active families, several further CAZyme groups showed widespread developmental regulation in fruiting bodies, for which the substrates in fruiting bodies are less clear. These make very interesting groups indicating potentially novel cell wall components, however, they are hard to place in context of fruiting body development as of now.

GH79 and 88 - An orthogroup containing GH79 $\beta$-glucuronidases (C. cinerea protein ID: 470689) (Table 26), showed developmental expression in several species, consistent with reports of Krizsan et al(Krizsán et al. 2019). Low amounts of glucuronic acid was reported in $N$. crassa cell walls(Verdín et al. 2019), but the role of GH79 enzymes in cell wall biosynthesis or remodeling are unknown. On a related note, an orthogroup of GH88 glucuronyl hydrolases was detected (C. cinerea protein ID: 384105)(Table 26). GH88 genes are generally linked to pectin degradation by wood-decay fungi(van den Brink and de Vries 2011); their potential role in fruiting bodies is not known.

GH27 - Similarly, two CDE orthogroups (represented by Armosto1_254810 and Armosto1_269656) of GH27 enzymes with alpha-galactosidase or a- $N$ acetylgalactosaminidase activity were detected (Table 26). This family is discussed mostly in the context of lignocellulose degradation(Kabel et al. 2017), to our knowledge it has not been reported in fruiting body transcriptomes before. As a tendency, GH27 encoding genes were often upregulated late in the development of the containing species. Their role in fruiting body formation is not known.

GT18 - The GT18 family, which contains $\alpha-1,3(6)$-mannosylglycoprotein $\beta-1,6-\mathrm{N}$-acetylglucosaminyltransferases showed a patchy distribution in the examined species, being completely missing from the genomes of $C$. cinerea and $A$. ostoyae. However, it showed a strong upregulation in primordia relative to vegetative mycelium and constant high expression in fruiting bodies of $A$. bisporus, $A$. ampla, S. commune, $C$. aegerita and $P$. ostreatus (not in M. kentingensis and L. bicolor) (Table 26, S. commune protein ID: Schco3_2633734). This indicates fruiting body specific functions for this family, possibly in chitin synthesis, although its exact role remains unknown. To our best knowledge, the role of the GT18 family in modifying the fungal cell wall has not been described in any species. GH72 - Contrary to our expectations, members of the GH72 family did not appear to be developmentally relevant in Agaricomycetes. This family contains cell wall transglycosylases 
bioRxiv preprint doi: https://doi.org/10.1101/2021.12.09.471732; this version posted December 10,2021 . The copyright holder for this preprint (which was not certified by peer review) is the author/funder, who has granted bioRxiv a license to display the preprint in perpetuity. It is made available under aCC-BY-NC-ND 4.0 International license.

Running head: fruiting body genomics

3133 that crosslink glycoproteins to cell wall components in the Ascomycota(Kar et al. 2019), and 3134 therefore we expected it to be developmentally expressed in Basidiomycota as well. Their 3135 expression is considerable but largely constant in Agaricomycetes fruiting bodies. This could 3136 indicate a difference between Asco- and Basidiomycota cell wall composition, or that they 3137 act during fruiting body development irrespective of their constant expression.

3138 GH76 - In the Ascomycota, GH76 endo-1,6-alpha-mannosidases (Dfg5 and Dcw1 in S. 3139 cerevisiae) are required for normal cell wall synthesis. Their exact role may not be known 3140 with certainty, but they were hypothesized to be involved in the proper positioning of 3141 glycosylphosphatidylinositol-anchored cell wall proteins(Orlean 2012). To our best 3142 knowledge, the GH76 family has not yet been suggested to participate in fruiting body 3143 development in the Agaricomycetes. It is present 0-10 copies in the Agaricomycetes and 3144 shows predominantly mycelium-specific expression. We only found scattered expression 3145 dynamics in fruiting bodies, such as a peak in stipes of $P$. ostreatus

3146 (PleosPC15_2_1064904), but most genes are not developmentally regulated. Given that the 3147 family is missing in some species completely and the predominantly vegetative mycelium3148 specific expression, we hypothesize that the GH76 family does not participate, or only to a 3149 small extent, in cell wall assembly in Agaricomycete fruiting bodies.

3150 Pectinesterases - We detected a CDE orthogroup (represented by Schco3_2614029) that 3151 contains genes generally linked to pectin degradation. It contains pectinesterases that are 3152 developmentally regulated in 6/5 species (FC 2/4) (Table 26). Because pectin is not a known cell wall component in Agaricomycetes, speculating about the function of these genes is hard and may require more research.

\section{4.8.2.4. Multicopper oxidases and cupredoxins}

3156 Laccases catalyze the oxidation of diverse phenolic and non-phenolic compounds, which makes them suited to diverse (extra)cellular roles, from lignin degradation, pigment production to dye decolorization or fruiting body related functions(P 2006). Their biochemical properties provide laccases with significant potential in industrial applications. Despite very intense research on laccases (mostly in the context of lignocellulose decomposition), we still have very limited information on the precise role of laccases in fruiting body development.

Several reports of upregulation of laccase, or multicopper oxidase (MCO, the broader family in which laccases belong) genes or laccase activity in fruiting bodies of Agaricomycetes(Zhao and Kwan 1999; Ohga et al. 2000; Chen et al. 2003; Wang and Ng 2005; Madhavan et al. 2014; Sakamoto et al. 2015; Sipos et al. 2017b; Nagy et al. 2018) are available (reviewed in detail by(Kües et al. 2011)). Schizophyllum commune was reported to have $6 \mathrm{MCO}$ genes (two of which are laccases), of which 3 were reported to be upregulated in fruiting bodies(Madhavan et al. 2014) (Mco2, Mco3, Lcc1) by RT-PCR, which is consistent with later RNA-Seq data(Krizsán et al. 2019). Of these, Lcc1 showed the highest similarity to Fet3-type ferroxidases. Similarly, $P$. ostreatus laccases were reported to be expressed in a developmental stage-specific manner, with Lacc5 and Lacc12 specifically expressed in fruiting bodies and primordia, respectively, while other laccases had even or myceliumbiased expression patterns(Park et al. 2015; Jiao et al. 2018). Stage-specific MCO

3174 expression was reported in L. bicolor with specific laccase genes induced in fruiting bodies (Lcc2), ectomycorrhizae and free-living mycelium(Courty et al. 2009). These studies have linked laccase expression to two major functions, pigment production in either spores or fruiting bodies and fungal cell wall modification or cell-cell adhesion(Kües et al. 2011). Laccases were speculated to polymerize polyphenols in the intercellular spaces and thus 3179 contribute to the binding of neighboring hyphae to each other. The hypothesis of laccases 
bioRxiv preprint doi: https://doi.org/10.1101/2021.12.09.471732; this version posted December 10,2021 . The copyright holder for this preprint (which was not certified by peer review) is the author/funder, who has granted bioRxiv a license to display the preprint in perpetuity. It is made available under aCC-BY-NC-ND 4.0 International license.

Running head: fruiting body genomics

3180 being related to adhesion comes from Leatham and Stahmann(Leatham and Stahmann 3181 1981), but, to our knowledge, was never experimentally verified. Although not relevant to 3182 fruiting body formation, an interesting inverse relationship between mycelial laccase 3183 expression and flushes of fruiting body development was reported in several species, 3184 possibly related to stopping mycelium growth or nutrient acquisition when nutritional 3185 competence for fruiting is achieved (reviewed in(Kües et al. 2011)).

3186 Developmental studies indicated laccase function in fruiting body formation in 3187 Pleurotus spp(Das et al. 1997). and L. edodes(Nakade et al. 2011). In P. florida laccase 3188 gene silencing abolished fruiting body development and caused slower vegetative growth, 3189 whereas in L. edodes aerial hyphae formation was also reduced and hyphae developed 3190 thinner, non-layered cell walls upon the silencing of the Lcc1 laccase gene. Zhang et al 3191 reported faster vegetative growth and earlier fruiting in laccase (LCC1) overexpressing strains 3192 of $H$. marmoreus compared to wild type strains(Zhang et al. 2015a), although no clear 3193 hypothesis on how overexpression of a single structural gene could result in these 3194 phenotypes was presented.

$3195 \quad$ We here present annotations for multicopper oxidases, which include laccases, 3196 ascorbate oxidases and ferroxidases(Kües et al. 2011). The 11 genomes of Agaricomycetes 3197 examined here had 5-26 multicopper oxidases, a considerable proportion of which were 3198 developmentally regulated, for example, 13 out of 17 in $C$. cinerea (Table 27). We found 3199 three CDE laccase orthogroups; these comprised C. cinerea Lcc8, Lcc2 and their orthologs 3200 as well as reciprocal best hits of the $S$. cerevisiae ferroxidase Fet3. [Of note, we detected 3201 reciprocal best hits of the ferroxidase Fet3 in C. cinerea, whereas a previous report, based 3202 on phylogenetic analyses did not identify Fet3-type MCOs in this species(Kües et al. 2011); 3203 we note that this may arise from the limitations of our reciprocal best hit based search and 3204 that accurate classification of MCOs requires the examination of specific catalytic residues. 3205 Orthologs of $C$. cinerea Lcc2 (C. cinerea protein ID: 368135$)$ are also involved in 3206 morphogenesis in L. edodes (as Lcc1), and differentially expressed during Cryptococcus 3207 basidium formation(Liu et al. 2018; Merényi et al. 2021). We found that Lac4 from Volvariella 3208 volvacea, which was implicated in fruiting body development(Chen et al. 2003) showed 3209 highest similarity to the CDE orthogroup that comprised L. edodes Lcc1 (represented by $C$. 3210 cinerea 368135). In the examined Agaricomycetes, this group of genes is upregulated in 3211 caps ( $P$. ostreatus, $C$. cinerea), stipes (M. kentingensis) or primordia ( $A$. ampla), or shows 3212 flat expression curves (other species). The detected ferroxidase orthogroup was present in 7 3213 species, of which it was developmentally regulated in A. bisporus, A. ostoyae, $C$. cinerea, $L$. 3214 edodes and $P$. ostreatus ( $\mathrm{FC}>2$ ). A Fet3-like gene was also upregulated in $S$.

3215 commune(Madhavan et al. 2014), but this gene did not group into the detected orthogroup in 3216 our analysis. Ferroxidases are membrane proteins that catalyze the oxidation of $\mathrm{Fe}^{2+}$ to $\mathrm{Fe}^{3+}$ 3217 for iron uptake and have only weak laccase activities(Larrondo et al. 2003). Finally, $C$.

3218 cinerea 440170 is the orthologs of $C$. neoformans Lac1, which has been shown to be 3219 involved in melanin biosynthesis(Lee et al. 2019). This gene shows a strong expression peak in young fruiting body gills, suggesting that it is involved in producing spore pigments in C. cinerea.

In summary, experimental and gene expression evidence on the role of multicopper oxidases in fruiting body development is abundant and -omics data reveal marked developmental stage-specific expression of laccases. Hypotheses on their role either in pigment production or cell wall crosslinking/adhesion have been put forth, however, the available data do not allow us at the moment to select between these hypotheses, until more developmental studies and gene knockout phenotypes become available. 
bioRxiv preprint doi: https://doi.org/10.1101/2021.12.09.471732; this version posted December 10,2021 . The copyright holder for this preprint (which was not certified by peer review) is the author/funder, who has granted bioRxiv a license to display the preprint in perpetuity. It is made available under aCC-BY-NC-ND 4.0 International license.

Running head: fruiting body genomics

3228

3229

3230

3231

3232

3233

3234

3235

3236

3237

3238

3239

3240

3241

3242

3243

3244

3245

3246

3247

3248

3249

3250

3251

3252

3253

3254

3255

3256

3257

3258

3259

3260

3261

3262

3263

3264

3265

3266

3267

3268

3269

3270

3271

3272

3273

3274
In addition to bona fide MCOs, we identified four CDE orthogroup of proteins which belong to the Cupredoxin family, like laccases, but do not contain the typical multicopper oxidase domain. These might be highly diverged $\mathrm{MCO}$ genes or distant relatives of true MCOs with unknown functions. The five orthogroups are conserved across Agaricomycetes (C. cinerea protein ID: $356026,451850,441442$, 353997, 497434) (Table 27). One of these (C. cinerea protein ID: 356026 ) is FB-init is $A$. ostoyae, C. cinerea and $P$. ostreatus (not in $A$. ampla, $S$. commune and $M$. kentingensis), whereas a second (C. cinerea protein ID: 451850) was characteristically late upregulated in most species ( $A$. ampla, M. kentingensis, $P$. ostreatus, Pt. gracilis). Currently, it is impossible to speculate about the role of these proteins in fruiting body development.

\subsubsection{Expansins and cerato-platanins}

Fungal expansins (or expansin-like proteins) show similarity to plant expansins, proteins that modify the plant cell wall but do not show hydrolytic activity towards its polymers. Rather, they exert their action by loosening the cell wall structure, hence the name loosenins(QuirozCastañeda et al. 2011) and swollenins( $M$ et al. 2002a) for some members of the family. Fungal expansins with activities towards either the plant or fungal cell wall have been reported. Certain fungal expansins can bind chitin and facilitating the action of hydrolytic enzymes by separating cell wall chitin microfibrils. Two experimentally characterized Basidiomycota expansins are Loos1 from Bjerkandera adusta(Quiroz-Castañeda et al. 2011) and ScExlx1 from S. commune(Tovar-Herrera et al. 2015). Both were shown to bind chitin and facilitate the action of chitinases, therefore, it was concluded that they modify, but not hydrolyze the chitin polymer structure. Recent studies suggested that expansin-like proteins are involved in stipe cell elongation in $F$. velutipes( $Q$ et al. 2018) and $C$. cinerea(Niu et al. 2015) and were shown to have a role in stipe elongation, by loosening the chitin polymer structure (reviewed by(Liu et al. 2021)).

Several expansins show expression patterns compatible with a role in fruiting body development in previous transcriptome-based studies(Barsottini et al. 2013; Sipos et al. 2017b; Q et al. 2018; Almási et al. 2019; Krizsán et al. 2019). Based on analyses of 201 fungal genomes, Krizsan et al reported that expansins are enriched in the genomes of Agaricomycetes(Krizsán et al. 2019). The examined mushroom-forming fungi possess 9-27 expansin genes, with $C$. cinerea having 13 . Given the dual specificity of expansins, it is likely that several of these are involved in cellulose degradation. However, a significant proportion (19-85\%) of expansin genes are developmentally regulated in fruiting bodies, indicating roles in morphogenesis too. Expansins are a highly volatile group of genes that did not group into conserved orthogroups in our analysis (except two CDE orthogroups, see below), therefore, comparative analysis of their expression patterns across species is hardly possible. Within C. cinerea expansins show diverse expression peaks, in gills, cap or stipes with some genes highest expressed in vegetative mycelia too (Fig. 11). This could indicate tissue- and stagespecific roles for expansins in fruiting body formation. For example, four genes had a strong induction in stipes (C. cinerea protein ID: $451456,464389,359952,461698$ ), which is consistent with previous reports of their participation in stipe elongation(Liu et al. 2021). Three $C$. cinerea genes showed significant increase in expression from the vegetative mycelium to hyphal knots (C. cinerea protein ID: 461698, 286849, 496836). Despite the volatility of the family, we detected three putative expansin orthogroups (represented by $C$. cinerea 496836, 467907 and 461698) which were developmentally regulated in 12/10, 11/10 and 10/5 species (at FC>2/4) (Table 28). 
bioRxiv preprint doi: https://doi.org/10.1101/2021.12.09.471732; this version posted December 10,2021 . The copyright holder for this preprint (which was not certified by peer review) is the author/funder, who has granted bioRxiv a license to display the preprint in perpetuity. It is made available under aCC-BY-NC-ND 4.0 International license.

Running head: fruiting body genomics

3275 Cerato-platanins - Although initially thought to be related to hydrophobins, cerato-

3276 platanins share a common origin with expansins(Barsottini et al. 2013; Gaderer et al. 2014;

3277 Baccelli 2015; Luti et al. 2020). First described from the ascomycete Ceratocystis platani,

3278 they were initially and generally considered to be related to pathogenesis(Gaderer et al.

3279 2014; Baccelli 2015; Chen et al. 2017; Luti et al. 2020), however, there is piling evidence, so

3280 far only from transcriptome studies, that they might be important in fruiting body

3281 development as well. In such studies, cerato-platanin genes showed extensive expression

3282 dynamics in fruiting bodies, suggesting an activity on the fungal cell wall(Plaza et al. 2014b;

3283 Sipos et al. 2017b; Almási et al. 2019; Krizsán et al. 2019; Liu et al. 2020b), or in

3284 defense(Kunzler 2015). Structure-based biochemistry studies in Moniliophthora perniciosa

3285 showed that certain cerato-platanins are able to bind chitin and are active on the fungal cell

3286 wall(Barsottini et al. 2013). Like expansins, cerato-platanins are strongly enriched in the

3287 genomes of mushroom-forming fungi(Chen et al. 2017; Krizsán et al. 2019). There is,

3288 unfortunately, little information on their mechanistic role in fruiting body development, except

3289 a the study by de Barsottini(Barsottini et al. 2013), which provides proof that cerato-platanins

3290 can be active on chitin and the fungal cell wall, providing a biochemical link to their potential

3291 role in fruiting body development.

$3292 \quad$ Our analyses identified 1-8 cerato-platanins in the genomes of examined mushroom-

3293 forming fungi, which is in line with copy numbers reported recently(Chen et al. 2017). Like

3294 expansins, cerato-platanins are quite volatile and we identified only two conserved CDE

3295 orthogroups (C. cinerea protein ID: 439071, 544218) (Table 28), with most genes scattered

3296 in species-specific or smaller groups. The first showed opposing expression patterns in

3297 different species: in $C$. cinerea (protein ID: 544218) it was upregulated in caps relative to

3298 stipes, whereas in $A$. ostoyae and $M$. kentingensis it was upregulated in stipes relative to

3299 caps. In $P$. ostreatus and $M$. kentingensis it showed the highest expression in vegetative

3300 mycelia. In $A$. ampla and $S$. commune it showed a very strong induction (>1000-fold) in

3301 mature fruiting bodies.

$3302 \quad$ Expression data across species indicates that developmentally dynamic cerato-

3303 platanin expression was observed only in certain species, while not in others. For example,

3304 no developmentally regulated cerato-platanins were found in Ph. chrysosporium, $A$.

3305 bisporus, only a single in $M$. kentingensis, but several in A. ostoyae, C. cinerea, L. tigrinus,

3306 etc. This indicates that developmental expression of cerato-platanins may be a species-

3307 specific, rather than a conserved feature in mushroom-forming fungi.

3308

\subsubsection{Miscellaneous cell wall related families (HTP, DyP, AA3, AA5, GH76, GT8,} Slt2, Cfs1, Eln3, peptidases)

We identified several CDE gene families which are potentially linked to the fungal cell wall, however, evidence is lacking on their mechanistic roles and/or they could not be confidently ascribed to either chitin or glucan, and/or are not enzymatic proteins. These are discussed in this chapter, with noting that the widely observed developmental expression makes these protein families interesting, however, their assignment to the cell wall is preliminary. HTPs - Heme-thiolate peroxidases (HTPs) are fungal-specific proteins that were first described in $C$. aegerita (as haloperoxidase) and use $\mathrm{H}_{2} \mathrm{O}_{2}$ to modify substrates( $R$ et al. 2004). While their enzymatic properties have been quite thoroughly studied(M et al. 2015a), their biological roles are poorly known. A remarkable expansion of the family was noted in $A$. 
bioRxiv preprint doi: https://doi.org/10.1101/2021.12.09.471732; this version posted December $10,2021$. The copyright holder for this preprint (which was not certified by peer review) is the author/funder, who has granted bioRxiv a license to display the preprint in perpetuity. It is made available under aCC-BY-NC-ND 4.0 International license.

Running head: fruiting body genomics

3321 hypothesis that HTPs are linked to the litter decomposer lifestyle(Morin et al. 2012). In the 3322 context of fruiting bodies, HTPs were reported only in A. ostoyae(Sipos et al. 2017b) so far. 3323 We detected two CDE orthogroups of HTPs (represented by C. cinerea 479688, 3324 442333)(Table 29) as well as several other species-specific developmentally regulated HTP 3325 genes. The examined Agaricomycetes have 2-10 genes with an expansion to 23 genes in 3326 both $M$. kentingensis and $A$. bisporus as reported earlier(Morin et al. 2012). Most of the 3327 species had at least one HTP encoding gene that is strongly upregulated (up to 50 -fold) from 3328 vegetative mycelium to the first primordium stage. These observations establish HTPs as a 3329 potentially important morphogenetic gene family in fruiting body development with currently 3330 unknown function. We tentatively discuss this gene family under the chapter on cell wall 3331 modification, however, it should be mentioned that this is based on an assumption of a 3332 potential role of this family.

3333 DyPs - Dye decolorizing peroxidases (DyPs) are a group of fungal peroxidases that belong 3334 to the broader heme-containing peroxidase family. They are mostly discussed in the context 3335 of plant cell wall degradation by fungi(Floudas et al. 2012); to our knowledge, this is the first 3336 report of its potential role in fruiting body morphogenesis. We detected 0-5 copies (missing in 3337 A. ampla, S. commune and A. bisporus) and a patchy distribution in Agaricomycetes.

3338 However, in species that have DyP genes, these are often developmentally expressed. DyP 3339 genes show significant upregulation from vegetative mycelium to the first primordium stages in A. ostoyae, C. cinerea, C. aegerita, L. bicolor and P. ostreatus. In M. kentingensis all DyP genes show a marked stipe expression peak, whereas in Pt. gracilis, expression curves are flat. DyP genes grouped into a single CDE orthogroup (represented by C. cinerea 407604, Table 29). While some of the copy number variation is explained by the lignin-degrading ability of fungi(Almási et al. 2019), the developmental expression of DyP genes suggests morphogenetic roles as well, possibly in remodeling the cell wall, although this is based on assumptions distilled from the general activity of the family. It would be interesting to investigate if, similar to observations during lignin degradation, $\mathrm{H}_{2} \mathrm{O}_{2}$-generating enzyme gene expression is co-regulated with peroxidase expression patterns.

AA5_1 (GLOX) - Glyoxal oxidases (GLOX) in the AA5_1 family of auxiliary activities are $\mathrm{H}_{2} \mathrm{O}_{2}$ generating enzymes that facilitate the oxidative cleavage of lignin by peroxidases(Floudas et al. 2012). Surprisingly, GLOXs have also been reported to be upregulated in fruiting bodies in 6 species of Agaricomycetes(Krizsán et al. 2019) and were detected in fruiting bodies of Auricularia polytricha by protein mass spectrometry(Jia et al. 2017). Since lignin is obviously missing from fruiting bodies, they were speculated to contribute to cell wall remodeling(Krizsán et al. 2019). We found 1-22 GLOX genes in the examined Agaricomycetes, the fewest in the Schizophyllaceae (A. ampla, S. commune) possibly because of their reduced morphology or reduced lignin-degrading ability(Almási et al. 2019). Two CDE orthogroups (C. cinerea protein ID: 263775 and 440836 Table 29), and several species-specific developmentally regulated GLOX genes were found in our data. We found GLOX genes significantly upregulated in primordia relative to vegetative mycelium (FB-init) in A. ostoyae, A. ampla, C. cinerea, L. tigrinus, L. bicolor, S. commune, Ph. chrysosporium, $P$. ostreatus and $C$. aegerita (not in $M$. kentingensis, L. edodes and $A$. bisporus). Two of the three $C$. neoformans GLOX genes also showed significant upregulation during basidium development. These data indicate that GLOXs may be important morphogenetic factors in the Basidiomycota.

AA3_1 - cellobiose dehydrogenases in the AA3_1 family (GMC oxidoreductases) are known to donate electrons to LPMOs during lignocellulolysis in wood-decay fungi(Floudas et al. 2012). There are only two reports on the potential role of CDHs in fruiting body development, 
bioRxiv preprint doi: https://doi.org/10.1101/2021.12.09.471732; this version posted December 10,2021 . The copyright holder for this preprint (which was not certified by peer review) is the author/funder, who has granted bioRxiv a license to display the preprint in perpetuity. It is made available under aCC-BY-NC-ND 4.0 International license.

Running head: fruiting body genomics

3369 one is a comparative transcriptomics study(Krizsán et al. 2019) and the other is a report of 3370 the interaction between $\mathrm{CDH}$ and laccases in the formation of cinnabarinic acid, the red 3371 fruiting body pigment of Pycnoporus cinnabarinus(Temp and Eggert 1999). A CDE 3372 orthogroup of cellobiose dehydrogenase (CDH) genes (C. cinerea protein ID: 469460)(Table 3373 29) was developmentally regulated in all species in which it was present. Additional CDH 3374 genes (2-8 genes in Agaricomycetes) were developmentally regulated in a species-specific 3375 manner in several species, although expression levels were low in many cases. The 3376 biological significance of $\mathrm{CDH}$ in fruiting body development is currently unknown; based on previous studies it may be related to cell wall remodeling or pigment production.

SIt2 kinase family - A serine-threonine kinase orthologous to the MAP kinase involved in the cell wall integrity pathway of $S$. cerevisiae (SIt2) was detected as a CDE orthogroup ( $C$. cinerea protein ID: 413665). SIt2 is a terminal kinase in the pathway that phosphorylates downstream transcription factors (RIm1 and Swi4/6 in yeast)(Levin 2005). Although the gene is developmentally regulated in several species (Table 29), expression patterns are not consistent across taxa. Given the potentially pleiotropic role of this kinase, it is hard to guess its role in fruiting bodies, but extrapolations from its role in $S$. cerevisiae would suggest links to cell wall integrity, morphogenesis or the cell cycle.

3386 Cfs1 family - The orthogroup containing C. cinerea Cfs1 (C. cinerea protein ID: 447925), a cyclopropane fatty acid synthase, was developmentally regulated in 10/7 (FC>2/4) species (Table 29). Cfs1 was identified in a mutant screen of strains with developmental defects; its mutation leads to failures in the progression of hyphal knot development(Liu 2005) in C. cinerea (stalled at primary hyphal knot). The corresponding gene is highly induced $1 \mathrm{~h}$ after blue light exposure(Sakamoto et al. 2018). We discuss this gene under the cell wall, although some evidence suggests it may be more closely related to plasma membrane assembly. Structurally, Cfs 1 and related proteins are mycolic acid cyclopropane synthases, a family that occurs in 1-6 copies in the examined Agaricomycetes. Their function is not known, but presumably they are involved in the synthesis of long fatty acids (mycolic acid is not known to occur in fungi). In most examined species ( $A$. ampla, M. kentingensis, $C$. cinerea, $P$. ostreatus, $S$. commune), the gene is upregulated in primordia relative to vegetative mycelium, consistent with its phenotypic role in hyphal knot development. Eln3 family - This family is based on the Eln3 gene of $C$. cinerea, which was found in a REMI mutagenesis screen. The mutant strain displayed defects in stipe elongation and mutant stipe cells were disorganized and there were large gaps among stipe cells, whereas in the wild type strain stipe cells align closely with each other(Liu 2005). It has been hypothesized that Eln3 is involved in cell-cell connections, possibly adhesion.

We found that $C$. cinerea Eln3 (C. cinerea protein ID: 380322) formed a small CDE orthogroup present in 7 species and was developmentally regulated in 6 species (Table 29). In these species the gene was predominantly FB-init and stipe upregulated, in agreement with a previously reported role in stipe elongation(Arima et al. 2004). It should be noted that, in many species, upregulation in primordia is easy to confuse with stipe-specific upregulation, because primordia often comprise predominantly stipe tissue. In general, we found 7-8 genes the examined Agaricomycetes (only 4 in A. bisporus) with identical domain composition to Eln3. This does not guarantee identical function, nevertheless is indicative of the diversity of this family in mushrooms. These genes were often FB-init and showed stipe or cap-specific upregulation depending on the gene and the species. For example, in $C$. cinerea we found that 5 out of 6 genes were stipe-upregulated. In A. ostoyae we found four cap- and two stipe-upregulated, whereas in L. bicolor two cap- and one stipe-upregulated gene. The widespread developmental regulation of Eln3 and its homologs suggest 
bioRxiv preprint doi: https://doi.org/10.1101/2021.12.09.471732; this version posted December 10,2021 . The copyright holder for this preprint (which was not certified by peer review) is the author/funder, who has granted bioRxiv a license to display the preprint in perpetuity. It is made available under aCC-BY-NC-ND 4.0 International license.

Running head: fruiting body genomics

3417 morphogenetic functions, possibly in association with the cell wall. Based on its domain 3418 composition, we hypothesize it represents a cell wall active, possibly new CAZyme family 3419 not yet recognized by Cazy.org.

3420 GT8 - The GT8 family has 1-7 (most frequently 5) copies in the Agaricomycetes. The GT8

3421 family includes both $\mathrm{N}$-acetylglucosaminyltransferases linked to the modification of $\mathrm{N}$-linked

3422 glycans in the Golgi, and glycogenins involved in glycogen metabolism. Buser et al

3423 hypothesized that fruiting body-specific N-glycans generated by GT8 enzymes may

3424 contribute to adhesion in C. cinerea(Buser et al. 2010a). GT8 genes formed one CDE

3425 orthogroup, represented by $C$. cinerea 461263 FC2/4: $7 / 3$ species), which is closest to $S$.

3426 cerevisiae Glg1 glycogenin (discussed under carbohydrate metabolism). Of the other GT8

3427

3428

3429

3430

3431

3432

3433

3434

3435

3436

3437

3438

3439

3440

3441 genes $C$. cinerea (C. cinerea protein ID: 453097, =CcGnt1)(Buser et al. 2010a), A. ostoyae, L. tigrinus, $P$. ostreatus, $A$. ampla and $S$. commune had one FB-init gene each, however, these did not form CDE orthogroups. CcGnt2 (C. cinerea protein ID: 412828 ) was not expressed. Other species did not have developmentally regulated genes. Due to the variability of the family, it is hard to determine whether these are closer to glycogenins or $\mathrm{N}$ acetylglucosaminyltransferases.

$\mathrm{N}$-glycosylation - Mannosylation and N-glycosylation of proteins in the ER is a key process for cell wall synthesis. Berends et al provided a detailed view of the $\mathrm{N}$-glycosylation machinery in S. commune, $C$. cinerea and $L$. bicolor based on orthology to the $S$. cerevisiae $\mathrm{N}$-glycosylation pathway members and expression data in $S$. commune fruiting bodies and monokaryotic mycelium(Berends et al. 2009). They conclude that Agaricomycetes have the gene repertoire to produce oligomannose structures only, in contrast to some Ascomycota which can produce very complex modifications to proteins. Our expression results broadly agree with theirs in that we detected very low dynamics of corresponding genes (not shown) in the fruiting body transcriptomes analyzed here.

3442

\subsection{Cell surface and cell wall proteins}

3444 Secreted cell surface proteins fulfill diverse functions in the life of fungi, ranging from 3445 signaling, interactions with the environment, adhesion, cell wall modifications, but also 3446 communication with hosts in mutualistic or pathogenic interactions(Pellegrin et al. 2015; 3447 Essen et al. 2020). The fungal cell surface proteome and secretome profile of each species 3448 in a given environment is unique, making constituents of fungal communities recognizable by their extracellular protein profiles(Campbell et al. 2015). Secreted proteins have been the subject of intense research, mostly in the context of interactions between fungi and other organisms, adhesion (e.g. via GPI-anchored adhesins) of fungal cells to various surfaces(Essen et al. 2020; M et al. 2020b). Fruiting bodies, as environmentally exposed, 3dimensional multicellular structures, can be expected to display unique cell-surface attributes. In fact, cell surface proteins include some of the best-known fruiting bodyassociated proteins (e.g. hydrophobins, lectins, see below) or proteins related to defense (see these in a separate chapter). Conceivably, adhesion and/or cell-to-cell communication, two of the most important multicellular functions(Knoll 2011), could be realized via the action of secreted cell surface proteins in fruiting bodies, to just name a few of the diverse ways mushroom-forming fungi might make use of their cell surface protein repertoire. A significant open question in fruiting body biology is the nature and role of the extracellular matrix and the biochemical and communication events that take place in the interhyphal space. Cell 
bioRxiv preprint doi: https://doi.org/10.1101/2021.12.09.471732; this version posted December 10,2021 . The copyright holder for this preprint (which was not certified by peer review) is the author/funder, who has granted bioRxiv a license to display the preprint in perpetuity. It is made available under aCC-BY-NC-ND 4.0 International license.

Running head: fruiting body genomics

3462 surface proteins are undoubtedly important players in these processes, and therefore 3463 represent interesting targets of future research.

3464 While some cell surface proteins are the best examined fruiting body proteins (e.g.

3465 hydrophobins), the following inventory of conserved developmentally expressed genes

3466 shows that there is plenty of new insight to be learned about the cell surface proteome of

3467 Agaricomycetes fruiting bodies. In this chapter we discuss cell surface protein families in the

3468 context of CDE orthogroups detected in our meta-analysis of fruiting body transcriptomes.

\section{4.9.1. Hydrophobins}

3471 Hydrophobins are some of the best-known proteins in fruiting bodies. They confer

3472 hydrophobicity to hyphae, which they achieve by self-assembling into a water-repellent

3473 rodlet layer on the cell surface. Hydrophobins were hypothesized to line air channels in

3474 fruiting bodies(Lugones et al. 1999b), but also contribute to water sealing, which is important

3475 in fruiting bodies as they rely on water transported to them from the vegetative

3476 mycelium(Nehls and Dietz 2014). The family has extensively literature, in general(Wösten

3477

3478 2003; Linder et al. 2005; Bayry et al. 2012), in relation to fruiting body development(Ohm et al. 2010; Kües and Navarro-González 2015; Kim et al. 2016; Sammer et al. 2016; Y et al. 2019) or on potential uses in bioengineering(Wösten and Scholtmeijer 2015). We therefore concentrate on novel aspects detected in our data that have not or only rarely been reported before.

3482

3483

Basidiomycota hydrophobins mostly belong to class I hydrophobins(Sammer et al. 2016). On average 11-44 hydrophobin genes (only 6 in M. kentingensis) were detected in the examined Agaricomycetes, a significant proportion of which were developmentally regulated. They can be arranged into distinct groups based on expression patterns. Previous studies reported a near complete turnover of hydrophobin genes from vegetative mycelium to fruiting body, indicating that two distinct sets of hydrophobins exist(Ma et al. 2007; WW et al. 2008; Cheng et al. 2013a). We also observed that mycelium-expressed and fruiting bodyexpressed hydrophobins form distinct groups. For example, the numbers of hydrophobins showing mycelium- vs fruiting body specific expression was 6/5, 1/9, 10/4, 23/3 and 5/20 (at fold change 4/2) in Ph. chrysosporium, S. commune, P. ostreatus, A. ostoyae and $C$. cinerea, respectively. $A$. ostoyae further has two rhizomorph-specifically expressed hydrophobins, indicating morphogenesis-related diversification of the family. This is in line with observations in $F$. velutipes, where most hydrophobin genes were found to be primordium-upregulated(Kim et al. 2016). We also detected hydrophobin genes expressed during early development, but not later, indicating functions linked to primordium development, which are turned off during later development. These observations further substantiate the functional diversification, probably driven by morphogenesis and complexity.

Despite the large diversity and developmental expression of hydrophobins, only 2 CDE orthogroups were detected (C. cinerea protein ID: 99661, 419467) (Table 30), which were present in only a subset ( 11 and 8 species, respectively) of the examined species. This is due to the frequent duplication of hydrophobin genes(Mgbeahuruike et al. 2017), which quickly erases clear orthology relationships. CDE orthogroups did not show conserved expression patterns. Interestingly, M. kentingensis has only 6 hydrophobin genes, none of which were developmentally expressed in our reanalysis of data from $\mathrm{Ke}$ et al(Ke et al. 2020a). A possible explanation for this is that this species produces hygrophanous, rather than hydrophobic fruiting bodies, which absorb rather than repel water. The relatively low 
bioRxiv preprint doi: https://doi.org/10.1101/2021.12.09.471732; this version posted December 10,2021 . The copyright holder for this preprint (which was not certified by peer review) is the author/funder, who has granted bioRxiv a license to display the preprint in perpetuity. It is made available under aCC-BY-NC-ND 4.0 International license.

Running head: fruiting body genomics

3508 number of developmentally regulated hydrophobin genes in $A$. ostoyae may also be 3509 explained by the hygrophanous caps of this species. Hygrophanicity is a widespread trait in 3510 the Agaricales and might explain some of the differences in hydrophobin diversity and 3511 expression.

3512 Taken together, hydrophobins, as already established by previous research, are one 3513 of the best-known fruiting body-related structural cell surface proteins in the Agaricomycetes, 3514 with almost all species expressing developmental stage-specific suites of hydrophobin 3515 genes.

3516

\section{4.9.2. Lectins}

3518 Lectins will be also discussed in Chapter 10 on defense-related proteins, however, a role in 3519 defense has been proven for only a subset of the diverse lectin repertoires of mushroom3520 forming fungi; the remaining non-defense, or functionally unknown lectins are discussed in 3521 this chapter (even though not all lectins may be secreted or their secretion status is unclear 3522 at the moment, (Boulianne et al. 2000)). Lectins are proteins that specifically recognize and 3523 bind various carbohydrate moieties, mostly on the cell surface. This ability makes them 3524 suitable for multiple biological roles, from bacterial agglutination and cytotoxicity against 3525 mushroom grazers(Bleuler-Martínez et al. 2011; Kunzler 2015) to carbohydrate binding 3526 modules that fulfill roles related to the fungal cell wall (e.g. remodeling), adhesion, cell-to-cell communication or other functions in the extracellular matrix. Their carbohydrate binding ability makes them interesting in a wide range of biomedical fields(Hassan et al. 2015), including cancer research, which led to the broader lectin family rapidly expanding. Mushroom fruiting bodies remain one of the richest sources of lectins for applied research. However, regarding their physiological role for the fungi, boundaries between defenserelated and other lectins are far from clear at the moment(Bleuler-Martínez et al. 2011). Due to interest in their practical applications, there is often more information on the structure of mushroom lectins than on their roles in the fungus' life. Fruiting bodies express a diverse set of lectins (reviewed by( $A$ et al. 2013; Kobayashi et al. 2014; $Y$ and $H$ 2014)) and several of the well-known lectin families were first isolated from mushrooms. For example, the fungal fruit body lectin family was established based on the Xerocomus chrysenteron XCL protein, which was suggested to have insecticidal activity ( $C$ et al. 2004). Besides interest in their biotechnological applications, lectins have been in the focus of research on fruiting bodies. Early reports identified specific hyphal knot and primordium upregulated galectins (Cgl1 and Cgl2) in C. cinerea(Boulianne et al. 2000; Bertossa et al. 2004), which were initially postulated to be cell-cell adhesive or communication related molecules that may bind basidiolipids(Walser et al. 2005). Simultaneous silencing of the cgl1 and cgl2 genes did, however, not disturb fruiting, arguing against his hypothesis(MA et al. 2006). The lectins were rather shown to inhibit the development of insect and nematode larvae, in nematodes via binding to a specific $\mathrm{N}$-glycan epitope, suggesting a function in defense against predators(Butschi et al. 2010; Bleuler-Martínez et al. 2011). Both genes were found upregulated in primordia, relative to vegetative mycelium in recent RNA-Seq based studies $(\mathrm{cgl1}=$ C. cinerea 473274, cgl2 = C. cinerea 488611). Later, Wälti et al described cgl3 $(=C$. cinerea 539794), which resembles galectins, but binds chitooligosaccharides and had no effect on larval development of insects and nematodes(Wälti et al. 2008). Beta-glucan binding lectins of Serepindita indica may be involved in fungal cell wall remodeling at the plant-fungal interface(Wawra et al. 2019). Several other recent reports of individual lectins 
bioRxiv preprint doi: https://doi.org/10.1101/2021.12.09.471732; this version posted December 10,2021 . The copyright holder for this preprint (which was not certified by peer review) is the author/funder, who has granted bioRxiv a license to display the preprint in perpetuity. It is made available under aCC-BY-NC-ND 4.0 International license.

Running head: fruiting body genomics

3554 from a diverse set of medicinal or edible mushrooms is available but their detailed discussion 3555 is beyond the scope of this paper. Lectins are also involved in Ascomycota fruiting body 3556 (perithecium) formation and interestingly, the gene recently described in Podospora anserina showed highest similarity to Xerocomus lectins(Nowrousian and Cebula 2005). Several lectin families are overrepresented in mushroom-forming fungi(Krizsán et al. 2019), suggesting key roles in their life cycle.

'Lectomes' evolve fast (like other defense genes) both in terms of sequence and gene content. Most lectin families a phylogenetically patchily distributed in mushroom forming fungi, with the probable sole exception of ricin-B lectins, which occur in all examined species. It has been suggested that the fast gene turnover can contribute to the expression of defense gene cocktails by the fungus, which is advantageous and that horizontal gene transfer from bacteria can provide a mechanistic explanation for the phylogenetic patchiness of several lectin families(Moran et al. 2012; Kunzler 2015).

We here provide an overview of lectin copy numbers in the examined Agaricomycetes in the context of 8 main families, but note that more recent classifications(Lebreton et al. 2021) significantly reorganized lectin families and may provide a more nuanced view on lectin repertoires. Lectin copy numbers and expression patterns were summarized in the context of 6 Agaricomycetes species by Krizsan et al(Krizsán et al. 2019) with lectins showing the RicinB fold (which includes protease inhibitors too, see above and( $\mathrm{J}$ et al. 2016)) being most diverse and present in all species, whereas other families are more patchy. Nevertheless, all lectin families had developmentally regulated genes, often showing strong induction in primordium stages.

As noted earlier (e.g. (Kunzler 2015)), lectins are poorly conserved and hence mostly did not form conserved orthogroups in our analysis. Only two CDE orthogroups, all belonging to the RicinB family were detected (C. cinerea protein ID: 495335, 353931)(Table $31)$. Both orthogroups contained secreted proteins. Expression patterns were poorly conserved within these orthogroups, but we note that each had FB-init genes in several species, indicating that these secreted Ricin-B family members are involved in the initiation of fruiting body development.

\subsubsection{Other cell surface proteins (HsbA, Con6, PriA, AA7, CFEM, wax synthases,} fasciclins, peptidases, intradiol ring cleavage dioxygenase) fumigatus(KY et al. 2001). Detailed functional information is not available for either of these genes. The family contains GPI-anchored cell surface proteins that can bind to various surfaces and was circumscribed in the context of appressorium attachment and lignocellulose degradation in Ascomycetes. The common denominator between these circumstances may be the need for adhesion of hyphae to various surfaces, enabling secreted lytic enzymes to exert their action. In the context of fruiting bodies, the family was first reported in fruiting bodies of $A$. ostoyae(Sipos et al. 2017b). In our current analysis, we detected 0-3 members of the family in Agaricomycetes (missing from L. tigrinus, $P h$. chrysosporium, A. ampla. S. commune), all of which were developmentally expressed. The family did not form CDE orthogroups. Expression of members of this family showed significant dynamics (>100-fold), mostly with gill- or cap-specific expression peaks late during development. Functional analysis of this gene family in mushroom-forming fungi 
bioRxiv preprint doi: https://doi.org/10.1101/2021.12.09.471732; this version posted December 10,2021 . The copyright holder for this preprint (which was not certified by peer review) is the author/funder, who has granted bioRxiv a license to display the preprint in perpetuity. It is made available under aCC-BY-NC-ND 4.0 International license.

Running head: fruiting body genomics

3600 would be very interesting. If, as speculated above, the family is related to adhesion, then it 3601 may provide a missing link to adhesion in complex multicellularity in mushrooms.

3602 The Con6 protein family include small hydrophilic peptides that were first discovered as 3603 conidium-specific proteins in N. crassa and later shown in A. nidulans (ConF and ConJ) to 3604 be responsible for desiccation resistance of asexual spores(Suzuki et al. 2013). The family 3605 also includes the meiotically expressed Sch. pombe gene cum1. The Con6 family is 3606 conserved in Agaricomycetes, with 1-7 copies in the examined genomes, most of which are 3607 developmentally regulated. They showed two major expression patterns in all species: (i) 3608 strong induction in mature gills or combined gill+cap samples and/or (ii) upregulation in 3609 primordia relative to vegetative mycelia (Fig. 12, Supplementary Fig. 8). For some genes 3610 both for others only one of these expression patterns were observable. The family seems 3611 poorly conserved, nevertheless, they formed three CDE orthogroups (represented by $C$. 3612 cinerea 470234, 186809, S. commune 2624349)(Table 32). The gill-specific upregulation, 3613 combined with the conidium-specific expression of the gene family in filamentous 3614 Ascomycota, suggests that Con6 proteins may be involved in producing a spore-specific protein coating in these species. The orthogroup represented by $C$. cinerea 186809 showed upregulation in primordia of most species and has low expression in vegetative mycelia and later developmental stages. The strong upregulation of several Con6-encoding genes in primordia relative to vegetative mycelia in virtually all species suggests that it has general roles in fruiting bodies as well, possibly in regulating hydrophobicity/hydrophilicity of the cell surface, although this requires further research.

Wax synthases are long-chain alcohol O-fatty-acyltransferases that synthesize wax esters, a typical constituent of the cuticula of plants or the sebum of the animals(Li et al. 2008). To our best knowledge, only Krizsan et al(Krizsán et al. 2019) indicated developmental expression for this family previously.

We detected 4-16 wax synthases in the examined Agaricomycetes, the fewest in $A$. bisporus, but found no CDE orthogroups, suggesting a high duplication rate in the family. However, each species (except Pt. gracilis and A. bisporus) had 1-2 FB-init wax synthase genes, which suggests fruiting body specific roles for these genes. We observed considerable (>100-fold) upregulation compared to vegetative mycelia in many cases (Fig. 13, Supplementary Fig. 9). Given the widespread upregulation of wax synthases in mushroom-forming fungi, it is possible that they produce a wax-like layer on the surface of fruiting body cells. On plants, wax-coating of leaves serves as a defense system that inhibits penetration of pathogenic fungi and water-sealing(Lewandowska et al. 2020). Products of fungal wax synthases, although unknown at the moment, could have similar properties in fruiting bodies, worth investigating in the future.

PriA family - this family comprises secreted cell surface proteins first described as a primordium-induced protein in L. edodes (hence its name)( $S$ et al. 1992). The gene has been quite extensively studied in L. edodes( $T$ and $\mathrm{K} 2000$; $T$ et al. 2004a), where a link to zinc homeostasis has been suggested. It was also detected as developmentally regulated in F. velutipes(Liu et al. 2020b), P. eryngii(Fu et al. 2017), L. edodes(S et al. 1992) and in six species by Krizsan et al(Krizsán et al. 2019). The family also includes the virulence-related secreted adhesin $\mathrm{Cfl} 1$ of $C$. neoformans, which is highly induced during sexual development and has been thoroughly researched in C. neoformans(Wang et al. 2012, 2013). Cfl1 has been suggested to act as a morphogen that induces neighboring cells to phenocopy the producing cells' morphology(Wang et al. 2013). Cfl1 has 4 homologs in $C$. neoformans, which have stage-specific expression including Dha1, which is primarily expressed in basidia(Gyawali et al. 2017). The family is present in 0-4 copies in the Agaricomycetes and 
bioRxiv preprint doi: https://doi.org/10.1101/2021.12.09.471732; this version posted December $10,2021$. The copyright holder for this preprint (which was not certified by peer review) is the author/funder, who has granted bioRxiv a license to display the preprint in perpetuity. It is made available under aCC-BY-NC-ND 4.0 International license.

Running head: fruiting body genomics

3648 formed two CDE orthogroups. The first of these (C. cinerea protein ID: 359866)(Table 32)

3649 showed a very conserved, mycelium biased expression; it was highly expressed in

3650

3651

3652 vegetative mycelia and the first primordium stage, then virtually unexpressed in all later stages in A. ampla S. commune, M. kentingensis and $P$. ostreatus. In A. ostoyae expression

3653 dropped immediately after the mycelium sample. The second CDE orthogroup (represented by $C$. cinerea 419688) contains best hits of $C$. neoformans Dha1 and showed a peak expression in gills and caps of $C$. cinerea, $A$. bisporus, $C$. aegerita, $L$. edodes and late stages of $S$. commune and $A$. ampla. At the same time, these genes were significantly upregulated (up to 50-100x) in primordia relative to vegetative mycelium. In $L$. edodes, our RNA-Seq based observations are consistent with the first reports of the PriA gene as a primordium-upregulated one(S et al. 1992). The third orthogroup (represented by $S$. commune 2681702), was present in only 8 species, and was FB-init in A. ampla, A. ostoyae, $P$. ostreatus, $M$. kentingensis and $S$. commune (Table 32). In summary, the PriA family appears to be a very interesting family from the perspective of fruiting body development. In C. neoformans adhesive and signaling roles have been suggested for the family, although exact mechanisms of function are still unknown.

Aspartic peptidase A1 - This family is a versatile group of endopeptidases that has undergone a characteristic expansion in fungi(Revuelta et al. 2014). They were reported to be involved in pathogenesis, nutrition, cell wall maintenance and a range of other functions. The best researched family of A1 aspartic peptidases are yapsins, which are involved in cell wall assembly and remodeling, although their function is not clear; limited evidence suggests they proteolytically process secreted (cell wall) proteins(Gagnon-Arsenault et al. 2006; Gow et al. 2017; $M$ et al. 2021). Based on functional knowledge on yapsins, and the abundance of GPI-anchored A1 aspartic peptidases in fungi, we discuss this family in the cell surface section, despite almost certainly not all of them are related to the cell wall. The family has been reported to be upregulated in fruiting bodies by several studies previously(Rahmad et al. 2014; Song et al. 2018b; Krizsán et al. 2019). Aspartic peptidase A1 family was found as 15-60 copies in Agaricomycetes genomes. We detected 3 CDE orthogroups and several species-specifically developmentally expressed genes (Table 32), though these did not display strong expression conservation in the examined species. Nevertheless, the developmental expression of several members of this family, combined with evidence on yeast yapsins(Gagnon-Arsenault et al. 2006) and the secreted nature of these proteins suggest a cell wall related role for aspartic peptidases which may be worth investigating. Of note, we detected further CDE orthogroups of various peptidase families (M20, M28, M16, S10), which may also be involved in fruiting body development (Table 32), but our functional knowledge at the moment is too limited to speculate about their roles. Other peptidase families that were reported in mushroom-forming fungi include the metalloprotease M43 family, which was reported from $P$. ostreatus(Joh et al. 2004) and has a strong (>700x) induction upon fruiting in the data published by Merenyi et al(Merényi et al. 2021). AA7 family - One of the CDE orthogroups (represented by C. cinerea 501135)(Table 32) were top hits of Fusarium graminearum $\mathrm{ChitO}$, the only experimentally examined member of the family known to us(Heuts et al. 2007), which oxidises chitooligosaccharide species. AA7 comprises oligosaccharide oxidising flavo-enzymes which also contain a Berberine-like domain and produce $\mathrm{H}_{2} \mathrm{O}_{2}$ during their action. They have been speculated to be involved in the detoxification of lignocellulose degradation intermediates and in the production of various secondary metabolites(Levasseur et al. 2013). Based on the activity of their homologs and their expression in fruiting bodies, it is likely that these AA7 family members are active on chitin, although their exact roles remain obscure for now. 
bioRxiv preprint doi: https://doi.org/10.1101/2021.12.09.471732; this version posted December 10,2021 . The copyright holder for this preprint (which was not certified by peer review) is the author/funder, who has granted bioRxiv a license to display the preprint in perpetuity. It is made available under aCC-BY-NC-ND 4.0 International license.

Running head: fruiting body genomics

3696 CFEM domain proteins - We detected 2 CDE orthogroups of CFEM domain containing 3697 proteins (Table 32). Homologous CFEM domain proteins may be involved in defense, 3698 pathogenicity, host-pathogen interactions or iron uptake, as is the rbt5 family of Candida 3699 spp.(Gaurav Bairwa et al. 2017) In C. cinerea certain mycelium expressed CFEM domain 3700 proteins were reported to be involved in defense(Plaza et al. 2014b). The detected CDE 3701 orthogroups comprise cell surface proteins with unknown functions, though they might be 3702 involved in defense, however, evidence for that is missing at the moment.

3703 Fasciclins are adhesive cell surface proteins that facilitate the attachment of cells to various 3704 surfaces. The few fungal mentions include a report of a role in the adhesion of Magnaporthe 3705 oryzae to host surfaces(Liu et al. 2009) as well as a primordium-upregulated gene in $L$. 3706 edodes (Le.flp1)(Y et al. 2007). Recently, fasciclins were hypothesized to have been co3707 opted for fruiting body development independently in both the Agarico- and

3708 Pezizomycotina(Merényi et al. 2020b). We identified 2-8 fasciclin-encoding genes in the 3709 examined Agaricomycetes. All examined species have developmentally expressed fascicling genes, although these formed only small CDE orthogroups (Table 32). Several genes showed an upregulation in primordia relative to vegetative mycelium (as reported in $L$. edodes), suggesting that this family has widespread roles in mushroom formation, possibly by facilitating adhesion. We found that the sequence deposited by ( $Y$ et al. 2007) (Accession number: AB287432) as well as ascomycete homologs to which they analyzed are cupredoxins, not fasciclins, leaving doubts as to the identity of Le.flp1. Nevertheless, this does not undermine the observation that fasciclin encoding genes are widespread developmentally expressed genes in mushroom-forming fungi, probably with roles in fruiting body development.

Intradiol ring cleavage dioxygenases catalyze a ring-opening step in the degradation of aromatic compounds, and were hypothesized to be involved in xenobiotic detoxification or the utilization of aromatic compounds as carbon sources(Semana and Powlowski 2019). However, the physiological role of intradiol ring cleavage dioxygenases in fungi is not known. The only previous mention of a potential relationship to fruiting bodies reported developmental regulation in 4 out of 6 species(Krizsán et al. 2019). We identified 2-7 copies (28 in A. ostoyae) in the examined Agaricomycetes, with 1-3 developmentally expressed genes in most of the species that, however, did not form conserved orthogroups. Members of the family showed a significant upregulation in primordia relative to vegetative mycelium in A. ampla, S. commune, C. cinerea, A. ostoyae as well as a strong upregulation (>100fold) during late development of $C$. neoformans. Two genes show a very distinct upregulation in young fruiting body caps of $C$. cinerea. No developmentally expressed genes were found in Ph. chrysosporium and M. kentingensis. In summary, intradiol ring cleavage dioxygenases might be important genes during fruiting body development of several species; they might degrade aromatic compounds during fruiting, however, the identity of those compounds are

\subsection{Defense}

3737 Agaricomycete fruiting bodies (mushrooms) are, due to the concentration of biomass, 3738 attractive targets of predators, parasites and pathogens. Accordingly, these structures are known to be subject to grazing by slugs, to infestations with larvae of phorid and sciarid flies and to infections by bacteria and molds(Wood et al. 2004; Krivosheina 2008; Lakkireddy et 
Running head: fruiting body genomics

3742 considerable investment in terms of resources and energy, Agaricomycetes have evolved 3743 several strategies for the protection and defense of these structures(Künzler 2018). Such 3744 strategies include physical barriers(Varga et al. 2021) and chemical defense i.e. the 3745 production of molecules (toxins) impairing the growth, development and/or viability of antagonists(P 2008; FY and NP 2014; Stadler and Hoffmeister 2015).

Using saprobic Agaricomycete species, whose sexual cycles can be completed under laboratory conditions, it could be demonstrated that the production of these toxins occurs axenically i.e. in the absence of the antagonist(Plaza et al. 2014b; Tayyrov et al. 2019). This axenic toxin production is spatiotemporally regulated in that the toxin-encoding genes are expressed solely during fruiting body formation and in the fruiting body tissue. Some of these genes belong to the most highly upregulated genes of the entire genome during fruiting body formation. This expression pattern results in an efficient, constitutive protection of the fruiting body because at least some toxins are already in place in case the structure is attacked by an antagonist. It is unclear whether the chemical defense of the Agaricomycete fruiting body can be fortified by the induction of toxin production in response to environmental factors e.g. the presence of antagonists. Antagonist-specific induction of antibacterial and nematotoxic proteins upon challenge with bacteria and fungivorous nematodes, respectively, has recently been reported for the vegetative mycelium of $C$. cinerea(Kombrink et al. 2018; Tayyrov et al. 2019).

Toxins of Agaricomycete fruiting bodies include small molecules (secondary metabolites)(Kües et al. 2018), peptides (ribosomally or non-ribosomally synthesized) and proteins, all of which act by binding to and (in case of toxins with enzymatic activity) converting specific target molecules in the antagonists. In the following, we will summarize the current knowledge about the structure, function, biosynthesis, regulation and distribution of Agaricomycete fruiting body toxins. In accordance with the focus of this review on genomics, we restrict our summary to compounds where the biosynthetic genes have been identified. We also make references to available gene expression data, but it must be noted that most toxin-encoding genes evolve fast and thus they do not easily conform to orthologybased categorizations that are central to the rest of this paper.

Small molecules - The structurally related alkaloids ibotenic acid and muscimol of the fly agaric Amanita muscaria are two well-known examples of defensive secondary metabolites of Agaricomycete fruiting bodies(Michelot and Melendez-Howell 2003). The toxicity of these compounds toward flies gave rise to the name of the producer fungus. Their bioactivity is due to their structural similarity to the neurotransmitters glutamate and GABA and their binding to respective receptors in the brains of both invertebrates and vertebrates. The recent discovery of the biosynthetic gene cluster for the compounds revealed that their biosynthesis initiates from glutamate and makes it possible to identify additional Amanita species producing these compounds on a genetic basis(Obermaier and Müller 2020). Another psychotropic alkaloid produced by Agaricomycete fruiting bodies is psilocybin produced by several Psilocybe species(J et al. 2017; Torrens-Spence et al. 2018). This tryptophan derivative is an agonist of serotonergic receptors and is currently tested for treatment of depressions(Tullis 2021). The transcriptional analysis of the recently identified biosynthetic genes confirms its fruiting body-specific production( $R$ et al. 2020). The blueing reaction of psilocybin-producing fruiting bodies upon injury has recently been demonstrated to be due to enzyme-mediated oxidative oligomerization of psilocybin suggesting that at least part of the defense function of this compound may be based on optical deterrence(Lenz et al. 2020). Wound-activation by injury (oxidation) has been reported for 
bioRxiv preprint doi: https://doi.org/10.1101/2021.12.09.471732; this version posted December $10,2021$. The copyright holder for this preprint (which was not certified by peer review) is the author/funder, who has granted bioRxiv a license to display the preprint in perpetuity. It is made available under aCC-BY-NC-ND 4.0 International license.

Running head: fruiting body genomics

3789 other secondary metabolites of Agaricomycete fruiting bodies(Stadler and Sterner 1998; P

3790

3791

3792

3793

3794

3795

3796

3797

3798

3799

3800

3801

3802

3803

3804

3805

3806

3807

3808

3809

3810

3811

3812

3813

3814

3815

3816

3817

3818

3819

3820

3821

3822

3823

3824

3825

3826

3827

3828

3829

3830

3831

3832

3833

3834

3835

3836
2008) but the genetic basis of these compounds and reactions is often not known.

Defense peptides - The bicyclic octapeptide a-amanitin produced by some Amanita, Galerina, Conocybe and Lepiota species is probably the most famous toxin of Agaricomycete fruiting bodies(Walton 2018). The thermal stability and the very low lethal dose $(0.1 \mathrm{mg} / \mathrm{kg}$ body weight) of the peptide have led to the common names 'death cap' (Amanita phalloides) or 'destroying angel' (Amanita bisporigera) of a-amanitin producers. Upon ingestion, the peptide is released from the fruiting body tissue and able to passively enter the intestinal epithelial cells where it binds and inactivates the essential enzyme RNA polymerase II in the nucleus( $X$ et al. 2018). The fungus protects itself from the toxin by expressing an insensitive variant of the target enzyme(PA et al. 1978). In 2007, Walton and coworkers demonstrated that $\alpha$-amanitin and its relative phallacidin are derived from ribosomally synthesized precursor peptides with common flanking sequences (MSDIN) by the action of a dual function prolyloligopeptidase-macrocyclase (POPB)(Hallen et al. 2007; H et al. 2012). Genome surveys of above Agaricomycete species revealed a large family of peptide precursor genes differing in the core peptide sequence and, thus, coding for $a-$ amanitin- and phallacidin-related, but structurally different peptides, referred to as cycloamanides, whose function is unknown(Pulman et al. 2016; $\mathrm{H}$ et al. 2018; He et al. 2020; Zhou et al. 2021). Localization of POPB expression confirmed the fruiting bodyspecific expression of a-amanitin and phallacidin(Luo et al. 2010; $\mathrm{CH}$ et al. 2018).

Above described secondary metabolites and peptides have received considerable attention, mainly due to their activity towards humans. During the last two decades of research on fungal defense, it has become evident, however, that a large part of the chemical defense of Agaricomycete fruiting bodies, in particular against invertebrate predators and parasites, is based on protein toxins( $\mathrm{M}$ et al. 2002b; J et al. 2016; Tayyrov et al. 2018). These toxins have received less attention because their activity is usually lost upon boiling and is specific for a particular group of invertebrates. In addition, based on their fruiting body-specific expression, some of these proteins have been implicated in fruiting body formation rather than defense (see the example of $C$. cinerea galectins CGL1 and CGL2 mentioned above), which has delayed their recognition and functional analysis as toxins(S et al. 2007a; A et al. 2019a). Most of these protein toxins are stored intracellularly and released upon ingestion of the fruiting body tissue by fungivores(Kunzler 2015; Künzler 2018). Once released, they interact with the digestive tract of the fungivore. Characterized classes of protein toxins from Agaricomycete fruiting bodies include lectins(Goldstein and Winter 2007; Bleuler-Martínez et al. 2011; A et al. 2013), protease inhibitors(S et al. 2015; Sabotič et al. 2019), pore-forming toxins (PFTs)(Anderluh and Maček 2003; JM et al. 2010; Panevska et al. 2020), ribotoxins(Tayyrov et al. 2019; Ragucci et al. 2021) and biotin-binding proteins(Y et al. 2009; $S$ et al. 2012a).

Defense related lectins - Fruiting body lectins have long been used as tools in glycobiology without addressing their biological function( $Y$ and $H$ 2014). In the meantime, it has been established that these proteins bind to specific glycoepitopes on the epithelial cells of the digestive tract of the fungivore which can ultimately lead to disruption of the structure and function of this tissue(Stutz et al. 2015). The mechanism of lectin-mediated toxicity is not clear yet but, in case of lectins without additional functional domain (hololectins), the bioactivity appears to depend on the crosslinking of protein-bound glycoepitopes on the epithelial cell surface(S et al. 2017), whereas in case of lectins with additional functional domains (chimerolectins), the bioactivity appears to depend on binding to lipid-bound glycoepitopes and endocytosis(T et al. 2011; Juillot et al. 2016). Fruiting body lectins can 
bioRxiv preprint doi: https://doi.org/10.1101/2021.12.09.471732; this version posted December $10,2021$. The copyright holder for this preprint (which was not certified by peer review) is the author/funder, who has granted bioRxiv a license to display the preprint in perpetuity. It is made available under aCC-BY-NC-ND 4.0 International license.

Running head: fruiting body genomics

3837 adopt various folds and carbohydrate specificities amongst which the RicinB-like ( $\beta$-trefoil) 3838 fold and fucoside-binding, respectively, are most common. A prototypic example for such a 3839 fruiting body lectin is the hololectin CCL2 (C. cinerea protein ID: 408852) from the model 3840 Agaricomycete Coprinopsis cinerea which is present in other Agaricomycete species, adopts 3841 a RicinB-like fold, forms a dimer, binds to a specific fucoside present on nematode N-glycan 3842 cores and is toxic towards bacterivorous and fungivorous nematodes(Schubert et al. 2012; S 3843 et al. 2017; Tayyrov et al. 2018). A summary of diverse entomo- and nematotoxic lectin have 3844 been described from fruiting bodies has been given by Sabotic et al ( $\mathrm{J}$ et al. 2016). Our data 3845 confirm previous reports on the upregulation (>150-fold) of CCL1 (C. cinerea protein ID: 3846 456849) and CCL2 (C. cinerea protein ID: 408852) in primordia (Table 33).

3847 The RicinB-like (beta-trefoil) fold is also adopted by protease-inhibitors isolated from

3848 Agaricomycete fruiting bodies( $\mathrm{J}$ et al. 2016). These proteins were shown to inhibit serine and 3849 cysteine proteases of various insect larvae. A prototypic example for this type of fruiting body 3850 toxin is Clitocypin, an insecticidal cysteine-protease inhibitor isolated from the 3851 Agaricomycete Clitocybe nebularis(J et al. 2011; Šmid et al. 2015). Of the examined 3852 species, the genomes of $C$. aegerita and L. bicolor encode 1 and 20 putative clitocypins, 3853 respectively. Our data identified a putatively defense-related RicinB protein orthogroup 3854 (represented by C. cinerea 485770)(Table 33). Another example is the six-bladed beta3855 propeller lectin Tectonin2 from L. bicolor whose production is induced in fruiting bodies and 3856 ectomycorrhizae(Martin et al. 2008). This lectin forms tetramers harboring 24 binding sites 3857 for O-methylated glycoepitopes and exhibits toxicity towards nematodes and agglutination of 3858 bacteria displaying respective glycoepitopes on their cell surfaces(Wohlschlager et al. 2014; 3859 Sommer et al. 2018). We detected tectonin homologs in the genomes of $L$. bicolor and $C$. 3860 aegerita.

Pore-forming toxins - A common and probably very ancient class of protein toxins of Agaricomycete fruiting bodies are pore-forming toxins (PFTs)(Peraro and van der Goot 2015). These toxins are divided into $\alpha$ - and $\beta$-PFTs depending on whether the predominant secondary structure motif is an $\alpha$-helix or $\beta$-sheet. Within the producing cell, these proteins are usually monomeric and soluble, but upon release and binding to a target cell membrane, they undergo a dramatic conformational change. This change in conformation leads to oligomerization and insertion of the protein into the membrane. The result of this complex process is a proteinaceous pore that leads to lysis of the target cell. Among PFTs occurring in Agaricomycete fruiting bodies, members of the aerolysin and Membrane Attack $\underline{\text { Complex }}$

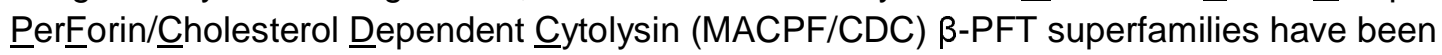
characterized most extensively(Reboul et al. 2016; Cirauqui et al. 2017). Examples for aerolysins are the hemolytic Laetiporus sulphureus lectin (LSL)(JM et al. 2010), a homologous and recently identified nematotoxic protein, CC1G_11805 (C. cinerea protein ID: 546868) and its two paralogs, from C. cinerea(Plaza et al. 2014b) and the hemolytic flammutoxin from $F$. velutipes(T et al. 2004b). Pri1, a primordium-expressed gene in $C$. aegerita (Agrae_CAA7262647) also belongs to pore-forming toxins(Fernandez Espinar and Labarère 1997). The MACPF/CDC superfamily is represented by the insecticidal pleurotolysin complex, consisting of PlyA and PlyB, from the edible mushroom $P$. ostreatus(K et al. 2014; $M$ et al. 2015b; $N$ et al. 2015; A et al. 2019a). The main representatives of the two $\beta$-PFT superfamilies differ in the specificity determinant of the target membrane and the dimensions of the formed membrane pore. Whereas LSL likely binds to lactose-containing glycoepitopes (as part of glycolipids or GPI-anchored glycoproteins) on the target membrane via an $\mathrm{N}$-terminal RicinB-type( $\beta$-trefoil) lectin domain and forms homomeric transmembrane $\beta$-barrels (pores) of seven or eight protomers with an 
bioRxiv preprint doi: https://doi.org/10.1101/2021.12.09.471732; this version posted December 10,2021 . The copyright holder for this preprint (which was not certified by peer review) is the author/funder, who has granted bioRxiv a license to display the preprint in perpetuity. It is made available under aCC-BY-NC-ND 4.0 International license.

Running head: fruiting body genomics

3885 inner diameter of $\sim 4 \mathrm{~nm}$ (I et al. 2011; Szczesny et al. 2011), pleurotolysin PlyA binds to 3886 sphingophospholipids (sphingomyelins) of cholesterol-rich membranes and directs PlyB to 3887 the formation of transmembrane $\beta$-barrels (pores) consisting of 13 heteromeric protomers 3888 and an inner diameter of $\sim 8 \mathrm{~nm}$ (Novak et al. 2020).

3889

Ribotoxins are ribonucleases cleaving a single phosphodiester bond in the universally conserved sarcin/ricin loop (SRL) of 23S or 28S rRNA in intact prokaryotic or eukaryotic ribosomes, respectively( $\mathrm{M}$ et al. 2017b). Upon this action, the ribosome is unable to interact with translation elongation factors and translation is stalled. The SRL is named after $a$-sarcin, one of the best characterized ribotoxins secreted by the ascomycetous mold Aspergillus giganteus( $\mathrm{M}$ et al. 2017b). Other fungal ribotoxins have been isolated from ascomycetous entomopathogens and are generally highly active against insects(A et al. 2009; $M$ et al. 2017a). Recently, the first basidiomycetous ribotoxin, ageritin (Agt1), from fruiting bodies of the edible mushroom C. aegerita was isolated(Landi et al. 2017). Heterologous expression of the respective cDNA in Escherichia coli and phylogenetic analysis showed that the protein is widespread but patchy among Agaricomycetes and displays entomotoxic activity similar to its ascomycetous counterparts(A et al. 2019b). In contrast to its ascomycetous counterparts, however, ageritin does not contain a canonical signal peptide for secretion suggesting that the protein, as most protein toxins of Agaricomycete fruiting bodies, is stored intracellularly(Baglivo et al. 2020). In order to avoid damage to the ribosomes of the producing fungal cell, the protein might be sequestered into a specific subcellular compartment which might involve N-terminal truncation of the protein(Ragucci et al. 2021). The agt1 gene (C. aegerita CAA7259527) was induced considerably in primordia relative to vegetative mycelium in $C$. aegerita, based on data produced by Orban et al (Orban et al. 2021).

Tamavadins - Finally, defense proteins of some Agaricomycete fruiting bodies, similar to animals and bacteria, include proteins that are able to avidly bind and thus sequester biotin at equimolar concentrations(Michael Green 1990). The prototype of these proteins, avidin, is thought to protect the eggs of birds from bacterial infections, but the protein and its relatives also exhibit broad and strong insecticidal activity(JT et al. 2010). A family of avidin-related biotin-binding proteins, tamavidin (called Tam1 and Tam2), was isolated from the edible mushroom Pleurotus cornucopiae (Tamogitake mushroom) and shown to possess very strong entomotoxic and nematotoxic activity(Y et al. 2009; S et al. 2012a). Amongst the fruiting body protein toxins tested against two fungivorous nematode species, tamavidin was the most effective one(Tayyrov et al. 2018). It is unclear how the producing cells shield their own biotin against this cytoplasmic protein.

Taken together, this data shows that the chemical armory of Agaricomycete fruiting bodies is very diverse, in terms of compound classes, molecular targets and antagonist specificity of the toxins, but three common themes can be recognized: (1) Toxin-encoding genes are phylogenetically patchy, with each family present in only a subset of species (2) protein toxins provide a large part of the chemical defense of these structures; and (3) intracellularly stored toxins directed against nematodes and arthropods are predominant. Latter finding may reflect the use of Agaricomycete fruiting bodies as habitat by these organisms(Ruess and Lussenhop 2005; Krivosheina 2008). It has even been postulated that some mycophagous Drosophila flies exploit fruiting body nematotoxins to purge themselves from insect-parasitic nematodes(JA et al. 2017).

3930 
bioRxiv preprint doi: https://doi.org/10.1101/2021.12.09.471732; this version posted December 10,2021 . The copyright holder for this preprint (which was not certified by peer review) is the author/funder, who has granted bioRxiv a license to display the preprint in perpetuity. It is made available under aCC-BY-NC-ND 4.0 International license.

Running head: fruiting body genomics

3931

3932

3933

3934

3935

3936

3937

3938

3939

3940

3941

3942

3943

3944

3945

3946

3947

3948

3949

3950

3951

3952

3953

3954

3955

3956

3957

3958

3959

3960

3961

3962

3963

3964

3965

3966

3967

3968

3969

3970

3971

3972

3973

3974

3975

3976

3977

\subsection{Secondary metabolism and natural product biosynthesis}

Secondary metabolites are one of the most important classes of molecules in chemical warfare and developmental signaling in fungi(Spiteller 2015; Keller 2019; Gressler et al. 2021). Based on recent genome surveys it appears that the fungal secondary metabolome is extremely diverse and is a largely untapped resource of natural products and bioactive molecules(Keller 2019). This may be particularly true for the Agaricomycetes, despite several lines of evidence for a large diversity of secondary metabolites produced by Agaricomycetes(Spiteller 2015; Gressler et al. 2021). From the perspective of fruiting body formation, it is important to note that secondary metabolites can serve defense or repellent purposes in fruiting bodies (see chapter on Defense) but can also influence developmental processes. For example, the deletion of a polyketide synthase gene resulted in malformed perithecia of Sordaria macrospora(D and M 2014). The lack of coprinoferrin production in $C$. cinerea resulted in the loss of fruiting body formation(Tsunematsu et al. 2019).

Due to differences in basic genome organization between Asco- and Basidiomycota, the latter possess fewer secondary metabolism gene clusters, yet, according to a recent study some developmentally regulated genes formed 'clusters' in the genomes of 8 species and some of these 'clusters' overlapped with predicted biosynthetic gene clusters that might encode secondary metabolism genes(Merényi et al. 2021).

The number of functionally characterized secondary metabolism genes and linked natural products of Agaricomycetes is rapidly increasing. For example, ArmB and orsellinic acid in Armillaria spp(Lackner et al. 2013), LpaA and laetiporic acid in Laetiporus sp(Seibold et al. 2020), or St.pks1 and strobilurin in Strobilurus tenacellus(Nofiani et al. 2018) as well as numerous terpene synthases have been circumscribed in the past decade (reviewed in more detail by(Gressler et al. 2021)). Strobilurin derivatives are available commercially and are the world's most sold fungicides(DW et al. 2002). Siderophore-type secondary metabolites, coprinoferrin, ferrichrome A and basidioferrin in C. cinerea, Omphalotus olearius and Ceriporiopsis subvermispora, respectively, and their putative synthesizing genes were also identified. In $C$. cinerea cfp1 (C. cinerea protein ID: 354233) was reported to be responsible for coprinoferrin production(Tsunematsu et al. 2019), whereas in O. olearius a cluster composed of fso1, omo1 and ato1( $\mathrm{K}$ et al. 2005). Coprinoferrin proved necessary for fruiting body development, as the cfp1 mutant did not, only in the presence of exogenously administered coprinoferrin produce fruiting bodies. Contrary to expectations based on Ascomycota knowledge, deleting a homolog (C. cinerea protein ID: 357031) of $A$. nidulans LaeA did not compromise coprinoferrin production(Tsunematsu et al. 2019). Despite recent advances in understanding Agaricomycetes secondary metabolism, given the number of putative secondary metabolism genes encoded by fungal genomes, we are likely hardly scratching the surface of natural product diversity in mushroom-forming fungi.

Since many secondary metabolites specifically occur in fruiting bodies of Agaricomycetes, it is expected that their biosynthetic gene clusters may be developmentally regulated. However, secondary metabolism related genes evolve fast, which does not make them easily detectable in the orthology-based framework we use here. Nevertheless, we identified several secondary metabolism related genes that showed shared and conserved developmental expression profiles in the examined Agaricomycetes, although the natural products they produce are unknown in most cases.

Polyketide synthases (PKS) are large (often >1500 amino acid) multi-domain enzymes that produce diverse secondary metabolites. They are widely distributed in fungi and are subject to intense research, mostly in the Ascomycota. Polyketide synthases and 
bioRxiv preprint doi: https://doi.org/10.1101/2021.12.09.471732; this version posted December $10,2021$. The copyright holder for this preprint (which was not certified by peer review) is the author/funder, who has granted bioRxiv a license to display the preprint in perpetuity. It is made available under aCC-BY-NC-ND 4.0 International license.

Running head: fruiting body genomics

3978 the few examples of known natural products of the Basidiomycota have recently been 3979 reviewed(Gressler et al. 2021). Basidiomycete PKSs mostly belong to type 1, which is 3980 divided into highly-, partially- and non-reducing PKSs. Hybrid nonribosomal peptide 3981 synthase-PKS genes have been shown to underlie fungal bioluminescence(Ke et al. 2020a; 3982 Gressler et al. 2021).

3983 We identified orthologs of previously described siderophore biosynthesis related 3984 genes in the examined Agaricomycetes. Members of the biosynthetic cluster described from 3985 O. olearius( $\mathrm{K}$ et al. 2005) seem to be conserved, with fso1 being orthologous to $C$. cinerea 3986 cfp1 (C. cinerea protein ID: 354233), whereas L-ornithine N(5)-monooxygenase omo1 and 3987 the $\mathrm{N}$-acyltransferase ato1 seem single-copy in Agaricomycetes genomes (represented by 3988 C. cinerea 492586 and 161175). These genes were not developmentally regulated and had 3989 highest expression in vegetative mycelia. We further identified a single CDE orthogroup of 3990 polyketide synthases (represented by $C$. cinerea 446258 , Table 34 ), which was developmentally regulated in $11 / 8$ species $(\mathrm{FC}>2 / 4)$. These genes were stipe upregulated (albeit weakly) in most species (C. cinerea, $P$. ostreatus, M. kentingensis, A. ostoyae), although their expression also increased in mature caps of $P$. ostreatus, $A$. ostoyae, $M$. kentingensis relative to younger cap stages and in mature fruiting bodies of $C$. aegerita, $A$. ampla and $S$. commune relative to earlier stages. The $C$. cinerea gene shows peak expression in meiotic gill tissues, whereas in $P$. ostreatus and $A$. ostoyae, where gills and caps were sampled separately like in $C$. cinerea, highest expression was observed in caps but not in gills. The $C$. cinerea gene in this orthogroup is the best hit of Aspergillus fumigatus $\mathrm{PksB}$, which is a polyketide synthase involved in spore pigment (melanin) production. Based on this similarity, we hypothesize that these genes are also involved in pigment production in Agaricomycetes.

Terpene synthases have been a focus of secondary metabolism studies in mushroom-forming fungi. They encode highly promiscuous enzymes that can generate a large diversity of metabolites(Slot and Gluck-Thaler 2019) and thus provide the basis for fast adaptive evolution. Slot and Gluck-Thaler showed that morphologically complex fungi harbor more terpene synthases than simpler counterparts(Slot and Gluck-Thaler 2019). Similarly, terpene synthases were found to be strongly overrepresented in the genomes of Agaricomycetes compared to other fungi (FDR corrected $p$-value $<10^{-125}$ ) (Krizsán et al. 2019). Twelve sesquiterpene synthases (Fla1-12) were identified in F. velutipes(Tao et al. 2016), eleven in C. aegerita (Agr1-Agr11)(Zhang et al. 2020, 2021a) and in Omphalotus olearius (Omp1-10)(GT et al. 2012) and five (Cop1-Cop6) in C. cinerea(Agger et al. 2009), with information on the potential metabolite products in many cases (e.g. viridiflorene/viridiflorol, $\Delta^{6}$-protoilludene, $\mathrm{Y}$-cadinene, germacrene).

Agger et al identified six genes, Cop1 to Cop6, related to terpene biosynthesis in $C$. cinerea(Agger et al. 2009). Of the genes reported by Agger et al, orthologs of Cop2 and Cop4 appear interesting from the perspective of mushroom development. Orthologs of Cop1 show an upregulation in primordia relative to vegetative mycelium in $C$. aegerita, $A$. ampla (only FC>2), L. bicolor, Ph. chrysosporium and were cap-upregulated in A. ostoyae, $P$. ostreatus, $L$. edodes and $M$. kentingensis. On the other hand, orthologs of Cop4 (C. cinerea protein ID: 30510) were FB-init in C. cinerea, C. aegerita, $A$. ampla, L. bicolor, L. edodes and $P$. ostreatus (Table 34). Cop2 (C. cinerea protein ID: 443805) is only present in C. cinerea, in all species, suggesting it functions during fruiting body initiation. Products of these genes were reported to as $\delta$-cadinene and germacrene $A$, but the functions of these natural products remain unknown. 
bioRxiv preprint doi: https://doi.org/10.1101/2021.12.09.471732; this version posted December 10,2021 . The copyright holder for this preprint (which was not certified by peer review) is the author/funder, who has granted bioRxiv a license to display the preprint in perpetuity. It is made available under aCC-BY-NC-ND 4.0 International license.

Running head: fruiting body genomics

4026

4027

4028

4029

4030

4031

4032

4033

4034

4035

4036

4037

4038

4039

4040

4041

4042

4043

4044

4045

4046

4047

4048

4049

4050

4051

4052

4053

4054

4055

4056

4057

4058

4059

4060

4061

4062

4063

4064

4065

4066

4067

4068

4069

4070

4071

We found 13-28 (63 in A. ostoyae) putative terpene synthases in the examined Agaricomycetes genomes. Of these, several were developmentally regulated, showing one of two most typical expression profiles: (i) highest expression in vegetative mycelium or (ii) a strong induction in primordia and other fruiting stages relative to vegetative mycelium. The latter expression profile is reminiscent of that of typical defense genes (e.g. pore-forming toxins, see above), but it is compatible with several other putative functions as well. Terpene synthases formed three CDE orthogroups (Table 34), two of these correspond to $C$. cinerea Cop1 (C. cinerea protein ID: 469738) and Cop4 (C. cinerea protein ID: 30510, contains $C$. aegerita Agr4 also), discussed above. The third CDE orthogroup (represented by $C$. cinerea 488324 ) is developmentally regulated in $8 / 6$ species, but shows mixed expression profiles.

Taken together, terpene synthases might participate in important fruiting body functions, including events around the initiation of primordium development, yet their exact functions, whether the production of defense/repellent metabolites and/or compounds for communication, remain unknown for the moment.

Phenylalanine ammonia-lyases - We detected two highly conserved CDE orthogroups of phenylalanine ammonia-lyases (PAL), one of which (C. cinerea protein ID: 386671 , ortholog of pal1 of Pleurotus) is developmentally regulated in $11 / 9$ species, the other (C. cinerea protein ID: 361541 , ortholog of pal2 of Pleurotus(Hou et al. 2019)) is in 8/5 (out of 9 species in which present) (Table 34). PAL enzymes are best known from plants, where they are part of the phenylpropanoid pathway, which is involved in the production of diverse secondary metabolites, associated with defense, lignin and flavonoid biosynthesis(Dixon et al. 2002). In fungi, phenylalanine ammonia-lyases are discussed mostly in the context of catabolism of phenylalanine to cinnamic acid(Hyun et al. 2011), although some reports of the production of diverse compounds have been published(Hyun et al. 2011). Recently, an association with fruiting body formation has been reported in $F$. velutipes(Yun et al. 2015) and P. ostreatus (Hou et al. 2019).

Our analyses revealed two PAL-encoding genes in most of the examined Agaricomycetes (up to 4 in A. ostoyae). Expression profiles of genes within the two CDE orthogroups were not conserved, although several genes showed an upregulation in primordia (e.g. both genes in C. cinerea). At the moment the physiological role of PALs is unknown, to our best knowledge, in any fungus. They could be involved in the production of defense-related metabolites, as in plants, or other fruiting body related functionalities.

\subsection{Cytoskeleton}

A plastic cytoskeleton is a key factor for eukaryotic cell shape regulation and has been named one of the prerequisites for diversification of size, form and function in multicellular eukaryotes(Knoll 2011). It is involved in the regulation of diverse morphogenetic processes in fungi, including hyphal growth, conidiation, biofilm formation, dimorphic switching, to name a few(Riquelme et al. 2018). In line with this, although at a somewhat limited scale, evidence is available for the role of cytoskeleton rearrangement in fruiting body development.

Evidence from Gene Ontology analyses pointed at differential cytoskeletal gene expression in $P$. microcarpus(Pereira et al. 2017). Microscopy observations provided evidence for a key role of cytoskeletal elements during the development of mushroom-forming fungi. For example, longitudinally oriented microtubules were observed in the sterigmata during spore formation in $C$. cinerea, along which Golgi vesicles carry carbohydrates to the developing spore and spore wall(Kües and Liu 2000). 
bioRxiv preprint doi: https://doi.org/10.1101/2021.12.09.471732; this version posted December $10,2021$. The copyright holder for this preprint (which was not certified by peer review) is the author/funder, who has granted bioRxiv a license to display the preprint in perpetuity. It is made available under aCC-BY-NC-ND 4.0 International license.

Running head: fruiting body genomics

4072 In terms of expression levels, we found only limited dynamics in cytoskeletal proteins, 4073 likely reflecting the need for continuous supply of its components for the cell. Cheng et al 4074 reported an upregulation of tubulin and tubulin-specific chaperone genes in primordium 4075 stages of $C$. cinerea(Cheng et al. 2013a) using 5'-SAGE technology. Although newer data 4076 did not confirm these observations(Muraguchi et al. 2015; Krizsán et al. 2019), both recent RNA-Seq studies reported upregulation of these genes in stipes of $C$. cinerea.

Following these lines of evidence, we annotated the main cytoskeletal protein groups

4079

4080

4081

4082

4083

4084

4085

4086

4087

4088

4089

4090

4091

4092

4093

4094

4095

4096

4097

4098

4099

4100

4101

4102

4103

4104

4105

4106

4107

4108

4109

4110

4111

4112

4113

4114

4115

4116

4117

4118

4119 in C. cinerea based on homology and domain composition and checked their orthologs' expression patterns across mushroom-forming fungi. We grouped 67 cytoskeletal and associated protein coding genes into the following categories (Table 35): actin, actin nucleation, actin filament organization, actin-based motor protein, microtubule, microtubule heterodimerization, microtubule-based motor protein, regulation of microtubule-based motor proteins. Copy numbers in these categories agreed with previous annotations, including 2 copies of formins per species and a tubulin gene duplication in the common ancestor of Basidiomycota(Zhao et al. 2014). Of cytoskeletal genes, only kinesin genes and the Dynactin complex showed consistent expression dynamics. In both cases, expression peaks were observed in young fruiting body gills and stipes in $C$. cinerea (Fig. 14, Supplementary Fig. 10).

Tubulin gene expression (alpha1-, alpha2-, beta-tubulin and tubulin chaperone) was previously reported to be regulated during early fruiting events of $C$. cinerea, with upregulation in stage 1 primordia based on 5'-SAGE data(Cheng et al. 2013a). Our data on the corresponding genes revealed that the three of these, alpha1- $(C$. cinerea protein ID: 176636), beta-tubulin (C. cinerea protein ID: 393528 ) and tubulin chaperone (C. cinerea protein ID: 413977 ) had constant expression in primordia, but show expression peak in young fruiting body stipes, whereas alpha2-tubulin (C. cinerea protein ID: 359665) was upregulated in young fruiting body caps. These expression patterns were not consistent across species (Table 35) and possibly reflect the fast stipe elongation of $C$. cinerea.

Septins - Septins form a highly conserved family of proteins involved in morphogenesis, development and in generating cell asymmetries in fungi and in eukaryotes in general(Gladfelter 2006; Khan et al. 2015). They have been shown to be responsible for morphogenesis in a number of model fungi, such as bud emergence in $S$. cerevisiae, septum formation in hyphae or appressorium formation in Magnaporthe oryzae, among others(Gladfelter 2006). Their broad involvement in morphogenesis and limited evidence from fruiting body forming fungi suggest that septins have important, although to date underappreciated, roles in fruiting body development. For example, they have been reported in stipe elongation in C. cinerea(Shioya et al. 2013).

We annotated septins based on their domain composition: the combination of two IPR domain terms (IPR030379, IPR016491) are diagnostic for fungal septins. Most mushroom-forming fungi have 7 septin genes (C. cinerea protein ID: 367587,416864 [cdc3, = A. nidulans AspE, =AspB], 103773, 415573 [top hit of $A$. nidulans AspE], 103809, 455217, 481878 ), with some having only 6 while a few having 8 , which is similar to the septin repertoires seen in filamentous fungi and yeasts(Douglas et al. 2005).

Septins are highly variable genes, and did not form conserved orthogroups, but nevertheless maintain some functional conservation in mushroom-forming fungi. Two orthogroups of septins showed consistent upregulation at the initiation of fruiting body development, in the very first primordium stages in all species in which they are present(Krizsán et al. 2019; Merényi et al. 2021). One of these is the top hit of AspE of $A$. nidulans, which is involved in conidiation and spore production(Hernández-Rodríguez et al. 
bioRxiv preprint doi: https://doi.org/10.1101/2021.12.09.471732; this version posted December 10,2021 . The copyright holder for this preprint (which was not certified by peer review) is the author/funder, who has granted bioRxiv a license to display the preprint in perpetuity. It is made available under aCC-BY-NC-ND 4.0 International license.

Running head: fruiting body genomics

4120 2014). Septin expression seems to be highly induced in stipes in C. cinerea, $M$.

4121 kentingensis, $A$. ostoyae, but following a different expression tendency in $P$. ostreatus

4122 (mostly in primordia) and $A$. bisporus (mostly in young fruiting bodies). The stipe induced

4123 septins included $C$ c.cdc3 of $C$. cinerea, which has been shown to be involved in stipe

4124 elongation(Shioya et al. 2013). The ortholog of $C$. cinerea cdc3 was also mostly stipe-

4125 induced in A. bisporus(卢园萍 et al. 2019). Muraguchi et al reported a downregulation of

4126 septin expression from the 4_OhrPri primordia (approximately coinciding with stage 2

4127 primordia of Krizsan et al and Kues 2000(Kües 2000; Krizsán et al. 2019)) to the 5_12hrPri

4128 stage of $C$. cinerea(Muraguchi et al. 2015).

4129 The limited diversity of septins raises the question how they can fulfill the diverse

4130 roles they have in fungal morphogenesis. Although most septins had peak expression in

4131 stipes of mushroom-forming fungi (except in certain species) their expression level was

4132 dynamically changing in other tissues and from one developmental stage to the other as

4133 well, suggesting that there is still a long way to uncover their roles in fruiting body

4134 development. Understanding their protein-protein interaction networks is a promising future

4135 avenue of research.

4136 4.13. Ubiquitin-proteasome system

4137 4.13.1. Cop9 signalosome

4138 The Cop9 signalosome is a protein complex conserved across all eukaryotes, which

4139 catalyzes the deactivation of cullin-RING ubiquitin ligase (CRL) complexes by removing the

4140 Nedd8 protein from the cullin subunit of the complex (deneddylation reaction). As such, it is

4141 an important component of various signal transduction pathways and is involved primarily in

4142 multicellular developmental processes. In fungi, it was found dispensable for vegetative

4143 growth, but necessary for sexual fruiting body formation(Busch et al. 2003; Braus et al.

4144 2010), secondary metabolism and it was reported to interact with the Velvet complex(Kress

4145 et al. 2012; Meister et al. 2019). Recognition of the COP9 signalosome (CSN) as an

4146 important player in fruiting body formation started with genetic screens that identified CsnE

4147 and $\mathrm{CsnD}$, which displayed identical defects in fruiting body initiation in $A$. nidulans(Busch et

4148 al. 2003, 2007). The $A$. nidulans Cop9 complex represents an 8-subunit, stereotypical

4149 complex, whereas that of other model fungi ( $N$. crassa, $S$. cerevisiae or $S$. pombe) possess

4150 fewer subunits and are not typical(Braus et al. 2010). Agaricomycetes genomes appear to

4151 encode a typical 8-subunit CSN (Table 36). Neither we nor previous studies found significant

4152 expression dynamics of any subunit of the CSN in mushroom-forming fungi. It is not known

4153 whether the complex is involved in fruiting body development as it is in Ascomycota,

4154 nevertheless, it will be an interesting target for future experiments, especially because it is

4155 involved in light regulation in $N$. crassa and can interact with the Velvet complex, two key

4156 factors that regulate Basidiomycota development also.

\section{4.13.2. Protein ubiquitination}

4158 Covalent protein modification by the ubiquitin-proteasome system is a widely conserved

4159 regulatory machinery that functions in several essential processes in eukaryotes and was

4160 recently detected as a potentially very interesting function during fruiting body

4161 development(Krizsán et al. 2019). The regulatory potential of the ubiquitin-proteasome

4162 system stems from its ability to attach the 76-amino acid ubiquitin protein to lysines of target 
bioRxiv preprint doi: https://doi.org/10.1101/2021.12.09.471732; this version posted December 10,2021 . The copyright holder for this preprint (which was not certified by peer review) is the author/funder, who has granted bioRxiv a license to display the preprint in perpetuity. It is made available under aCC-BY-NC-ND 4.0 International license.

Running head: fruiting body genomics

4163 proteins, which either destines them for degradation by the proteasome or modifies their 4164 activity, cellular localization or DNA-binding ability(LC et al. 2007; Liu and Xue 2011; Finley 4165 et al. 2012). Ubiquitination can modify the activity of transcription factors, which provides an 4166 additional layer of transcriptional regulation(Freiman and Tjian 2003).

$4167 \quad$ The protein ubiquitination machinery is composed of E1 ubiquitin-activating, E2 4168 ubiquitin-conjugating enzymes and E3 ubiquitin ligases, which attach the ubiquitin to target 4169 proteins. While E1 and E2 enzymes are generic components of the system and are present 4170 in limited numbers in eukaryotic genomes (Table 37), ubiquitin ligases provide the substrate 4171 specificity and come in many subtypes and display considerable diversity due to 4172 interchangeable protein components, of which hundreds may exist in the genomes of some 4173 species(Hutchins et al. 2013; Zheng and Shabek 2017). The most diverse such parts are F4174 box and BTB/POZ domain proteins, both of which can confer substrate-specificity to the E3 4175 complexes( $R$ et al. 2003) and RING-type zinc finger proteins, which are scaffold proteins 4176 linking target proteins with E2 enzymes(Metzger et al. 2012). These three gene families 4177 were found to be strongly expanded in mushroom-forming fungi, with frequent tissue-specific 4178 developmental regulation seen in RNA-Seq data(Almási et al. 2019; Krizsán et al. 2019) 4179 (see also Table 37). The expansion stems from Agaricomycetes-specific duplication events 4180 and a correlation between fruiting body complexity and F-Box, BTB/POZ and RING-type zinc 4181 finger copy number and expression has been suggested(Krizsán et al. 2019), which could 4182 explain the evolution of increasingly complex mushroom morphologies in the class.

The widespread cellular roles of ubiquitination suggests that it should be instrumental during fruiting body morphogenesis too. In line with this, the protein ubiquitination system was found differentially expressed in F. velutipes fruiting bodies(M et al. 2006; Liu et al. $2020 \mathrm{~b}$ ) and in caps of the same species subjected to differential $\mathrm{CO}_{2}$ treatments(Yan et al. 2019). In recent comparative transcriptomics analyses, we provided evidence for the developmental regulation of several components, mostly those that confer substrate specificity to E3 ligase complexes, in multiple Agaricomycetes species, including $C$. cinerea, A. ostoyae, A. ampla, S. commune or P. ostreatus, among others(Almási et al. 2019; Krizsán et al. 2019; Merényi et al. 2021). BTB domain-containing proteins, a family of E3 proteins, were reported to be upregulated in fruiting bodies in Antrodia cinnamomea (Lu et al. 2014). The combination of a remarkable gene family expansion with developmental regulation suggests that the ubiquitin-proteasome system could play a pivotal role in fruiting body development, although mechanistic details of the process remain to be understood. (reviewed in(Riquelme et al. 2018)). However, a similar expansion of F-Box domains, as seen in Agaricomycetes, has not been reported.

Hereafter, we present an annotation of components of the protein ubiquitination machinery in selected mushroom-forming fungi, with particular emphasis on $C$. cinerea genes and comparisons across species. In this presentation, we follow the classification of E3 ubiquitin ligases used by Hutchins et al(Hutchins et al. 2013) and identify protein diversity of each protein family based on Interpro domain HMM-s. Results of the annotation along with orthologous genes and conservation levels are shown in Supplementary table 4. A limitation of this approach is that the presence of a defining domain in the protein does not in all the cases, indicate a function in the ubiquitination machinery. For example, BTB/POZ domains, which contribute to expanding the diversity of E3 ligase complexes( $R$ et al. 2003) transcription regulating proteins(Stogios et al. 2005)). 
bioRxiv preprint doi: https://doi.org/10.1101/2021.12.09.471732; this version posted December $10,2021$. The copyright holder for this preprint (which was not certified by peer review) is the author/funder, who has granted bioRxiv a license to display the preprint in perpetuity. It is made available under aCC-BY-NC-ND 4.0 International license.

Running head: fruiting body genomics

4211 Several components of the ubiquitination machinery are very conserved in 4212 Agaricomycetes genomes, including E1 (on average 3 genes) and E2 enzymes (19-24

4213 genes), Cullin genes (4-10 genes) and deubiquitinating enzyme-encoding genes (23-27

4214 genes) (Supplementary table 4). Most of these genes show no or modest expression

4215 dynamics, which is not surprising given that the ubiquitination machinery is needed

4216 constantly in the cell. Of the E1 enzymes, only S. cerevisiae Uba3 orthologs (C. cinerea

4217 protein ID: 485679), which belong to the neddylation pathway, are developmentally

4218 regulated to some extent (8/3 species at $\mathrm{FC}=2 / 4)$. In $C$. cinerea 14 of the $23 \mathrm{E2}$ enzymes

4219 and half (13 of 26 ) of deubiquitinating enzymes are developmentally regulated, potentially

4220 indicating functional diversification of E2 enzymes and deubiquitinating enzymes during

4221 development (Supplementary table 5). We found that none of the cullin genes in C. cinerea

4222 are developmentally regulated. Significantly larger dynamics can be observed in E3 ligase

4223 components, which are discussed in detail below.

4224

4225

4226

4227

4228

4229

4230

4231

4232

4233

4234

4235

4236

4237

4238

4239

4240

4241

4242

4243

4244

4245

4246

4247

4248

4249

4250

4251

4252

4253

4254

4255

4256

4257

4258

RING-type Zinc finger proteins - RING-domain containing proteins, named after RING (really interesting new gene), are E3 ubiquitin ligases that confer interchangeability to the ligase complexes(Deshaies and Joazeiro 2009). Eukaryotic genomes contain large numbers of RING-domain proteins, which, along with other E3 ligases, enable the ubiquitin system to selectively ubiquitinate proteins. RING-domain proteins are generally poorly known in filamentous fungi, though it was recently noticed that they are strongly overrepresented in Agaricomycetes(Krizsán et al. 2019). The $m f b A c$ (=Le.MFB1) gene of $L$. edodes (Lenedo1_1172639) described as a protein with adhesive properties by Kondoh et al(Kondoh et al. 1995) also belongs to this gene family.

The examined Agaricomycetes possess 81-148 RING-domain containing proteins (Table 37), consistent with a previous broader sampling across 200 species. On average, 30-50 RING-domain proteins were developmentally regulated in the examined species(Almási et al. 2019; Krizsán et al. 2019), representing a significant pool of potentially important regulators. RING-domain proteins are highly variable, with most of them not showing clear orthology across species. Nevertheless, we found eight CDE orthogroups which displayed conserved developmental expression, mostly at FC>2 (Table 37). For example, one of these (represented by $C$. cinerea 378120) showed a very distinct peak in gills of $C$. cinerea, $A$. ostoyae, $P$. ostreatus and caps (containing gills) of $M$. kentingensis, $L$. edodes as well as mature fruiting bodies of $S$. commune and $A$. ampla. Another CDE orthogroup (represented by $C$. cinerea 440772) was upregulated in gill tissues of mature fruiting bodies in $P$. ostreatus, A. ostoyae, M. kentingensis, L. bicolor and mature fruiting bodies of $S$. commune and $A$. ampla, but not in $A$. bisporus, $L$. edodes or $C$. cinerea. These two orthogroups coincide (partly) with meiosis and sporulation, so they could be involved in these, or other gill-specific processes. Genes in another CDE orthogroup (C. cinerea protein ID: 543850) showed an abrupt, although modest upregulation at the initiation of fruiting body development and expression peak in mature fruiting bodies or gills therein in C. cinerea, $A$. ostoyae, C. aegerita, A. ampla, L. bicolor, L. tigrinus, Ph. chrysosporium, P. ostreatus and $S$. commune (not in L. edodes).

F-box proteins - F-box proteins were recently identified as a potentially key group of proteins in fruiting body development(Krizsán et al. 2019). They are generally responsible for providing target specificity to the ubiquitination machinery, by binding and docking substrate proteins to Cullin E3 ubiquitin ligase complexes. Some F-box proteins interact with multiple target proteins while others only with a single protein(Jonkers and Rep 2009). Similarly, some F-Box proteins function independently of the SCF complex(Jonkers and Rep 2009), allowing for additional, yet poorly known regulatory functions to emerge. For example, F-box 
bioRxiv preprint doi: https://doi.org/10.1101/2021.12.09.471732; this version posted December 10,2021 . The copyright holder for this preprint (which was not certified by peer review) is the author/funder, who has granted bioRxiv a license to display the preprint in perpetuity. It is made available under aCC-BY-NC-ND 4.0 International license.

Running head: fruiting body genomics

4259 proteins can act as transcriptional co-regulators in plants(Chae et al. 2008) and are present 4260 in large numbers in plant genomes (up to 700-1000 genes(Xu et al. 2009)), providing the 4261 possibility to assemble an enormous diversity of alternative ubiquitin ligase complexes.

4262 The F-box family is highly expanded in mushroom-forming fungi and is composed of 4263 fast-evolving proteins with little similarity to each other. Agaricomycetes possess 69 to $4264 \sim 1200 \mathrm{~F}$-box proteins in their genomes, whereas other filamentous fungi and yeasts possess 4265 up to 90 and 20 such genes(Krizsán et al. 2019), respectively. This represents a strong 4266 enrichment in Agaricomycetes genomes $\left(\mathrm{P}<10^{-140}\right)$, considering that the number of other 4267 proteins is not proportionately higher in this group than in other fungi. F-box protein encoding 4268 genes often showed tissue-specific expression and their numbers showed a positive correlation with the complexity level of the fruiting bodies(Krizsán et al. 2019).

F-Box proteins are highly variable and form few conserved orthogroups, compared to their diversity in fungal genomes. However, we found eleven orthogroups that showed some level of conservation and consistent developmental regulation across species (Table 37). These included orthologs of $A$. nidulans GrrA (C. cinerea protein ID: 358351), which is needed for meiosis and ascospore maturation(S et al. 2006a; Riquelme et al. 2018), are conserved across Agaricomycetes and developmentally regulated in five species (FC>2), indicating that some F-Box proteins are conserved despite the general volatility of the family. Due to this high variability, F-box proteins are hard to examine systematically using a comparative transcriptomics approach. Rather, they likely often fulfill species- or cladespecific roles, which could be studied either with more densely sampled transcriptomic data, or reverse genetics approaches.

BTB/POZ domain proteins - Proteins that contain the BTB/POZ motif, a proteinprotein interaction (homodimerization) domain, can possess diverse cellular functions, the most frequent of which are regulation of chromatin structure (thus, transcription) and protein ubiquitination, as members of E3 ubiquitin ligase complexes(S et al. 1994; R et al. 2003). They are poorly known in fungi.

In animals, plants as well as fungi outside the Agaricomycetes, BTB domains are mostly fused to other conserved protein domains(Stogios et al. 2005) (in animals, mostly to zinc fingers(S et al. 1994)). In contrast, we observed that in the Agaricomycetes, less than $10 \%$ of BTB/POZ domain proteins associate with any other domains and virtually never with DNA binding transcription factor domains, indicating that the gene family expansion in mushroom-forming fungi is concerned with proteins that only contain the BTB/POZ domain. This domain composition suggests that the BTB/POZ family is primarily involved in E3 ligases in Agaricomycetes, therefore we discuss this family under the chapter on protein ubiquitination.

We found $8 \mathrm{CDE}$ orthogroups with some level of conserved developmental expression in Agaricomycetes (Table 37). Members of two (C. cinerea protein ID: 379603, 449824) of these orthogroups are co-expressed in stages or tissues in which meiosis and sporulation occurs: gills of $A$. ostoyae and $P$. ostreatus, mature fruiting bodies of $P h$. chrysosporium, A. ampla and S. commune, mature caps of $M$. kentingensis, L. bicolor, $L$. edodes (but not developmentally regulated in $C$. cinerea). Another CDE orthogroup ( $C$. cinerea protein ID: 459680 ) was upregulated in stipes of $C$. cinerea, $A$. ostoyae, $M$. kentingensis, $L$. edodes (but not in $P$. ostreatus and $A$. bisporus), primordium stages of $A$. ampla and $S$. commune and during early development (primordia + young fruiting bodies) of L. bicolor. A fourth CDE orthogroup, which contains reciprocal best hits to the putative E3 ubiquitin ligase protein btb1 of Sch. pombe (C. cinerea protein ID: 545078), but contains also 
bioRxiv preprint doi: https://doi.org/10.1101/2021.12.09.471732; this version posted December 10,2021 . The copyright holder for this preprint (which was not certified by peer review) is the author/funder, who has granted bioRxiv a license to display the preprint in perpetuity. It is made available under aCC-BY-NC-ND 4.0 International license.

Running head: fruiting body genomics

4306 domains that suggest it regulates chromatin condensation and thus gene expression. 4307 Members of this orthogroup were upregulated typically late during development.

\section{4.14. Other gene groups}

\section{4.14.1. Transporters}

4310 Among the conserved developmentally expressed genes (CDE orthogroups) we detected 49 4311 putatively transport-related gene families. Many of these genes belong to the Major 4312 Facilitator Superfamily or ABC transporters, fast-evolving gene families that are represented 4313 by hundreds of genes in fungal genomes. Transporters have been reported in transcriptomic 4314 studies of several mushroom-forming species, both in fruiting body(Song et al. 2018b; 4315 Krizsán et al. 2019) and ectomycorrhiza formation(Xu et al. 2015). Due to the fast evolution 4316 of these families, orthology to genes of Ascomycota model systems and even within the 4317 Agaricomycetes is lacking in most cases, making it hard to infer their functions.

4318 Nevertheless, some conserved transporter genes were detected, including orthologs of $S$. 4319 cerevisiae Pns1 (choline transporter, C. cinerea 355745), A. nidulans MepA (ammonium 4320 permease, C. cinerea 538158), Sch. pombe Kha1 (plasma membrane potassium ion/proton symporter, C. cinerea 449705), S. cerevisiae Emp70 (endosomal transport protein, $C$. 496395), among others (see Table 38). In the absence of functional annotations for the vast majority of these genes, we list the genes in Table 38, but do not go into detailed analyses, because it is unlikely that we can arrive at any reasonable speculation about their function.

Notable CDE orthogroups include an MFS transporter $C$. cinerea 465489, which was developmentally regulated in $9 / 5$ species $(\mathrm{FC}>2 / 4)$ and $\mathrm{FB}$-init in $C$. cinerea, $A$. ostoyae, $P$. ostreatus, $S$. commune (in the latter at $\mathrm{FC}>2$ only). A phosphate transporter CDE orthogroup (C. cinerea protein ID: 30849) was FB-init in C. cinerea, C. aegerita, A. ostoyae, A. ampla, S. commune, M. kentingensis and, at FC>2 in L. bicolor (not in L. edodes) (Table 38). A group of zinc-regulated transceptors (transporter + receptor, C. cinerea protein ID: 455616) orthologous to $S$. cerevisiae Zrt1 was also detected. In summary, transporters can be expected to fulfill key functions in fruiting bodies, in moving around goods, signal molecules, modulating osmolarity to name a few, however, information about these in fruiting bodies is still limited.

Aquaporins are water and solute-transporting membrane channels that are integral to several different aspects of the fungal lifestyle. They were reported to be overexpressed in primordia and stipes of $F$. filiformis, relative to other tissues and developmental stages(Liu et al. 2020b). They were also reported to be upregulated in L. bicolor fruiting bodies and ectomycorrhizae(Xu et al. 2015).

We found 2-11 aquaporin genes in the examined Agaricomycetes and observed conserved aquaporin expression in two aquaporin orthogroups. One of these $(C$. cinerea protein ID: 373141) contains putative orthologs of yeast Fps1, an aquaglyceroporin that has suggested roles in acetate uptake or glycerol transport(Nehls and Dietz 2014), not water uptake (see under chapter on Acetyl CoA). It is worth noting that aquaglyceroporins were also differentially regulated in L. bicolor fruiting bodies(Xu et al. 2015) and that these proteins were shown to have a capacity to transport various solutes, including $\mathrm{NH}_{3}$ and glycerol. This led Xu et al to hypothesize that these genes could be involved in osmosis regulation or signaling(Xu et al. 2015), which is different from the conclusion we make above on acetate transport. 
bioRxiv preprint doi: https://doi.org/10.1101/2021.12.09.471732; this version posted December 10,2021 . The copyright holder for this preprint (which was not certified by peer review) is the author/funder, who has granted bioRxiv a license to display the preprint in perpetuity. It is made available under aCC-BY-NC-ND 4.0 International license.

Running head: fruiting body genomics

$4351 \quad$ The other orthogroup (represented by S. commune 2501677) comprises stipe4352 specific aquaporin genes, which show similar expression trajectories in A. bisporus, $A$. 4353 ostoyae, $M$ kentingensis and $P$. ostreatus, as well as in an external $F$. filiformis dataset(Liu et 4354 al. 2020b). In all of these species, the genes showed expression peaks in stipes, mostly in 4355 young and mature fruiting bodies. It is notable that this pattern is seen in pileate-stipitate 4356 species and has been noted in a previous 3-species comparison of transcriptomes(Plaza et 4357 al. 2014b). Species without separate cap and stipe tissues either do not have this gene (Pt. gracilis) or they do, but the gene is not developmentally regulated (S. commune, Ph. chrysosporium). The current genome assembly of $C$. cinerea does not contain an orthologue in this group, which might be because it became dispensable for the unique developmental trajectory of this species or because the genome assembly is incomplete at this genomic region. The marked stipe expression peak suggests a widespread role of water transport during mushroom development. Further, the increasing expression parallel with developmental progression could indicate a role in water supply needed for cellular expansion. On a related note, $C$. neoformans also shows an upregulation of an aquaporin gene (Cryne_JEC21_1_3081) late in its development.

A consequence of growth by cell expansion is that growth rate is limited by the speed of water transport, not cell division and CW material deposition(Roper and Seminara 2019). Therefore, aquaporin-facilitated water transport might be a selected trait in many mushroomforming fungi that undergo fast growth. Although unknown at this moment, if fruiting body expansion is mediated by locally produced osmolytes (e.g. by breaking down glycogen), then that could produce a gradient that draws water towards the expanding tissues. This may be further facilitated by water channels formed by aquaporins. Finally, it is possible that other physical solutions, e.g. inter-hyphal water passageways also help transporting (e.g. via surface tension) water in the fruiting body.

The shared stipe upregulation of aquaporins can shed light on the origins of the typical mushroom (pileate-stipitate) morphology. All examined stipitate Agaricales species showed a stipe-specific aquaporin expression, which supports a single origin of pileatestipitate fruiting bodies in the Agaricales. On the other hand, L. tigrinus, which evolved pileate-stipitate fruiting bodies independently in the Polyporales, does not show stipe-specific aquaporin expression.

\section{4.14.2. Stress response genes}

4383 Diverse stress-related genes were detected among CDE orthogroups. Based on functional 4384 annotations and orthology relationships, many of these seem to be related to protection from 4385 oxidative stress, either through direct protection by their protein products (peroxiredoxins) or 4386 via the produced metabolites (ergothioneine) that protect from oxidative stress. We found 4387 evidence for all three pathways of reactive oxygen detoxification, the peroxiredoxin pathway, 4388 cytoplasmic catalases and glutathione peroxidases(Breitenbach et al. 2015), being active in 4389 fruiting bodies. The dynamic expression of these genes may point to the existence of an 4390 oxidative environment in fruiting bodies. However, it is currently unknown in which 4391 tissues/stages and in what cellular contexts this may arise. One possibility is that the action 4392 of cell wall modifying enzymes (e.g. peroxidases), which require an oxidative environment $4393\left(\mathrm{H}_{2} \mathrm{O}_{2}\right)$, results in excess reactive oxygen species, though this has not been confirmed yet 4394 and remains a hypothesis for now. Below we discuss the main stress-related CDE 4395 orthogroups which we detected in the developmental transcriptomes of Agaricomycetes. 
bioRxiv preprint doi: https://doi.org/10.1101/2021.12.09.471732; this version posted December $10,2021$. The copyright holder for this preprint (which was not certified by peer review) is the author/funder, who has granted bioRxiv a license to display the preprint in perpetuity. It is made available under aCC-BY-NC-ND 4.0 International license.

Running head: fruiting body genomics

$4396 \quad$ Peroxiredoxins - Peroxiredoxins are antioxidant proteins that can reduce diverse 4397 reactive oxygen species (ROS), including $\mathrm{H}_{2} \mathrm{O}_{2}$, and are one of the three cellular pathways 4398 of ROS detoxification in fungi. They have also been reported to be involved in signal 4399 transduction. The function of peroxiredoxins are not known in fruiting bodies and we 4400 identified a single report of peroxiredoxin upregulation in fruiting bodies, in

4401 Termitomyces(Rahmad et al. 2014). Another gene from Antrodia camphorata was cloned 4402 and biochemically characterized(Wen et al. 2007). We found orthologs of peroxiredoxin Tpx1 4403 of Sch. pombe and Tsa1 of S. cerevisiae (C. cinerea protein ID: 468305) to be 4404 developmentally regulated in 12/8 species ( $\mathrm{FC}>2 / 4)$ (Table 39). Tpx1 has been 4405 characterized as the $\mathrm{H}_{2} \mathrm{O}_{2}$ sensor that relays the redox signal to the transcription factor Pap1 4406 by inducing the formation of intramolecular disulfide bonds in Pap1(Jara et al. 2007).

4407 Orthologs of Tpx1 are upregulated mostly in gill and cap tissues of mature fruiting bodies in 4408 the examined species. Orthologs of the Pap1 transcription factor (represented by C. cinerea 4409 378471) are expressed constantly in mushroom-forming fungi. Orthologs of yeast Ahp1 4410 peroxiredoxin (C. cinerea protein ID: 444300) were also developmentally regulated in the 4411 majority of species (10/4 species at $\mathrm{FC}=2 / 4$, Table 39$)$. This gene preferentially eliminates 4412 organic peroxides, rather than $\mathrm{H}_{2} \mathrm{O}_{2}$ and relays signal to the Cad1 transcription factor in $S$. 4413 cerevisiae(Iwai et al. 2010). In C. cinerea this gene shows a very strong induction in young 4414 fruiting body gills, whereas in other species no evidence for a transcript enrichment in gill 4415 tissues was found. Finally, we detected orthologs of $S$. cerevisiae Dot5 (C. cinerea protein 4416 ID: 379600) (Table 39), a thioredoxin (thiol-specific peroxidase) that is involved in the 4417 detoxification of peroxides(Cha et al. 2003; Izawa et al. 2003), but was also reported to be 4418 involved in the regulation of telomere length. Thioredoxins, together with peroxiredoxins 4419 (thioredoxin peroxidase) make up the thioredoxin system, which acts similarly to the 4420 glutaredoxin system in ROS scavenging. This orthogroup showed a characteristic 4421 upregulation in primordia relative to vegetative mycelium, then lower expression in late 4422 developmental stages in C. cinerea, L. edodes, C. aegerita, A. ostoyae and A. ampla, but 4423 not in other species.

$4424 \quad$ Ergothionine biosynthesis - We also detected orthologs of the ergothioneine 4425 biosynthesis protein Egt1 of $S$. pombe. Ergothioneine is a histidine-derived antioxidant 4426 metabolite known to confer beneficial health effects to mushroom fruiting bodies(Weigand4427 Heller et al. 2012; Kalaras et al. 2017). For eukaryotic cells, it might be needed for growth 4428 and survival under oxidative stress conditions, for example during conidial survival and 4429 germination(MH et al. 2012; Sheridan et al. 2016). Ergothioneine is produced via a two-step 4430 reaction pathway in S. pombe(Pluskal et al. 2014), comprising Egt1 then Egt2. Both are 4431 conserved in C. cinerea (C. cinerea protein ID: 356399 and 258252, respectively, Table 39), 4432 however, only the first gene is developmentally regulated in the majority of the species, the 4433 other is not. Expression patterns of the Egt1 orthologs varied, in certain species they are FB4434 init, while in others it showed stipe-upregulation (A. ostoyae, L. bicolor, L. edodes, L. tigrinus, $4435 M$. kentingensis, but not $C$. cinerea). How this can be explained is not clear, nevertheless, 4436 our data provide some evidence for the upregulation of ergothioneine biosynthesis in fruiting 4437 bodies. Orthologs of Sch. pombe Egt2, which catalyzes the second step of ergothioneine 4438 production (C. cinerea protein ID: 258252) were developmentally regulated in a more 4439 restricted set of species.

4440 Dehydro-D-arabinono-1;4-lactone - Two orthogroups that make up the 4441 biosynthesis pathway of dehydro-D-arabinono-1;4-lactone were developmentally expressed 4442 in several species. Dehydro-D-arabinono-1;4-lactone (previously known as D4443 erythroascorbate) is a protective metabolite against oxidative stress and is produced by a 
bioRxiv preprint doi: https://doi.org/10.1101/2021.12.09.471732; this version posted December 10,2021 . The copyright holder for this preprint (which was not certified by peer review) is the author/funder, who has granted bioRxiv a license to display the preprint in perpetuity. It is made available under aCC-BY-NC-ND 4.0 International license.

Running head: fruiting body genomics

4444 pathway comprising Ara1, Ara2 and Alo1 in S. cerevisiae(K et al. 2006). Of these, Ara2 and 4445 Alo1 have clear orthologs in Agaricomycetes (represented by C. cinerea 365550 and 4446 432313, respectively)(Table 39), whereas Ara1 does not. Both Ara2 and Alo1 orthologs are 4447 strongly upregulated in fruiting bodies. ALO1 orthologs showed a clear upregulation in stipe 4448 tissues of C. cinerea, A. ostoyae and P. ostreatus (but not in L. bicolor or L. edodes), 4449 whereas in S. commune, $A$. ampla and L. edodes it is upregulated in primordia and stays 4450 high throughout fruiting body development. In $C$. aegerita, the gene is strongly upregulated 4451 in the first primordium stage, then expression drops to low levels.

$4452 \quad$ Catalases - Two catalase orthogroups (represented by C. cinerea 472224 and 4453 420157)(Table 39) were developmentally regulated in the majority of species. Catalases are involved in $\mathrm{H}_{2} \mathrm{O}_{2}$ scavenging and thus protection against reactive oxygen species, but also in other pathways (such as beta-oxidation, see above), so their assignment to oxidative stress response is less certain than that of the above-mentioned genes.

Glutathione system - The glutathione system is composed of (i) glutathione, a 3amino acid redox-active metabolite(Pócsi et al. 2004; Sato et al. 2009); (ii) glutathione peroxidases, which reduce $\mathrm{H}_{2} \mathrm{O}_{2}$; (iii) glutathione reductases, which convert oxidized glutathione back to its non-oxidized form; as well as (iv) glutaredoxins, which repair oxidatively damaged proteins. In $S$. cerevisiae glutathione is produced by a two-step reaction from L-cysteine and L-glutamate by the enzymes GSH1 and GSH2(CM 2001). We annotated members of this pipeline in $C$. cinerea based on orthology information to components of the glutathione system in S. cerevisiae(Pócsi et al. 2004; Sato et al. 2009)( Table 39). We identified single-copy orthologs of two proteins in the glutathione synthesis pathway, glutathione reductase, glutathione peroxidase. Glutaredoxins are present, on average, as 5-6 distinct genes in Agaricomycetes genomes, except for Pt. gracilis and $M$. kentingensis, which harbor 9 and 11 glutaredoxin genes in their genomes, respectively. Unlike the other antioxidant systems, the expression patterns in the glutathione system show only moderate conservation across species, being developmentally regulated in 3-6 species (Table 39).

Glutathione-S-transferases are also involved in defense against oxidative damage. They represent a diverse gene family also involved in other cellular detoxification tasks, such as the neutralization of xenobiotics, drugs or pesticides(M et al. 2009b). Leon-Ramirez et al hypothesized that in $U$. maydis 'basidiocarps' glutathione-S-transferases are involved in the protection against oxidizing intracellular conditions generated by some enzymes, such as NAPDH oxidases (CG et al. 2017). Therefore, we only marginally treat them under this chapter, noting that any glutathione-S-transferase orthogroup, also by virtue of the lack of clear orthology to functionally characterized genes from model species, could be related to any of the possible functions of this family, not necessarily protection from oxidative damage. Glutathione S-transferases are a diverse gene family in fungi, with 20-49 genes (on average 32 ) in the genomes of Agaricomycetes. In each of the species, $~ 50 \%$ of glutathione Stransferase encoding genes (up to $70 \%$ in Coprinopsis) were developmentally regulated, indicating that these play important roles in fruiting body development, however, due to the general lack of conservation of these genes, these did not form CDE orthogroups. Interestingly, each species had genes that showed a marked upregulation (FC>4) at the initiation of fruiting body development. 
bioRxiv preprint doi: https://doi.org/10.1101/2021.12.09.471732; this version posted December 10,2021 . The copyright holder for this preprint (which was not certified by peer review) is the author/funder, who has granted bioRxiv a license to display the preprint in perpetuity. It is made available under aCC-BY-NC-ND 4.0 International license.

Running head: fruiting body genomics

4488

4489

4490

4491

4492

4493

4494

4495

4496

4497

4498

4499

4500

4501

4502

4503

4504

4505

4506

4507

4508

4509

4510

4511

4512

4513

4514

4515

4516

4517

4518

4519

4520

4521

4522

4523

4524

4525

4526

4527

4528

4529

4530

4531

4532

4533

\subsubsection{Unannotated genes}

Genes that encode proteins with no known conserved domain (Pfam or InterPro) signatures, referred to as unannotated genes here, are abundant in fungal genomes and represent some of the most exciting gene families. At the same time, they represent a challenge to link to fruiting body development, due to the complete lack of functional clues. In general, few literature reports are available on unannotated genes, possibly due to the difficulty of tackling their functions. An exception from this is a report of the enrichment of unannotated genes in mature peridioles of Pisolithus microcarpus fruiting bodies(Pereira et al. 2017). An example of unannotated genes that turn out to be developmentally relevant is the LPMO-like X325 gene family(Labourel et al. 2020), which was circumscribed just recently and appears to be related to the cell wall (see chapter 4.8.2.1).

We identified 176 CDE orthogroups of unannotated genes (Supplementary Table 6). These included, for example, Spc14 and Spc33 of S. commune, which are involved in the formation of the septal pore cap(Van Peer et al. 2010), are unannotated proteins but form conserved, single-copy gene families in the Agaricomycetes (represented by $C$. cinerea 419716 and 473658 ) that was developmentally regulated in 10/6 and 12/5 species, respectively. In $C$. cinerea alone, we identified 32 FB-init unannotated genes, several of which showed very significant fold changes (up to 150-200x).

This category of genes included Ctg1 of L. edodes and Cc.Ctg1 of C. cinerea. Ctg1 was originally identified in $L$. edodes as a target of the pre-mRNA splicing factor Cdc5 and was later shown to influence stipe elongation in C. cinerea(Nakazawa et al. 2008). In the latter study knockdown experiments were unsuccessful, whereas overexpression $C$. cinerea mutants showed accelerated stipe elongation(Nakazawa et al. 2009). In our data Ctg1 orthologs formed a CDE orthogroup (represented by C. cinerea 528942) which was developmentally regulated in 10/8 species (FC>2/4)(Supplementary Table 6). Despite the conservation of developmental regulation, expression profiles were only partially conserved across species. For example, the gene was upregulated in mature fruiting bodies in $C$. aegerita, $A$. ampla, C. cinerea, $L$. edodes, $P$. ostreatus, whereas in other species (e.g. $A$. ostoyae), it showed upregulation in primordia relative to vegetative mycelium. Overall, CTG1 orthologs seem to be a very exciting group of developmental genes, with no conserved domains and no mechanistic information on how they might contribute to fruiting body formation. Its role in stipe elongation needs to be conserved in other species, if expression profile conservation correlates with phenotype, we may expect other phenotypic effects in other species.

In summary, unannotated genes may contain very interesting, novel gene families involved in fruiting body development, yet circumscribing their functions remains challenging at the moment and will require extensive genetic manipulation techniques.

\subsubsection{Functionally poorly known genes}

In this group of genes we placed ones that had some (mostly automatic) annotations, but we could not clearly link them to cellular or fruiting-related processes. Thus, this category differs from unannotated genes in that unannotated genes are completely devoid of all (even automated) annotations. Altogether, we assigned 182 CDE orthogroups to this category (Supplementary Table 7). Some of these genes are discussed briefly below, but we note that many more interesting, fruiting-related genes are potentially hidden among the 182 orthogroups, however, clarifying their roles is left for future studies. 
bioRxiv preprint doi: https://doi.org/10.1101/2021.12.09.471732; this version posted December $10,2021$. The copyright holder for this preprint (which was not certified by peer review) is the author/funder, who has granted bioRxiv a license to display the preprint in perpetuity. It is made available under aCC-BY-NC-ND 4.0 International license.

Running head: fruiting body genomics

$4534 \quad$ Cytochrome p450 family - We discuss cytochrome p450s in this category, because 4535 they participate in diverse functions, from secondary metabolite biosynthesis (as part of gene 4536 clusters, e.g.(Nofiani et al. 2018)) to unknown cellular pathways mediating stipe 4537 elongation(Muraguchi and Kamada 2000). We detected 8 CDE orthogroups of cytochrome 4538 p450s, most of which are functionally unknown. Among the cytochrome p450s, C. cinerea 4539 Eln2 should be mentioned. This gene was identified associated with a dominant mutation 4540 that confers an elongationless stipe phenotype(Muraguchi and Kamada 2000). Eln2 was 4541 reported to be a constitutively expressed cytochrome p450. In accordance with this, we did 4542 not find considerable expression dynamics on the orthogroup containing eln2 (represented 4543 by C. cinerea 381558). However, the developmentally regulated orthogroups may be worthy 4544 targets of functional analyses in the future.

$4545 \quad$ GT25 family - This family includes enzymes with diverse activities

4546 (lipopolysaccharide $\beta$-1,4-galactosyltransferase, $\beta$-1,3- or $\beta$-1,2-glucosyltransferase). In 4547 bacteria, GT25 are responsible for the biosynthesis of the membrane component 4548 lipopolysaccharides(Bertani and Ruiz 2018) ('endotoxins'). Most known GT25 enzymes are 4549 of bacterial origin, we found no functional study of fungal genes to date. The examined 4550 Agaricomycetes had mostly a single gene encoding GT25, which showed sporulation4551 associated expression peaks in most species: young fruiting body gills in $C$. cinerea, fruiting 4552 body gills in $P$. ostreatus, fruiting body cap in $L$. edodes and $C$. aegerita, mature fruiting 4553 bodies in A. ampla and S. commune, etc.. GT25 encoding genes showed very low 4554 expression in general, in many cases only detectable in the peak expressing stage/tissue. 4555 The broader orthogroup (C. cinerea protein ID: 501720) that contains the detected GT25 4556 genes is specific to the Basidiomycota (result not shown), which could indicate bacterial 4557 origin into the phylum. The function of these proteins in fruiting bodies is currently unknown, 4558 however, expression peaks coincident with sporulation suggest they are related to late 4559 developmental events.

4560 UstYa-like mycotoxin biosynthesis proteins - this gene family was first reported to 4561 be involved in the synthesis of the ribosomally synthesised peptide toxin ustiloxin in the 4562 ascomycete Ustilaginoidea virens(T et al. 2015). This family of genes was found to 4563 participate in the production of other toxic cyclic peptides, collectively named 'dikaritins', in 4564 plant pathogenic fungi (Ding et al. 2016; Vogt and Künzler 2019). Biochemically, they are 4565 oxidases of ribosomally produced peptides, including ustiloxins but also alpha-pheromones 4566 of $S$. cerevisiae (collectively Kex2-processed repeat proteins, KEPs)(Umemura 2020). Using 4567 a simple domain-based search, we detected 2-16 (28 in A. ostoyae) genes in the examined 4568 Agaricomycetes. Of these, each species had one to multiple genes that showed an induction 4569 (>4fold) in primordia relative to vegetative mycelia (except $C$. cinerea, in which two genes 4570 were induced in young fruiting body caps), and high expression in all subsequent fruiting 4571 body stages, suggesting that their protein product has an increased abundance in fruiting 4572 bodies. Although the role of these genes in the Basidiomycota remains unknown for now, we 4573 speculate they may be responsible for the production of toxic compounds, possibly with a 4574 role in defense.

4575 Carbonic anhydrases (CA) - convert $\mathrm{CO}_{2}$ to $\mathrm{HCO}_{3}{ }^{-}$which is then used in various 4576 ways by eukaryotic cells. CAs have been shown to be involved in fruiting body formation: CA 4577 knockout strains of the ascomycete $S$. macrospora showed slower vegetative growth and 4578 delayed fruiting body formation or defects in ascospore germination(Elleuche and Pöggeler $45792009,2010)$. The delay in fruiting body formation suggests a lower rate of $\mathrm{HCO}_{3}{ }^{-}$buildup 4580 necessary for some as yet unknown downstream processes, that, however, can be made up 4581 in longer time. Similar roles of CAs have not yet been reported in Basidiomycota, however, 
bioRxiv preprint doi: https://doi.org/10.1101/2021.12.09.471732; this version posted December 10,2021 . The copyright holder for this preprint (which was not certified by peer review) is the author/funder, who has granted bioRxiv a license to display the preprint in perpetuity. It is made available under aCC-BY-NC-ND 4.0 International license.

Running head: fruiting body genomics

4582 they would be worth investigating as many CAs appeared developmentally regulated in 4583 fruiting bodies. We found 2-9 CA encoding gene in the examined Agaricomycetes and that 4584 each species had at least one developmentally regulated carbonic anhydrase gene, 4585 although clear patterns of expression conservation were not noticeable. We found one CDE 4586 orthogroup (C. cinerea protein ID: 450047 ) that was widely developmentally regulated in 4587 mushroom-forming fungi (8/5 species at FC2/4), showing diverse expression peaks in 4588 different species. It should be noted that CA expression levels potentially react also to ambient $\mathrm{CO}_{2}$ concentrations, so some of the expression variation we encountered may be due to the growth conditions used during fruiting.

CipB gene family - This category of genes included the cipB gene reported from $C$. cinerea(Nakazawa et al. 2009). The cipB gene was first identified as an interacting partner of the splicing factor cdc5 in L. edodes(Nakazawa et al. 2008). We found that the orthogroup that contained cipB orthologs (represented by $C$. cinerea 401749) was developmentally regulated in 10/2 species ( $F C>2 / 4)$, indicating that these genes have widespread although moderate expression dynamics (Supplementary Table 7). Based on conserved domain annotations, cipB genes are transmembrane ATP synthases located in the mitochondria; its contribution to fruiting body formation is not known at the moment.

\subsubsection{Ferric reductase superfamily proteins and iron metabolism}

4600 The Ferric Reductase Domain Superfamily comprises a conserved group of proteins that 4601 include ferric reductases and NAPDH oxidases, both of which are located in the plasma 4602 membrane(Zhang et al. 2013). Ferric reductases are part of a reductive iron uptake system which, in many model fungi, are involved in maintaining iron and potentially also copper homeostasis(Gaurav Bairwa et al. 2017). These genes have mostly been studied in the context of iron acquisition of pathogenic fungi from their hosts. Agaricomycetes don't share clear orthology with experimentally characterized ferric reductases of the Ascomycota (reviewed in(Gaurav Bairwa et al. 2017))(cite Hegedus et al 2021). Nevertheless, we identified eight ferric reductase orthogroups, of which two were developmentally regulated in multiple species (Table 40), suggesting that ferric reductases have conserved roles in fruiting body development, although their function in the process is unknown. One of these was developmentally expressed in 9/5 species (represented by $C$. cinerea $354442, F C>2 / 4$ ) contains genes orthologous to $S$. cerevisiae FRE7, which might be involved in copper homeostasis in S. cerevisiae(C et al. 2000). Another orthogroup, which contains orthologs of A. nidulans NoxA and N. crassa nox-1 (represented by C. cinerea 260659), was developmentally regulated in 5/1 species. This gene is a NADPH oxidase, which has been shown to be involved in developmental signaling and tissue differentiation(Nguyen et al. 2017).

The Ferric reductase superfamily also includes NADPH oxidases (NOXs)(Zhang et al. 2013), which are reactive oxygen-generating cell membrane proteins involved in differentiation-related signaling processes and tissue patterning(Blackstone). NOX genes and their regulator NoxR were reported to have been lost repeatedly in yeast lineages, underscoring their role in multicellularity(Nguyen et al. 2017). There is evidence for NOXs regulating sexual, but not asexual or vegetative development in $A$. nidulans(T et al. 2003) and G. lucidum and there is expression evidence in H. marmoreus(Zhang et al. 2015b). We identified two putative NOX orthogroups in Agaricomycetes (C. cinerea protein ID: 461481, 260659). A third (represented by $C$. cinerea 152927) was interpreted as a NOX by LeónRamírez et al(CG et al. 2017), but appears to be a ferric reductase. Despite clear reports of 
bioRxiv preprint doi: https://doi.org/10.1101/2021.12.09.471732; this version posted December 10,2021 . The copyright holder for this preprint (which was not certified by peer review) is the author/funder, who has granted bioRxiv a license to display the preprint in perpetuity. It is made available under aCC-BY-NC-ND 4.0 International license.

Running head: fruiting body genomics

4628 NOXs being induced in sexual structures of Ascomycota(T et al. 2003), we did not find 4629 evidence for consistent induction of these genes in fruiting bodies, rather, expression 4630 patterns vary among fruiting body forming fungi. NOX genes show modest expression 4631 dynamics in mushroom forming fungi $(2<\mathrm{FC}<4$, Table 40$)$. C. cinerea 448679 appears to be 4632 a single-copy homolog of noxR that is conserved among mushroom-forming fungi. Members 4633 of this orthogroup are not developmentally expressed.

4634

\section{A synoptic model of fruiting body development}

4636 This study aimed to provide a catalog of conserved fruiting body development genes based

4637 on a literature review and a meta-analysis of developmental transcriptomes of 12

4638 mushroom-forming fungi. Based on sequence analyses of genes showing developmentally

4639 expression during fruiting body morphogenesis in these 12 species, we identified 921

4640 conserved developmentally expressed orthogroups and several gene families. To more

4641 broadly explore pathways linked to these genes, as well as genes that are known to be

4642 involved in fruiting but do not show significant expression dynamics, we analyzed further 558

4643 gene families. As a result, altogether 1479 genes of $C$. cinerea, and the orthologs of these in

464411 other species are treated in this paper. These allowed us to identify developmental

4645 expression patterns that are shared across species. For most of the conserved

4646 developmental orthogroups, we provided broad functional classifications and speculations

4647 on their probable role in fruiting body development, although elucidating their exact function

4648 and molecular mechanisms of their action remains for future studies. In this chapter we

4649 synthesize these data into an updated, synoptic model of fruiting body development in the

4650 Agaricomycetes (Fig. 15). This model strongly builds on work by previous scholars, who

4651 attempted to provide overviews of developmental and/or molecular mechanisms of

4652 mushroom formation. In a series of papers Moore provided detailed descriptions of

4653 developmental events based on morphological and biochemical analyses(Moore 2005).

4654 Later, Kues summarized knowledge on the life history of $C$. cinerea and details of its

4655 developmental biology(Kües 2000; Kües and Liu 2000). More recently, de Plaza et al, Ohm

4656 et al and Muraguchi et al provided examples of transcription networks active during

4657 development and a molecular overview of $C$. cinerea development based on gene ontology, 4658 respectively(Ohm et al. 2011; Plaza et al. 2014b; Muraguchi et al. 2015). This paper further

4659 elaborates these models with more detail, and a selective presentation of only those

4660 processes that, based on gene expression patterns are conserved across twelve

4661 Agaricomycetes species. We hereafter discuss developmental events in three phases: early

4662 events (primordium formation and cell proliferation), growth by cell expansion and late

4663 events (sexual processes, sporogenesis).

\section{5.1. Early events: primordium formation and cell proliferation}

4665 Expression patterns of developmentally regulated gene groups outline several distinct

4666 processes and a temporal sequence of cellular events in fruiting bodies. The first in this

4667 sequence and probably the most interesting is the initiation of fruiting body development,

4668 which involves the rearrangement of hyphal branching patterns, and the light-dependent

4669 formation of a primary, then a compact, secondary hyphal knot(Kües 2000; Liu 2005;

4670 Sakamoto et al. 2018). Hyphal knot formation represents a transition from loosely arranged

4671 hyphae with fractal dimensions to true complex multicellular structures(Nagy et al. 2018). 
bioRxiv preprint doi: https://doi.org/10.1101/2021.12.09.471732; this version posted December 10,2021 . The copyright holder for this preprint (which was not certified by peer review) is the author/funder, who has granted bioRxiv a license to display the preprint in perpetuity. It is made available under aCC-BY-NC-ND 4.0 International license.

Running head: fruiting body genomics

4672 Fruiting body initiation has been a subject of intense research. Several gene expression 4673 changes have been described in this transition, including light-regulated(Sakamoto et al. 4674 2018), defense (e.g. galectins, pore-forming toxins(Plaza et al. 2014b), lectins(Boulianne et 4675 al. 2000; Walser et al. 2005)), fatty acid biosynthesis(Liu 2005), transcriptional regulation 4676 (TFs, velvet proteins(Ohm et al. 2011; Plaza et al. 2014b)), cell surface (hydrophobins, PriA, 4677 fasciclins(S et al. 1992; Y et al. 2007)), cell wall (Kre9/Knh1, laccases(Kües et al. 2011)) as 4678 well as others(Liu 2005; Hou et al. 2019). We update these observations by several 4679 functionalities in this work.

$4680 \quad$ The largest gene group that shows an upregulation in primordium samples is related 4681 to mitotic cell division (See Fig. 3 in chapter 4.1.1 above), which coincides with tissue 4682 differentiation in the primordium and produces the final number of cells that makes up the 4683 fruiting body. Ribosomal and chromatin-remodeling related gene (e.g. histones) expression 4684 show very similar expression profiles in primordia (see Fig. 4 and 10 in chapter 4.1 .2 and 4685 4.5.4.1), suggesting that novel protein synthesis and chromatin remodeling are required cell 4686 proliferation/division in primordia. Chromatin remodeling might be required for mitotic 4687 chromosome condensation and/or for rearranging chromatin accessibility for fruiting-specific 4688 trans regulatory factors. We term this the proliferative phase of development, which, in $C$. 4689 cinerea, encompasses hyphal knots to stage 2 primordia (Fig. 15). This pattern of mitotic, 4690 histone and ribosomal gene expression has been observed in most species, suggesting that 4691 fruiting body development can, in general, be divided into proliferative phase and growth by 4692 cell division. However, some species (e.g. A. bisporus(Craig et al. 1977), see chapter 4.1.3) 4693 might not fully conform to this pattern and we expect non-Agaricales species (e.g.

4694 Polyporales, bracket fungi) to not follow this rule.

While the vegetative mycelium is entangled in the substrate and sensitive to bacterial and fungal competitors, fruiting bodies grow above ground and are, thus, more exposed to more extreme environmental conditions and biotic stress (animals grazers or infections). Accordingly, It has been reported that mycelial- and fruiting body-expressed defense gene repertoires show little overlap, indicating a dramatic switch in defense strategy upon fruiting(Plaza et al. 2014b). Defense genes generally show little conservation and high phylogenetic patchiness and did not form conserved orthogroups in our analyses. We, therefore, discussed them in the context of broader gene families (for detailed reviews see(Kunzler 2015; Künzler 2018)). Putative secondary metabolism and oxylipin-related genes might also serve defense and/or antimicrobial purposes.

Probably as a response to differences in environment compared to the substrate, such as higher temperature or humidity fluctuations, several cell surface protein-encoding and fatty acid metabolism genes showed induction in primordia. These included hydrophobins, cerato-platanins, wax synthases and the Con6 family, among others. Hydrophobins probably help retaining water or provide protection from excess water aboveground(Lugones et al. 1999b). Wax synthases and Con6 proteins, although haven't been examined to date, might act in a similar way. We infer changes in cell membrane composition in primordia from expression changes in genes influencing membrane composition. For example, fatty acid desaturases related to linoleic acid biosynthesis are 4715 fruiting bodies reported before(SHAW 1967; Song et al. 2018a), with potentially to oxylipin 4716 synthesis too(A et al. 2020)). Putative stress-related gene expression changes 4717 (peroxiredoxins and ergothioneine-biosynthesis genes) might also correspond to genetically 4718 encoded mechanisms for coping with adverse above-ground conditions. 
bioRxiv preprint doi: https://doi.org/10.1101/2021.12.09.471732; this version posted December 10,2021 . The copyright holder for this preprint (which was not certified by peer review) is the author/funder, who has granted bioRxiv a license to display the preprint in perpetuity. It is made available under aCC-BY-NC-ND 4.0 International license.

Running head: fruiting body genomics

$4719 \quad$ Cell wall remodeling emerges as a potentially important process in fruiting body 4720 initiation both in this study and previous ones (e.g.(Liu et al. 2021)). Fruiting body-specific 4721 cell wall architectures exist and are probably produced by a diverse suite of lytic and cell wall 4722 remodeling enzymes that are induced in primordia (Fig. 15). From the array of conserved 4723 orthogroups, it appears that glucan remodeling is more important than chitin modification. 4724 Additionally, oxidative enzyme genes (HTPs, DyP, MCOs, AA5_1) seem to be important in 4725 primordium development, although their functions have not been elucidated, yet. Cell wall 4726 related gene families show conservation at the gene family level, not always at the level of 4727 strict CDE orthogroups. Therefore, the complete array of cell wall remodeling enzymes 4728 induced in primordia is much broader in any species than shown on Fig. 15. Several cell wall 4729 remodeling related gene families described from the Ascomycota were developmentally 4730 regulated in the Agaricomycetes as well (e.g. GH16/17, Kre9/Knh1), whereas some, that are 4731 central to Ascomycota cell wall remodeling showed constant expression (e.g. GH72) or are 4732 missing in the Agaricomycetes (e.g. GH76). Further, it appears that dynamic expression is 4733 mostly observed in cell wall-active enzyme genes, not in upstream (e.g. ER-localized) components of the cell wall assembly line.

Although the light-dependency of fruiting body initiation differs from species to species(Sakamoto 2018), we detected some orthogroups that probably correspond to light reception. These include phytochromes, the $C$. cinerea Dst2 family and potentially PAS domain containing proteins. The latter is particularly interesting, given that homologs in $N$. crassa regulate the white collar complex and mediate adaptation to changing light intensities during the day(Fuller et al. 2015).

The initiation of fruiting body development represents a transition from fractal-like to complex multicellularity. Accordingly, several complex multicellular processes, such as cellto-cell communication, adhesion and the regulation of differentiation(Knoll 2011; Nagy et al. 2018) should turn on in primordia. Like for previous studies, decoding these aspects proved difficult for us too. We expect regulatory factors, such as transcription factors, kinases, phosphatases, potentially F-box proteins or non-proteinogenic factors (e.g. small RNA) to participate in cellular differentiation. We and previous work detected some transcription factor, kinase, NOX, pheromone, F-box orthogroups upregulated in primordia. However, these generally did not show significant conservation of expression, so the main regulatory mechanisms of cellular differentiation in fruiting bodies remain an enigma. It further remains to be established if differentiation mechanisms described from plants and animals, such as morphogens, hormone-like substances (e.g. oxylipins) or highly conserved gene regulatory circuits exist in fungi. Similarly, adhesion-related proteins are mostly unknown at the moment. Fasciclins and cell wall modification (e.g. by laccases) have been speculated to be related to adhesion(Nagy et al. 2018; Merényi et al. 2020b), though glucan remodeling enzymes, wax synthases, the carbohydrate-binding properties of lectins or species-specific

4757 mechanisms could also be key and more research is needed to elucidate what genes glue 4758 hyphae together in 3-dimension fruiting bodies.

\subsection{Transition to growth by expansion}

4760 At the stage 2 primordium stage (following nomenclature in C. cinerea, Fig. 15), cell and 4761 tissue differentiation, cell proliferation has largely completed. This is reflected in the 4762 downregulation of mitotic, ribosomal and chromatin-remodeling genes. At this point, the 4763 fruiting body transitions to a different developmental mechanism, which we term 'growth by 4764 cell expansion'. Growth by cell expansion is a well-known fungal trait and was already noted 
Running head: fruiting body genomics

4765 by de Bary in 1887(Bary et al. 1887), however, the molecular details have remained

4766 unknown. The phenomenon is marginally similar to fruit ripening in plants, but the

4767 mechanisms are probably completely different, though both involve cell wall loosening,

4768 remodeling and cell expansion.

$4769 \quad$ Based on analyses of developmental transcriptomes, we detected what we believe is

4770 a plausible molecular mechanism for growth by expansion. This involves the upregulation of

4771 membrane biosynthesis genes and the rewiring of basic metabolic pathways for the

4772 production of excess Acetyl-CoA (see chapter 4.2), which is the starting point of the

4773 biosynthesis of membrane components (e.g. ergosterol). Upregulation of genes involved in

4774 sterol transport, ergosterol biosynthesis correlates with the rewiring of basic metabolism. At

4775 the same time, aquaporin gene expression increases in stipes, which probably supports

4776 water transport driving the expansion of cells via turgor. Turgor manipulation requires an

4777 osmolyte, however, despite speculations of trehalose or mannitol serving as osmolytes, its

4778 identity remain unresolved; the expression data analyzed here did not provide unequivocal

4779 evidence for any given osmotically active molecule in fruiting bodies. Trehalose, glycogen

4780 and mannitol metabolism genes are upregulated at this stage, but many of them in gills,

4781 which suggest an involvement in spore storage material production, rather than turgor

4782 manipulation.

4783

4784

4785

4786

4787

4788

4789

For enabling cell volume increases, the fungus deploys a diverse and mostly species-specific array of cell wall remodeling enzymes. Cell expansion also probably follows different mechanisms in each tissue. For example, according to the model of stipe elongation in C. cinerea proposed by Liu et al(Liu et al. 2021), CE4 and GH30 genes may contribute to the rigidification of the cell wall in the stipe base, causing it to be more resistant to loosening by lytic enzymes than the stipe apex, thereby reducing its elongation potential. It is reasonable to assume that similar mechanisms underlie the differential expansion potential of different tissues within the fruiting body. We detected a large suite of conserved CAZyme orthogroups upregulated in elongating stipes (Fig. 15), whereas only one conserved orthogroup (GH55) was found in expanding caps. Since caps are composed of multiple tissues, it is possible that RNA-Seq techniques with higher resolution (e.g. singlecell RNA-Seq) are needed to uncover conserved tissue-specific cell wall remodeling gene expression.

What regulatory genes underlie growth by cell expansion will need to be determined in future studies. We detected conserved orthogroups of several developmentally expressed regulators (transcription factors, RING-type zinc fingers, F-box, BTB and RNA-binding proteins) but most showed tissue-specific expression patterns and no expression profile clearly suggested involvement in regulating cell expansion in general. Interestingly, the Exp1 transcription factor reported to be responsible for cap expansion in $C$. cinerea(Muraguchi et al. 2008b) was more frequently upregulated in stipes than in caps across the examined species. We detected a red light-sensing phytochrome orthogroup upregulated in stipes, which we hypothesize is responsible directing stipe growth away from closed spaces towards the open air.

\subsection{Late events: meiosis and spore production}

4807 The young and mature fruiting body (in C. cinerea nomenclature) stages are the scene for 4808 most sexual events, including meiosis and spore production. Accordingly, meiotic genes as 4809 well as several genes involved in DNA replication/repair, chromatin remodeling, ribosome 4810 assembly, which showed a peak in the proliferative phase in primordia, show a very sharp 
bioRxiv preprint doi: https://doi.org/10.1101/2021.12.09.471732; this version posted December 10,2021 . The copyright holder for this preprint (which was not certified by peer review) is the author/funder, who has granted bioRxiv a license to display the preprint in perpetuity. It is made available under aCC-BY-NC-ND 4.0 International license.

Running head: fruiting body genomics

4811 expression peak associated with meiosis (see chapter 4.1.1, 4.1.2 and 4.5.4). We found 4812 several conserved orthogroups of BTB/POZ proteins and RING-type zinc fingers with 4813 expression peaks in gills; these might regulate key events associated with meiosis. A 4814 conserved transcriptional co-repressor, Cag1 of $C$. cinerea should be mentioned here. 4815 Although this gene and its orthologs did not show significant expression dynamics, its 4816 deletion caused abnormalities in gill development(Masuda et al. 2016).

$4817 \quad$ Genes associated with storage carbohydrate metabolism were upregulated in gills in 4818 most species, which is consistent with a rich body of literature reporting glycogen fluxes in 4819 fruiting bodies(Ji and Moore 1993; Kües 2000). These genes included ones related to 4820 glycogen mobilization, trehalose and mannitol metabolism. We found evidence for glycogen synthesis (Gsy1 orthologs), suggesting that this carbohydrate is packaged into spores.

Finally, in $C$. cinerea gills we detected an ortholog of the $A$. nidulans pigmentproducing polyketide synthase ( $\mathrm{PksB}$ ), this gene might be involved in spore melanin production, although in other species this orthogroup showed different expression dynamics.

\section{5}

\section{Outstanding questions related to fruiting body} morphogenesis

4827 Despite the advances in understanding cellular events of fruiting body morphogenesis, 4828 several significant questions remain open and await further research. We below outline eight key questions that we think are instrumental to develop a better understanding of fungal fruiting body formation and complex multicellularity. This represents a subjective assessment of significant challenges of fruiting body research, there may be other perspectives on remaining key challenges to be solved.

1. Although new information on fruiting body morphogenesis accumulates at a steady pace, several questions related to multicellular organization remain open. Fruiting bodies are complex multicellular structures and thus display complex developmental programs involving tissue differentiation and cell type specification. Besides a developmental program, adhesion and cell-to-cell communication are two key traits for complex multicellularity(Knoll 2011). While we are starting to understand transcriptional networks governing tissue differentiation (see chapter 4.5.1), we still know very little about the genes/proteins mediating hypha-hypha adhesion, communication or whether an extracellular matrix (other than the cell wall) exists in mushroom-forming fungi. Hypotheses have been put forth on adhesion (via laccases or fasciclins), however, these have not yet been tested experimentally. Tissue differentiation results in distinct cell types within the fruiting body, however, our current understanding of cell type diversity in mushroom-forming fungi is limited. All current estimates for cell type diversity are based on morphology, which probably represent underestimates; technologies like single-cell RNA-Seq might provide more insights into cell type classification.

2. Regarding regulatory genes, it is an open question whether 'master' regulators of fruiting body development or tissue differentiation exists, similarly to animal and plant developmental regulator systems(Meyerowitz 2002). Developmental processes are often organized modularly and hierarchically so that the resulting modules have a high level of autonomy. Such autonomy of mushroom development, to our best knowledge, has rarely been documented. An exception from this is monokaryotic fruiting, the process of forming basidiocarps on monokaryotic cultures without mating in certain species (e.g. (Herzog et al. 
bioRxiv preprint doi: https://doi.org/10.1101/2021.12.09.471732; this version posted December 10,2021 . The copyright holder for this preprint (which was not certified by peer review) is the author/funder, who has granted bioRxiv a license to display the preprint in perpetuity. It is made available under aCC-BY-NC-ND 4.0 International license.

Running head: fruiting body genomics

4856 underlying regulatory networks. Moore et al characterized fruiting body morphogenesis as 4857 being made up of developmental subroutines that are somewhat independent of each other 4858 and that makes the whole process resilient to a certain degree of developmental 4859 imprecision(Loftus et al. 2020).

4860 3. Transcriptional changes during the initiation of fruiting are significant. Most studies 4861 with a sufficient number of time points sampled (e.g.(Plaza et al. 2014b; Muraguchi et al.

4862 2015; Sipos et al. 2017b; Almási et al. 2019; Krizsán et al. 2019; Merényi et al. 2021; Zhang 4863 et al. 2021d)) (Ruytinx et al in prep) found the largest number of differentially expressed 4864 genes at the transition from vegetative mycelium to the hyphal knot/primordium stage. 4865 However, whether this transcriptional rewiring event is purely a result of transcription factor 4866 activity or whether there are more general changes, for example, in chromatin accessibility 4867 or epigenetics, is unknown at the moment. Assays such as ATAC-Seq(Buenrostro et al. 4868 2015) or ChIP-Seq (see e.g.(Vonk and Ohm 2021)) could provide insight into the magnitude 4869 of transcriptional versus chromatin-related rearrangement during fruiting body initiation. 4870 Similarly, posttranslational modification of proteins may be a significant regulatory 4871 mechanism in fruiting body morphogenesis(Pelkmans et al. 2017a). Gene methylation, an 4872 important developmental mechanism in animals, seems to be missing in fungi, on the other 4873 hand(Bewick et al. 2019).

4874 4. Understanding tissue specificity of genes and encoded proteins is key to 4875 uncovering mechanisms of tissue differentiation and predicting gene function. The bulk 4876 expression studies and a few studies involving fluorescent protein tagging revealed 4877 numerous examples of tissue-specifically expressed genes; however, more systematic 4878 studies will be needed to understand tissue-specific expression and the mechanisms that 4879 generate it.

48805 . We identified altogether 176 conserved developmentally expressed orthogroups of 4881 unannotated genes. These genes encode proteins that contain no known protein domains, 4882 therefore, they lack even automated functional annotations. Unannotated genes are 4883 common in fungal genomes and represent an enigma for mycology. Their conservation 4884 implies that they have important functions these are, however, unknown in the vast majority 4885 of cases (see Spc genes of S. commune for an exception, chapter 4.14.3). Therefore, 4886 unannotated genes might represent a future treasure trove for developmental biology.

6. Growth by cell expansion is a widely conserved trait of mushroom-forming fungi. Above we provided evidence for this process involving excess Ac-CoA production, the upregulation of membrane biosynthetic processes and cell wall remodeling genes (chapter $4.2,4.3)$. However, the identity of the osmolyte that drives turgor increase in fruiting bodies remains unknown and its identification represents a significant challenge for future research.

7. Although not strictly morphogenetic, how the fruiting body is supported by the 4894 known that the directionality of the transport of goods in the mycelium switches as fruiting 4895 body development is initiated(Herman et al. 2020). Also, it has recently been suggested that 4896 secondary metabolite biosynthesis during fruiting body development depends also on the mycelium(Orban et al. 2021). Thus, transcriptional and metabolic reprogramming has to affect the entire fungal colony and not only the part where primordia emerge. The feeding and nutrient transport mechanisms as well as regulation of fruiting in relation to nutrient availability are exciting avenues of research, but beyond the primarily morphogenetic scope of the present paper.

8. While useful for discovering shared aspects of fruiting body morphogenesis, the comparative approach used by us, ignores species-specific aspects of morphology, 
bioRxiv preprint doi: https://doi.org/10.1101/2021.12.09.471732; this version posted December 10,2021 . The copyright holder for this preprint (which was not certified by peer review) is the author/funder, who has granted bioRxiv a license to display the preprint in perpetuity. It is made available under aCC-BY-NC-ND 4.0 International license.

Running head: fruiting body genomics

4904 ecological adaptation, gene repertoires and expression dynamics. Non-conserved aspects of 4905 development are certainly important, particularly for understanding the biology of individual 4906 cultivated species and will deserve further study in the future. However, given the available 4907 information, making functional inferences for taxonomically restricted or species-specific 4908 genes is even harder than for conserved genes, therefore, we here restrict ourselves to the 4909 discussion of the latter.

\section{Conclusions}

4911 The development of mushroom fruiting bodies is one of the most complex morphogenetic 4912 processes in fungi. A significant body of previous research has focused on understanding 4913 the underlying genetics and has made significant progress in cataloging genes related to the 4914 initiation, morphogenesis, defense, pigmentation of fruiting bodies, among others. However, 4915 available knowledge is still patchy and lacks key details in multiple aspects, such as 4916 conserved events in fruiting body morphogenesis and corresponding genes or key regulators 4917 of major differentiation steps.

$4918 \quad$ This study aimed at providing a catalog of conserved fruiting body development 4919 genes based on a literature review and a meta-analysis of developmental transcriptomes of 492012 mushroom-forming fungi. We identified gene orthogroups that show conserved 4921 developmental expression across multiple species. Despite some limitations of the genomic 4922 and transcriptomic approach used here, this study provided novel insights (such as gene 4923 families, orthogroups, conserved expression profiles) into the morphogenesis of mushroom 4924 fruiting bodies. We hope these data reveal some aspects of the core genetic program of 4925 agaricomycete fruiting body morphogenesis and will provide a roadmap for functional 4926 analyses of fruiting body development. We are aware that the summary provided here is still an incomplete survey of developmental genes in fruiting body forming fungi. Comparisons at finer scales, such as within economically relevant genera, denser sampling of time points during development or at better spatial resolution (e.g. single-cell RNA-Seq) could yield additional insights. Joint analyses of gene expression patterns with gene phylogenies or with gene family copy number dynamics, functional genomics assays, the inference of gene coexpression networks or population genomics (e.g. GWAS analyses) among others, could all provide more detailed views on the synergy between evolutionary changes in gene content, expression regulation and morphogenesis. We anticipate that with advances in functional genomics of Agaricomycetes, it will become easier to conduct such studies and shed light on conserved and species-specific aspects of fruiting body morphogenesis.

\section{Acknowledgements}

4938 We are grateful to all friends and colleagues who, over the years, inspired our work on 4939 fruiting body development through collaborations or discussions. We appreciate the help of 4940 Levente Karaffa in making sense of Acetyl-CoA production and basic metabolism. We 4941 appreciate the permission of Francis Martin and Jason Tsai to use photographs of $L$. bicolor 4942 and M. kentingensis in Fig. 1, respectively. We are grateful to Annegret Kohler for providing 4943 pre-publication access to the transcriptome of $L$. bicolor. This work was supported by the 4944 European Research Council (grant no. 758161 to L.G.N.), the "Momentum" program of the 4945 Hungarian Academy of Sciences (contract No. LP2019-13/2019 to L.G.N.), the Hungarian 4946 National Research, Development, and Innovation Office (contract No. GINOP-2.3.2-15- 
bioRxiv preprint doi: https://doi.org/10.1101/2021.12.09.471732; this version posted December $10,2021$. The copyright holder for this preprint (which was not certified by peer review) is the author/funder, who has granted bioRxiv a license to display the preprint in perpetuity. It is made available under aCC-BY-NC-ND 4.0 International license.

Running head: fruiting body genomics

4947 2016-00052), the Szeged Scientists Academy under the sponsorship of the Hungarian 4948 Ministry of Innovation and Technology (FEIF/646-4/2021-ITM_SZERZ, to B.Sz., A. Cs. And

4949 L.G.N.) and with the professional support of the Doctoral Student Scholarship Program of

4950 the Co-operative Doctoral Program of the Ministry of Innovation and Technology financed

4951 from the National Research, Development and Innovation Fund (KDP-17-4/PALY-2021

4952 , to Cs.F.). T.V. was supported by the National Talent Programme (NTP-NFTÖ-21-B-0074)

4953 of the Hungarian Government. FH gratefully acknowledges funding from the German

4954 Research Foundation (Deutsche Forschungsgemeinschaft, DFG) under grant HE 7849/3-1.

\section{References}

4956 (PDF) The Igraph Software Package for Complex Network ResearchAvailable from https://www.researchgate.net/publication/221995787_The_lgraph_Software_Package_f or_Complex_Network_Research.

4960

4961

4962

2. Stem and cap morphogenesis in Coprinopsis fruit bodies.

A B., K V., R T., J P., D V., N F.-D., R F. 2005. The Aspergillus nidulans phytochrome FphA represses sexual development in red light. Curr. Biol. 15:1833-1838.

A B., R C., M M., L L.-V., L R., AG P., M P., L R. 2018. Transposon-associated epigenetic silencing during Pleurotus ostreatus life cycle. DNA Res. 25:451-464.

A I., J H. 2005. Photosensing fungi: phytochrome in the spotlight. Curr. Biol. 15.

A O., F H., M R. 2020. Volatilomes of Cyclocybe aegerita during different stages of monokaryotic and dikaryotic fruiting. Biol. Chem. 401:995-1004.

A P., V H., M S., M N., Š M., I P., S P., M Z., N R., P M., P V., J R., K S. 2019a. Poreforming protein complexes from Pleurotus mushrooms kill western corn rootworm and Colorado potato beetle through targeting membrane ceramide phosphoethanolamine. Sci. Rep. 9.

A T., S A., R H., E V., S A., P L., P M., M R., F H., M K. 2019b. Heterologous Production and Functional Characterization of Ageritin, a Novel Type of Ribotoxin Highly Expressed during Fruiting of the Edible Mushroom Agrocybe aegerita. Appl. Environ. Microbiol. 85.

A V., E H.-G., M O., AL M., M B. 2009. Solution structure of hirsutellin A--new insights into the active site and interacting interfaces of ribotoxins. FEBS J. 276:2381-2390.

A V., SM B., A I., Varrot A., Basheer S.M., Imberty A. 2013. Fungal lectins: structure, function and potential applications. Curr. Opin. Struct. Biol. 23:678-685.

AA F., DI J., CC F., MH G., SR J., JA G., F T. 1991. Protein geranylgeranyltransferase of Saccharomyces cerevisiae is specific for Cys-Xaa-Xaa-Leu motif proteins and requires the CDC43 gene product but not the DPR1 gene product. Proc. Natl. Acad. Sci. U. S. A. 88:4448-4452.

Agger S., Lopez-Gallego F., Schmidt-Dannert C. 2009. Diversity of sesquiterpene synthases in the basidiomycete Coprinus cinereus. Mol. Microbiol. 72:1181-1195.

Ahmed Y.L., Gerke J., Park H.-S., Bayram Ö., Neumann P., Ni M., Dickmanns A., Kim S.C., Yu J.-H., Braus G.H., Ficner R. 2013. The Velvet Family of Fungal Regulators Contains a DNA-Binding Domain Structurally Similar to NF-\$K\$B. PLOS Biol. 11:e1001750.

AL L., TK L., R B. 1999. Characterization of the Wsc1 protein, a putative receptor in the stress response of Saccharomyces cerevisiae. Genetics. 152:1487-1499.

Almási É., Sahu N., Krizsán K., Bálint B., Kovács G.M., Kiss B., Cseklye J., Drula E., Henrissat B., Nagy I., Chovatia M., Adam C., LaButti K., Lipzen A., Riley R., Grigoriev I. V., Nagy L.G. 2019. Comparative genomics reveals unique wood-decay strategies and fruiting body development in the Schizophyllaceae. New Phytol.

AM M., CS M., OP S., DS M., SN A., ME Z. 2009. Meiotic localization of Mre11 and Rad50 in wild type, spo11-1, and MRN complex mutants of Coprinus cinereus. Chromosoma. 118:471-486.

AN C., MD C. 2010. Basidiosporogenesis, meiosis, and post-meiotic mitosis in the ectomycorrhizal fungus Pisolithusmicrocarpus. Fungal Genet. Biol. 47:477-483. 
bioRxiv preprint doi: https://doi.org/10.1101/2021.12.09.471732; this version posted December $10,2021$. The copyright holder for this preprint (which was not certified by peer review) is the author/funder, who has granted bioRxiv a license to display the preprint in perpetuity. It is made available under aCC-BY-NC-ND 4.0 International license.

Running head: fruiting body genomics

4998

4999

5000

5001

5002

5003

5004

5005

5006

5007

5008

5009

5010

5011

5012

5013

5014

5015

5016

5017

5018

5019

5020

5021

5022

5023

5024

5025

5026

5027

5028

5029

5030

5031

5032

5033

5034

5035

5036

5037

5038

5039

5040

5041

5042

5043

5044

5045

5046

5047

5048

5049

5050

5051

5052
Anderluh G., Maček P. 2003. Dissecting the Actinoporin Pore-Forming Mechanism. Structure. 11:1312-1313.

Ando Y., Nakazawa T., Oka K., Nakahori K., Kamada T. 2013. Cc.snf5, a gene encoding a putative component of the SWI/SNF chromatin remodeling complex, is essential for sexual development in the agaricomycete Coprinopsis cinerea. Fungal Genet. Biol. 50:82-89.

Arima T., Yamamoto M., Hirata A., Kawano S., Kamada T. 2004. The eln3 gene involved in fruiting body morphogenesis of Coprinus cinereus encodes a putative membrane protein with a general glycosyltransferase domain. Fungal Genet. Biol. 41:805-812.

Arroyo J., Farkaš V., Sanz A.B., Cabib E. 2016. 'Strengthening the fungal cell wall through chitin-glucan cross-links: effects on morphogenesis and cell integrity".' Cell. Microbiol. 18:1239-1250.

Arsuffi G., Braybrook S.A. 2018. Acid growth: an ongoing trip. J. Exp. Bot. 69:137-146.

Ashikari T., Nakamura N., Tanaka Y., Kiuchi N., Shibano Y., Tanaka T., Amachi T., Yoshizumi H. 1986. Rhizopus raw-starch-degrading glucoamylase: Its cloning and expression in yeast. Agric. Biol. Chem. 50:957-964.

ASM S., N S.-T., B L., RA O., PM H., K S., JJP B., A van P. 2020. Telomere-to-telomere assembled and centromere annotated genomes of the two main subspecies of the button mushroom Agaricus bisporus reveal especially polymorphic chromosome ends. Sci. Rep. 10.

B F., M F., I N. 2001. Ethanol catabolism in Aspergillus nidulans: a model system for studying gene regulation. Prog. Nucleic Acid Res. Mol. Biol. 69:149-204.

Baccelli I. 2015. Cerato-platanin family proteins: one function for multiple biological roles? Front. Plant Sci. 5:769.

Baglivo I., Ragucci S., D'Incecco P., Landi N., Russo R., Faoro F., Pedone P. V, Maro A. Di. 2020. Gene Organization, Expression, and Localization of Ribotoxin-Like Protein Ageritin in Fruiting Body and Mycelium of Agrocybe aegerita. Int. J. Mol. Sci. 2020, Vol. 21, Page 7158. 21:7158.

Bai Y., Wang Y., Liu X., Zhao J., Kang L.Q., Liu Z., Yuan S. 2020. Heterologous expression and characterization of a novel chitin deacetylase, CDA3, from the mushroom Coprinopsis cinerea. Int. J. Biol. Macromol. 150:536-545.

Baker L.G., Specht C.A., Donlin M.J., Lodge J.K. 2007. Chitosan, the Deacetylated Form of Chitin, Is Necessary for Cell Wall Integrity in Cryptococcus neoformans. Eukaryot. Cell. 6:855.

Banks I.R., Specht C.A., Donlin M.J., Gerik K.J., Levitz S.M., Lodge J.K. 2005. A Chitin Synthase and Its Regulator Protein Are Critical for Chitosan Production and Growth of the Fungal Pathogen Cryptococcus neoformans. Eukaryot. Cell. 4:1902.

Barsottini M.R. de O., de Oliveira J.F., Adamoski D., Teixeira P.J.P.L., do Prado P.F. V, Tiezzi H.O., Sforça M.L., Cassago A., Portugal R. V, de Oliveira P.S.L., Zeri A.C. de M., Dias S.M.G., Pereira G.A.G., Ambrosio A.L.B. 2013. Functional Diversification of Cerato-Platanins in Moniliophthora perniciosa as Seen by Differential Expression and Protein Function Specialization. http://dx.doi.org/10.1094/MPMI-05-13-0148-R. 26:1281-1293.

Bartoš O., Röslein J., Kotusz J., Paces J., Pekárik L., Petrtýl M., Halačka K., Štefková Kašparová E., Mendel J., Boroń A., Juchno D., Leska A., Jablonska O., Benes V., Šídová M., Janko K. 2019. The Legacy of Sexual Ancestors in Phenotypic Variability, Gene Expression, and Homoeolog Regulation of Asexual Hybrids and Polyploids. Mol. Biol. Evol. 36:1902-1920.

Bary A. de, Bary A. de, Balfour I.B., Garnsey H.E.F., Garnsey H.E.F. 1887. Comparative morphology and biology of the Fungi, Mycetozoa and bacteria. Oxford: Clarendon press,.

Bayram Ö., Krappmann S., Ni M., Bok J.W., Helmstaedt K., Valerius O., Braus-Stromeyer S., Kwon N.-J., Keller N.P., Yu J.-H., Braus G.H. 2008. VelB/VeA/LaeA Complex Coordinates Light Signal with Fungal Development and Secondary Metabolism. Science (80-. ). 320:1504-1506. 
bioRxiv preprint doi: https://doi.org/10.1101/2021.12.09.471732; this version posted December $10,2021$. The copyright holder for this preprint (which was not certified by peer review) is the author/funder, who has granted bioRxiv a license to display the preprint in perpetuity. It is made available under aCC-BY-NC-ND 4.0 International license.

Running head: fruiting body genomics

5053

5054

5055

5056

5057

5058

5059

5060

5061

5062

5063

5064

5065

5066

5067

5068

5069

5070

5071

5072

5073

5074

5075

5076

5077

5078

5079

5080

5081

5082

5083

5084

5085

5086

5087

5088

5089

5090

5091

5092

5093

5094

5095

5096

5097

5098

5099

5100

5101

5102

5103

5104

5105

5106

5107
Bayry J., Aimanianda V., Latge P. 2012. Hydrophobins-Unique Fungal Proteins. PLoS Pathog. 8:4.

Berends E., Ohm R.A., De Jong J.F., Rouwendal G., Wösten H.A.B., Lugones L.G., Bosch D. 2009. Genomic and biochemical analysis of $\mathrm{N}$ glycosylation in the mushroomforming basidiomycete Schizophyllum commune. Appl. Environ. Microbiol. 75:46484652.

Berendsen R.L., Kalkhove S.I.C., Lugones L.G., Baars J.J.P., Wösten H.A.B., Bakker P.A.H.M. 2013. Effects of the mushroom-volatile 1-octen-3-ol on dry bubble disease. Appl. Microbiol. Biotechnol. 97:5535-5543.

Bertani B., Ruiz N. 2018. Function and biogenesis of lipopolysaccharides. EcoSal Plus. 8.

Bertossa R.C., Kües U., Aebi M., Künzler M. 2004. Promoter analysis of cgl2, a galectin encoding gene transcribed during fruiting body formation in Coprinopsis cinerea (Coprinus cinereus). Fungal Genet. Biol. 41:1120-1131.

Bewick A.J., Hofmeister B.T., Powers R.A., Mondo S.J., Grigoriev I. V., James T.Y., Stajich J.E., Schmitz R.J. 2019. Diversity of cytosine methylation across the fungal tree of life. Nat. Ecol. Evol. 3:479-490.

Bieszke J.A., Braun E.L., Bean L.E., Kang S., Natvig D.O., Borkovich K.A. 1999. The nop-1 gene of Neurospora crassa encodes a seven transmembrane helix retinal-binding protein homologous to archaeal rhodopsins. Proc. Natl. Acad. Sci. U. S. A. 96:8034.

Blackstone N.W. Redox control and the evolution of multicellularity. .

Bleuler-Martínez S., Butschi A., Garbani M., Wîlti M.A., Wohlschlager T., Potthoff E., Sabotia J., Pohleven J., Lüthy P., Hengartner M.O., Aebi M., Künzler M. 2011. A lectinmediated resistance of higher fungi against predators and parasites. Mol. Ecol. 20:3056-3070.

Bojunga N., Kötter P., Entian K.-D. 1998. The succinate/fumarate transporter Acr1p of Saccharomyces cerevisiae is part of the gluconeogenic pathway and its expression is regulated by Cat8p. Mol. Gen. Genet. MGG 1998 2605. 260:453-461.

Boontawon T., Nakazawa T., Horii M., Tsuzuki M., Kawauchi M., Sakamoto M., Honda Y. 2021. Functional analyses of Pleurotus ostreatus pcc1 and clp1 using CRISPR/Cas9. Fungal Genet. Biol. 154:103599.

Borkovich K.A., Alex L.A., Yarden O., Freitag M., Turner G.E., Read N.D., Seiler S., BellPedersen D., Paietta J., Plesofsky N., Plamann M., Goodrich-Tanrikulu M., Schulte U., Mannhaupt G., Nargang F.E., Radford A., Selitrennikoff C., Galagan J.E., Dunlap J.C., Loros J.J., Catcheside D., Inoue H., Aramayo R., Polymenis M., Selker E.U., Sachs M.S., Marzluf G.A., Paulsen I., Davis R., Ebbole D.J., Zelter A., Kalkman E.R., O'Rourke R., Bowring F., Yeadon J., Ishii C., Suzuki K., Sakai W., Pratt R. 2004. Lessons from the genome sequence of Neurospora crassa: tracing the path from genomic blueprint to multicellular organism. Microbiol. Mol. Biol. Rev. 68:1-108.

Boulianne R.P., Liu Y., Aebi M., Lu B.C., Kües U. 2000. Fruiting body development in Coprinus cinereus: regulated expression of two galectins secreted by a non-classical pathwayThe GenBank accession number for the sequence reported in this paper is AF130360. Microbiology. 146:1841-1853.

Braus G.H., Irniger S., Bayram Ö. 2010. Fungal development and the COP9 signalosome. Curr. Opin. Microbiol. 13:672-676.

Breitenbach M., Weber M., Rinnerthaler M., Karl T., Breitenbach-Koller L. 2015. Oxidative Stress in Fungi: Its Function in Signal Transduction, Interaction with Plant Hosts, and Lignocellulose Degradation. Biomolecules. 5:318.

van den Brink J., de Vries R.P. 2011. Fungal enzyme sets for plant polysaccharide degradation. Appl. Microbiol. Biotechnol. 2011 916. 91:1477-1492.

Brodhun F., Feussner I. 2011. Oxylipins in fungi. FEBS J. 278:1047-1063.

Brown J.L., Bussey H. 1993. The yeast KRE9 gene encodes an O glycoprotein involved in cell surface beta-glucan assembly. Mol. Cell. Biol. 13:6346-6356.

Brown N.A., Schrevens S., Van Dijck P., Goldman G.H. 2018. Fungal G-protein-coupled receptors: Mediators of pathogenesis and targets for disease control. Nat. Microbiol.

Buenrostro J.D., Wu B., Chang H.Y., Greenleaf W.J. 2015. ATAC-seq: A method for 
bioRxiv preprint doi: https://doi.org/10.1101/2021.12.09.471732; this version posted December $10,2021$. The copyright holder for this preprint (which was not certified by peer review) is the author/funder, who has granted bioRxiv a license to display the preprint in perpetuity. It is made available under aCC-BY-NC-ND 4.0 International license.

Running head: fruiting body genomics

5108

5109

5110

5111

5112

5113

5114

5115

5116

5117

5118

5119

5120

5121

5122

5123

5124

5125

5126

5127

5128

5129

5130

5131

5132

5133

5134

5135

5136

5137

5138

5139

5140

5141

5142

5143

5144

5145

5146

5147

5148

5149

5150

5151

5152

5153

5154

5155

5156

5157

5158

5159

5160

5161

5162 assaying chromatin accessibility genome-wide. Curr. Protoc. Mol. Biol. 2015:21.29.1-21.29.9.

Buhian W.P., Bensmihen S. 2018. Mini-Review: Nod Factor Regulation of Phytohormone Signaling and Homeostasis During Rhizobia-Legume Symbiosis. Front. Plant Sci. $0: 1247$.

Burns C., Stajich J.E., Rechtsteiner A., Casselton L., Hanlon S.E., Wilke S.K., Savytskyy O.P., Gathman A.C., Lilly W.W., Lieb J.D., Zolan M.E., Pukkila P.J. 2010. Analysis of the Basidiomycete Coprinopsis cinerea Reveals Conservation of the Core Meiotic Expression Program over Half a Billion Years of Evolution. PLOS Genet. 6:e1001135.

Busch S., Eckert S.E., Krappmann S., Braus G.H. 2003. The COP9 signalosome is an essential regulator of development in the filamentous fungus Aspergillus nidulans. Mol. Microbiol. 49:717-730.

Busch S., Schwier E.U., Nahlik K., Bayram Ö., Helmstaedt K., Draht O.W., Krappmann S., Valerius O., Lipscomb W.N., Braus G.H. 2007. An eight-subunit COP9 signalosome with an intact JAMM motif is required for fungal fruit body formation. Proc. Natl. Acad. Sci. 104:8089-8094.

Buser R., Lazar Z., Käser S., Künzler M., Aebi M. 2010a. Identification, characterization, and biosynthesis of a novel $\mathrm{N}$-glycan modification in the fruiting body of the basidiomycete Coprinopsis cinerea. J. Biol. Chem. 285:10715-10723.

Buser R., Lazar Z., Käser S., Künzler M., Aebi M. 2010b. Identification, Characterization, and Biosynthesis of a Novel $N$-Glycan Modification in the Fruiting Body of the Basidiomycete Coprinopsis cinerea. J. Biol. Chem. 285:10715-10723.

Butschi A., Titz A., Wälti M.A., Olieric V., Paschinger K., Nöbauer K., Guo X., Seeberger P.H., Wilson I.B.H., Aebi M., Hengartner M.O., Künzler M. 2010. Caenorhabditis elegans N-glycan Core $\beta$-galactoside Confers Sensitivity towards Nematotoxic Fungal Galectin CGL2. PLoS Pathog. 6:e1000717.

C B., L D., C M.-D., A L., C S.-B., P K., D F., L P., JP S. 2004. A new lectin family with structure similarity to actinoporins revealed by the crystal structure of Xerocomus chrysenteron lectin XCL. J. Mol. Biol. 344:1409-1420.

C G., M K., VR I., PO B., DR W. 2000. Identification of the copper regulon in Saccharomyces cerevisiae by DNA microarrays. J. Biol. Chem. 275:32310-32316.

C V.-F., C C., A K., E M., R B., J P., E D., P C., A W., RP de V., B H., F M. 2014. Genomic and transcriptomic analysis of Laccaria bicolor CAZome reveals insights into polysaccharides remodelling during symbiosis establishment. Fungal Genet. Biol. 72:168-181.

Caldwell G.A., Naider F., Becker J.M. 1995. Fungal lipopeptide mating pheromones: A model system for the study of protein prenylation. Microbiol. Rev. 59:406-422.

Caligiuri M., Connolly T., Beach D. 1997. Ran1 functions to control the Cdc10/Sct1 complex through Puc1. Mol. Biol. Cell. 8:1117.

Campbell L.T., Padula M.P., Harry E., Carter D.A., Campbell L.T., Padula M.P., Harry E., Carter D.A. 2015. You are what you secrete: extracellular proteins and virulence in Cryptococcus. Microbiol. Aust. 36:93-95.

Cao L., Chan C., Lee C., Wong S.S., Yuen K. 1998. MP1 Encodes an Abundant and Highly Antigenic Cell Wall Mannoprotein in the Pathogenic Fungus Penicillium marneffei. Infect. Immun. 66:966.

Carman G.M., Han G.-S. 2011. Regulation of Phospholipid Synthesis in the Yeast Saccharomyces cerevisiae. Annu. Rev. Biochem. 80:859.

Carmichael J.B., Provost P., Ekwall K., Hobman T.C., JB C., P P., K E., TC H. 2004. ago1 and dcr1, two core components of the RNA interference pathway, functionally diverge from rdp1 in regulating cell cycle events in Schizosaccharomyces pombe. Mol. Biol. Cell. 15:1425-1435.

Ceccaroli P., Buffalini M., Saltarelli R., Barbieri E., Polidori E., Ottonello S., Kohler A., Tisserant E., Martin F., Stocchi V. 2011. Genomic profiling of carbohydrate metabolism in the ectomycorrhizal fungus Tuber melanosporum. New Phytol. 189:751-764.

CG L.-R., JL C.-P., D M.-S., A S.-A., ET A.-C., J R.-H. 2017. Transcriptomic analysis of 
bioRxiv preprint doi: https://doi.org/10.1101/2021.12.09.471732; this version posted December $10,2021$. The copyright holder for this preprint (which was not certified by peer review) is the author/funder, who has granted bioRxiv a license to display the preprint in perpetuity. It is made available under aCC-BY-NC-ND 4.0 International license.

Running head: fruiting body genomics

5163 basidiocarp development in Ustilago maydis (DC) Cda. Fungal Genet. Biol. 101:34-45.

5164

5165

5166

5167

5168

5169

5170

5171

5172

5173

5174

5175

5176

5177

5178

5179

5180

5181

5182

5183

5184

5185

5186

5187

5188

5189

5190

5191

5192

5193

5194

5195

5196

5197

5198

5199

5200

5201

5202

5203

5204

5205

5206

5207

5208

5209

5210

5211

5212

5213

5214

5215

5216

5217
CH Z., JP Z., WQ D., TH L., ZD J. 2018. Molecular cloning and the expression pattern of AePOPB involved in the \$a $\$$-amanitin biosynthesis in Amanita exitialis fruiting bodies. Gene. 662:123-130.

Cha M.-K., Choi Y.-S., Hong S.-K., Kim W.-C., No K.T., Kim I.-H. 2003. Nuclear Thiol Peroxidase as a Functional Alkyl-hydroperoxide Reductase Necessary for Stationary Phase Growth of Saccharomyces cerevisiae*. J. Biol. Chem. 278:24636-24643.

Chae E., Tan Q.K.-G., Hill T.A., Irish V.F. 2008. An Arabidopsis F-box protein acts as a transcriptional co-factor to regulate floral development. Development. 135:1235-1245.

Chakraborty T.K., Basu D., Das N., Sengupta S., Mukherjee M. 2004. The mannitol cycle in Pleurotus ostreatus (Florida). FEMS Microbiol. Lett. 236:307-311.

Chang J., Chan P.L., Xie Y., Ma K.L., Cheung M.K., Kwan H.S. 2019a. Modified recipe to inhibit fruiting body formation for living fungal biomaterial manufacture. PLoS One. 14:e0209812.

Chang Y., Desirò A., Na H., Sandor L., Lipzen A., Clum A., Barry K., Grigoriev I. V., Martin F.M., Stajich J.E., Smith M.E., Bonito G., Spatafora J.W. 2019b. Phylogenomics of Endogonaceae and evolution of mycorrhizas within Mucoromycota. New Phytol. 222:511-525.

Chen D.D., Shi L., Yue S.N., Zhang T.J., Wang S.L., Liu Y.N., Ren A., Zhu J., Yu H.S., Zhao M.W. 2019. The SIt2-MAPK pathway is involved in the mechanism by which target of rapamycin regulates cell wall components in Ganoderma lucidum. Fungal Genet. Biol. 123:70-77.

Chen H., Kovalchuk A., Keriö S., Asiegbu F.O. 2017. Distribution and bioinformatic analysis of the cerato-platanin protein family in Dikarya. http://dx.doi.org/10.3852/13-115. 105:1479-1488.

Chen J., Li J.-M., Tang Y.-J., Ma K., Li B., Zeng X., Liu X.-B., Li Y., Yang Z.-L., Xu W.-N., Xie B.-G., Liu H.-W., Guo S.-X. 2020. Genome-wide analysis and prediction of genes involved in the biosynthesis of polysaccharides and bioactive secondary metabolites in high-temperature-tolerant wild Flammulina filiformis. BMC Genomics 2020 211. 21:116.

Chen L., Gong Y., Cai Y., Liu W., Zhou Y., Xiao Y., Xu Z., Liu Y., Lei X., Wang G., Guo M., Ma X., Bian Y. 2016. Genome Sequence of the Edible Cultivated Mushroom Lentinula edodes (Shiitake) Reveals Insights into Lignocellulose Degradation. PLoS One. 11:e0160336.

Chen S., Ma D., Ge W., Buswell J.A. 2003. Induction of laccase activity in the edible straw mushroom, Volvariella volvacea. FEMS Microbiol. Lett. 218:143-148.

Chen S., Xu J., Liu C., Zhu Y., Nelson D.R., Zhou S., Li C., Wang L., Guo X., Sun Y., Luo H., Li Y., Song J., Henrissat B., Levasseur A., Qian J., Li J., Luo X., Shi L., He L., Xiang L., Xu X., Niu Y., Li Q., Han M. V, Yan H., Zhang J., Chen H., Lv A., Wang Z., Liu M., Schwartz D.C., Sun C. 2012. Genome sequence of the model medicinal mushroom Ganoderma lucidum. Nat. Commun. 2012 31. 3:1-9.

Chen Y., Zhang Y., Siewers V., Nielsen J. 2015. Ach1 is involved in shuttling mitochondrial acetyl units for cytosolic C2 provision in Saccharomyces cerevisiae lacking pyruvate decarboxylase. FEMS Yeast Res. 15:1-8.

Cheng C.K., Au C.H., Wilke S.K., Stajich J.E., Zolan M.E., Pukkila P.J., Kwan H.S. 2013a. 5'-Serial Analysis of Gene Expression studies reveal a transcriptomic switch during fruiting body development in Coprinopsis cinerea. BMC Genomics. 14:195.

Cheng C.K., Au C.H., Wilke S.K., Stajich J.E., Zolan M.E., Pukkila P.J., Kwan H.S. 2013b. 5'-Serial Analysis of Gene Expression studies reveal a transcriptomic switch during fruiting body development in Coprinopsis cinerea. BMC Genomics. 14:195.

Chitarra G.S., Abee T., Rombouts F.M., Posthumus M.A., Dijksterhuis J. 2004. Germination of Penicillium paneum conidia is regulated by 1-octen-3-ol, a volatile self-inhibitor. Appl. Environ. Microbiol. 70:2823-2829.

Chiu E.Y.H., Lin Y.H., Wu W., Song Q., Chang P.F.L., Gao L.Y., Chou C.C., Ueng P.P. 2010. Alternative splicing and genetic diversity of the white collar-1 (wc-1) gene in 
bioRxiv preprint doi: https://doi.org/10.1101/2021.12.09.471732; this version posted December $10,2021$. The copyright holder for this preprint (which was not certified by peer review) is the author/funder, who has granted bioRxiv a license to display the preprint in perpetuity. It is made available under aCC-BY-NC-ND 4.0 International license.

Running head: fruiting body genomics

$5218 \quad$ cereal Phaeosphaeria pathogens. Eur. J. Plant Pathol.

5219

5220

5221

5222

5223

5224

5225

5226

5227

5228

5229

5230

5231

5232

5233

5234

5235

5236

5237

5238

5239

5240

5241

5242

5243

5244

5245

5246

5247

5248

5249

5250

5251

5252

5253

5254

5255

5256

5257

5258

5259

5260

5261

5262

5263

5264

5265

5266

5267

5268

5269

5270

5271

5272

Cirauqui N., Abriata L.A., van der Goot F.G., Dal Peraro M. 2017. Structural, physicochemical and dynamic features conserved within the aerolysin pore-forming toxin family. Sci. Reports 2017 71. 7:1-12.

CM B., PJ E. 2010. Sterol regulatory element binding proteins in fungi: hypoxic transcription factors linked to pathogenesis. Eukaryot. Cell. 9:352-359.

CM G. 2001. Role of the glutathione/glutaredoxin and thioredoxin systems in yeast growth and response to stress conditions. Mol. Microbiol. 39:533-541.

Cohen-Kupiec R., Broglie K.E., Friesem D., Broglie R.M., Chet I. 1999. Molecular characterization of a novel $\$ \beta \$$-1,3-exoglucanase related to mycoparasitism of Trichoderma harzianum. Gene. 226:147-154.

Combet E., Henderson J., Eastwood D.C., Burton K.S. 2006. Eight-carbon volatiles in mushrooms and fungi: properties, analysis, and biosynthesis. Mycoscience. 47:317326.

Combet E., Henderson J., Eastwood D.C., Burton K.S. 2009. Influence of sporophore development, damage, storage, and tissue specificity on the enzymic formation of volatiles in mushrooms (agaricus bisporus). J. Agric. Food Chem. 57:3709-3717.

Corrochano L.M. 2011. Fungal photobiology: a synopsis. IMA Fungus 201121 . 2:25-28.

Corrochano L.M. 2019. Light in the Fungal World: From Photoreception to Gene Transcription and beyond. Annu. Rev. Genet. 53:149-170.

Costa Ana S.M.B.;Thomas D.J.I. $\square$;Eastwoo. D.S.B. $\square$;Baile. A.M. $\square$;Foste. G.D. $\square$;Mill. P.R. $\square$;Challe. M.P.. 2009. Quantifiable Downregulation of Endogenous Genes in Agaricus bisporus Mediated by Expression of RNA Hairpins. J. Microbiol. Biotechnol. 19:271-276.

Costachel C., Coddeville B., Latgé J.-P., Fontaine T. 2005. Glycosylphosphatidylinositolanchored Fungal Polysaccharide in Aspergillus fumigatus. J. Biol. Chem. 280:3983539842.

Courty P.E., Hoegger P.J., Kilaru S., Kohler A., Buée M., Garbaye J., Martin F., Kües U. 2009. Phylogenetic analysis, genomic organization, and expression analysis of multicopper oxidases in the ectomycorrhizal basidiomycete Laccaria bicolor. New Phytol. 182:736-750.

Couturier M., Ladevèze S., Sulzenbacher G., Ciano L., Fanuel M., Moreau C., Villares A., Cathala B., Chaspoul F., Frandsen K.E., Labourel A., Herpoël-Gimbert I., Grisel S., Haon M., Lenfant N., Rogniaux H., Ropartz D., Davies G.J., Rosso M.-N., Walton P.H., Henrissat B., Berrin J.-G. 2018. Lytic xylan oxidases from wood-decay fungi unlock biomass degradation. Nat. Chem. Biol. 2017 143. 14:306-310.

Craig G.D., Gull K., Wood D.A. 1977. Stipe elongation in Agaricus bisporus. Microbiology. 102:337-347.

Crestini C., Kovac B., Giovannozzi-Sermanni G. 1996. Production and isolation of chitosan by submerged and solid-state fermentation from Lentinus edodes. Biotechnol. Bioeng. 50:207-210.

Cummings W.J., Merino S.T., Young K.G., Li L., Johnson C.W., Sierra E.A., Zolan M.E. 2002. The Coprinus cinereus adherin Rad9 functions in Mre11-dependent DNA repair, meiotic sister-chromatid cohesion, and meiotic homolog pairing. Proc. Natl. Acad. Sci. 99:14958-14963.

D'Souza C.A., Heitman J. 2001. Conserved cAMP signaling cascades regulate fungal development and virulence. FEMS Microbiol. Rev. 25:349-364.

D S., M N. 2014. The polyketide synthase gene pks4 is essential for sexual development and regulates fruiting body morphology in Sordaria macrospora. Fungal Genet. Biol. 68:48-59.

Dai Y., Sun L., Yin X., Gao M., Zhao Y., Jia P., Yuan X., Fu Y., Li Y. 2019. Pleurotus eryngii Genomes Reveal Evolution and Adaptation to the Gobi Desert Environment. Front. Microbiol. 0:2024.

Dang Y., Yang Q., Xue Z., Liu Y. 2011. RNA Interference in Fungi: Pathways, Functions, and Applications. Eukaryot. Cell. 10:1148. 
bioRxiv preprint doi: https://doi.org/10.1101/2021.12.09.471732; this version posted December $10,2021$. The copyright holder for this preprint (which was not certified by peer review) is the author/funder, who has granted bioRxiv a license to display the preprint in perpetuity. It is made available under aCC-BY-NC-ND 4.0 International license.

Running head: fruiting body genomics

5273 Darwiche R., Mène-Saffrané L., Gfeller D., Asojo O.A., Schneiter R. 2017. The pathogen-

5274

5275

5276

5277

5278

5279

5280

5281

5282

5283

5284

5285

5286

5287

5288

5289

5290

5291

5292

5293

5294

5295

5296

5297

5298

5299

5300

5301

5302

5303

5304

5305

5306

5307

5308

5309

5310

5311

5312

5313

5314

5315

5316

5317

5318

5319

5320

5321

5322

5323

5324

5325

5326

5327 related yeast protein Pry1, a member of the CAP protein superfamily, is a fatty acidbinding protein. J. Biol. Chem. 292:8304.

Das N., Sengupta S., Mukherjee M. 1997. Importance of laccase in vegetative growth of Pleurotus florida. Appl. Environ. Microbiol. 63:4120-4122.

Dekker N., Speijer D., Grün C.H., Van Den Berg M., De Haan A., Hochstenbach F. 2004. Role of the a-glucanase Agn1p in fission-yeast cell separation. Mol. Biol. Cell. 15:39033914.

Deshaies R.J., Joazeiro C.A.P. 2009. RING Domain E3 Ubiquitin Ligases. https://doi.org/10.1146/annurev.biochem.78.101807.093809. 78:399-434.

Deveau A., Kohler A., Frey-Klett P., Martin F. 2008. The major pathways of carbohydrate metabolism in the ectomycorrhizal basidiomycete Laccaria bicolor S238N. New Phytol. 180:379-390.

Díaz-Valderrama J.R., Aime M.C. 2016. The cacao pathogen Moniliophthora roreri (Marasmiaceae) possesses biallelic $A$ and $B$ mating loci but reproduces clonally. Heredity (Edinb). 116:491-501.

Dijkgraaf G.J.P., Brown J.L., Bussey H. 1996. The KNHI Gene of Saccharomyces cerevisiae is a Functional Homolog of KREO. 12:683-692.

Ding W., Liu W.-Q., Jia Y., Li Y., van der Donk W.A., Zhang Q. 2016. Biosynthetic investigation of phomopsins reveals a widespread pathway for ribosomal natural products in Ascomycetes. Proc. Natl. Acad. Sci. 113:3521-3526.

Dixon R.A., Achnine L., Kota P., Liu C.J., Reddy M.S.S., Wang L. 2002. The phenylpropanoid pathway and plant defence - A genomics perspective. Mol. Plant Pathol. 3:371-390.

Douglas L.M., Alvarez F.J., McCreary C., Konopka J.B. 2005. Septin function in yeast model systems and pathogenic fungi. Eukaryot. Cell. 4:1503-1512.

Dressaire E., Yamada L., Song B., Roper M. 2016. Mushrooms use convectively created airflows to disperse their spores. Proc. Natl. Acad. Sci. U. S. A.

Duan M., Bao H., Bau T. 2021. Analyses of transcriptomes and the first complete genome of Leucocalocybe mongolica provide new insights into phylogenetic relationships and conservation. Sci. Reports 2021 111. 11:1-12.

Dueñas-Santero E., Martín-Cuadrado A.B., Fontaine T., Latgé J.P., del Rey F., de Aldana C.V. 2010. Characterization of glycoside hydrolase family 5 proteins in Schizosaccharomyces pombe. Eukaryot. Cell. 9:1650-1660.

DW B., JM C., JR G., AA H., M H., B P.-D. 2002. The strobilurin fungicides. Pest Manag. Sci. 58:649-662.

Eastwood D.C., Herman B., Noble R., Dobrovin-Pennington A., Sreenivasaprasad S., Burton K.S. 2013. Environmental regulation of reproductive phase change in Agaricus bisporus by 1-octen-3-ol, temperature and CO2. Fungal Genet. Biol. 55:54-66.

Eis C., Nidetzky B. 1999. Characterization of trehalose phosphorylase from Schizophyllum commune. Biochem. J. 341:385.

Elleuche S., Pöggeler S. 2009. \$ \$\$-Carbonic Anhydrases Play a Role in Fruiting Body Development and Ascospore Germination in the Filamentous Fungus Sordaria macrospora. PLoS One. 4:e5177.

Elleuche S., Pöggeler S. 2010. Carbonic anhydrases in fungi. Microbiology. 156:23-29.

Emms D.M., Kelly S. 2015. OrthoFinder: solving fundamental biases in whole genome comparisons dramatically improves orthogroup inference accuracy. Genome Biol. 16:157.

Endo H., Kajiwara S., Tsunoka O., Shishido K. 1994. A novel cDNA, priBc, encoding a protein with a Zn(II)2Cys6 zinc cluster DNA-binding motif, derived from the basidiomycete Lentinus edodes. Gene. 139:117-121.

Essen L.-O., Vogt M.S., Mösch H.-U. 2020. Diversity of GPI-anchored fungal adhesins. Biol. Chem. 401:1389-1405.

F T., I G., S V. 2005. Ejection mechanics and trajectory of the ascospores of Gibberella zeae (anamorph Fuarium graminearum). Fungal Genet. Biol. 42:528-533. 
bioRxiv preprint doi: https://doi.org/10.1101/2021.12.09.471732; this version posted December $10,2021$. The copyright holder for this preprint (which was not certified by peer review) is the author/funder, who has granted bioRxiv a license to display the preprint in perpetuity. It is made available under aCC-BY-NC-ND 4.0 International license.

Running head: fruiting body genomics

5328

5329

5330

5331

5332

5333

5334

5335

5336

5337

5338

5339

5340

5341

5342

5343

5344

5345

5346

5347

5348

5349

5350

5351

5352

5353

5354

5355

5356

5357

5358

5359

5360

5361

5362

5363

5364

5365

5366

5367

5368

5369

5370

5371

5372

5373

5374

5375

5376

5377

5378

5379

5380

5381

5382
F W., X S., X D., J Z., C D. 2017. DASH-type cryptochromes regulate fruiting body development and secondary metabolism differently than $\mathrm{CmWC}-1$ in the fungus Cordyceps militaris. Appl. Microbiol. Biotechnol. 101:4645-4657.

Fakas S. 2017. Lipid biosynthesis in yeasts: A comparison of the lipid biosynthetic pathway between the model nonoleaginous yeast Saccharomyces cerevisiae and the model oleaginous yeast Yarrowia lipolytica. Eng. Life Sci. 17:292-302.

Fäldt J., Jonsell M., Nordlander G., Borg-Karlson A.K. 1999. Volatiles of bracket fungi Fomitopsis pinicola and Fomes fomentarius and their functions as insect attractants. J. Chem. Ecol. 25:567-590.

Fang H., Zhang W., Niu X., Liu Z., Lu C., Wei H., Yuan S. 2014. Stipe wall extension of Flammulina velutipes could be induced by an expansin-like protein from Helix aspersa. Fungal Biol. 118:1-11.

Fernandez Espinar M.-T., Labarère J. 1997. Cloning and sequencing of the Aa-Pri1 gene specifically expressed during fruiting initiation in the edible mushroom Agrocybe aegerita, and analysis of the predicted amino-acid sequence. Curr. Genet. 1997326. 32:420-424.

Finley D., Ulrich H.D., Sommer T., Kaiser P. 2012. The Ubiquitin-Proteasome System of Saccharomyces cerevisiae. Genetics. 192:319.

Fischer M., Cox J., Davis D.J., Wagner A., Taylor R., Huerta A.J., Money N.P. 2004. New information on the mechanism of forcible ascospore discharge from Ascobolus immersus. Fungal Genet. Biol. 41:698-707.

FJ A., JB K. 2007. Identification of an N-acetylglucosamine transporter that mediates hyphal induction in Candida albicans. Mol. Biol. Cell. 18:965-975.

Floudas D., Binder M., Riley R., Barry K., Blanchette R.A., Henrissat B., Martínez A.T., Otillar R., Spatafora J.W., Yadav J.S., Aerts A., Benoit I., Boyd A., Carlson A., Copeland A., Coutinho P.M., Vries R.P. de, Ferreira P., Findley K., Foster B., Gaskell J., Glotzer D., Górecki P., Heitman J., Hesse C., Hori C., Igarashi K., Jurgens J.A., Kallen N., Kersten P., Kohler A., Kües U., Kumar T.K.A., Kuo A., LaButti K., Larrondo L.F., Lindquist E., Ling A., Lombard V., Lucas S., Lundell T., Martin R., McLaughlin D.J., Morgenstern I., Morin E., Murat C., Nagy L.G., Nolan M., Ohm R.A.,

Patyshakuliyeva A., Rokas A., Ruiz-Dueñas F.J., Sabat G., Salamov A., Samejima M., Schmutz J., Slot J.C., John F. St., Stenlid J., Sun H., Sun S., Syed K., Tsang A., Wiebenga A., Young D., Pisabarro A., Eastwood D.C., Martin F., Cullen D., Grigoriev I. V., Hibbett D.S. 2012. The Paleozoic Origin of Enzymatic Lignin Decomposition Reconstructed from 31 Fungal Genomes. Science (80-. ). 336:1715-1719.

Foster J., Nakata P.A. 2014. An oxalyl-CoA synthetase is important for oxalate metabolism in Saccharomyces cerevisiae. FEBS Lett. 588:160-166.

Foulongne-Oriol M., Taskent O., Kües U., Sonnenberg A.S.M., van Peer A.F., Giraud T. 2021. Mating-Type Locus Organization and Mating-Type Chromosome Differentiation in the Bipolar Edible Button Mushroom Agaricus bisporus. Genes (Basel). 12:1079.

François J., Parrou J.L. 2001. Reserve carbohydrates metabolism in the yeast Saccharomyces cerevisiae. FEMS Microbiol. Rev. 25:125-145.

Fraser J.A., Hsueh Y.-P., Findley K.M., Heitman J. 2014. Evolution of the Mating-Type Locus: The Basidiomycetes. Sex in Fungi. Washington, DC, USA: ASM Press. p. 1934.

Frawley D., Bayram Ö. 2020. The pheromone response module, a mitogen-activated protein kinase pathway implicated in the regulation of fungal development, secondary metabolism and pathogenicity. Fungal Genet. Biol. 144:103469.

Freihorst D., Fowler T.J., Bartholomew K., Raudaskoski M., Horton J.S., Kothe E. 2016. 13 The Mating-Type Genes of the Basidiomycetes. In: Wendland J., editor. Growth, Differentiation and Sexuality. Cham: Springer International Publishing. p. 329-349.

Freiman R.N., Tjian R. 2003. Regulating the Regulators: Lysine Modifications Make Their Mark. Cell. 112:11-17.

Freitag M. 2017. Histone Methylation by SET Domain Proteins in Fungi. Annu. Rev. Microbiol. 71:413-439. 
bioRxiv preprint doi: https://doi.org/10.1101/2021.12.09.471732; this version posted December $10,2021$. The copyright holder for this preprint (which was not certified by peer review) is the author/funder, who has granted bioRxiv a license to display the preprint in perpetuity. It is made available under aCC-BY-NC-ND 4.0 International license.

Running head: fruiting body genomics

5383 Froehlich A.C., Chen C.H., Belden W.J., Madeti C., Roenneberg T., Merrow M., Loros J.J., 5384 5385 5386 5387 5388 5389 5390 Dunlap J.C. 2010. Genetic and molecular characterization of a cryptochrome from the filamentous fungus Neurospora crassa. Eukaryot. Cell. 9:738-750.

Fu Y., Dai Y., Yang C., Wei P., Song B., Yang Y., Sun L., Zhang Z.-W.W., Li Y. 2017. Comparative Transcriptome Analysis Identified Candidate Genes Related to Bailinggu Mushroom Formation and Genetic Markers for Genetic Analyses and Breeding. Sci. Reports 2017 71. 7:1-12.

Fuller K.K., Loros J.J., Dunlap J.C. 2015. Fungal photobiology: visible light as a signal for stress, space and time. Curr. Genet. 61:275.

FY L., NP K. 2014. Spatial and temporal control of fungal natural product synthesis. Nat. Prod. Rep. 31:1277-1286.

G S., AS S., LJ van G. 2013. Development and growth of fruit bodies and crops of the button mushroom, Agaricus bisporus. Fungal Biol. 117:697-707.

G V.-K., B W., SJ H., Z L., H Z., M S., VG E. 2010. An oxidative enzyme boosting the enzymatic conversion of recalcitrant polysaccharides. Science. 330:219-222.

Gaderer R., Bonazza K., Seidl-Seiboth V. 2014. Cerato-platanins: A fungal protein family with intriguing properties and application potential. Appl. Microbiol. Biotechnol. 98:4795-4803.

Gagnon-Arsenault I., Tremblay J., Bourbonnais Y. 2006. Fungal yapsins and cell wall: a unique family of aspartic peptidases for a distinctive cellular function. FEMS Yeast Res. 6:966-978.

Gao W., Qu J., Zhang J., Sonnenberg A., Chen Q., Zhang Y., Huang C. 2018. A genetic linkage map of Pleurotus tuoliensis integrated with physical mapping of the de novo sequenced genome and the mating type loci. BMC Genomics. 19:18.

Garcia-Rubio R., de Oliveira H.C., Rivera J., Trevijano-Contador N. 2020. The Fungal Cell Wall: Candida, Cryptococcus, and Aspergillus Species. Front. Microbiol. 0:2993.

Garcia-Santamarina S., Probst C., Festa R.A., Ding C., Smith A.D., Conklin S.E., Brander S., Kinch L.N., Grishin N. V, Franz K.J., Riggs-Gelasco P., Leggio L. Lo, Johansen K.S., Thiele D.J. 2020. A lytic polysaccharide monooxygenase-like protein functions in fungal copper import and meningitis. Nat. Chem. Biol. 2020 163. 16:337-344.

Gaurav Bairwa, Jung W.H., W. Kronstad J. 2017. Iron acquisition in fungal pathogens of humans. Metallomics. 9:215-227.

GB A., L K., OU S., V S.-S. 2015. Molecular diversity of LysM carbohydrate-binding motifs in fungi. Curr. Genet. 61:103-113.

Gehrmann T., Pelkmans J.F., Lugones L.G., Wösten H.A.B., Abeel T., Reinders M.J.T. 2016. Schizophyllum commune has an extensive and functional alternative splicing repertoire. Sci. Rep. 6.

Gehrmann T., Pelkmans J.F., Ohm R.A., Vos A.M., Sonnenberg A.S.M.M., Baars J.J.P.P., Wösten H.A.B.B., Reinders M.J.T.T., Abeel T. 2018. Nucleus-specific expression in the multinuclear mushroom-forming fungus Agaricus bisporus reveals different nuclear regulatory programs. 115:4429-4434.

Gerecke E.E., Zolan M.E. 2000. An mre11 mutant of Coprinus cinereus has defects in meiotic chromosome pairing, condensation and synapsis. Genetics. 154:1125.

Gessler N.N., Filippovich S.Y., Bachurina G.P., Kharchenko E.A., Groza N. V., Belozerskaya T.A. 2017. Oxylipins and oxylipin synthesis pathways in fungi. Appl. Biochem. Microbiol. 53:628-639.

Gilberti M., Baruffini E., Donnini C., Dallabona C. 2018. Pathological alleles of MPV17 modeled in the yeast Saccharomyces cerevisiae orthologous gene SYM1 reveal their inability to take part in a high molecular weight complex. PLoS One. 13.

Gladfelter A.S. 2006. Control of filamentous fungal cell shape by septins and formins. Nat. Rev. Microbiol. 2006 43. 4:223-229.

GM G., K R., MK O. 2008. The CAP superfamily: cysteine-rich secretory proteins, antigen 5, and pathogenesis-related 1 proteins--roles in reproduction, cancer, and immune defense. Endocr. Rev. 29:865-897.

Gold M.H., Cheng T.M. 1979. Conditions for fruit body formation in the white rot 
bioRxiv preprint doi: https://doi.org/10.1101/2021.12.09.471732; this version posted December $10,2021$. The copyright holder for this preprint (which was not certified by peer review) is the author/funder, who has granted bioRxiv a license to display the preprint in perpetuity. It is made available under aCC-BY-NC-ND 4.0 International license.

Running head: fruiting body genomics

5438

5439

5440

5441

5442

5443

5444

5445

5446

5447

5448

5449

5450

5451

5452

5453

5454

5455

5456

5457

5458

5459

5460

5461

5462

5463

5464

5465

5466

5467

5468

5469

5470

5471

5472

5473

5474

5475

5476

5477

5478

5479

5480

5481

5482

5483

5484

5485

5486

5487

5488

5489

5490

5491

5492 basidiomycete Phanerochaete chrysosporium. Arch. Microbiol. 121:37-41.

Goldstein I.J., Winter H.C. 2007. Mushroom Lectins. Compr. Glycosci. From Chem. to Syst. Biol. 3-4:601-621.

Gong W., Xie C., Zhou Y., Zhu Z., Wang Y., Peng Y. 2020. A Resequencing-Based Ultradense Genetic Map of Hericium erinaceus for Anchoring Genome Sequences and Identifying Genetic Loci Associated With Monokaryon Growth. Front. Microbiol. 0:3129.

Gonzalez-Hilarion S., Paulet D., Lee K.T., Hon C.C., Lechat P., Mogensen E., Moyrand F., Proux C., Barboux R., Bussotti G., Hwang J., Coppée J.Y., Bahn Y.S., Janbon G. 2016. Intron retention-dependent gene regulation in Cryptococcus neoformans. Sci. Rep.

Goodrich-Tanrikulu M., Howe K., Stafford A., Nelson M.A. 1998. Changes in fatty acid composition of Neurospora crassa accompany sexual development and ascospore germination. Microbiology. 144:1713-1720.

Gow N.A.R., Latge J.-P., Munro C.A. 2017. The Fungal Cell Wall: Structure, Biosynthesis, and Function. Microbiol. Spectr. 5.

Grau-Bové X., Ruiz-Trillo I., Irimia M. 2018. Origin of exon skipping-rich transcriptomes in animals driven by evolution of gene architecture. Genome Biol.

Grąz M., Jarosz-Wilkołazka A., Janusz G., Mazur A., Wielbo J., Koper P., Żebracki K., Kubik-Komar A. 2017. Transcriptome-based analysis of the saprophytic fungus Abortiporus biennis - response to oxalic acid. Microbiol. Res. 199:79-88.

Gressler M., Löhr N.A., Schäfer T., Lawrinowitz S., Seibold P.S., Hoffmeister D. 2021. Mind the mushroom: natural product biosynthetic genes and enzymes of Basidiomycota. Nat. Prod. Rep. 38:702-722.

Grützmann K., Szafranski K., Pohl M., Voigt K., Petzold A., Schuster S. 2014. Fungal alternative splicing is associated with multicellular complexity and virulence: A genomewide multi-species study. DNA Res. 21:27-39.

GT W., MB Q., S C., F L.-G., C S.-D. 2012. Draft genome of Omphalotus olearius provides a predictive framework for sesquiterpenoid natural product biosynthesis in Basidiomycota. Chem. Biol. 19:772-783.

Gupta D.K., Rühl M., Mishra B., Kleofas V., Hofrichter M., Herzog R., Pecyna M.J., Sharma R., Kellner H., Hennicke F., Thines M. 2018. The genome sequence of the commercially cultivated mushroom Agrocybe aegerita reveals a conserved repertoire of fruiting-related genes and a versatile suite of biopolymer-degrading enzymes. BMC Genomics. 19:1-13.

Gyawali R., Upadhyay S., Way J., Lin X. 2017. A Family of Secretory Proteins Is Associated with Different Morphotypes in Cryptococcus neoformans. Appl Env. Microbiol. 83.

H L., HE H.-A., JS S.-C., JD W. 2012. Ribosomal biosynthesis of \$a-amanitin in Galerina marginata. Fungal Genet. Biol. 49:123-129.

H L., HT C. 2009. Enhanced expression of chitinase during the autolysis of mushroom in Coprinellus congregatus. J. Microbiol. 47:225-228.

H L., Q C., Y L., X L., R S., HE H.-A., ZL Y. 2018. The MSDIN family in amanitin-producing mushrooms and evolution of the prolyl oligopeptidase genes. IMA Fungus. 9:225-242.

H L., Q W., Y H., L C., C H., C J., Y L., Y D., Z K., JR X. 2016. Genome-wide A-to-I RNA editing in fungi independent of ADAR enzymes. Genome Res. 26:499-509.

H M., D S., JE S., V B. 2015. Deciphering the uniqueness of Mucoromycotina cell walls by combining biochemical and phylogenomic approaches. Environ. Microbiol. 17:16491662.

H S., S K. 2003. A stearoyl-CoA-specific Delta 9 fatty acid desaturase from the basidiomycete Lentinula edodes. Biosci. Biotechnol. Biochem. 67:2431-2437.

H S., S K. 2005. Cloning and functional characterization of a Delta12 fatty acid desaturase gene from the basidiomycete Lentinula edodes. Mol. Genet. Genomics. 273:336-341.

Ha B., Kim S., Kim M., Ro H.S. 2018. Activation of the mating pheromone response pathway of Lentinula edodes by synthetic pheromones. Mycobiology. 46:407-415.

Haber J.E. 2012. Mating-Type Genes and MAT Switching in Saccharomyces cerevisiae. Genetics. 191:33-64.

Hallen H.E., Luo H., Scott-Craig J.S., Walton J.D. 2007. Gene family encoding the major 
bioRxiv preprint doi: https://doi.org/10.1101/2021.12.09.471732; this version posted December $10,2021$. The copyright holder for this preprint (which was not certified by peer review) is the author/funder, who has granted bioRxiv a license to display the preprint in perpetuity. It is made available under aCC-BY-NC-ND 4.0 International license.

Running head: fruiting body genomics

5493

5494

5495

5496

5497

5498

5499

5500

5501

5502

5503

5504

5505

5506

5507

5508

5509

5510

5511

5512

5513

5514

5515

5516

5517

5518

5519

5520

5521

5522

5523

5524

5525

5526

5527

5528

5529

5530

5531

5532

5533

5534

5535

5536

5537

5538

5539

5540

5541

5542

5543

5544

5545

5546

5547 toxins of lethal Amanita mushrooms. Proc. Natl. Acad. Sci. 104:19097-19101.

Han S., Adams T.H. 2001. Complex control of the developmental regulatory locus brlA in Aspergillus nidulans. Mol. Genet. Genomics. 266:260-270.

Han S.E., Kwon H. Bin, Lee S.B., Yi B.Y., Murayama I., Kitamoto Y., Byun M.O. 2003. Cloning and characterization of a gene encoding trehalose phosphorylase (TP) from Pleurotus sajor-caju. Protein Expr. Purif. 30:194-202.

Hartl L., Zach S., Seidl-Seiboth V. 2011. Fungal chitinases: diversity, mechanistic properties and biotechnological potential. Appl. Microbiol. Biotechnol. 2011 932. 93:533-543.

Hassan M.A., Rouf R., Tiralongo E., May T.W., Tiralongo J. 2015. Mushroom lectins: specificity, structure and bioactivity relevant to human disease. Int J Mol Sci. 16:78027838.

Hastrup A.C.S., III F.G., Lebow P.K., Jensen B. 2012. Enzymatic oxalic acid regulation correlated with wood degradation in four brown-rot fungi. Int. Biodeterior. Biodegrad. , Vol. 75, 2012; pp. 109-114. . 75:109-114.

He Z., Long P., Fang F., Li S., Zhang P., Chen Z. 2020. Diversity of MSDIN family members in amanitin-producing mushrooms and the phylogeny of the MSDIN and prolyl oligopeptidase genes. BMC Genomics 2020 211. 21:1-18.

Heneghan M.N., Costa A.M.S.B., Challen M.P., Mills P.R., Bailey A., Foster G.D. 2007. A comparison of methods for successful triggering of gene silencing in Coprinus cinereus. Mol. Biotechnol. 2007 353. 35:283-296.

Herman K.C., Wösten H.A.B., Fricker M.D., Bleichrodt R.J. 2020. Growth induced translocation effectively directs an amino acid analogue to developing zones in Agaricus bisporus. Fungal Biol. 124:1013-1023.

Hernández-Rodríguez Y., Masuo S., Johnson D., Orlando R., Smith A., Couto-Rodriguez M., Momany M. 2014. Distinct Septin Heteropolymers Co-Exist during Multicellular Development in the Filamentous Fungus Aspergillus nidulans. PLoS One. 9:e92819.

Herranz S., Rodríguez J.M., Bussink H.J., Sánchez-Ferrero J.C., Arst H.N., Peñalva M.A., Vincent O. 2005. Arrestin-related proteins mediate $\mathrm{pH}$ signaling in fungi. Proc. Natl. Acad. Sci. U. S. A. 102:12141-12146.

Herzog R., Solovyeva I., Rühl M., Thines M., Hennicke F. 2016. Dikaryotic fruiting body development in a single dikaryon of Agrocybe aegerita and the spectrum of monokaryotic fruiting types in its monokaryotic progeny. Mycol. Prog. 15:947-957.

Heuts D.P.H.M., Janssen D.B., Fraaije M.W. 2007. Changing the substrate specificity of a chitooligosaccharide oxidase from Fusarium graminearum by model-inspired sitedirected mutagenesis. FEBS Lett. 581:4905-4909.

Hibbett D.S. 2004. Trends in morphological evolution in homobasidiomycetes inferred using maximum likelihood: A comparison of binary and multistate approaches. Syst. Biol. 53:889-903.

HM H., DE N., J G., MS S. 2009. Evolutionary roles of upstream open reading frames in mediating gene regulation in fungi. Annu. Rev. Microbiol. 63:385-409.

Holighaus G., Rohlfs M. 2019. Volatile and non-volatile fungal oxylipins in fungusinvertebrate interactions. Fungal Ecol. 38:28-36.

Hong Jai-Sik;Kim T.-Y. 1988. Contents of Free-Sugars \& Free-Sugaralcohols in Pleurotus ostreatus, Lentinus edods \& Agaricus bisporus. Korean J. Food Sci. Technol. 20:459462.

Hou L., Wang L., Wu X., Gao W., Zhang J., Huang C. 2019. Expression patterns of two pal genes of Pleurotus ostreatus across developmental stages and under heat stress. BMC Microbiol. 19:231.

Hou Z., Chen Q., Zhao M., Huang C., Wu X. 2020. Genome-wide characterization of the Zn(II)2Cys6 zinc cluster-encoding gene family in Pleurotus ostreatus and expression analyses of this family during developmental stages and under heat stress. PeerJ. 8:e9336.

Houbraken J., Dyer P.S. 2015. Induction of the Sexual Cycle in Filamentous Ascomycetes. :23-46.

Huang X., Zhang R., Qiu Y., Wu H., Xiang Q., Yu X., Zhao K., Zhang X., Chen Q., Penttinen 
bioRxiv preprint doi: https://doi.org/10.1101/2021.12.09.471732; this version posted December $10,2021$. The copyright holder for this preprint (which was not certified by peer review) is the author/funder, who has granted bioRxiv a license to display the preprint in perpetuity. It is made available under aCC-BY-NC-ND 4.0 International license.

Running head: fruiting body genomics

5548

5549

5550

5551

5552

5553

5554

5555

5556

5557

5558

5559

5560

5561

5562

5563

5564

5565

5566

5567

5568

5569

5570

5571

5572

5573

5574

5575

5576

5577

5578

5579

5580

5581

5582

5583

5584

5585

5586

5587

5588

5589

5590

5591

5592

5593

5594

5595

5596

5597

5598

5599

5600

5601

5602
P., Gu Y. 2020. RNA-seq Profiling Showed Divergent Carbohydrate-Active Enzymes (CAZymes) Expression Patterns in Lentinula edodes at Brown Film Formation Stage Under Blue Light Induction. Front. Microbiol. 0:1044.

Huber S., Lottspeich F., Kämper J. 2002. A gene that encodes a product with similarity to dioxygenases is highly expressed in teliospores of Ustilago maydis. Mol. Genet. Genomics 2002 2676. 267:757-771.

Hung R., Lee S., Bennett J.W. 2014. The effects of low concentrations of the enantiomers of mushroom alcohol (1-octen-3-ol) on Arabidopsis thaliana. Mycology. 5:73-80.

Hutchins A.P., Liu S., Diez D., Miranda-Saavedra D. 2013. The Repertoires of Ubiquitinating and Deubiquitinating Enzymes in Eukaryotic Genomes. Mol. Biol. Evol. 30:1172-1187.

Hyun M.W., Yun Y.H., Kim J.Y., Kim S.H. 2011. Fungal and plant phenylalanine ammonialyase. Mycobiology. 39:257-265.

I A., I A., B de las R., R M., I R.-C., M M., P G., H T., IJ G., B P.-A., JM M. 2011. Highresolution structural insights on the sugar-recognition and fusion tag properties of a versatile $\$ \beta \$$-trefoil lectin domain from the mushroom Laetiporus sulphureus. Glycobiology. 21:1349-1361.

Ismaya W.T., Tjandrawinata R.R., Rachmawati H. 2020. Lectins from the Edible Mushroom Agaricus bisporus and Their Therapeutic Potentials. Molecules. 25.

Iwai K., Naganuma A., Kuge S. 2010. Peroxiredoxin Ahp1 Acts as a Receptor for Alkylhydroperoxides to Induce Disulfide Bond Formation in the Cad1 Transcription Factor *. J. Biol. Chem. 285:10597-10604.

Izawa S., Kuroki N., Inoue Y. 2003. Nuclear thioredoxin peroxidase Dot5 in Saccharomyces cerevisiae: roles in oxidative stress response and disruption of telomeric silencing. Appl. Microbiol. Biotechnol. 2004 641. 64:120-124.

J F., F B., D H. 2017. Enzymatic Synthesis of Psilocybin. Angew. Chem. Int. Ed. Engl. $56: 12352-12355$.

J P., J B., L V., A L., A C., B S., J K., J S. 2011. Basidiomycete Clitocybe nebularis is rich in lectins with insecticidal activities. Appl. Microbiol. Biotechnol. 91:1141-1148.

J R.-R., M H., C K., S M., R F. 2010. Fungi, hidden in soil or up in the air: light makes a difference. Annu. Rev. Microbiol. 64:585-610.

J S., RA O., M K. 2016. Entomotoxic and nematotoxic lectins and protease inhibitors from fungal fruiting bodies. Appl. Microbiol. Biotechnol. 100:91-111.

JA C., RP O., SJ P., EA H. 2017. Olfactory Preferences of the Parasitic Nematode Howardula aoronymphium and its Insect Host Drosophila falleni. J. Chem. Ecol. 43:362-373.

JA D., NF L., SP J. 2000. A role for Saccharomyces cerevisiae histone H2A in DNA repair. Nature. 408:1001-1004.

James T.Y. 2015. Why mushrooms have evolved to be so promiscuous: Insights from evolutionary and ecological patterns. Fungal Biol. Rev. 29:167-178.

James T.Y., Lee M., van Diepen L.T.A. 2011. A Single mating-type locus composed of homeodomain genes promotes nuclear migration and heterokaryosis in the white-rot fungus phanerochaete chrysosporium. Eukaryot. Cell. 10:249-261.

Jara M., Vivancos A.P., Calvo I.A., Moldón A., Sansó M., Hidalgo E. 2007. The Peroxiredoxin Tpx1 Is Essential as a H2O2 Scavenger during Aerobic Growth in Fission Yeast. https://doi.org/10.1091/mbc.e06-11-1039. 18:2288-2295.

Ji J., Moore D. 1993. Glycogen metabolism in relation to fruit body maturation in Coprinus cinereus. Mycol. Res. 97:283-289.

Jia D., Wang B., Li X., Peng W., Zhou J., Tan H., Tang J., Huang Z., Tan W., Gan B., Yang Z., Zhao J. 2017. Proteomic Analysis Revealed the Fruiting-Body Protein Profile of Auricularia polytricha. Curr. Microbiol. 2017 748. 74:943-951.

Jiao X., Li G., Wang Y., Nie F., Cheng X., Abdullah M., Lin Y., Cai Y. 2018. Molecules | Free Full-Text | Systematic Analysis of the Pleurotus ostreatus Laccase Gene (PoLac) Family and Functional Characterization of PoLac2 Involved in the Degradation of Cotton-Straw Lignin | HTML. Mol. 2018, Vol. 23, Page 880. 23:880.

Jin L., Li G., Yu D., Huang W., Cheng C., Liao S., Wu Q., Zhang Y. 2017. Transcriptome 
bioRxiv preprint doi: https://doi.org/10.1101/2021.12.09.471732; this version posted December $10,2021$. The copyright holder for this preprint (which was not certified by peer review) is the author/funder, who has granted bioRxiv a license to display the preprint in perpetuity. It is made available under aCC-BY-NC-ND 4.0 International license.

Running head: fruiting body genomics

5603

5604

5605

5606

5607

5608

5609

5610

5611

5612

5613

5614

5615

5616

5617

5618

5619

5620

5621

5622

5623

5624

5625

5626

5627

5628

5629

5630

5631

5632

5633

5634

5635

5636

5637

5638

5639

5640

5641

5642

5643

5644

5645

5646

5647

5648

5649

5650

5651

5652

5653

5654

5655

5656

5657 analysis reveals the complexity of alternative splicing regulation in the fungus Verticillium dahliae. BMC Genomics. 18:130.

JM C., EL H., C A., R B., G B., ET C., KR C., MC C., SS D., SR E., DG F., JE H., BC H., K K., CJ K., SR M., RS N., J P., MS S., M S., S W., ED W. 2012. Saccharomyces Genome Database: the genomics resource of budding yeast. Nucleic Acids Res. 40.

JM M., H T., D S., IJ G. 2010. Laetiporus sulphureus lectin and aerolysin protein family. Adv. Exp. Med. Biol. 677:67-80.

JM S., H M. 1998. Cloning and characterization of NADP-mannitol dehydrogenase cDNA from the button mushroom, Agaricus bisporus, and its expression in response to $\mathrm{NaCl}$ stress. Appl. Environ. Microbiol. 64:4689-4696.

Joh J.-H.H., Kim B.-G.G., Kong W.-S.S., Yoo Y.-B.B., Kim N.-K.K., Park H.-R.R., Cho B.G.G., Lee C.-S.S. 2004. Cloning and developmental expression of a metzincin family metalloprotease cDNA from oyster mushroom Pleurotus ostreatus. FEMS Microbiol. Lett. 239:57-62.

Jones S.K., Bennett R.J. 2011. Fungal Mating Pheromones: Choreographing the Dating Game. Fungal Genet. Biol. 48:668-676.

de Jong J.F., Deelstra H.J., Wösten H.A.B., Lugones L.G. 2006. RNA-Mediated Gene Silencing in Monokaryons and Dikaryons of Schizophyllum commune. Appl. Environ. Microbiol. 72:1267.

De Jong J.F., Ohm R.A., De Bekker C., Wösten H.A.B., Lugones L.G. 2010. Inactivation of ku80 in the mushroom-forming fungus Schizophyllum commune increases the relative incidence of homologous recombination. FEMS Microbiol. Lett. 310:91-95.

Jonkers W., Rep M. 2009. Lessons from fungal F-Box proteins. Eukaryot. Cell. 8:677-695.

Jorgensen P., Nishikawa J.L., Breitkreutz B.J., Tyers M. 2002. Systematic identification of pathways that couple cell growth and division in yeast. Science (80-. ). 297:395-400.

JT C., NP M., EP B., LA M. 2010. The use of biotin-binding proteins for insect control. J. Econ. Entomol. 103:497-508.

Juillot S., Cott C., Madl J., Claudinon J., van der Velden N.S.J., Künzler M., Thuenauer R., Römer W. 2016. Uptake of Marasmius oreades agglutinin disrupts integrin-dependent cell adhesion. Biochim. Biophys. Acta. 1860:392.

K A., K F., TA S., E H., R K., K G. 2006. NAD+-specific D-arabinose dehydrogenase and its contribution to erythroascorbic acid production in Saccharomyces cerevisiae. FEBS Lett. 580:6428-6434.

K O., M B., G V., M D.S., K S., P M. 2014. Fungal MACPF-like proteins and aegerolysins: bicomponent pore-forming proteins? Subcell. Biochem. 80:271-291.

K S., H Y., Y O., S F., E T., S H. 1998. Production of trehalose synthase from a basidiomycete, Grifola frondosa, in Escherichia coli. Appl. Microbiol. Biotechnol. 50:193-198.

K W., K E., L A., T A., H A. 2005. Characterization of the ferrichrome A biosynthetic gene cluster in the homobasidiomycete Omphalotus olearius. FEMS Microbiol. Lett. 249:157-163.

Kabel M.A., Jurak E., Mäkelä M.R., de Vries R.P. 2017. Occurrence and function of enzymes for lignocellulose degradation in commercial Agaricus bisporus cultivation. Appl. Microbiol. Biotechnol. 2017 10111. 101:4363-4369.

Kalaras M.D., Richie J.P., Calcagnotto A., Beelman R.B., MD K., JP R., A C., RB B. 2017. Mushrooms: A rich source of the antioxidants ergothioneine and glutathione. Food Chem. 233:429-433.

Káldi K., González B.H., Brunner M., K K., BH G., M B. 2006. Transcriptional regulation of the Neurospora circadian clock gene wc-1 affects the phase of circadian output. EMBO Rep. 7:199-204.

Kamada T., Sano H., Nakazawa T., Nakahori K. 2010. Regulation of fruiting body photomorphogenesis in Coprinopsis cinerea. Fungal Genet. Biol.

Kamada T., Takemaru T., Prosser J.I., Gooday G.W. 1991. Right and left handed helicity of chitin microfibrils in stipe cells inCoprinus cinereus. Protoplasma 1991 1651. 165:6470 . 
bioRxiv preprint doi: https://doi.org/10.1101/2021.12.09.471732; this version posted December $10,2021$. The copyright holder for this preprint (which was not certified by peer review) is the author/funder, who has granted bioRxiv a license to display the preprint in perpetuity. It is made available under aCC-BY-NC-ND 4.0 International license.

Running head: fruiting body genomics

5658

5659

5660

5661

5662

5663

5664

5665

5666

5667

5668

5669

5670

5671

5672

5673

5674

5675

5676

5677

5678

5679

5680

5681

5682

5683

5684

5685

5686

5687

5688

5689

5690

5691

5692

5693

5694

5695

5696

5697

5698

5699

5700

5701

5702

5703

5704

5705

5706

5707

5708

5709

5710

5711

5712

Kar B., Patel P., Ao J., Free S.J. 2019. Neurospora crassa family GH72 glucanosyltransferases function to crosslink cell wall glycoprotein N-linked galactomannan to cell wall lichenin. Fungal Genet. Biol. 123:60-69.

Katsimpouras C., Dedes G., Thomaidis N.S., Topakas E. 2019. A novel fungal GH30 xylanase with xylobiohydrolase auxiliary activity. Biotechnol. Biofuels 2019 121. 12:114.

Käufer N.F., Potashkin J. 2000. Analysis of the splicing machinery in fission yeast: a comparison with budding yeast and mammals. Nucleic Acids Res. 28:3003.

Ke H.-M., Lee H.-H., Lin C.-Y.I., Liu Y.-C., Lu M.R., Hsieh J.-W.A., Chang C.-C., Wu P.-H., Lu M.J., Li J.-Y., Shang G., Lu R.J.-H., Nagy L.G., Chen P.-Y., Kao H.-W., Tsai I.J. 2020a. Mycena genomes resolve the evolution of fungal bioluminescence. Proc. Natl. Acad. Sci. 117:31267-31277.

Ke H.-M., Lee H.-H., Lin C.-Y.I., Liu Y.-C., Lu M.R.M.J.M.R., Hsieh J.-W.A., Chang C.-C., Wu P.-H., Lu M.R.M.J.M.R., Li J.-Y., Shang G., Lu R.J.-H., Nagy L.G., Chen P.-Y., Kao H.-W., Tsai I.J. 2020b. Mycena genomes resolve the evolution of fungal bioluminescence. Proc. Natl. Acad. Sci. 117:31267-31277.

Keller N.P. 2019. Fungal secondary metabolism: regulation, function and drug discovery. Nat. Rev. Microbiol. 17:167.

Khan A., McQuilken M., Gladfelter A.S. 2015. Septins and Generation of Asymmetries in Fungal Cells. http://dx.doi.org/10.1146/annurev-micro-091014-104250. 69:487-503.

Kim H. II, Lee C.S., Park Y.J. 2016. Further characterization of hydrophobin genes in genome of Flammulina velutipes. Mycoscience. 57:320-325.

Kim J.Y., Kim D.Y., Park Y.J., Jang M.J. 2020. Transcriptome analysis of the edible mushroom Lentinula edodes in response to blue light. PLoS One.

Kim K.-H., Kang Y.M., Im C.H., Ali A., Kim S.Y., Je H.-J., Kim M.-K., Rho H.S., Lee H.S., Kong W.-S., Ryu J.-S. 2014. Identification and Functional Analysis of Pheromone and Receptor Genes in the B3 Mating Locus of Pleurotus eryngii. PLoS One. 9:e104693.

Kipreos E.T., Pagano M. 2000. The F-box protein family. Genome Biol. 2000 15. 1:1-7.

Kitamoto Y., Kobayashi A., Mori N., Ohga S. 2001. Metabolic function of glycogen phosphorylase and trehalose phosphorylase in fruit-body formation of Flammulina velutipes. Mycoscience. 42:143-147.

Kiyama R., Furutani Y., Kawaguchi K., Nakanishi T. 2018. Genome sequence of the cauliflower mushroom Sparassis crispa (Hanabiratake) and its association with beneficial usage. Sci. Reports 2018 81. 8:1-11.

Klis F.M., Boorsma A., De Groot P.W.J. 2006. Cell wall construction in Saccharomyces cerevisiae. Yeast. 23:185-202.

Knabe N., Jung E.M., Freihorst D., Hennicke F., Horton J.S., Kothe E. 2013. A central role for ras 1 in morphogenesis of the basidiomycete Schizophyllum commune. Eukaryot. Cell. 12:941-952.

Knoll A.H. 2011. The Multiple Origins of Complex Multicellularity. Earth Planet. Sci. 39:217239.

Knoll F. Untersuehungen über Längenwachstum und Geotropismus der Fruehtkörperstiele von Coprinus stiriaeus. .

Kobayashi Y., Tateno H., Ogawa H., Yamamoto K., Hirabayashi J. 2014. Comprehensive List of Lectins: Origins, Natures, and Carbohydrate Specificities. Methods Mol. Biol. 1200:555-577.

Koch R.A., Yoon G.M., Aryal U.K., Lail K., Amirebrahimi M., LaButti K., Lipzen A., Riley R., Barry K., Henrissat B., Grigoriev I. V., Herr J.R., Aime M.C. 2021. Symbiotic nitrogen fixation in the reproductive structures of a basidiomycete fungus. Curr. Biol. 31:39053914.e6.

Kohler A., Kuo A., Nagy L.G., Morin E., Barry K.W., Buscot F., Canbäck B., Choi C., Cichocki N., Clum A., Colpaert J., Copeland A., Costa M.D., Doré J., Floudas D., Gay G., Girlanda M., Henrissat B., Herrmann S., Hess J., Högberg N., Johansson T., Khouja H.-R.R., Labutti K., Lahrmann U., Levasseur A., Lindquist E.A., Lipzen A., Marmeisse R., Martino E., Murat C., Ngan C.Y., Nehls U., Plett J.M., Pringle A., Ohm R.A., Perotto 
bioRxiv preprint doi: https://doi.org/10.1101/2021.12.09.471732; this version posted December $10,2021$. The copyright holder for this preprint (which was not certified by peer review) is the author/funder, who has granted bioRxiv a license to display the preprint in perpetuity. It is made available under aCC-BY-NC-ND 4.0 International license.

Running head: fruiting body genomics

5713

5714

5715

5716

5717

5718

5719

5720

5721

5722

5723

5724

5725

5726

5727

5728

5729

5730

5731

5732

5733

5734

5735

5736

5737

5738

5739

5740

5741

5742

5743

5744

5745

5746

5747

5748

5749

5750

5751

5752

5753

5754

5755

5756

5757

5758

5759

5760

5761

5762

5763

5764

5765

5766

5767
S., Peter M., Riley R., Rineau F., Ruytinx J., Salamov A., Shah F., Sun H., Tarkka M., Tritt A., Veneault-Fourrey C., Zuccaro A., Tunlid A., Grigoriev I. V, Hibbett D.S., Martin F., Martin F. 2015. Convergent losses of decay mechanisms and rapid turnover of symbiosis genes in mycorrhizal mutualists. Nat. Genet. 47:410-415.

Kombrink A., Tayyrov A., Essig A., Stöckli M., Micheller S., Hintze J., van Heuvel Y., Dürig N., Lin C., Kallio P.T., Aebi M., Künzler M. 2018. Induction of antibacterial proteins and peptides in the coprophilous mushroom Coprinopsis cinerea in response to bacteria. ISME J. 2018 133. 13:588-602.

Konada L., Aricthota S., Vadla R., Haldar D. 2018. Fission Yeast Sirtuin Hst4 Functions in Preserving Genomic Integrity by Regulating Replisome Component Mcl1. Sci. Reports 2018 81. 8:1-17.

Kondoh O., Muto A., Kajiwara S., Takagi J., Saito Y., Shishido K. 1995. A fruiting bodyspecific cDNA, mfbAc, from the mushroom Lentinus edodes encodes a high-molecularweight cell-adhesion protein containing an Arg-Gly-Asp motif. Gene. 154:31-37.

Kraakman L.S., Griffioen G., Zerp S., Groeneveld P., Thevelein J.M., Mager W.H., Planta R.J. 1993. Growth-related expression of ribosomal protein genes in Saccharomyces cerevisiae. Mol. Gen. Genet. MGG 1993 2391. 239:196-204.

Kress M.R. von Z., Harting R., Bayram Ö., Christmann M., Irmer H., Valerius O., Schinke J., Goldman G.H., Braus G.H. 2012. The COP9 signalosome counteracts the accumulation of cullin SCF ubiquitin E3 RING ligases during fungal development. Mol. Microbiol. 83:1162-1177.

Krijgsheld P., Bleichrodt R., van Veluw G.J., Wang F., Müller W.H., Dijksterhuis J., Wösten H.A.B. 2013. Development in aspergillus. Stud. Mycol.

Krivosheina N.P. 2008. Macromycete fruit bodies as a habitat for dipterans (Insecta, Diptera). Entomol. Rev. 2008 887. 88:778-792.

Krizsán K., Almási É., Merényi Z., Sahu N., Virágh M., Kószó T., Mondo S., Kiss B., Bálint B., Kües U., Barry K., Cseklye J., Hegedüs B., Henrissat B., Johnson J., Lipzen A., Ohm R.A., Nagy I., Pangilinan J., Yan J., Xiong Y., Grigoriev I. V, Hibbett D.S., Nagy L.G. 2019. Transcriptomic atlas of mushroom development reveals conserved genes behind complex multicellularity in fungi. Proc. Natl. Acad. Sci. U. S. A. 116:7409-7418.

Kück U., Radchenko D., Teichert I. 2019. STRIPAK, a highly conserved signaling complex, controls multiple eukaryotic cellular and developmental processes and is linked with human diseases. Biol. Chem. 400:1005-1022.

Kuees U. 2015. From two to many: multiple mating types in Basidiomycetes. Fungal Biol. Rev. 29:126-166.

Kues U. 2000. Life history and developmental processes in the basidiomycete Coprinus cinereus. Microbiol Mol Biol Rev. 64:316-353.

Kües U. 2000. Life History and Developmental Processes in the Basidiomycete Coprinus cinereus. Microbiol. Mol. Biol. Rev.

Kües U. 2015. From two to many: Multiple mating types in Basidiomycetes. Fungal Biol. Rev. 29:126-166.

Kues U., Granado J.D., Hermann R., Boulianne R.P., Kertesz-Chaloupkova K., Aebi M. 1998. The A mating type and blue light regulate all known differentiation processes in the basidiomycete Coprinus cinereus. Mol Gen Genet. 260:81-91.

Kües U., Khonsuntia W., Subba S., Dörnte B. 2018. Volatiles in Communication of $<$ Emphasis Type="Italic">Agaricomycetes</Emphasis >. Physiol. Genet.:149-212.

Kues U., Liu Y. 2000. Fruiting body production in basidiomycetes. Appl. Microbiol. Biotechnol.

Kües U., Liu Y. 2000. Fruiting body production in basidiomycetes. Appl. Microbiol. Biotechnol. 54:141-152.

Kües U., Navarro-González M. 2015. How do Agaricomycetes shape their fruiting bodies? 1. Morphological aspects of development. Fungal Biol. Rev. 29:63-97.

Kües U., Rühl M., U K., M R. 2011. Multiple multi-copper oxidase gene families in basidiomycetes - what for? Curr. Genomics. 12:72-94.

Kues U., Walser P.J., Klaus M.J., Aebi M. 2002. Influence of activated A and B mating-type 
bioRxiv preprint doi: https://doi.org/10.1101/2021.12.09.471732; this version posted December $10,2021$. The copyright holder for this preprint (which was not certified by peer review) is the author/funder, who has granted bioRxiv a license to display the preprint in perpetuity. It is made available under aCC-BY-NC-ND 4.0 International license.

Running head: fruiting body genomics

5768

5769

5770

5771

5772

5773

5774

5775

5776

5777

5778

5779

5780

5781

5782

5783

5784

5785

5786

5787

5788

5789

5790

5791

5792

5793

5794

5795

5796

5797

5798

5799

5800

5801

5802

5803

5804

5805

5806

5807

5808

5809

5810

5811

5812

5813

5814

5815

5816

5817

5818

5819

5820

5821

5822 pathways on developmental processes in the basidiomycete Coprinus cinereus. Mol Genet Genomics. 268:262-271.

Kuhad R.C., Rosin I. V, Moore D. 1987. A possible relation between cyclic-AMP levels and glycogen mobilization in Coprinus cinereus. Trans. Br. Mycol. Soc. 88:229-236.

Kumar M.J., Jamaluddin M.S., Natarajan K., Kaur D., Datta A. 2000. The inducible Nacetylglucosamine catabolic pathway gene cluster in Candida albicans: Discrete Nacetylglucosamine-inducible factors interact at the promoter of NAG1. Proc. Natl. Acad. Sci. 97:14218-14223.

Kunzler M. 2015. Hitting the sweet spot-glycans as targets of fungal defense effector proteins. Molecules. 20:8144-8167.

Künzler M. 2018. How fungi defend themselves against microbial competitors and animal predators. PLOS Pathog. 14:e1007184.

Kuratani M., Tanaka K., Terashima K., Muraguchi H., Nakazawa T., Nakahori K., Kamada T. 2010. The dst2 gene essential for photomorphogenesis of Coprinopsis cinerea encodes a protein with a putative FAD-binding-4 domain. Fungal Genet. Biol.

KY Y., CM C., KM C., PC W., XY C., AS L., L C. 2001. Characterization of AFMP1: a novel target for serodiagnosis of aspergillosis. J. Clin. Microbiol. 39:3830-3837.

L A.-F., C C., C L., V A., V S.-S., E M., JP L. 2011. Functional analysis of the fungal/plant class chitinase family in Aspergillus fumigatus. Fungal Genet. Biol. 48:418-429.

L L., EE G., ME Z. 1999. Homolog pairing and meiotic progression in Coprinus cinereus. Chromosoma. 108:384-392.

Labourel A., Frandsen K.E.H., Zhang F., Brouilly N., Grisel S., Haon M., Ciano L., Ropartz D., Fanuel M., Martin F., Navarro D., Rosso M.-N., Tandrup T., Bissaro B., Johansen K.S., Zerva A., Walton P.H., Henrissat B., Leggio L. Lo, Berrin J.-G. 2020. A fungal family of lytic polysaccharide monooxygenase-like copper proteins. Nat. Chem. Biol. 2020 163. 16:345-350.

Lackner G., Bohnert M., Wick J., Hoffmeister D. 2013. Assembly of Melleolide Antibiotics Involves a Polyketide Synthase with Cross-Coupling Activity. Chem. Biol. 20:11011106.

Lakkireddy K., Khonsuntia W., Kües U. 2020. Mycoparasite Hypomyces odoratus infests Agaricus xanthodermus fruiting bodies in nature. AMB Express. 10.

Landi N., Pacifico S., Ragucci S., Iglesias R., Piccolella S., Amici A., Di Giuseppe A.M.A., Di Maro A. 2017. Purification, characterization and cytotoxicity assessment of Ageritin: The first ribotoxin from the basidiomycete mushroom Agrocybe aegerita. Biochim. Biophys. Acta - Gen. Subj. 1861:1113-1121.

Langner T., Göhre V. 2015. Fungal chitinases: function, regulation, and potential roles in plant/pathogen interactions. Curr. Genet. 2015 622. 62:243-254.

Larrondo L.F., Salas L., Melo F., Vicuña R., Cullen D. 2003. A Novel Extracellular Multicopper Oxidase from Phanerochaete chrysosporium with Ferroxidase Activity. Appl. Environ. Microbiol. 69:6257-6263.

Lau A.Y.-T., Cheng X., Cheng C.K., Nong W., Cheung M.K., Chan R.H.-F., Hui J.H.L., Kwan H.S. 2018. Discovery of microRNA-like RNAs during early fruiting body development in the model mushroom Coprinopsis cinerea. bioRxiv.

Lavrijssen B., Baars J.P., Lugones L.G., Scholtmeijer K., Sedaghat Telgerd N., Sonnenberg A.S.M., van Peer A.F. 2020. Interruption of an MSH4 homolog blocks meiosis in metaphase I and eliminates spore formation in Pleurotus ostreatus. PLoS One. 15:e0241749.

LC T., X W., A A., Z C., J T.-F., H Y., NP P., BS C., C C.-C., H E.-B., P T., SG C., HJ K., T M., X J., PP P. 2007. Ubiquitination regulates PTEN nuclear import and tumor suppression. Cell. 128:141-156.

Leatham G.F., Stahmann M.A. 1981. Studies on the Laccase of Lentinus edodes: Specificity, Localization and Association with the Development of Fruiting Bodies. Microbiology. 125:147-157.

Lebreton A., Bonnardel F., Dai Y.-C., Imberty A., Martin F.M., Lisacek F. 2021. A Comprehensive Phylogenetic and Bioinformatics Survey of Lectins in the Fungal 
bioRxiv preprint doi: https://doi.org/10.1101/2021.12.09.471732; this version posted December $10,2021$. The copyright holder for this preprint (which was not certified by peer review) is the author/funder, who has granted bioRxiv a license to display the preprint in perpetuity. It is made available under aCC-BY-NC-ND 4.0 International license.

Running head: fruiting body genomics

5823

5824

5825

5826

5827

5828

5829

5830

5831

5832

5833

5834

5835

5836

5837

5838

5839

5840

5841

5842

5843

5844

5845

5846

5847

5848

5849

5850

5851

5852

5853

5854

5855

5856

5857

5858

5859

5860

5861

5862

5863

5864

5865

5866

5867

5868

5869

5870

5871

5872

5873

5874

5875

5876

5877
Kingdom. J. Fungi. 7:453.

Lee D., Jang E.H., Lee M., Kim S.W., Lee Y., Lee K.T., Bahna Y.S. 2019. Unraveling melanin biosynthesis and signaling networks in cryptococcus neoformans. MBio. 10.

Lee K.T., So Y.S., Yang D.H., Jung K.W., Choi J., Lee D.G., Kwon H., Jang J., Wang L.L., Cha S., Meyers G.L., Jeong E., Jin J.H., Lee Y., Hong J., Bang S., Ji J.H., Park G., Byun H.J., Park S.W., Park Y.M., Adedoyin G., Kim T., Averette A.F., Choi J.S., Heitman J., Cheong E., Lee Y.H., Bahn Y.S. 2016. Systematic functional analysis of kinases in the fungal pathogen Cryptococcus neoformans. Nat. Commun. 7.

Lee Y.Y., de Ulzurrun G.V.D., Schwarz E.M., Stajich J.E., Hsueh Y.P. 2021. Genome sequence of the oyster mushroom Pleurotus ostreatus strain PC9. G3 Genes, Genomes, Genet. 11.

Lenz C., Wick J., Braga D., García-Altares M., Lackner G., Hertweck C., Gressler M., Hoffmeister D. 2020. Injury-Triggered Blueing Reactions of Psilocybe "Magic" Mushrooms. Angew. Chemie Int. Ed. 59:1450-1454.

Lesage G., Bussey H. 2006. Cell Wall Assembly in Saccharomyces cerevisiae. Microbiol. Mol. Biol. Rev. 70:317.

Levasseur A., Drula E., Lombard V., Coutinho P.M., Henrissat B. 2013. Expansion of the enzymatic repertoire of the CAZy database to integrate auxiliary redox enzymes. Biotechnol. Biofuels. 6.

Levasseur A., Lomascolo A., Chabrol O., Ruiz-Dueñas F.J., Boukhris-Uzan E., Piumi F., Kües U., Ram A.F.J., Murat C., Haon M., Benoit I., Arfi Y., Chevret D., Drula E., Kwon M.J., Gouret P., Lesage-Meessen L., Lombard V., Mariette J., Noirot C., Park J., Patyshakuliyeva A., Sigoillot J.C., Wiebenga A., Wösten H.A.B., Martin F., Coutinho P.M., de Vries R.P., Martínez A.T., Klopp C., Pontarotti P., Henrissat B., Record E. 2014. The genome of the white-rot fungus Pycnoporus cinnabarinus: A basidiomycete model with a versatile arsenal for lignocellulosic biomass breakdown. BMC Genomics. 15:486.

Levin D.E. 2005. Cell Wall Integrity Signaling in Saccharomyces cerevisiae. Microbiol. Mol. Biol. Rev. 69:262.

Lewandowska M., Keyl A., Feussner I. 2020. Wax biosynthesis in response to danger: its regulation upon abiotic and biotic stress. New Phytol. 227:698-713.

Li F., Wu X., Lam P., Bird D., Zheng H., Samuels L., Jetter R., Kunst L., F L., X W., P L., D B., H Z., L S., R J., L K., Li F., Wu X., Lam P., Bird D., Zheng H., Samuels L., Jetter R., Kunst L. 2008. Identification of the Wax Ester Synthase/Acyl-Coenzyme

A:Diacylglycerol Acyltransferase WSD1 Required for Stem Wax Ester Biosynthesis in Arabidopsis. Plant Physiol. 148:97-107.

Liao Y., Smyth G.K., Shi W. 2014. featureCounts: an efficient general purpose program for assigning sequence reads to genomic features. Bioinformatics. 30:923-930.

Linder M.B., Szilvay G.R., Nakari-Setälä T., Penttilä M.E. 2005. Hydrophobins: the proteinamphiphiles of filamentous fungi. FEMS Microbiol. Rev. 29:877-896.

Liu C., Bi J., Kang L., Zhou J., Liu X., Liu Z., Yuan S. 2021. The molecular mechanism of stipe cell wall extension for mushroom stipe elongation growth. Fungal Biol. Rev. 35:14-26.

Liu H., Li Y., Chen D., Qi Z., Wang Q., Wang J., Jiang C., Xu J.-R. 2017. A-to-I RNA editing is developmentally regulated and generally adaptive for sexual reproduction in Neurospora crassa. Proc. Natl. Acad. Sci. 114:E7756--E7765.

Liu J., Shang X., Liu J., Tan Q. 2016. Changes in trehalose content, enzyme activity and gene expression related to trehalose metabolism in Flammulina velutipes under heat shock. Microbiology. 162:1274-1285.

Liu L., He G.J., Chen L., Zheng J., Chen Y., Shen L., Tian X., Li E., Yang E., Liao G., Wang L. 2018. Genetic basis for coordination of meiosis and sexual structure maturation in cryptococcus neoformans. Elife.

Liu T.B., Chen G.Q., Min H., Lin F.C. 2009. MoFLP1, encoding a novel fungal fasciclin-like protein, is involved in conidiation and pathogenicity in Magnaporthe oryzae. J. Zhejiang Univ. Sci. B. 10:434-444. 
bioRxiv preprint doi: https://doi.org/10.1101/2021.12.09.471732; this version posted December $10,2021$. The copyright holder for this preprint (which was not certified by peer review) is the author/funder, who has granted bioRxiv a license to display the preprint in perpetuity. It is made available under aCC-BY-NC-ND 4.0 International license.

Running head: fruiting body genomics

5878

5879

5880

5881

5882

5883

5884

5885

5886

5887

5888

5889

5890

5891

5892

5893

5894

5895

5896

5897

5898

5899

5900

5901

5902

5903

5904

5905

5906

5907

5908

5909

5910

5911

5912

5913

5914

5915

5916

5917

5918

5919

5920

5921

5922

5923

5924

5925

5926

5927

5928

5929

5930

5931

5932
Liu T.B., Xue C. 2011. The ubiquitin-proteasome system and F-box proteins in pathogenic fungi. Mycobiology. 39:243-248.

Liu X.-B. Bin, Xia E.-H.H., Li M., Cui Y.-Y.Y., Wang P.-M.M., Zhang J.-X.X., Xie B.-G.G., Xu J.-P.P., Yan J.-J.J., Li J., Nagy L.G., Yang Z.L. 2020a. Transcriptome data reveal conserved patterns of fruiting body development and response to heat stress in the mushroomforming fungus Flammulina filiformis. PLoS One. 15:e0239890.

Liu X. Bin, Xia E.H., Li M., Cui Y.Y., Wang P.M., Zhang J.X., Xie B.G., Xu J.P., Yan J.J., Li J., Nagy L.G., Yang Z.L. 2020b. Transcriptome data reveal conserved patterns of fruiting body development and response to heat stress in the mushroomforming fungus Flammulina filiformis. PLoS One.

Liu Y. 2005. An Essential Gene for Fruiting Body Initiation in the Basidiomycete Coprinopsis cinerea Is Homologous to Bacterial Cyclopropane Fatty Acid Synthase Genes. Genetics. 172:873-884.

Loftus M.G., Sánchez C., Moore D., Robson G., Trinci T. 2020. A 21st century miniguide to sporophore morphogenesis and development in Agaricomycetes and their biotechnological potential. .

López-Mondéjar R., Catalano V., Kubicek C.P., Seidl V. 2009. The \$ \$-Nacetylglucosaminidases NAG1 and NAG2 are essential for growth of Trichoderma atroviride on chitin. FEBS J. 276:5137-5148.

Lu B.C., Gallo N., Kües U., BC L., N G., U K. 2003. White-cap mutants and meiotic apoptosis in the basidiomycete Coprinus cinereus. Fungal Genet. Biol. 39:82-93.

Lu M.-Y.J., Fan W.-L., Wang W.-F., Chen T., Tang Y.-C., Chu F.-H., Chang T.-T., Wang S.Y., Li M.-Y., Chen Y.-H., Lin Z.-S., Yang K.-J., Chen S.-M., Teng Y.-C., Lin Y.-L., Shaw J.-F., Wang T.-F., Li W.-H. 2014. Genomic and transcriptomic analyses of the medicinal fungus Antrodia cinnamomea for its metabolite biosynthesis and sexual development. Proc. Natl. Acad. Sci. U. S. A. 111:E4743.

Lu Y.P., Liao J.H., Guo Z.J., Cai Z.X., Chen M.Y. 2020. Genome Survey and Transcriptome Analysis on Mycelia and Primordia of Agaricus blazei. Biomed Res. Int. 2020.

Lugones L.G., Scholtmeijer K., Klootwijk R., Wessels J.G.H. 1999a. Introns are necessary for mRNA accumulation in Schizophyllum commune. Mol. Microbiol. 32:681-689.

Lugones L.G., Wösten H.A.B., Birkenkamp K.U., Sjollema K.A., Zagers J., Wessels J.G.H. 1999b. Hydrophobins line air channels in fruiting bodies of Schizophyllum commune and Agaricus bisporus. Mycol. Res. 103:635-640.

Luo H., Hallen-Adams H.E., Scott-Craig J.S., Walton J.D. 2010. Colocalization of Amanitin and a Candidate Toxin-Processing Prolyl Oligopeptidase in Amanita Basidiocarps. Eukaryot. Cell. 9:1891.

Luti S., Sella L., Quarantin A., Pazzagli L., Baccelli I. 2020. Twenty years of research on cerato-platanin family proteins: clues, conclusions, and unsolved issues. Fungal Biol. Rev. 34:13-24.

Luyten K., Albertyn J., Skibbe W.F., Prior B.A., Ramos J., Thevelein J.M., Hohmann S. 1995. Fps1, a yeast member of the MIP family of channel proteins, is a facilitator for glycerol uptake and efflux and is inactive under osmotic stress. EMBO J. 14:1360.

M C., S P., RP A., C G., C L. 1999. The lactate-proton symport of Saccharomyces cerevisiae is encoded by JEN1. J. Bacteriol. 181:2620-2623.

M F., J K., B F. 2003. Relationships between the ethanol utilization (alc) pathway and unrelated catabolic pathways in Aspergillus nidulans. Eur. J. Biochem. 270:3555-3564.

M H., H K., MJ P., R U. 2015a. Fungal unspecific peroxygenases: heme-thiolate proteins that combine peroxidase and cytochrome p450 properties. Adv. Exp. Med. Biol. 851:341-368.

M H., SM T., SJ M., RA H., KL H., CT B., E O. 2020a. Bacterial Brown Pit, a New Disease of Edible Mushrooms Caused by Mycetocola sp. Plant Dis. 104:1445-1454.

M K., S D., F M., AG P. 2009a. RNA silencing in the model mycorrhizal fungus Laccaria bicolor: gene knock-down of nitrate reductase results in inhibition of symbiosis with Populus. Environ. Microbiol. 11:1878-1896.

M L., B Ž., D H., K L., R T., V M. 2021. Systematic Comparison of Cell Wall-Related Proteins 
bioRxiv preprint doi: https://doi.org/10.1101/2021.12.09.471732; this version posted December $10,2021$. The copyright holder for this preprint (which was not certified by peer review) is the author/funder, who has granted bioRxiv a license to display the preprint in perpetuity. It is made available under aCC-BY-NC-ND 4.0 International license.

Running head: fruiting body genomics

5933

5934

5935

5936

5937

5938

5939

5940

5941

5942

5943

5944

5945

5946

5947

5948

5949

5950

5951

5952

5953

5954

5955

5956

5957

5958

5959

5960

5961

5962

5963

5964

5965

5966

5967

5968

5969

5970

5971

5972

5973

5974

5975

5976

5977

5978

5979

5980

5981

5982

5983

5984

5985

5986

5987 of Different Yeasts. J. fungi (Basel, Switzerland). 7:1-19.

M L., G G., V S., A K., R R., W T., J M., M O. 2010. C terminus of Nce102 determines the structure and function of microdomains in the Saccharomyces cerevisiae plasma membrane. Eukaryot. Cell. 9:1184-1192.

M M., AA N., M D., JP J., E G. 2009b. The fungal glutathione S-transferase system. Evidence of new classes in the wood-degrading basidiomycete Phanerochaete chrysosporium. Cell. Mol. Life Sci. 66:3711-3725.

M N.-L., C G.M. 1981. The cell walls of Agaricus bisporus and Agaricus campestris fruiting body hyphae. Can. J. Microbiol. 27:779-787.

M N., N K., M S., P M., G A., K S. 2015b. Fungal aegerolysin-like proteins: distribution, activities, and applications. Appl. Microbiol. Biotechnol. 99:601-610.

M O., P M., F B., JG G., Á M.-D.-P., L G.-O. 2017a. Characterization of a new toxin from the entomopathogenic fungus Metarhizium anisopliae: the ribotoxin anisoplin. Biol. Chem. 398:135-142.

M O., R L.-G., JC L.-R., Á M.-D.-P., M O., M M.-L., J L., JG G., L G.-O. 2017b. Fungal Ribotoxins: A Review of Potential Biotechnological Applications. Toxins (Basel). 9.

M S., M P., S H., J P., B S., E N., A B., M W., M P. 2002a. Swollenin, a Trichoderma reesei protein with sequence similarity to the plant expansins, exhibits disruption activity on cellulosic materials. Eur. J. Biochem. 269:4202-4211.

M S., P C., M B., M B., M M.-N., JP L., I M. 2020b. GPI Anchored Proteins in Aspergillus fumigatus and Cell Wall Morphogenesis. Curr. Top. Microbiol. Immunol. 425:167-186.

M W., V T., L P., L C., D F. 2002b. Proteins as active compounds involved in insecticidal activity of mushroom fruitbodies. J. Econ. Entomol. 95:603-607.

M Y., M K., S I., G T., M O., M S. 2008. Isolation and characterization of a gene coding for chitin deacetylase specifically expressed during fruiting body development in the basidiomycete Flammulina velutipes and its expression in the yeast Pichia pastoris. FEMS Microbiol. Lett. 289:130-137.

M Y., S S., K S., G T., S I., M O., M S., Yamada M., Sakuraba S., Shibata K., Taguchi G., Inatomi S., Okazaki M., Shimosaka M. 2006. Isolation and analysis of genes specifically expressed during fruiting body development in the basidiomycete Flammulina velutipes by fluorescence differential display. FEMS Microbiol. Lett. 254:165-172.

Ma A., Shan L., Wang N., Zheng L., Chen L., Xie B. 2007. Characterization of a Pleurotus ostreatus fruiting body-specific hydrophobin gene, Po.hyd. J. Basic Microbiol. 47:317324.

Ma G., Yang W., Zhao L., Pei F., Fang D., Hu Q. 2018. A critical review on the health promoting effects of mushrooms nutraceuticals. Food Sci. Hum. Wellness. 7:125-133.

MA W., C V., RM B., A G., M A., M K., Wälti M.A., Villalba C., Buser R.M., Grünler A., Aebi M., Künzler M. 2006. Targeted gene silencing in the model mushroom Coprinopsis cinerea (Coprinus cinereus) by expression of homologous hairpin RNAs. Eukaryot. Cell. 5:732-744.

Ma X., Fan X., Wang G., Xu R., Yan L., Zhou Y., Gong Y., Xiao Y., Bian Y. 2021. Enhanced Expression of Thaumatin-like Protein Gene (LeTLP1) Endows Resistance to Trichoderma atroviride in Lentinula edodes. Life. 11:863.

Madhavan S., Krause K., Jung E.-M., Kothe E. 2014. Differential regulation of multi-copper oxidases in Schizophyllum commune during sexual development. Mycol. Prog. 2014 134. 13:1199-1206.

Mäkelä M.R., Sietiö O.-M., Vries R.P. de, Timonen S., Hildén K. 2014. Oxalate-Metabolising Genes of the White-Rot Fungus Dichomitus squalens Are Differentially Induced on Wood and at High Proton Concentration. PLoS One. 9:e87959.

Mäkinen M., Kuuskeri J., Laine P., Smolander O.-P., Kovalchuk A., Zeng Z., Asiegbu F.O., Paulin L., Auvinen P., Lundell T. 2019. Genome description of Phlebia radiata 79 with comparative genomics analysis on lignocellulose decomposition machinery of phlebioid fungi. BMC Genomics 2019 201. 20:1-22.

Martin F., Aerts A., Ahrén D., Brun A., Danchin E.G.J., Duchaussoy F., Gibon J., Kohler A., Lindquist E., Pereda V., Salamov A., Shapiro H.J., Wuyts J., Blaudez D., Buée M., 
bioRxiv preprint doi: https://doi.org/10.1101/2021.12.09.471732; this version posted December $10,2021$. The copyright holder for this preprint (which was not certified by peer review) is the author/funder, who has granted bioRxiv a license to display the preprint in perpetuity. It is made available under aCC-BY-NC-ND 4.0 International license.

Running head: fruiting body genomics

5988

5989

5990

5991

5992

5993

5994

5995

5996

5997

5998

5999

6000

6001

6002

6003

6004

6005

6006

6007

6008

6009

6010

6011

6012

6013

6014

6015

6016

6017

6018

6019

6020

6021

6022

6023

6024

6025

6026

6027

6028

6029

6030

6031

6032

6033

6034

6035

6036

6037

6038

6039

6040

6041

6042

Brokstein P., Canbäck B., Cohen D., Courty P.E., Coutinho P.M., Delaruelle C., Detter J.C., Deveau A., DiFazio S., Duplessis S., Fraissinet-Tachet L., Lucic E., Frey-Klett P., Fourrey C., Feussner I., Gay G., Grimwood J., Hoegger P.J., Jain P., Kilaru S., Labbé J., Lin Y.C., Legué V., Le Tacon F., Marmeisse R., Melayah D., Montanini B., Muratet M., Nehls U., Niculita-Hirzel H., Secq M.P.O.-L., Peter M., Quesneville H., Rajashekar B., Reich M., Rouhier N., Schmutz J., Yin T., Chalot M., Henrissat B., Kües U., Lucas S., Van de Peer Y., Podila G.K., Polle A., Pukkila P.J., Richardson P.M., Rouzé P., Sanders I.R., Stajich J.E., Tunlid A., Tuskan G., Grigoriev I. V. 2008. The genome of Laccaria bicolor provides insights into mycorrhizal symbiosis. Nature. 452:88-92.

Martinez D., Challacombe J., Morgenstern I., Hibbett D., Schmoll M., Kubicek C.P., Ferreira P., Ruiz-Duenas F.J., Martinez A.T., Kersten P., Hammel K.E., Wymelenberg A. Vanden, Gaskell J., Lindquist E., Sabat G., BonDurant S.S., Larrondo L.F., Canessa P., Vicuna R., Yadav J., Doddapaneni H., Subramanian V., Pisabarro A.G., Lavín J.L., Oguiza J.A., Master E., Henrissat B., Coutinho P.M., Harris P., Magnuson J.K., Baker S.E., Bruno K., Kenealy W., Hoegger P.J., Kües U., Ramaiya P., Lucas S., Salamov A., Shapiro H., Tu H., Chee C.L., Misra M., Xie G., Teter S., Yaver D., James T., Mokrejs M., Pospisek M., Grigoriev I. V., Brettin T., Rokhsar D., Berka R., Cullen D. 2009a. Genome, transcriptome, and secretome analysis of wood decay fungus Postia placenta supports unique mechanisms of lignocellulose conversion. Proc. Natl. Acad. Sci. 106:1954-1959.

Martinez D., Challacombe J., Morgenstern I., Hibbett D., Schmoll M., Kubicek C.P., Ferreira P., Ruiz-Duenas F.J., Martinez A.T., Kersten P., Hammel K.E., Vanden Wymelenberg A., Gaskell J., Lindquist E., Sabat G., Splinter BonDurant S., Larrondo L.F., Canessa P., Vicuna R., Yadav J., Doddapaneni H., Subramanian V., Pisabarro A.G., Lavín J.L., Oguiza J.A., Master E., Henrissat B., Coutinho P.M., Harris P., Magnuson J.K., Baker S.E., Bruno K., Kenealy W., Hoegger P.J., Kües U., Ramaiya P., Lucas S., Salamov A., Shapiro H., Tu H., Chee C.L., Misra M., Xie G., Teter S., Yaver D., James T., Mokrejs M., Pospisek M., Grigoriev I. V, Brettin T., Rokhsar D., Berka R., Cullen D. 2009b. Genome, transcriptome, and secretome analysis of wood decay fungus Postia placentasupports unique mechanisms of lignocellulose conversion. Proc. Natl. Acad. Sci. 106:1954-1959.

Martinez D., Larrondo L.F., Putnam N., Sollewijn Gelpke M.D., Huang K., Chapman J., Helfenbein K.G., Ramaiya P., Detter J.C., Larimer F., Coutinho P.M., Henrissat B., Berka R., Cullen D., Rokhsar D. 2004. Genome sequence of the lignocellulose degrading fungus Phanerochaete chrysosporium strain RP78. Nat. Biotechnol. 22:695700.

Masuda R., Iguchi N., Tukuta K., Nagoshi T., Kemuriyama K., Muraguchi H. 2016. The Coprinopsis cinerea Tup1 homologue Cag1 is required for gill formation during fruiting body morphogenesis. Biol. Open. 5:1844-1852.

Mata J., Lyne R., Burns G., Bähler J. 2002. The transcriptional program of meiosis and sporulation in fission yeast. Nat. Genet. 2002 321. 32:143-147.

Matityahu A., Hadar Y., Dosoretz C.G., Belinky P.A. 2008. Gene silencing by RNA interference in the white rot fungus Phanerochaete chrysosporium. Appl. Environ. Microbiol. 74:5359-5365.

Meister C., Thieme K.G., Thieme S., Köhler A.M., Schmitt K., Valerius O., Braus G.H. 2019. COP9 Signalosome Interaction with UspA/Usp15 Deubiquitinase Controls VeAMediated Fungal Multicellular Development. Biomol. 2019, Vol. 9, Page 238. 9:238.

Meng L., Chou T., Jiang S., Wang L., Zhu M., Mukhtar I., Xie B., Wang W. 2020. Characterization and expression pattern analysis of pheromone receptor-like genes in Winter Mushroom Flammulina filiformis. Arch. Microbiol. 202:2671-2678.

Meng L., Lyu X., Shi L., Wang Q., Wang L., Zhu M., Mukhtar I., Xie B., Wang W. 2021. The transcription factor FvHmg1 negatively regulates fruiting body development in Winter Mushroom Flammulina velutipes. Gene. 785:145618.

Merényi Z., Prasanna A.N., Wang Z., Kovács K., Hegedüs B., Bálint B., Papp B., Townsend J.P., Nagy L.G. 2020a. Unmatched level of molecular convergence among deeply 
bioRxiv preprint doi: https://doi.org/10.1101/2021.12.09.471732; this version posted December $10,2021$. The copyright holder for this preprint (which was not certified by peer review) is the author/funder, who has granted bioRxiv a license to display the preprint in perpetuity. It is made available under aCC-BY-NC-ND 4.0 International license.

Running head: fruiting body genomics

6043

6044

6045

6046

6047

6048

6049

6050

6051

6052

6053

6054

6055

6056

6057

6058

6059

6060

6061

6062

6063

6064

6065

6066

6067

6068

6069

6070

6071

6072

6073

6074

6075

6076

6077

6078

6079

6080

6081

6082

6083

6084

6085

6086

6087

6088

6089

6090

6091

6092

6093

6094

6095

6096

6097 divergent complex multicellular fungi. Mol. Biol. Evol. 37:2228-2240.

Merényi Z., Prasanna A.N., Wang Z., Kovács K., Hegedüs B., Bálint B., Papp B., Townsend J.P., Nagy L.G. 2020b. Unmatched Level of Molecular Convergence among Deeply Divergent Complex Multicellular Fungi. Mol. Biol. Evol. 37:2228-2240.

Merényi Z., Virágh M., Gluck-Thaler E., Slot J.C., Kiss B., Varga T., Geösel A., Hegedüs B., Bálint B., Nagy L.G. 2021. Gene age predicts the transcriptional landscape of sexual morphogenesis in multicellular fungi. bioRxiv.:2021.06.04.447176.

Metzger M.B., Hristova V.A., Weissman A.M. 2012. HECT and RING finger families of E3 ubiquitin ligases at a glance. J. Cell Sci. 125:531-537.

Meyerowitz E.M. 2002. Comparative genomics. Plants compared to animals: The broadest comparative study of development. Science (80-. ). 295:1482-1485.

Mgbeahuruike A.C., Kovalchuk A., Asiegbu F.O. 2017. Comparative genomics and evolutionary analysis of hydrophobins from three species of wood-degrading fungi. http://dx.doi.org/10.3852/13-077. 105:1471-1478.

MH B., V B.-P., D M., L E. 2012. The Neurospora crassa mutant Nc $\$ \Delta \$$ Egt-1 identifies an ergothioneine biosynthetic gene and demonstrates that ergothioneine enhances conidial survival and protects against peroxide toxicity during conidial germination. Fungal Genet. Biol. 49:160-172.

Michael Green N. 1990. [5] Avidin and streptavidin. Methods Enzymol. 184:51-67.

Michelot D., Melendez-Howell L.M. 2003. Amanita muscaria: chemistry, biology, toxicology, and ethnomycology. Mycol. Res. 107:131-146.

Middleton R., Gao D., Thomas A., Singh B., Au A., Wong J.J.-L., Bomane A., Cosson B., Eyras E., Rasko J.E.J., Ritchie W. 2017. IRFinder: assessing the impact of intron retention on mammalian gene expression. Genome Biol. 2017 181. 18:1-11.

Min B., Kim S., Oh Y.-L., Kong W.-S., Park H., Cho H., Jang K.-Y., Kim J.-G., Choi I.-G. 2018. Genomic discovery of the hypsin gene and biosynthetic pathways for terpenoids in Hypsizygus marmoreus. BMC Genomics 2018 191. 19:1-12.

Min B., Yoon H., Park J., Oh Y.-L., Kong W.-S., Kim J.-G., Choi I.-G. 2020. Unusual genome expansion and transcription suppression in ectomycorrhizal Tricholoma matsutake by insertions of transposable elements. PLoS One. 15:e0227923.

Miyauchi S., Kiss E., Kuo A., Drula E., Kohler A., Sánchez-García M., Morin E., Andreopoulos B., Barry K.W., Bonito G., Buée M., Carver A., Chen C., Cichocki N., Clum A., Culley D., Crous P.W., Fauchery L., Girlanda M., Hayes R.D., Kéri Z., LaButti K., Lipzen A., Lombard V., Magnuson J., Maillard F., Murat C., Nolan M., Ohm R.A., Pangilinan J., Pereira M. de F., Perotto S., Peter M., Pfister S., Riley R., Sitrit Y., Stielow J.B., Szöllősi G., Žifčáková L., Štursová M., Spatafora J.W., Tedersoo L., Vaario L.M., Yamada A., Yan M., Wang P., Xu J., Bruns T., Baldrian P., Vilgalys R., Dunand C., Henrissat B., Grigoriev I. V, Hibbett D., Nagy L.G., Martin F.M. 2020. Largescale genome sequencing of mycorrhizal fungi provides insights into the early evolution of symbiotic traits. Nat. Commun.

Miyazaki Y., Jojima T., Ono T., Yamazaki T., Shishido K. 2004. A cDNA homologue of Schizosaccharomyces pombe cdc5 + from the mushroom Lentinula edodes: Characterization of the cDNA and its expressed product. Biochim. Biophys. Acta - Gene Struct. Expr. 1680:93-102.

Mol P.C., Vermeulen C.A., Wessels J.G.H. 1990. Diffuse extension of hyphae in stipes of Agaricus bisporus may be based on a unique wall structure. Mycol. Res. 94:480-488.

Mol P.C., Wessels J.G.H. 1990. Differences in wall structure between substrate hyphae and hyphae of fruit-body stipes in Agaricus bisporus. Mycol. Res. 94:472-479.

Mollapour M., Piper P.W. 2007. Hog1 Mitogen-Activated Protein Kinase Phosphorylation Targets the Yeast Fps1 Aquaglyceroporin for Endocytosis, Thereby Rendering Cells Resistant to Acetic Acid. Mol. Cell. Biol. 27:6446.

Moore D. 2005. Principles of Mushroom Developmental Biology. .

Moore D. (David). 2013. Coprinopsis $\square$ : an autobiography. :208.

MOORE D., ELHITI M.M.Y., BUTLER R.D. 1979. MORPHOGENENSIS OF THE CARPOPHORE OF COPRINUS CINEREUS. New Phytol. 83:695-722. 
bioRxiv preprint doi: https://doi.org/10.1101/2021.12.09.471732; this version posted December $10,2021$. The copyright holder for this preprint (which was not certified by peer review) is the author/funder, who has granted bioRxiv a license to display the preprint in perpetuity. It is made available under aCC-BY-NC-ND 4.0 International license.

Running head: fruiting body genomics

6098

6099

6100

6101

6102

6103

6104

6105

6106

6107

6108

6109

6110

6111

6112

6113

6114

6115

6116

6117

6118

6119

6120

6121

6122

6123

6124

6125

6126

6127

6128

6129

6130

6131

6132

6133

6134

6135

6136

6137

6138

6139

6140

6141

6142

6143

6144

6145

6146

6147

6148

6149

6150

6151

6152
Moran Y., Fredman D., Szczesny P., Grynberg M., Technau U. 2012. Recurrent horizontal transfer of bacterial toxin genes to eukaryotes. Mol. Biol. Evol. 29:2223-2230.

Morin-Crini N., Lichtfouse E., Torri G., Crini G. 2019. Applications of chitosan in food, pharmaceuticals, medicine, cosmetics, agriculture, textiles, pulp and paper, biotechnology, and environmental chemistry. Environ. Chem. Lett. 2019 174. 17:16671692.

Morin E., Kohler A., Baker A.R., Foulongne-Oriol M., Lombard V., Nagy L.G., Ohm R.A., Patyshakuliyeva A., Brun A., Aerts A.L., Bailey A.M., Billette C., Coutinho P.M., Deakin G., Doddapaneni H., Floudas D., Grimwood J., Hildén K., Kües U., Labutti K.M., Lapidus A., Lindquist E.A., Lucas S.M., Murat C., Riley R.W., Salamov A.A., Schmutz J., Subramanian V., Wösten H.A.B., Xu J., Eastwood D.C., Foster G.D., Sonnenberg A.S.M., Cullen D., de Vries R.P., Lundell T., Hibbett D.S., Henrissat B., Burton K.S., Kerrigan R.W., Challen M.P., Grigoriev I. V., Martin F. 2012. Genome sequence of the button mushroom Agaricus bisporus reveals mechanisms governing adaptation to a humic-rich ecological niche. Proc. Natl. Acad. Sci. U. S. A. 109:17501-17506.

Morton N., Hammond J.B.W., Dickerson A.G. 1985. The Agaricus bisporus mannitol pathway during sporophore growth. Trans. Br. Mycol. Soc. 85:671-675.

Munir E., Yoon J.J., Tokimatsu T., Hattori T., Shimada M. 2001. A physiological role for oxalic acid biosynthesis in the wood-rotting basidiomycete Fomitopsis palustris. Proc. Natl. Acad. Sci. 98:11126-11130.

Muraguchi H., Abe K., Nakagawa M., Nakamura K., Yanagi S.O., H M., K A., M N., K N., SO Y. 2008a. Identification and characterisation of structural maintenance of chromosome 1 (smc1) mutants of Coprinopsis cinerea. Mol. Genet. Genomics. 280:223-232.

Muraguchi H., Fujita T., Kishibe Y., Konno K., Ueda N., Nakahori K., Yanagi S.O., Kamada T., H M., T F., Y K., K K., N U., K N., SO Y., T K., Muraguchi H., Fujita T., Kishibe Y., Konno K., Ueda N., Nakahori K., Yanagi S.O., Kamada T. 2008b. The exp1 gene essential for pileus expansion and autolysis of the inky cap mushroom Coprinopsis cinerea (Coprinus cinereus) encodes an HMG protein. Fungal Genet. Biol. 45:890-896.

Muraguchi H., Kamada T. 1998. The ich1 gene of the mushroom Coprinus cinereus is essential for pileus formation in fruiting. Development. 125:3133-3141.

Muraguchi H., Kamada T. 2000. A mutation in the eln2 gene encoding a cytochrome P450 of Coprinus cinereus affects mushroom morphogenesis. Fungal Genet. Biol. 29:49-59.

Muraguchi H., Umezawa K., Niikura M., Yoshida M., Kozaki T., Ishii K., Sakai K., Shimizu M., Nakahori K., Sakamoto Y., Choi C., Ngan C.Y., Lindquist E., Lipzen A., Tritt A., Haridas S., Barry K., Grigoriev I. V, Pukkila P.J. 2015. Strand-specific RNA-seq analyses of fruiting body development in Coprinopsis cinerea. PLoS One. 10.

Murata Y., Fujii M., Zolan M.E., Kamada T. 1998. Molecular analysis of pcc1, a gene that leads to A-regulated sexual morphogenesis in Coprinus cinereus. Genetics. 149:17531761.

N L., SC K., I F., RH L., CF R., TT C.-D., BA S., O K., DA T., SM E., I V., JA T., T H., K O., EM H., RK T., JC W., M T., HR S., MA D. 2015. Conformational changes during pore formation by the perforin-related protein pleurotolysin. PLoS Biol. 13.

N T., S Y., A O., H H., Takeshita N., Yamashita S., Ohta A., Horiuchi H. 2006. Aspergillus nidulans class $\mathrm{V}$ and $\mathrm{VI}$ chitin synthases CsmA and CsmB, each with a myosin motorlike domain, perform compensatory functions that are essential for hyphal tip growth. Mol. Microbiol. 59:1380-1394.

Nagy L.G. 2017. Evolution: Complex Multicellular Life with 5,500 Genes. Curr. Biol. 27:R609-R612.

Nagy L.G., Kovács G.M., Krizsán K. 2018. Complex multicellularity in fungi: evolutionary convergence, single origin, or both? Biol. Rev.

Nagy L.G., Ohm R.A., Kovács G.M., Floudas D., Riley R., Gácser A., Sipiczki M., Davis J.M., Doty S.L., de Hoog G.S., Lang B.F., Spatafora J.W., Martin F.M., Grigoriev I. V, Hibbett D.S. 2014. Latent homology and convergent regulatory evolution underlies the repeated emergence of yeasts. 5:4471.

Nakade K., Watanabe H., Sakamoto Y., Sato T. 2011. Gene silencing of the Lentinula 
bioRxiv preprint doi: https://doi.org/10.1101/2021.12.09.471732; this version posted December $10,2021$. The copyright holder for this preprint (which was not certified by peer review) is the author/funder, who has granted bioRxiv a license to display the preprint in perpetuity. It is made available under aCC-BY-NC-ND 4.0 International license.

Running head: fruiting body genomics

6153

6154

6155

6156

6157

6158

6159

6160

6161

6162

6163

6164

6165

6166

6167

6168

6169

6170

6171

6172

6173

6174

6175

6176

6177

6178

6179

6180

6181

6182

6183

6184

6185

6186

6187

6188

6189

6190

6191

6192

6193

6194

6195

6196

6197

6198

6199

6200

6201

6202

6203

6204

6205

6206

6207 edodes lcc1 gene by expression of a homologous inverted repeat sequence. Microbiol. Res. 166:484-493.

Nakazawa T., Ando Y., Hata T., Nakahori K., T N., Y A., T H., K N. 2016. A mutation in the Cc.arp9 gene encoding a putative actin-related protein causes defects in fruiting initiation and asexual development in the agaricomycete Coprinopsis cinerea. Curr. Genet. 62:565-574.

Nakazawa T., Ando Y., Kitaaki K., Nakahori K., Kamada T. 2011. Efficient gene targeting in $\delta \mathrm{Cc} . \mathrm{ku} 70$ or $\mathrm{\delta C}$.lig4 mutants of the agaricomycete coprinopsis cinerea. Fungal Genet. Biol. 48:939-946.

Nakazawa T., Kaneko S., Miyazaki Y., Jojima T., Yamazaki T., Katsukawa S., Shishido K. 2008. Basidiomycete Lentinula edodes CDC5 and a novel interacting protein CIPB bind to a newly isolated target gene in an unusual manner. Fungal Genet. Biol. 45:818-828.

Nakazawa T., Kaneko S., Murata H., Kamada T., Shishido K. 2009. The homologue of Lentinula edodes ctg1, a target for CDC5 and its interacting partner CIPB, from Coprinopsis cinerea is involved in fruitingbody morphogenesis of $\mathrm{C}$. cinerea. Mycoscience. 50:331-342.

Nakazawa T., Morimoto R., Wu H., Kodera R., Sakamoto M., Honda Y. 2019. Dominant effects of gat1 mutations on the ligninolytic activity of the white-rot fungus Pleurotus ostreatus. Fungal Biol. 123:209-217.

Nakazawa T., Tatsuta Y., Fujita T., Nakahori K., Kamada T. 2010. Mutations in the Cc.rmt1 gene encoding a putative protein arginine methyltransferase alter developmental programs in the basidiomycete Coprinopsis cinerea. Curr. Genet. 56:361-367.

Namekawa S.H., Iwabata K., Sugawara H., Hamada F.N., Koshiyama A., Chiku H., Kamada T., Sakaguchi K. 2005. Knockdown of LIM15/DMC1 in the mushroom Coprinus cinereus by double-stranded RNA-mediated gene silencing. Microbiology. 151:36693678.

Nara T., Hamada F., Namekawa S., Sakaguchi K. 2001. Strand Exchange Reaction in Vitro and DNA-Dependent ATPase Activity of Recombinant LIM15/DMC1 and RAD51 Proteins from Coprinus cinereus. Biochem. Biophys. Res. Commun. 285:92-97.

Nehls U., Dietz S. 2014. Fungal aquaporins: cellular functions and ecophysiological perspectives. Appl. Microbiol. Biotechnol. 2014 9821. 98:8835-8851.

Nguyen D.V., Roret T., Fernandez-Gonzalez A., Kohler A., Morel-Rouhier M., Gelhaye E., Sormani R. 2020. Target of Rapamycin pathway in the white-rot fungus Phanerochaete chrysosporium. PLoS One. 15.

Nguyen T.A., Cissé O.H., Yun Wong J., Zheng P., Hewitt D., Nowrousian M., Stajich J.E., Jedd G. 2017. Innovation and constraint leading to complex multicellularity in the Ascomycota. 8:14444.

Nickerson K.W., Atkin A.L., Hornby J.M., KW N., AL A., JM H. 2006. Quorum sensing in dimorphic fungi: farnesol and beyond. Appl. Environ. Microbiol. 72:3805-3813.

Niu X., Liu C.-C., Xiong Y.-J., Yang M.-M., Ma F., Liu Z.-H., Yuan S. 2016. The Modes of Action of Chilll, a Chitinase from Mushroom Coprinopsis cinerea, Shift with Changes in the Length of GlcNAc Oligomers. J. Agric. Food Chem. 64:6958-6968.

Niu X., Liu Z., Zhou Y., Wang J., Zhang W., Yuan S. 2015. Stipe cell wall architecture varies with the stipe elongation of the mushroom Coprinopsis cinerea. Fungal Biol. 119:946956.

Noble R., Dobrovin-Pennington A., Hobbs P.J., Pederby J., Rodger J.P.A. 2009. Volatile C8 compounds and pseudomonads influence primordium formation of Agaricus bisporus. Mycologia. 101:583-591.

Nofiani R., de Mattos-Shipley K., Lebe K.E., Han L.-C., Iqbal Z., Bailey A.M., Willis C.L., Simpson T.J., Cox R.J. 2018. Strobilurin biosynthesis in Basidiomycete fungi. Nat. Commun. 2018 91. 9:1-11.

Novak M., Krpan T., Panevska A., Shewell L.K., Day C.J., Jennings M.P., Guella G., Sepčić K. 2020. Binding specificity of ostreolysin A6 towards Sf9 insect cell lipids. Biochim. Biophys. Acta - Biomembr. 1862:183307.

Nowrousian M., Cebula P. 2005. The gene for a lectin-like protein is transcriptionally 
bioRxiv preprint doi: https://doi.org/10.1101/2021.12.09.471732; this version posted December $10,2021$. The copyright holder for this preprint (which was not certified by peer review) is the author/funder, who has granted bioRxiv a license to display the preprint in perpetuity. It is made available under aCC-BY-NC-ND 4.0 International license.

Running head: fruiting body genomics

6208

6209

6210

6211

6212

6213

6214

6215

6216

6217

6218

6219

6220

6221

6222

6223

6224

6225

6226

6227

6228

6229

6230

6231

6232

6233

6234

6235

6236

6237

6238

6239

6240

6241

6242

6243

6244

6245

6246

6247

6248

6249

6250

6251

6252

6253

6254

6255

6256

6257

6258

6259

6260

6261

6262 activated during sexual development, but is not essential for fruiting body formation in the filamentous fungus Sordaria macrospora. BMC Microbiol. 5:64.

NY S., JM L., GJ V., HH O., JD P., ME Z. 1997. Isolation and characterization of rad51 orthologs from Coprinus cinereus and Lycopersicon esculentum, and phylogenetic analysis of eukaryotic recA homologs. Curr. Genet. 31:144-157.

O'Connor E., Doyle S., Amini A., Grogan H., Fitzpatrick D.A. 2021. Transmission of mushroom virus $X$ and the impact of virus infection on the transcriptomes and proteomes of different strains of Agaricus bisporus. Fungal Biol. 125:704-717.

O'Connor E., McGowan J., McCarthy C.G.P., Amini A., Grogan H., Fitzpatrick D.A. 2019. Whole genome sequence of the commercially relevant mushroom strain Agaricus bisporus var. bisporus ARP23. G3 Genes, Genomes, Genet. 9:3057-3066.

Obermaier S., Müller M. 2020. Ibotenic Acid Biosynthesis in the Fly Agaric Is Initiated by Glutamate Hydroxylation. Angew. Chemie Int. Ed. 59:12432-12435.

Ohga S., Cho N.-S., Thurston C.F., Wood D.A. 2000. Transcriptional regulation of laccase and cellulase in relation to fruit body formation in the mycelium of Lentinula edodes on a sawdust-based substrate. Mycoscience. 41:149-153.

Ohm R.A., Aerts D., Wösten H.A.B., Lugones L.G. 2013. The blue light receptor complex WC-1/2 of Schizophyllum communeis involved in mushroom formation and protection against phototoxicity. Environ. Microbiol. 15:943-955.

Ohm R.A., de Jong J.F., de Bekker C., Wösten H.A.B., Lugones L.G. 2011. Transcription factor genes of Schizophyllum commune involved in regulation of mushroom formation. Mol. Microbiol. 81:1433-1445.

Ohm R.A., Jong J.F. de, Lugones L.G., Aerts A., Kothe E., Stajich J.E., Vries R.P. de, Record E., Levasseur A., Baker S.E., Bartholomew K.A., Coutinho P.M., Erdmann S., Fowler T.J., Gathman A.C., Lombard V., Henrissat B., Knabe N., Kües U., Lilly W.W., Lindquist E., Lucas S., Magnuson J.K., Piumi F., Raudaskoski M., Salamov A., Schmutz J., Schwarze F.W.M.R., vanKuyk P.A., Horton J.S., Grigoriev I. V, Wösten H.A.B., de Jong J.F., Lugones L.G., Aerts A., Kothe E., Stajich J.E., de Vries R.P., Record E., Levasseur A., Baker S.E., Bartholomew K.A., Coutinho P.M., Erdmann S., Fowler T.J., Gathman A.C., Lombard V., Henrissat B., Knabe N., Kües U., Lilly W.W., Lindquist E., Lucas S., Magnuson J.K., Piumi F., Raudaskoski M., Salamov A., Schmutz J., Schwarze F.W.M.R., vanKuyk P.A., Horton J.S., Grigoriev I. V, Wösten H.A.B. 2010. Genome sequence of the model mushroom Schizophyllum commune. Nat. Biotechnol. 2010 289. 28:957-963.

Ohm R.A., Riley R., Salamov A., Min B., Choi I.G., Grigoriev I. V. 2014. Genomics of wooddegrading fungi. Fungal Genet. Biol. 72:82-90.

Ojeda-López M., Chen W., Eagle C.E., Gutiérrez G., Jia W.L., Swilaiman S.S., Huang Z., Park H.S., Yu J.H., Cánovas D., Dyer P.S. 2018. Evolution of asexual and sexual reproduction in the aspergilli. Stud. Mycol.

de Oliveira E.S., Junges Â., Sbaraini N., Andreis F.C., Thompson C.E., Staats C.C., Schrank A. 2018. Molecular evolution and transcriptional profile of GH3 and GH20 $\$ \beta \$-\mathrm{N}-$ acetylglucosaminidases in the entomopathogenic fungus Metarhizium anisopliae. Genet. Mol. Biol. 41:843-857.

Orban A., Weber A., Herzog R., Hennicke F., Rühl M. 2021. Transcriptome of different fruiting stages in the cultivated mushroom Cyclocybe aegerita suggests a complex regulation of fruiting and reveals enzymes putatively involved in fungal oxylipin biosynthesis. BMC Genomics $2021221.22: 1-23$.

Orlean P. 2012. Architecture and Biosynthesis of the Saccharomyces cerevisiae Cell Wall. Genetics. 192:775.

P B. 2006. Fungal laccases - occurrence and properties. FEMS Microbiol. Rev. 30:215-242.

P S. 2008. Chemical defence strategies of higher fungi. Chemistry. 14:9100-9110.

PA H., AC V., JF A. 1978. The insensitivity of mushroom nuclear RNA polymerase activity to inhibition by amatoxins. Arch. Microbiol. 118:317-319.

Palmer G.E., Horton J.S. 2006. Mushrooms by magic: making connections between signal transduction and fruiting body development in the basidiomycete fungus Schizophyllum 
bioRxiv preprint doi: https://doi.org/10.1101/2021.12.09.471732; this version posted December $10,2021$. The copyright holder for this preprint (which was not certified by peer review) is the author/funder, who has granted bioRxiv a license to display the preprint in perpetuity. It is made available under aCC-BY-NC-ND 4.0 International license.

Running head: fruiting body genomics

6263

6264

6265

6266

6267

6268

6269

6270

6271

6272

6273

6274

6275

6276

6277

6278

6279

6280

6281

6282

6283

6284

6285

6286

6287

6288

6289

6290

6291

6292

6293

6294

6295

6296

6297

6298

6299

6300

6301

6302

6303

6304

6305

6306

6307

6308

6309

6310

6311

6312

6313

6314

6315

6316

6317 commune. FEMS Microbiol. Lett. 262:1-8.

Palmieri F., Estoppey A., House G.L., Lohberger A., Bindschedler S., Chain P.S.G., Junier P. 2019. Oxalic acid, a molecule at the crossroads of bacterial-fungal interactions. Adv. Appl. Microbiol.:1-29.

Panevska A., Skočaj M., Modic Š., Razinger J., Sepčić K. 2020. Aegerolysins from the fungal genus Pleurotus - Bioinsecticidal proteins with multiple potential applications. J. Invertebr. Pathol.:107474.

Park M., Kim M., Kim S., Ha B., Ro H.-S.S. 2015. Differential expression of laccase genes in Pleurotus ostreatus and biochemical characterization of laccase isozymes produced in Pichia pastoris. Mycobiology. 43:280.

Park S.G., il Yoo S., Ryu D.S., Lee H., Ahn Y.J., Ryu H., Ko J., Hong C.P. 2017. Long-read transcriptome data for improved gene prediction in Lentinula edodes. Data Br. 15:454458.

Park Y.-J., Baek J.H., Lee S., Kim C., Rhee H., Kim H., Seo J.-S., Park H.-R., Yoon D.-E., Nam J.-Y., Kim H.-I., Kim J.-G., Yoon H., Kang H.-W., Cho J.-Y., Song E.-S., Sung G.H., Yoo Y.-B., Lee C.-S., Lee B.-M., Kong W.-S. 2014. Whole Genome and Global Gene Expression Analyses of the Model Mushroom Flammulina velutipes Reveal a High Capacity for Lignocellulose Degradation. PLoS One. 9:e93560.

Park Y.-J., Lee C.-S., Kong W.-S. 2019. Genomic Insights into the Fungal Lignocellulolytic Machinery of Flammulina rossica. Microorganisms. 7.

Patel P.K., Free S.J. 2019. The Genetics and Biochemistry of Cell Wall Structure and Synthesis in Neurospora crassa, a Model Filamentous Fungus. Front. Microbiol. $0: 2294$.

Patyshakuliyeva A., Jurak E., Kohler A., Baker A., Battaglia E., De Bruijn W., Burton K.S., Challen M.P., Coutinho P.M., Eastwood D.C., Gruben B.S., Mäkelä M.R., Martin F., Nadal M., van den Brink J., Wiebenga A., Zhou M., Henrissat B., Kabel M., Gruppen H., De Vries R.P. 2013. Carbohydrate utilization and metabolism is highly differentiated in Agaricus bisporus. BMC Genomics. 14.

Van Peer A.F., Wang F., Van Driel K.G.A., De Jong J.F., Van Donselaar E.G., Müller W.H., Boekhout T., Lugones L.G., Wösten H.A.B., Peer A.F. Van, Wang F., Driel K.G.A. Van, Jong J.F. De, Donselaar E.G. Van, Müller W.H., Boekhout T., Lugones L.G., Wösten H.A.B. 2010. The septal pore cap is an organelle that functions in vegetative growth and mushroom formation of the wood-rot fungus Schizophyllum commune. Environ. Microbiol. 12:833-844.

Peisker K., Chiabudini M., Rospert S. 2010. The ribosome-bound Hsp70 homolog Ssb of Saccharomyces cerevisiae. Biochim. Biophys. Acta - Mol. Cell Res. 1803:662-672.

Pelkmans J.F. 2016. Environmental signaling and regulation of mushroom formation. .

Pelkmans J.F., Patil M.B., Gehrmann T., Reinders M.J.T., Wösten H.A.B., Lugones L.G. 2017a. Transcription factors of schizophyllum commune involved in mushroom formation and modulation of vegetative growth. Sci. Rep. 7:1-11.

Pelkmans J.F., Patil M.B., Gehrmann T., Reinders M.J.T.T., Wösten H.A.B.B., Lugones L.G. 2017b. Transcription factors of schizophyllum commune involved in mushroom formation and modulation of vegetative growth. Sci. Rep. 7:1-11.

Pelkmans J.F., Vos A.M., Scholtmeijer K., Hendrix E., Baars J.J.P., Gehrmann T., Reinders M.J.T., Lugones L.G., Wösten H.A.B. 2016. The transcriptional regulator c2h2 accelerates mushroom formation in Agaricus bisporus. Appl. Microbiol. Biotechnol. 100:7151-7159.

Pellegrin C., Morin E., Martin F.M., Veneault-Fourrey C. 2015. Comparative Analysis of Secretomes from Ectomycorrhizal Fungi with an Emphasis on Small-Secreted Proteins. Front. Microbiol. 6:1278.

Peñalva M.A., Tilburn J., Bignell E., Arst H.N. 2008. Ambient pH gene regulation in fungi: making connections. Trends Microbiol. 16:291-300.

Peraro M.D., van der Goot F.G. 2015. Pore-forming toxins: ancient, but never really out of fashion. Nat. Rev. Microbiol. 2015 142. 14:77-92.

Pereira M. de F., Campos A.N. da R., Anastacio T.C., Morin E., Brommonschenkel S.H., 
bioRxiv preprint doi: https://doi.org/10.1101/2021.12.09.471732; this version posted December $10,2021$. The copyright holder for this preprint (which was not certified by peer review) is the author/funder, who has granted bioRxiv a license to display the preprint in perpetuity. It is made available under aCC-BY-NC-ND 4.0 International license.

Running head: fruiting body genomics

6318

6319

6320

6321

6322

6323

6324

6325

6326

6327

6328

6329

6330

6331

6332

6333

6334

6335

6336

6337

6338

6339

6340

6341

6342

6343

6344

6345

6346

6347

6348

6349

6350

6351

6352

6353

6354

6355

6356

6357

6358

6359

6360

6361

6362

6363

6364

6365

6366

6367

6368

6369

6370

6371

6372

Martin F., Kohler A., Costa M.D. 2017. The transcriptional landscape of basidiosporogenesis in mature Pisolithus microcarpus basidiocarp. BMC Genomics 2017 181. 18:1-16.

Plagemann I., Zelena K., Arendt P., Ringel P.D., Krings U., Berger R.G. 2013. LOXPsa1, the first recombinant lipoxygenase from a basidiomycete fungus. J. Mol. Catal. B Enzym. 87:99-104.

Plaza D.F., Lin C.-W.W., van der Velden N.S.J., Aebi M., Kunzler M., Künzler M., Kunzler M., Künzler M. 2014a. Comparative transcriptomics of the model mushroom Coprinopsis cinerea reveals tissue-specific armories and a conserved circuitry for sexual development. BMC Genomics. 15:492.

Plaza D.F., Lin C.W., van der Velden N.S., Aebi M., Kunzler M. 2014b. Comparative transcriptomics of the model mushroom Coprinopsis cinerea reveals tissue-specific armories and a conserved circuitry for sexual development. BMC Genomics. 15:492.

Pluskal T., Ueno M., Yanagida M. 2014. Genetic and Metabolomic Dissection of the Ergothioneine and Selenoneine Biosynthetic Pathway in the Fission Yeast, S. pombe, and Construction of an Overproduction System. PLoS One. 9:97774.

Pócsi I., Prade R.A., Penninckx M.J. 2004. Glutathione, Altruistic Metabolite in Fungi. Adv. Microb. Physiol. 49:1-76.

Poeta M. Del, Nimrichter L., Rodrigues M.L., Luberto C. 2014. Synthesis and Biological Properties of Fungal Glucosylceramide. PLoS Pathog. 10.

Pöggeler S., Nowrousian M., Kück U. 2006a. 16 Fruiting-Body Development in Ascomycetes. .

Pöggeler S., Nowrousian M., Kück U. 2006b. Fruiting-Body Development in Ascomycetes. In: Kües U., Fischer R., editors. Growth, Differentiation and Sexuality. Berlin, Heidelberg: Springer Berlin Heidelberg. p. 325-355.

Polonio Á., Fernández-Ortuño D., de Vicente A., Pérez-García A. 2021. A haustorialexpressed lytic polysaccharide monooxygenase from the cucurbit powdery mildew pathogen Podosphaera xanthii contributes to the suppression of chitin-triggered immunity. Mol. Plant Pathol. 22:580-601.

Prasanna A.N., Gerber D., Kijpornyongpan T., Aime M.C., Doyle V.P., Nagy L.G. 2020. Model choice, missing data, and taxon sampling impact phylogenomic inference of deep basidiomycota relationships. Syst. Biol.

Primig M., Williams R.M., Winzeler E.A., Tevzadze G.G., Conway A.R., Hwang S.Y., Davis R.W., Esposito R.E. 2000. The core meiotic transcriptome in budding yeasts. Nat. Genet. 2000 264. 26:415-423.

Pulman J.A., Childs K.L., Sgambelluri R.M., Walton J.D. 2016. Expansion and diversification of the MSDIN family of cyclic peptide genes in the poisonous agarics Amanita phalloides and A. bisporigera. BMC Genomics 2016 171. 17:1-14.

Q H., X H., I M., L G., R H., L F., J Y., Y T., B C., B X. 2018. Identification and Expression Patterns of fvexpl1, an Expansin-Like Protein-Encoding Gene, Suggest an Auxiliary Role in the Stipe Morphogenesis of Flammulina velutipes. J. Microbiol. Biotechnol. 28:622-629.

Q Z., L X., S Y., Q Z., X W., L W., Z H., Y Y. 2020. NGT1 Is Essential for NAcetylglucosamine-Mediated Filamentous Growth Inhibition and HXK1 Functions as a Positive Regulator of Filamentous Growth in Candida tropicalis. Int. J. Mol. Sci. 21:118.

Qi Y., Chen H., Zhang M., Wen Q., Qiu L., Shen J. 2019. Identification and expression analysis of Pofst3 suggests a role during Pleurotus ostreatus primordia formation. Fungal Biol. 123:200-208.

Qi Y., Sun X., Ma L., Wen Q., Qiu L., Shen J. 2020. Identification of two Pleurotus ostreatus blue light receptor genes (PoWC-1 and PoWC-2) and in vivo confirmation of complex PoWC-12 formation through yeast two hybrid system. Fungal Biol. 124:8-14.

Quiroz-Castañeda R.E., Martínez-Anaya C., Cuervo-Soto L.I., Segovia L., Folch-Mallol J.L. 2011. Loosenin, a novel protein with cellulose-disrupting activity from Bjerkandera adusta. Microb. Cell Factories 2011 101. 10:1-9. 
bioRxiv preprint doi: https://doi.org/10.1101/2021.12.09.471732; this version posted December $10,2021$. The copyright holder for this preprint (which was not certified by peer review) is the author/funder, who has granted bioRxiv a license to display the preprint in perpetuity. It is made available under aCC-BY-NC-ND 4.0 International license.

Running head: fruiting body genomics

6373 R D., J F., S D., M G., D H. 2020. S-Adenosyl-I-Methionine Salvage Impacts Psilocybin

6374

6375

6376

6377

6378

6379

6380

6381

6382

6383

6384

6385

6386

6387

6388

6389

6390

6391

6392

6393

6394

6395

6396

6397

6398

6399

6400

6401

6402

6403

6404

6405

6406

6407

6408

6409

6410

6411

6412

6413

6414

6415

6416

6417

6418

6419

6420

6421

6422

6423

6424

6425

6426

6427 Formation in "Magic" Mushrooms. Chembiochem. 21:1364-1371.

R G., S W., S A., J Y., DA W. 2003. BTB/POZ domain proteins are putative substrate adaptors for cullin 3 ubiquitin ligases. Mol. Cell. 12:783-790.

R U., J N., K S., J S., M H. 2004. Novel haloperoxidase from the agaric basidiomycete Agrocybe aegerita oxidizes aryl alcohols and aldehydes. Appl. Environ. Microbiol. 70:4575-4581.

Ragucci S., Landi N., Russo R., Valletta M., Pedone P.V., Chambery A., Maro A. Di. 2021. Ageritin from Pioppino Mushroom: The Prototype of Ribotoxin-Like Proteins, a Novel Family of Specific Ribonucleases in Edible Mushrooms. Toxins 2021, Vol. 13, Page 263. 13:263.

Rahmad N., Al-Obaidi J.R., Rashid N.M., Zean N., Yusoff M.H.Y., Shaharuddin N., Jamil N.A., Saleh N. 2014. Comparative proteomic analysis of different developmental stages of the edible mushroom Termitomyces heimii. Biol. Res. 47:30.

Rahman F., Hassan M., Hanano A., Fitzpatrick D.A., McCarthy C.G.P., Murphy D.J. 2018. Evolutionary, structural and functional analysis of the caleosin/peroxygenase gene family in the Fungi. BMC Genomics 2018 191. 19:1-24.

Raudaskoski M., Kothe E. 2010. Basidiomycete mating type genes and pheromone signaling. Eukaryot Cell. 9:847-859.

Rayle D.L., Cleland R.E. 1992. The Acid Growth Theory of auxin-induced cell elongation is alive and well. Plant Physiol. 99:1271-1274.

Reboul C.F., Whisstock J.C., Dunstone M.A. 2016. Giant MACPF/CDC pore forming toxins: A class of their own. Biochim. Biophys. Acta - Biomembr. 1858:475-486.

Reinhold R., Krüger V., Meinecke M., Schulz C., Schmidt B., Grunau S.D., Guiard B., Wiedemann N., Laan M. van der, Wagner R., Rehling P., Dudek J. 2012. The ChannelForming Sym1 Protein Is Transported by the TIM23 Complex in a PresequenceIndependent Manner. Mol. Cell. Biol. 32:5009.

Rennemeier C., Schwab M., Lermann U., Albert C., Kämmerer U., Frambach T., Morschhäuser J., Dietl J., Staib P. 2011. Seminal plasma protects human spermatozoa and pathogenic yeasts from capture by dendritic cells. Hum. Reprod. 26:987-999.

Reschka E.J., Nordzieke S., Valerius O., Braus G.H., Pöggeler S., J. Reschka E., Nordzieke S., Valerius O., Braus G.H., Pöggeler S. 2018. A novel STRIPAK complex component mediates hyphal fusion and fruiting-body development in filamentous fungi. Mol. Microbiol. 110:513-532.

Revuelta M. V, van Kan J.A.L., Kay J., ten Have A. 2014. Extensive Expansion of A1 Family Aspartic Proteinases in Fungi Revealed by Evolutionary Analyses of 107 Complete Eukaryotic Proteomes. Genome Biol. Evol. 6:1480-1494.

Riley R., Salamov A.A., Brown D.W., Nagy L.G., Floudas D., Held B.W., Levasseur A., Lombard V., Morin E., Otillar R., Lindquist E.A., Sun H., LaButti K.M., Schmutz J., Jabbour D., Luo H., Baker S.E., Pisabarro A.G., Walton J.D., Blanchette R.A., Henrissat B., Martin F., Cullen D., Hibbett D.S., Grigoriev I. V. 2014. Extensive sampling of basidiomycete genomes demonstrates inadequacy of the white-rot/brownrot paradigm for wood decay fungi. Proc. Natl. Acad. Sci. U. S. A. 111:9923-9928.

Riquelme M., Aguirre J., Bartnicki-García S., Braus G.H., Feldbrügge M., Fleig U., Hansberg W., Herrera-Estrella A., Kämper J., Kück U., Mouriño-Pérez R.R., Takeshita N., Fischer R. 2018. Fungal Morphogenesis, from the Polarized Growth of Hyphae to Complex Reproduction and Infection Structures. Microbiol. Mol. Biol. Rev. 82:e00068-17.

Riquelme M., Challen M.P., Casselton L.A., Brown A.J. 2005. The origin of multiple B mating specificities in Coprinus cinereus. Genetics. 170:1105-1119.

Robinson M.D., McCarthy D.J., Smyth G.K. 2010. edgeR: a Bioconductor package for differential expression analysis of digital gene expression data. Bioinformatics. 26:139140.

Rodenburg S.Y.A., Terhem R.B., Veloso J., Stassen J.H.M., Van Kan J.A.L. 2018. Functional analysis of mating type genes and transcriptome analysis during fruiting body development of botrytis cinerea. MBio. 9. 
bioRxiv preprint doi: https://doi.org/10.1101/2021.12.09.471732; this version posted December $10,2021$. The copyright holder for this preprint (which was not certified by peer review) is the author/funder, who has granted bioRxiv a license to display the preprint in perpetuity. It is made available under aCC-BY-NC-ND 4.0 International license.

Running head: fruiting body genomics

6428 van Roermund C.W.T., Waterham H.R., ljlst L., Wanders R.J.A. 2003. Fatty acid metabolism

6429

6430

6431

6432

6433

6434

6435

6436

6437

6438

6439

6440

6441

6442

6443

6444

6445

6446

6447

6448

6449

6450

6451

6452

6453

6454

6455

6456

6457

6458

6459

6460

6461

6462

6463

6464

6465

6466

6467

6468

6469

6470

6471

6472

6473

6474

6475

6476

6477

6478

6479

6480

6481

6482 in Saccharomyces cerevisiae. Cell. Mol. Life Sci. C. 2003 609. 60:1838-1851.

Román E., Arana D.M., Nombela C., Alonso-Monge R., Pla J. 2007. MAP kinase pathways as regulators of fungal virulence. Trends Microbiol. 15:181-190.

Romano J.D., Michaelis S. 2001. Topological and mutational analysis of Saccharomyces cerevisiae Ste14p, founding member of the isoprenylcysteine carboxyl methyltransferase family. Mol. Biol. Cell. 12:1957-1971.

Roper M., Seminara A. 2019. Mycofluidics: The Fluid Mechanics of Fungal Adaptation. https://doi.org/10.1146/annurev-fluid-122316-045308. 51:511-538.

Royse D.J., Baars J., Tan Q. 2017. Current Overview of Mushroom Production in the World. Edible and Medicinal Mushrooms. .

Ruess L., Lussenhop J. 2005. Trophic Interactions of Fungi and Animals. :581-598.

S B.-M., K S., R S., M C., JM M., M H., M S., A V., M K. 2017. Dimerization of the fungal defense lectin CCL2 is essential for its toxicity against nematodes. Glycobiology. 27:486-500.

S B.-M., S S., M A., M K. 2012a. Biotin-binding proteins in the defense of mushrooms against predators and parasites. Appl. Environ. Microbiol. 78:8485-8487.

S B., J P., I V., K R., F P., T T., P M., A S., K S. 2007a. Ostreolysin enhances fruiting initiation in the oyster mushroom (Pleurotus ostreatus). Mycol. Res. 111:1431-1436.

S C., FE N., A V., S T.-M., RM R.-V. 2012b. Two distinct RNA-dependent RNA polymerases are required for initiation and amplification of RNA silencing in the basal fungus Mucor circinelloides. Mol. Microbiol. 83:379-394.

S C., J D., M E., J M., D B., PO B., I H. 1998a. The transcriptional program of sporulation in budding yeast. Science. 282:699-705.

S H., J S., H M., U B., J S. 2001. The crystallographic structure of the mannitol 2dehydrogenase NADP+ binary complex from Agaricus bisporus. J. Biol. Chem. 276:27555-27561.

S K., K Y., K H., H M., T S., T K., K S., Kajiwara S., Yamaoka K., Hori K., Miyazawa H., Saito T., Kanno T., Shishido K. 1992. Isolation and sequence of a developmentally regulated putative novel gene, priA, from the basidiomycete Lentinus edodes. Gene. 114:173-178.

S K., N J., B M., S B., RA P., GH B. 2006a. The Aspergillus nidulans F-box protein GrrA links SCF activity to meiosis. Mol. Microbiol. 61:76-88.

S K., T S., S I., Y H., T O. 1993. A mutant allele skt5 affecting protoplast regeneration and killer toxin resistance has double mutations in its wild-type structural gene in Saccharomyces cerevisiae. Biosci. Biotechnol. Biochem. 57:1391-1393.

S N., M L., H B. 1998b. Isolation of Candida glabrata homologs of the Saccharomyces cerevisiae KRE9 and KNH1 genes and their involvement in cell wall beta-1,6-glucan synthesis. J. Bacteriol. 180:5020-5029.

S O., H M., T T., Y Y., F H., K G., T N., K A. 2006b. Novel hydrophobic surface binding protein, HsbA, produced by Aspergillus oryzae. Appl. Environ. Microbiol. 72:24072413.

S O., Y T., A W., M I. 2019. Carbohydrate-binding property of a cell wall integrity and stress response component (WSC) domain of an alcohol oxidase from the rice blast pathogen Pyricularia oryzae. Enzyme Microb. Technol. 125:13-20.

S P., F D., S B., C J., M C. 2004. Ady2p is essential for the acetate permease activity in the yeast Saccharomyces cerevisiae. Yeast. 21:201-210.

S P., U K. 2004. A WD40 repeat protein regulates fungal cell differentiation and can be replaced functionally by the mammalian homologue striatin. Eukaryot. Cell. 3:232-240.

S Y., S S., Q Y., X F., Z J., X Y. 2014. Biochemical characterization of the first fungal glycoside hydrolyase family $3 \$ \beta \$-\mathrm{N}$-acetylglucosaminidase from Rhizomucor miehei. J. Agric. Food Chem. 62:5181-5190.

S Z., D G., GG P., JL C., FA L. 1994. The BTB domain, found primarily in zinc finger proteins, defines an evolutionarily conserved family that includes several developmentally regulated genes in Drosophila. Proc. Natl. Acad. Sci. U. S. A. 
bioRxiv preprint doi: https://doi.org/10.1101/2021.12.09.471732; this version posted December $10,2021$. The copyright holder for this preprint (which was not certified by peer review) is the author/funder, who has granted bioRxiv a license to display the preprint in perpetuity. It is made available under aCC-BY-NC-ND 4.0 International license.

Running head: fruiting body genomics

6483

6484

6485

6486

6487

6488

6489

6490

6491

6492

6493

6494

6495

6496

6497

6498

6499

6500

6501

6502

6503

6504

6505

6506

6507

6508

6509

6510

6511

6512

6513

6514

6515

6516

6517

6518

6519

6520

6521

6522

6523

6524

6525

6526

6527

6528

6529

6530

6531

6532

6533

6534

6535

6536

6537
91:10717-10721.

S Z., E S., K I., S S. 2007b. Identification of a novel bifunctional delta12/delta15 fatty acid desaturase from a basidiomycete, Coprinus cinereus TD\#822-2. FEBS Lett. 581:315319.

S Ž., J P., J K., J S. 2015. \$ß\$-Trefoil structure enables interactions between lectins and protease inhibitors that regulate their biological functions. J. Biochem. 158:83-90.

Sabotič J., Renko M., Kos J. 2019. \$及\$-Trefoil Protease Inhibitors Unique to Higher Fungi. Acta Chim. Slov. 66:28-36.

Sakamoto Y. 2018. Influences of environmental factors on fruiting body induction, development and maturation in mushroom-forming fungi. Fungal Biol. Rev.

Sakamoto Y., Minato K., Nagai M., Mizuno M., Sato T. 2005. Characterization of the Lentinula edodes exg2 gene encoding a lentinan-degrading exo-\$\$\$-1,3-glucanase. Curr. Genet. 2005 483. 48:195-203.

Sakamoto Y., Nakade K., Sato S., Yoshida K., Miyazaki K., Natsume S., Konno N. 2017a. Lentinula edodes Genome Survey and Postharvest Transcriptome Analysis. Appl. Environ. Microbiol. 83.

Sakamoto Y., Nakade K., Sato S., Yoshida K., Miyazaki K., Natsume S., Konno N. 2017b. Lentinula edodes genome survey and postharvest transcriptome analysis. Appl. Environ. Microbiol. 83.

Sakamoto Y., Nakade K., Sato T. 2009. Characterization of the post-harvest changes in gene transcription in the gill of the Lentinula edodes fruiting body. Curr. Genet. 55:409423.

Sakamoto Y., Nakade K., Yoshida K., Natsume S., Miyazaki K., Sato S., van Peer A.F., Konno N. 2015. Grouping of multicopper oxidases in Lentinula edodes by sequence similarities and expression patterns. AMB Express. 5.

Sakamoto Y., Sato S., Ito M., Ando Y., Nakahori K., Muraguchi H. 2018. Blue light exposure and nutrient conditions influence the expression of genes involved in simultaneous hyphal knot formation in Coprinopsis cinerea. Microbiol. Res. 217:81-90.

Sakamoto Y., Watanabe H., Nagai M., Nakade K., Takahashi M., Sato T. 2006. Lentinula edodes tlg1 encodes a thaumatin-like protein that is involved in lentinan degradation and fruiting body senescence. Plant Physiol. 141:793-801.

Sammer D., Krause K., Gube M., Wagner K., Kothe E. 2016. Hydrophobins in the Life Cycle of the Ectomycorrhizal Basidiomycete Tricholoma vaccinum. PLoS One. 11:e0167773.

Sampedro J., Cosgrove D.J. 2005. The expansin superfamily. Genome Biol. 2005 612. 6:111.

Sánchez-Arreguin J.A., Cabrera-Ponce J.L., León-Ramírez C.G., Camargo-Escalante M.O., Ruiz-Herrera J. 2020. Analysis of the photoreceptors involved in the light-depending basidiocarp formation in Ustilago maydis. Arch. Microbiol. 202:93-103.

Sánchez-García M., Ryberg M., Khan F.K., Varga T., Nagy L.G., Hibbett D.S. 2020. Fruiting body form, not nutritional mode, is the major driver of diversification in mushroomforming fungi. Proc. Natl. Acad. Sci. U. S. A. 117:32528-32534.

Sánchez-Pulido L., Martín-Belmonte F., Valencia A., Alonso M.A. 2002. MARVEL: a conserved domain involved in membrane apposition events. Trends Biochem. Sci. 27:599-601.

Sánchez-Vallet A., Mesters J.R., Thomma B.P.H.J. 2015. The battle for chitin recognition in plant-microbe interactions. FEMS Microbiol. Rev. 39:171-183.

Sano H., Kaneko S., Sakamoto Y., Sato T., Shishido K. 2009. The basidiomycetous mushroom Lentinula edodes white collar-2 homolog PHRB, a partner of putative bluelight photoreceptor PHRA, binds to a specific site in the promoter region of the $L$. edodes tyrosinase gene. Fungal Genet. Biol.

Sato I., Shimizu M., Hoshino T., Takaya N. 2009. The Glutathione System of Aspergillus nidulans Involves a Fungus-specific Glutathione S-Transferase. J. Biol. Chem. 284:8042.

Sato M., Kurahashi A., Nishibori K., Fujimori F. 2015. Development of a transformation system for the edible mushroom Grifola frondosa: Demonstrating heterologous gene 
bioRxiv preprint doi: https://doi.org/10.1101/2021.12.09.471732; this version posted December $10,2021$. The copyright holder for this preprint (which was not certified by peer review) is the author/funder, who has granted bioRxiv a license to display the preprint in perpetuity. It is made available under aCC-BY-NC-ND 4.0 International license.

Running head: fruiting body genomics

6538

6539

6540

6541

6542

6543

6544

6545

6546

6547

6548

6549

6550

6551

6552

6553

6554

6555

6556

6557

6558

6559

6560

6561

6562

6563

6564

6565

6566

6567

6568

6569

6570

6571

6572

6573

6574

6575

6576

6577

6578

6579

6580

6581

6582

6583

6584

6585

6586

6587

6588

6589

6590

6591

6592 expression and RNAi-mediated gene silencing. Mycoscience. 56:364-372.

SB I., H Q., M A., T W., Y O. 1999. Prenylation of Rho1p is required for activation of yeast 1, 3-beta-glucan synthase. J. Biol. Chem. 274:38119-38124.

Schneiter R., Pietro A. Di. 2013. The CAP protein superfamily: function in sterol export and fungal virulence. Biomol. Concepts. 4:519-525.

Schubert D., Raudaskoski M., Knabe N., Kothe E. 2006. Ras GTPase-activating protein Gap1 of the homobasidiomycete Schizophyllum commune regulates hyphal growth orientation and sexual development. Eukaryot. Cell. 5:683-695.

Schubert M., Bleuler-Martinez S., Butschi A., Wälti M.A., Egloff P., Stutz K., Yan S., Wilson I.B.H., Hengartner M.O., Aebi M., Allain F.H.T., Künzler M. 2012. Plasticity of the $\$ \beta \$-$ trefoil protein fold in the recognition and control of invertebrate predators and parasites by a fungal defence system. PLoS Pathog. 8.

Schulz T.A., Prinz W.A. 2007. Sterol Transport In Yeast and the Oxysterol Binding Protein Homologue (OSH) Family. Biochim. Biophys. Acta. 1771:769.

Schwalb M.N. 1974. Effect of adenosine 3',5'-cyclic monophosphate on the morphogenesis of fruit bodies of Schizophyllum commune. Arch. Microbiol. 96:17-20.

Sebastian J., Rouissi T., Brar S.K. 2020. Fungal chitosan: prospects and challenges. Handb. Chitin Chitosan.:419-452.

Seibold P.S., Lenz C., Gressler M., Hoffmeister D. 2020. The Laetiporus polyketide synthase LpaA produces a series of antifungal polyenes. J. Antibiot. 2020 7310. 73:711-720.

Seitz L.C., Tang K., Cummings W.J., Zolan M.E. 1996. The Rad9 Gene of Coprinus Cinereus Encodes a Proline-Rich Protein Required for Meiotic Chromosome Condensation and Synapsis. Genetics. 142:1105.

Semana P., Powlowski J. 2019. Four aromatic intradiol ring cleavage dioxygenases from aspergillus niger. Appl. Environ. Microbiol. 85.

SH N., FN H., K S. 2004. [Latest frontiers of meiosis research in Coprinus cinereus]. Seikagaku. 76:1450-1454.

Shahinian S., Bussey H. 2000. \$\$-1,6-Glucan synthesis in Saccharomyces cerevisiae. Mol. Microbiol. 35:477-489.

SHAW R. 1967. Fatty Acids of Fruiting Bodies of Basidiomycetes. Nat. 19672135071. 213:86-87.

Sheridan K.J., Lechner B.E., Keeffe G.O., Keller M.A., Werner E.R., Lindner H., Jones G.W., Haas H., Doyle S. 2016. Ergothioneine Biosynthesis and Functionality in the Opportunistic Fungal Pathogen, Aspergillus fumigatus. Sci. Reports 2016 61. 6:1-17.

Shim D., Park S.G., Kim K., Bae W., Lee G.W., Ha B.S., Ro H.S., Kim M., Ryoo R., Rhee S.K., Nou I.S., Koo C.D., Hong C.P., Ryu H. 2016. Whole genome de novo sequencing and genome annotation of the world popular cultivated edible mushroom, Lentinula edodes. J. Biotechnol. 223:24-25.

Shioya T., Nakamura H., Ishii N., Takahashi N., Sakamoto Y., Ozaki N., Kobayashi M., Okano K., Kamada T., Muraguchi H., T S., H N., N I., N T., Y S., N O., M K., K O., T K., H M. 2013. The Coprinopsis cinerea septin Cc.Cdc3 is involved in stipe cell elongation. Fungal Genet. Biol. 58-59:80-90.

Šimočková M., Holič R., Tahotná D., Patton-Vogt J., Griač P. 2008. Yeast Pgc1p (YPL206c) controls the amount of phosphatidylglycerol via a phospholipase C-type degradation mechanism. J. Biol. Chem. 283:17107-17115.

Sipos G., Prasanna A.N., Walter M.C., O’Connor E., Bálint B., Krizsán K., Kiss B., Hess J., Varga T., Slot J., Riley R., Bóka B., Rigling D., Barry K., Lee J., Mihaltcheva S., LaButti K., Lipzen A., Waldron R., Moloney N.M., Sperisen C., Kredics L., Vágvölgyi C., Patrignani A., Fitzpatrick D., Nagy I., Doyle S., Anderson J.B., Grigoriev I. V., Güldener U., Münsterkötter M., Nagy L.G. 2017a. Genome expansion and lineage-specific genetic innovations in the forest pathogenic fungi Armillaria. Nat. Ecol. Evol. 2017112. 1:1931-1941.

Sipos G., Prasanna A.N., Walter M.C., O’Connor E., Bálint B., Krizsán K., Kiss B., Hess J., Varga T., Slot J., Riley R., Bóka B., Rigling D., Barry K., Lee J., Mihaltcheva S., LaButti K., Lipzen A., Waldron R., Moloney N.M., Sperisen C., Kredics L., Vágvölgyi C., 
bioRxiv preprint doi: https://doi.org/10.1101/2021.12.09.471732; this version posted December $10,2021$. The copyright holder for this preprint (which was not certified by peer review) is the author/funder, who has granted bioRxiv a license to display the preprint in perpetuity. It is made available under aCC-BY-NC-ND 4.0 International license.

Running head: fruiting body genomics

6593

6594

6595

6596

6597

6598

6599

6600

6601

6602

6603

6604

6605

6606

6607

6608

6609

6610

6611

6612

6613

6614

6615

6616

6617

6618

6619

6620

6621

6622

6623

6624

6625

6626

6627

6628

6629

6630

6631

6632

6633

6634

6635

6636

6637

6638

6639

6640

6641

6642

6643

6644

6645

6646

6647
Patrignani A., Fitzpatrick D., Nagy I., Doyle S., Anderson J.B., Grigoriev I. V, Güldener U., Münsterkötter M., Nagy L.G. 2017b. Genome expansion and lineage-specific genetic innovations in the forest pathogenic fungi Armillaria. Nat. Ecol. Evol. 1:19311941.

Slot J.C., Gluck-Thaler E. 2019. Metabolic gene clusters, fungal diversity, and the generation of accessory functions. Curr. Opin. Genet. Dev. 58-59:17-24.

Šmid I., Rotter A., Gruden K., Brzin J., Buh Gašparič M., Kos J., Žel J., Sabotič J. 2015. Clitocypin, a fungal cysteine protease inhibitor, exerts its insecticidal effect on Colorado potato beetle larvae by inhibiting their digestive cysteine proteases. Pestic. Biochem. Physiol. 122:59-66.

Soanes D.M., Chakrabarti A., Paszkiewicz K.H., Dawe A.L., Talbot N.J. 2012. Genome-wide Transcriptional Profiling of Appressorium Development by the Rice Blast Fungus Magnaporthe oryzae. PLOS Pathog. 8:e1002514.

Sommer R., Makshakova O.N., Wohlschlager T., Hutin S., Marsh M., Titz A., Künzler M., Varrot A. 2018. Crystal Structures of Fungal Tectonin in Complex with O-Methylated Glycans Suggest Key Role in Innate Immune Defense. Structure. 26:391-402.e4.

Song C.H., Cho K.Y., Nair N.G., Vine J. 2018a. Growth Stimulation and Lipid Synthesis in Lentinus Edodes. https://doi.org/10.1080/00275514.1989.12025782. 81:514-522.

Song H.-Y., Kim D.-H., Kim J.-M. 2018b. Comparative transcriptome analysis of dikaryotic mycelia and mature fruiting bodies in the edible mushroom Lentinula edodes. Sci. Rep. 8.

Sonnenberg A.S.M., Sedaghat-Telgerd N., Lavrijssen B., Ohm R.A., Hendrickx P.M., Scholtmeijer K., Baars J.J.P., van Peer A. 2020. Telomere-to-telomere assembled and centromere annotated genomes of the two main subspecies of the button mushroom Agaricus bisporus reveal especially polymorphic chromosome ends. Sci. Reports 2020 101. 10:1-15.

Specht C.A. 1995. Isolation of the Ba and B $\beta$ mating-type loci of Schizophyllum commune. Curr. Genet. 28:374-379.

Spiteller P. 2015. Chemical ecology of fungi. Nat. Prod. Rep. 32:971-993.

Stadler M., Hoffmeister D. 2015. Fungal natural products-the mushroom perspective. Front. Microbiol. 0:127.

Stadler M., Sterner O. 1998. Production of bioactive secondary metabolites in the fruit bodies of macrofungi as a response to injury. Phytochemistry. 49:1013-1019.

Stajich J.E., Berbee M.L., Blackwell M., Hibbett D.S., James T.Y., Spatafora J.W., Taylor J.W. 2009. The Fungi. Curr. Biol. 19:R840--R845.

Stajich J.E., Wilke S.K., Ahrén D., Au C.H., Birren B.W., Borodovsky M., Burns C., Canbäck B., Casselton L.A., Cheng C.K., Deng J., Dietrich F.S., Fargo D.C., Farman M.L., Gathman A.C., Goldberg J., Guigó R., Hoegger P.J., Hooker J.B., Huggins A., James T.Y., Kamada T., Kilaru S., Kodira C., Kües U., Kupfer D., Kwan H.S., Lomsadze A., Li W., Lilly W.W., Ma L.-J., Mackey A.J., Manning G., Martin F., Muraguchi H., Natvig D.O., Palmerini H., Ramesh M.A., Rehmeyer C.J., Roe B.A., Shenoy N., Stanke M., Ter-Hovhannisyan V., Tunlid A., Velagapudi R., Vision T.J., Zeng Q., Zolan M.E., Pukkila P.J. 2010. Insights into evolution of multicellular fungi from the assembled chromosomes of the mushroom Coprinopsis cinerea (Coprinus cinereus). Proc. Natl. Acad. Sci. U. S. A. 107:11889-94.

Steinegger M., Söding J. 2017. MMseqs2 enables sensitive protein sequence searching for the analysis of massive data sets. Nat. Biotechnol. 35:1026-1028.

Stogios P.J., Downs G.S., Jauhal J.J.S., Nandra S.K., Privé G.G. 2005. Sequence and structural analysis of BTB domain proteins. Genome Biol. 2005 610. 6:1-18.

Strijbis K., Distel B. 2010. Intracellular Acetyl Unit Transport in Fungal Carbon Metabolism. Eukaryot. Cell. 9:1809.

Stutz K., Kaech A., Aebi M., Künzler M., Hengartner M.O. 2015. Disruption of the C. elegans Intestinal Brush Border by the Fungal Lectin CCL2 Phenocopies Dietary Lectin Toxicity in Mammals. PLoS One. 10:e0129381.

Sun J., Ma Y., Xu X., Liu Z., Zou L. 2020. Molecular cloning and bioinformatics analyses of a 
bioRxiv preprint doi: https://doi.org/10.1101/2021.12.09.471732; this version posted December $10,2021$. The copyright holder for this preprint (which was not certified by peer review) is the author/funder, who has granted bioRxiv a license to display the preprint in perpetuity. It is made available under aCC-BY-NC-ND 4.0 International license.

Running head: fruiting body genomics

6648

6649

6650

6651

6652

6653

6654

6655

6656

6657

6658

6659

6660

6661

6662

6663

6664

6665

6666

6667

6668

6669

6670

6671

6672

6673

6674

6675

6676

6677

6678

6679

6680

6681

6682

6683

6684

6685

6686

6687

6688

6689

6690

6691

6692

6693

6694

6695

6696

6697

6698

6699

6700

6701

6702
GH3 beta-glucosidase from Auricularia heimuer. http://mc.manuscriptcentral.com/tbeq. 34:850-859.

Sunagawa M., Magae Y. 2005. Isolation of genes differentially expressed during the fruit body development of Pleurotus ostreatus by differential display of RAPD. FEMS Microbiol. Lett. 246:279-284.

Suzuki S., Sarikaya Bayram Ö., Bayram Ö., Braus G.H. 2013. conF and conJ contribute to conidia germination and stress response in the filamentous fungus Aspergillus nidulans. Fungal Genet. Biol. 56:42-53.

Swamy S., Uno I., Ishikawa T. 1985. Regulation of cyclic AMP metabolism by the incompatibility factors in Coprinus cinereus. J. Gen. Microbiol. 131:3211-3217.

Szczesny P., lacovache I., Muszewska A., Ginalski K., van der Goot F.G., Grynberg M. 2011. Extending the Aerolysin Family: From Bacteria to Vertebrates. PLoS One. 6:e20349.

Szeto C.Y., Leung G.S., Kwan H.S., CY S., GS L., HS K. 2007. Le.MAPK and its interacting partner, Le.DRMIP, in fruiting body development in Lentinula edodes. Gene. 393:8793.

T H., T S., H E. 2004a. Isolation of genes specifically expressed in the fruit body of the edible basidiomycete Lentinula edodes. Biosci. Biotechnol. Biochem. 68:468-472.

T I., K S. 2000. Decreased zinc ion accumulation by the basidiomycete Lentinus edodes over-expressing L. edodes PriA gene. FEMS Microbiol. Lett. 193:111-115.

T L.-O., H R.-R., J A. 2003. Reactive oxygen species generated by microbial NADPH oxidase NoxA regulate sexual development in Aspergillus nidulans. Mol. Microbiol. 50:1241-1255.

T T., N N., M U., T K., G T., M M., K A. 2015. Ustiloxins, fungal cyclic peptides, are ribosomally synthesized in Ustilaginoidea virens. Bioinformatics. 31:981-985.

T T., Y M., K C., K O., N K., N S.-T., N D., Y H., K T. 2004b. Protein sequence analysis, cloning, and expression of flammutoxin, a pore-forming cytolysin from Flammulina velutipes. Maturation of dimeric precursor to monomeric active form by carboxylterminal truncation. J. Biol. Chem. 279:54161-54172.

T W., A B., K Z., SC V., U A. dem K., P G., S B.-M., MO H., M A., M K. 2011. Nematotoxicity of Marasmius oreades agglutinin (MOA) depends on glycolipid binding and cysteine protease activity. J. Biol. Chem. 286:30337-30343.

Tamaki H. 2007. Glucose-stimulated cAMP-protein kinase a pathway in yeast Saccharomyces cerevisiae. J. Biosci. Bioeng. 104:245-250.

Tan Y.H., Moore D. High concentrations of mannitol in the shiitake mushroom Lentinula edodes. .

Tang L., Jian H., Song C., Bao D., Shang X., Wu D., Tan Q., Zhang X. 2013. Transcriptome analysis of candidate genes and signaling pathways associated with light-induced brown film formation in Lentinula edodes. Appl. Microbiol. Biotechnol. 97:4977-4989.

Tang X., Ding X., Hou Y. 2020. Comparative analysis of transcriptomes revealed the molecular mechanism of development of Tricholoma matsutake at different stages of fruiting bodies. Food Sci. Biotechnol. 2020 297. 29:939-951.

Tao Q., Ma K., Yang Y., Wang K., Chen B., Huang Y., Han J., Bao L., Liu X.-B., Yang Z., Yin W.-B., Liu H. 2016. Bioactive Sesquiterpenes from the Edible Mushroom Flammulina velutipes and Their Biosynthetic Pathway Confirmed by Genome Analysis and Chemical Evidence. J. Org. Chem. 81:9867-9877.

Tao Y., Xie B., Yang Z., Chen Z., Chen B., Deng Y., Jiang Y., van Peer A.F. 2013. Identification and expression analysis of a new glycoside hydrolase family 55 exo-beta1,3-glucanase-encoding gene in Volvariella volvacea suggests a role in fruiting body development. Gene. 527:154-160.

Tatebe H., Shiozaki K. 2017. Evolutionary conservation of the components in the tor signaling pathways. Biomolecules. 7.

Taylor J.W., Ellison C.E. 2010. Mushrooms: Morphological complexity in the fungi. Proc. Natl. Acad. Sci. 107:11655-11656.

Tayyrov A., Schmieder S.S., Bleuler-Martinez S., Plaza D.F., Künzlera M. 2018. Toxicity of 
bioRxiv preprint doi: https://doi.org/10.1101/2021.12.09.471732; this version posted December $10,2021$. The copyright holder for this preprint (which was not certified by peer review) is the author/funder, who has granted bioRxiv a license to display the preprint in perpetuity. It is made available under aCC-BY-NC-ND 4.0 International license.

Running head: fruiting body genomics

6703

6704

6705

6706

6707

6708

6709

6710

6711

6712

6713

6714

6715

6716

6717

6718

6719

6720

6721

6722

6723

6724

6725

6726

6727

6728

6729

6730

6731

6732

6733

6734

6735

6736

6737

6738

6739

6740

6741

6742

6743

6744

6745

6746

6747

6748

6749

6750

6751

6752

6753

6754

6755

6756

6757 potential fungal defense proteins towards the fungivorous nematodes Aphelenchus avenae and Bursaphelenchus okinawaensis. Appl. Environ. Microbiol. 84.

Tayyrov A., Stanley C.E., Azevedo S., Künzler M. 2019. Combining microfluidics and RNAsequencing to assess the inducible defensome of a mushroom against nematodes. BMC Genomics 2019 201. 20:1-13.

Teichert I., Dahlmann T.A., Kuck U., Nowrousian M., Kück U., Nowrousian M. 2017. RNA Editing During Sexual Development Occurs in Distantly Related Filamentous Ascomycetes. Genome Biol Evol. 9:855-868.

Teichert I., Steffens E.K., Schnaß N., Fränzel B., Krisp C., Wolters D.A., Kück U. 2014. PRO40 Is a Scaffold Protein of the Cell Wall Integrity Pathway, Linking the MAP Kinase Module to the Upstream Activator Protein Kinase C. PLoS Genet. 10:e1004582.

Telzrow C.L., Nichols C.B., Castro-Lopez N., Wormley F.L., Alspaugh J.A. 2019. A fungal arrestin protein contributes to cell cycle progression and pathogenesis. MBio. 10.

Temp U., Eggert C. 1999. Novel interaction between laccase and cellobiose dehydrogenase during pigment synthesis in the white rot fungus Pycnoporus cinnabarinus. Appl. Environ. Microbiol. 65:389-395.

Terashima K., Yuki K., Muraguchi H., Akiyama M., Kamada T. 2005. The dst1 gene involved in mushroom photomorphogenesis of Coprinus cinereus encodes a putative photoreceptor for blue light. Genetics.

Thammahong A., Puttikamonkul S., Perfect J.R., Brennan R.G., Cramer R.A. 2017. Central Role of the Trehalose Biosynthesis Pathway in the Pathogenesis of Human Fungal Infections: Opportunities and Challenges for Therapeutic Development. Microbiol. Mol. Biol. Rev. 81.

TM H., JW B., MD A., Y R.-D., C S., NP K. 2008. RNA silencing gene truncation in the filamentous fungus Aspergillus nidulans. Eukaryot. Cell. 7:339-349.

Tong X., Zhang H., Wang F., Xue Z., Cao J., Peng C., Guo J. 2020. Comparative transcriptome analysis revealed genes involved in the fruiting body development of Ophiocordyceps sinensis. PeerJ.

Torrens-Spence M.P., Liu C.-T., Pluskal T., Chung Y.K., Weng J.-K. 2018. Monoamine Biosynthesis via a Noncanonical Calcium-Activatable Aromatic Amino Acid Decarboxylase in Psilocybin Mushroom. ACS Chem. Biol. 13:3343-3353.

Tovar-Herrera O.E., Batista-García R.A., Sánchez-Carbente M.D.R., Iracheta-Cárdenas M.M., Arévalo-Niño K., Folch-Mallol J.L. 2015. A novel expansin protein from the whiterot fungus Schizophyllum commune. PLoS One. 10.

Tsitsigiannis D.I., Keller N.P. 2007. Oxylipins as developmental and host-fungal communication signals. Trends Microbiol.

Tsunematsu Y., Takanishi J., Asai S., Masuya T., Nakazawa T., Watanabe K. 2019. Genomic Mushroom Hunting Decrypts Coprinoferrin, A Siderophore Secondary Metabolite Vital to Fungal Cell Development. Org. Lett. 21:7582-7586.

Tullis P. 2021. How ecstasy and psilocybin are shaking up psychiatry. Nature. 589:506-509.

U K., Kues U., Kües U. 2000. Life history and developmental processes in the basidiomycete Coprinus cinereus. Microbiol. Mol. Biol. Rev. 64:316-353.

Umemura M. 2020. Peptides derived from Kex2-processed repeat proteins are widely distributed and highly diverse in the Fungi kingdom. Fungal Biol. Biotechnol. 7:11.

Uno I., Ishikawa T. 1973. Purification and identification of the fruiting-inducing substances in Coprinus macrorhizus. J. Bacteriol. 113:1240-1248.

Varga T., Földi C., Bense V., Nagy L.G. 2021. Developmental innovations promote species diversification in mushroom-forming fungi. bioRxiv.:2021.03.10.434564.

Varga T., Krizsán K., Földi C., Dima B., Sánchez-García M., Sánchez-Ramírez S., Szöllösi G.J., Szarkándi J.G., Papp V., Albert L., Andreopoulos W., Angelini C., Antonín V., Barry K.W., Bougher N.L., Buchanan P., Buyck B., Bense V., Catcheside P., Chovatia M., Cooper J., Dämon W., Desjardin D., Finy P., Geml J., Haridas S., Hughes K., Justo A., Karasiński D., Kautmanova I., Kiss B., Kocsubé S., Kotiranta H., LaButti K.M., Lechner B.E., Liimatainen K., Lipzen A., Lukács Z., Mihaltcheva S., Morgado L.N., Niskanen T., Noordeloos M.E., Ohm R.A., Ortiz-Santana B., Ovrebo C., Rácz N., Riley 
bioRxiv preprint doi: https://doi.org/10.1101/2021.12.09.471732; this version posted December $10,2021$. The copyright holder for this preprint (which was not certified by peer review) is the author/funder, who has granted bioRxiv a license to display the preprint in perpetuity. It is made available under aCC-BY-NC-ND 4.0 International license.

Running head: fruiting body genomics

6758

6759

6760

6761

6762

6763

6764

6765

6766

6767

6768

6769

6770

6771

6772

6773

6774

6775

6776

6777

6778

6779

6780

6781

6782

6783

6784

6785

6786

6787

6788

6789

6790

6791

6792

6793

6794

6795

6796

6797

6798

6799

6800

6801

6802

6803

6804

6805

6806

6807

6808

6809

6810

6811

6812
R., Savchenko A., Shiryaev A., Soop K., Spirin V., Szebenyi C., Tomšovský M., Tulloss R.E., Uehling J., Grigoriev I. V., Vágvölgyi C., Papp T., Martin F.M., Miettinen O., Hibbett D.S., Nagy L.G. 2019. Megaphylogeny resolves global patterns of mushroom evolution. Nat. Ecol. Evol.

Veeneman B.A., Shukla S., Dhanasekaran S.M., Chinnaiyan A.M., Nesvizhskii A.I. 2016. Two-pass alignment improves novel splice junction quantification. Bioinformatics. 32:43-49.

Venter P., Kock J.L.F., Kumar G.S., Botha A., Coetzee D.J., Botes P.J., Bhatt R.K., Falck J.R., Schewe T., Nigam S. 1997. Production of 3R-hydroxy-polyenoic fatty acids by the yeast Dipodascopsis uninucleata. Lipids. 32:1277-1283.

Verdín J., Sánchez-León E., Rico-Ramírez A.M., Martínez-Núñez L., Fajardo-Somera R.A., Riquelme M. 2019. Off the wall: The rhyme and reason of Neurospora crassa hyphal morphogenesis. Cell Surf. 5:100020.

Virágh M., Merényi Z., Csernetics Á., Földi C., Sahu N., Liu X.-B., Hibbett D.S., Nagy L.G. 2021. Evolutionary Morphogenesis of Sexual Fruiting Bodies in Basidiomycota: Toward a New Evo-Devo Synthesis. Microbiol. Mol. Biol. Rev. 86.

Vogt E., Künzler M. 2019. Discovery of novel fungal RiPP biosynthetic pathways and their application for the development of peptide therapeutics. Appl. Microbiol. Biotechnol. 2019 10314. 103:5567-5581.

Vonk P.J., Ohm R.A. 2018. The role of homeodomain transcription factors in fungal development. Fungal Biol. Rev. 32:219-230.

Vonk P.J., Ohm R.A. 2021. H3K4me2 ChIP-Seq reveals the epigenetic landscape during mushroom formation and novel developmental regulators of Schizophyllum commune. Sci. Reports 2021 111. 11:1-15.

W G., Y W., C X., Y Z., Z Z., Y P. 2020. Whole genome sequence of an edible and medicinal mushroom, Hericium erinaceus (Basidiomycota, Fungi). Genomics. 112:2393-2399.

W Z., X W., Y Z.Z., Z L., W Z., X N., Y Z.Z., S P., Y Z.Z., S Y., Zhang W., Wu X., Zhou Y., Liu Z., Zhang W., Niu X., Zhao Y., Pei S., Zhao Y., Yuan S. 2014. Characterization of stipe elongation of the mushroom Coprinopsis cinerea. Microbiology. 160:1893-1902.

Walser P.J., Kües U., Aebi M., Künzler M. 2005. Ligand interactions of the Coprinopsis cinerea galectins. Fungal Genet. Biol.

Wälti M.A., Walser P.J., Thore S., Grünler A., Bednar M., Künzler M., Aebi M. 2008. Structural Basis for Chitotetraose Coordination by CGL3, a Novel Galectin-Related Protein from Coprinopsis cinerea. J. Mol. Biol. 379:146-159.

Walton J. 2018. Ecology and Evolution of the <Emphasis Type $=$ "Italic" $>$ Amanita $</$ Emphasis $>$ Cyclic Peptide Toxins. Cycl. Pept. Toxins Amanita Other Poisonous Mushrooms.:93-130.

Wang H.X., Ng T.B. 2005. Purification of a laccase from fruiting bodies of the mushroom Pleurotus eryngii. Appl. Microbiol. Biotechnol. 2005 695. 69:521-525.

Wang L., Gao W., Wu X., Zhao M., Qu J., Huang C., Zhang J. 2018a. Genome-Wide Characterization and Expression Analyses of Pleurotus ostreatus MYB Transcription Factors during Developmental Stages and under Heat Stress Based on de novo Sequenced Genome. Int. J. Mol. Sci. 19.

Wang L., Tian X., Gyawali R., Lin X. 2013. Fungal adhesion protein guides community behaviors and autoinduction in a paracrine manner. Proc Natl Acad Sci U S A. 110:11571-11576.

Wang L., Zhai B., Lin X. 2012. The Link between Morphotype Transition and Virulence in Cryptococcus neoformans. PLoS Pathog. 8:e1002765.

Wang R., Ma P., Li C., Xiao L., Liang Z., Dong J. 2019. Combining transcriptomics and metabolomics to reveal the underlying molecular mechanism of ergosterol biosynthesis during the fruiting process of Flammulina velutipes. BMC Genomics 2019 201. 20:1-12.

Wang Y., Niu X., Guo X., Yu H., Liu Z., Zhang Z., Yuan S. 2018b. Heterologous expression, characterization and possible functions of the chitin deacetylases, Cda1 and Cda2, from mushroom Coprinopsis cinerea. Glycobiology. 28:318-332.

Wang Z., Li N., Li J., Dunlap J.C., Trail F., Townsend J.P. 2016. The fast-evolving phy-2 
bioRxiv preprint doi: https://doi.org/10.1101/2021.12.09.471732; this version posted December $10,2021$. The copyright holder for this preprint (which was not certified by peer review) is the author/funder, who has granted bioRxiv a license to display the preprint in perpetuity. It is made available under aCC-BY-NC-ND 4.0 International license.

Running head: fruiting body genomics

6813

6814

6815

6816

6817

6818

6819

6820

6821

6822

6823

6824

6825

6826

6827

6828

6829

6830

6831

6832

6833

6834

6835

6836

6837

6838

6839

6840

6841

6842

6843

6844

6845

6846

6847

6848

6849

6850

6851

6852

6853

6854

6855

6856

6857

6858

6859

6860

6861

6862

6863

6864

6865

6866

6867 gene modulates sexual development in response to light in the model fungus Neurospora crassa. MBio. 7.

Wannet W.J.B., Aben E.M.J., Van Der Drift C., Van Griensven L.J.L.D., Vogels G.D., Op Den Camp H.J.M. 1999. Trehalose phosphorylase activity and carbohydrate levels during axenic fruiting in three Agaricus bisporus strains. Curr. Microbiol. 39:205-210.

Watcharawipas A., Watanabe D., Takagi H. 2018. Sodium Acetate Responses in Saccharomyces cerevisiae and the Ubiquitin Ligase Rsp5. Front. Microbiol. 9.

Wawra S., Fesel P., Widmer H., Neumann U., Lahrmann U., Becker S., Hehemann J.-H., Langen G., Zuccaro A. 2019. FGB1 and WSC3 are in planta-induced \$B\$-glucanbinding fungal lectins with different functions. New Phytol. 222:1493-1506.

Weiberg A., Wang M., Lin F.M., Zhao H., Zhang Z., Kaloshian I., Huang H. Da, Jin H. 2013. Fungal small RNAs suppress plant immunity by hijacking host RNA interference pathways. Science (80-. ). 342:118-123.

Weigand-Heller A.B.J., Kris-Etherton P.M., Beelman R.B. 2012. The bioavailability of ergothioneine from mushrooms (Agaricus bisporus) and the acute effects on antioxidant capacity and biomarkers of inflammation. Prev. Med. (Baltim). 54:S75--S78.

WELLS T.K., HAMMOND J.B.W., DICKERSON A.G. 1987. VARIATIONS IN ACTIVITIES OF GLYCOGEN PHOSPHORYLASE AND TREHALASE DURING THE PERIODIC FRUITING OF THE EDIBLE MUSHROOM AGARICUS BISPORUS (LANGE) IMBACH. New Phytol. 105:273-280.

Wen J., Zhang Z., Gong L., Xun H., Li J., Qi B., Wang Q., Li X., Li Y., Liu B. 2019. Transcriptome Changes during Major Developmental Transitions Accompanied with Little Alteration of DNA Methylome in Two Pleurotus Species. Genes (Basel). 10.

Wen L., Huang H.-M., Juang R.-H., Lin C.-T. 2007. Biochemical characterization of 1-Cys peroxiredoxin from Antrodia camphorata. Appl. Microbiol. Biotechnol. 2006736. 73:1314-1322.

Whiteford J.R., Thurston C.F. 2000. The molecular genetics of cultivated mushrooms. Adv. Microb. Physiol. 42:1-23.

Wilinski D., Buter N., Klocko A.D., Lapointe C.P., Selker E.U., Gasch A.P., Wickens M. 2017. Recurrent rewiring and emergence of RNA regulatory networks. Proc. Natl. Acad. Sci. 114:E2816--E2825.

Wirth S., Freihorst D., Krause K., Kothe E. 2021. What role might non-mating receptors play in schizophyllum commune? J. Fungi. 7.

Wohlschlager T., Butschi A., Grassi P., Sutov G., Gauss R., Hauck D., Schmieder S.S., Knobel M., Titz A., Dell A., Haslam S.M., Hengartner M.O., Aebi M., Kun $\square$ zler M. 2014. Methylated glycans as conserved targets of animal and fungal innate defense. Proc. Natl. Acad. Sci. U. S. A. 111:E2787-E2796.

Wood W.F., Clark T.J., Bradshaw D.E., Foy B.D., Largent D.L., Thompson B.L. 2004. Clitolactone: A Banana Slug Antifeedant from Clitocybe flaccida. Mycologia. 96:23.

Wösten H.A.B. 2003. Hydrophobins: Multipurpose Proteins. http://dx.doi.org/10.1146/annurev.micro.55.1.625. 55:625-646.

Wösten H.A.B., Scholtmeijer K. 2015. Applications of hydrophobins: current state and perspectives. Appl. Microbiol. Biotechnol. 2015 994. 99:1587-1597.

Wu B., Gaskell J., Zhang J., Toapanta C., Ahrendt S., Grigoriev I. V, Blanchette R.A., Schilling J.S., Master E., Cullen D., Hibbett D.S. 2019. Evolution of substrate-specific gene expression and RNA editing in brown rot wood-decaying fungi. ISME J. 2019136. 13:1391-1403.

Wu B., Xu Z., Knudson A., Carlson A., Chen N., Kovaka S., LaButti K., Lipzen A., Pennachio C., Riley R., Schakwitz W., Umezawa K., Ohm R.A., Grigoriev I. V, Nagy L.G., Gibbons J., Hibbett D. 2018. Genomics and Development of Lentinus tigrinus $\square$ : A White-Rot Wood-Decaying Mushroom with Dimorphic Fruiting Bodies. Genome Biol. Evol. 10:3250-3261.

Wu F.-L., Zhang G., Ren A., Dang Z.-H., Shi L., Jiang A.-L., Zhao M.-W., FL W., G Z., A R., ZH D., L S., AL J., MW Z. 2016. The pH-responsive transcription factor PacC regulates mycelial growth, fruiting body development, and ganoderic acid biosynthesis in 
bioRxiv preprint doi: https://doi.org/10.1101/2021.12.09.471732; this version posted December $10,2021$. The copyright holder for this preprint (which was not certified by peer review) is the author/funder, who has granted bioRxiv a license to display the preprint in perpetuity. It is made available under aCC-BY-NC-ND 4.0 International license.

Running head: fruiting body genomics

6868

6869

6870

6871

6872

6873

6874

6875

6876

6877

6878

6879

6880

6881

6882

6883

6884

6885

6886

6887

6888

6889

6890

6891

6892

6893

6894

6895

6896

6897

6898

6899

6900

6901

6902

6903

6904

6905

6906

6907

6908

6909

6910

6911

6912

6913

6914

6915

6916

6917

6918

6919

6920

6921

6922

Ganoderma lucidum. Mycologia. 108:1104-1113.

Wu L., van Peer A., Song W., Wang H., Chen M., Tan Q., Song C., Zhang M., Bao D. 2013. Cloning of the Lentinula edodes $B$ mating-type locus and identification of the genetic structure controlling B mating. Gene. 531:270-278.

Wu T., Hu C., Xie B., Wei S., Zhang L., Zhu Z., Zhang Z., Li S. 2020a. A putative transcription factor LFC1 negatively regulates development and yield of winter mushroom. Appl. Microbiol. Biotechnol. 2020 10413. 104:5827-5844.

Wu T., Zhang Z., Hu C., Zhang L., Wei S., Li S. 2020b. A WD40 Protein Encoding Gene Fvcpc2 Positively Regulates Mushroom Development and Yield in Flammulina velutipes. Front. Microbiol. 11:498.

WW C., KT N., RS S., CH A., HS K. 2008. Gene expression studies of the dikaryotic mycelium and primordium of Lentinula edodes by serial analysis of gene expression. Mycol. Res. 112:950-964.

Wymelenberg A. Vanden, Gaskell J., Mozuch M., Kersten P., Sabat G., Martinez D., Cullen D. 2009. Transcriptome and Secretome Analyses of Phanerochaete chrysosporium Reveal Complex Patterns of Gene Expression. Appl. Environ. Microbiol. 75:4058.

X C., JH H., YY L., PT W.L., HS K., Cheng X., Hui J.H.L., Lee Y.Y., Wan Law P.T., Kwan H.S. 2015. A "developmental hourglass" in fungi. Mol. Biol. Evol. 32:1556-1566.

X L., L F., C W., P C. 2018. Cryo-EM structure of a mammalian RNA polymerase II elongation complex inhibited by \$a $\$$-amanitin. J. Biol. Chem. 293:7189-7194.

X L., S D. 2009. Molecular characterization of a new acetyl xylan esterase (AXEII) from edible straw mushroom Volvariella volvacea with both de-O-acetylation and de-Nacetylation activity. FEMS Microbiol. Lett. 295:50-56.

Xie C., Gong W., Zhu Z., Yan L., Hu Z., Peng Y. 2018. Comparative transcriptomics of Pleurotus eryngii reveals blue-light regulation of carbohydrate-active enzymes (CAZymes) expression at primordium differentiated into fruiting body stage. Genomics. 110:201-209.

Xie Y., Chang J., Kwan H.S. 2020. Carbon metabolism and transcriptome in developmental paths differentiation of a homokaryotic Coprinopsis cinerea strain. Fungal Genet. Biol. 143:103432.

Xu G., Ma H., Nei M., Kong H. 2009. Evolution of F-box genes in plants: Different modes of sequence divergence and their relationships with functional diversification. Proc. Natl. Acad. Sci. 106:835-840.

Xu H., Navarro-Ródenas A., Cooke J.E.K., Zwiazek J.J., H X., A N.-R., JE C., JJ Z., Xu H., Navarro-Ródenas A., Cooke J.E.K., Zwiazek J.J. 2015. Transcript profiling of aquaporins during basidiocarp development in Laccaria bicolor ectomycorrhizal with Picea glauca. Mycorrhiza 2015 261. 26:19-31.

Xue C., Hsueh Y.P., Heitman J. 2008. Magnificent seven: Roles of G protein-coupled receptors in extracellular sensing in fungi. FEMS Microbiol. Rev.

Y F., A O.-U., T G., Y P., NO K. 2015. Involvement of a caleosin in lipid storage, spore dispersal, and virulence in the entomopathogenic filamentous fungus, Beauveria bassiana. Environ. Microbiol. 17:4600-4614.

Y K., H K. 2014. Fungal lectins: a growing family. Methods Mol. Biol. 1200:15-38.

Y M., S K., M S., K S., T Y., M N., K B., Miyazaki Y., Kaneko S., Sunagawa M., Shishido K., Yamazaki T., Nakamura M., Babasaki K. 2007. The fruiting-specific Le.flp1 gene, encoding a novel fungal fasciclin-like protein, of the basidiomycetous mushroom Lentinula edodes. Curr. Genet. 51:367-375.

Y S., K N., N K., Sakamoto Y., Nakade K., Konno N. 2011. Endo-\$ß\$-1,3-glucanase GLU1, from the fruiting body of Lentinula edodes, belongs to a new glycoside hydrolase family. Appl. Environ. Microbiol. 77:8350-8354.

Y S., T I., T S., Sakamoto Y., Irie T., Sato T. 2005. Isolation and characterization of a fruiting body-specific exo-beta-1,3-glucanase-encoding gene, exg1, from Lentinula edodes. Curr. Genet. 47:244-252.

Y T., M T., J S., S U., Y K., N O., M I., T Y. 2009. Tamavidins--novel avidin-like biotin-binding proteins from the Tamogitake mushroom. FEBS J. 276:1383-1397. 
bioRxiv preprint doi: https://doi.org/10.1101/2021.12.09.471732; this version posted December $10,2021$. The copyright holder for this preprint (which was not certified by peer review) is the author/funder, who has granted bioRxiv a license to display the preprint in perpetuity. It is made available under aCC-BY-NC-ND 4.0 International license.

Running head: fruiting body genomics

6923

6924

6925

6926

6927

6928

6929

6930

6931

6932

6933

6934

6935

6936

6937

6938

6939

6940

6941

6942

6943

6944

6945

6946

6947

6948

6949

6950

6951

6952

6953

6954

6955

6956

6957

6958

6959

6960

6961

6962

6963

6964

6965

6966

6967

6968

6969

6970

6971

6972

6973

6974

6975

6976

6977
Y T., R C., J Y., Y L., Z T., H S., B X. 2019. A hydrophobin gene, Hyd9, plays an important role in the formation of aerial hyphae and primordia in Flammulina filiformis. Gene. 706:84-90.

Y T., S T., T K., T J. 2013. Molecular characterization of a lipoxygenase from the basidiomycete mushroom Pleurotus ostreatus. Biosci. Biotechnol. Biochem. 77:38-45.

Y W., X Z., W L. 2018. De novo transcriptomic analysis during Lentinula edodes fruiting body growth. Gene. 641:326-334.

Yamada-Okabe T., Sakamori Y., Mio T., Yamada-Okabe H. 2001. Identification and characterization of the genes for $\mathrm{N}$-acetylglucosamine kinase and $\mathrm{N}$ acetylglucosamine-phosphate deacetylase in the pathogenic fungus Candida albicans. Eur. J. Biochem. 268:2498-2505.

Yamagishi K., Kimura T., Suzuki M., Shinmoto H. 2002. Suppression of fruit-body formation by constitutively active G-protein \$a-subunits ScGP-A and ScGP-C in the homobasidiomycete Schizophyllum commune. Microbiology. 148:2797-2809.

Yamagishi K., Kimura T., Suzuki M., Shinmoto H., Yamaki K.J. 2004. Elevation of intracellular cAMP levels by dominant active heterotrimeric $\mathrm{G}$ protein alpha subunits ScGP-A and ScGP-C in homobasidiomycete, Schizophyllum commune. Biosci. Biotechnol. Biochem. 68:1017-1026.

Yan J.-J.J., Tong Z.-J.J., Liu Y.-Y.Y., Li Y.-N.N., Zhao C., Mukhtar I., Tao Y.-X.X., Chen B.Z.Z., Deng Y.-J.J., Xie B.-G.G. 2019. Comparative transcriptomics of Flammulina filiformis suggests a high $\mathrm{CO} 2$ concentration inhibits early pileus expansion by decreasing cell division control pathways. Int. J. Mol. Sci. 20:5923.

Ye S.-Q., Zou Y., Zheng Q.-W., Liu Y.-L., Li R.-R., Lin J.-F., Guo L.-Q. 2021. TMT-MS/MS proteomic analysis of the carbohydrate-active enzymes in the fruiting body of Pleurotus tuoliensis during storage. J. Sci. Food Agric. 101:1879-1891.

Yin G., Padhi S., Lee S., Hung R., Zhao G., Bennett J.W. 2015. Effects of Three Volatile Oxylipins on Colony Development in Two Species of Fungi and on Drosophila Larval Metamorphosis. Curr. Microbiol. 71:347-356.

Yoo S., Lee H.-Y., Markkandan K., Moon S., Ahn Y.J., Ji S., Ko J., Kim S.-J., Ryu H., Hong C.P. 2019. Comparative transcriptome analysis identified candidate genes involved in mycelium browning in Lentinula edodes. 20.

Yu Z., Armant O., Fischer R. 2016. Fungi use the SakA (HogA) pathway for phytochromedependent light signalling. Nat. Microbiol. 2016 15. 1:1-7.

Yu Z., Fischer R. 2018. Light sensing and responses in fungi. Nat. Rev. Microbiol. 2018171. 17:25-36.

Yun Y.H., Koo J.S., Kim S.H., Kong W.S. 2015. Cloning and expression analysis of phenylalanine ammonia-lyase gene in the mycelium and fruit body of the edible mushroom Flammulina velutipes. Mycobiology. 43:327-332.

Zawirska-Wojtasiak R. 2004. Optical purity of (R)-(-)-1-octen-3-ol in the aroma of various species of edible mushrooms. Food Chem. 86:113-118.

Zeng X., Ling H., Yang J., Chen J., Guo S. 2018. Proteome analysis provides insight into the regulation of bioactive metabolites in Hericium erinaceus. Gene. 666:108-115.

Zhang C., Chen X., Lee R.T.C., Rehka T., Maurer-Stroh S., Rühl M. 2021a. Bioinformaticsaided identification, characterization and applications of mushroom linalool synthases. Commun. Biol. 4:1-11.

Zhang C., Chen X., Orban A., Shukal S., Birk F., Too H.-P., Rühl M. 2020. Agrocybe aegerita Serves As a Gateway for Identifying Sesquiterpene Biosynthetic Enzymes in Higher Fungi. ACS Chem. Biol. 15:1268-1277.

Zhang J., Chen H., Chen M., Ren A., Huang J., Wang H., Zhao M., Feng Z. 2015a. Cloning and functional analysis of a laccase gene during fruiting body formation in Hypsizygus marmoreus. Microbiol. Res. 179:54-63.

Zhang J., Hao H., Liu H., Wang Q., Chen M., Feng Z., Chen H. 2021b. Genetic and functional analysis of the $\mathrm{Zn}$ (II)2Cys6 transcription factor HADA-1 in Hypsizygus marmoreus. Appl. Microbiol. Biotechnol. 2021 1057. 105:2815-2829.

Zhang J., Ren A., Chen H., Zhao M., Shi L., Chen M., Wang H., Feng Z. 2015b. 
bioRxiv preprint doi: https://doi.org/10.1101/2021.12.09.471732; this version posted December $10,2021$. The copyright holder for this preprint (which was not certified by peer review) is the author/funder, who has granted bioRxiv a license to display the preprint in perpetuity. It is made available under aCC-BY-NC-ND 4.0 International license.

Running head: fruiting body genomics

6978

6979

6980

6981

6982

6983

6984

6985

6986

6987

6988

6989

6990

6991

6992

6993

6994

6995

6996

6997

6998

6999

7000

7001

7002

7003

7004

7005

7006

7007

7008

7009

7010

7011

7012

7013

7014

7015

7016

7017

7018

7019

7020

7021

7022

7023

7024

7025

7026

7027

7028

7029

7030

7031

7032
Transcriptome Analysis and Its Application in Identifying Genes Associated with Fruiting Body Development in Basidiomycete Hypsizygus marmoreus. PLoS One.

10:e0123025.

Zhang J., Shen N., Li C., Xiang X., Liu G., Gui Y., Patev S., Hibbett D.S., Barry K., Andreopoulos W., Lipzen A., Riley R., He G., Yan M., Grigoriev I. V., Shan Kwan H., Kit Cheung M., Bian Y., Xiao Y. 2021c. Population genomics provides insights into the genetic basis of adaptive evolution in the mushroom-forming fungus Lentinula edodes. J. Adv. Res.

Zhang L., Gong W., Li C., Shen N., Gui Y., Bian Y., Kwan H.S., Cheung M.K., Xiao Y. 2021d. RNA-Seq-based high-resolution linkage map reveals the genetic architecture of fruiting body development in shiitake mushroom, Lentinula edodes. Comput. Struct. Biotechnol. J. 19:1641-1653.

Zhang X., Krause K.-H., Xenarios I., Soldati T., Boeckmann B. 2013. Evolution of the Ferric Reductase Domain (FRD) Superfamily: Modularity, Functional Diversification, and Signature Motifs. PLoS One. 8:e58126.

Zhang Z.-M., Wu W.-W., Li G.-K. 2008. A GC--MS Study of the Volatile Organic Composition of Straw and Oyster Mushrooms During Maturity and its Relation to Antioxidant Activity. J. Chromatogr. Sci. 46:690-696.

Zhao C., Waalwijk C., de Wit P.J.G.M., Tang D., van der Lee T. 2013. RNA-Seq analysis reveals new gene models and alternative splicing in the fungal pathogen Fusarium graminearum. BMC Genomics 2013 141. 14:1-16.

Zhao J., Kwan H.S. 1999. Characterization, molecular cloning, and differential expression analysis of laccase genes from the edible mushroom Lentinula edodes. Appl. Environ. Microbiol. 65:4908-4913.

Zhao Z., Liu H., Luo Y., Zhou S., An L., Wang C., Jin Q., Zhou M., Xu J.R. 2014. Molecular evolution and functional divergence of tubulin superfamily in the fungal tree of life. Sci. Rep. 4.

Zheng N., Shabek N. 2017. Ubiquitin Ligases: Structure, Function, and Regulation. https://doi.org/10.1146/annurev-biochem-060815-014922. 86:129-157.

Zhou J., Kang L., Liu C., Niu X., Wang X., Liu H., Zhang W., Liu Z., Latgé J.-P., Yuan S. 2019. Chitinases Play a Key Role in Stipe Cell Wall Extension in the Mushroom Coprinopsis cinerea. Appl. Environ. Microbiol. 85.

Zhou S., Li X., Lüli Y., Li X., Chen Z.H., Yuan P., Yang Z.L., Li G., Luo H. 2021. Novel Cyclic Peptides from Lethal Amanita Mushrooms through a Genome-Guided Approach. J. Fungi. 7:204.

Zhou S., Ma F., Zhang X., Zhang J. 2016a. Carbohydrate changes during growth and fruiting in Pleurotus ostreatus. Fungal Biol. 120:852-861.

Zhou S., Ma F., Zhang X., Zhang J., S Z., F M., X Z., J Z., Zhou S., Ma F., Zhang X., Zhang J., S Z., F M., X Z., J Z. 2016b. Carbohydrate changes during growth and fruiting in Pleurotus ostreatus. Fungal Biol. 120:852-861.

Zhou S., Zhang J., Ma F., Tang C., Tang Q., Zhang X. 2018. Investigation of lignocellulolytic enzymes during different growth phases of Ganoderma lucidum strain G0119 using genomic, transcriptomic and secretomic analyses. PLoS One. 13:e0198404.

Zhou Y., Chen L., Fan X., Bian Y. 2014. De Novo Assembly of Auricularia polytricha Transcriptome Using Illumina Sequencing for Gene Discovery and SSR Marker Identification. PLoS One. 9:e91740.

Zhou Y., Kang L., Niu X., Wang J., Liu Z., Yuan S. 2016c. Purification, characterization and physiological significance of a chitinase from the pilei of Coprinopsis cinerea fruiting bodies. FEMS Microbiol. Lett. 363:1-7.

Zhu W., Hu J., Li Y., Yang B., Guan Y., Xu C., Chen F., Chi J., Bao Y. 2019. Comparative Proteomic Analysis of Pleurotus ostreatus Reveals Great Metabolic Differences in the Cap and Stipe Development and the Potential Role of $\mathrm{Ca} 2+$ in the Primordium Differentiation. Int. J. Mol. Sci. 2019, Vol. 20, Page 6317. 20:6317.

Zhu Y., Luo H., Zhang X., Song J., Sun C., Ji A., Xu J., Chen S. 2014. Abundant and Selective RNA-Editing Events in the Medicinal Mushroom Ganoderma lucidum. 
bioRxiv preprint doi: https://doi.org/10.1101/2021.12.09.471732; this version posted December 10, 2021. The copyright holder for this preprint (which was not certified by peer review) is the author/funder, who has granted bioRxiv a license to display the preprint in perpetuity. It is made available under aCC-BY-NC-ND 4.0 International license.

Running head: fruiting body genomics

7033

7034

7035

7036

7037

7038

7039

7040

7041

7042

7043

7044

7045

7046

7047

7048

7049
Genetics. 196:1047.

Zhu Y., Zhuang L., Goodell B., Cao J., Mahaney J. 2016. Iron sequestration in brown-rot fungi by oxalate and the production of reactive oxygen species (ROS). Int. Biodeterior. Biodegradation. 109:185-190.

卢园萍, 郭仲杰, 蔡志欣, 施肖枋, 陈美元, 廖剑华, 王泽生, Yuan-Ping L.U., Zhong-Jie G.U.O., Zhi-Xin C.A.I., Xiao-Kun S.H.I., Mei-Yuan C., Jian-Hua L., Ze-Sheng W. 2019.

双孢蘑菇Septin基因Ab.Cdc3的克隆与分析. 菌物学报. 37:1635-1642.

\section{Figures}
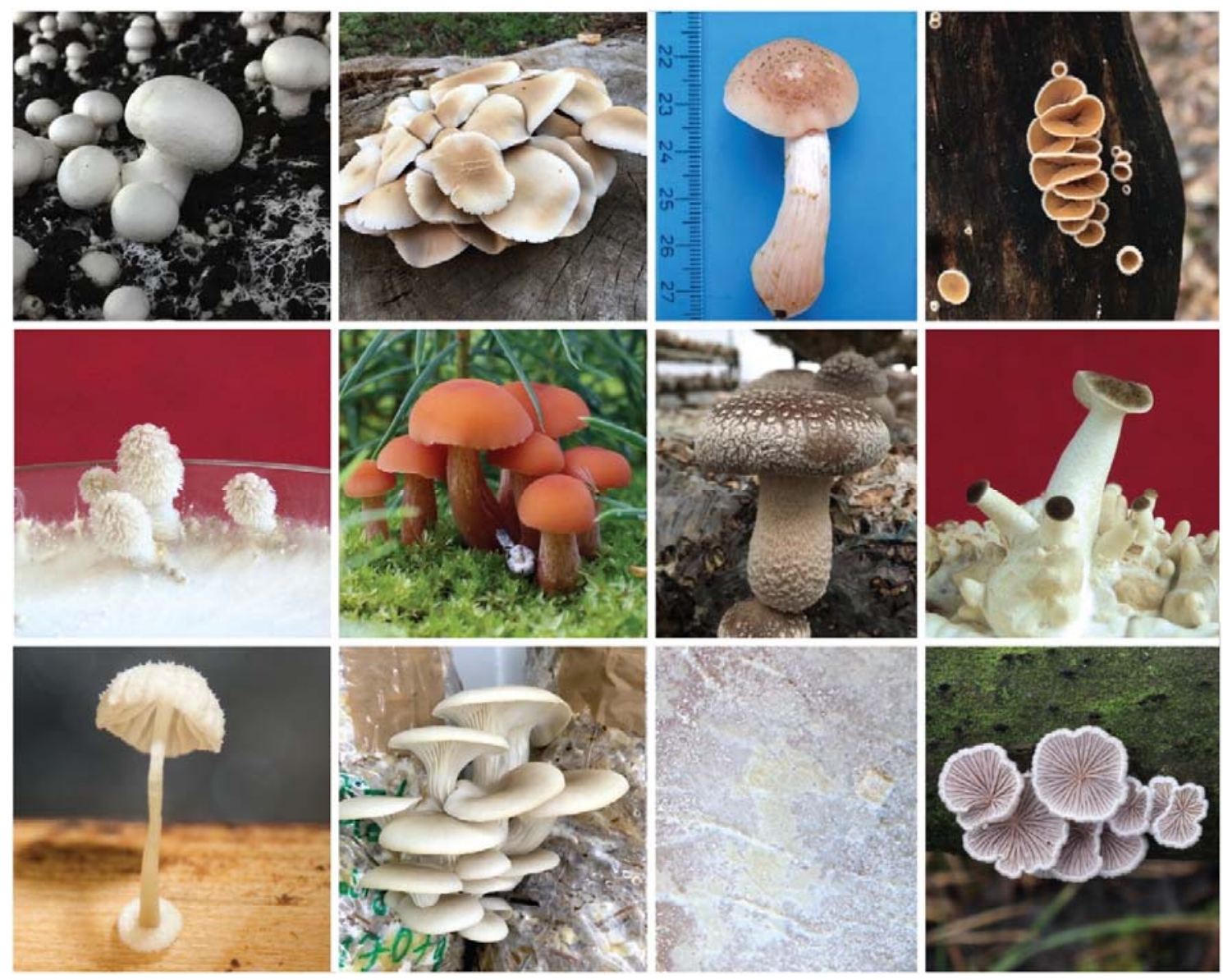

Fig. 1. Species used in this study. Top row left to right: Agaricus bisporus, Cyclocybe aegerita, Armillaria ostoyae, Auriculariopsis ampla; Middle row: Coprinopsis cinerea, Laccaria bicolor, Lentinula edodes, Lentinus tigrinus; Bottom row: Mycena kentingensis, Phanerochaete chrysosporium, Pleurotus ostreatus, Schizophyllum commune 
bioRxiv preprint doi: https://doi.org/10.1101/2021.12.09.471732; this version posted December 10,2021 . The copyright holder for this preprint (which was not certified by peer review) is the author/funder, who has granted bioRxiv a license to display the preprint in perpetuity. It is made available under aCC-BY-NC-ND 4.0 International license.

Running head: fruiting body genomics

A
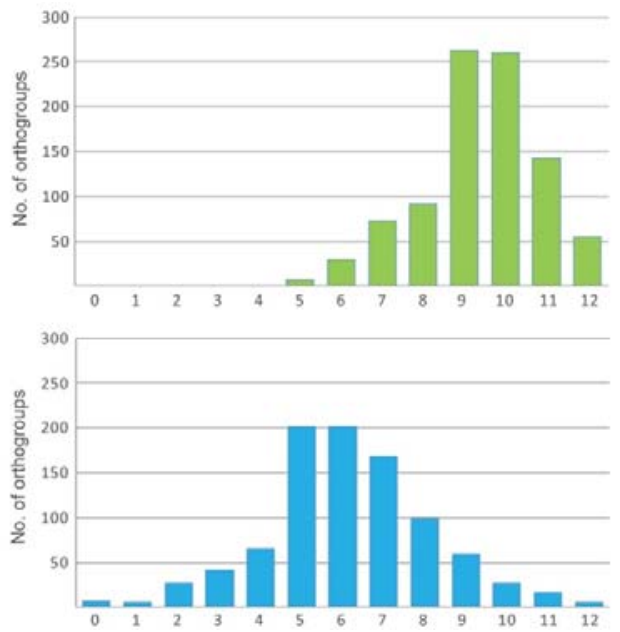

B

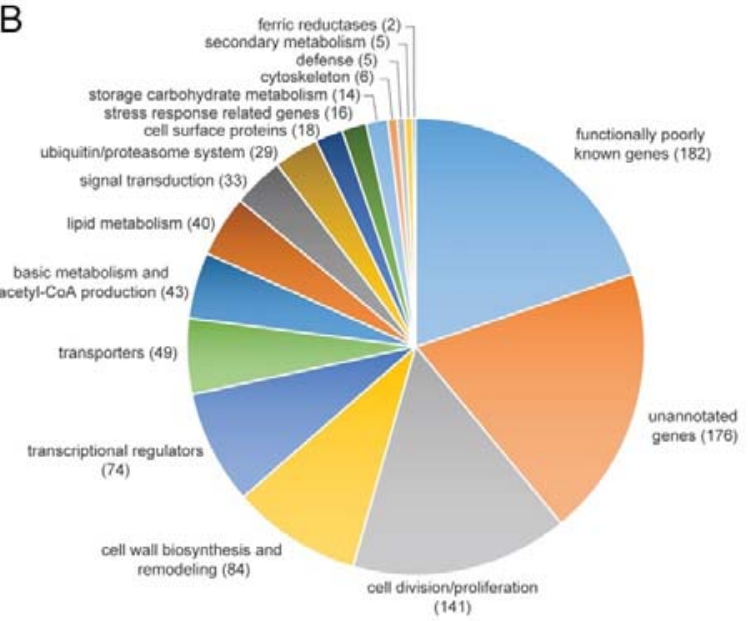

Fig. 2. The distribution of conserved developmentally regulated orthogroups across species.

A The number of orthogroups as a function of the number of species in which the orthogroup is developmentally regulated at fold change $>2$ (top) and fold change $>4$ (bottom). Horizontal axis represent the number of species. B Numbers of genes in each of the functional categories discussed in the paper.

7056 
bioRxiv preprint doi: https://doi.org/10.1101/2021.12.09.471732; this version posted December 10,2021 . The copyright holder for this preprint (which was not certified by peer review) is the author/funder, who has granted bioRxiv a license to display the preprint in perpetuity. It is made available under aCC-BY-NC-ND 4.0 International license.

Running head: fruiting body genomics
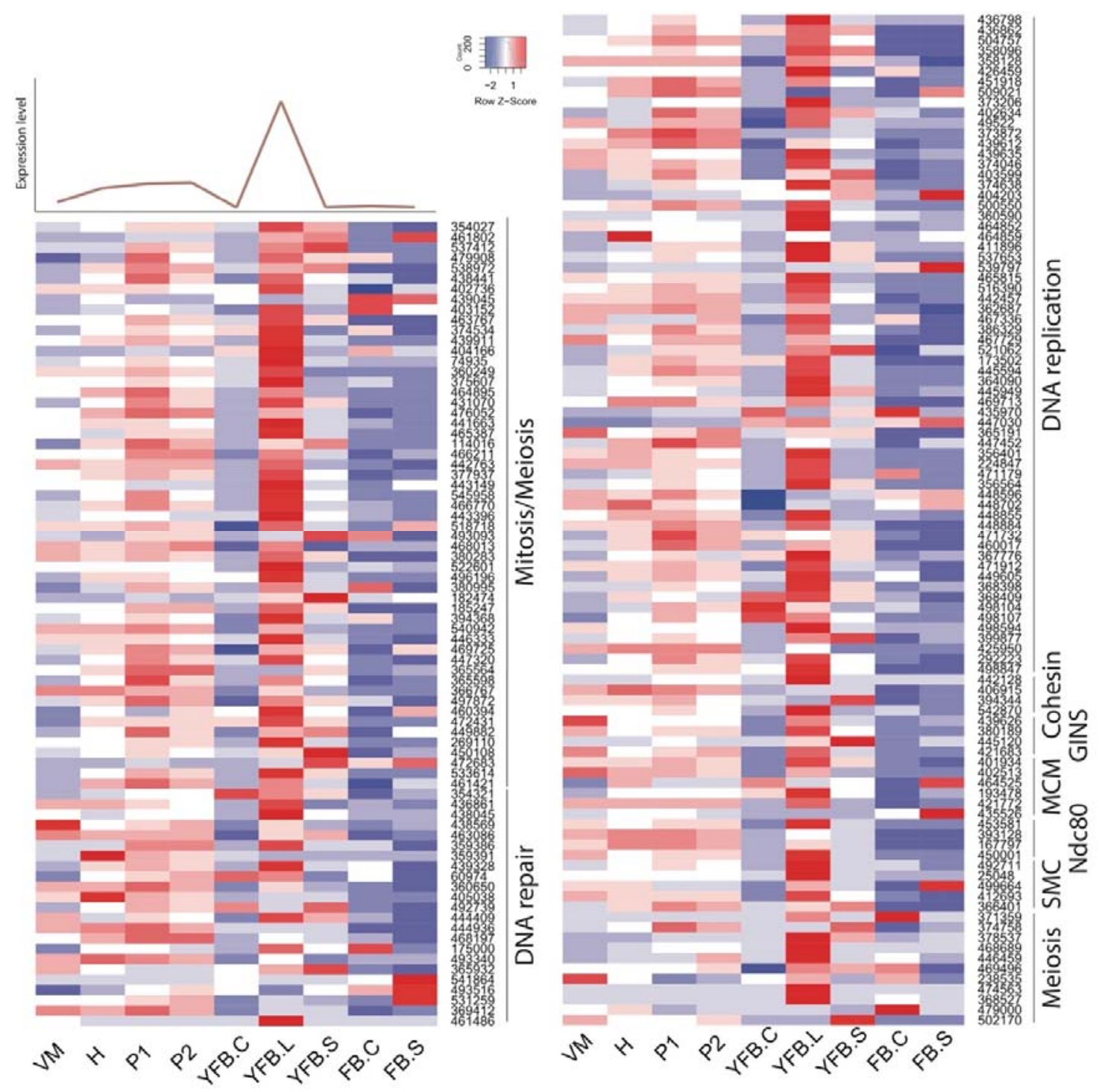

Fig. 3 Expression heatmap of DNA replication, repair, mitosis and meiosis related genes in C. cinerea. A model expression trajectory, illustrating the two peaks characteristic of most examined species, is shown above the left panel. Well-delimited complexes mentioned in the paper are shown separately. Genes are denoted by Protein IDs. Blue and red colors represent low and high expression, respectively. Developmental stages are abbreviated as follows: VM - vegetative mycelium, $\mathrm{H}$ - hyphal knot, $\mathrm{P} 1$ - stage 1 primordium, $\mathrm{P} 2$ - stage 2 primordium, YFB.C - young fruiting body cap, YFB.L - young fruiting body gills, YFB.S young fruiting body stipe, FB.C - mature fruiting body cap (including gills), FB.S - mature 
bioRxiv preprint doi: https://doi.org/10.1101/2021.12.09.471732; this version posted December 10,2021 . The copyright holder for this preprint (which was not certified by peer review) is the author/funder, who has granted bioRxiv a license to display the preprint in perpetuity. It is made available under aCC-BY-NC-ND 4.0 International license.

Running head: fruiting body genomics
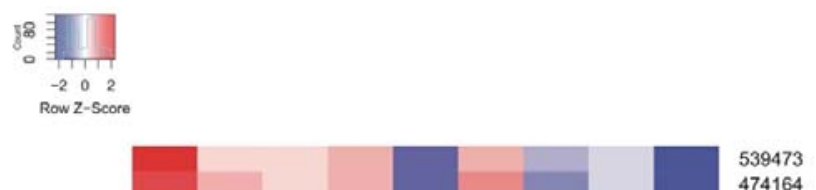

430785

447700

374411

130885

405648

401275

398047

462840

398174

437986

381804

375577

385517

430789

165767
442748

412005

385868

385868
429262

398594

494449

416494

464129

394207

394207
475978

475978
539347

441325

412254

441652

412936

446338

426937

404670

403137

475518

423325

467768

467322

424304

64051

367451

366589

438977

472703

426850

493053

420962

16780

493413

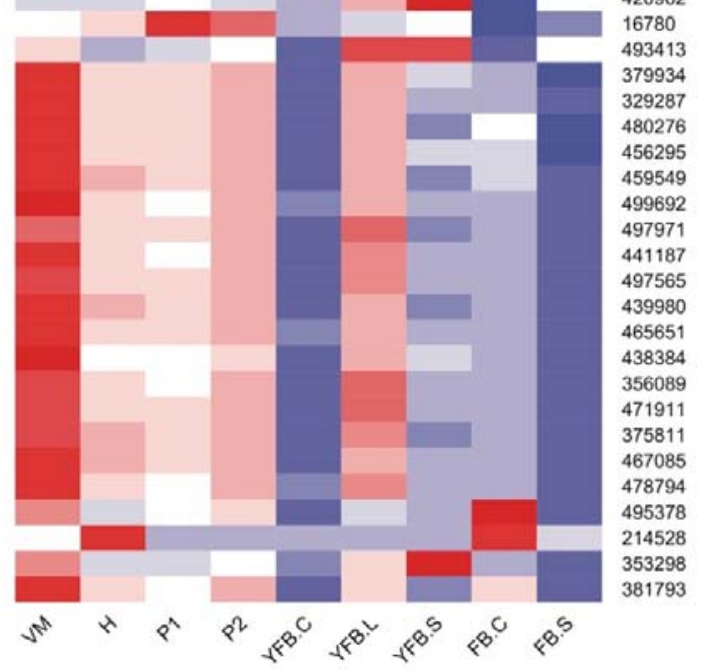

Fig. 4. Expression heatmap of ribosomal protein encoding genes in C. cinerea. Genes are denoted by Protein IDs. Blue and red colors represent low and high expression, respectively. 
bioRxiv preprint doi: https://doi.org/10.1101/2021.12.09.471732; this version posted December 10, 2021. The copyright holder for this preprint (which was not certified by peer review) is the author/funder, who has granted bioRxiv a license to display the preprint in perpetuity. It is made available under aCC-BY-NC-ND 4.0 International license.

Running head: fruiting body genomics

knot, P1 - stage 1 primordium, P2 - stage 2 primordium, YFB.C - young fruiting body cap, YFB.L - young fruiting body gills, YFB.S - young fruiting body stipe, FB.C - mature fruiting body cap (including gills), FB.S - mature fruiting body stipe.

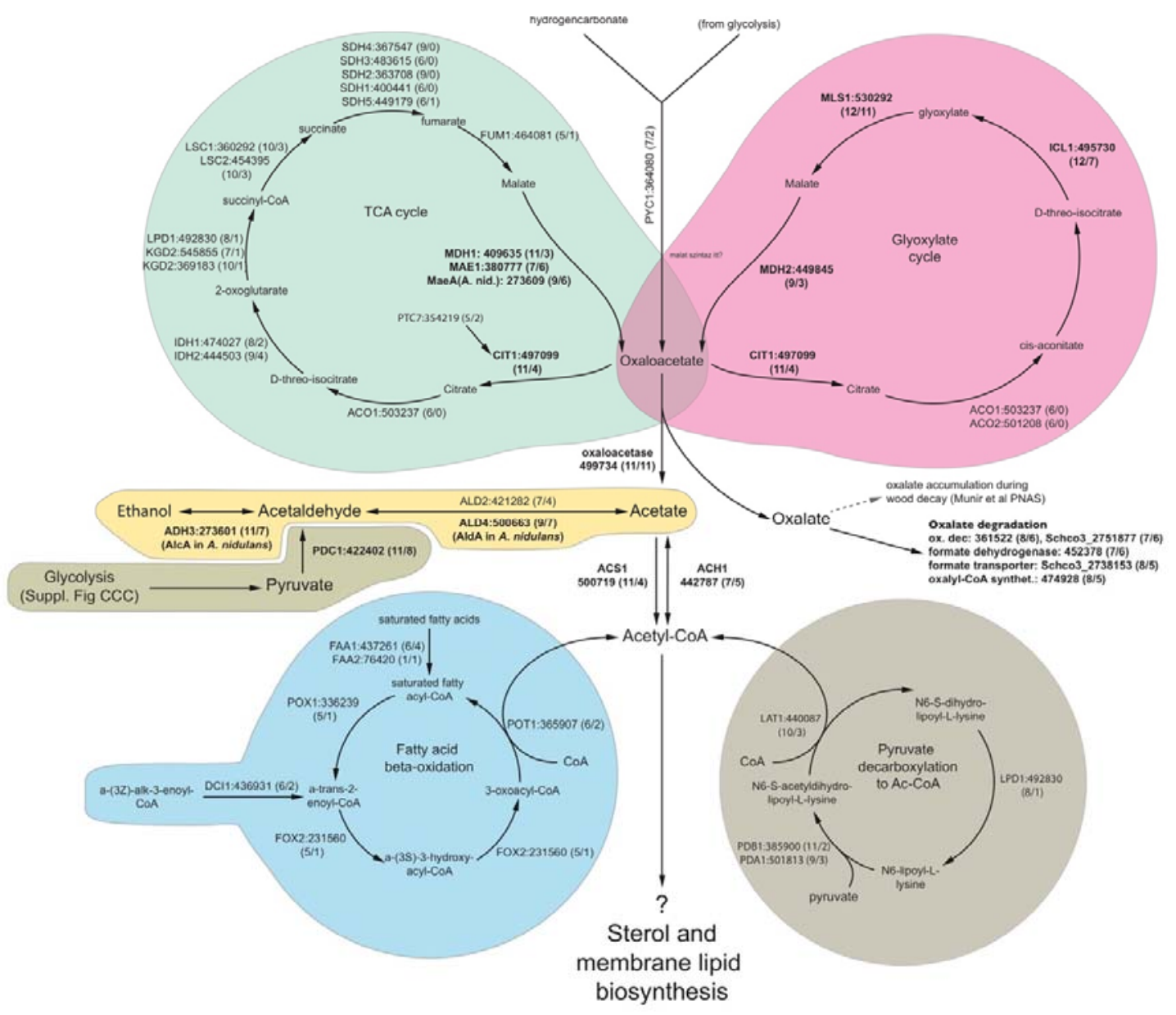

Fig. 5. Basic metabolic pathways of $C$. cinerea. Genes in the pathways are denoted by the parentheses the number of species in which the gene was developmentally regulated at fold change $>2 />4$. Pathways are colored differently for clarity. 
bioRxiv preprint doi: https://doi.org/10.1101/2021.12.09.471732; this version posted December 10, 2021. The copyright holder for this preprint (which was not certified by peer review) is the author/funder, who has granted bioRxiv a license to display the preprint in perpetuity. It is made available under aCC-BY-NC-ND 4.0 International license.

Running head: fruiting body genomics

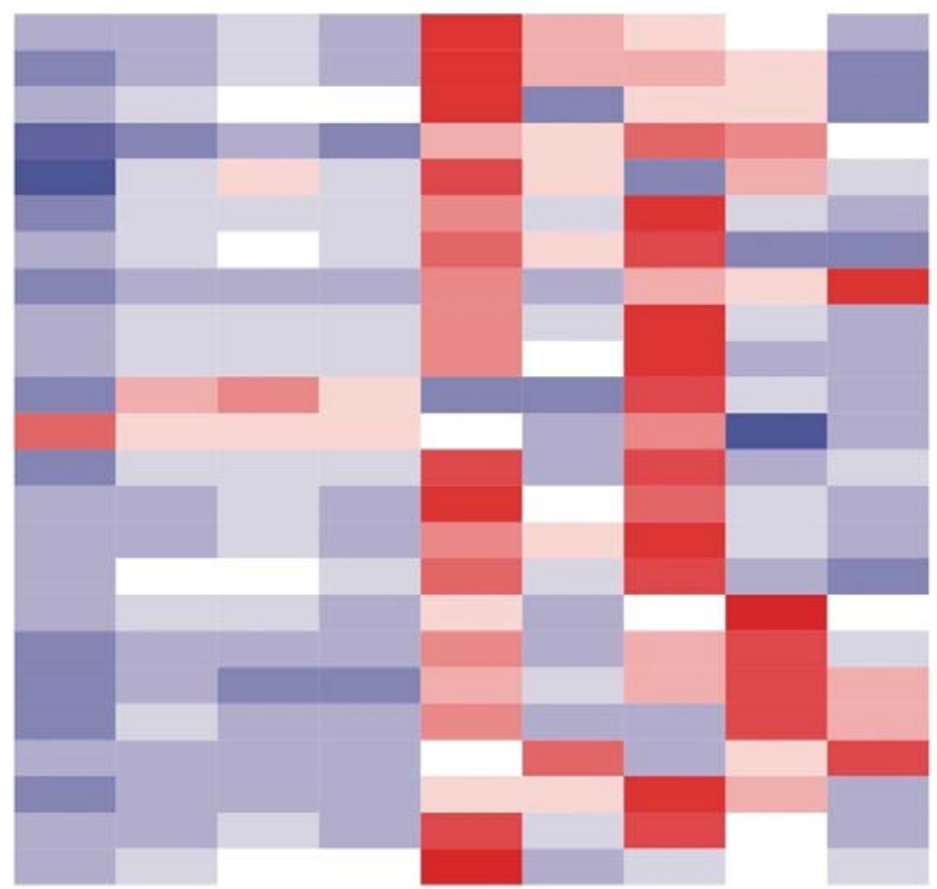

358591

420341

438368

462226

544612

360799

535364

489517

17387

43445

355838

366787

439474

397292

357271

459666

366442

494314

355558

413057

539004

373683

496056

460395

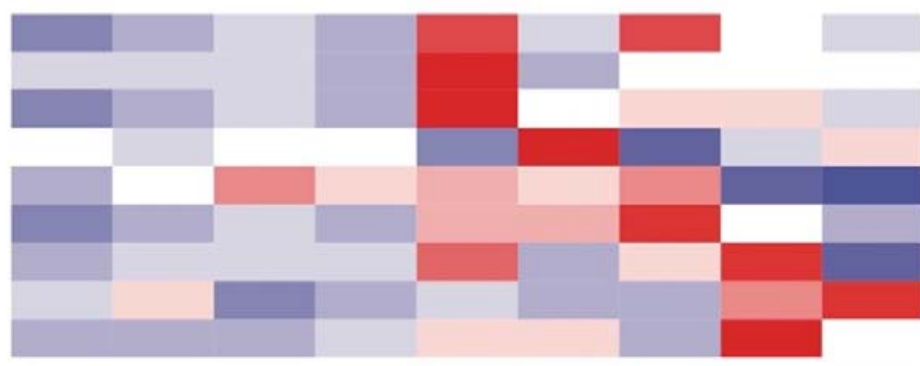

429678

420050

441179

1859

456289

546344

379742

372474

388282

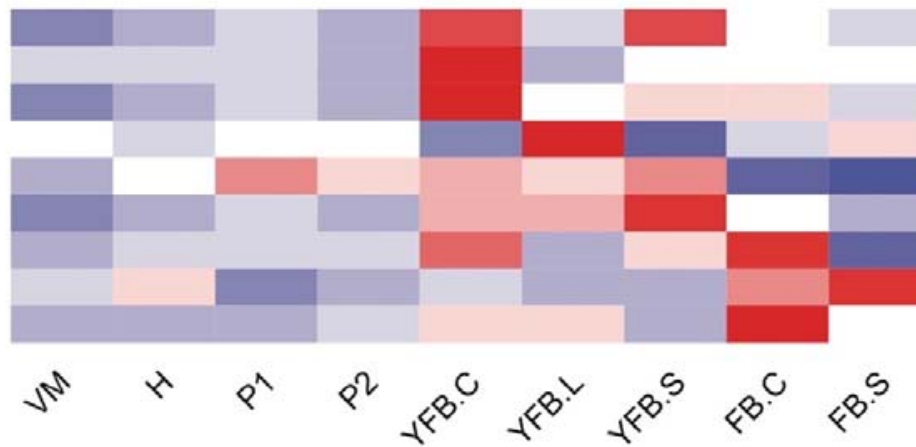

429678

420050

441179

1859

456289

546344

379742

372474

388282

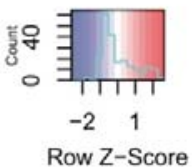

Fig. 6. Expression heatmap of ergosterol and sphingolipid biosynthesis related genes in $C$. cinerea. Genes are denoted by Protein IDs. Blue and red colors represent low and high expression, respectively. Developmental stages are abbreviated as follows: VM - vegetative mycelium, $\mathrm{H}$ - hyphal knot, P1 - stage 1 primordium, P2 - stage 2 primordium, YFB.C young fruiting body cap, YFB.L - young fruiting body gills, YFB.S - young fruiting body stipe, FB.C - mature fruiting body cap (including gills), FB.S - mature fruiting body stipe. 
bioRxiv preprint doi: https://doi.org/10.1101/2021.12.09.471732; this version posted December 10, 2021. The copyright holder for this preprint (which was not certified by peer review) is the author/funder, who has granted bioRxiv a license to display the preprint in perpetuity. It is made available under aCC-BY-NC-ND 4.0 International license.

Running head: fruiting body genomics

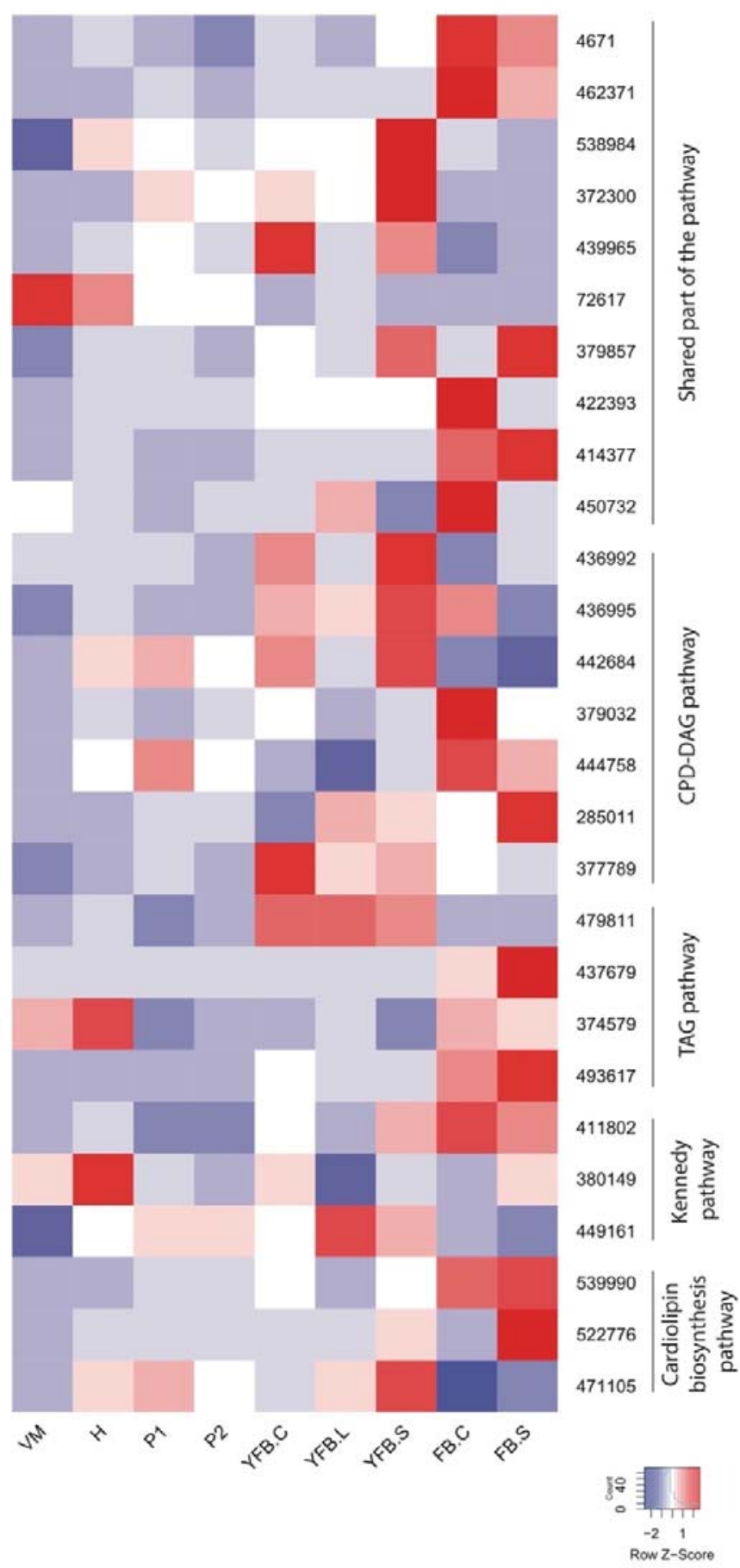

Fig. 7. Expression heatmap of membrane phospholipid and fatty acid biosynthetic genes in C. cinerea. Genes are denoted by Protein IDs. Blue and red colors represent low and high expression, respectively. Developmental stages are abbreviated as follows: VM - vegetative mycelium, $\mathrm{H}$ - hyphal knot, P1 - stage 1 primordium, P2 - stage 2 primordium, YFB.C young fruiting body cap, YFB.L - young fruiting body gills, YFB.S - young fruiting body stipe, FB.C - mature fruiting body cap (including gills), FB.S - mature fruiting body stipe. 
bioRxiv preprint doi: https://doi.org/10.1101/2021.12.09.471732; this version posted December 10,2021 . The copyright holder for this preprint (which was not certified by peer review) is the author/funder, who has granted bioRxiv a license to display the preprint in perpetuity. It is made available under aCC-BY-NC-ND 4.0 International license.

Running head: fruiting body genomics

7099

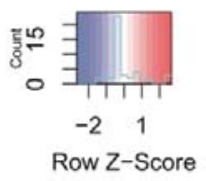

$$
\text { narreso }
$$

Fig. 8 Expression heatmap of putatively linoleic acid biosynthesis related fatty acid desaturase genes in C. cinerea. Genes are denoted by Protein IDs. Blue and red colors represent low and high expression, respectively. Developmental stages are abbreviated as follows: VM - vegetative mycelium, $\mathrm{H}$ - hyphal knot, $\mathrm{P} 1$ - stage 1 primordium, P2 - stage 2 primordium, YFB.C - young fruiting body cap, YFB.L - young fruiting body gills, YFB.S young fruiting body stipe, FB.C - mature fruiting body cap (including gills), FB.S - mature 
bioRxiv preprint doi: https://doi.org/10.1101/2021.12.09.471732; this version posted December 10,2021 . The copyright holder for this preprint (which was not certified by peer review) is the author/funder, who has granted bioRxiv a license to display the preprint in perpetuity. It is made available under aCC-BY-NC-ND 4.0 International license.

Running head: fruiting body genomics

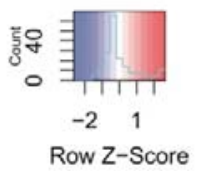

Fig. 9. Expression heatmap of storage carbohydrate metabolism genes in C. cinerea. Genes are denoted by Protein IDs. Blue and red colors represent low and high expression, respectively. Developmental stages are abbreviated as follows: VM - vegetative mycelium, $\mathrm{H}$ - hyphal knot, $\mathrm{P} 1$ - stage 1 primordium, P2 - stage 2 primordium, YFB.C - young fruiting body cap, YFB.L - young fruiting body gills, YFB.S - young fruiting body stipe, FB.C mature fruiting body cap (including gills), FB.S - mature fruiting body stipe. 
bioRxiv preprint doi: https://doi.org/10.1101/2021.12.09.471732; this version posted December 10,2021 . The copyright holder for this preprint (which was not certified by peer review) is the author/funder, who has granted bioRxiv a license to display the preprint in perpetuity. It is made available under aCC-BY-NC-ND 4.0 International license.

Running head: fruiting body genomics

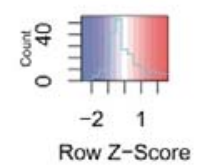

Fig. 10 Expression heatmap of chromatin remodeling related genes (histones, histone chaperones, histone deacethylases) in C. cinerea. Genes are denoted by Protein IDs. Blue and red colors represent low and high expression, respectively. Developmental stages are abbreviated as follows: VM - vegetative mycelium, $\mathrm{H}$ - hyphal knot, $\mathrm{P} 1$ - stage 1 primordium, P2 - stage 2 primordium, YFB.C - young fruiting body cap, YFB.L - young fruiting body gills, YFB.S - young fruiting body stipe, FB.C - mature fruiting body cap (including gills), FB.S - mature fruiting body stipe. 
bioRxiv preprint doi: https://doi.org/10.1101/2021.12.09.471732; this version posted December 10,2021 . The copyright holder for this preprint (which was not certified by peer review) is the author/funder, who has granted bioRxiv a license to display the preprint in perpetuity. It is made available under aCC-BY-NC-ND 4.0 International license.

Running head: fruiting body genomics

7126

7127

7128

7129

7130

7131

7132

7133
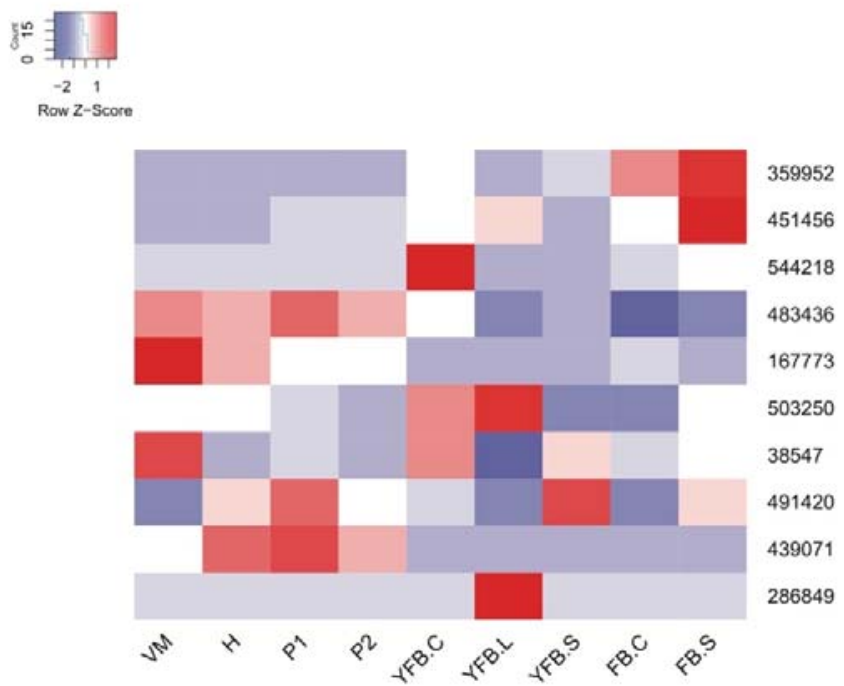

Fig. 11. Expression heatmap of expansin-encoding genes in C. cinerea. Genes are denoted by Protein IDs. Blue and red colors represent low and high expression, respectively.

Developmental stages are abbreviated as follows: $\mathrm{VM}$ - vegetative mycelium, $\mathrm{H}$ - hyphal knot, P1 - stage 1 primordium, P2 - stage 2 primordium, YFB.C - young fruiting body cap, YFB.L - young fruiting body gills, YFB.S - young fruiting body stipe, FB.C - mature fruiting body cap (including gills), FB.S - mature fruiting body stipe.

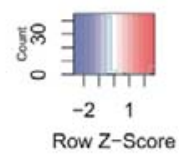

Fig. 12. Expression heatmap of Con6 family cell surface protein-encoding genes in $C$. cinerea. Genes are denoted by Protein IDs. Blue and red colors represent low and high expression, respectively. Developmental stages are abbreviated as follows: VM - vegetative mycelium, $\mathrm{H}$ - hyphal knot, $\mathrm{P} 1$ - stage 1 primordium, $\mathrm{P} 2$ - stage 2 primordium, YFB.C young fruiting body cap, YFB.L - young fruiting body gills, YFB.S - young fruiting body stipe, FB.C - mature fruiting body cap (including gills), FB.S - mature fruiting body stipe. 
bioRxiv preprint doi: https://doi.org/10.1101/2021.12.09.471732; this version posted December 10,2021 . The copyright holder for this preprint (which was not certified by peer review) is the author/funder, who has granted bioRxiv a license to display the preprint in perpetuity. It is made available under aCC-BY-NC-ND 4.0 International license.

Running head: fruiting body genomics
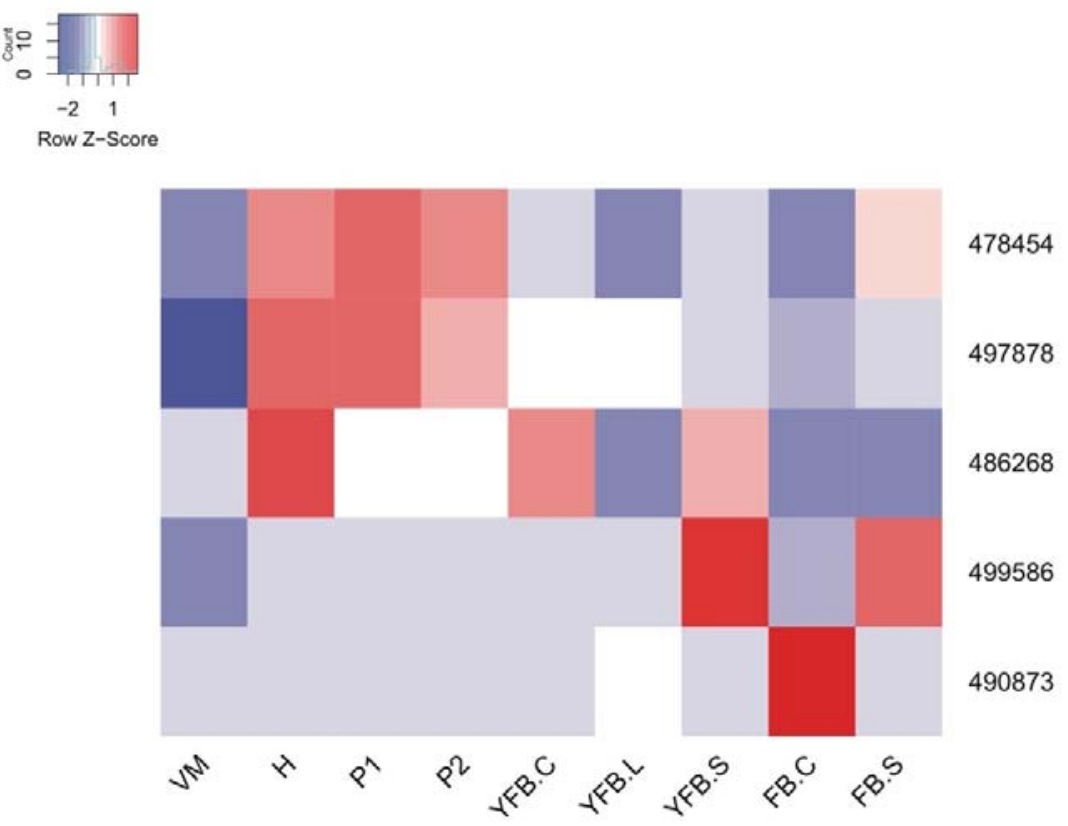

Fig. 13. Expression heatmap of putative wax synthase encoding genes in C. cinerea. Genes are denoted by Protein IDs. Blue and red colors represent low and high expression, respectively. Developmental stages are abbreviated as follows: VM - vegetative mycelium, $\mathrm{H}$ - hyphal knot, $\mathrm{P} 1$ - stage 1 primordium, $\mathrm{P} 2$ - stage 2 primordium, YFB.C - young fruiting body cap, YFB.L - young fruiting body gills, YFB.S - young fruiting body stipe, FB.C mature fruiting body cap (including gills), FB.S - mature fruiting body stipe.
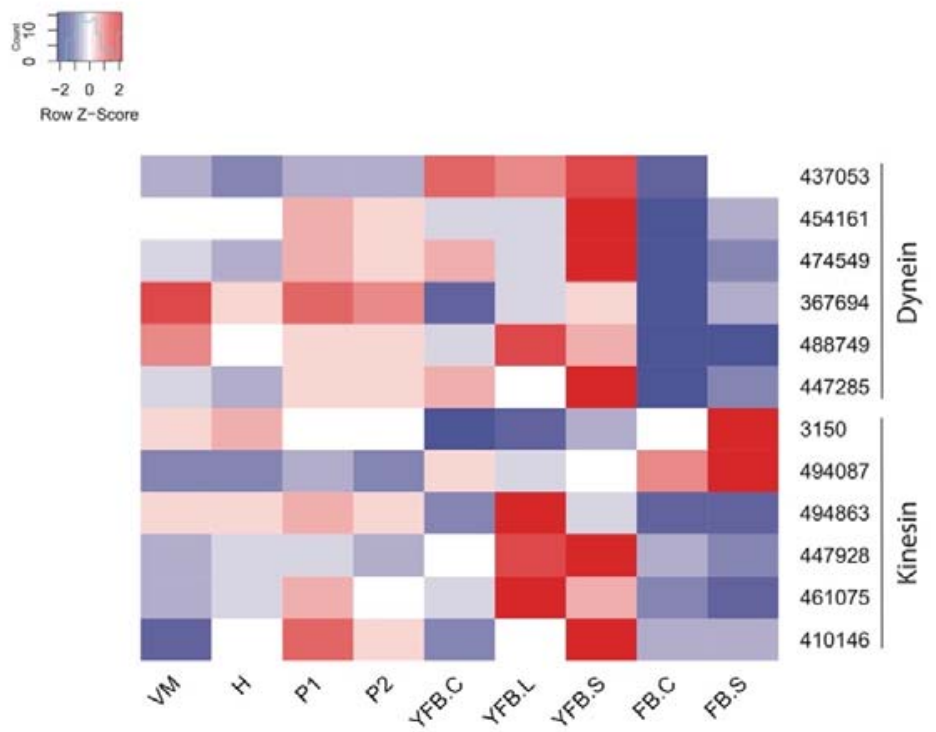

Fig. 14. Expression heatmap of kinesin genes and members of the Dynactin complex in $C$. cinerea. Genes are denoted by Protein IDs. Blue and red colors represent low and high expression, respectively. Developmental stages are abbreviated as follows: VM - vegetative mycelium, $\mathrm{H}$ - hyphal knot, $\mathrm{P} 1$ - stage 1 primordium, $\mathrm{P} 2$ - stage 2 primordium, YFB.C young fruiting body cap, YFB.L - young fruiting body gills, YFB.S - young fruiting body stipe, FB.C - mature fruiting body cap (including gills), FB.S - mature fruiting body stipe. 
bioRxiv preprint doi: https://doi.org/10.1101/2021.12.09.471732; this version posted December 10,2021 . The copyright holder for this preprint (which was not certified by peer review) is the author/funder, who has granted bioRxiv a license to display the preprint in perpetuity. It is made available under aCC-BY-NC-ND 4.0 International license.

Running head: fruiting body genomics

7156

7157

7158

7159

7160

7161

7162

7163

7164

7165

7166

7167

7168

7169

7170

7171

7172

7173

7174

7175

7176

7177

7178

7179

7180

7181

7182

7183

7184
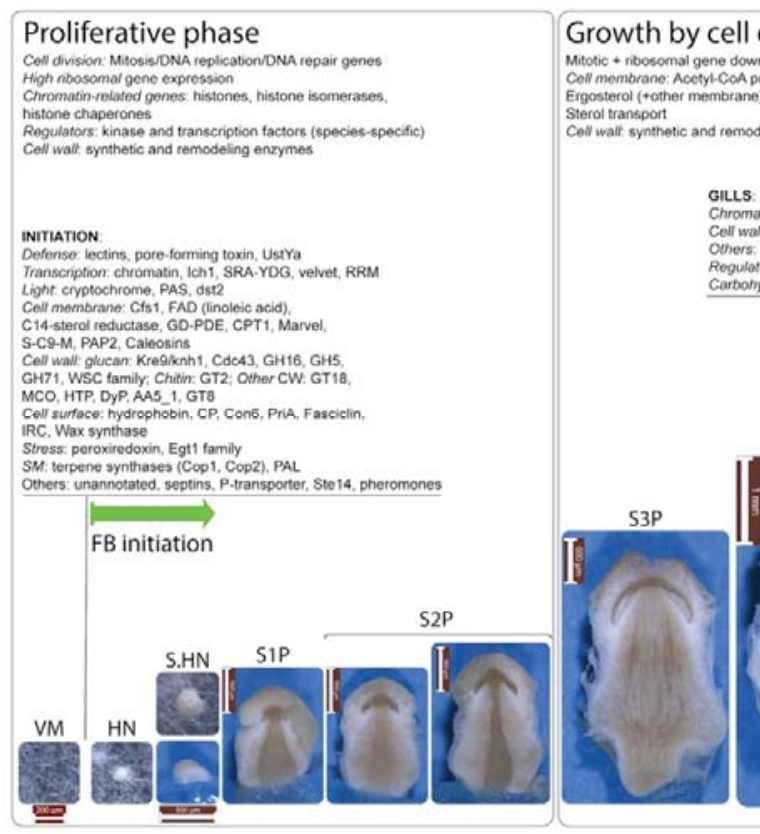

Fig. 15: The most important, conserved genes belonging to each phase are shown - genes with limited expression conservation or species-specific expression are not depicted.

[footnote: *pigment patterns vary by species] TP: trehalose phosphorylase, PKS: pyloketide synthase; OSH: Oxysterol Binding Protein Homologue; AQP: aquaporins; EXP: expansins; MCO: multicopper oxidase; RRM: RNA recognition motif family proteins; PAP2:

diacylglycerol pyrophosphate phosphatases; FAD: fatty acid desaturase; GD-PDE:

Glycerophosphodiester phosphodiesterases; S-C9-M: sphingolipid C9-methyltransferases;

IRC: intradiol ring cleavage dioxygenase; PAL: phenylalanine ammonia-lyase; SM:

secondary metabolism; HTP: heme-thiolate peroxidase; CP: cerato-platanin. genes

associated with cap autolysis are not shown on this Figure, because it is a species-specific

trait of $C$. cinerea. Note that light induction schemes are not shown on this Figure, as this varies from species to species.

\section{Table Legends}

Supplementary Table 1. Table summarizing ortholog protein ID for all discussed orthogroups in twelve Agaricomycetes.

Supplementary Table 2. Summary of read numbers and alignments in the analyzed fruiting body transcriptomes.

Supplementary Table 3. Summary of developmental expression dynamics of CDE orthogroups of genes related to alternative splicing across 12 species. Protein ID of a representative protein is given follows by the number of species in which the orthogroup is developmentally regulated at fold change 2 and 4 (FC>2 and $F C>4$, respectively). Putative function and ortholog in S. cerevisiae (if any) are also given. Abbreviations: 0-gene absent, 1-gene present but not developmentally regulated, 2 - developmentally regulated at fold change $>2$, 4- developmentally regulated at fold change $>4$. 
bioRxiv preprint doi: https://doi.org/10.1101/2021.12.09.471732; this version posted December 10, 2021. The copyright holder for this preprint

(which was not certified by peer review) is the author/funder, who has granted bioRxiv a license to display the preprint in perpetuity. It is made available under aCC-BY-NC-ND 4.0 International license.

Running head: fruiting body genomics

7185

7186 Supplementary table 4. Copy number distributions of genes related to protein ubiquitination

7187

7188

7189

7190

7191

7192

7193

7194

7195

7196

7197

7198

7199

7200

7201

7202

7203

7204

7205

7206

and the proteasome in twelve examined Agaricomycetes.

Supplementary table 5 . Genes related to protein ubiquitination and the proteasome in $C$. cinerea.

Supplementary Table 6 . Summary of developmental expression dynamics of CDE orthogroups of unannotated genes across 12 species. Protein ID of a representative protein is given follows by the number of species in which the orthogroup is developmentally regulated at fold change 2 and 4 (FC>2 and FC>4, respectively). Putative function and ortholog in S. cerevisiae (if any) are also given. Abbreviations: 0-gene absent, 1-gene present but not developmentally regulated, 2 - developmentally regulated at fold change $>2$, 4 - developmentally regulated at fold change $>4$.

Supplementary Table 7. Summary of developmental expression dynamics of CDE orthogroups of functionally poorly known genes across 12 species. Protein ID of a representative protein is given follows by the number of species in which the orthogroup is developmentally regulated at fold change 2 and 4 (FC>2 and $F C>4$, respectively). Putative function and ortholog in S. cerevisiae (if any) are also given. Abbreviations: 0-gene absent, 1 -gene present but not developmentally regulated, 2 - developmentally regulated at fold change $>2$, 4- developmentally regulated at fold change $>4$. 
Table 1. Summary of developmental expression dynamics of CDE orthogroups of cell divisionrelated genes across 12 species. Protein ID of a representative protein is given follows by the number of species in which the orthogroup is developmentally regulated at fold change 2 and 4 (FC>2 and FC>4, respectively). Putative function and ortholog in S. cerevisiae (if any) are also given. Abbreviations: 0-gene absent, 1-gene present but not developmentally regulated, 2 developmentally regulated at fold change $>2$, 4- developmentally regulated at fold change $>4$. Species names are abbreviated as: $\mathrm{Cc}-C$. cinerea, $\mathrm{Aa}-A$. ampla, Ab - A. bisporus, Ao $-A$. ostoyae, $\mathrm{Ca}-C$. aegerita, $\mathrm{Lb}-\mathrm{L}$. bicolor, Le - L. edodes, $\mathrm{Lt}-\mathrm{L}$. tigrinus, Mk $-M$. kentingensis, $\mathrm{Pc}$ - Ph. chrysosporium, Po-P. ostreatus, Sc - S. commune.

\begin{tabular}{|c|c|c|c|}
\hline \multirow[b]{2}{*}{ ID } & \multicolumn{2}{|c|}{ Fold change } & \multirow[b]{2}{*}{ Putative function } \\
\hline & $>2$ & $>4$ & \\
\hline $\begin{array}{l}\text { S.c. } \\
205281 \\
9\end{array}$ & 6 & 5 & $\begin{array}{l}\text { DNA endonuclease involved in } \\
\text { DNA excision repair }\end{array}$ \\
\hline $\begin{array}{l}\text { A.o. } \\
268345\end{array}$ & 7 & 5 & $\begin{array}{l}\text { DNA recombination and repair } \\
\text { protein Rad51-like }\end{array}$ \\
\hline 365932 & 11 & 3 & $\begin{array}{l}\text { DNA recombination and repair } \\
\text { protein Rad51-like }\end{array}$ \\
\hline $\begin{array}{l}\text { S.c. } \\
246364 \\
5\end{array}$ & 7 & 4 & DNA repair protein $\operatorname{Rad} 52 / 59 / 22$ \\
\hline 468197 & 7 & 4 & $\begin{array}{l}\text { Postreplication repair E3 ubiquitin- } \\
\text { protein ligase RAD18 }\end{array}$ \\
\hline 493516 & 9 & 6 & $\begin{array}{l}\text { DNA repair and recombination } \\
\text { protein RDH54 }\end{array}$ \\
\hline 439328 & 5 & 3 & DNA repair protein RAD59 \\
\hline 175000 & 10 & 4 & $\begin{array}{l}\text { DNA mismatch repair protein } \\
\text { MSH6 }\end{array}$ \\
\hline 354321 & 10 & 5 & $\begin{array}{l}\text { DNA repair and recombination } \\
\text { protein RAD54 }\end{array}$ \\
\hline 60974 & 9 & 6 & $\begin{array}{l}\text { ATP-dependent DNA helicase } \\
\text { SRS2 }\end{array}$ \\
\hline 438569 & 8 & 5 & Nucleoside diphosphate kinase \\
\hline 405038 & 11 & 6 & Flap endonuclease 1 \\
\hline 531259 & 10 & 5 & DNA repair protein RAD5 \\
\hline 492739 & 11 & 7 & $\begin{array}{l}\text { Double-strand break repair protein } \\
\text { MRE11 }\end{array}$ \\
\hline 438045 & 12 & 5 & Ribonuclease $\mathrm{H} 2$ subunit $\mathrm{A}$ \\
\hline 444936 & 7 & 3 & DNA repair protein RAD50 \\
\hline 541864 & 11 & 7 & $\begin{array}{l}\text { DNA mismatch repair protein } \\
\text { MSH2 }\end{array}$ \\
\hline 493340 & 8 & 3 & $\begin{array}{l}\text { Serine/threonine-protein kinase } \\
\text { RAD53 }\end{array}$ \\
\hline 360650 & 11 & 6 & AP endonuclease 1 \\
\hline 444409 & 9 & 5 & BRCT domain containing protein \\
\hline 463086 & 9 & 5 & DNA damage checkpoint protein 1 \\
\hline 461486 & 8 & 2 & DNA repair metallo-beta-lactamase \\
\hline 436861 & 8 & 2 & DNA repair protein crb2 \\
\hline 369412 & 8 & 3 & $\begin{array}{l}\text { Kinetochore-associated protein } \\
\text { DSN1 }\end{array}$ \\
\hline 359386 & 9 & 6 & Ribonuclease $\mathrm{H} 2$ subunit $\mathrm{B}$ \\
\hline
\end{tabular}

$S$. cerevisiae
ortholog Ce Aa Ab Ao Ca Lb Le Lt Mk Pc Po Sc

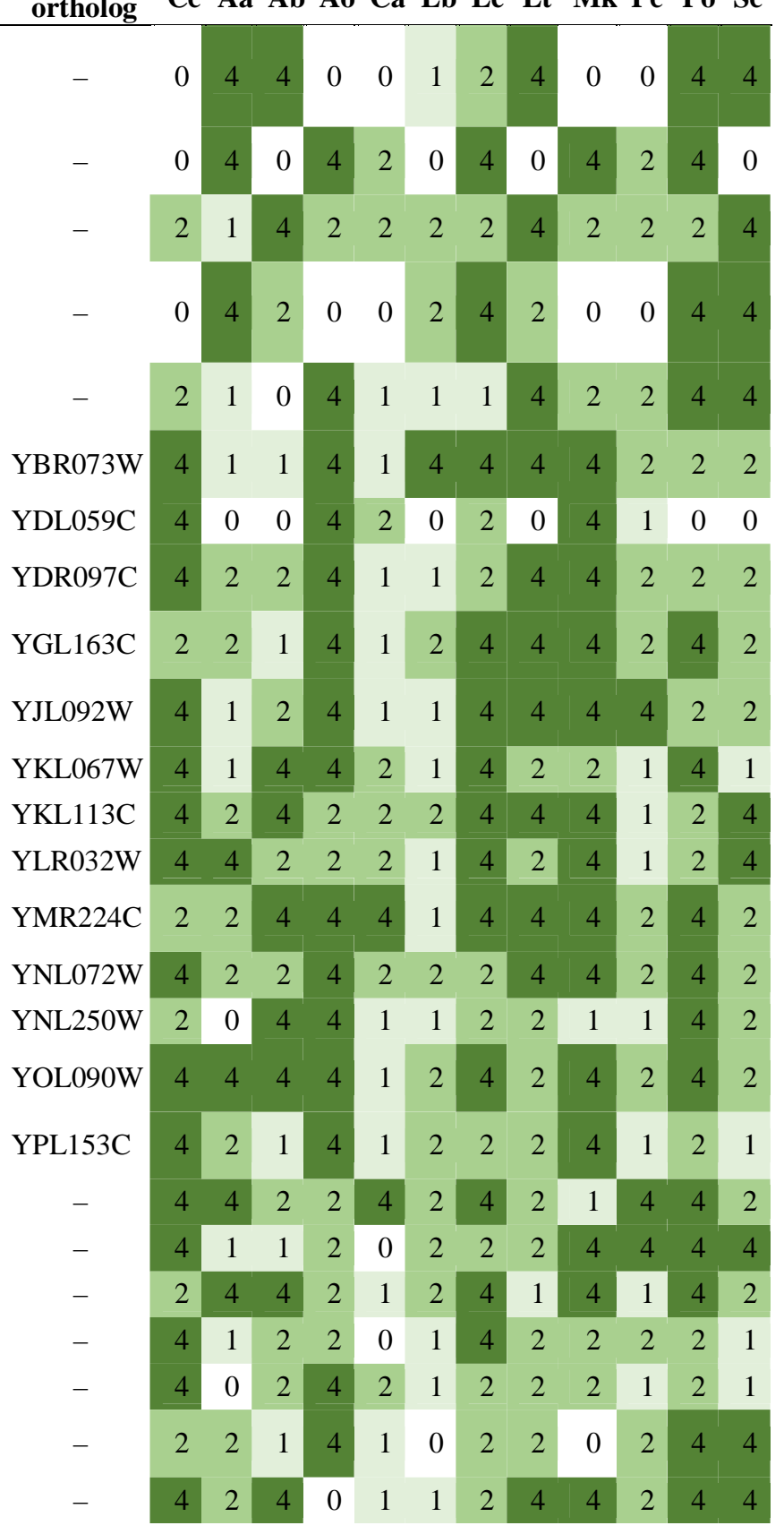




\begin{tabular}{|c|c|c|c|}
\hline 359391 & 11 & 7 & UV-damage endonuclease \\
\hline 498107 & 10 & 9 & Cell division control protein 6 \\
\hline 442457 & 10 & 4 & Checkpoint protein hus 1 \\
\hline 402634 & 12 & 5 & $\begin{array}{l}\text { Chromatin assembly factor } 1 \\
\text { subunit A }\end{array}$ \\
\hline 504757 & 10 & 5 & $\begin{array}{l}\text { Chromosome segregation in meiosis } \\
\text { protein } 3\end{array}$ \\
\hline 451918 & 12 & 7 & Kinesin \\
\hline 404203 & 9 & 7 & $\mathrm{~N}$-acetyltransferase ECO1 \\
\hline 509021 & 6 & 3 & DNA helicase \\
\hline 498847 & 8 & 4 & $\begin{array}{l}\text { Origin recognition complex } \\
\text { associated protein }\end{array}$ \\
\hline 465815 & 7 & 3 & Polynucleotide 3'-phosphatase \\
\hline 373872 & 10 & 6 & Topoisomerase 1-associated factor \\
\hline 358128 & 7 & 2 & Replication factor A protein 1 \\
\hline 435970 & 11 & 5 & $\begin{array}{l}\text { Serine/threonine-protein kinase } \\
\text { KIN3 }\end{array}$ \\
\hline 362687 & 9 & 3 & DNA polymerase alpha subunit $B$ \\
\hline 436862 & 9 & 5 & Kinesin-like protein KIP1 \\
\hline 364090 & 10 & 6 & Condensin complex subunit 2 \\
\hline 224847 & 11 & 7 & Replication factor $\mathrm{C}$ subunit 5 \\
\hline 374046 & 11 & 8 & Proliferating cell nuclear antigen \\
\hline 439635 & 10 & 8 & Pachytene checkpoint protein 2 \\
\hline 439612 & 12 & 10 & $\begin{array}{l}\text { Deoxyuridine 5'-triphosphate } \\
\text { nucleotidohydrolase }\end{array}$ \\
\hline 445594 & 11 & 4 & Cell division control protein 7 \\
\hline 445949 & 7 & 4 & $\begin{array}{l}\text { DNA polymerase delta catalytic } \\
\text { subunit }\end{array}$ \\
\hline 467336 & 12 & 8 & MutS protein homolog 5 \\
\hline 368409 & 10 & 5 & DNA ligase 1 \\
\hline 356564 & 10 & 7 & $\begin{array}{l}\text { DDK kinase regulatory subunit } \\
\text { DBF4 }\end{array}$ \\
\hline 469713 & 11 & 6 & Condensin complex subunit 3 \\
\hline 425950 & 11 & 6 & $\begin{array}{l}\text { Ribonucleoside-diphosphate } \\
\text { reductase large chain } 1\end{array}$ \\
\hline 436798 & 12 & 8 & $\begin{array}{l}\text { Uncharacterized ATP-dependent } \\
\text { helicase IRC5 }\end{array}$ \\
\hline 358096 & 12 & 9 & $\begin{array}{l}\text { ATP-dependent DNA helicase } \\
\text { MER3 }\end{array}$ \\
\hline 521062 & 11 & 4 & $\begin{array}{l}\text { Minichromosome maintenance } \\
\text { protein } 10\end{array}$ \\
\hline 411896 & 8 & 3 & $\begin{array}{l}\text { ATP-dependent DNA helicase } \\
\text { MPH1 }\end{array}$ \\
\hline 448596 & 10 & 4 & DNA primase small subunit \\
\hline 386329 & 12 & 10 & $\begin{array}{l}\text { Ribonucleoside-diphosphate } \\
\text { reductase small chain } 1\end{array}$ \\
\hline 368398 & 11 & 7 & $\begin{array}{l}\text { Mitotic spindle checkpoint } \\
\text { component MAD2 }\end{array}$ \\
\hline 373206 & 12 & 9 & Replication factor $\mathrm{C}$ subunit 2 \\
\hline 90 & 7 & 3 & $\begin{array}{l}\text { Structural maintenance of } \\
\text { chromosomes protein } 4\end{array}$ \\
\hline 500550 & 9 & 7 & Cell division control protein 45 \\
\hline
\end{tabular}

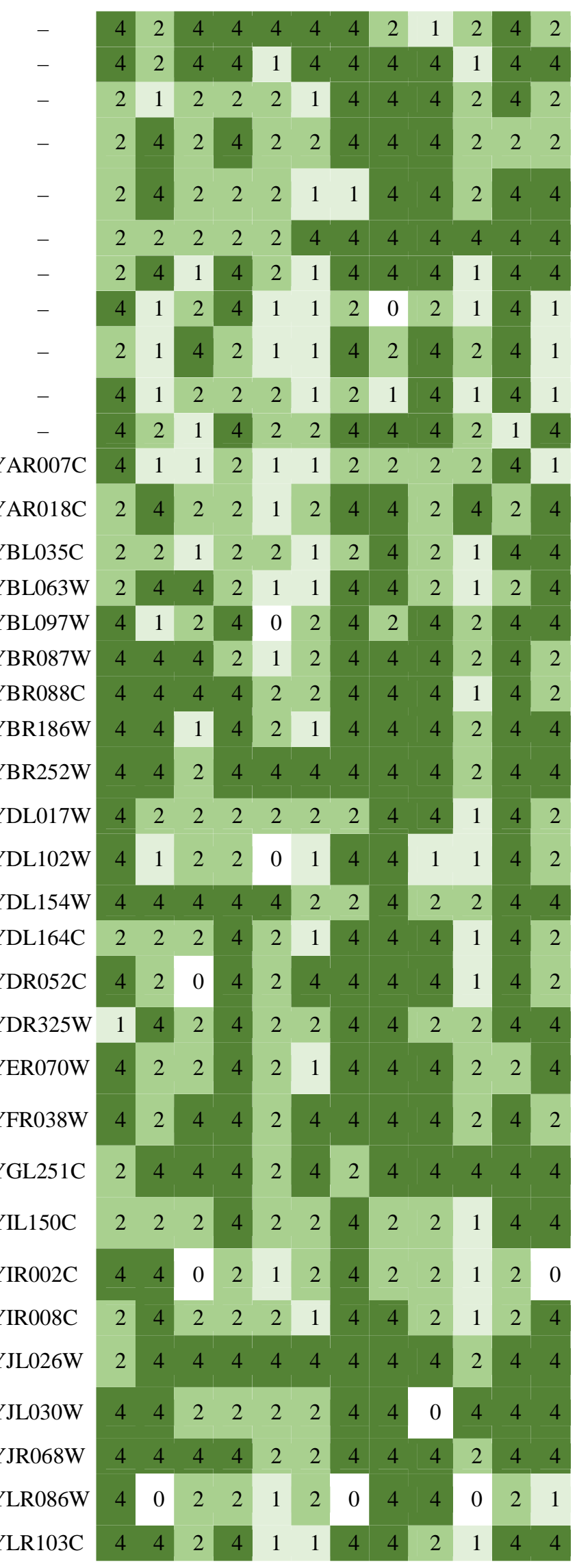




\begin{tabular}{|c|c|c|c|}
\hline 471179 & 12 & 9 & G2/mitotic-specific cyclin-4 \\
\hline 448884 & 9 & 6 & DNA topoisomerase 3 \\
\hline 399877 & 8 & 1 & FACT complex subunit POB3 \\
\hline 360590 & 11 & 8 & $\begin{array}{l}\text { Chromosome transmission fidelity } \\
\text { protein } 18\end{array}$ \\
\hline 173502 & 11 & 7 & ATP-dependent helicase SGS1 \\
\hline 449605 & 10 & 2 & $\begin{array}{l}\text { DNA polymerase epsilon catalytic } \\
\text { subunit A }\end{array}$ \\
\hline 471912 & 11 & 6 & Replication factor $\mathrm{C}$ subunit 3 \\
\hline 498594 & 12 & 6 & Thymidylate synthase \\
\hline 403599 & 10 & 3 & ATP-dependent helicase ULS1 \\
\hline 448855 & 6 & 3 & Replication factor $\mathrm{C}$ subunit 1 \\
\hline 356401 & 10 & 7 & $\begin{array}{l}\text { ATP-dependent DNA helicase } \\
\text { CHL1 }\end{array}$ \\
\hline 498104 & 11 & 7 & $\begin{array}{l}\text { DNA polymerase alpha-binding } \\
\text { protein }\end{array}$ \\
\hline 467729 & 12 & 0 & DNA polymerase epsilon subunit $B$ \\
\hline 448702 & 7 & 2 & ATP-dependent DNA ligase \\
\hline 537653 & 10 & 3 & $\begin{array}{l}\text { E3 ubiquitin-protein ligase substrate } \\
\text { receptor MMS22 }\end{array}$ \\
\hline 49522 & 10 & 5 & $\begin{array}{l}\text { Mitochondrial morphogenesis } \\
\text { protein SLD7 }\end{array}$ \\
\hline 292223 & 11 & 5 & Alpha-actinin-binding protein \\
\hline 426459 & 9 & 2 & $\begin{array}{l}\text { Chromosome transmission fidelity } \\
\text { protein } 8\end{array}$ \\
\hline 367776 & 10 & 5 & DNA helicase \\
\hline 374638 & 10 & 5 & DNA helicase \\
\hline 447030 & 10 & 6 & DNA helicase \\
\hline 460017 & 10 & 5 & DNA polymerase delta subunit 3 \\
\hline 464859 & 12 & 10 & DNA replication associated protein \\
\hline 447452 & 10 & 6 & DNA replication factor \\
\hline 471732 & 11 & 8 & Formin \\
\hline 365191 & 11 & 5 & Replication factor A protein 3 \\
\hline 464852 & 9 & 4 & Telomere binding protein \\
\hline 539797 & 10 & 7 & TPX2 domain-containing protein \\
\hline 394344 & 10 & 1 & Cohesin subunit SCC3 \\
\hline 442128 & 7 & 1 & Sister chromatid cohesion protein 1 \\
\hline 542870 & 8 & 3 & $\begin{array}{l}\text { Structural maintenance of } \\
\text { chromosomes protein } 1\end{array}$ \\
\hline 406915 & 11 & 2 & $\begin{array}{l}\text { Struc } \\
\text { chrol }\end{array}$ \\
\hline 445120 & 10 & 1 & $\begin{array}{l}\text { DNA replication complex GINS } \\
\text { protein SLD5 }\end{array}$ \\
\hline 380189 & 8 & 3 & $\begin{array}{l}\text { DNA replication complex GINS } \\
\text { protein PSF1 }\end{array}$ \\
\hline 3 & 10 & 4 & $\begin{array}{l}\text { DNA replication complex GINS } \\
\text { protein PSF2 }\end{array}$ \\
\hline 439626 & 9 & 5 & $\begin{array}{l}\text { DNA replication complex GINS } \\
\text { protein PSF3 }\end{array}$ \\
\hline 193478 & 9 & 3 & $\begin{array}{l}\text { DNA replication licensing factor } \\
\text { MCM2 }\end{array}$ \\
\hline
\end{tabular}

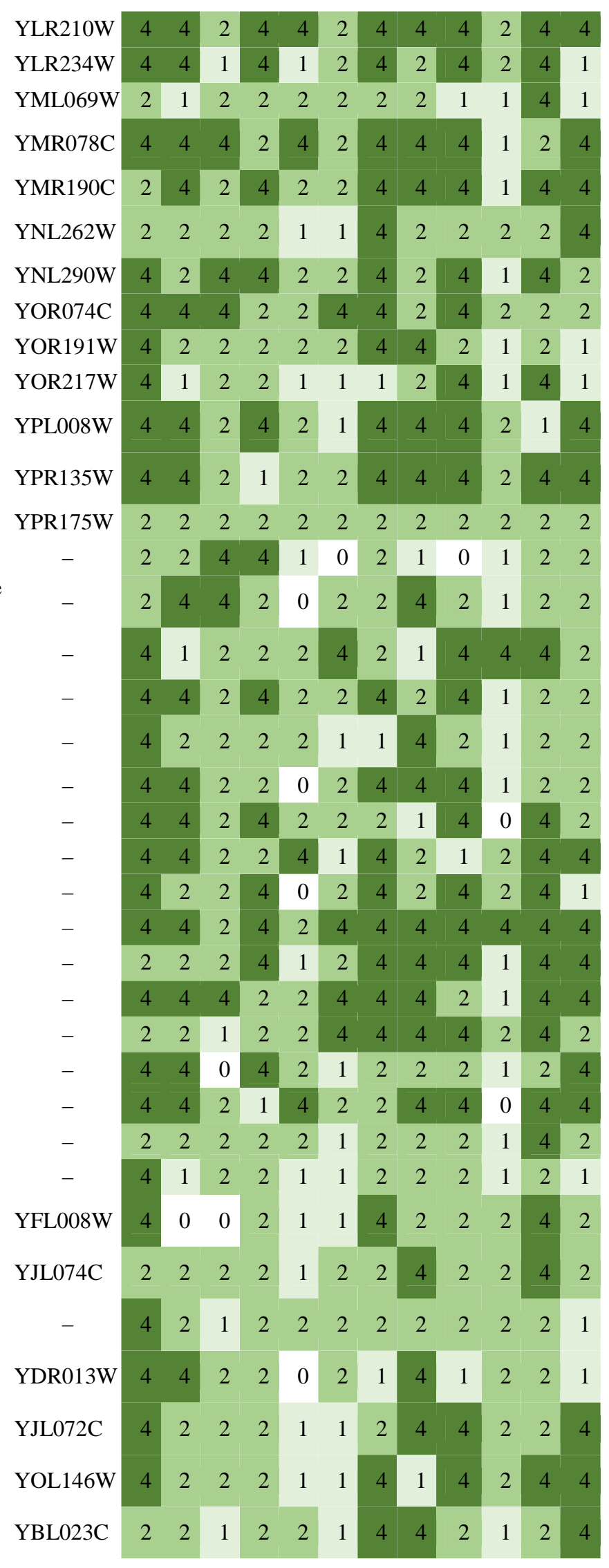




\begin{tabular}{|c|c|c|c|c|c|c|c|c|c|c|c|c|c|c|c|c|}
\hline 435526 & 11 & 5 & $\begin{array}{l}\text { DNA replication licensing factor } \\
\text { MCM7 }\end{array}$ & YBR202W & 2 & 2 & 4 & 2 & 2 & 1 & 4 & 4 & 2 & 2 & 4 & 4 \\
\hline 164525 & 9 & 5 & $\begin{array}{l}\text { DNA replication licensing factor } \\
\text { MCM3 }\end{array}$ & YEL032W & 2 & 4 & 2 & 2 & 2 & 1 & 4 & 4 & 1 & 1 & 4 & 4 \\
\hline 21772 & 10 & 4 & $\begin{array}{l}\text { DNA replication licensing factor } \\
\text { MCM6 }\end{array}$ & YGL201C & 2 & 2 & 1 & 4 & 1 & 2 & 4 & 4 & 2 & 2 & 4 & 2 \\
\hline 01934 & 10 & 6 & $\begin{array}{l}\text { Minichromosome maintenance } \\
\text { protein } 5\end{array}$ & YLR274W & 4 & 4 & 1 & 2 & 2 & 2 & 4 & 4 & 2 & 1 & 4 & 4 \\
\hline 402513 & 9 & 3 & $\begin{array}{l}\text { DNA replication licensing factor } \\
\text { MCM4 }\end{array}$ & YPR019W & 4 & 2 & 0 & 4 & 2 & 1 & 2 & 4 & 2 & 1 & 2 & 2 \\
\hline 50001 & 11 & 5 & Kinetochore protein NUF2 & - & 4 & 2 & 2 & 4 & 2 & 2 & 4 & 4 & 2 & 1 & 4 & 2 \\
\hline 393128 & 11 & 2 & Kinetochore protein NDC80 & YIL144W & 2 & 2 & 2 & 4 & 2 & 1 & 2 & 4 & 2 & 2 & 2 & 2 \\
\hline 167797 & 10 & 2 & Kinetochore protein SPC24 & - & 2 & 4 & 2 & 2 & 2 & 1 & 2 & 2 & 4 & 1 & 2 & 2 \\
\hline 453581 & 9 & 6 & Kinetochore protein SPC25 & - & 4 & 2 & 2 & 4 & 1 & 1 & 4 & 4 & 4 & 1 & 4 & 2 \\
\hline 366401 & 12 & 5 & E3 SUMO-protein ligase MMS21 & - & 2 & 4 & 2 & 2 & 2 & 2 & 4 & 4 & 4 & 2 & 4 & 2 \\
\hline 492711 & 10 & 6 & $\begin{array}{l}\text { Non-structural maintenance of } \\
\text { chromosomes element } 1\end{array}$ & - & 4 & 4 & 0 & 4 & 2 & 1 & 2 & 2 & 4 & 2 & 4 & 4 \\
\hline 412693 & 9 & 2 & $\begin{array}{l}\text { ural maintenance of } \\
\text { ne element } 4\end{array}$ & YDL105W & 2 & 2 & 2 & 0 & 2 & 1 & 2 & 4 & 2 & 1 & 4 & 2 \\
\hline 25048 & 8 & 5 & $\begin{array}{l}\text { Structur } \\
\text { chromo }\end{array}$ & YOL034W & 4 & 2 & 0 & 4 & 1 & 2 & 1 & 2 & 4 & 1 & 4 & 4 \\
\hline 499664 & 10 & 4 & $\begin{array}{l}\text { ural maintenance of } \\
\text { ne element } 3\end{array}$ & - & 2 & 2 & 1 & 4 & 2 & 4 & 2 & 2 & 4 & 2 & 4 & 1 \\
\hline 446459 & 9 & 6 & Meiotic nuclear division protein 1 & - & 4 & 1 & 2 & 4 & 0 & 2 & 4 & 2 & 4 & 1 & 4 & 4 \\
\hline $\begin{array}{l}\text { A.o. } \\
269495\end{array}$ & 7 & 7 & $\begin{array}{l}\text { Meiotic recombination protein } \\
\text { DMC1 }\end{array}$ & - & 0 & 4 & 0 & 4 & 4 & 4 & 4 & 0 & 4 & 4 & 0 & 0 \\
\hline 468689 & 10 & 8 & DNA helicase & - & 4 & 2 & 0 & 4 & 4 & 4 & 4 & 0 & 4 & 2 & 4 & 4 \\
\hline 371359 & 12 & 12 & $\mathrm{PHO}$ & YDL127W & 4 & 4 & 4 & 4 & 4 & 4 & 4 & 4 & 4 & 4 & 4 & 4 \\
\hline 502170 & 12 & 11 & MutS protein homolog 4 & YFL003C & 4 & 4 & 2 & 4 & 4 & 4 & 4 & 4 & 4 & 4 & 4 & 4 \\
\hline 238535 & 12 & 10 & Homologous-pai & YGL033W & 2 & 4 & 2 & 4 & 4 & 4 & 4 & 4 & 4 & 4 & 4 & 4 \\
\hline 368527 & 10 & 7 & Protein NAM8 & YHR086W & 4 & 2 & 1 & 4 & 4 & 4 & 4 & 2 & 4 & 2 & 4 & 1 \\
\hline 479000 & 11 & 8 & $\begin{array}{l}\text { Meiotic sister chromatid } \\
\text { recombination protein } 1\end{array}$ & YML128C & 4 & 2 & 2 & 4 & 4 & 4 & 4 & 2 & 4 & 1 & 4 & 4 \\
\hline 374758 & 9 & 4 & Spindle assembly checkpoint kinase & YPL209C & 2 & 2 & 4 & 4 & 1 & 2 & 4 & 4 & 1 & 2 & 2 & 1 \\
\hline $\begin{array}{l}\text { S.c. } \\
85873\end{array}$ & 9 & 7 & Nuclear fusion protein KAR5 & - & 0 & 4 & 2 & 4 & 1 & 2 & 4 & 4 & 0 & 4 & 4 & 4 \\
\hline 474563 & 6 & 4 & Holl & - & 4 & 0 & 0 & 4 & 2 & 2 & 0 & 1 & 0 & 4 & 4 & 0 \\
\hline 469496 & 6 & 2 & Meiosis specific protein & - & 4 & 1 & 2 & 1 & 2 & 1 & 1 & 2 & 4 & 1 & 2 & 1 \\
\hline 378537 & 12 & 12 & Meiotic coiled-coil protein & - & 4 & 4 & 4 & 4 & 4 & 4 & 4 & 4 & 4 & 4 & 4 & 4 \\
\hline 185247 & 7 & 0 & n ARP9 & - & 2 & 1 & 2 & 2 & 0 & 1 & 2 & 2 & 1 & 2 & 2 & 1 \\
\hline 447320 & 10 & 7 & $\begin{array}{l}\text { Altered inheritance of mitochondria } \\
\text { protein } 44\end{array}$ & - & 4 & 4 & 2 & 4 & 1 & 2 & 1 & 4 & 4 & 2 & 4 & 4 \\
\hline 465387 & 8 & 4 & Checkpoint protein RAD24 & - & 4 & 2 & 1 & 4 & 0 & 2 & 2 & 4 & 4 & 1 & 2 & 1 \\
\hline $\begin{array}{l}\text { S.c. } \\
257047 \\
6\end{array}$ & 4 & 1 & DNA polymerase IV & - & 2 & 1 & 0 & 2 & 2 & 1 & 1 & 0 & 0 & 1 & 4 & 1 \\
\hline 461421 & 8 & 2 & G1/S-specific cyclin CLN3 & - & 1 & 2 & 2 & 4 & 1 & 1 & 2 & 2 & 4 & 1 & 2 & 2 \\
\hline 404166 & 11 & 7 & Holliday junction resolvase YEN1 & - & 4 & 4 & 2 & 2 & 4 & 2 & 2 & 4 & 4 & 4 & 4 & 1 \\
\hline 182474 & 10 & 7 & M-phase inducer phosphatase & - & 4 & 4 & 2 & 2 & 1 & 2 & 4 & 4 & 4 & 1 & 4 & 4 \\
\hline 215276 & 11 & 6 & $\begin{array}{l}\text { Mediator of replication checkpoint } \\
\text { protein } 1\end{array}$ & - & 4 & 2 & 2 & 4 & 4 & 2 & 2 & 4 & 4 & 2 & 4 & 0 \\
\hline 472431 & 9 & 7 & Meiosis-specific protein SPO11 & - & 4 & 4 & 1 & 4 & 2 & 4 & 4 & 1 & 0 & 2 & 4 & 4 \\
\hline
\end{tabular}




\begin{tabular}{|c|c|c|c|c|c|c|c|c|c|c|c|c|c|c|c|c|}
\hline 461802 & 11 & 2 & Protein SFI1 & - & 2 & 2 & 2 & 4 & 1 & 2 & 2 & 2 & 2 & 2 & 4 & \\
\hline 269110 & 5 & 4 & DNA helicase & - & 4 & 4 & 0 & 4 & 0 & 0 & 4 & 0 & 0 & 0 & 0 & \\
\hline 65598 & 11 & 5 & $\begin{array}{l}\text { Serine/threonine-protein kinase } \\
\text { Haspin homolog ALK1 }\end{array}$ & - & 2 & 4 & 2 & 2 & 2 & 1 & 4 & 4 & 4 & 2 & 2 & \\
\hline 166770 & 10 & 4 & $\begin{array}{l}\text { Serine/threonine-protein kinase } \\
\text { MPS1 }\end{array}$ & - & 4 & 2 & 2 & 2 & 2 & 2 & 4 & 4 & 2 & 1 & 4 & \\
\hline 469725 & 10 & 4 & $\begin{array}{l}\text { Spindle assembly checkpoint } \\
\text { component MAD3 }\end{array}$ & - & 2 & 4 & 2 & 2 & 2 & 1 & 4 & 4 & 2 & 2 & 4 & 1 \\
\hline $\begin{array}{l}\text { S.c. } \\
250315 \\
8\end{array}$ & 8 & 7 & Uracil-DNA glycosylase & - & 0 & 4 & 4 & 4 & 2 & 4 & 4 & 0 & 4 & 0 & 0 & \\
\hline 442763 & 9 & 3 & $\begin{array}{l}\text { Kinetochore-associated protein } \\
\text { MTW1 }\end{array}$ & $\begin{array}{l}\text { YAL034W } \\
-A\end{array}$ & 4 & 1 & 2 & 1 & 2 & 1 & 2 & 4 & 4 & 2 & 2 & \\
\hline 476052 & 8 & 3 & $\begin{array}{l}\text { Origin recognition complex subunit } \\
2\end{array}$ & YBR060C & 4 & 1 & 1 & 2 & 2 & 2 & 4 & 4 & 1 & 2 & 2 & 1 \\
\hline 377937 & 7 & 5 & $\begin{array}{l}\text { Anaphase-promoting complex } \\
\text { subunit } 11\end{array}$ & YDL008W & 4 & 1 & 4 & 4 & 0 & 2 & 4 & 1 & 2 & 1 & 4 & 1 \\
\hline 518718 & 5 & 0 & $\begin{array}{l}\text { General transcription and DNA } \\
\text { repair factor IIH subunit TFB1 }\end{array}$ & YDR311W & 2 & 1 & 2 & 1 & 2 & 1 & 1 & 1 & 1 & 1 & 2 & 2 \\
\hline 479908 & 8 & 2 & $\begin{array}{l}\text { Tyrosine-protein phosphatase } \\
\text { CDC14 }\end{array}$ & YFR028C & 2 & 2 & 2 & 4 & 1 & 1 & 2 & 1 & 2 & 1 & 4 & 2 \\
\hline 394368 & 9 & 5 & $\begin{array}{l}\text { Structural maintenance of } \\
\text { chromosomes protein } 2\end{array}$ & YFR031C & 4 & 0 & 2 & 2 & 1 & 2 & 4 & 4 & 4 & 1 & 2 & 4 \\
\hline 74935 & 8 & 5 & $\begin{array}{l}\text { Spindle pole body component } \\
\text { SPC105 }\end{array}$ & YGL093W & 4 & 2 & 2 & 4 & 2 & 1 & 0 & 4 & 4 & 1 & 4 & 1 \\
\hline 545958 & 12 & 7 & APC/C activator protein CDC20 & YGL116W & 2 & 4 & 2 & 2 & 2 & 4 & 4 & 4 & 4 & 4 & 4 & 2 \\
\hline 538972 & 9 & 3 & $\begin{array}{l}\text { Checkpoint serine/threonine-protein } \\
\text { kinase BUB } 1\end{array}$ & YGR188C & 2 & 4 & 2 & 2 & 1 & 1 & 4 & 4 & 1 & 2 & 2 & \\
\hline 439189 & 10 & 5 & Cell division control protein 6 & YJL194W & 2 & 4 & 2 & 4 & 2 & 1 & 2 & 4 & 4 & 2 & 4 & 1 \\
\hline 497872 & 5 & 0 & $\begin{array}{l}\text { Anaphase-promoting complex } \\
\text { subunit CDC16 }\end{array}$ & YKL022C & 2 & 2 & 1 & 2 & 1 & 1 & 2 & 1 & 2 & 1 & 1 & 1 \\
\hline 354027 & 9 & 3 & DNA primase large subunit & YKL045W & 2 & 4 & 1 & 2 & 1 & 1 & 2 & 4 & 4 & 2 & 2 & 2 \\
\hline 402736 & 5 & 1 & Inner kinetochore subunit MIF2 & YKL089W & 1 & 1 & 1 & 2 & 2 & 1 & 1 & 1 & 4 & 1 & 2 & 2 \\
\hline 439911 & 8 & 4 & Condensin complex subunit 1 & YLR272C & 4 & 1 & 2 & 2 & 1 & 1 & 4 & 4 & 2 & 2 & 4 & 1 \\
\hline 360249 & 8 & 6 & Uracil-DNA glycosylase & YML021C & 4 & 1 & 4 & 2 & 4 & 1 & 4 & 2 & 4 & 0 & 4 & 1 \\
\hline 403152 & 10 & 6 & $\begin{array}{l}\text { Cell cycle serine/threonine-protein } \\
\text { kinase CDC5/MSD2 }\end{array}$ & YMR001C & 4 & 2 & 1 & 2 & 1 & 2 & 4 & 4 & 4 & 4 & 4 & 2 \\
\hline 449882 & 11 & 4 & DNA topoisomerase 2 & YNL088W & 2 & 2 & 4 & 2 & 2 & 2 & 4 & 4 & 2 & 1 & 4 & 2 \\
\hline 441663 & 9 & 5 & $\begin{array}{l}\text { DNA polymerase alpha catalytic } \\
\text { subunit A }\end{array}$ & YNL102W & 4 & 4 & 1 & 2 & 0 & 1 & 4 & 4 & 2 & 2 & 4 & 2 \\
\hline 439045 & 11 & 8 & PHO85 cyclin-1 & YNL289W & 4 & 4 & 4 & 4 & 4 & 4 & 4 & 2 & 1 & 2 & 4 & 2 \\
\hline 375607 & 11 & 6 & Cell division control protein 31 & YOR257W & 4 & 2 & 2 & 2 & 1 & 4 & 4 & 4 & 4 & 2 & 4 & 2 \\
\hline 533614 & 12 & 11 & G2/mitotic-specific cyclin-2 & YPR119W & 4 & 4 & 2 & 4 & 4 & 4 & 4 & 4 & 4 & 4 & 4 & 4 \\
\hline 380995 & 10 & 8 & Kinesin-like protein KAR3 & YPR141C & 4 & 2 & 4 & 4 & 0 & 4 & 4 & 4 & 4 & 2 & 4 & 1 \\
\hline 438441 & 10 & 6 & $\begin{array}{l}\text { Anaphase spindle elongation protein } \\
1\end{array}$ & - & 2 & 2 & 1 & 2 & 0 & 4 & 4 & 4 & 4 & 2 & 4 & 4 \\
\hline 114016 & 9 & 6 & Chromo/chromo shadow domain & - & 4 & 2 & 4 & 4 & 2 & 1 & 2 & 1 & 4 & 1 & 4 & 4 \\
\hline 522601 & 7 & 3 & DASH complex subunit Dam1 & - & 4 & 1 & 1 & 4 & 2 & 2 & 2 & 4 & 2 & 1 & 0 & 1 \\
\hline 472683 & 7 & 3 & DNA replication regulator SLD2 & - & 4 & 1 & 0 & 2 & 1 & 2 & 4 & 2 & 0 & 1 & 4 & 2 \\
\hline 380283 & 7 & 4 & DNA replication regulator SLD3 & - & 2 & 1 & 2 & 4 & 0 & 2 & 4 & 1 & 4 & 1 & 4 & 0 \\
\hline 463767 & 7 & 1 & Dynactin subunit 3 & - & 2 & 1 & 1 & 2 & 2 & 2 & 0 & 2 & 4 & 1 & 2 & 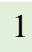 \\
\hline 466211 & 10 & 5 & Kinetochore protein & - & 2 & 1 & 2 & 2 & 2 & 2 & 4 & 4 & 4 & 1 & 4 & 4 \\
\hline 468013 & 3 & 2 & Mitotic-spindle organizing protein 1 & - & 4 & 1 & 0 & 2 & 0 & 1 & 1 & 0 & 0 & 1 & 4 & 1 \\
\hline
\end{tabular}




\begin{tabular}{|c|c|c|c|}
\hline 537412 & 9 & 1 & Nuclear rim protein 1 \\
\hline 431070 & 12 & 10 & $\begin{array}{l}\text { Anaphase-promoting complex } \\
\text { subunit } 4\end{array}$ \\
\hline 365564 & 9 & 7 & CCCh-type zinc finger protein \\
\hline 540942 & 8 & 1 & $\begin{array}{l}\text { DNA damage response (DDR) and } \\
\text { DNA repair protein }\end{array}$ \\
\hline $\begin{array}{l}\text { A.o. } \\
259630\end{array}$ & 4 & 3 & DNA helicase \\
\hline 446333 & 9 & 4 & DNA helicase \\
\hline 450108 & 7 & 0 & DNA helicase \\
\hline 460394 & 12 & 10 & $\operatorname{Rad} 21 / \operatorname{Rec} 8$-like protein \\
\hline $\begin{array}{l}\text { A.o. } \\
254302\end{array}$ & 8 & 5 & Ribonuclease $\mathrm{H} 2$; subunit $\mathrm{C}$ \\
\hline 374534 & 9 & 1 & Separase \\
\hline 496196 & 5 & 4 & SET domain protein \\
\hline 366767 & 10 & 5 & RecQ genome instability protein \\
\hline 493093 & 12 & 6 & Sporulation-specific protein 12 \\
\hline 443396 & 10 & 3 & Ku80 protein \\
\hline 443149 & 7 & 3 & Ku70 protein \\
\hline
\end{tabular}

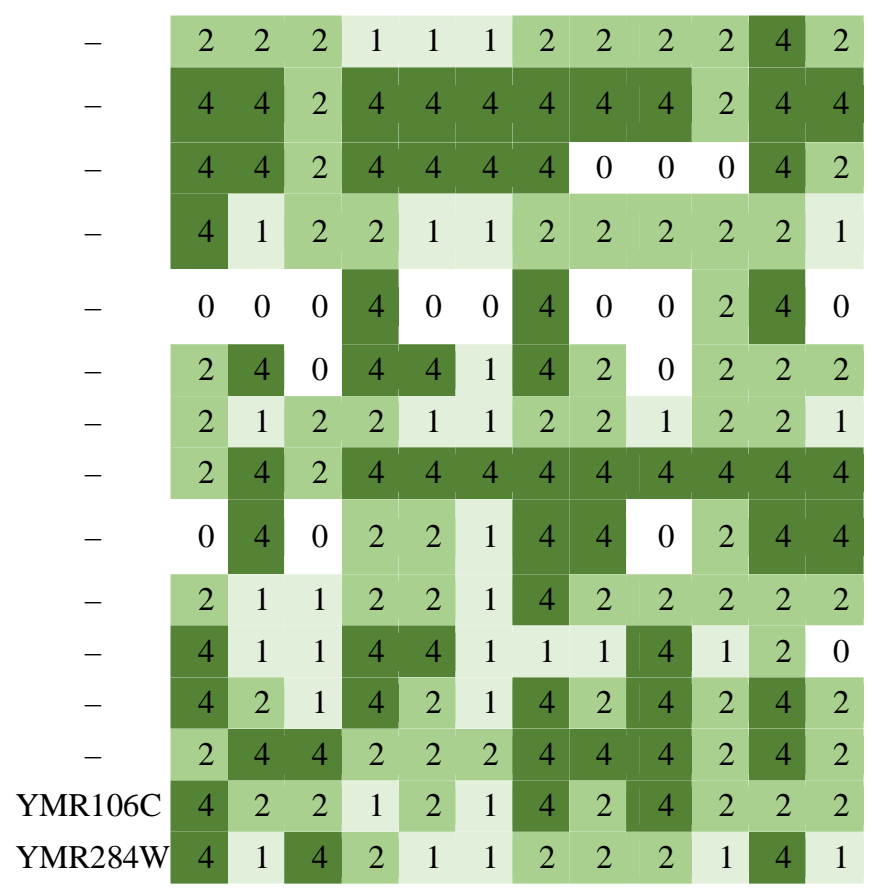

Table 2. Summary of developmental expression dynamics of CDE orthogroups of ribosomal genes across 12 species. Protein ID of a representative protein is given follows by the number of species in which the orthogroup is developmentally regulated at fold change 2 and 4 (FC>2 and $F C>4$, respectively). Putative function and ortholog in $S$. cerevisiae (if any) are also given. Abbreviations: 0-gene absent, 1-gene present but not developmentally regulated, 2 - developmentally regulated at fold change $>2$, 4- developmentally regulated at fold change $>4$. Species names are abbreviated as: $\mathrm{Cc}-C$. cinerea, $\mathrm{Aa}-A$. ampla, $\mathrm{Ab}-\mathrm{A}$. bisporus, $\mathrm{Ao}-\mathrm{A}$. ostoyae, $\mathrm{Ca}-\mathrm{C}$. aegerita, $\mathrm{Lb}-\mathrm{L}$. bicolor, $\mathrm{Le}-$ L. edodes, $\mathrm{Lt}-$ L. tigrinus, $\mathrm{Mk}-\mathrm{M}$. kentingensis, $\mathrm{Pc}-\mathrm{Ph}$. chrysosporium, $\mathrm{Po}-\mathrm{P}$. ostreatus, Sc - S. commune.

\begin{tabular}{lccll} 
& \multicolumn{3}{c}{ Fold change } & \\
\cline { 2 - 3 } ID & $>\mathbf{2}$ & $\mathbf{> 4}$ & Putative function \\
\hline 423325 & 10 & 3 & 60S ribosomal protein L28e \\
405648 & 10 & 2 & 60S ribosomal protein L19-B \\
412936 & 10 & 2 & 40S ribosomal protein S8-A \\
478794 & 11 & 3 & 60S ribosomal protein L23-A \\
366589 & 9 & 5 & 60S ribosomal protein L4-A \\
497971 & 7 & 5 & 40S ribosomal protein S11-B \\
464129 & 8 & 4 & 40S ribosomal protein S6-B \\
430785 & 8 & 2 & 60S ribosomal protein L21-A \\
467322 & 9 & 5 & 40S ribosomal protein S14-A \\
493413 & 9 & 1 & transport of pre-60S ribosomal \\
403137 & 11 & 4 & 60S ribosomal protein L31-A \\
398174 & 9 & 3 & 40S ribosomal protein S16-B \\
412254 & 8 & 2 & 60S ribosomal protein L35-B \\
493053 & 10 & 4 & Putatively involved in
\end{tabular}

\begin{tabular}{|c|c|c|c|c|c|c|c|c|c|c|c|c|}
\hline \multirow{2}{*}{$\begin{array}{c}S . \\
\text { cerevisiae } \\
\text { ortholog }\end{array}$} & \multicolumn{12}{|c|}{ Species } \\
\hline & $\mathrm{Cc}$ & Aa & Ab & Ao & $\mathbf{C a}$ & Lb & Le & $\mathbf{L t}$ & Mk & Pc & Po & Sc \\
\hline - & 4 & 2 & 2 & 2 & 2 & 4 & 2 & 2 & 4 & 1 & 2 & 1 \\
\hline YBL027W & 4 & 2 & 2 & 2 & 2 & 4 & 2 & 2 & 2 & 1 & 2 & 1 \\
\hline YBL072C & 4 & 2 & 2 & 2 & 1 & 2 & 2 & 2 & 2 & 2 & 4 & 1 \\
\hline YBL087C & 4 & 2 & 2 & 2 & 2 & 4 & 2 & 2 & 4 & 2 & 2 & 1 \\
\hline YBR031W & 4 & 2 & 4 & 2 & 0 & 4 & 4 & 2 & 4 & 1 & 2 & 1 \\
\hline YBR048W & 4 & 2 & 4 & 0 & 2 & 4 & 0 & 1 & 4 & 1 & 4 & 1 \\
\hline YBR181C & 4 & 2 & 4 & 2 & 1 & 4 & 2 & 1 & 4 & 1 & 2 & 1 \\
\hline YBR191W & 4 & 2 & 2 & 2 & 2 & 1 & 4 & 1 & 0 & 2 & 2 & 1 \\
\hline YCR031C & 4 & 2 & 4 & 2 & 2 & 4 & 2 & 1 & 4 & 1 & 4 & 1 \\
\hline YCR072C & 4 & 2 & 2 & 2 & 1 & 2 & 2 & 2 & 2 & 1 & 2 & 1 \\
\hline YDL075W & 4 & 2 & 4 & 2 & 2 & 4 & 2 & 2 & 4 & 2 & 2 & 1 \\
\hline YDL083C & 4 & 2 & 2 & 2 & 2 & 2 & 2 & 1 & 4 & 1 & 4 & 1 \\
\hline YDL136W & 4 & 2 & 2 & 2 & 2 & 4 & 2 & 1 & 0 & 1 & 2 & 1 \\
\hline YDL208W & 4 & 2 & 2 & 4 & 2 & 2 & 2 & 2 & 4 & 1 & 4 & 1 \\
\hline
\end{tabular}




\begin{tabular}{|c|c|c|c|}
\hline & & & ridylation of rRNA \\
\hline 64051 & 9 & 4 & 40S ribosomal protein $\mathrm{S} 13$ \\
\hline 381793 & 10 & 4 & $\begin{array}{l}60 \mathrm{~S} \text { acidic ribosomal protein } \mathrm{P} 2- \\
\text { beta }\end{array}$ \\
\hline 459549 & 8 & 2 & 60S ribosomal protein L12-B \\
\hline 404670 & 11 & 4 & 40S ribosomal protein S17-B \\
\hline 442748 & 8 & 2 & 40S ribosomal protein S18-A \\
\hline 499692 & 10 & 5 & 60S ribosomal protein L27-B \\
\hline 429262 & 9 & 4 & 60S ribosomal protein L34-A \\
\hline 439980 & 9 & 3 & 40S ribosomal protein $\mathrm{S} 24-\mathrm{A}$ \\
\hline 329287 & 10 & 5 & 40S ribosomal protein S26-B \\
\hline 426937 & 9 & 2 & 60S ribosomal protein L2-A \\
\hline $\begin{array}{l}\text { S.c. } \\
274266 \\
7\end{array}$ & 10 & 4 & $60 \mathrm{~S}$ ribosomal protein $\mathrm{L} 7 \mathrm{~A}$ \\
\hline 441325 & 10 & 3 & $60 S$ ribosomal protein $\mathrm{L} 28$ \\
\hline 475518 & 10 & 4 & 40S ribosomal protein $\mathrm{S} 2$ \\
\hline 437986 & 9 & 4 & 60S ribosomal protein L1-B \\
\hline 480276 & 8 & 3 & 40S ribosomal protein $\mathrm{S} 25-\mathrm{A}$ \\
\hline 475978 & 10 & 3 & 60S ribosomal protein L26-B \\
\hline 130885 & 9 & 3 & 60S ribosomal protein L11-B \\
\hline 495378 & 5 & 0 & 40S ribosomal protein $\mathrm{S} 23-\mathrm{A}$ \\
\hline 379934 & 9 & 3 & 60S ribosomal protein L24-B \\
\hline 494449 & 10 & 4 & 40S ribosomal protein $\mathrm{S} 0-\mathrm{A}$ \\
\hline 467085 & 10 & 3 & 60S ribosomal protein L14-B \\
\hline 165767 & 9 & 3 & 40S ribosomal protein $\mathrm{S} 20$ \\
\hline 438384 & 6 & 1 & 40S ribosomal protein S27-B \\
\hline 214528 & 6 & 6 & $\begin{array}{l}\text { Protein involved in rRNA- } \\
\text { processing }\end{array}$ \\
\hline 441187 & 9 & 4 & 60S ribosomal protein L42-B \\
\hline 441652 & 7 & 3 & 40S ribosomal protein S4-B \\
\hline 497565 & 8 & 2 & 60S ribosomal protein L39 \\
\hline 375577 & 9 & 5 & $40 \mathrm{~S}$ ribosomal protein S22-A \\
\hline 385517 & 8 & 4 & 60S ribosomal protein L43-B \\
\hline 446338 & 10 & 3 & 40S ribosomal protein $\mathrm{S} 5$ \\
\hline 472703 & 8 & 2 & 60 S ribosomal protein L8-B \\
\hline 374411 & 7 & 2 & 60S ribosomal protein L22-A \\
\hline 471911 & 7 & 4 & $\begin{array}{l}\text { Ubiquitin-40S ribosomal protein } \\
\text { S31 }\end{array}$ \\
\hline 447700 & 7 & 3 & 60S ribosomal protein L37-A \\
\hline 353298 & 7 & 2 & 40S ribosomal protein S28-B \\
\hline 398047 & 7 & 3 & $60 \mathrm{~S}$ ribosomal protein $\mathrm{L} 38$ \\
\hline 367451 & 7 & 3 & $60 \mathrm{~S}$ acidic ribosomal protein $\mathrm{P} 0$ \\
\hline 398594 & 9 & 3 & 40S ribosomal protein S29-A \\
\hline 385868 & 10 & 4 & 60S ribosomal protein L6-B \\
\hline
\end{tabular}

\begin{tabular}{|c|c|c|c|c|c|c|c|c|c|c|c|c|}
\hline YDR064W & 4 & 2 & 4 & 2 & 1 & 4 & 2 & 2 & 2 & 1 & 4 & 1 \\
\hline YDR382W & 4 & 2 & 2 & 2 & 2 & 4 & 2 & 2 & 4 & 1 & 4 & 1 \\
\hline YDR418W & 4 & 2 & 2 & 2 & 1 & 4 & 2 & 1 & 2 & 1 & 2 & 0 \\
\hline YDR447C & 4 & 2 & 4 & 2 & 2 & 4 & 2 & 1 & 4 & 2 & 2 & 2 \\
\hline YDR450W & 4 & 2 & 2 & 2 & 0 & 4 & 2 & 1 & 0 & 1 & 2 & 2 \\
\hline YDR471W & 4 & 2 & 2 & 2 & 2 & 4 & 4 & 1 & 4 & 2 & 4 & 1 \\
\hline $\begin{array}{l}\text { YER056C- } \\
\text { A }\end{array}$ & 4 & 2 & 2 & 2 & 2 & 4 & 4 & 1 & 0 & 2 & 4 & 1 \\
\hline YER074W & 4 & 0 & 0 & 2 & 2 & 4 & 2 & 2 & 2 & 1 & 4 & 2 \\
\hline YER131W & 4 & 2 & 4 & 2 & 2 & 4 & 2 & 2 & 4 & 1 & 4 & 1 \\
\hline $\begin{array}{l}\text { YFR031C- } \\
\text { A }\end{array}$ & 4 & 2 & 2 & 2 & 2 & 4 & 2 & 1 & 0 & 2 & 2 & 1 \\
\hline YGL076C & 0 & 2 & 4 & 2 & 2 & 4 & 4 & 2 & 4 & 2 & 2 & 1 \\
\hline YGL103W & 4 & 2 & 2 & 2 & 2 & 4 & 2 & 1 & 4 & 2 & 2 & 1 \\
\hline YGL123W & 4 & 2 & 4 & 2 & 2 & 4 & 2 & 2 & 4 & 1 & 2 & 1 \\
\hline YGL135W & 4 & 2 & 2 & 2 & 2 & 4 & 2 & 1 & 4 & 1 & 4 & 1 \\
\hline YGR027C & 4 & 2 & 4 & 2 & 2 & 4 & 2 & 1 & 0 & 1 & 2 & 1 \\
\hline YGR034W & 4 & 2 & 2 & 0 & 2 & 4 & 4 & 2 & 2 & 2 & 2 & 1 \\
\hline YGR085C & 4 & 2 & 4 & 2 & 2 & 4 & 2 & 2 & 0 & 1 & 2 & 1 \\
\hline YGR118W & 2 & 1 & 2 & 2 & 1 & 2 & 1 & 1 & 0 & 2 & 1 & 1 \\
\hline YGR148C & 4 & 2 & 2 & 2 & 2 & 4 & 2 & 0 & 4 & 1 & 2 & 1 \\
\hline YGR214W & 4 & 2 & 4 & 2 & 2 & 4 & 2 & 2 & 4 & 1 & 2 & 1 \\
\hline YHL001W & 4 & 2 & 2 & 2 & 2 & 4 & 2 & 2 & 4 & 1 & 2 & 1 \\
\hline YHL015W & 4 & 2 & 2 & 2 & 2 & 4 & 2 & 2 & 0 & 1 & 4 & 1 \\
\hline YHR021C & 4 & 1 & 1 & 2 & 0 & 2 & 2 & 1 & 2 & 1 & 2 & 1 \\
\hline YHR087W & 4 & 1 & 4 & 4 & 1 & 1 & 4 & 4 & 1 & 1 & 4 & 0 \\
\hline YHR141C & 4 & 2 & 1 & 2 & 2 & 4 & 2 & 1 & 4 & 2 & 4 & 1 \\
\hline YHR203C & 4 & 2 & 0 & 2 & 0 & 4 & 2 & 1 & 4 & 1 & 2 & 1 \\
\hline YJL189W & 4 & 1 & 2 & 2 & 0 & 4 & 2 & 2 & 0 & 2 & 2 & 1 \\
\hline YJL190C & 4 & 2 & 4 & 2 & 2 & 4 & 4 & 1 & 4 & 1 & 2 & 1 \\
\hline $\begin{array}{l}\text { YJR094W- } \\
\text { A }\end{array}$ & 4 & 2 & 0 & 0 & 0 & 4 & 2 & 2 & 4 & 2 & 4 & 1 \\
\hline YJR123W & 4 & 2 & 4 & 2 & 2 & 4 & 2 & 1 & 2 & 2 & 2 & 1 \\
\hline YLL045C & 2 & 2 & 4 & 2 & 2 & 2 & 2 & 1 & 0 & 1 & 4 & 1 \\
\hline YLR061W & 2 & 2 & 4 & 2 & 2 & 1 & 2 & 1 & 0 & 1 & 4 & 1 \\
\hline YLR167W & 4 & 0 & 4 & 2 & 1 & 4 & 2 & 1 & 4 & 1 & 2 & 1 \\
\hline YLR185W & 4 & 0 & 2 & 2 & 0 & 4 & 2 & 1 & 4 & 1 & 2 & 1 \\
\hline YLR264W & 4 & 2 & 2 & 2 & 0 & 4 & 2 & 1 & 0 & 2 & 0 & 0 \\
\hline YLR325C & 4 & 2 & 2 & 0 & 0 & 4 & 2 & 1 & 0 & 2 & 4 & 1 \\
\hline YLR340W & 4 & 2 & 1 & 2 & 1 & 2 & 4 & 1 & 2 & 1 & 4 & 1 \\
\hline YLR388W & 4 & 2 & 2 & 2 & 0 & 4 & 2 & 2 & 0 & 2 & 4 & 1 \\
\hline YLR448W & 4 & 2 & 4 & 2 & 2 & 4 & 2 & 2 & 0 & 2 & 4 & 1 \\
\hline
\end{tabular}




\begin{tabular}{|c|c|c|c|}
\hline 474164 & 9 & 5 & 40S ribosomal protein S1-B \\
\hline 424304 & 8 & 4 & 60S ribosomal protein L13-B \\
\hline 456295 & 8 & 2 & 60S ribosomal protein L36-A \\
\hline 375811 & 8 & 3 & 60S ribosomal protein L20-A \\
\hline 412005 & 9 & 3 & $60 \mathrm{~S}$ ribosomal protein L9-B \\
\hline 416494 & 5 & 2 & 40S ribosomal protein $\mathrm{S} 3$ \\
\hline 438977 & 9 & 0 & $\begin{array}{l}\text { Ribosome-bound, Hsp70-type } \\
\text { chaperone }\end{array}$ \\
\hline 462840 & 8 & 4 & 60S ribosomal protein L18-B \\
\hline 21836 & 6 & 0 & $\begin{array}{l}\text { Putatively involved in nucleolar } \\
\text { processing of pre- } 18 \mathrm{~S} \text { ribosomal } \\
\text { RNA }\end{array}$ \\
\hline 381804 & 8 & 2 & 40S ribosomal protein $\mathrm{S} 15$ \\
\hline 465651 & 10 & 4 & 40S ribosomal protein S19-A \\
\hline 426850 & 7 & 4 & 60S ribosomal protein L3 \\
\hline 401275 & 9 & 6 & 40S ribosomal protein $\mathrm{S} 12$ \\
\hline 430789 & 10 & 4 & 40S ribosomal protein S9-A \\
\hline 467768 & 8 & 5 & 60S ribosomal protein L5 \\
\hline 356089 & 10 & 4 & 60S ribosomal protein L33-A \\
\hline 539347 & 5 & 3 & $60 S$ ribosomal protein L29 \\
\hline 394207 & 7 & 5 & $60 S$ ribosomal protein L30 \\
\hline 539473 & 7 & 2 & $60 \mathrm{~S}$ ribosomal protein L16-A \\
\hline
\end{tabular}

\begin{tabular}{|c|c|c|c|c|c|c|c|c|c|c|c|}
\hline YML063W & 4 & 2 & 4 & 2 & 2 & 4 & 4 & 1 & 2 & 1 & 4 \\
\hline YMR142C & 4 & 2 & 0 & 2 & 2 & 4 & 0 & 4 & 2 & 1 & 4 \\
\hline YMR194W & 4 & 2 & 2 & 2 & 0 & 4 & 2 & 2 & U & 1 & \\
\hline YMR242C & 4 & 2 & 4 & 2 & 0 & 4 & 2 & 1 & $?$ & 1 & \\
\hline YNL067W & 4 & 2 & 2 & 2 & 2 & 4 & 2 & 1 & 2 & 1 & 4 \\
\hline YNL178W & 4 & 2 & 0 & 0 & 1 & 4 & $?$ & 1 & 2 & 1 & \\
\hline YNL209W & 2 & 2 & 2 & 2 & 2 & 1 & 2 & 2 & $?$ & 1 & \\
\hline YNL301C & 4 & 2 & 4 & 2 & 1 & 4 & 2 & 1 & 2 & 1 & 4 \\
\hline YNL308C & 2 & 1 & 2 & 2 & 2 & 1 & 1 & 1 & 2 & 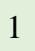 & 2 \\
\hline YOL040C & 4 & 2 & 2 & 2 & 0 & 4 & 2 & 1 & 2 & 1 & 2 \\
\hline YOL121C & 4 & 2 & 4 & 2 & 2 & 4 & 2 & 2 & 4 & 1 & 2 \\
\hline YOR063W & 4 & 2 & 2 & 0 & 2 & 4 & 0 & 1 & 4 & 1 & 4 \\
\hline YOR369C & 4 & 2 & 1 & 4 & 0 & 4 & 4 & 2 & 4 & 2 & 1 \\
\hline YPL081W & 4 & 2 & 4 & 2 & 2 & 4 & 2 & 2 & 4 & 1 & 2 \\
\hline YPL131W & 4 & 2 & 4 & 2 & 2 & 4 & 4 & 1 & 0 & 1 & 4 \\
\hline YPL143W & 4 & 2 & 2 & 0 & 2 & 4 & 2 & 2 & 4 & $?$ & 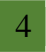 \\
\hline $\begin{array}{l}\text { YFR032C- } \\
\text { A }\end{array}$ & 4 & 2 & 0 & 0 & 0 & 4 & 4 & 1 & 0 & 2 & 0 \\
\hline YGL030W & 4 & 2 & 0 & 2 & 0 & 4 & 2 & 1 & 4 & 1 & 2 \\
\hline YIL133C & 4 & 2 & 2 & 0 & 2 & 4 & 2 & 1 & 0 & 1 & 2 \\
\hline
\end{tabular}

Table 3. Summary of developmental expression dynamics of CDE orthogroups of genes related to Acetyl-CoA production and basic metabolism across 12 species. Protein ID of a representative protein is given follows by the number of species in which the orthogroup is developmentally regulated at fold change 2 and 4 ( $F C>2$ and $F C>4$, respectively). Putative function and ortholog in S. cerevisiae (if any) are also given. Abbreviations: 0-gene absent, 1-gene present but not developmentally regulated, 2 - developmentally regulated at fold change $>2$, 4- developmentally regulated at fold change $>4$. Species names are abbreviated as: $\mathrm{Cc}-C$. cinerea, $\mathrm{Aa}-A$. ampla, $\mathrm{Ab}-A$. bisporus, $\mathrm{Ao}-A$. ostoyae, $\mathrm{Ca}-C$. aegerita, $\mathrm{Lb}$ - L. bicolor, Le - L. edodes, Lt - L. tigrinus, Mk - M. kentingensis, $\mathrm{Pc}-P h$. chrysosporium, $\mathrm{Po}-\mathrm{P}$. ostreatus, $\mathrm{Sc}-\mathrm{S}$. commune.

\begin{tabular}{|c|c|c|c|}
\hline \multirow[b]{2}{*}{ ID } & \multicolumn{2}{|c|}{ Fold change } & \multirow[b]{2}{*}{ Putative function } \\
\hline & $>2$ & $>4$ & \\
\hline 500663 & 9 & 7 & $\begin{array}{l}\text { Aldehyde dehydrogenase } 5 \text {, } \\
\text { mitochondrial }\end{array}$ \\
\hline 500719 & 11 & 4 & Acetyl-coenzyme A synthetase 2 \\
\hline 273601 & 11 & 7 & $\begin{array}{l}\text { Alcohol dehydrogenase } 3 \text {, } \\
\text { mitochondrial }\end{array}$ \\
\hline 421282 & 7 & 4 & Aldehyde dehydrogenase \\
\hline 450047 & 8 & 5 & Carbonic anhydrase \\
\hline 355051 & 9 & 5 & Ceramide synthase \\
\hline 82352 & 9 & 6 & $\begin{array}{l}\text { Uncharacterized TLC domain- } \\
\text { containing protein YPR114W }\end{array}$ \\
\hline 503618 & 9 & 3 & $\begin{array}{l}\text { 3-methyl-2-oxobutanoate } \\
\text { hydroxymethyltransferase }\end{array}$ \\
\hline 443766 & 8 & 1 & Dephospho-CoA kinase CAB5 \\
\hline
\end{tabular}

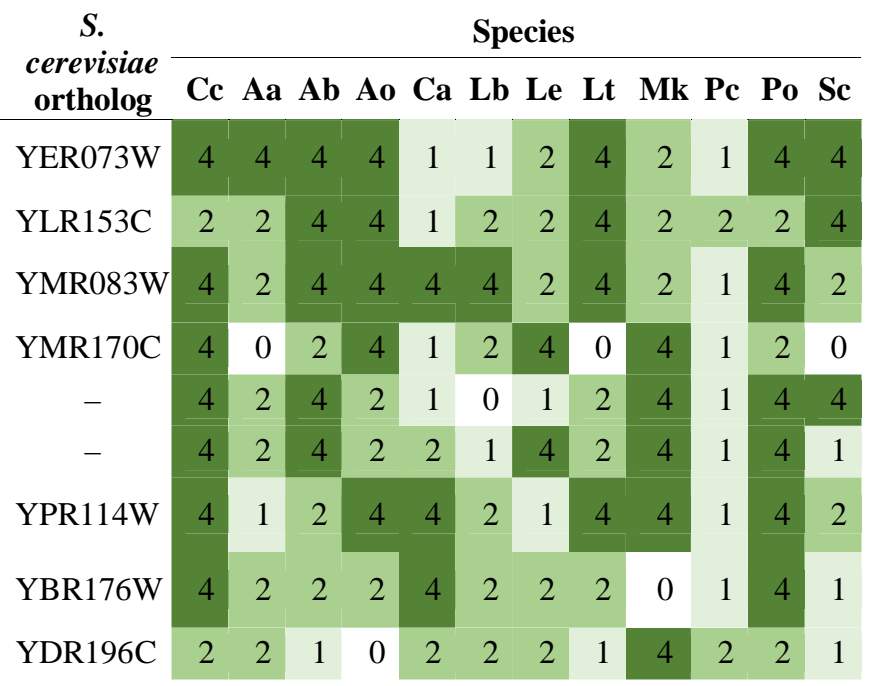




\begin{tabular}{|c|c|c|c|c|c|c|c|c|c|c|c|c|c|c|c|c|}
\hline 474787 & 6 & 1 & Pantothenate kinase $\mathrm{CAB} 1$ & YDR531W & 4 & 1 & 2 & 0 & 0 & 2 & 2 & 1 & 1 & 1 & 2 & 2 \\
\hline 1914 & 6 & 2 & $\begin{array}{l}\text { Phosphopantetheine } \\
\text { adenylyltransferase }\end{array}$ & C & 1 & 1 & 0 & 2 & 4 & 0 & 2 & 1 & 2 & 1 & 4 & 2 \\
\hline 282840 & 5 & 2 & 2-dehydropantoate 2-reductase & YHR063C & 4 & 1 & 1 & 2 & 2 & 1 & 1 & 1 & 2 & 1 & 4 & 1 \\
\hline 500145 & 6 & 3 & Pantoate--beta-alanine ligase & YIL145C & 4 & 1 & 0 & 1 & 4 & 1 & 2 & 1 & 2 & 1 & 2 & 4 \\
\hline 374749 & 8 & 1 & Coenzyme A biosynthesis protein 3 & YKL088W & 2 & 2 & 4 & 2 & 1 & 2 & 2 & 2 & 0 & 1 & 2 & 1 \\
\hline 453548 & 8 & 4 & Pyruvate kinase 1 & YAL038W & 4 & 2 & 4 & 4 & 1 & 1 & 2 & 2 & 1 & 1 & 4 & 2 \\
\hline 499160 & 9 & 5 & Glucose-6-phosphate isomerase & YBR196C & 4 & 1 & 4 & 2 & 2 & 4 & 2 & 2 & 4 & 1 & 4 & 1 \\
\hline 365495 & 5 & 2 & Glucokinase-1 & YCL040W & 4 & 2 & 1 & 1 & 2 & 2 & 1 & 1 & 1 & 1 & 4 & 1 \\
\hline 442066 & 7 & 4 & Phosphoglycerate kinase & YCR012W & 4 & 1 & 2 & 4 & 1 & 1 & 2 & 4 & 2 & 1 & 4 & 1 \\
\hline 173684 & 9 & 5 & Triosephosphate isomerase & YDR050C & 4 & 1 & 4 & 2 & 2 & 2 & 4 & 4 & 2 & 1 & 4 & 1 \\
\hline 402119 & 8 & 3 & Hexokinase-2 & YGL253W & 2 & 2 & 4 & 4 & 1 & 2 & 2 & 1 & 2 & 1 & 4 & 1 \\
\hline 245737 & 9 & 4 & Enolase 1 & YGR254W & 4 & 1 & 4 & 2 & 2 & 2 & 2 & 4 & 2 & 1 & 4 & 1 \\
\hline 264276 & 7 & 5 & $\begin{array}{l}\text { le-3-phosphate } \\
\text { e } 1\end{array}$ & YJL052W & 4 & 1 & 4 & 2 & 0 & 2 & 4 & 4 & 1 & 1 & 4 & 1 \\
\hline 546961 & 8 & 4 & Fructose-bisphosphate aldolase & YKL060C & 4 & 1 & 4 & 2 & 2 & 1 & 4 & 2 & 2 & 1 & 4 & 1 \\
\hline 422402 & 11 & 8 & Pyruvate decarboxylase isozyme 1 & YLR044C & 4 & 2 & 4 & 4 & 4 & 2 & 4 & 4 & 4 & 1 & 4 & 2 \\
\hline 436876 & 9 & 6 & Fructose-1,6-bisphosphatase & YLR377C & 4 & 1 & 4 & 4 & 2 & 2 & 2 & 4 & 0 & 1 & 4 & 4 \\
\hline 462738 & 8 & 1 & $\begin{array}{l}\text { ATP-dependent 6- } \\
\text { phosphofructokinase subunit beta }\end{array}$ & YMR205C & 2 & 1 & 4 & 2 & 2 & 1 & 2 & 2 & 2 & 1 & 2 & 1 \\
\hline 495730 & 12 & 7 & Isocitrate lyase & YER065C & 2 & 4 & 2 & 4 & 4 & 2 & 2 & 4 & 4 & 4 & 2 & 4 \\
\hline 530292 & 12 & 11 & Malate & YIR031C & 4 & 4 & 4 & 4 & 4 & 2 & 4 & 4 & 4 & 4 & 4 & 4 \\
\hline 501208 & 6 & 0 & lehydratase, & YJL200C & 2 & 2 & 2 & 0 & 2 & 1 & 2 & 2 & 1 & 1 & 0 & 1 \\
\hline 37 & 12 & 7 & $\begin{array}{l}\text { Succinate/fumarate mitochondrial } \\
\text { transporter }\end{array}$ & YJR095W & 4 & 4 & 2 & 4 & 4 & 2 & 2 & 4 & 4 & 2 & 2 & 4 \\
\hline 493553 & 10 & 5 & Phosphoenolpyruvate carboxykinase & YKR097W & 4 & 2 & 0 & 4 & 2 & 2 & 1 & 4 & 2 & 2 & 4 & 4 \\
\hline 449845 & 9 & 3 & Malate dehydrogenase & - & 1 & 1 & 4 & 2 & 2 & 2 & 2 & 4 & 2 & 1 & 4 & 2 \\
\hline 442593 & 10 & 4 & ATP & - & 4 & 2 & 2 & 4 & 2 & 4 & 2 & 2 & 2 & 1 & 4 & 1 \\
\hline 443703 & 10 & 5 & $\begin{array}{l}\text { carnitine } \mathrm{O}- \\
\text { ase }\end{array}$ & YAR035W & 4 & 1 & 4 & 4 & 2 & 2 & 1 & 4 & 4 & 2 & 2 & 2 \\
\hline 442787 & 7 & 5 & Acetyl-CoA hydrolase & YBL015W & 4 & 4 & 0 & 4 & 1 & 4 & 2 & 0 & 0 & 0 & 2 & 4 \\
\hline 441597 & 7 & 6 & Accumulation of dyads protein 2 & YCR010C & 4 & 1 & 4 & 4 & 0 & 4 & 4 & 1 & 2 & 1 & 4 & 1 \\
\hline 373141 & 10 & 8 & Aquaglycerol po & YFL054C & 4 & 4 & 4 & 4 & 2 & 2 & 4 & 0 & 4 & 0 & 4 & 4 \\
\hline 357888 & 10 & 2 & $\begin{array}{l}\text { loxaloacetate } \\
\text { ein }\end{array}$ & YKL120W & 2 & 1 & 2 & 2 & 4 & 2 & 2 & 1 & 2 & 2 & 4 & 2 \\
\hline 375404 & 11 & 6 & lic acid transporter protein & YKL217W & 4 & 2 & 4 & 4 & 2 & 4 & 2 & 2 & 4 & 1 & 4 & 2 \\
\hline 147126 & 9 & 3 & Protein SYM1 & YLR251W & 2 & 2 & 4 & 2 & 1 & 1 & 4 & 4 & 2 & 1 & 2 & 2 \\
\hline 470219 & 9 & 3 & $\begin{array}{l}\text { Carnitine O-acetyltransferase, } \\
\text { mitochondrial }\end{array}$ & YML042W & 2 & 1 & 0 & 2 & 4 & 4 & 2 & 2 & 1 & 2 & 4 & 2 \\
\hline 474886 & 9 & 3 & Citrate/oxoglutarate carrier protein & YMR241W & 4 & 1 & 4 & 2 & 0 & 2 & 2 & 2 & 2 & 1 & 4 & 2 \\
\hline 543155 & 7 & 6 & Acetate kinase & - & 4 & 2 & 0 & 4 & 4 & 0 & 0 & 4 & 0 & 1 & 4 & 4 \\
\hline 534888 & 9 & 5 & Malate transporter & - & 2 & 4 & 4 & 4 & 2 & 0 & 2 & 2 & 0 & 1 & 4 & 4 \\
\hline $\begin{array}{l}\text { S.c. } \\
273815 \\
3\end{array}$ & 8 & 5 & Formate transporter & - & 0 & 1 & 4 & 4 & 4 & 0 & 4 & 2 & 2 & 1 & 4 & 2 \\
\hline 474928 & 8 & $\mathrm{~J}$ & Oxalate-CoA ligase & - & 4 & 0 & 2 & 4 & 2 & 1 & 4 & 4 & 2 & 1 & 4 & 1 \\
\hline 452378 & 9 & 7 & Formate dehydrogenase 1 & YOR388C & 4 & 4 & 1 & 4 & 4 & 0 & 4 & 2 & 4 & 1 & 4 & 0 \\
\hline
\end{tabular}




\begin{tabular}{|c|c|c|c|}
\hline 361522 & 10 & 8 & Oxalate decarboxylase \\
\hline $\begin{array}{l}\text { S.c. } \\
275187 \\
7\end{array}$ & 6 & 5 & Oxalate decarboxylase \\
\hline 499734 & 11 & 11 & Oxaloacetase \\
\hline 385900 & 11 & 2 & $\begin{array}{l}\text { Pyruvate dehydrogenase E1 } \\
\text { component subunit beta, } \\
\text { mitochondrial }\end{array}$ \\
\hline 501813 & 9 & 3 & $\begin{array}{l}\text { Pyruvate dehydrogenase E1 } \\
\text { component subunit alpha, } \\
\text { mitochondrial }\end{array}$ \\
\hline 440087 & 10 & 3 & $\begin{array}{l}\text { Dihydrolipoyllysine-residue } \\
\text { acetyltransferase component of } \\
\text { pyruvate dehydrogenase complex, } \\
\text { mitochondrial }\end{array}$ \\
\hline 273609 & 9 & 6 & NADP-dependent malic enzyme \\
\hline 364080 & 7 & 2 & Pyruvate carboxylase 2 \\
\hline 369183 & 10 & 1 & $\begin{array}{l}\text { Dihydrolipoyllysine-residue } \\
\text { succinyltransferase, mitochondrial }\end{array}$ \\
\hline 492830 & 8 & 1 & $\begin{array}{l}\text { Dihydrolipoyl dehydrogenase, } \\
\text { mitochondrial }\end{array}$ \\
\hline 454395 & 10 & 3 & $\begin{array}{l}\text { Succinate--CoA ligase subunit beta, } \\
\text { mitochondrial }\end{array}$ \\
\hline 354219 & 5 & 2 & $\begin{array}{l}\text { Protein phosphatase } 2 \mathrm{C} \text { homolog } 7 \text {, } \\
\text { mitochondrial }\end{array}$ \\
\hline 545855 & 7 & 1 & $\begin{array}{l}\text { 2-oxoglutarate dehydrogenase, } \\
\text { mitochondrial }\end{array}$ \\
\hline 380777 & 7 & 6 & $\begin{array}{l}\text { NAD-dependent malic enzyme, } \\
\text { mitochondrial }\end{array}$ \\
\hline 409635 & 11 & 3 & $\begin{array}{l}\text { Malate dehydrogenase, } \\
\text { mitochondrial }\end{array}$ \\
\hline 483615 & 6 & 0 & $\begin{array}{l}\text { Succinate dehydrogenase } \\
\text { cytochrome b subunit, } \\
\text { mitochondrial }\end{array}$ \\
\hline 400441 & 6 & 0 & $\begin{array}{l}\text { Succinate dehydrogenase } \\
\text { flavoprotein subunit, mitochondrial }\end{array}$ \\
\hline 363708 & 8 & 0 & $\begin{array}{l}\text { Succinate dehydrogenase iron-sulfur } \\
\text { subunit, mitochondrial }\end{array}$ \\
\hline 367547 & 9 & 0 & $\begin{array}{l}\text { Mitochondrial inner membrane } \\
\text { protein SHH4 }\end{array}$ \\
\hline 503237 & 6 & 0 & Aconitate hydratase, mitochondrial \\
\hline 474027 & 8 & 2 & $\begin{array}{l}\text { Isocitrate dehydrogenase subunit } 1 \text {, } \\
\text { mitochondrial }\end{array}$ \\
\hline 497099 & 11 & 4 & Citrate synthase, mitochondrial \\
\hline 449179 & 6 & 1 & $\begin{array}{l}\text { Succinate dehydrogenase assembly } \\
\text { factor } 2 \text {, mitochondrial }\end{array}$ \\
\hline 444503 & 9 & 4 & $\begin{array}{l}\text { Isocitrate dehydrogenase subunit } 2 \text {, } \\
\text { mitochondrial }\end{array}$ \\
\hline 360292 & 10 & 3 & $\begin{array}{l}\text { Succinate--CoA ligase subunit } \\
\text { alpha, mitochondrial }\end{array}$ \\
\hline 464081 & 6 & 2 & Fumarate hydratase, mitochondrial \\
\hline
\end{tabular}

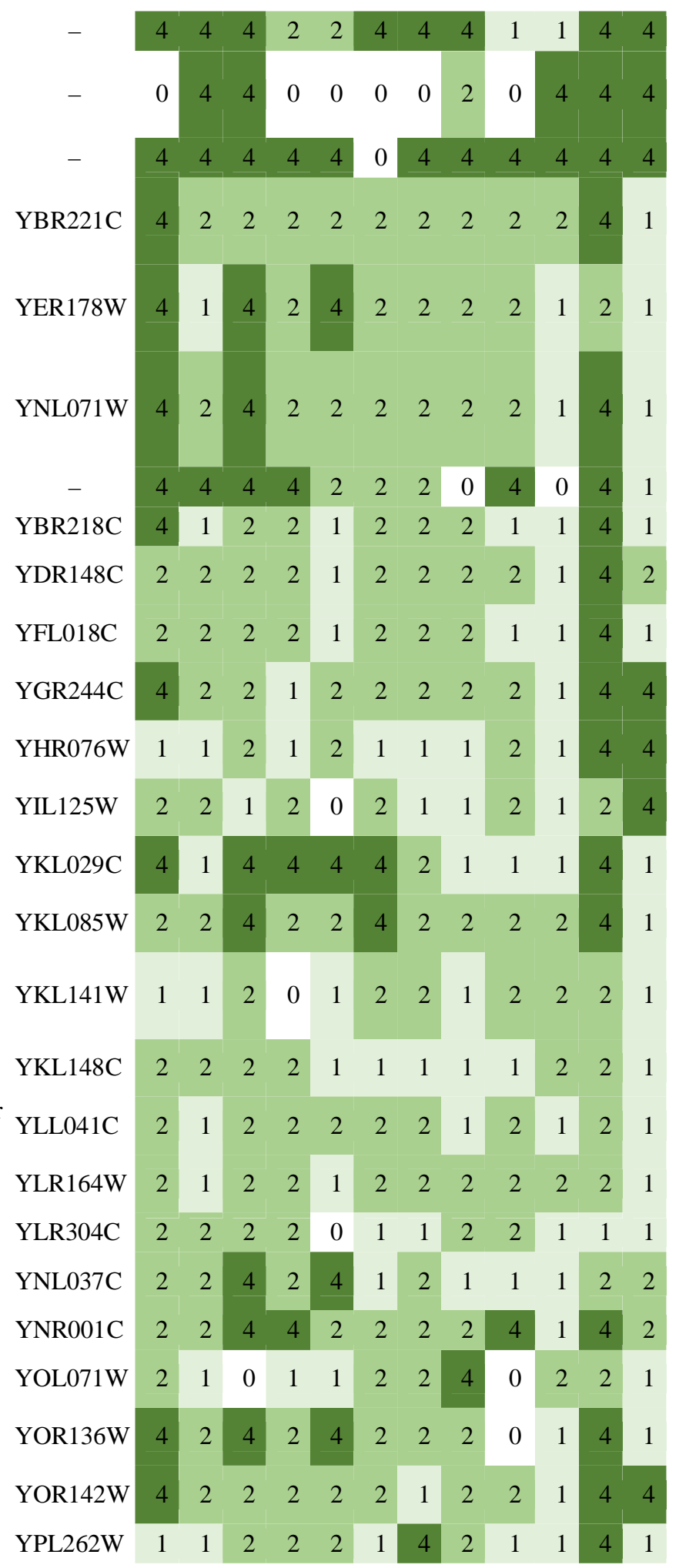

Table 4. Summary of developmental expression dynamics of CDE orthogroups of fatty acid betaoxidation related genes across 12 species. Protein ID of a representative protein is given follows by the number of species in which the orthogroup is developmentally regulated at fold change 2 and 4 (FC>2 and FC>4, respectively). Putative function and ortholog in S. cerevisiae (if any) are also 
given. Abbreviations: 0-gene absent, 1-gene present but not developmentally regulated, 2 developmentally regulated at fold change $>2$, 4- developmentally regulated at fold change $>4$. Species names are abbreviated as: $\mathrm{Cc}-C$. cinerea, $\mathrm{Aa}-A$. ampla, Ab-A. bisporus, Ao - A. ostoyae, $\mathrm{Ca}-\mathrm{C}$. aegerita, $\mathrm{Lb}-$ L. bicolor, Le - L. edodes, Lt - L. tigrinus, $\mathrm{Mk}-\mathrm{M}$. kentingensis, $\mathrm{Pc}$ - Ph. chrysosporium, Po-P. ostreatus, $\mathrm{Sc}-\mathrm{S}$. commune.

\begin{tabular}{|c|c|c|c|c|c|c|c|c|c|c|c|c|c|c|c|c|}
\hline \multirow[b]{2}{*}{ ID } & \multicolumn{2}{|c|}{ Fold change } & \multirow[b]{2}{*}{ Putative function } & \multirow{2}{*}{$\begin{array}{l}\text { S. } \\
\text { cerevisiae } \\
\text { ortholog }\end{array}$} & \multicolumn{12}{|c|}{ Species } \\
\hline & $>2$ & 4 & & & Ce & Aa & $\mathbf{A b}$ & Ao & Ca & $\mathbf{L b}$ & Le & $\mathbf{L t}$ & Mk & Pc & Po & Sc \\
\hline 437115 & 7 & 4 & General amino acid permease AGP2 & YBR132C & 4 & 2 & 4 & 1 & 4 & 2 & 4 & 0 & 1 & 0 & 1 & 2 \\
\hline 336239 & 6 & 1 & Acyl-coenzyme A oxidase & YGL205W & 1 & 1 & 1 & 2 & 2 & 1 & 2 & 2 & 1 & 1 & 4 & 2 \\
\hline 438072 & 10 & 5 & Acyl-CoA-binding protein & YGR037C & 4 & 2 & 4 & 4 & 2 & 2 & 2 & 4 & 2 & 1 & 4 & 1 \\
\hline 365907 & 6 & 2 & $\begin{array}{l}\text { 3-ketoacyl-CoA thiolase, } \\
\text { peroxisomal }\end{array}$ & YIL160C & 4 & 1 & 2 & 2 & 4 & 2 & 0 & 1 & 2 & 1 & 1 & 1 \\
\hline 231560 & 5 & 1 & $\begin{array}{l}\text { Peroxisomal hydratase- } \\
\text { dehydrogenase-epimerase }\end{array}$ & YKR009C & 2 & 1 & 1 & 4 & 2 & 1 & 2 & 1 & 2 & 1 & 1 & 1 \\
\hline 354296 & 6 & 2 & Aspartate aminotransferase & YLR027C & 2 & 2 & 4 & 0 & 0 & 2 & 1 & 1 & 1 & 1 & 4 & 2 \\
\hline 4337 & 8 & 3 & $\begin{array}{l}\text { Peroxisomal 2,4-dienoyl-CoA } \\
\text { reductase SPS19 [(3E)-enoyl-CoA- } \\
\text { producing] }\end{array}$ & YNL202W & 4 & 1 & 4 & 2 & 2 & 4 & 1 & 1 & 2 & 1 & 2 & 2 \\
\hline 374386 & 7 & 1 & $\begin{array}{l}\text { Peroxisomal membrane protein } \\
\text { PMP } 27\end{array}$ & YOL147C & 2 & 1 & 2 & 2 & 2 & 2 & 1 & 1 & 2 & 1 & 4 & 1 \\
\hline 436931 & 6 & 2 & $\begin{array}{l}\text {,5)-Delta(2,4)-dienoyl-CoA } \\
\text { ase }\end{array}$ & YOR180C & 2 & 1 & 2 & 1 & 0 & 4 & 1 & 2 & 0 & 1 & 4 & 2 \\
\hline 401814 & 5 & 2 & $\begin{array}{l}\text { somal long-chain fatty acid } \\
\text { protein } 2\end{array}$ & YPL147W & 4 & 1 & 1 & 2 & 4 & 1 & 1 & 1 & 2 & 1 & 2 & 1 \\
\hline 395825 & 8 & 3 & $\begin{array}{l}\text { Peroxisomal adenine nucleotide } \\
\text { transporter } 1\end{array}$ & YPR128C & 4 & 1 & 2 & 2 & 1 & 4 & 2 & 2 & 2 & 1 & 4 & 1 \\
\hline 437261 & 6 & 4 & $\begin{array}{l}\text { Long-chain-fatty-acid--CoA ligase } \\
1\end{array}$ & YOR317W & 2 & 1 & 4 & 4 & 2 & 1 & 4 & 1 & 0 & 1 & 4 & 1 \\
\hline
\end{tabular}

Table 5. Summary of developmental expression dynamics of CDE orthogroups of ergosterol and sphingolipid biosynthesis related genes across 12 species. Protein ID of a representative protein is given follows by the number of species in which the orthogroup is developmentally regulated at fold change 2 and 4 (FC>2 and FC>4, respectively). Putative function and ortholog in S. cerevisiae (if any) are also given. Abbreviations: 0-gene absent, 1-gene present but not developmentally regulated, 2 - developmentally regulated at fold change $>2,4$ - developmentally regulated at fold change $>4$. Species names are abbreviated as: $\mathrm{Cc}-C$. cinerea, $\mathrm{Aa}-A$. ampla, $\mathrm{Ab}-A$. bisporus, $\mathrm{Ao}-A$. ostoyae, $\mathrm{Ca}-C$. aegerita, $\mathrm{Lb}-$ L. bicolor, $\mathrm{Le}-$ L. edodes, $\mathrm{Lt}-\mathrm{L}$. tigrinus, $\mathrm{Mk}-\mathrm{M}$. kentingensis, $\mathrm{Pc}-\mathrm{Ph}$. chrysosporium, $\mathrm{Po}-\mathrm{P}$. ostreatus, $\mathrm{Sc}-\mathrm{S}$. commune.

\begin{tabular}{|c|c|c|c|c|c|c|c|c|c|c|c|c|c|c|c|c|}
\hline \multirow[b]{2}{*}{ ID } & \multicolumn{2}{|c|}{ Fold change } & \multirow[b]{2}{*}{ Putative function } & \multirow{2}{*}{$\begin{array}{c}\text { S. } \\
\text { cerevisiae } \\
\text { ortholog }\end{array}$} & \multicolumn{12}{|c|}{ Species } \\
\hline & $>2$ & $>4$ & & & Cc & $\mathbf{A a}$ & $\mathbf{A b}$ & Ao & $\mathrm{Ca}$ & $\mathbf{L b}$ & Le & $\mathbf{L t}$ & Mk & Pc & Po & Sc \\
\hline 488426 & 9 & 3 & Transcription factor & - & 4 & 1 & 0 & 4 & 2 & 2 & 4 & 2 & 2 & 2 & 2 & 1 \\
\hline \multicolumn{17}{|c|}{ Late pathway } \\
\hline 366787 & 9 & 4 & Ergosterol biosynthetic protein 28 & YER044C & 2 & 1 & 2 & 2 & 4 & 2 & 4 & 4 & 2 & 1 & 4 & 1 \\
\hline 366442 & 11 & 6 & $\begin{array}{l}\text { Sterol-4-alpha-carboxylate } 3 \text { - } \\
\text { dehydrogenase, decarboxylating }\end{array}$ & YGL001C & 4 & 2 & 4 & 4 & 2 & 4 & 2 & 4 & 2 & 2 & 4 & 1 \\
\hline 544612 & 10 & 7 & $\operatorname{Delta}(24(24(1)))$-sterol reductase & YGL012W & 4 & 2 & 4 & 2 & 0 & 4 & 4 & 4 & 2 & 4 & 4 & 1 \\
\hline 496056 & 10 & 7 & Methylsterol monooxygenase & YGR060W & 4 & 1 & 4 & 2 & 4 & 4 & 2 & 4 & 2 & 4 & 4 & 1 \\
\hline 489517 & 9 & 6 & Squalene monooxygenase & YGR175C & 4 & 1 & 2 & 2 & 0 & 4 & 4 & 4 & 4 & 2 & 4 & 1 \\
\hline
\end{tabular}




$\begin{array}{lccl}397292 & 11 & 7 & \begin{array}{l}\text { Lanosterol 14-alpha demethylase } \\ \text { NADPH--cytochrome P450 } \\ \text { reductase }\end{array} \\ 420341 & 8 & 5 & \text { Lanosterol synthase } \\ 494314 & 7 & 4 & \text { Delta(7)-sterol 5(6)-desaturase } \\ 462226 & 8 & 4 & \text { 3-keto-steroid reductase } \\ 373683 & 10 & 7 & \text { Sterol 24-C-methyltransferase } \\ 357271 & 10 & 6 & \text { Cytochrome P450 61 } \\ 17387 & 11 & 7 & \text { C-8 sterol isomerase } \\ 535364 & 10 & 3 & \text { Delta(14)-sterol reductase }\end{array}$

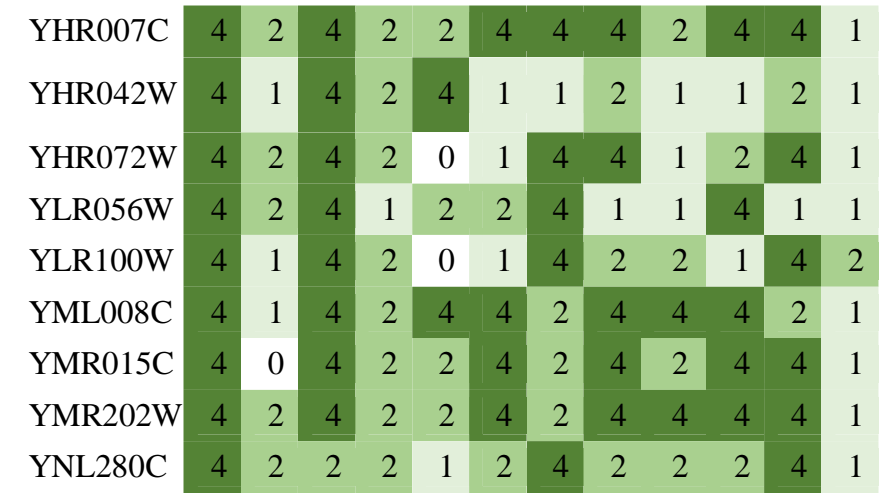

Mevalonate pathway

\begin{tabular}{|c|c|c|c|}
\hline 358591 & 7 & 2 & Cystathionine beta-lyase \\
\hline 43445 & 9 & 6 & Squalene synthase \\
\hline 459666 & 9 & 5 & Farnesyl pyrophosphate synthase \\
\hline 360799 & 8 & 2 & $\begin{array}{l}\text { 3-hydroxy-3-methylglutaryl- } \\
\text { coenzyme A reductase } 2\end{array}$ \\
\hline 439474 & 12 & 5 & $\begin{array}{l}\text { Hydroxymethylglutaryl-CoA } \\
\text { synthase }\end{array}$ \\
\hline 355838 & 12 & 10 & Phosphomevalonate kinase \\
\hline 438368 & 8 & 2 & $\begin{array}{l}\text { Diphosphomevalonate } \\
\text { decarboxylase }\end{array}$ \\
\hline 355558 & 7 & 0 & Acetyl-CoA acetyltransferase \\
\hline 460395 & 6 & 2 & $\begin{array}{l}\text { Isopentenyl-diphosphate Delta- } \\
\text { isomerase }\end{array}$ \\
\hline 539004 & 12 & 7 & $\begin{array}{l}\text { 3-oxo-5-alpha-steroid 4- } \\
\text { dehydrogenase }\end{array}$ \\
\hline
\end{tabular}

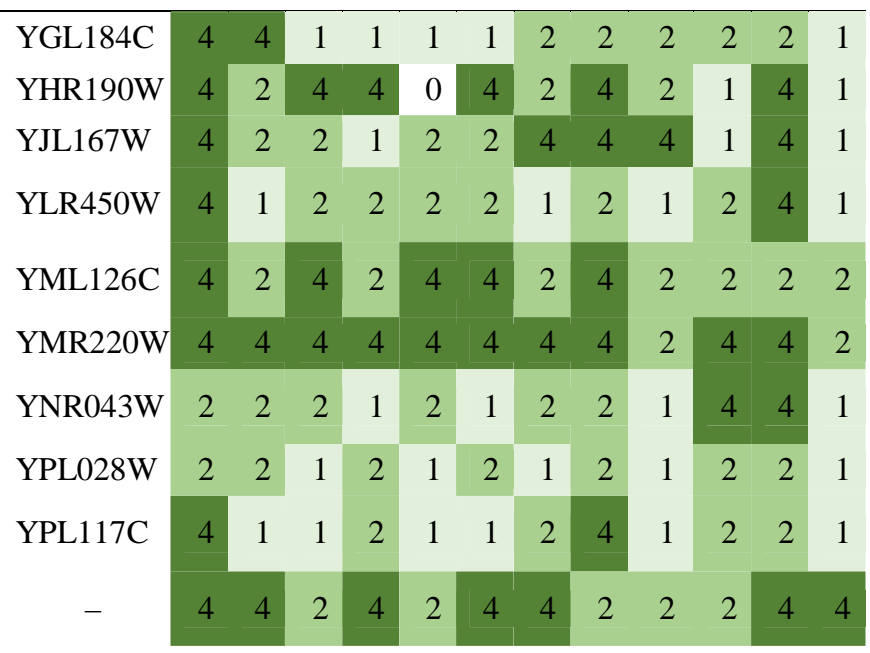

Sphingolipid biosynthesis

\begin{tabular}{|c|c|c|c|c|c|c|c|c|c|c|c|c|c|c|c|c|}
\hline 388282 & 9 & 5 & Alkaline ceramidase YPC1 & YBR183W & 4 & 2 & 4 & 4 & 2 & 2 & 1 & 4 & 2 & 1 & 4 & 1 \\
\hline 429678 & 6 & 1 & Serine palmitoyltransferase 2 & YDR062W & 4 & 1 & 2 & 1 & 2 & 2 & 1 & 2 & 1 & 1 & 2 & 1 \\
\hline 372474 & 7 & 1 & Sphingosine-1-phosphate lyase & YDR294C & 2 & 1 & 2 & 4 & 2 & 1 & 2 & 2 & 1 & 1 & 2 & 1 \\
\hline 379742 & 7 & 0 & Sphingolipid C4-hydroxylase SUR2 & YDR297W & 2 & 1 & 1 & 2 & 0 & 2 & 2 & 1 & 2 & 1 & 2 & 2 \\
\hline 456289 & 8 & 0 & Ceramide synthase LAG1 & YHL003C & 1 & 2 & 0 & 2 & 2 & 1 & 2 & 2 & 2 & 1 & 2 & 2 \\
\hline 420050 & 6 & 3 & $\begin{array}{l}\text { Dihydrosphingosine 1-phosphate } \\
\text { phosphatase LCB3 }\end{array}$ & YJL134W & 4 & 1 & 1 & 4 & 1 & 2 & 2 & 1 & 1 & 1 & 4 & 2 \\
\hline 441179 & 7 & 2 & $\begin{array}{l}\text { Ceramide very long chain fatty acid } \\
\text { hydroxylase SCS7 }\end{array}$ & YMR272C & 4 & 1 & 2 & 2 & 2 & 4 & 2 & 1 & 1 & 1 & 2 & 1 \\
\hline 546344 & 6 & 3 & Serine palmitoyltransferase 1 & YMR296C & 4 & 1 & 2 & 1 & 4 & 1 & 2 & 1 & 2 & 1 & 4 & 1 \\
\hline 1859 & 6 & 2 & Sphingoid long chain base kinase 4 & YOR171C & 4 & 1 & 1 & 1 & 1 & 2 & 4 & 2 & 2 & 1 & 2 & 1 \\
\hline
\end{tabular}

Table 6. Summary of developmental expression dynamics of CDE orthogroups of sterol transport related genes across 12 species. Protein ID of a representative protein is given follows by the number of species in which the orthogroup is developmentally regulated at fold change 2 and 4 (FC>2 and FC>4, respectively). Putative function and ortholog in S. cerevisiae (if any) are also given. Abbreviations: 0-gene absent, 1-gene present but not developmentally regulated, 2 developmentally regulated at fold change $>2$, 4- developmentally regulated at fold change $>4$. Species names are abbreviated as: $\mathrm{Cc}-C$. cinerea, $\mathrm{Aa}-A$. ampla, Ab-A. bisporus, $\mathrm{Ao}-A$. ostoyae, $\mathrm{Ca}-C$. aegerita, $\mathrm{Lb}-\mathrm{L}$. bicolor, $\mathrm{Le}-\mathrm{L}$. edodes, $\mathrm{Lt}-\mathrm{L}$. tigrinus, Mk $-M$. kentingensis, $\mathrm{Pc}$ - Ph. chrysosporium, Po - P. ostreatus, Sc - S. commune. 


\begin{tabular}{|c|c|c|c|c|c|c|c|c|c|c|c|c|c|c|c|c|}
\hline \multirow[b]{2}{*}{ ID } & \multicolumn{2}{|c|}{ Fold change } & \multirow[b]{2}{*}{ Putative function } & \multirow{2}{*}{$\begin{array}{c}S . \\
\text { cerevisiae } \\
\text { ortholog }\end{array}$} & \multicolumn{12}{|c|}{ Species } \\
\hline & $>2$ & $>4$ & & & $\mathrm{Cc}$ & Aa & $\mathbf{A b}$ & Ao & Ca & Lb & Le & $\mathbf{L t}$ & Mk & $\mathbf{P c}$ & Po & Sc \\
\hline 470387 & 7 & 0 & Prefoldin subunit 2 & - & 4 & 4 & 4 & 4 & 1 & 2 & 4 & 0 & 1 & 0 & 4 & 0 \\
\hline 475807 & 7 & 5 & $\begin{array}{l}\text { phosphatidyli } \\
\text { n }\end{array}$ & DL046W & 2 & 1 & 4 & 4 & 0 & 4 & 1 & 1 & 4 & 1 & 4 & 2 \\
\hline 378276 & 6 & 1 & $\begin{array}{l}\text { Oxysterol-binding protein homolog } \\
7\end{array}$ & YHR001W & 4 & 1 & 2 & 2 & 1 & 1 & 1 & 1 & 2 & 1 & 2 & 2 \\
\hline 494709 & 7 & 1 & $\begin{array}{l}\text { NPC intracellular sterol transporter } \\
\text { 1-related protein } 1\end{array}$ & YPL006W & 4 & 2 & 1 & 2 & 2 & 1 & 2 & 1 & 1 & 1 & 2 & 2 \\
\hline 7232 & 10 & 2 & $\begin{array}{l}\text { Oxysterol-binding protein homolog } \\
4\end{array}$ & PL145C & 4 & 2 & 2 & 4 & 2 & 1 & 2 & 2 & 2 & 1 & 2 & 2 \\
\hline 478741 & 6 & 2 & SCP2 sterol transport protein family & - & 2 & 1 & 2 & 4 & 1 & 2 & 0 & 1 & 2 & 1 & 4 & 1 \\
\hline
\end{tabular}

Table 7. Summary of developmental expression dynamics of CDE orthogroups of membrane phoshpolipid and fatty acid biosynthesis genes genes across 12 species. Protein ID of a representative protein is given follows by the number of species in which the orthogroup is developmentally regulated at fold change 2 and 4 (FC>2 and $F C>4$, respectively). Putative function and ortholog in S. cerevisiae (if any) are also given. Abbreviations: 0-gene absent, 1-gene present but not developmentally regulated, 2 - developmentally regulated at fold change $>2$, 4developmentally regulated at fold change $>4$. Species names are abbreviated as: $\mathrm{Cc}-C$. cinerea, $\mathrm{Aa}-A$. ampla, $\mathrm{Ab}-A$. bisporus, Ao - A. ostoyae, $\mathrm{Ca}-C$. aegerita, $\mathrm{Lb}-$ L. bicolor, Le $-L$. edodes, $\mathrm{Lt}-$ L. tigrinus, $\mathrm{Mk}-M$. kentingensis, $\mathrm{Pc}-\mathrm{Ph}$. chrysosporium, Po $-P$. ostreatus, $\mathrm{Sc}-\mathrm{S}$. commune.

Fold change

ID $>2>4$ Putative function
S. cerevisiae
ortholog Ce Aa Ab Ao Ca Lb Le Lt Mk Pc Po Sc

Cardiolipin biosynthesis pathway

\begin{tabular}{|c|c|c|c|c|c|c|c|c|c|c|c|c|c|c|c|c|}
\hline 539990 & 4 & 0 & $\begin{array}{l}\text { Phosphatidylglycerophosphatase } \\
\text { GEP4, mitochondrial }\end{array}$ & YHR100C & 2 & 2 & 1 & 0 & 1 & 1 & 1 & 2 & 0 & 1 & 2 & 1 \\
\hline 471105 & 9 & 3 & $\begin{array}{l}\text { CDP-diacylglycerol--glycerol-3- } \\
\text { phosphate 3-phosphatidyltransferase }\end{array}$ & YCL004W & 2 & 1 & 2 & 2 & 2 & 1 & 2 & 1 & 4 & 4 & 4 & 2 \\
\hline 522776 & 6 & 1 & Cardiolipin synthase & YDL142C & 4 & 2 & 1 & 0 & 1 & 2 & 2 & 1 & 0 & 1 & 2 & 2 \\
\hline \multicolumn{17}{|c|}{ CPD-DAG pathway } \\
\hline 442684 & 8 & 1 & Phosphatidate cytidylyltransferase & YBR029C & 2 & 2 & 2 & 1 & 2 & 2 & 1 & 1 & 2 & 1 & 4 & 2 \\
\hline 436995 & 8 & 1 & $\begin{array}{l}\text { CDP-diacylglycerol-serine O- } \\
\text { phosphatidyltransferase }\end{array}$ & YER026C & 4 & 1 & 2 & 2 & 0 & 2 & 2 & 2 & 1 & 1 & 2 & 2 \\
\hline 444758 & 11 & 4 & $\begin{array}{l}\text { Phosphatidylethanolamine N- } \\
\text { methyltransferase }\end{array}$ & YGR157W & 4 & 2 & 2 & 4 & 2 & 2 & 2 & 4 & 1 & 2 & 4 & 2 \\
\hline 379032 & 7 & 1 & $\begin{array}{l}\text { Phosphatidylserine decarboxylase } \\
\text { proenzyme } 2\end{array}$ & YGR170W & 2 & 1 & 2 & 2 & 2 & 1 & 1 & 2 & 2 & 1 & 4 & 1 \\
\hline 285011 & 9 & 6 & $\begin{array}{l}\text { Phosphatidyl-N- } \\
\text { methylethanolamine N- } \\
\text { methyltransferase }\end{array}$ & YJR073C & 4 & 1 & 4 & 0 & 4 & 2 & 2 & 4 & 4 & 2 & 4 & 1 \\
\hline 377789 & 7 & 2 & $\begin{array}{l}\text { Phosphatidylserine decarboxylase } \\
\text { proenzyme } 1 \text {, mitochondrial }\end{array}$ & YNL169C & 4 & 1 & 2 & 4 & 0 & 1 & 2 & 2 & 1 & 1 & 2 & 2 \\
\hline 436992 & 6 & 2 & $\begin{array}{l}\text { CDP-diacylglycerol-inositol 3- } \\
\text { phosphatidyltransferase }\end{array}$ & YPR113W & 4 & 1 & 2 & 2 & 2 & 2 & 1 & 1 & 1 & 1 & 4 & 1 \\
\hline \multicolumn{17}{|c|}{ Kennedy pathway } \\
\hline 449161 & 7 & 3 & $\begin{array}{l}\text { Choline-phosphate } \\
\text { cytidylyltransferase }\end{array}$ & YGR202C & 4 & 1 & 0 & 4 & 0 & 1 & 4 & 2 & 0 & 2 & 2 & 2 \\
\hline 411802 & 3 & 1 & Choline kinase & YLR133W & 4 & 1 & 1 & 1 & 1 & 1 & 1 & 1 & 0 & 1 & 2 & 2 \\
\hline 380149 & 10 & 5 & Cholinephosphotransferase 1 & YNL130C & 4 & 2 & 4 & 2 & 2 & 4 & 2 & 2 & 1 & 1 & 4 & 4 \\
\hline
\end{tabular}


Shared part of the pathway

\begin{tabular}{|c|c|c|c|c|c|c|c|c|c|c|c|c|c|c|c|c|}
\hline 76420 & 1 & 1 & $\begin{array}{l}\text { Long-chain-fatty-acid--CoA ligase } \\
2\end{array}$ & YER015W & 4 & 0 & 0 & 0 & 0 & 0 & 0 & 0 & 0 & 0 & 0 & 0 \\
\hline 439965 & 6 & 4 & Elongation of fatty acids protein 2 & YCR034W & 4 & 1 & 4 & 1 & 4 & 2 & 1 & 2 & 0 & 2 & 4 & 1 \\
\hline 462371 & 5 & 0 & $\begin{array}{l}\text { 1-acyl-sn-glycerol-3-phosphate } \\
\text { acyltransferase }\end{array}$ & YDL052C & 2 & 1 & 1 & 1 & 2 & 1 & 2 & 1 & 2 & 1 & 2 & 1 \\
\hline 379857 & 9 & 3 & Acetyl-CoA carboxylase & YNR016C & 4 & 2 & 1 & 4 & 0 & 2 & 2 & 4 & 1 & 2 & 2 & 2 \\
\hline 414 & 7 & 6 & $\begin{array}{l}\text { Glycerol-3-phosphate } \\
\text { dehydrogenase }[\mathrm{NAD}(+)] 2 \text {, } \\
\text { mitochondrial }\end{array}$ & YOL & 4 & 1 & 4 & 4 & 2 & 1 & 4 & 1 & 4 & 1 & 4 & 1 \\
\hline 372300 & 9 & 4 & Lysophospholipid acyltransferase & YOR175C & 4 & 2 & 4 & 2 & 2 & 2 & 2 & 1 & 4 & 1 & 4 & 1 \\
\hline 538984 & 9 & 5 & bunit alpha & YPL2 & 4 & 2 & 1 & 4 & 2 & 2 & 4 & 4 & 1 & 1 & 4 & 2 \\
\hline 72617 & 4 & 4 & Fatty acid desaturase & - & 4 & 1 & 1 & 4 & 0 & 1 & 1 & 4 & 0 & 0 & 0 & 4 \\
\hline 4671 & 6 & 0 & $\begin{array}{l}\text { Glycerol-3-phosphate O- } \\
\text { acyltransferase } 1\end{array}$ & YBL011W & 2 & 1 & 2 & 2 & 1 & 1 & 2 & 1 & 2 & 1 & 2 & 1 \\
\hline 450732 & 8 & 3 & $\begin{array}{l}\text { NADPH-dependent } 1 \text { - } \\
\text { acyldihydroxyacetone phosphate } \\
\text { reductase }\end{array}$ & YIL124W & 2 & 1 & 2 & 4 & 2 & 1 & 4 & 1 & 2 & 2 & 4 & 1 \\
\hline 422393 & 10 & 6 & Fatty acid desaturase & - & 4 & 2 & 4 & 2 & 4 & 4 & 2 & 4 & 1 & 1 & 2 & 4 \\
\hline 437261 & 6 & 4 & $\begin{array}{l}\text { Long-chain-fatty-acid--CoA ligase } \\
1\end{array}$ & YOR317W & 2 & 1 & 4 & 4 & 2 & 1 & 4 & 1 & 0 & 1 & 4 & 1 \\
\hline \multicolumn{17}{|c|}{ TAG pathway } \\
\hline 437679 & 4 & 0 & $\begin{array}{l}\text { Phosphatidic acid phosphohydrolase } \\
1\end{array}$ & - & 2 & 1 & 1 & 2 & 1 & 1 & 1 & 2 & 1 & 1 & 1 & 2 \\
\hline 493617 & 3 & 1 & $\begin{array}{l}\text { Phospholipid:diacylglycerol } \\
\text { acyltransferase }\end{array}$ & YNR008W & 4 & 1 & 1 & 2 & 1 & 1 & 1 & 1 & 1 & 1 & 2 & 1 \\
\hline 374579 & 7 & 2 & Sterol O-acyltransferase 2 & - & 2 & 1 & 2 & 2 & 0 & 2 & 1 & 1 & 4 & 1 & 4 & 2 \\
\hline 479811 & 6 & 2 & Diacylglycerol O-acyltransferase 1 & YOR245C & 1 & 1 & 1 & 4 & 1 & 2 & 2 & 1 & 2 & 1 & 4 & 2 \\
\hline
\end{tabular}

Table 8. Summary of developmental expression dynamics of CDE orthogroups of linoleic acid biosynthesis related genes across 12 species. Protein ID of a representative protein is given follows by the number of species in which the orthogroup is developmentally regulated at fold change 2 and 4 (FC>2 and FC>4, respectively). Putative function and ortholog in S. cerevisiae (if any) are also given. Abbreviations: 0-gene absent, 1-gene present but not developmentally regulated, 2 developmentally regulated at fold change $>2$, 4- developmentally regulated at fold change $>4$. Species names are abbreviated as: $\mathrm{Cc}-C$. cinerea, $\mathrm{Aa}-A$. ampla, Ab-A. bisporus, Ao - A. ostoyae, $\mathrm{Ca}-\mathrm{C}$. aegerita, $\mathrm{Lb}-$ L. bicolor, Le - L. edodes, Lt - L. tigrinus, Mk - M. kentingensis, $\mathrm{Pc}$ - Ph. chrysosporium, Po-P. ostreatus, $\mathrm{Sc}-\mathrm{S}$. commune.

\begin{tabular}{lccl} 
& \multicolumn{2}{c}{ Fold change } & \\
\cline { 2 - 3 } ID & $\mathbf{> 2}$ & $\mathbf{> 4}$ & Putative function \\
\hline $\begin{array}{l}\text { S.c. } \\
251100\end{array}$ & 10 & 3 & Fatty acid desaturase \\
2 & & & \\
363743 & 9 & 7 & Fatty acid desaturase \\
422976 & 8 & 8 & Fatty acid desaturase \\
471478 & 9 & 7 & Fatty acid desaturase \\
493933 & 11 & 9 & Fatty acid desaturase \\
422393 & 10 & 6 & Fatty acid desaturase
\end{tabular}

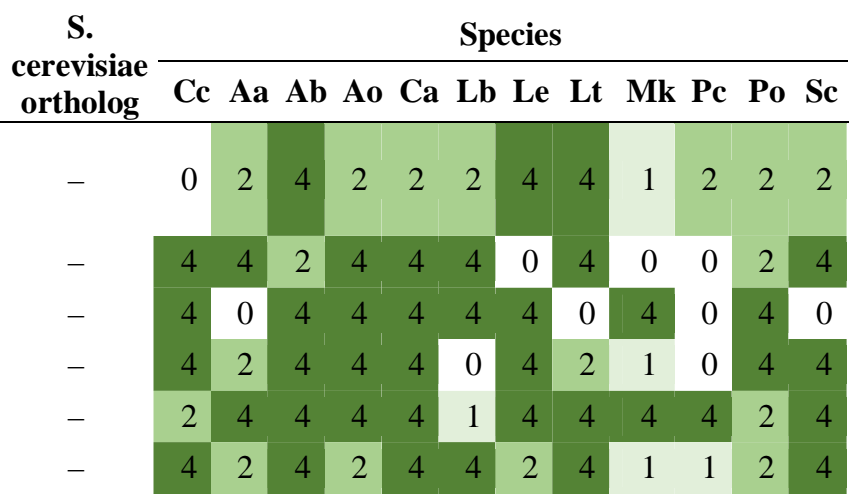


Table 9. Summary of developmental expression dynamics of CDE orthogroups of other lipid metabolism related genes across 12 species. Protein ID of a representative protein is given follows by the number of species in which the orthogroup is developmentally regulated at fold change 2 and 4 (FC>2 and FC>4, respectively). Putative function and ortholog in S. cerevisiae (if any) are also given. Abbreviations: 0-gene absent, 1-gene present but not developmentally regulated, 2 developmentally regulated at fold change $>2$, 4- developmentally regulated at fold change $>4$. Species names are abbreviated as: $\mathrm{Cc}-C$. cinerea, $\mathrm{Aa}-A$. ampla, Ab-A. bisporus, Ao - A. ostoyae, $\mathrm{Ca}-\mathrm{C}$. aegerita, Lb - L. bicolor, Le - L. edodes, Lt - L. tigrinus, Mk $-M$. kentingensis, Pc - Ph. chrysosporium, Po - P. ostreatus, Sc - S. commune.

\begin{tabular}{|c|c|c|c|c|c|c|c|c|c|c|c|c|c|c|c|c|}
\hline \multirow[b]{2}{*}{ ID } & \multicolumn{2}{|c|}{ Fold change } & \multirow[b]{2}{*}{ Putative function } & \multirow{2}{*}{$\begin{array}{c}S . \\
\text { cerevisiae } \\
\text { ortholog }\end{array}$} & \multicolumn{12}{|c|}{ Species } \\
\hline & $>2$ & $>4$ & & & $\mathrm{Cc}$ & Aa & $\mathbf{A b}$ & Ao & $\mathbf{C a}$ & Lb & Le & Lt & Mk & $\mathbf{P c}$ & Po & Sc \\
\hline 20997 & 12 & 11 & $\begin{array}{l}\text { Glycerophosphodiester } \\
\text { phosphodiesterase }\end{array}$ & - & 4 & 4 & 4 & 4 & 4 & 4 & 4 & 4 & 4 & 2 & 4 & 4 \\
\hline 380219 & 6 & 6 & $\begin{array}{l}\text { Diacylglycerol pyrophosphate } \\
\text { phosphatases }\end{array}$ & - & 4 & 4 & 0 & 4 & 0 & 0 & 0 & 0 & 4 & 0 & 4 & 4 \\
\hline 376602 & 9 & 9 & Caleosin & - & 4 & 4 & 1 & 0 & 4 & 4 & 0 & 4 & 4 & 4 & 4 & 4 \\
\hline 365330 & 12 & 9 & Sphingolipid C9-methyltransferase & - & 4 & 2 & 2 & 4 & 4 & 4 & 4 & 4 & 2 & 4 & 4 & 4 \\
\hline 395241 & 11 & 8 & Sphingolipid C9-methyltransferase & - & 4 & 2 & 4 & 4 & 2 & 4 & 4 & 4 & 4 & 2 & 4 & 1 \\
\hline 444998 & 8 & 6 & Marvel domain protein & - & 4 & 1 & 0 & 4 & 1 & 1 & 4 & 2 & 4 & 2 & 4 & 4 \\
\hline 462849 & 10 & 5 & Marvel domain protein & - & 4 & 1 & 0 & 2 & 2 & 2 & 4 & 2 & 4 & 4 & 2 & 4 \\
\hline
\end{tabular}

Table 10. Summary of developmental expression dynamics of CDE orthogroups of storage carbohydrate metabolism genes across 12 species. Protein ID of a representative protein is given follows by the number of species in which the orthogroup is developmentally regulated at fold change 2 and 4 (FC>2 and FC>4, respectively). Putative function and ortholog in S. cerevisiae (if any) are also given. Abbreviations: 0-gene absent, 1-gene present but not developmentally regulated, 2 - developmentally regulated at fold change $>2$, 4- developmentally regulated at fold change $>4$. Species names are abbreviated as: $\mathrm{Cc}-C$. cinerea, $\mathrm{Aa}-A$. ampla, $\mathrm{Ab}-\mathrm{A}$. bisporus, $\mathrm{Ao}-A$. ostoyae, $\mathrm{Ca}-C$. aegerita, $\mathrm{Lb}-$ L. bicolor, Le - L. edodes, $\mathrm{Lt}-\mathrm{L}$. tigrinus, $\mathrm{Mk}-\mathrm{M}$. kentingensis, $\mathrm{Pc}-$ Ph. chrysosporium, Po - P. ostreatus, $\mathrm{Sc}-\mathrm{S}$. commune.

\begin{tabular}{|c|c|c|c|c|c|c|c|c|c|c|c|c|c|c|c|c|}
\hline \multirow[b]{2}{*}{ ID } & \multicolumn{2}{|c|}{ Fold change } & \multirow[b]{2}{*}{ Putative function } & \multirow{2}{*}{$\begin{array}{c}S . \\
\text { cerevisiae } \\
\text { ortholog }\end{array}$} & \multicolumn{12}{|c|}{ Species } \\
\hline & $>2$ & $>4$ & & & $\mathrm{Cc}$ & Aa & $\mathbf{A b}$ & Ao & $\mathbf{C a}$ & Lb & Le & Lt & Mk & $\mathbf{P c}$ & Po & Sc \\
\hline 360850 & 9 & 8 & Alcohol dehydrogenase & - & 4 & 1 & 4 & 4 & 4 & 4 & 4 & 2 & 4 & 1 & 4 & 1 \\
\hline 369180 & 10 & 7 & Sorbitol dehydrogenase & - & 2 & 4 & 4 & 4 & 0 & 4 & 2 & 4 & 4 & 1 & 2 & 4 \\
\hline 455149 & 11 & 6 & CBM21 domain containing protein & - & 4 & 2 & 2 & 4 & 4 & 2 & 2 & 2 & 4 & 1 & 4 & 4 \\
\hline 441344 & 7 & 2 & Glucoamylase & - & 2 & 4 & 0 & 2 & 1 & 0 & 0 & 2 & 4 & 2 & 2 & 1 \\
\hline 365699 & 8 & 5 & 1,4-alpha-glucan-branching enzyme & YEL011W & 4 & 1 & 2 & 4 & 2 & 4 & 4 & 1 & 0 & 1 & 4 & 2 \\
\hline 496146 & 2 & 0 & $\begin{array}{l}\text { Serine/threonine-protein } \\
\text { phosphatase controlling glycogen } \\
\text { metabolism }\end{array}$ & YER133W & 2 & 1 & 1 & 0 & 1 & 2 & 1 & 1 & 1 & 1 & 1 & 1 \\
\hline 457685 & 9 & 5 & Glycogen synthase & YFR015C & 4 & 2 & 2 & 4 & 1 & 2 & 4 & 1 & 4 & 1 & 4 & 2 \\
\hline 473268 & 6 & 3 & Glucoamylase & YIL099W & 4 & 0 & 2 & 4 & 0 & 1 & 2 & 0 & 4 & 0 & 2 & 0 \\
\hline 461263 & 7 & 3 & Glycogenin & YJL137C & 4 & 1 & 2 & 4 & 2 & 1 & 2 & 1 & 1 & 1 & 4 & 2 \\
\hline 359312 & 9 & 5 & $\begin{array}{l}\text { UTP-glucose-1-phosphate } \\
\text { uridylyltransferase }\end{array}$ & YKL035W & 4 & 2 & 2 & 4 & 2 & 4 & 4 & 1 & 2 & 1 & 4 & 1 \\
\hline
\end{tabular}




\begin{tabular}{|c|c|c|c|}
\hline 497782 & 7 & 5 & Phosphoglucomutase 2 \\
\hline 538513 & 2 & 1 & Cyclin-dependent protein kinase \\
\hline 495987 & 9 & 7 & Glycogen phosphorylase \\
\hline 405214 & 10 & 6 & Glycogen debranching enzyme \\
\hline 495249 & 10 & 7 & $\begin{array}{l}\text { Glycoside hydrolase family } 13 \\
\text { alpha-amylase }\end{array}$ \\
\hline 544130 & 11 & 7 & CBM21 domain containing protein \\
\hline 429272 & 4 & 4 & Mannitol dehydrogenase \\
\hline 478544 & 8 & 2 & $\begin{array}{l}\text { Alpha,alpha-trehalose-phosphate } \\
\text { synthase }\end{array}$ \\
\hline 437212 & 10 & 2 & Neutral alpha-trehalase \\
\hline 473004 & 8 & 4 & Trehalose-phosphatase \\
\hline 503099 & 7 & 3 & $\begin{array}{l}\text { Trehalose synthase complex } \\
\text { regulatory subunit TSL1 }\end{array}$ \\
\hline 376383 & 9 & 6 & Trehalase \\
\hline 540817 & 11 & 6 & Trehalose phosphorylase \\
\hline
\end{tabular}

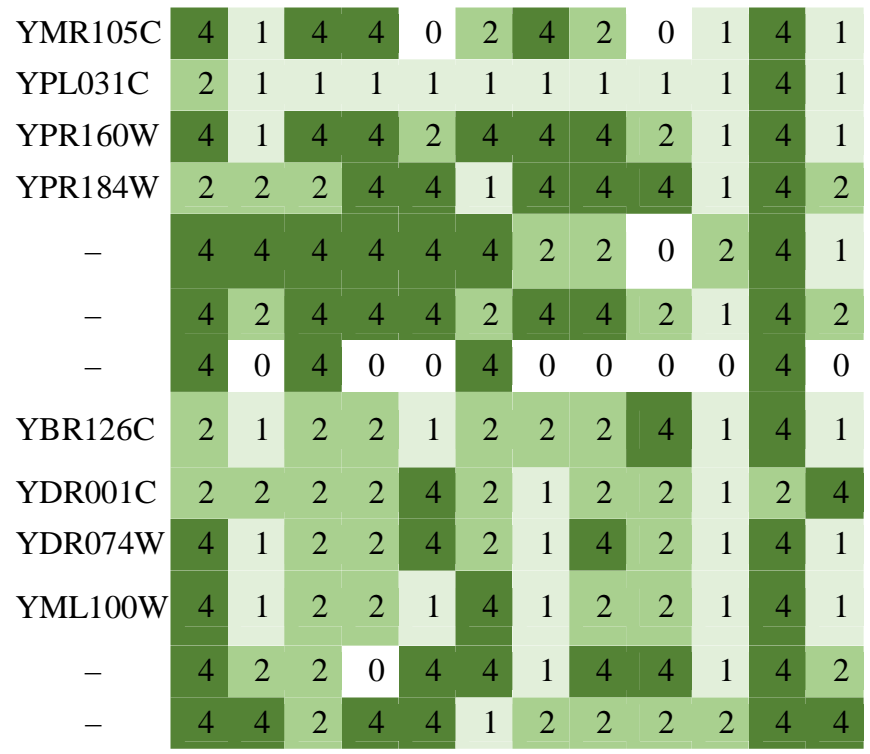

Table 11. Summary of developmental expression dynamics of CDE orthogroups of transcriptional regulator genes across 12 species. Protein ID of a representative protein is given follows by the number of species in which the orthogroup is developmentally regulated at fold change 2 and 4 (FC>2 and FC>4, respectively). Putative function and ortholog in S. cerevisiae (if any) are also given. Abbreviations: 0-gene absent, 1-gene present but not developmentally regulated, 2 developmentally regulated at fold change $>2$, 4- developmentally regulated at fold change $>4$. Species names are abbreviated as: $\mathrm{Cc}-C$. cinerea, $\mathrm{Aa}-A$. ampla, Ab-A. bisporus, Ao - A. ostoyae, $\mathrm{Ca}-C$. aegerita, Lb - L. bicolor, Le - L. edodes, Lt - L. tigrinus, Mk - M. kentingensis, Pc - Ph. chrysosporium, Po-P. ostreatus, $\mathrm{Sc}-\mathrm{S}$. commune.

\begin{tabular}{|c|c|c|c|}
\hline \multirow[b]{2}{*}{ ID } & \multicolumn{2}{|c|}{ Fold change } & \multirow[b]{2}{*}{ Putative function } \\
\hline & $>2$ & $>4$ & \\
\hline 421729 & 9 & 5 & $\begin{array}{l}\text { Transcription factor, } C \text {. cinerea } \\
\text { exp1 }\end{array}$ \\
\hline 395717 & 7 & 7 & Transcription factor \\
\hline 404567 & 9 & 7 & Transcription factor \\
\hline 437393 & 11 & 9 & Transcription factor \\
\hline 446227 & 9 & 8 & Transcription factor \\
\hline 491825 & 10 & 4 & Transcription factor \\
\hline 494418 & 10 & 8 & Transcription factor \\
\hline 497620 & 12 & 9 & Transcription factor \\
\hline $\begin{array}{l}\text { S.c. } \\
252058 \\
3\end{array}$ & 7 & 6 & Transcription factor \\
\hline 8610 & 10 & 6 & $\begin{array}{l}\text { Transcription factor, C. cinerea } \\
\text { Cc.wc2 }\end{array}$ \\
\hline 148855 & 10 & 6 & Transcription factor, L. edodes PriB \\
\hline 493627 & 10 & 9 & $\begin{array}{l}\text { Transcription factor, S. commune } \\
\text { Hom } 1\end{array}$ \\
\hline 449111 & 7 & 6 & Transcription factor \\
\hline 495715 & 12 & 12 & $\begin{array}{l}\text { Transcription factor, } S \text {. commune } \\
\text { Tea } 1\end{array}$ \\
\hline
\end{tabular}

$S$. cerevisiae
ortholog Ce Aa Ab Ao Ca Lb Le Lt Mk Pc Po Sc

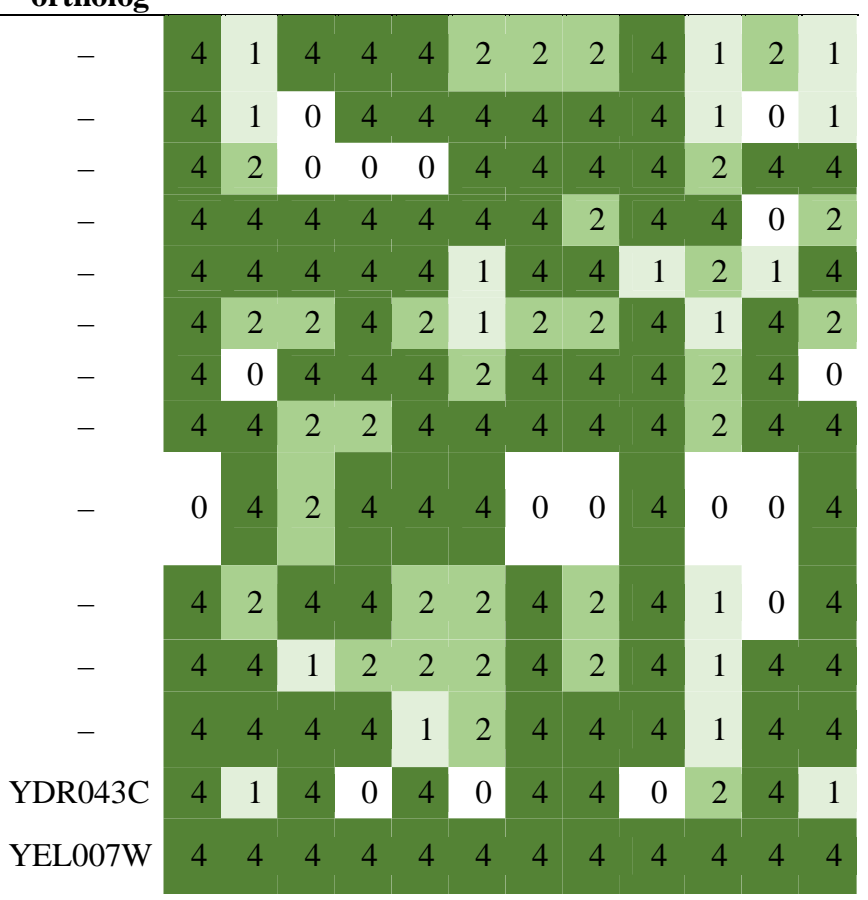




\begin{tabular}{|c|c|c|c|c|c|c|c|c|c|c|c|c|c|c|c|c|}
\hline 458586 & 10 & 3 & $\begin{array}{l}\text { Transcription-related RNA-binding } \\
\text { protein }\end{array}$ & YGR159C & 2 & 2 & 2 & 4 & 2 & 2 & 4 & 2 & 2 & 1 & 4 & 1 \\
\hline 482097 & 10 & 7 & Transcription factor & YPR015C & 2 & 4 & 4 & 2 & 4 & 4 & 0 & 2 & 4 & 1 & 4 & 4 \\
\hline 155160 & 9 & 6 & Transcription initiation factor & - & 4 & 2 & 2 & 4 & 4 & 4 & 4 & 2 & 4 & 1 & 0 & 0 \\
\hline 449345 & 9 & 5 & Transcription factor & - & 4 & 2 & 1 & 4 & 4 & 4 & 2 & 2 & 2 & 1 & 4 & 1 \\
\hline 463573 & 9 & 5 & $\begin{array}{l}\text { Transcription factor, S. commune } \\
\text { Fst } 3\end{array}$ & - & 4 & 1 & 2 & 4 & 2 & 4 & 2 & 2 & 4 & 1 & 4 & 1 \\
\hline 365344 & 9 & 6 & $\begin{array}{l}\text { Transcription factor, S. commune } \\
\text { Fst } 4\end{array}$ & - & 4 & 2 & 2 & 4 & 4 & 4 & 2 & 1 & 4 & 1 & 4 & 1 \\
\hline 211795 & 10 & 5 & Transcription factor & - & 4 & 4 & 2 & 4 & 2 & 4 & 2 & 2 & 4 & 1 & 2 & 1 \\
\hline 498166 & 10 & 6 & $\begin{array}{l}\text { Possibly related to transcription } \\
\text { elongation factors }\end{array}$ & - & 2 & 4 & 4 & 2 & 1 & 1 & 4 & 4 & 4 & 2 & 2 & 4 \\
\hline 508285 & 11 & 4 & $\begin{array}{l}\text { Potentially involved in in pre- } \\
\text { mRNA 3' end processing }\end{array}$ & - & 4 & 2 & 2 & 4 & 0 & 2 & 2 & 2 & 4 & 2 & 2 & 4 \\
\hline 497197 & 7 & 6 & Rho termination factor & - & 4 & 1 & 0 & 4 & 0 & 2 & 4 & 4 & 4 & 4 & 0 & 0 \\
\hline 142396 & 10 & 7 & Transcription factor & - & 4 & 1 & 4 & 2 & 4 & 4 & 4 & 4 & 4 & 2 & 0 & 2 \\
\hline 351135 & 7 & 5 & Transcription factor & - & 4 & 2 & 4 & 4 & 0 & 4 & 2 & 1 & 0 & 1 & 4 & 1 \\
\hline 357193 & 9 & 7 & Transcription factor & - & 4 & 1 & 4 & 4 & 2 & 1 & 4 & 4 & 4 & 1 & 4 & 2 \\
\hline 359157 & 10 & 6 & Transcription factor & - & 4 & 2 & 2 & 4 & 4 & 2 & 2 & 4 & 4 & 1 & 4 & 1 \\
\hline 360688 & 11 & 9 & Transcription factor & - & 4 & 4 & 4 & 4 & 2 & 4 & 4 & 4 & 4 & 1 & 2 & 4 \\
\hline 367840 & 8 & 7 & Transcription factor & - & 4 & 1 & 4 & 4 & 1 & 4 & 4 & 2 & 4 & 1 & 4 & 1 \\
\hline 374546 & 8 & 5 & Transcription factor & - & 4 & 2 & 4 & 4 & 0 & 4 & 0 & 2 & 2 & 1 & 4 & 1 \\
\hline 411205 & 9 & 7 & Transcription factor & - & 4 & 4 & 4 & 4 & 2 & 4 & 1 & 2 & 4 & 1 & 4 & 1 \\
\hline 416057 & 10 & 4 & Transcription factor & - & 2 & 2 & 2 & 4 & 2 & 1 & 4 & 4 & 2 & 2 & 0 & 4 \\
\hline 440363 & 12 & 7 & Transcription factor & - & 4 & 2 & 2 & 4 & 4 & 4 & 4 & 2 & 4 & 2 & 4 & 2 \\
\hline 441820 & 6 & 5 & Transcription factor & - & 4 & 1 & 4 & 4 & 2 & 4 & 1 & 1 & 1 & 1 & 4 & 1 \\
\hline 442154 & 10 & 7 & Transcription factor & - & 4 & 4 & 4 & 4 & 2 & 1 & 2 & 4 & 2 & 4 & 0 & 4 \\
\hline 443979 & 10 & 8 & Transcription factor & - & 4 & 4 & 4 & 4 & 0 & 2 & 4 & 4 & 0 & 2 & 4 & 4 \\
\hline 451915 & 7 & 6 & Transcription factor & - & 4 & 0 & 2 & 0 & 4 & 4 & 0 & 4 & 4 & 4 & 0 & 0 \\
\hline 452897 & 9 & 8 & Transcription factor & - & 4 & 4 & 2 & 4 & 4 & 4 & 0 & 0 & 1 & 4 & 4 & 4 \\
\hline 465631 & 7 & 6 & Transcription factor & - & 4 & 2 & 4 & 4 & 0 & 4 & 4 & 0 & 4 & 0 & 0 & 1 \\
\hline 492675 & 7 & 4 & Transcription factor & - & 4 & 2 & 4 & 2 & 1 & 4 & 1 & 0 & 1 & 1 & 4 & 2 \\
\hline 500056 & 10 & 6 & Transcription factor & - & 4 & 2 & 2 & 4 & 2 & 4 & 1 & 4 & 4 & 1 & 4 & 2 \\
\hline $\begin{array}{l}\text { S.c. } \\
261437 \\
1\end{array}$ & 7 & 7 & Transcription factor & - & 0 & 4 & 0 & 4 & 0 & 0 & 4 & 0 & 4 & 4 & 4 & 4 \\
\hline $\begin{array}{l}\text { S.c. } \\
270530 \\
0\end{array}$ & 6 & 5 & Transcription factor & - & 0 & 4 & 0 & 4 & 0 & 2 & 4 & 4 & 0 & 4 & 0 & 1 \\
\hline 438877 & 9 & 5 & Transcription related protein & - & 2 & 4 & 2 & 4 & 4 & 2 & 4 & 2 & 1 & 1 & 4 & 1 \\
\hline 471238 & 10 & 10 & $\begin{array}{l}\text { RNA binding protein; distantly } \\
\text { related to yeast PAB } 1\end{array}$ & - & 4 & 4 & 0 & 4 & 4 & 0 & 4 & 4 & 4 & 4 & 4 & 4 \\
\hline 369621 & 10 & 4 & Transcription factor, $C$. cinerea dst 1 & - & 4 & 2 & 4 & 4 & 2 & 1 & 2 & 2 & 2 & 1 & 4 & 2 \\
\hline 538904 & 8 & 6 & $\begin{array}{l}\text { Transcription factor, S. commune } \\
\text { Gat1 }\end{array}$ & - & 4 & 2 & 4 & 4 & 1 & 4 & 4 & 1 & 4 & 1 & 2 & 1 \\
\hline 355613 & 9 & 3 & $\begin{array}{l}\text { Transcription factor, } S \text {. commune } \\
\text { Hom } 2\end{array}$ & - & 4 & 1 & 4 & 2 & 4 & 2 & 2 & 2 & 2 & 1 & 1 & 2 \\
\hline 413307 & 7 & 5 & $\begin{array}{l}\text { Transcription related Mynd-type } \\
\text { zinc finger }\end{array}$ & - & 4 & 4 & 4 & 4 & 0 & 4 & 1 & 2 & 0 & 1 & 2 & 1 \\
\hline
\end{tabular}


Table 12. Summary of developmental expression dynamics of CDE orthogroups of RNA-binding protein encoding genes across 12 species. Protein ID of a representative protein is given follows by the number of species in which the orthogroup is developmentally regulated at fold change 2 and 4 (FC>2 and FC $>4$, respectively). Putative function and ortholog in S. cerevisiae (if any) are also given. Abbreviations: 0-gene absent, 1-gene present but not developmentally regulated, 2 developmentally regulated at fold change $>2$, 4- developmentally regulated at fold change $>4$. Species names are abbreviated as: $\mathrm{Cc}-C$. cinerea, $\mathrm{Aa}-A$. ampla, Ab-A. bisporus, Ao - A. ostoyae, $\mathrm{Ca}-\mathrm{C}$. aegerita, $\mathrm{Lb}-$ L. bicolor, Le - L. edodes, $\mathrm{Lt}-$ L. tigrinus, $\mathrm{Mk}-M$. kentingensis, $\mathrm{Pc}$ - Ph. chrysosporium, Po - P. ostreatus, Sc - S. commune.

\begin{tabular}{|c|c|c|c|c|c|c|c|c|c|c|c|c|c|c|c|c|}
\hline \multirow[b]{2}{*}{ ID } & \multicolumn{2}{|c|}{ Fold change } & \multirow[b]{2}{*}{ Putative function } & \multirow{2}{*}{$\begin{array}{c}S . \\
\text { cerevisiae } \\
\text { ortholog }\end{array}$} & \multicolumn{12}{|c|}{ Species } \\
\hline & $>2$ & $>4$ & & & $\mathrm{Cc}$ & Aa & $\mathbf{A b}$ & Ao & $\mathbf{C a}$ & $\mathbf{L b}$ & Le & $\mathbf{L t}$ & Mk & $\mathbf{P c}$ & Po & Sc \\
\hline 357717 & 12 & 12 & RNA binding domain protein & - & 4 & 4 & 4 & 4 & 4 & 4 & 4 & 4 & 4 & 4 & 4 & 4 \\
\hline 354109 & 8 & 6 & mRNA-binding Pumilio protein & YLL013C & 4 & 4 & 1 & 4 & 0 & 2 & 2 & 4 & 0 & 1 & 4 & 4 \\
\hline 444698 & 10 & 3 & RNA binding domain protein & - & 2 & 2 & 2 & 2 & 0 & 2 & 4 & 1 & 4 & 2 & 4 & 2 \\
\hline 541323 & 11 & 10 & $\begin{array}{l}\text { Armadillo domain containing RNA } \\
\text { binding protein }\end{array}$ & - & 4 & 4 & 4 & 4 & 4 & 2 & 4 & 4 & 4 & 1 & 4 & 4 \\
\hline 356106 & 10 & 5 & RNA binding domain protein & - & 4 & 2 & 2 & 4 & 4 & 2 & 2 & 2 & 4 & 1 & 4 & 1 \\
\hline 424800 & 12 & 11 & RNA binding domain protein & - & 4 & 4 & 2 & 4 & 4 & 4 & 4 & 4 & 4 & 4 & 4 & 4 \\
\hline 193545 & 11 & 11 & RNA binding domain protein & - & 4 & 4 & 4 & 4 & 4 & 4 & 4 & 4 & 4 & 4 & 0 & 4 \\
\hline
\end{tabular}

Table 13. Summary of developmental expression dynamics of CDE orthogroups of RNA interference related genes across 12 species. Protein ID of a representative protein is given follows by the number of species in which the orthogroup is developmentally regulated at fold change 2 and 4 (FC>2 and FC>4, respectively). Putative function and ortholog in S. cerevisiae (if any) are also given. Abbreviations: 0-gene absent, 1-gene present but not developmentally regulated, 2 developmentally regulated at fold change $>2$, 4- developmentally regulated at fold change $>4$. Species names are abbreviated as: $\mathrm{Cc}-C$. cinerea, $\mathrm{Aa}-A$. ampla, Ab-A. bisporus, Ao - A. ostoyae, $\mathrm{Ca}-\mathrm{C}$. aegerita, Lb - L. bicolor, Le - L. edodes, Lt - L. tigrinus, Mk - M. kentingensis, Pc - Ph. chrysosporium, Po-P. ostreatus, Sc-S. commune.

\begin{tabular}{lccll} 
& \multicolumn{3}{c}{ Fold change } & \\
\cline { 2 - 3 } ID & $>\mathbf{2}$ & $>\mathbf{4}$ & Putative function \\
\hline 155983 & 6 & 2 & RNA-dependent RNA polymerase \\
498671 & 4 & 1 & RNA-dependent RNA polymerase \\
438955 & 4 & 2 & Dicer-like protein \\
464843 & 2 & 1 & RNA-dependent RNA polymerase \\
366047 & 6 & 1 & Dicer-like protein \\
473372 & 8 & 0 & AGO-like protein \\
495272 & 6 & 4 & Argonaute-like protein \\
& & & Argonaute-like proteins with high \\
502116 & 9 & 6 & similarity to A. nidulans rsdA and $S$ \\
& & & pombe ago1 \\
363313 & 7 & 1 & Orthologs of QDE-2 \\
440860 & 5 & 1 & RNA-dependent RNA polymerase \\
496192 & 5 & 1 & RNA-dependent RNA polymerase \\
498410 & 5 & 1 & RNA-dependent RNA polymerase
\end{tabular}

$S$. cerevisiae
ortholog Ce Aa Ab Ao Ca Lb Le Lt Mk Pc Po Sc

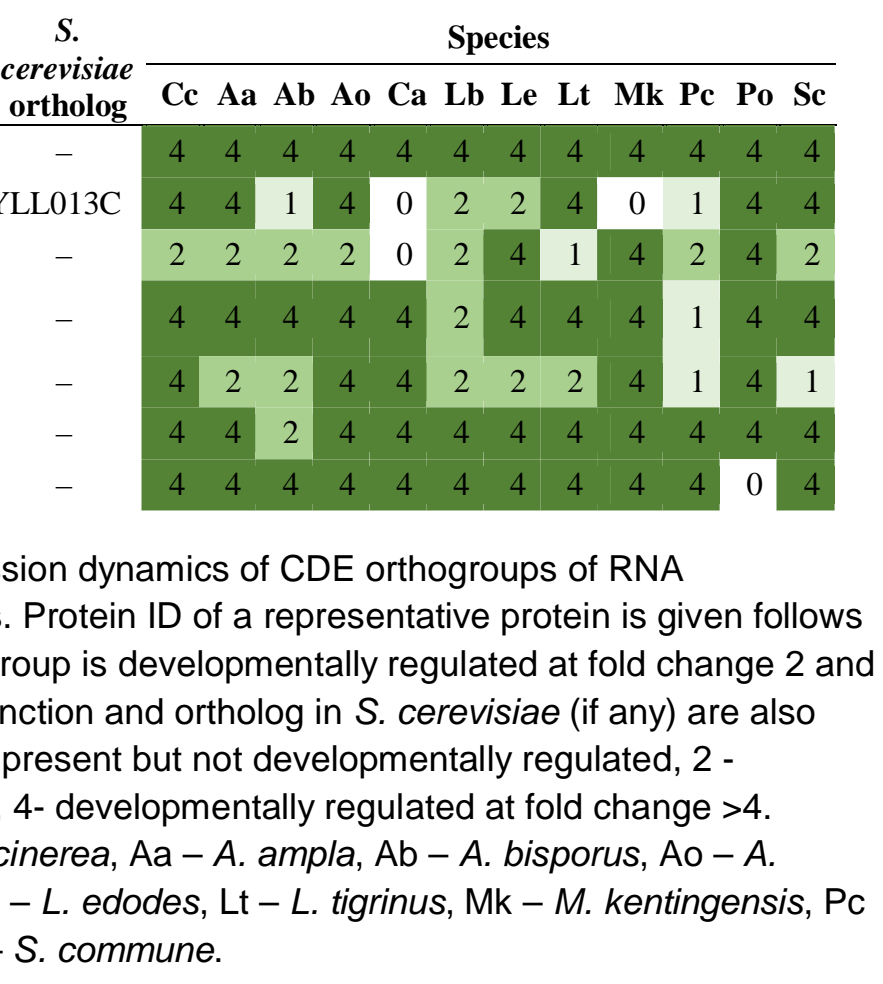

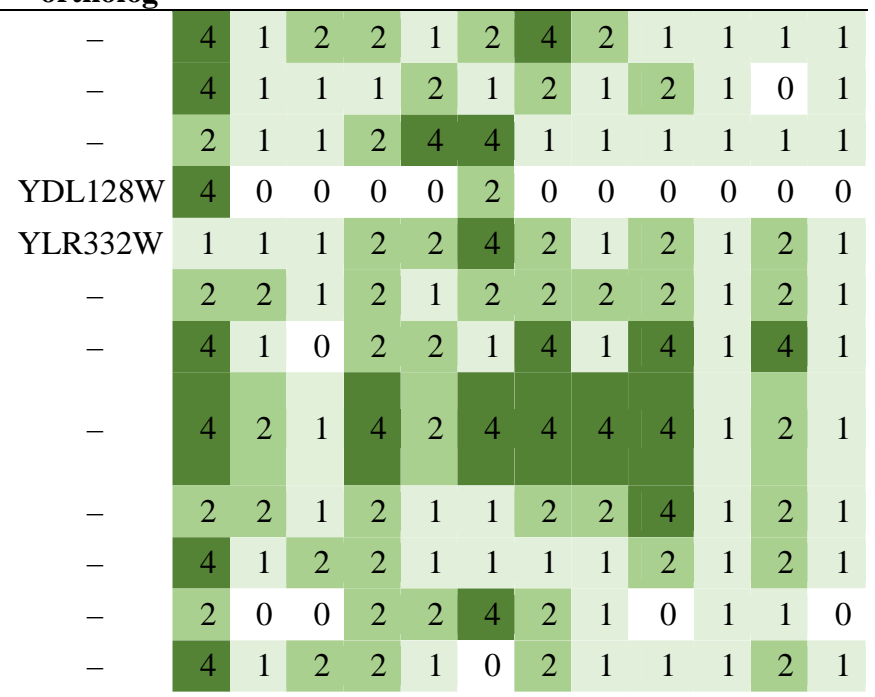




$\begin{array}{llll}355234 & 1 & 1 & \text { AGO ortholog } \\ 368472 & 1 & 0 & \text { Argonaute-like protein } \\ 465906 & 1 & 0 & \text { Argonaute-like protein } \\ 444647 & 3 & 0 & \text { Dicer-like protein } \\ 124704 & 1 & 1 & \text { RNA-dependent RNA polymerase } \\ 444494 & 1 & 1 & \text { RNA-dependent RNA polymerase }\end{array}$

$\begin{array}{lllllllllllll}- & 4 & 0 & 0 & 0 & 0 & 0 & 0 & 0 & 0 & 0 & 0 & 0 \\ - & 2 & 0 & 0 & 0 & 0 & 0 & 0 & 0 & 0 & 0 & 0 & 0 \\ - & 2 & 0 & 0 & 0 & 0 & 0 & 0 & 0 & 0 & 0 & 0 & 0 \\ - & 2 & 1 & 1 & 1 & 1 & 1 & 1 & 1 & 2 & 1 & 2 & 1 \\ - & 4 & 0 & 0 & 0 & 0 & 0 & 0 & 0 & 0 & 0 & 0 & 0 \\ - & 4 & 0 & 0 & 0 & 0 & 0 & 0 & 0 & 0 & 0 & 0 & 0\end{array}$

Table 14. Summary of developmental expression dynamics of CDE orthogroups of chromatinrelated genes across 12 species. Protein ID of a representative protein is given follows by the number of species in which the orthogroup is developmentally regulated at fold change 2 and 4 (FC>2 and FC>4, respectively). Putative function and ortholog in S. cerevisiae (if any) are also given. Abbreviations: 0-gene absent, 1-gene present but not developmentally regulated, 2 developmentally regulated at fold change $>2$, 4- developmentally regulated at fold change $>4$. Species names are abbreviated as: $\mathrm{Cc}-C$. cinerea, $\mathrm{Aa}-A$. ampla, Ab-A. bisporus, Ao - A. ostoyae, $\mathrm{Ca}-\mathrm{C}$. aegerita, Lb - L. bicolor, Le - L. edodes, Lt - L. tigrinus, Mk - M. kentingensis, Pc - Ph. chrysosporium, Po-P. ostreatus, Sc - S. commune.

\begin{tabular}{|c|c|c|c|}
\hline \multirow[b]{2}{*}{ ID } & \multicolumn{2}{|c|}{ Fold change } & \multirow[b]{2}{*}{ Putative function } \\
\hline & $>2$ & $>4$ & \\
\hline 58294 & 10 & 0 & Actin-related protein 4 \\
\hline 485296 & 9 & 0 & $\begin{array}{l}\text { Protein arginine } \mathrm{N}- \\
\text { methyltransferase } 1\end{array}$ \\
\hline 498885 & 11 & 8 & $\begin{array}{l}\text { Histone H2A.Z-specific chaperone } \\
\text { CHZ1 }\end{array}$ \\
\hline 493226 & 10 & 7 & Histone isomerase \\
\hline 455597 & 9 & 9 & $\begin{array}{l}\text { NAD-dependent histone deacetylase } \\
\text { HST3 }\end{array}$ \\
\hline 545797 & 11 & 9 & C. cinerea Ich1 gene \\
\hline 392307 & 11 & 5 & HIRA-interacting protein 3 \\
\hline 384835 & 8 & 6 & Poly(ADP-ribose) polymerase \\
\hline 459336 & 9 & 5 & Poly(ADP-ribose) polymerase \\
\hline 473747 & 8 & 6 & High mobility group protein \\
\hline 66389 & 10 & 4 & Suppressor protein STM1 \\
\hline 264120 & 2 & 0 & SET domain protein \\
\hline 170659 & 1 & 0 & SET domain protein \\
\hline 456684 & 3 & 0 & SET domain protein \\
\hline 497003 & 1 & 0 & SET domain protein \\
\hline 365798 & 2 & 0 & $\begin{array}{l}\text { C. cinerea Snf5 family chromatin } \\
\text { remodeling protein }\end{array}$ \\
\hline 252667 & 8 & 2 & $\begin{array}{l}\text { Histone acetyltransferase type B } \\
\text { subunit } 2\end{array}$ \\
\hline 518613 & 10 & 4 & $\begin{array}{l}\text { Histone acetyltransferase complex } \\
\text { member }\end{array}$ \\
\hline 420113 & 7 & 4 & $\begin{array}{l}\text { Histone acetyltransferase type B } \\
\text { catalytic subunit }\end{array}$ \\
\hline 457726 & 8 & 4 & Histone acethytransferase \\
\hline 430366 & 6 & 0 & Histone deacetylase \\
\hline 415916 & 4 & 0 & Histone deacethylase \\
\hline
\end{tabular}

$S$. cerevisiae
ortholog Ce Aa Ab Ao Ca Lb Le Lt Mk Pc Po Sc

$\begin{array}{llllllllllllll}\text { YBR034C } & 2 & 1 & 2 & 2 & 2 & 2 & 2 & 2 & 2 & 1 & 2 & 1\end{array}$

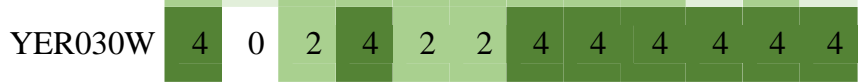

\begin{tabular}{l|l|l|l|l|l|l|l|l|l|l|l|l|} 
YML074C & 4 & 2 & 4 & 4 & 4 & 2 & 2 & 4 & 4 & 1 & 4 & 1
\end{tabular}

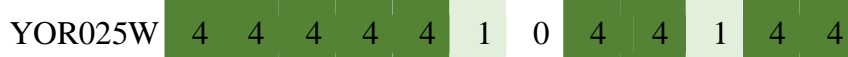

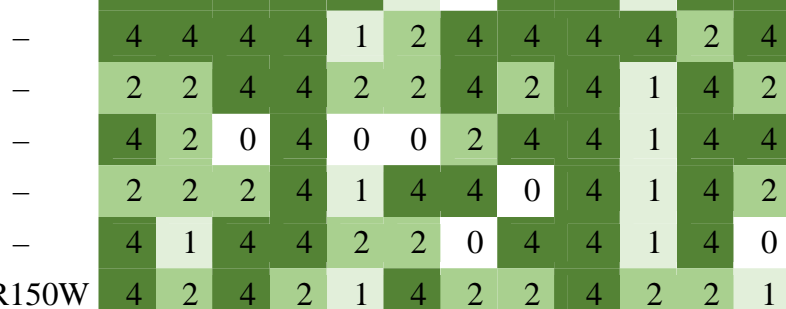

\begin{tabular}{llll|l|l|l|l|l|l|l|l|l|l} 
YLR150W & 4 & 2 & 4 & 2 & 1 & 4 & 2 & 2 & 4 & 2 & 2 & 1
\end{tabular}

$\begin{array}{lllllllllllllll}\text { YJL168C } & 1 & 1 & 1 & 1 & 1 & 1 & 2 & 1 & 1 & 1 & 2 & 1\end{array}$

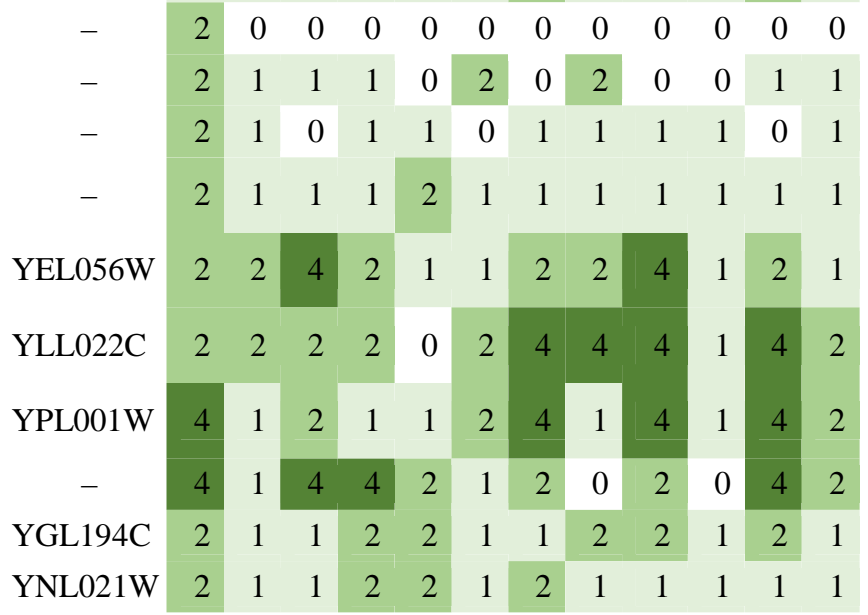




\begin{tabular}{|c|c|c|c|c|c|c|c|c|c|c|c|c|c|c|c|c|}
\hline 354601 & 6 & 2 & Histone deacetylase & YNL330C & 4 & 2 & 2 & 2 & 1 & 1 & 2 & 1 & 1 & 1 & 4 & 1 \\
\hline 52602 & 5 & 3 & Histone deacetylase & YPL116W & 4 & 1 & 1 & 4 & 1 & 1 & 4 & 2 & 0 & 2 & 1 & 1 \\
\hline 364572 & 5 & 0 & Histone deacetylase & - & 1 & 1 & 2 & 2 & 1 & 1 & 2 & 1 & 2 & 1 & 2 & 1 \\
\hline 465141 & 8 & 2 & Histone deacetylase & - & 2 & 4 & 4 & 2 & 2 & 1 & 2 & 1 & 2 & 1 & 1 & 2 \\
\hline 363625 & 8 & 6 & Agaricales-specific histone group & - & 4 & 0 & 0 & 0 & 4 & 4 & 2 & 4 & 4 & 2 & 4 & 0 \\
\hline 154410 & 11 & 6 & Centromere-specific histone variant & - & 2 & 4 & 2 & 2 & 2 & 4 & 2 & 4 & 4 & 1 & 4 & 4 \\
\hline 182362 & 11 & 5 & Histone & - & 4 & 4 & 2 & 2 & 2 & 2 & 2 & 2 & 4 & 0 & 4 & 4 \\
\hline 473916 & 8 & 6 & Histone & - & 2 & 0 & 4 & 4 & 1 & 4 & 0 & 2 & 4 & 0 & 4 & 4 \\
\hline 393767 & 9 & 5 & Histone $\mathrm{H} 2 \mathrm{~B}$ & - & 1 & 1 & 4 & 4 & 2 & 2 & 4 & 4 & 0 & 2 & 2 & 4 \\
\hline 361866 & 5 & 4 & Histone H2B.2 & YBL002W & 2 & 0 & 0 & 4 & 0 & 0 & 4 & 4 & 4 & 0 & 0 & 0 \\
\hline 418275 & 10 & 7 & Histone H2A.2 & YBL003C & 2 & 4 & 4 & 4 & 2 & 4 & 4 & 0 & 0 & 2 & 4 & 4 \\
\hline 401831 & 9 & 6 & Histone $\mathrm{H} 4$ & YBR009C & 1 & 4 & 4 & 2 & 1 & 4 & 2 & 4 & 4 & 1 & 2 & 4 \\
\hline 546355 & 4 & 1 & Histone H3 & YBR010W & 2 & 0 & 1 & 2 & 1 & 1 & 1 & 2 & 4 & 1 & 1 & 1 \\
\hline 284515 & 11 & 2 & Histone H2A.Z & YOL012C & 2 & 2 & 4 & 2 & 2 & 2 & 2 & 2 & 4 & 2 & 2 & 1 \\
\hline 467558 & 10 & 9 & Histone $\mathrm{H} 1$ & YPL127C & 4 & 4 & 4 & 4 & 4 & 4 & 4 & 4 & 1 & 2 & 0 & 4 \\
\hline 320458 & 0 & 0 & Histone & - & 1 & 0 & 0 & 0 & 0 & 0 & 0 & 0 & 0 & 0 & 0 & 0 \\
\hline 324852 & 0 & 0 & Histone & - & 1 & 0 & 0 & 0 & 0 & 0 & 0 & 0 & 0 & 0 & 0 & 0 \\
\hline 380437 & 1 & 1 & Histone & - & 4 & 0 & 0 & 0 & 0 & 0 & 0 & 0 & 0 & 0 & 0 & 0 \\
\hline 385598 & 1 & 1 & Histone & - & 4 & 0 & 0 & 0 & 0 & 0 & 0 & 0 & 0 & 0 & 0 & 0 \\
\hline 446837 & 1 & 0 & Histone & - & 2 & 0 & 0 & 0 & 0 & 0 & 0 & 0 & 0 & 0 & 0 & 0 \\
\hline 492876 & 1 & 0 & Histone & - & 2 & 0 & 0 & 0 & 0 & 0 & 0 & 0 & 0 & 0 & 0 & 0 \\
\hline 503008 & 1 & 0 & Histone & - & 2 & 0 & 0 & 0 & 0 & 0 & 0 & 0 & 0 & 0 & 0 & 0 \\
\hline 452576 & 4 & 0 & $\begin{array}{l}\text { AMP-activated protein } \\
\text { serine/threonine kinase alpha } \\
\text { subunit Ssp2 }\end{array}$ & YDR477W & 2 & 1 & 1 & 2 & 1 & 1 & 2 & 1 & 2 & 1 & 1 & 1 \\
\hline 360033 & 4 & 1 & $\begin{array}{l}\text { 5'-AMP-activated protein kinase } \\
\text { subunit gamma }\end{array}$ & YGL115W & 1 & 1 & 4 & 1 & 1 & 1 & 2 & 2 & 1 & 1 & 2 & 1 \\
\hline 377394 & 8 & 1 & $\begin{array}{l}\text { Histone-lysine N-methyltransferase, } \\
\text { H3 lysine- } 79 \text { specific }\end{array}$ & YDR440W & 2 & 1 & 1 & 4 & 2 & 1 & 2 & 2 & 2 & 1 & 2 & 2 \\
\hline 511209 & 9 & 8 & SRA-YDG domain protein & - & 2 & 4 & 4 & 4 & 4 & 4 & 4 & 0 & 0 & 0 & 4 & 4 \\
\hline
\end{tabular}

Table 15. Summary of developmental expression dynamics of CDE orthogroups of signal transduction related genes across 12 species. Protein ID of a representative protein is given follows by the number of species in which the orthogroup is developmentally regulated at fold change 2 and 4 (FC>2 and FC>4, respectively). Putative function and ortholog in $S$. cerevisiae (if any) are also given. Abbreviations: 0-gene absent, 1-gene present but not developmentally regulated, 2 developmentally regulated at fold change $>2$, 4- developmentally regulated at fold change $>4$. Species names are abbreviated as: $\mathrm{Cc}-C$. cinerea, $\mathrm{Aa}-A$. ampla, Ab $-A$. bisporus, Ao $-A$. ostoyae, $\mathrm{Ca}-$ C. aegerita, Lb - L. bicolor, Le - L. edodes, Lt - L. tigrinus, Mk - M. kentingensis, Pc - Ph. chrysosporium, Po-P. ostreatus, Sc - S. commune.

\begin{tabular}{|c|c|c|c|c|c|c|c|c|c|c|c|c|c|c|c|c|}
\hline \multirow[b]{2}{*}{ ID } & \multicolumn{2}{|c|}{ Fold change } & \multirow[b]{2}{*}{ Putative function } & \multirow{2}{*}{$\begin{array}{c}S . \\
\text { cerevisiae } \\
\text { ortholog }\end{array}$} & \multicolumn{12}{|c|}{ Species } \\
\hline & $>2$ & $>4$ & & & $\mathrm{Ce}$ & Aa & $\mathbf{A b}$ & Ao & $\mathbf{C a}$ & $\mathbf{L b}$ & Le 1 & $\mathbf{L t}$ & Mk & $\mathbf{P c}$ & Po & Sc \\
\hline $\begin{array}{l}\text { S.c. } \\
264367 \\
1\end{array}$ & 7 & 6 & Protein kinase related to mating & - & 1 & 2 & 4 & 2 & 4 & 4 & 4 & 1 & 1 & 1 & 4 & 4 \\
\hline 363127 & 8 & 7 & Alcohol dehydrogenase & YMR318C & 4 & 1 & 0 & 4 & 4 & 0 & 4 & 4 & 2 & 4 & 0 & 4 \\
\hline
\end{tabular}




\begin{tabular}{|c|c|c|c|c|c|c|c|c|c|c|c|c|c|c|c|c|}
\hline 398037 & 9 & 7 & Linoleate diol synthase & - & 4 & 1 & 4 & 2 & 2 & 4 & 4 & 0 & 4 & 4 & 0 & 4 \\
\hline 423716 & 10 & 5 & Linoleate diol synthase & - & 4 & 4 & 2 & 4 & 0 & 2 & 4 & 2 & 1 & 2 & 4 & 2 \\
\hline kinase & & & & & & & & & & & & & & & & \\
\hline 357261 & 8 & 7 & Serine-threonine kinase & - & $\overline{4}$ & $\overline{4}$ & 0 & $\overline{4}$ & 0 & 2 & 0 & 4 & U & $\overline{4}$ & $\overline{4}$ & $\overline{4}$ \\
\hline 397547 & 7 & 5 & Serine-threonine kinase & - & 4 & 0 & 0 & 4 & 4 & 0 & 0 & 2 & 4 & 2 & 0 & 4 \\
\hline 405337 & 12 & 11 & Serine-threonine kinase & - & 4 & 4 & 2 & 4 & 4 & 4 & 4 & 4 & 4 & 4 & 4 & 4 \\
\hline 441896 & 10 & 8 & Serine-threonine kinase & - & 4 & 0 & 2 & 4 & 4 & 4 & 4 & 2 & 4 & 4 & 4 & 0 \\
\hline 456276 & 10 & 8 & Serine-threonine kinase & - & 2 & 4 & 2 & 4 & 4 & 4 & 4 & 1 & 4 & 1 & 4 & 4 \\
\hline 493180 & 9 & 4 & Serine-threonine kinase & - & 4 & 2 & 2 & 4 & 1 & 2 & 1 & 2 & 2 & 1 & 4 & 4 \\
\hline 538093 & 11 & 8 & Serine-threonine kinase & - & 2 & 4 & 2 & 4 & 2 & 4 & 4 & 4 & 0 & 4 & 4 & 4 \\
\hline 462137 & 11 & 9 & Serine-threonine kinase & YDR247W & 2 & 2 & 4 & 4 & 4 & 4 & 4 & 4 & 4 & 1 & 4 & 4 \\
\hline 449305 & 10 & 4 & $\begin{array}{l}\text { P38 mitogen-activated protein } \\
\text { kinase HOG1 }\end{array}$ & YLR113W & 4 & 1 & 2 & 2 & 2 & 4 & 4 & 2 & 2 & 1 & 4 & 2 \\
\hline 451611 & 10 & 6 & Serine-threonine kinase & YLR248W & 4 & 2 & 1 & 4 & 4 & 4 & 2 & 2 & 2 & 1 & 4 & 4 \\
\hline 448193 & 10 & 6 & Serine-threonine kinase & - & 2 & 4 & 1 & 4 & 1 & 2 & 4 & 4 & 4 & 2 & 4 & 2 \\
\hline 359429 & 6 & 5 & Serine-threonine kinase & - & 4 & 0 & 0 & 4 & 1 & 4 & 2 & 0 & 4 & 0 & 4 & 0 \\
\hline 448807 & 6 & 5 & Serine-threonine kinase & - & 4 & 0 & 4 & 2 & 4 & 0 & 0 & 4 & 4 & 1 & 0 & 0 \\
\hline 470972 & 8 & 6 & Serine-threonine kinase & - & 4 & 4 & 0 & 2 & 0 & 4 & 1 & 4 & 2 & 4 & 0 & 4 \\
\hline
\end{tabular}

Table 16. Summary of developmental expression dynamics of CDE orthogroups of pheromone precursor genes across 12 species. Protein ID of a representative protein is given follows by the number of species in which the orthogroup is developmentally regulated at fold change 2 and 4 (FC>2 and $F C>4$, respectively). Putative function and ortholog in $S$. cerevisiae (if any) are also given. Abbreviations: 0-gene absent, 1-gene present but not developmentally regulated, 2 developmentally regulated at fold change $>2$, 4- developmentally regulated at fold change $>4$. Species names are abbreviated as: $\mathrm{Cc}-C$. cinerea, $\mathrm{Aa}-A$. ampla, $\mathrm{Ab}-A$. bisporus, $\mathrm{Ao}-A$. ostoyae, $\mathrm{Ca}-\mathrm{C}$. aegerita, Lb - L. bicolor, Le - L. edodes, Lt - L. tigrinus, Mk - M. kentingensis, Pc - Ph. chrysosporium, Po-P. ostreatus, $\mathrm{Sc}-\mathrm{S}$. commune.

\begin{tabular}{|c|c|c|c|}
\hline \multirow[b]{2}{*}{ ID } & \multicolumn{2}{|c|}{ Fold change } & \multirow[b]{2}{*}{ Putative function } \\
\hline & $>2$ & $>4$ & \\
\hline 153902 & 2 & 0 & $\begin{array}{l}\text { Protein-S-isoprenylcysteine O- } \\
\text { methyltransferase }\end{array}$ \\
\hline 116060 & 1 & 1 & Mating pheromone precursor \\
\hline 446846 & 1 & 1 & Mating pheromone precursor \\
\hline 446955 & 1 & 1 & Mating pheromone precursor \\
\hline 446956 & 1 & 1 & Mating pheromone precursor \\
\hline 446958 & 0 & 0 & Mating pheromone precursor \\
\hline 446959 & 1 & 1 & Mating pheromone precursor \\
\hline 446966 & 0 & 0 & Mating pheromone precursor \\
\hline 356637 & 1 & 1 & $\begin{array}{l}\text { Pheromone sensing GPCR Ste } 3 \\
\text { homolog }\end{array}$ \\
\hline 394943 & 4 & 2 & $\begin{array}{l}\text { Pheromone sensing GPCR Ste } 3 \\
\text { homolog }\end{array}$ \\
\hline 477564 & 5 & 3 & $\begin{array}{l}\text { Pheromone sensing GPCR Ste } 3 \\
\text { homolog }\end{array}$ \\
\hline 377901 & 7 & 3 & CAAX prenyl protease 2 \\
\hline
\end{tabular}

$S$. cerevisiae
ortholog

\begin{tabular}{|c|c|c|c|c|c|c|c|c|c|c|c|c|}
\hline YDR410C & 2 & 1 & 0 & 1 & 2 & 1 & 1 & 1 & 1 & 1 & 1 & 1 \\
\hline - & 4 & 0 & 0 & 0 & 0 & 0 & 0 & 0 & 0 & 0 & 0 & 0 \\
\hline- & 4 & 0 & 0 & 0 & 0 & 0 & 0 & 0 & 0 & 0 & 0 & 0 \\
\hline - & 4 & 0 & 0 & 0 & 0 & 0 & 0 & 0 & 0 & 0 & 0 & 0 \\
\hline - & 4 & 0 & 0 & 0 & 0 & 0 & 0 & 0 & 0 & 0 & 0 & 0 \\
\hline - & 1 & 0 & 0 & 0 & 0 & 0 & 0 & 0 & 0 & 0 & 0 & 0 \\
\hline- & 4 & 0 & 0 & 0 & 0 & 0 & 0 & 0 & 0 & 0 & 0 & 0 \\
\hline- & 1 & 0 & 0 & 0 & 0 & 0 & 0 & 0 & 0 & 0 & 0 & 0 \\
\hline - & 4 & 0 & 0 & 0 & 0 & 0 & 0 & 0 & 0 & 0 & 0 & 0 \\
\hline - & 4 & 0 & 0 & 4 & 2 & 1 & 0 & 0 & 0 & 0 & 2 & 0 \\
\hline- & 4 & 0 & 2 & 0 & 4 & 0 & 0 & 2 & 0 & 0 & 4 & 0 \\
\hline - & 4 & 1 & 2 & 1 & 1 & 2 & 4 & 1 & 4 & 1 & 2 & 2 \\
\hline
\end{tabular}




\begin{tabular}{|c|c|c|c|c|c|c|c|c|c|c|c|c|c|c|c|c|}
\hline 203704 & 6 & 3 & $\begin{array}{l}\text { Pheromone sensing GPCR Ste3 } \\
\text { homolog }\end{array}$ & - & 4 & 2 & 0 & 4 & 4 & 2 & 1 & 0 & 1 & 1 & 0 & 2 \\
\hline 465723 & 8 & 4 & $\begin{array}{l}\text { Pheromone sensing GPCR Ste3 } \\
\text { homolog }\end{array}$ & - & 2 & 1 & 4 & 4 & 2 & 1 & 4 & 2 & 1 & 1 & 4 & 2 \\
\hline 486965 & 5 & 3 & $\begin{array}{l}\text { Pheromone sensing GPCR Ste } 3 \\
\text { homolog }\end{array}$ & - & 2 & 0 & 0 & 4 & 0 & 4 & 0 & 2 & 1 & 1 & 4 & 0 \\
\hline 491483 & 3 & 2 & $\begin{array}{l}\text { Protein farnesyltransferase subunit } \\
\text { beta }\end{array}$ & YDL090C & 4 & 1 & 1 & 1 & 4 & 1 & 1 & 1 & 1 & 1 & 2 & 1 \\
\hline 437675 & 5 & 0 & $\begin{array}{l}\text { Pheromone-processing } \\
\text { carboxypeptidase KEX1 }\end{array}$ & YGL203C & 2 & 1 & 1 & 1 & 2 & 2 & 2 & 1 & 1 & 1 & 2 & 1 \\
\hline 545529 & 5 & 1 & Dipeptidyl aminopeptidase B & YHR028C & 1 & 1 & 4 & 2 & 1 & 1 & 1 & 2 & 2 & 1 & 2 & 1 \\
\hline 419650 & 6 & 1 & CAAX prenyl protease 1 ste 24 & YJR117W & 4 & 2 & 2 & 1 & 2 & 1 & 1 & 2 & 1 & 1 & 2 & 1 \\
\hline 473005 & 8 & 2 & $\begin{array}{l}\text { Protein } \\
\text { farnesyltransferase/geranylgeranyltr } \\
\text { ansferase type-1 subunit alpha }\end{array}$ & YKL019W & 4 & 2 & 1 & 4 & 2 & 2 & 2 & 2 & 1 & 1 & 2 & 1 \\
\hline 540181 & 10 & 4 & Alpha-factor-transporting ATPase & YKL209C & 4 & 4 & 2 & 2 & 2 & 4 & 2 & 2 & 4 & 1 & 1 & 2 \\
\hline 358206 & 8 & 2 & A-factor-processing enzyme & YLR389C & 2 & 1 & 1 & 2 & 2 & 4 & 4 & 1 & 2 & 1 & 2 & 2 \\
\hline 448165 & 5 & 2 & Putative kexin & YNL238W & 1 & 2 & 2 & 4 & 1 & 1 & 1 & 1 & 4 & 1 & 1 & 2 \\
\hline 106286 & 7 & 2 & $\begin{array}{l}\text { Pheromone sensing GPCR Ste3 } \\
\text { homolog }\end{array}$ & - & 4 & 1 & 2 & 4 & 2 & 0 & 2 & 2 & 2 & 0 & 0 & 1 \\
\hline 152667 & 9 & 9 & Homolog of S. cerevisiae Ste14 & - & 4 & 0 & 4 & 4 & 4 & 4 & 4 & 4 & 1 & 4 & 4 & 0 \\
\hline
\end{tabular}

Table 17. Summary of developmental expression dynamics of CDE orthogroups of conserved signal transduction pathway genes across 12 species. Protein ID of a representative protein is given follows by the number of species in which the orthogroup is developmentally regulated at fold change 2 and 4 (FC>2 and FC>4, respectively). Putative function and ortholog in S. cerevisiae (if any) are also given. Abbreviations: 0-gene absent, 1-gene present but not developmentally regulated, 2 - developmentally regulated at fold change $>2$, 4- developmentally regulated at fold change $>4$. Species names are abbreviated as: $\mathrm{Cc}-C$. cinerea, $\mathrm{Aa}-A$. ampla, $\mathrm{Ab}-A$. bisporus, Ao - A. ostoyae, $\mathrm{Ca}-C$. aegerita, $\mathrm{Lb}-$ L. bicolor, Le - L. edodes, $\mathrm{Lt}-\mathrm{L}$. tigrinus, $\mathrm{Mk}-M$. kentingensis, $\mathrm{Pc}-\mathrm{Ph}$. chrysosporium, $\mathrm{Po}-\mathrm{P}$. ostreatus, $\mathrm{Sc}-\mathrm{S}$. commune.

\begin{tabular}{|c|c|c|c|c|c|c|c|c|c|c|c|c|c|c|c|c|}
\hline \multirow[b]{2}{*}{ ID } & \multicolumn{2}{|c|}{ Fold change } & \multirow[b]{2}{*}{ Putative function } & \multirow{2}{*}{$\begin{array}{c}S . \\
\text { cerevisiae } \\
\text { ortholog }\end{array}$} & \multicolumn{12}{|c|}{ Species } \\
\hline & $>2$ & $>4$ & & & $\mathrm{Cc}$ & Aa & $\mathbf{A b}$ & Ao & $\mathbf{C a}$ & $\mathbf{L b}$ & Le & $\mathbf{L t}$ & Mk & Pc & Po & Sc \\
\hline \multicolumn{17}{|l|}{ cAMP } \\
\hline 465055 & 4 & 0 & $\begin{array}{l}\text { Phosphatidylinositol phosphate } \\
\text { phosphatase }\end{array}$ & - & 2 & 1 & 2 & 1 & 1 & 1 & 2 & 1 & 2 & 1 & 1 & 1 \\
\hline 462760 & 6 & 0 & $\begin{array}{l}\text { GTPase involved in G-protein } \\
\text { signaling in adenylate cyclase } \\
\text { activation }\end{array}$ & - & 2 & 1 & 2 & 2 & 1 & 2 & 1 & 2 & 1 & 1 & 2 & 1 \\
\hline 539854 & 10 & 3 & $\begin{array}{l}\text { Low-affinity cyclic AMP } \\
\text { phosphodiesterase }\end{array}$ & YGL248W & 4 & 4 & 2 & 2 & 1 & 2 & 4 & 2 & 2 & 1 & 2 & 2 \\
\hline 494851 & 7 & 2 & $\begin{array}{l}\text { Phosphatidylinositol phosphate } \\
\text { phosphatase }\end{array}$ & YKL212W & 2 & 2 & 4 & 2 & 1 & 1 & 2 & 1 & 2 & 1 & 4 & 1 \\
\hline 175104 & 5 & 1 & $\begin{array}{l}\text { High-affinity cyclic AMP } \\
\text { phosphodiesterase }\end{array}$ & YOR360C & 1 & 1 & 4 & 2 & 1 & 2 & 1 & 1 & 1 & 1 & 2 & 2 \\
\hline 45965 & 7 & 2 & Protein kinase A catalytic subunit 2 & YPL203W & 4 & 2 & 2 & 2 & 1 & 1 & 1 & 2 & 2 & 1 & 4 & 1 \\
\hline 361063 & 6 & 1 & Protein kinase A catalytic subunit 1 & - & 2 & 1 & 4 & 1 & 2 & 1 & 1 & 2 & 2 & 1 & 2 & 1 \\
\hline 497554 & 2 & 0 & Adenylate cyclase & - & 1 & 1 & 1 & 2 & 1 & 1 & 1 & 1 & 2 & 1 & 1 & 1 \\
\hline 449966 & 1 & 1 & Carbonic anhydrase & - & 4 & 0 & 0 & 0 & 0 & 0 & 0 & 0 & 0 & 0 & 0 & 0 \\
\hline \multicolumn{17}{|c|}{ Cell wall stress } \\
\hline 453815 & 1 & 0 & 1-phosphatidylinositol 4-kinase & - & 2 & 1 & 1 & 1 & 1 & 1 & 1 & 1 & 1 & 1 & 1 & 1 \\
\hline
\end{tabular}




\begin{tabular}{|c|c|c|c|c|c|c|c|c|c|c|c|c|c|c|c|c|}
\hline & & & STT4 & & & & & & & & & & & & & \\
\hline 464132 & 4 & 2 & $\begin{array}{l}\text { 1-phosphatidylinositol-4-phosphate } \\
\text { 5-kinase }\end{array}$ & - & 4 & 1 & 1 & 1 & 2 & 2 & 1 & 1 & 1 & 1 & 4 & 1 \\
\hline 494847 & 2 & 0 & $\begin{array}{l}\text { Mitogen-activated protein kinase } \\
\text { kinase kinase BCK1 }\end{array}$ & - & 1 & 0 & 0 & 1 & 1 & 2 & 1 & 0 & 2 & 1 & 0 & 1 \\
\hline 186221 & 4 & 0 & $\begin{array}{l}\text { Mitogen-activated protein kinase } \\
\text { kinase MKK1 }\end{array}$ & - & 1 & 1 & 2 & 2 & 1 & 1 & 1 & 1 & 1 & 1 & 2 & 2 \\
\hline 466997 & 3 & 0 & $\begin{array}{l}\text { Rho family guanine nucleotide } \\
\text { exchange factor ROM } 2\end{array}$ & - & 2 & 1 & 1 & 1 & 0 & 1 & 2 & 1 & 2 & 1 & 1 & 1 \\
\hline 419097 & 5 & 1 & $\begin{array}{l}\text { Classical protein kinase } \mathrm{C} \text { alpha } \\
\text { type }\end{array}$ & YBL105C & 2 & 1 & 2 & 1 & 1 & 2 & 1 & 1 & 2 & 1 & 4 & 1 \\
\hline 373470 & 7 & 2 & $\begin{array}{l}\text { Cyclin-dependent serine/threonine- } \\
\text { protein kinase CDC } 28\end{array}$ & YBR160W & 2 & 1 & 1 & 0 & 1 & 4 & 2 & 2 & 2 & 1 & 4 & 2 \\
\hline 418410 & 5 & 1 & $\begin{array}{l}\text { 3-phosphoinositide dependent } \\
\text { protein kinase-3 }\end{array}$ & YDR490C & 2 & 2 & 1 & 1 & 2 & 1 & 1 & 1 & 2 & 1 & 4 & 1 \\
\hline 528086 & 6 & 0 & $\begin{array}{l}\text { Rho family GTPase RHO1, Ras } \\
\text { homolog gene family, member A }\end{array}$ & YPR165W & 2 & 1 & 2 & 2 & 1 & 2 & 1 & 1 & 2 & 1 & 2 & 1 \\
\hline 446030 & 7 & 1 & 1,3-beta-D-glucan synthase & YGR032W & 2 & 1 & 2 & 4 & 1 & 2 & 2 & 1 & 2 & 1 & 2 & 1 \\
\hline 498153 & 6 & 2 & Tyrosine protein phosphatase PTP3 & YER075C & 2 & 2 & 1 & 4 & 1 & 1 & 2 & 1 & 2 & 1 & 4 & 1 \\
\hline 413665 & 8 & 5 & $\begin{array}{l}\text { Mitogen-activated serine/threonine- } \\
\text { protein kinase SLT2 }\end{array}$ & YHR030C & 2 & 1 & 4 & 4 & 1 & 4 & 2 & 2 & 4 & 1 & 4 & 1 \\
\hline
\end{tabular}

\begin{tabular}{|c|c|c|c|c|c|c|c|c|c|c|c|c|c|c|c|c|}
\hline 474858 & 4 & 0 & $\begin{array}{l}\text { Mitogen-activated protein kinase } \\
\text { kinase kinase, osomolarity two- } \\
\text { component system, response } \\
\text { regulator SSK2 }\end{array}$ & - & 1 & 1 & 1 & 2 & 2 & 2 & 1 & 1 & 1 & 1 & 2 & 1 \\
\hline 494483 & 4 & 0 & $\begin{array}{l}\text { Rho family guanine nucleotide } \\
\text { exchange factor CDC } 24 \text {, cell } \\
\text { division control protein } 24\end{array}$ & - & 2 & 1 & 1 & 2 & 1 & 2 & 1 & 1 & 2 & 1 & 1 & 1 \\
\hline 446453 & 6 & 0 & Type $2 \mathrm{C}$ protein phosphatase $\mathrm{PTC} 1$ & YDL006W & 2 & 1 & 2 & 2 & 1 & 1 & 2 & 1 & 2 & 1 & 0 & 2 \\
\hline 356186 & 6 & 1 & $\begin{array}{l}\text { High osmolarity two-component } \\
\text { System, phosphorelay intermediate } \\
\text { protein YPD1 }\end{array}$ & YDL235C & 4 & 0 & 0 & 2 & 0 & 1 & 2 & 2 & 2 & 1 & 0 & 2 \\
\hline 484600 & 10 & 4 & G-protein beta-like WD40 protein & YMR116C & 4 & 2 & 4 & 2 & 2 & 1 & 2 & 2 & 4 & 2 & 4 & 1 \\
\hline 498153 & 6 & 2 & Tyrosine protein phosphatase PTP3 & YER075C & 2 & 2 & 1 & 4 & 1 & 1 & 2 & 1 & 2 & 1 & 4 & 1 \\
\hline 419006 & 5 & 0 & $\begin{array}{l}\text { Pheromone receptor transcription } \\
\text { factor }\end{array}$ & YMR043W & 2 & 1 & 1 & 2 & 1 & 2 & 2 & 2 & 0 & 1 & 1 & 0 \\
\hline \multicolumn{17}{|c|}{ Pheromone pathway } \\
\hline 16197 & 1 & 0 & GTPase-activating protein SST2 & - & 2 & 1 & 1 & 1 & 1 & 1 & 1 & 1 & 1 & 1 & 1 & 1 \\
\hline 370279 & 4 & 0 & $\begin{array}{l}\text { Mitogen-activated protein kinase } \\
\text { kinase }\end{array}$ & - & 1 & 2 & 2 & 1 & 2 & 1 & 1 & 2 & 1 & 1 & 1 & 1 \\
\hline 353910 & 1 & 0 & $\begin{array}{l}\text { Mitogen-activated protein kinase } \\
\text { kinase kinase STE11 }\end{array}$ & - & 1 & 1 & 0 & 2 & 1 & 1 & 1 & 1 & 1 & 1 & 0 & 1 \\
\hline 446910 & 3 & 0 & $\begin{array}{l}\text { Serine/threonine protein kinase } \\
\text { SKM1/CLA4, Serine/threonine- } \\
\text { protein kinase CLA4 }\end{array}$ & - & 1 & 1 & 1 & 2 & 2 & 1 & 1 & 1 & 0 & 1 & 2 & 1 \\
\hline 416655 & 3 & 0 & Rho family GTPase CDC42 & - & 2 & 1 & 1 & 0 & 1 & 1 & 2 & 1 & 1 & 1 & 2 & 1 \\
\hline 437458 & 3 & 0 & $\begin{array}{l}\text { Scaffold protein BEM1; } \\
\text { phosphatidylinositol-3-phosphate- } \\
\text { binding protein BEM1, bud } \\
\text { emergence protein } 1\end{array}$ & - & 2 & 1 & 2 & 1 & 1 & 1 & 1 & 1 & 2 & 1 & 1 & 1 \\
\hline 455590 & 2 & 0 & $\begin{array}{l}\text { The RA domain of Ste } 50 \text { adaptor } \\
\text { protein is required for delivery of } \\
\text { Ste } 11 \text { to the plasma membrane in } \\
\text { the filamentous growth signaling } \\
\text { pathway (???) }\end{array}$ & - & 2 & 1 & 1 & 1 & 0 & 1 & 1 & 1 & 1 & 1 & 2 & 1 \\
\hline
\end{tabular}




\begin{tabular}{|c|c|c|c|c|c|c|c|c|c|c|c|c|c|c|c|c|}
\hline 370180 & 4 & 0 & $\begin{array}{l}\text { Tyrosine/serine/threonine protein } \\
\text { phosphatase MSG5 }\end{array}$ & - & 2 & 1 & 1 & 1 & 2 & 1 & 1 & 1 & 2 & 1 & 2 & 1 \\
\hline 445505 & 5 & 1 & $\begin{array}{l}\text { Mitogen-activated serine/threonine- } \\
\text { protein kinase FUS3 }\end{array}$ & YBL016W & 1 & 2 & 2 & 1 & 1 & 1 & 2 & 4 & 1 & 1 & 2 & 1 \\
\hline 429295 & 6 & 0 & $\begin{array}{l}\text { Mitogen-activated protein kinase } \\
\text { kinase kinase kinase STE20, p21- } \\
\text { activated kinase } 1\end{array}$ & YHL007C & 2 & 1 & 2 & 2 & 0 & 2 & 1 & 1 & 2 & 1 & 2 & 1 \\
\hline 84056 & 4 & 2 & $\begin{array}{l}\text { Guanine nucleotide-binding protein } \\
\text { subunit gamma }\end{array}$ & YJR086W & 2 & 1 & 2 & 0 & 1 & 1 & 1 & 1 & 4 & 1 & 4 & 1 \\
\hline 449416 & 5 & 0 & $\begin{array}{l}\text { Guanine nucleotide-binding protein } \\
\text { GI/GS/GT subunit beta-1 }\end{array}$ & YOR212W & 2 & 1 & 2 & 2 & 1 & 1 & 1 & 1 & 2 & 1 & 2 & 1 \\
\hline 498153 & 6 & 2 & Tyrosine protein phosphatase PTP3 & YER075C & 2 & 2 & 1 & 4 & 1 & 1 & 2 & 1 & 2 & 1 & 4 & 1 \\
\hline 419006 & 5 & 0 & $\begin{array}{l}\text { Pheromone receptor transcription } \\
\text { factor }\end{array}$ & YMR043W & 2 & 1 & 1 & 2 & 1 & 2 & 2 & 2 & 0 & 1 & 1 & 0 \\
\hline 376911 & 8 & 1 & $\begin{array}{l}\text { Homeodomain family transcription } \\
\text { factor STE12 }\end{array}$ & - & 4 & 1 & 2 & 2 & 1 & 2 & 2 & 2 & 2 & 1 & 0 & 2 \\
\hline Starvatic & patl & & & & & & & & & & & & & & & \\
\hline 356859 & 4 & 2 & SHO1 osmosensor & - & 4 & 1 & 2 & 1 & 1 & 2 & 1 & 1 & 1 & 1 & 4 & 1 \\
\hline 197636 & 2 & 1 & Signaling mucin MSB2 & - & 2 & 0 & 1 & 0 & 1 & 0 & 0 & 1 & 4 & 0 & 0 & 0 \\
\hline 389009 & 7 & 0 & 14-3-3 family protein BMH1 & YDR099W & 1 & 2 & 2 & 1 & 2 & 2 & 1 & 2 & 2 & 1 & 2 & 1 \\
\hline 498153 & 6 & 2 & Tyrosine protein phosphatase PTP3 & YER075C & 2 & 2 & 1 & 4 & 1 & 1 & 2 & 1 & 2 & 1 & 4 & 1 \\
\hline 376911 & 8 & 1 & $\begin{array}{l}\text { Homeodomain family transcription } \\
\text { factor STE12 }\end{array}$ & - & 4 & 1 & 2 & 2 & 1 & 2 & 2 & 2 & 2 & 1 & 0 & 2 \\
\hline
\end{tabular}

\begin{tabular}{|c|c|c|c|}
\hline 407528 & 6 & 3 & SEA complex subunit Seh1 \\
\hline 365555 & 4 & 0 & Protein kinase A regulatory subunit \\
\hline 434690 & 10 & 4 & $\begin{array}{l}\text { Phosphoinositide } \mathrm{PI} 4,5 \mathrm{P}(2) \text { binding } \\
\text { Protein, forms a complex with } \\
\text { Slm2p }\end{array}$ \\
\hline 502431 & 5 & 1 & $\begin{array}{l}\text { COPII-coated vesicle component } \\
\text { Sec13 }\end{array}$ \\
\hline 466474 & 8 & 6 & FK506-binding protein 1 \\
\hline 81628 & 6 & 0 & Gtr1/RagA G protein Gtr1 \\
\hline 417412 & 2 & 0 & $\begin{array}{l}\text { Component of a membrane-bound } \\
\text { complex containing the Tor } 2 p \\
\text { kinase }\end{array}$ \\
\hline 498449 & 1 & 0 & $\begin{array}{l}\text { DEP domain containing 5; subunit } \\
\text { of SEACIT (GATOR1) }\end{array}$ \\
\hline 438057 & 4 & 1 & Gtr1/RagA G protein Gtr2 \\
\hline 467184 & 4 & 1 & $\begin{array}{l}\text { Guanyl-nucleotide exchange factor, } \\
\text { HOPS complex subunit Vam6 }\end{array}$ \\
\hline 440799 & 4 & 0 & $\begin{array}{l}\text { Nitrogen permease regulator 2-like } \\
\text { protein; subunit of SEACIT } \\
\text { (GATOR1) }\end{array}$ \\
\hline 366098 & 2 & 0 & $\begin{array}{l}\text { Nitrogen permease regulator 3-like } \\
\text { protein; subunit of SEACIT } \\
\text { (GATOR1) }\end{array}$ \\
\hline 374354 & 4 & 1 & $\begin{array}{l}\text { Protein required for the transport of } \\
\text { Gap1p }\end{array}$ \\
\hline 493094 & 1 & 1 & Ras homolog \\
\hline 517273 & 3 & 0 & $\begin{array}{l}\text { Subunit of SEACAT, a subcomplex } \\
\text { of the SEA complex; Mtc1p }\end{array}$ \\
\hline 367284 & 0 & 0 & $\begin{array}{l}\text { Subunit of SEACAT, a subcomplex } \\
\text { of the SEA complex; Sea } 4 p\end{array}$ \\
\hline 440869 & 2 & 0 & Subunit of TORC1 \\
\hline
\end{tabular}

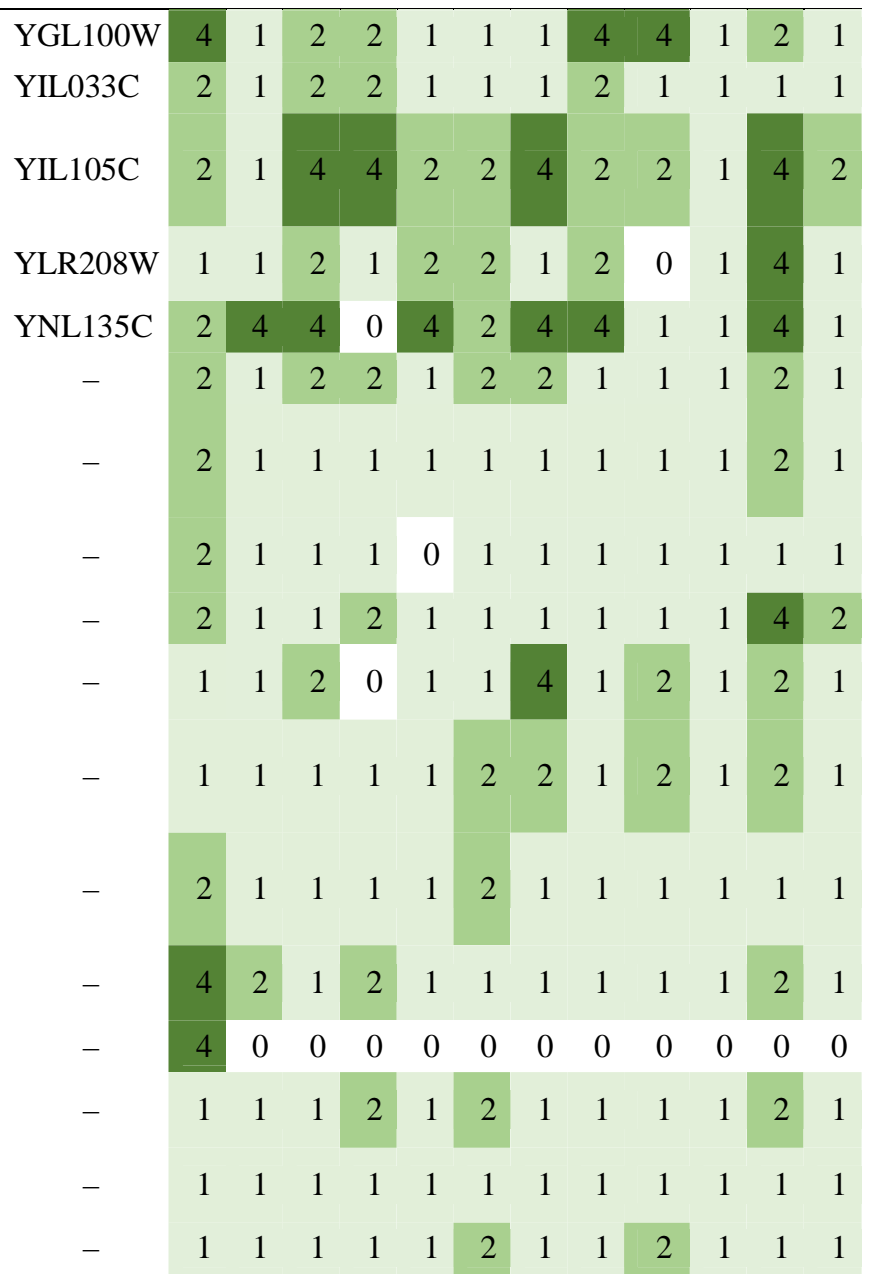




\begin{tabular}{llll}
495775 & 3 & 1 & $\begin{array}{l}\text { Subunit of TORC2, RICTOR } \\
\text { homolog }\end{array}$ \\
439934 & 2 & 0 & $\begin{array}{l}\text { Target of rapamycin } \\
23387\end{array}$ \\
\hline & 0 & 0 & $\begin{array}{l}\text { Tuberous sclerosis 2 protein } \\
\text { homolog }\end{array}$
\end{tabular}

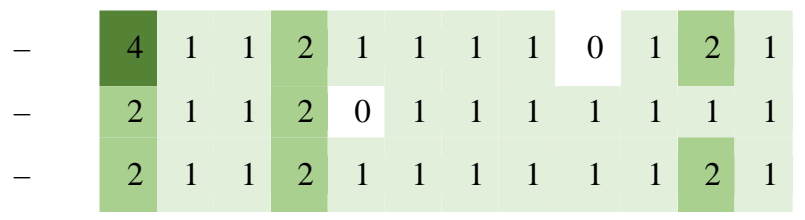

Table 18. Summary of developmental expression dynamics of CDE orthogroups of G-protein coupled receptor genes across 12 species. Protein ID of a representative protein is given follows by the number of species in which the orthogroup is developmentally regulated at fold change 2 and 4 ( $F C>2$ and $F C>4$, respectively). Putative function and ortholog in $S$. cerevisiae (if any) are also given. Abbreviations: 0-gene absent, 1-gene present but not developmentally regulated, 2 developmentally regulated at fold change $>2$, 4- developmentally regulated at fold change $>4$. Species names are abbreviated as: $\mathrm{Cc}-C$. cinerea, $\mathrm{Aa}-A$. ampla, Ab-A. bisporus, Ao $-A$. ostoyae, $\mathrm{Ca}-C$. aegerita, $\mathrm{Lb}-\mathrm{L}$. bicolor, $\mathrm{Le}-\mathrm{L}$. edodes, $\mathrm{Lt}-\mathrm{L}$. tigrinus, $\mathrm{Mk}-\mathrm{M}$. kentingensis, $\mathrm{Pc}$ - Ph. chrysosporium, Po-P. ostreatus, Sc - S. commune.

\begin{tabular}{|c|c|c|c|}
\hline \multirow[b]{2}{*}{ ID } & \multicolumn{2}{|c|}{ Fold change } & \multirow[b]{2}{*}{ Putative function } \\
\hline & $>2$ & $>4$ & \\
\hline 283463 & 6 & 0 & G-protein coupled receptor \\
\hline 263790 & 4 & 3 & G-protein coupled receptor \\
\hline 485193 & 6 & 0 & G-protein coupled receptor \\
\hline 379008 & 0 & 0 & G-protein coupled receptor \\
\hline 489342 & 1 & 1 & G-protein coupled receptor \\
\hline 229833 & 11 & 4 & $\begin{array}{l}\text { G-protein involved in signal } \\
\text { transduction }\end{array}$ \\
\hline 479562 & 11 & 7 & Regulator of G-protein signaling \\
\hline $\begin{array}{l}\text { S.c. } \\
249031 \\
2\end{array}$ & 9 & 6 & Putative arrestin \\
\hline 473465 & 10 & 5 & Putative arrestin \\
\hline 472370 & 11 & 7 & Ras superfamily protein \\
\hline
\end{tabular}

$S$.

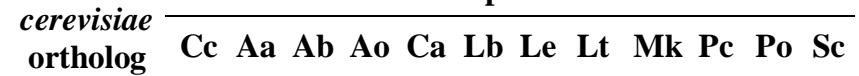

\begin{tabular}{c|ccccccccccccc} 
YKL039W & 2 & 1 & 2 & 2 & 1 & 1 & 2 & 1 & 2 & 1 & 2 & 1 \\
- & 4 & 1 & 0 & 4 & 1 & 4 & 0 & 0 & 0 & 0 & 0 & 2 \\
- & 2 & 2 & 1 & 2 & 2 & 1 & 1 & 2 & 1 & 1 & 2 & 1 \\
- & 1 & 0 & 0 & 0 & 0 & 0 & 0 & 0 & 0 & 0 & 0 & 0 \\
- & 4 & 0 & 0 & 0 & 0 & 0 & 0 & 0 & 0 & 0 & 0 & 0 \\
\hline & 2 & 1 & 4 & 2 & 2 & 4 & 2 & 2 & 4 & 2 & 4 & 2 \\
- & 4 & 4 & 4 & 4 & 2 & 2 & 4 & 1 & 2 & 2 & 4 & 4 \\
\hline & & & 4 & 2 & 4 & 4 & 4 & 1 & 2 & 0 & 4 & 4 & 2 \\
- & 4 & 4 & 4 & 2 & 1 & 2 & 2 & 2 & 4 & 1 & 4 & 2 \\
- & 4 & 2 & 2 & 4 & 4 & 4 & 1 & 4 & 4 & 2 & 4 & 2
\end{tabular}

Table 19. Summary of developmental expression dynamics of CDE orthogroups of velvet protein encoding genes across 12 species. Protein ID of a representative protein is given follows by the number of species in which the orthogroup is developmentally regulated at fold change 2 and 4 (FC>2 and FC>4, respectively). Putative function and ortholog in S. cerevisiae (if any) are also given. Abbreviations: 0-gene absent, 1-gene present but not developmentally regulated, 2 developmentally regulated at fold change $>2$, 4- developmentally regulated at fold change $>4$. Species names are abbreviated as: $\mathrm{Cc}-C$. cinerea, $\mathrm{Aa}-A$. ampla, $\mathrm{Ab}-\mathrm{A}$. bisporus, $\mathrm{Ao}-A$. ostoyae, $\mathrm{Ca}-\mathrm{C}$. aegerita, $\mathrm{Lb}-\mathrm{L}$. bicolor, Le - L. edodes, $\mathrm{Lt}-\mathrm{L}$. tigrinus, $\mathrm{Mk}-\mathrm{M}$. kentingensis, $\mathrm{Pc}$ - Ph. chrysosporium, Po - P. ostreatus, Sc - S. commune.

\begin{tabular}{|c|c|c|c|}
\hline \multirow[b]{2}{*}{ ID } & \multicolumn{2}{|c|}{ Fold change } & \multirow[b]{2}{*}{ Putative funct } \\
\hline & $>2$ & $>4$ & \\
\hline 365674 & 4 & 1 & Velvet factor \\
\hline 355684 & 3 & 3 & Velvet factor \\
\hline 374867 & 6 & 1 & Velvet factor \\
\hline 385561 & 8 & 5 & Velvet factor \\
\hline
\end{tabular}

\begin{tabular}{|c|c|c|c|c|c|c|c|c|c|c|c|c|}
\hline \multirow{2}{*}{$\begin{array}{c}S . \\
\text { cerevisiae } \\
\text { ortholog }\end{array}$} & \multicolumn{12}{|c|}{ Species } \\
\hline & $\mathrm{Cc}$ & $\mathbf{A a}$ & $\mathbf{A b}$ & Ao & $\mathbf{C a}$ & Lb & Le & $\mathbf{L t}$ & Mk & Pc & Po & Sc \\
\hline- & 4 & 1 & 1 & 1 & 1 & 1 & 2 & 1 & 2 & 1 & 2 & 1 \\
\hline - & 4 & 0 & 1 & 0 & 4 & 4 & 0 & 0 & 0 & 0 & 0 & 0 \\
\hline- & 2 & 1 & 2 & 1 & 2 & 2 & 2 & 1 & 4 & 0 & 1 & 1 \\
\hline- & 4 & 4 & 1 & 0 & 0 & 4 & 2 & 4 & 4 & 1 & 2 & 2 \\
\hline
\end{tabular}


Table 20. Summary of developmental expression dynamics of CDE orthogroups of STRIPAK complex protein genes across 12 species. Protein ID of a representative protein is given follows by the number of species in which the orthogroup is developmentally regulated at fold change 2 and 4 ( $F C>2$ and $F C>4$, respectively). Putative function and ortholog in $S$. cerevisiae (if any) are also given. Abbreviations: 0-gene absent, 1-gene present but not developmentally regulated, 2 developmentally regulated at fold change $>2$, 4- developmentally regulated at fold change $>4$. Species names are abbreviated as: $\mathrm{Cc}-C$. cinerea, $\mathrm{Aa}-A$. ampla, Ab - A. bisporus, Ao - A. ostoyae, $\mathrm{Ca}-\mathrm{C}$. aegerita, Lb - L. bicolor, Le - L. edodes, Lt - L. tigrinus, Mk - M. kentingensis, Pc - Ph. chrysosporium, Po - P. ostreatus, Sc - S. commune.

\begin{tabular}{|c|c|c|c|}
\hline \multirow[b]{2}{*}{ ID } & \multicolumn{2}{|c|}{ Fold change } & \multirow[b]{2}{*}{ Putative function } \\
\hline & $>2$ & $>4$ & \\
\hline 51761 & 1 & 0 & Forkhead-associated domain \\
\hline 439971 & 1 & 0 & $\begin{array}{l}\text { Protein phosphatase } \mathrm{PP} 2 \mathrm{~A} \\
\text { regulatory subunit A }\end{array}$ \\
\hline 280222 & 5 & 0 & $\begin{array}{l}\text { Serine/threonine-protein kinase } \\
\text { KIC1 }\end{array}$ \\
\hline 375927 & 4 & 0 & Factor arrest protein 11 \\
\hline 463106 & 5 & 2 & $\begin{array}{l}\text { Serine/threonine-protein } \\
\text { phosphatase PP2A-like PPG1 }\end{array}$ \\
\hline 499433 & 8 & 2 & MOB kinase activator \\
\hline 108726 & 5 & 0 & Striatin \\
\hline
\end{tabular}

\begin{tabular}{ccccccccccccccc}
$\begin{array}{c}\text { S. } \\
\text { cerevisiae } \\
\text { ortholog }\end{array}$ & \multicolumn{10}{c}{ Species } \\
\cline { 2 - 12 } & Cc & Aa & Ab & Ao & Ca & Lb & Le & Lt & Mk & Pc & Po & Sc \\
\hline- & 2 & 1 & 1 & 1 & 1 & 1 & 1 & 1 & 1 & 1 & 1 & 1 \\
YAL016W & 1 & 1 & 1 & 1 & 1 & 1 & 1 & 1 & 1 & 1 & 2 & 1 \\
YHR102W & 1 & 1 & 1 & 2 & 2 & 2 & 1 & 2 & 1 & 1 & 2 & 1 \\
YNL127W & 2 & 1 & 1 & 2 & 1 & 1 & 2 & 1 & 1 & 1 & 2 & 1 \\
YNR032W & 4 & 1 & 4 & 1 & 1 & 1 & 2 & 2 & 1 & 1 & 2 & 1 \\
- & 4 & 2 & 2 & 1 & 2 & 1 & 1 & 4 & 2 & 2 & 2 & 1 \\
- & 2 & 1 & 2 & 2 & 1 & 1 & 1 & 2 & 1 & 1 & 2 & 1
\end{tabular}

Table 21. Summary of developmental expression dynamics of CDE orthogroups of light sensing related genes across 12 species. Protein ID of a representative protein is given follows by the number of species in which the orthogroup is developmentally regulated at fold change 2 and 4 (FC>2 and FC>4, respectively). Putative function and ortholog in $S$. cerevisiae (if any) are also given. Abbreviations: 0-gene absent, 1-gene present but not developmentally regulated, 2 developmentally regulated at fold change $>2$, 4- developmentally regulated at fold change $>4$. Species names are abbreviated as: $\mathrm{Cc}-C$. cinerea, $\mathrm{Aa}-A$. ampla, Ab - A. bisporus, Ao - A. ostoyae, $\mathrm{Ca}-\mathrm{C}$. aegerita, $\mathrm{Lb}-$ L. bicolor, Le - L. edodes, Lt - L. tigrinus, $\mathrm{Mk}-M$. kentingensis, $\mathrm{Pc}$ - Ph. chrysosporium, Po-P. ostreatus, Sc - S. commune.

\begin{tabular}{|c|c|c|c|c|c|c|c|c|c|c|c|c|c|c|c|c|}
\hline \multirow[b]{2}{*}{ ID } & \multicolumn{2}{|c|}{ Fold change } & \multirow[b]{2}{*}{ Putative function } & \multirow{2}{*}{$\begin{array}{c}\text { S. } \\
\text { cerevisiae } \\
\text { ortholog }\end{array}$} & \multicolumn{12}{|c|}{ Species } \\
\hline & $>2$ & $>4$ & & & $\mathrm{Cc}$ & Aa & $\mathbf{A b}$ & Ao & $\mathbf{C a}$ & $\mathbf{L b}$ & Le & $\mathbf{L t}$ & Mk & Pc & Po & Sc \\
\hline 492962 & 9 & 7 & DASH-type cryptochrome & YOR386W & 4 & 1 & 2 & 4 & 4 & 0 & 4 & 2 & 4 & 4 & 4 & 1 \\
\hline 470504 & 7 & 2 & Phytochrome & - & 4 & 1 & 0 & 4 & 2 & 0 & 2 & 1 & 2 & 1 & 2 & 2 \\
\hline 361584 & 11 & 9 & $\begin{array}{l}\text { C. } \text { cinerea } \text { dst } 2 \text {, putative AA } 7 \\
\text { family member }\end{array}$ & - & 4 & 2 & 4 & 4 & 4 & 4 & 4 & 4 & 4 & 1 & 4 & 2 \\
\hline
\end{tabular}

Table 22. Summary of developmental expression dynamics of CDE orthogroups of chitin biosynthesis genes across 12 species. Protein ID of a representative protein is given follows by the number of species in which the orthogroup is developmentally regulated at fold change 2 and 4 (FC>2 and FC>4, respectively). Putative function and ortholog in S. cerevisiae (if any) are also given. Abbreviations: 0-gene absent, 1-gene present but not developmentally regulated, 2 developmentally regulated at fold change $>2,4$ - developmentally regulated at fold change $>4$. 
Species names are abbreviated as: $\mathrm{Cc}-C$. cinerea, $\mathrm{Aa}-A$. ampla, Ab - A. bisporus, Ao - A. ostoyae, $\mathrm{Ca}-C$. aegerita, $\mathrm{Lb}-$ L. bicolor, Le - L. edodes, Lt - L. tigrinus, Mk $-M$. kentingensis, $\mathrm{Pc}$ - Ph. chrysosporium, Po - P. ostreatus, Sc - S. commune.

\begin{tabular}{|c|c|c|c|c|c|c|c|c|c|c|c|c|c|c|c|c|}
\hline \multirow[b]{2}{*}{ ID } & \multicolumn{2}{|c|}{ Fold change } & \multirow[b]{2}{*}{ Putative function } & \multirow{2}{*}{$\begin{array}{c}S . \\
\text { cerevisiae } \\
\text { ortholog }\end{array}$} & \multicolumn{12}{|c|}{ Species } \\
\hline & $>2$ & $>4$ & & & $\mathrm{Ce}$ & Aa & $\mathbf{A b}$ & Ao & $\mathbf{C a}$ & Lb & Le 1 & $\mathbf{L t}$ & Mk & Pc & Po & Sc \\
\hline 371191 & 9 & 4 & $\begin{array}{l}\text { Glucosamine 6-phosphate N- } \\
\text { acetyltransferase }\end{array}$ & - & 4 & 2 & 4 & 4 & 1 & 2 & 2 & 2 & 2 & 1 & 4 & 1 \\
\hline 538650 & 6 & 0 & Chitin synthase regulator SKT5 & YBL061C & 2 & 1 & 2 & 2 & 2 & 1 & 1 & 2 & 2 & 1 & 1 & 1 \\
\hline 542039 & 8 & 1 & Chitin synthase 3 & YBR023C & 2 & 2 & 2 & 4 & 2 & 2 & 1 & 1 & 2 & 1 & 2 & 1 \\
\hline 357695 & 7 & 5 & $\begin{array}{l}\text { UDP-N-acetylglucosamine } \\
\text { pyrophosphorylase }\end{array}$ & YDL103C & 4 & 2 & 4 & 4 & 4 & 1 & 1 & 2 & 1 & 1 & 4 & 1 \\
\hline 478844 & 4 & 1 & $\begin{array}{l}\text { Phosphoacetylglucosamine mutase } \\
\text { involved in chitin precursor } \\
\text { synthesis }\end{array}$ & YEL058W & 4 & 1 & 1 & 2 & 2 & 1 & 1 & 1 & 1 & 1 & 2 & 1 \\
\hline 05 & 9 & 4 & $\begin{array}{l}\text { Glutamine-fructose-6-phosphate } \\
\text { aminotransferase }\end{array}$ & KL104C & 4 & 1 & 4 & 4 & 2 & 2 & 2 & 2 & 2 & 1 & 4 & 1 \\
\hline 439486 & 3 & 1 & Chitin synthase 1 & YNL192W & 4 & 1 & 1 & 1 & 1 & 2 & 1 & 1 & 1 & 1 & 2 & 1 \\
\hline 473500 & 4 & 2 & Cell wall related glycosyltransferase & YPL227C & 2 & 1 & 4 & 1 & 1 & 1 & 1 & 2 & 1 & 1 & 4 & 1 \\
\hline 38 & 7 & 1 & $\begin{array}{l}\text { Dolichol-phosphate } \\
\text { mannosyltransferase }\end{array}$ & YPR183W & 2 & 1 & 2 & 1 & 1 & 4 & 1 & 2 & 2 & 1 & 2 & 2 \\
\hline 372414 & 4 & 2 & Cell wall related glycosyltransferase & - & 2 & 4 & 0 & 1 & 2 & 0 & 4 & 1 & 0 & 1 & 0 & 1 \\
\hline 15443 & 1 & 1 & Chitin synthase & - & 4 & 0 & 0 & 0 & 0 & 0 & 0 & 0 & 0 & 0 & 0 & 0 \\
\hline 234699 & 3 & 0 & Chitin synthase & - & 1 & 1 & 2 & 0 & 1 & 1 & 1 & 1 & 2 & 1 & 1 & 2 \\
\hline 357091 & 5 & 1 & Chitin synthase & - & 1 & 1 & 1 & 2 & 2 & 2 & 1 & 1 & 2 & 1 & 4 & 1 \\
\hline 359180 & 12 & 10 & Chitin synthase & - & 4 & 4 & 4 & 4 & 2 & 4 & 4 & 4 & 2 & 4 & 4 & 4 \\
\hline 493091 & 8 & 2 & Chitin synthase & - & 2 & 1 & 4 & 2 & 2 & 2 & 2 & 1 & 2 & 1 & 4 & 1 \\
\hline 374670 & 3 & 0 & $\begin{array}{l}\text { Chitin synthase with myosin motor } \\
\text { domain }\end{array}$ & - & 2 & 1 & 0 & 2 & 1 & 1 & 1 & 1 & 1 & 1 & 2 & 1 \\
\hline 497826 & 5 & 0 & $\begin{array}{l}\text { Chitin synthase with myosin motor } \\
\text { domain }\end{array}$ & - & 2 & 1 & 2 & 2 & 1 & 2 & 1 & 1 & 2 & 1 & 1 & 1 \\
\hline 519767 & 11 & 6 & Glycosyltransferase 2 family protein & - & 4 & 2 & 4 & 4 & 2 & 4 & 2 & 4 & 2 & 1 & 4 & 2 \\
\hline
\end{tabular}

Table 23. Summary of developmental expression dynamics of CDE orthogroups of glucan biosynthesis related genes across 12 species. Protein ID of a representative protein is given follows by the number of species in which the orthogroup is developmentally regulated at fold change 2 and 4 (FC>2 and FC>4, respectively). Putative function and ortholog in S. cerevisiae (if any) are also given. Abbreviations: 0-gene absent, 1-gene present but not developmentally regulated, 2 developmentally regulated at fold change $>2$, 4- developmentally regulated at fold change $>4$. Species names are abbreviated as: $\mathrm{Cc}-C$. cinerea, $\mathrm{Aa}-A$. ampla, Ab-A. bisporus, Ao - A. ostoyae, $\mathrm{Ca}-\mathrm{C}$. aegerita, Lb - L. bicolor, Le - L. edodes, Lt - L. tigrinus, Mk $-M$. kentingensis, Pc - Ph. chrysosporium, Po-P. ostreatus, Sc - S. commune.

\begin{tabular}{|c|c|c|c|}
\hline \multirow[b]{2}{*}{ ID } & \multicolumn{2}{|c|}{ Fold change } & \multirow[b]{2}{*}{ Putative function } \\
\hline & $>2$ & $>4$ & \\
\hline 417746 & 12 & 4 & $\begin{array}{l}\text { Kre9/Knh1 family putative beta- } \\
\text { 1,6-glucan assembly protein }\end{array}$ \\
\hline 181364 & 4 & 0 & $\begin{array}{l}\text { Smi } 1 \text { family protein regulating } \\
\text { chitin deposition and cell wall } \\
\text { assembly }\end{array}$ \\
\hline
\end{tabular}

S. cerevisiae ortholog

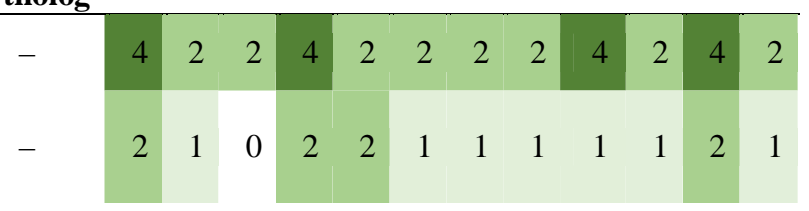




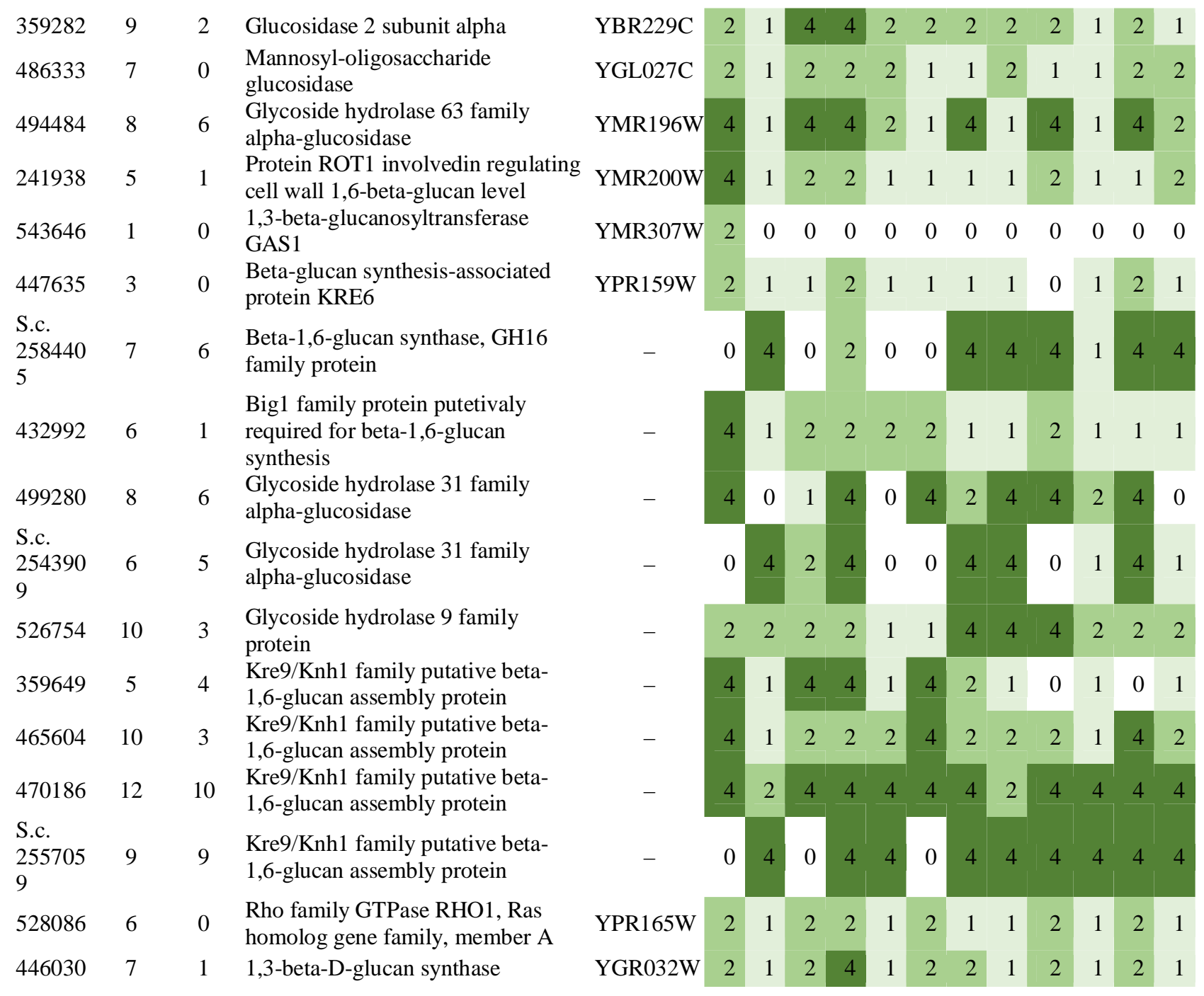

Table 24. Summary of developmental expression dynamics of CDE orthogroups of chitin remodeling genes across 12 species. Protein ID of a representative protein is given follows by the number of species in which the orthogroup is developmentally regulated at fold change 2 and 4 (FC>2 and FC>4, respectively). Putative function and ortholog in S. cerevisiae (if any) are also given. Abbreviations: 0-gene absent, 1-gene present but not developmentally regulated, 2 developmentally regulated at fold change $>2$, 4- developmentally regulated at fold change $>4$. Species names are abbreviated as: $\mathrm{Cc}-C$. cinerea, $\mathrm{Aa}-A$. ampla, Ab-A. bisporus, Ao-A. ostoyae, $\mathrm{Ca}-\mathrm{C}$. aegerita, $\mathrm{Lb}-$ L. bicolor, Le - L. edodes, Lt - L. tigrinus, Mk - M. kentingensis, Pc - Ph. chrysosporium, Po - P. ostreatus, Sc - S. commune.

\begin{tabular}{|c|c|c|c|}
\hline \multirow[b]{2}{*}{ ID } & \multicolumn{2}{|c|}{ Fold change } & \multirow[b]{2}{*}{ Putative function } \\
\hline & $>2$ & $>4$ & \\
\hline 360173 & 7 & 2 & CE4 chitin deacetylase \\
\hline 368217 & 10 & 9 & Glycoside hydrolase 18 , chitinase \\
\hline $\begin{array}{l}\text { S.c. } \\
85210\end{array}$ & 9 & 7 & Glycoside hydrolase 18 , chitinase \\
\hline 543586 & 7 & 2 & Glycoside hydrolase 18 , chitinase \\
\hline
\end{tabular}
\begin{tabular}{cccccccccccccc}
$\begin{array}{c}S . \\
\text { cerevisiae } \\
\text { ortholog }\end{array}$ & Cc & Aa & Ab & Ao & Ca & Lb & Le & Lt & Mk Pc & Po & Sc \\
\cline { 2 - 12 } & 4 & 1 & 2 & 4 & 0 & 1 & 2 & 2 & 0 & 1 & 2 & 2 \\
- & 4 & 4 & 4 & 4 & 4 & 0 & 4 & 4 & 2 & 4 & 0 & 4 \\
- & 0 & 4 & 4 & 2 & 4 & 0 & 4 & 4 & 4 & 2 & 4 & 1 \\
- & 4 & 1 & 2 & 2 & 2 & 1 & 2 & 2 & 1 & 1 & 4 & 1
\end{tabular} 


\begin{tabular}{|c|c|c|c|}
\hline 544873 & 6 & 4 & Polyamine transporter 2 \\
\hline 354859 & 10 & 4 & Endochitinase \\
\hline 355445 & 11 & 8 & CE4 chitin deacetylase 1 \\
\hline 413423 & 11 & 7 & CBM5/12 domain protein \\
\hline 375733 & 7 & 6 & CE4 chitin deacetylase \\
\hline 377259 & 10 & 4 & CE4 chitin deacetylase \\
\hline 379321 & 7 & 5 & CE4 chitin deacetylase \\
\hline 42776 & 2 & 1 & CE4 chitin deacetylase \\
\hline 440521 & 7 & 7 & CE4 chitin deacetylase \\
\hline 464952 & 1 & 1 & CE4 chitin deacetylase \\
\hline 502602 & 1 & 1 & CE4 chitin deacetylase \\
\hline 358869 & 8 & $J$ & Glycoside hydrolase 18 , chitinase \\
\hline 470416 & 9 & 6 & Glycoside hydrolase 18 , chitinase \\
\hline 488885 & 10 & 7 & Glycoside hydrolase 18 , chitinase \\
\hline 90984 & 1 & 1 & Glycoside hydrolase 18 , chitinase \\
\hline 91051 & 9 & 8 & Glycoside hydrolase 18 , chitinase \\
\hline 446427 & 8 & 6 & $\begin{array}{l}\text { Glycoside hydrolase } 20 \text { family } \\
\text { putative } \beta-1,6-\mathrm{N} \text { - } \\
\text { acetylglucosaminidase }\end{array}$ \\
\hline 464567 & 9 & 6 & $\begin{array}{l}\text { Glycoside hydrolase } 20 \text { family } \\
\text { putative } \beta-1,6-\mathrm{N} \text { - } \\
\text { acetylglucosaminidase }\end{array}$ \\
\hline 520359 & 10 & 7 & $\begin{array}{l}\text { Glycoside hydrolase family } 18 \\
\text { chitinase }\end{array}$ \\
\hline 356467 & 7 & 4 & $\begin{array}{l}\text { GlcN6P deaminase (Nag1 family) } \\
\text { involved in cell wall biogenesis }\end{array}$ \\
\hline 454418 & 9 & 6 & X325 family LPMO-like protein \\
\hline
\end{tabular}

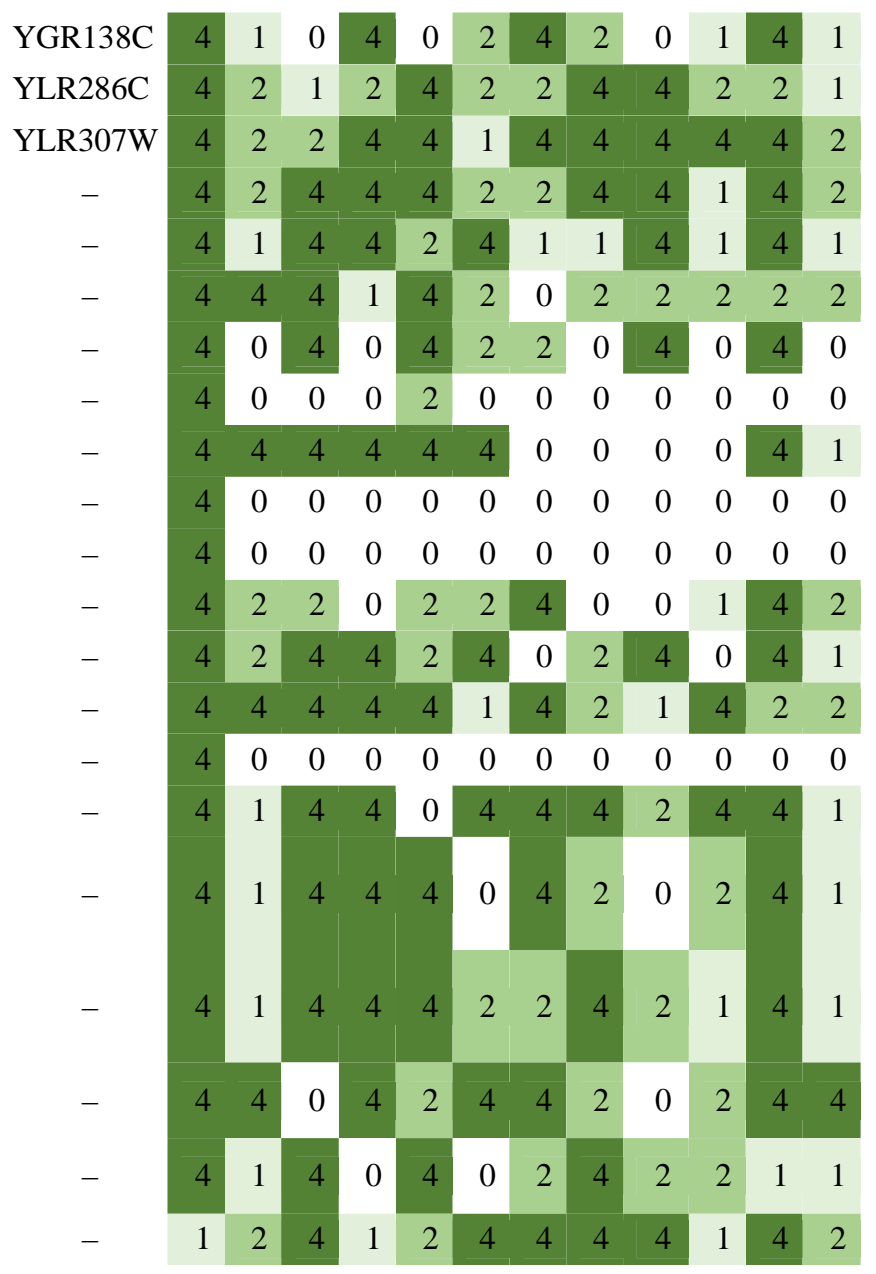

Table 25. Summary of developmental expression dynamics of CDE orthogroups of glucan remodeling related genes across 12 species. Protein ID of a representative protein is given follows by the number of species in which the orthogroup is developmentally regulated at fold change 2 and 4 ( $F C>2$ and $F C>4$, respectively). Putative function and ortholog in $S$. cerevisiae (if any) are also given. Abbreviations: 0-gene absent, 1-gene present but not developmentally regulated, 2 developmentally regulated at fold change $>2,4$ - developmentally regulated at fold change $>4$. Species names are abbreviated as: $\mathrm{Cc}-C$. cinerea, $\mathrm{Aa}-A$. ampla, $\mathrm{Ab}-\mathrm{A}$. bisporus, $\mathrm{Ao}-\mathrm{A}$. ostoyae, $\mathrm{Ca}-\mathrm{C}$. aegerita, $\mathrm{Lb}-\mathrm{L}$. bicolor, Le - L. edodes, $\mathrm{Lt}-\mathrm{L}$. tigrinus, Mk - M. kentingensis, $\mathrm{Pc}$ - Ph. chrysosporium, Po - P. ostreatus, $\mathrm{Sc}-\mathrm{S}$. commune.

\begin{tabular}{|c|c|c|c|c|c|c|c|c|c|c|c|c|c|c|c|c|}
\hline \multirow[b]{2}{*}{ ID } & \multicolumn{2}{|c|}{ Fold change } & \multirow[b]{2}{*}{ Putative function } & \multirow{2}{*}{$\begin{array}{c}S . \\
\text { cerevisiae } \\
\text { ortholog }\end{array}$} & \multicolumn{12}{|c|}{ Species } \\
\hline & $>2$ & $>4$ & & & $\mathrm{Cc}$ & Aa & $\mathbf{A b}$ & Ao & $\mathbf{C a}$ & $\mathbf{L b}$ & Le & $\mathbf{L t}$ & Mk & Pc & Po & Sc \\
\hline $\begin{array}{l}\text { S.c. } \\
268654 \\
4\end{array}$ & 9 & 9 & $\begin{array}{l}\text { Beta-1,6-glucan synthase, GH16 } \\
\text { family protein }\end{array}$ & 8 & 0 & 4 & 0 & 4 & 0 & 4 & 4 & 4 & 4 & 4 & 4 & 4 \\
\hline 546300 & 10 & 10 & Beta-glucanase; GH5 family & - & 1 & 4 & 4 & 4 & 0 & 4 & 4 & 4 & 4 & 4 & 4 & 4 \\
\hline 464399 & 10 & 8 & $\begin{array}{l}\text { GH71 alpha-1,3-glucanase/beta } \\
\text { glucuronidase family protein }\end{array}$ & - & 4 & 4 & 4 & 4 & 4 & 4 & 2 & 0 & 4 & 0 & 4 & 2 \\
\hline 373418 & 9 & 7 & $\begin{array}{l}\text { Glycoside hydrolase } 3 \text { family } \\
\text { protein }\end{array}$ & - & 4 & 1 & 4 & 4 & 4 & 2 & 4 & 0 & 4 & 0 & 2 & 4 \\
\hline 449960 & 6 & 2 & Probable glycosidase CRH2 & YEL040W & 4 & 2 & 2 & 1 & 2 & 1 & 1 & 1 & 4 & 2 & 1 & 1 \\
\hline
\end{tabular}




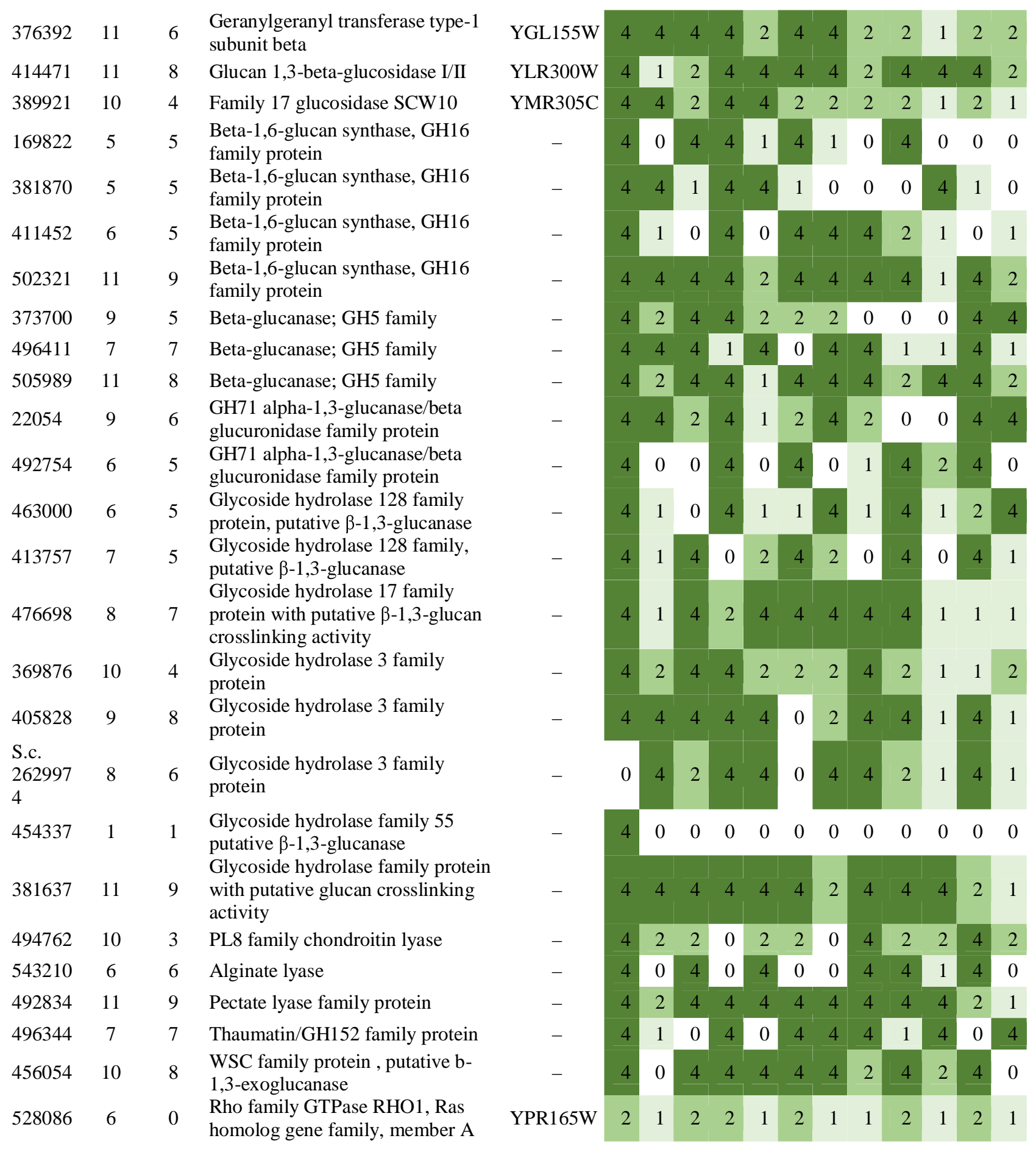

Table 26. Summary of developmental expression dynamics of CDE orthogroups of miscellaneous cell wall remodeling genes across 12 species. Protein ID of a representative protein is given follows by the number of species in which the orthogroup is developmentally regulated at fold change 2 and 4 (FC>2 and $F C>4$, respectively). Putative function and ortholog in S. cerevisiae (if any) are also given. Abbreviations: 0-gene absent, 1-gene present but not developmentally regulated, 2 developmentally regulated at fold change $>2$, 4- developmentally regulated at fold change $>4$. Species names are abbreviated as: Cc $-C$. cinerea, $\mathrm{Aa}-A$. ampla, Ab-A. bisporus, Ao $-A$. 
ostoyae, $\mathrm{Ca}-$ C. aegerita, Lb - L. bicolor, Le - L. edodes, Lt - L. tigrinus, Mk - M. kentingensis, Pc - Ph. chrysosporium, Po - P. ostreatus, Sc - S. commune.

\begin{tabular}{|c|c|c|c|c|c|c|c|c|c|c|c|c|c|c|c|c|}
\hline \multirow[b]{2}{*}{ ID } & \multicolumn{2}{|c|}{ Fold change } & \multirow[b]{2}{*}{ Putative function } & \multirow{2}{*}{$\begin{array}{c}S . \\
\text { cerevisiae } \\
\text { ortholog }\end{array}$} & \multicolumn{12}{|c|}{ Species } \\
\hline & $>2$ & $>4$ & & & $\mathrm{Cc}$ & Aa & $\mathbf{A b}$ & Ao & $\mathbf{C a}$ & Lb & Le & $\mathbf{L t}$ & Mk & $\mathbf{P c}$ & Po & Sc \\
\hline 384105 & 9 & 6 & Glucunoryl hydrolase; GH88 & - & 2 & 4 & 4 & 4 & 4 & 2 & 1 & 4 & 2 & 1 & 4 & 1 \\
\hline $\begin{array}{l}\text { A.o. } \\
254810\end{array}$ & 7 & 5 & Glycoside hydrolase; family 27 & - & 0 & 4 & 0 & 4 & 2 & 0 & 4 & 0 & 4 & 2 & 4 & 0 \\
\hline $\begin{array}{l}\text { A.o. } \\
269656\end{array}$ & 8 & 6 & Glycoside hydrolase; family 27 & - & 0 & 4 & 4 & 1 & 4 & 2 & 4 & 4 & 4 & 0 & 0 & 2 \\
\hline $\begin{array}{l}\text { S.c. } \\
261402 \\
9\end{array}$ & 7 & 6 & Pectinesterase & - & 0 & 4 & 4 & 1 & 1 & 2 & 1 & 4 & 1 & 4 & 4 & 4 \\
\hline 470689 & 9 & 8 & Beta-glucuronidase & - & 4 & 0 & 4 & 4 & 2 & 4 & 4 & 4 & 4 & 1 & 4 & 0 \\
\hline $\begin{array}{l}\text { S.c. } \\
263373 \\
4\end{array}$ & 5 & 4 & $\begin{array}{l}\text { GT18 } \alpha-1,3(6)- \\
\text { mannosylglycoprotein } \beta-1,6-\mathrm{N}- \\
\text { acetyl-glucosaminyltransferase }\end{array}$ & - & 0 & 4 & 4 & 0 & 1 & 2 & 0 & 0 & 1 & 0 & 4 & 4 \\
\hline
\end{tabular}

Table 27. Summary of developmental expression dynamics of CDE orthogroups of multicopper oxidase genes across 12 species. Protein ID of a representative protein is given follows by the number of species in which the orthogroup is developmentally regulated at fold change 2 and 4 (FC>2 and FC>4, respectively). Putative function and ortholog in $S$. cerevisiae (if any) are also given. Abbreviations: 0-gene absent, 1-gene present but not developmentally regulated, 2 developmentally regulated at fold change $>2$, 4- developmentally regulated at fold change $>4$. Species names are abbreviated as: $\mathrm{Cc}-C$. cinerea, $\mathrm{Aa}-A$. ampla, $\mathrm{Ab}-A$. bisporus, Ao $-A$. ostoyae, $\mathrm{Ca}-\mathrm{C}$. aegerita, $\mathrm{Lb}-\mathrm{L}$. bicolor, Le - L. edodes, $\mathrm{Lt}-\mathrm{L}$. tigrinus, $\mathrm{Mk}-\mathrm{M}$. kentingensis, $\mathrm{Pc}$ - Ph. chrysosporium, Po - P. ostreatus, Sc - S. commune.

\begin{tabular}{|c|c|c|c|c|c|c|c|c|c|c|c|c|c|c|c|c|}
\hline \multirow[b]{2}{*}{ ID } & \multicolumn{2}{|c|}{ Fold change } & \multirow[b]{2}{*}{ Putative function } & \multirow{2}{*}{$\begin{array}{c}S . \\
\text { cerevisiae } \\
\text { ortholog }\end{array}$} & \multicolumn{12}{|c|}{ Species } \\
\hline & $>2$ & $>4$ & & & $\mathrm{Cc}$ & Aa & $\mathbf{A b}$ & Ao & $\mathbf{C a}$ & Lb & Le & $\mathbf{L t}$ & Mk & Pc & Po & Sc \\
\hline 408308 & 0 & 0 & C. cinerea laccase lcc1 & - & 1 & 0 & 0 & 0 & 0 & 0 & 0 & 0 & 0 & 0 & 0 & 0 \\
\hline 378517 & 0 & 0 & C. cinerea laccase lcc15 & - & 1 & 0 & 0 & 0 & 0 & 0 & 0 & 0 & 0 & 0 & 0 & 0 \\
\hline 443425 & 1 & 1 & C. cinerea laccase lcc5 & - & 4 & 0 & 0 & 0 & 0 & 0 & 0 & 0 & 0 & 0 & 0 & 0 \\
\hline 467038 & 0 & 0 & C. cinerea laccase lcc6 & - & 1 & 0 & 0 & 0 & 0 & 0 & 0 & 0 & 0 & 0 & 0 & 0 \\
\hline 89107 & 0 & 0 & C. cinerea laccase lcc7 & - & 1 & 0 & 0 & 0 & 0 & 0 & 0 & 0 & 0 & 0 & 0 & 0 \\
\hline 368135 & 10 & 4 & C. cinerea laccase lcc15 & - & 4 & 4 & 4 & 2 & 2 & 2 & 0 & 2 & 2 & 0 & 4 & 2 \\
\hline 353997 & 9 & 4 & Cupredoxin & - & 4 & 1 & 4 & 4 & 2 & 2 & 1 & 2 & 4 & 1 & 2 & 2 \\
\hline 501548 & 5 & 3 & $\begin{array}{l}\text { Iron transport multicopper oxidase } \\
\text { FET3 }\end{array}$ & YMR058W & 4 & 0 & 4 & 4 & 1 & 0 & 2 & 0 & 0 & 1 & 2 & 0 \\
\hline 266892 & 2 & 1 & C. cinerea laccase lcc 3 & - & 2 & 0 & 0 & 0 & 0 & 0 & 4 & 0 & 0 & 0 & 0 & 0 \\
\hline 440170 & 2 & 2 & C. cinerea laccase lcc8 & - & 4 & 0 & 4 & 0 & 0 & 0 & 0 & 0 & 0 & 0 & 0 & 0 \\
\hline $\begin{array}{l}\text { A.o. } \\
257754\end{array}$ & 7 & 7 & Cupredoxin & - & 0 & 0 & 1 & 4 & 4 & 1 & 4 & 4 & 4 & 4 & 4 & 0 \\
\hline 356026 & 9 & 7 & Cupredoxin & - & 4 & 1 & 0 & 4 & 4 & 4 & 4 & 2 & 4 & 2 & 4 & 1 \\
\hline 409847 & 5 & 3 & Cupredoxin & - & 4 & 4 & 0 & 0 & 2 & 4 & 0 & 0 & 0 & 0 & 1 & 2 \\
\hline 441442 & 9 & 4 & Cupredoxin & - & 4 & 1 & 1 & 4 & 0 & 2 & 4 & 2 & 4 & 2 & 2 & 2 \\
\hline 451850 & 6 & 5 & Cupredoxin & - & 4 & 2 & 4 & 4 & 1 & 0 & 0 & 0 & 4 & 1 & 4 & 0 \\
\hline 497434 & 6 & 5 & Cupredoxin & - & 4 & 0 & 0 & 1 & 4 & 4 & 4 & 0 & 4 & 0 & 2 & 0 \\
\hline
\end{tabular}


Table 28. Summary of developmental expression dynamics of CDE orthogroups of expansin genes across 12 species. Protein ID of a representative protein is given follows by the number of species in which the orthogroup is developmentally regulated at fold change 2 and 4 (FC>2 and $F C>4$, respectively). Putative function and ortholog in $S$. cerevisiae (if any) are also given. Abbreviations: 0-gene absent, 1-gene present but not developmentally regulated, 2 - developmentally regulated at fold change $>2$, 4- developmentally regulated at fold change $>4$. Species names are abbreviated as: $\mathrm{Cc}-$ C. cinerea, $\mathrm{Aa}-\mathrm{A}$. ampla, $\mathrm{Ab}-\mathrm{A}$. bisporus, Ao - A. ostoyae, $\mathrm{Ca}-\mathrm{C}$. aegerita, $\mathrm{Lb}-\mathrm{L}$. bicolor, $\mathrm{Le}-$ L. edodes, Lt - L. tigrinus, $\mathrm{Mk}-M$. kentingensis, $\mathrm{Pc}-\mathrm{Ph}$. chrysosporium, $\mathrm{Po}-\mathrm{P}$. ostreatus, Sc - S. commune.

\begin{tabular}{lccll} 
& \multicolumn{3}{c}{ Fold change } & \\
\cline { 2 - 3 } ID & $\mathbf{> 2}$ & $\mathbf{> 4}$ & Putative function \\
\hline 439071 & 6 & 5 & Cerato-platanin \\
544218 & 8 & 8 & Cerato-platanin \\
461698 & 10 & 5 & Expansin \\
467907 & 11 & 10 & Expansin \\
496836 & 12 & 10 & Expansin
\end{tabular}

\begin{tabular}{|c|c|c|c|c|c|c|c|c|c|c|c|c|}
\hline \multirow{2}{*}{$\begin{array}{c}S . \\
\text { cerevisiae } \\
\text { ortholog }\end{array}$} & \multicolumn{12}{|c|}{ Species } \\
\hline & $\mathrm{Cc}$ & Aa & Ab & Ao & $\mathrm{Ca}$ & Lb & Le & $\mathbf{L t}$ & Mk & Pc & Po & Sc \\
\hline- & 4 & 4 & 0 & 4 & 4 & 0 & 2 & 0 & 0 & 0 & 0 & 4 \\
\hline- & 4 & 0 & 0 & 4 & 0 & 4 & 4 & 4 & 4 & 1 & 4 & 4 \\
\hline- & 4 & 2 & 4 & 2 & 4 & 4 & 4 & 1 & 0 & 2 & 2 & 2 \\
\hline- & 4 & 4 & 2 & 4 & 0 & 4 & 4 & 4 & 4 & 4 & 4 & 4 \\
\hline- & 4 & 4 & 4 & 4 & 4 & 2 & 4 & 4 & 4 & 2 & 4 & 4 \\
\hline
\end{tabular}

Table 29. Summary of developmental expression dynamics of CDE orthogroups of miscellaneous cell wall related genes across 12 species. Protein ID of a representative protein is given follows by the number of species in which the orthogroup is developmentally regulated at fold change 2 and 4 (FC>2 and FC>4, respectively). Putative function and ortholog in S. cerevisiae (if any) are also given. Abbreviations: 0-gene absent, 1-gene present but not developmentally regulated, 2 developmentally regulated at fold change $>2$, 4- developmentally regulated at fold change $>4$. Species names are abbreviated as: Cc $-C$. cinerea, Aa - A. ampla, Ab-A. bisporus, Ao - A. ostoyae, $\mathrm{Ca}-\mathrm{C}$. aegerita, $\mathrm{Lb}-$ L. bicolor, Le - L. edodes, $\mathrm{Lt}-$ L. tigrinus, $\mathrm{Mk}-M$. kentingensis, $\mathrm{Pc}$ - Ph. chrysosporium, Po - P. ostreatus, Sc - S. commune.

\begin{tabular}{|c|c|c|c|c|c|c|c|c|c|c|c|c|c|c|c|c|}
\hline \multirow[b]{2}{*}{ ID } & \multicolumn{2}{|c|}{ Fold change } & \multirow[b]{2}{*}{ Putative function } & \multirow{2}{*}{$\begin{array}{c}S . \\
\text { cerevisiae } \\
\text { ortholog }\end{array}$} & \multicolumn{12}{|c|}{ Species } \\
\hline & $>2$ & $>4$ & & & Cc & Aa & $\mathbf{A b}$ & Ao & $\mathbf{C a}$ & $\mathbf{L b}$ & Le & $\mathbf{L t}$ & Mk & Pc & Po & Sc \\
\hline 380322 & 6 & 4 & C. cinerea $\mathrm{E} \ln 3$ & - & 4 & 0 & 4 & 4 & 1 & 4 & 2 & 0 & 2 & 0 & 0 & 0 \\
\hline 407604 & 5 & 5 & Dye decolorising peroxidase & - & 4 & 0 & 0 & 4 & 4 & 4 & 0 & 0 & 0 & 0 & 4 & 0 \\
\hline 447925 & 10 & 7 & C. cinerea $\mathrm{Cfs} 1$ & - & 4 & 4 & 4 & 2 & 1 & 1 & 4 & 4 & 4 & 2 & 4 & 2 \\
\hline 469460 & 8 & 6 & Cellobiose dehydrogenase & - & 4 & 0 & 0 & 4 & 2 & 1 & 4 & 4 & 4 & 2 & 4 & 0 \\
\hline 442333 & 11 & 7 & Chloroperoxidase & - & 4 & 2 & 4 & 4 & 4 & 2 & 4 & 2 & 4 & 1 & 4 & 2 \\
\hline 479688 & 10 & 7 & Chloroperoxidase & - & 4 & 4 & 0 & 4 & 2 & 2 & 4 & 2 & 4 & 1 & 4 & 4 \\
\hline 263775 & 9 & 7 & Glyoxal oxidase & - & 4 & 0 & 2 & 4 & 4 & 2 & 0 & 4 & 4 & 4 & 4 & 0 \\
\hline 440836 & 10 & 10 & Glyoxal oxidase & - & 4 & 4 & 1 & 4 & 4 & 0 & 4 & 4 & 4 & 4 & 4 & 4 \\
\hline 413665 & 8 & 5 & $\begin{array}{l}\text { Mitogen-activated serine/threonine- } \\
\text { protein kinase SLT2 }\end{array}$ & YHR030C & 2 & 1 & 4 & 4 & 1 & 4 & 2 & 2 & 4 & 1 & 4 & 1 \\
\hline
\end{tabular}

Table 30. Summary of developmental expression dynamics of CDE orthogroups of hydrophobin genes across 12 species. Protein ID of a representative protein is given follows by the number of species in which the orthogroup is developmentally regulated at fold change 2 and 4 (FC>2 and FC $>4$, respectively). Putative function and ortholog in $S$. cerevisiae (if any) are also given. Abbreviations: 0-gene absent, 1-gene present but not developmentally regulated, 2 - 
developmentally regulated at fold change $>2$, 4- developmentally regulated at fold change $>4$. Species names are abbreviated as: $\mathrm{Cc}-C$. cinerea, $\mathrm{Aa}-A$. ampla, Ab - A. bisporus, Ao - A. ostoyae, $\mathrm{Ca}-\mathrm{C}$. aegerita, $\mathrm{Lb}-$ L. bicolor, Le - L. edodes, $\mathrm{Lt}-$ L. tigrinus, $\mathrm{Mk}-M$. kentingensis, $\mathrm{Pc}$ - Ph. chrysosporium, Po-P. ostreatus, Sc - S. commune.

\begin{tabular}{|c|c|c|c|c|c|c|c|c|c|c|c|c|c|c|c|c|}
\hline \multirow[b]{2}{*}{ ID } & \multicolumn{2}{|c|}{ Fold change } & \multirow[b]{2}{*}{ Putative function } & \multirow{2}{*}{$\begin{array}{c}S . \\
\text { cerevisiae } \\
\text { ortholog }\end{array}$} & \multicolumn{12}{|c|}{ Species } \\
\hline & $>2$ & $>4$ & & & Cc & Aa & $\mathbf{A b}$ & Ao & $\mathbf{C a}$ & $\mathbf{L b}$ & Le & $\mathbf{L t}$ & Mk & Pc & Po & Sc \\
\hline 99661 & 7 & 7 & Hydrophobin & - & 1 & 4 & 0 & 0 & 4 & 1 & 4 & 4 & 0 & 4 & 4 & 4 \\
\hline 419467 & 6 & 6 & Hydrophobin & - & 4 & 4 & 4 & 4 & 0 & 4 & 1 & 0 & 0 & 1 & 0 & 4 \\
\hline
\end{tabular}

Table 31. Summary of developmental expression dynamics of genes belonging to conserved lectin orthogroups across 12 species. Protein ID of a representative protein is given follows by the number of species in which the orthogroup is developmentally regulated at fold change 2 and 4 (FC>2 and $\mathrm{FC}>4$, respectively). Putative function and ortholog in $S$. cerevisiae (if any) are also given.

Abbreviations: 0-gene absent, 1-gene present but not developmentally regulated, 2 developmentally regulated at fold change $>2$, 4- developmentally regulated at fold change $>4$. Species names are abbreviated as: Cc - C. cinerea, Aa - A. ampla, Ab-A. bisporus, Ao - A. ostoyae, $\mathrm{Ca}-\mathrm{C}$. aegerita, $\mathrm{Lb}-$ L. bicolor, Le - L. edodes, Lt - L. tigrinus, $\mathrm{Mk}-M$. kentingensis, $\mathrm{Pc}$ - Ph. chrysosporium, Po - P. ostreatus, Sc - S. commune.

\begin{tabular}{|c|c|c|c|c|c|c|c|c|c|c|c|c|c|c|c|c|}
\hline \multirow[b]{2}{*}{ ID } & \multicolumn{2}{|c|}{ Fold change } & \multirow[b]{2}{*}{ Putative function } & \multirow{2}{*}{$\begin{array}{c}S . \\
\text { cerevisiae } \\
\text { ortholog }\end{array}$} & \multicolumn{12}{|c|}{ Species } \\
\hline & $>2$ & $>4$ & & & $\mathrm{Cc}$ & Aa & $\mathbf{A b}$ & Ao & $\mathrm{Ca}$ & $\mathbf{L b}$ & Le & $\mathbf{L t}$ & Mk & Pc & Po & Sc \\
\hline 353931 & 8 & 7 & RicinB lectin protein & - & 4 & 4 & $\overline{0}$ & 4 & 4 & 0 & 4 & 0 & 0 & 2 & 4 & 4 \\
\hline 495335 & 9 & 9 & RicinB lectin protein & - & 4 & 1 & 4 & 4 & 4 & 4 & 0 & 4 & 4 & 1 & 4 & 4 \\
\hline
\end{tabular}

Table 32. Summary of developmental expression dynamics of CDE orthogroups of cell surface protein encoding genes across 12 species. Protein ID of a representative protein is given follows by the number of species in which the orthogroup is developmentally regulated at fold change 2 and 4 (FC>2 and FC>4, respectively). Putative function and ortholog in $S$. cerevisiae (if any) are also given. Abbreviations: 0-gene absent, 1-gene present but not developmentally regulated, 2 developmentally regulated at fold change $>2,4$ - developmentally regulated at fold change $>4$. Species names are abbreviated as: $\mathrm{Cc}-C$. cinerea, $\mathrm{Aa}-A$. ampla, Ab-A. bisporus, Ao - A. ostoyae, $\mathrm{Ca}-C$. aegerita, $\mathrm{Lb}-$ L. bicolor, Le - L. edodes, Lt - L. tigrinus, Mk $-M$. kentingensis, Pc - Ph. chrysosporium, Po-P. ostreatus, $\mathrm{Sc}-\mathrm{S}$. commune.

\begin{tabular}{lccl} 
& \multicolumn{2}{c}{ Fold change } & \\
\cline { 2 - 3 } ID & $>\mathbf{2}$ & $\mathbf{> 4}$ & Putative function \\
\hline 493867 & 6 & 1 & Fasciclin \\
186809 & 6 & 5 & Con6 family cell surface protein \\
$\begin{array}{l}\text { S.c. } \\
262434\end{array}$ & 5 & 5 & Con6 family cell surface protein \\
9 & & & \\
361136 & 7 & 4 & Fasciclin \\
437384 & 7 & 5 & Fasciclin \\
419688 & 7 & 5 & PriA family protein \\
470234 & 6 & 6 & Con6 family cell surface protein
\end{tabular}

\begin{tabular}{cccccccccccccc}
\multirow{10}{*}{$\begin{array}{c}S . \\
\text { cerevisiae } \\
\text { ortholog }\end{array}$} & Cc & Aa & Ab & Ao & Ca & Lb & Le & Lt & Mk Pc & Po & Sc \\
\hline YOR100C & 2 & 2 & 2 & 2 & 1 & 1 & 1 & 1 & 1 & 1 & 2 & 4 \\
- & 4 & 2 & 0 & 4 & 0 & 0 & 4 & 1 & 0 & 1 & 4 & 4 \\
- & 0 & 4 & 0 & 0 & 0 & 0 & 4 & 0 & 4 & 0 & 4 & 4 \\
- & & & & & & & & & & & & \\
- & 4 & 1 & 2 & 4 & 2 & 2 & 0 & 4 & 0 & 1 & 4 & 1 \\
- & 1 & 4 & 4 & 1 & 4 & 2 & 4 & 4 & 0 & 1 & 2 & 0 \\
- & 4 & 2 & 4 & 0 & 4 & 1 & 4 & 1 & 0 & 2 & 0 & 4 \\
- & 1 & 4 & 4 & 0 & 0 & 0 & 4 & 0 & 4 & 0 & 4 & 4
\end{tabular}




$\begin{array}{llll}\begin{array}{l}359866 \\ \text { S.c. }\end{array} & 9 & 7 & \text { PriA family protein } \\ \begin{array}{l}268170 \\ 2\end{array} & 8 & 7 & \text { PriA family protein } \\ 501135 & 9 & 9 & \text { AA7 chitooligosaccharide oxidase } \\ 513409 & 9 & 7 & \text { AA7 chitooligosaccharide oxidase } \\ \text { A.o. } & 6 & 5 & \text { Aspartic peptidase } \\ \begin{array}{l}269613 \\ 442743\end{array} & 10 & 10 & \text { Aspartic peptidase } \\ \begin{array}{l}\text { S.c. } \\ 75642\end{array} & 9 & 9 & \text { Aspartic peptidase } \\ 367835 & 10 & 8 & \text { Peptidase M20 } \\ \begin{array}{l}\text { S.c. } \\ 254761\end{array} & 8 & 7 & \text { Serine carboxypeptidase } \\ 6 & & & \\ \text { S.c. } & & & \\ 264576 & 8 & 7 & \text { Serine carboxypeptidase } \\ 1 & & & \\ 462300 & 10 & 7 & \text { Serine carboxypeptidase S28 }\end{array}$

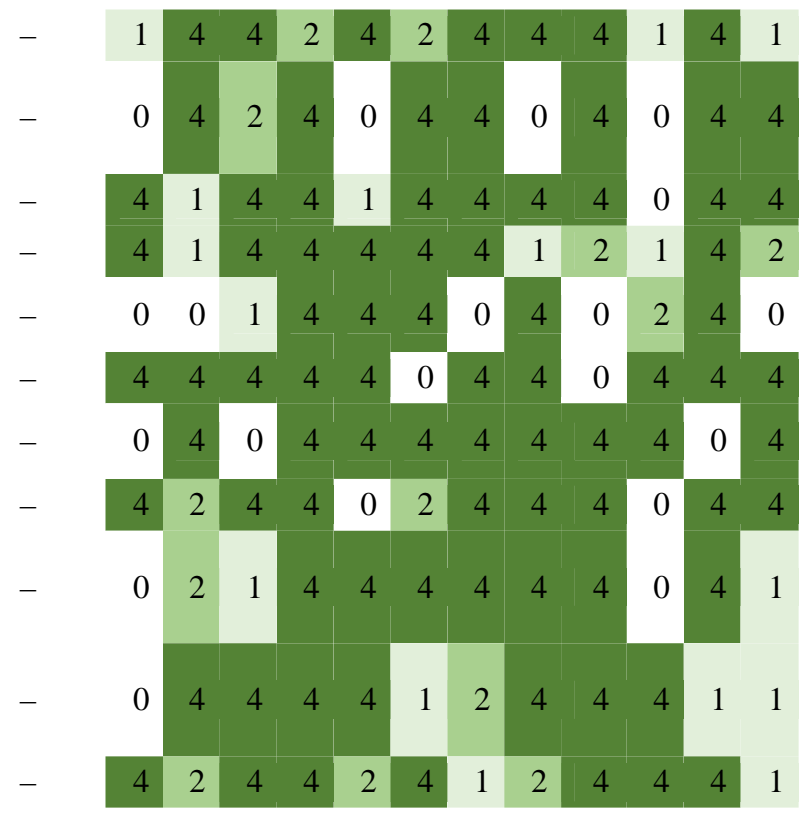

Table 33. Summary of developmental expression dynamics of CDE orthogroups of defense-related genes across 12 species. Protein ID of a representative protein is given follows by the number of species in which the orthogroup is developmentally regulated at fold change 2 and 4 (FC>2 and $F C>4$, respectively). Putative function and ortholog in $S$. cerevisiae (if any) are also given.

Abbreviations: 0-gene absent, 1-gene present but not developmentally regulated, 2 developmentally regulated at fold change $>2$, 4- developmentally regulated at fold change $>4$. Species names are abbreviated as: $\mathrm{Cc}-C$. cinerea, $\mathrm{Aa}-A$. ampla, Ab-A. bisporus, Ao - A. ostoyae, $\mathrm{Ca}-\mathrm{C}$. aegerita, $\mathrm{Lb}-$ L. bicolor, Le - L. edodes, $\mathrm{Lt}-$ L. tigrinus, $\mathrm{Mk}-M$. kentingensis, $\mathrm{Pc}$ - Ph. chrysosporium, Po-P. ostreatus, Sc - S. commune.

\begin{tabular}{|c|c|c|c|c|c|c|c|c|c|c|c|c|c|c|c|c|}
\hline \multirow[b]{2}{*}{ ID } & \multicolumn{2}{|c|}{ Fold change } & \multirow[b]{2}{*}{ Putative function } & \multirow{2}{*}{$\begin{array}{c}S . \\
\text { cerevisiae } \\
\text { ortholog }\end{array}$} & \multicolumn{12}{|c|}{ Species } \\
\hline & $>2$ & $>4$ & & & Cc & Aa & $\mathbf{A b}$ & Ao & $\mathbf{C a}$ & $\mathbf{L b}$ & Le & $\mathbf{L t}$ & Mk & Pc & Po & Sc \\
\hline 185637 & 9 & 5 & CFEM domain containing protein & - & 4 & 1 & 4 & 4 & 2 & 2 & 2 & 2 & 4 & 1 & 4 & 1 \\
\hline 439390 & 9 & 6 & CFEM domain containing protein & - & 4 & 4 & 4 & 4 & 2 & 2 & 2 & 4 & 0 & 1 & 1 & 4 \\
\hline 485770 & 7 & 5 & RicinB lectin protein & - & 4 & 2 & 4 & 0 & 0 & 1 & 4 & 2 & 0 & 0 & 4 & 4 \\
\hline
\end{tabular}

Table 34. Summary of developmental expression dynamics of CDE orthogroups of terpene synthase genes across 12 species. Protein ID of a representative protein is given follows by the number of species in which the orthogroup is developmentally regulated at fold change 2 and 4 (FC>2 and FC>4, respectively). Putative function and ortholog in $S$. cerevisiae (if any) are also given. Abbreviations: 0-gene absent, 1-gene present but not developmentally regulated, 2 developmentally regulated at fold change $>2$, 4- developmentally regulated at fold change $>4$. Species names are abbreviated as: $\mathrm{Cc}-C$. cinerea, $\mathrm{Aa}-A$. ampla, $\mathrm{Ab}-A$. bisporus, Ao $-A$. ostoyae, $\mathrm{Ca}-\mathrm{C}$. aegerita, Lb - L. bicolor, Le - L. edodes, Lt - L. tigrinus, Mk - M. kentingensis, Pc - Ph. chrysosporium, Po - P. ostreatus, Sc - S. commune.

Fold change

ID
$S$. cerevisiae ortholog
Species

$>2 \quad>4$ Putative function

Ce Aa Ab Ao Ca Lb Le Lt Mk Pc Po Sc




$\begin{array}{lccl}361541 & 8 & 5 & \text { Phenylalanine ammonia-lyase } \\ 386671 & 11 & 9 & \text { Phenylalanine ammonia-lyase } \\ 446258 & 11 & 8 & \text { Putative polyketide synthase } \\ 394772 & 4 & 2 & \text { Putative terpene synthase } \\ 443805 & 3 & 2 & \text { Putative terpene synthase } \\ 495900 & 1 & 1 & \text { Putative terpene synthase } \\ 124670 & 5 & 2 & \text { Putative terpene synthase } \\ 30510 & 9 & 8 & \text { Putative terpene synthase } \\ 469738 & 10 & 8 & \text { Putative terpene synthase } \\ 201523 & 1 & 1 & \text { Putative terpene synthase }\end{array}$

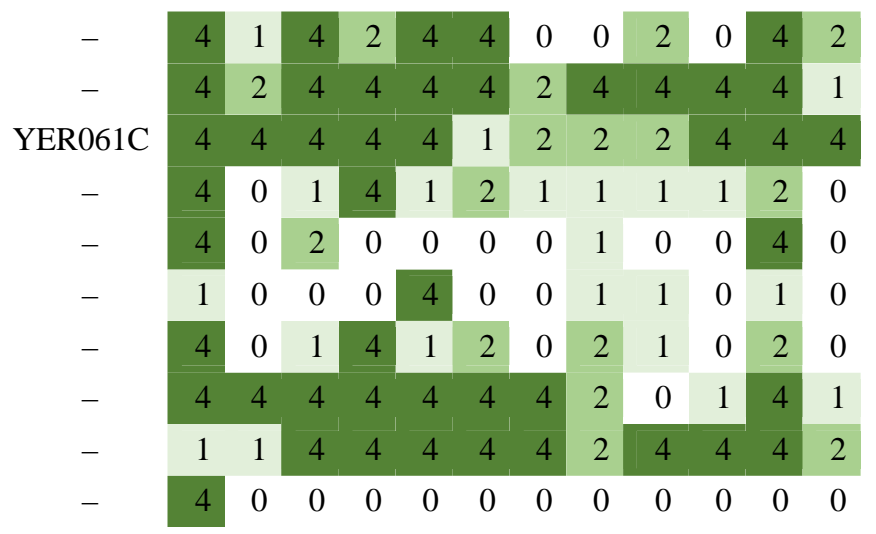

Table 35. Summary of developmental expression dynamics of CDE orthogroups of cytoskeletonrelated genes across 12 species. Protein ID of a representative protein is given follows by the number of species in which the orthogroup is developmentally regulated at fold change 2 and 4 (FC>2 and FC>4, respectively). Putative function and ortholog in S. cerevisiae (if any) are also given. Abbreviations: 0-gene absent, 1-gene present but not developmentally regulated, 2 developmentally regulated at fold change $>2$, 4- developmentally regulated at fold change $>4$. Species names are abbreviated as: $\mathrm{Cc}-C$. cinerea, $\mathrm{Aa}-A$. ampla, Ab-A. bisporus, $\mathrm{Ao}-A$. ostoyae, $\mathrm{Ca}-C$. aegerita, $\mathrm{Lb}-\mathrm{L}$. bicolor, $\mathrm{Le}-\mathrm{L}$. edodes, $\mathrm{Lt}-\mathrm{L}$. tigrinus, Mk $-M$. kentingensis, $\mathrm{Pc}$ - Ph. chrysosporium, Po - P. ostreatus, Sc - S. commune.

\begin{tabular}{|c|c|c|c|}
\hline \multirow[b]{2}{*}{ ID } & \multicolumn{2}{|c|}{ Fold change } & \multirow[b]{2}{*}{ Putative function } \\
\hline & $>2$ & $>4$ & \\
\hline 130811 & 3 & 1 & LisH domain protein \\
\hline 504216 & 9 & 1 & Misato tubulin \\
\hline 455153 & 4 & 0 & $\begin{array}{l}\text { Actin cytoskeleton-regulatory } \\
\text { complex protein PAN1 }\end{array}$ \\
\hline 496271 & 3 & 0 & Adenyl cyclase associated protein \\
\hline 356686 & 6 & 1 & Predicted Rho GTPase \\
\hline 381381 & 11 & 8 & $\begin{array}{l}\text { Agaricales-specific Rho GTPase } \\
\text { Activating Protein }\end{array}$ \\
\hline 472493 & 4 & 0 & Potential dynactin complex member \\
\hline 61699 & 5 & 1 & Actin \\
\hline 258718 & 5 & 1 & Myosin \\
\hline 473079 & 4 & 2 & Myosin \\
\hline 419142 & 2 & 0 & Myosin \\
\hline 461274 & 2 & 0 & Protein SLA2 \\
\hline 13965 & 2 & 0 & $\begin{array}{l}\text { Actin depolymerization related } \\
\text { protein }\end{array}$ \\
\hline 505644 & 6 & 2 & Cofilin-homolog protein \\
\hline 448482 & 6 & 3 & Fimbrin \\
\hline 59959 & 3 & 0 & Twinfilin \\
\hline 366601 & 4 & 0 & F-actin capping protein \\
\hline 466954 & 1 & 0 & Tropomyosin \\
\hline 443986 & 4 & 1 & Actin capping protein \\
\hline 370292 & 2 & 0 & Cofilin \\
\hline
\end{tabular}

$S$. cerevisiae $\quad$ Species cerevisiae
ortholog Cc Aa Ab Ao Ca Lb Le Lt Mk Pc Po Sc

\begin{tabular}{|c|c|c|c|c|c|c|c|c|c|c|c|c|}
\hline- & 4 & 1 & 1 & 2 & 0 & 2 & 1 & 1 & 0 & 1 & 0 & 1 \\
\hline- & 2 & 4 & 2 & 2 & 2 & 1 & 2 & 1 & 2 & 1 & 2 & 2 \\
\hline YIR006C & 2 & 1 & 2 & 1 & 1 & 2 & 1 & 1 & 1 & 1 & 2 & 1 \\
\hline YNL138W & 2 & 1 & 1 & 1 & 1 & 2 & 1 & 2 & 1 & 1 & 2 & 1 \\
\hline- & 2 & 1 & 2 & 1 & 1 & 2 & 2 & 1 & 4 & 1 & 2 & 1 \\
\hline- & 4 & 4 & 2 & 4 & 4 & 4 & 2 & 2 & 4 & 1 & 4 & 4 \\
\hline- & 2 & 1 & 1 & 2 & 1 & 1 & 1 & 2 & 2 & 1 & 1 & 1 \\
\hline YFL039C & 1 & 2 & 2 & 2 & 2 & 1 & 1 & 1 & 1 & 1 & 4 & 1 \\
\hline YHR023W & 2 & 1 & 1 & 1 & 1 & 2 & 2 & 2 & 1 & 1 & 4 & 1 \\
\hline YMR109W & 4 & 1 & 2 & 1 & 1 & 2 & 1 & 1 & 1 & 1 & 4 & 1 \\
\hline YOR326W & 2 & 1 & 1 & 1 & 1 & 1 & 1 & 1 & 1 & 1 & 2 & 1 \\
\hline- & 1 & 1 & 2 & 1 & 1 & 1 & 2 & 1 & 1 & 1 & 1 & 1 \\
\hline YCR088W & 2 & 1 & 1 & 1 & 1 & 1 & 1 & 1 & 1 & 1 & 2 & 1 \\
\hline YDR063W & 2 & 1 & 4 & 0 & 2 & 1 & 2 & 2 & 1 & 1 & 4 & 1 \\
\hline YDR129C & 4 & 1 & 4 & 0 & 2 & 1 & 1 & 2 & 2 & 1 & 4 & 1 \\
\hline YGR080W & 2 & 1 & 1 & 2 & 1 & 1 & 1 & 1 & 1 & 1 & 2 & 1 \\
\hline YIL034C & 2 & 1 & 2 & 2 & 1 & 1 & 1 & 1 & 0 & 1 & 2 & 1 \\
\hline YIL138C & 2 & 0 & 0 & 0 & 0 & 0 & 0 & 0 & 0 & 0 & 0 & 0 \\
\hline YKL007W & 4 & 1 & 2 & 1 & 1 & 1 & 1 & 1 & 2 & 1 & 2 & 1 \\
\hline YLL050C & 1 & 1 & 1 & 2 & 1 & 1 & 1 & 1 & 0 & 1 & 2 & 1 \\
\hline
\end{tabular}




\begin{tabular}{|c|c|c|c|}
\hline 484047 & 8 & 5 & Profilin \\
\hline 444584 & 5 & 0 & Actin filament binding protein \\
\hline 126636 & 3 & 0 & Coactosin \\
\hline 355823 & 0 & 0 & Villin/Gelsolin \\
\hline 376343 & 4 & 0 & Villin/Gelsolin \\
\hline 358108 & 3 & 1 & Arp2/3 complex member \\
\hline 130742 & 3 & 0 & Arp2/3 complex member \\
\hline 498732 & 4 & 1 & Arp2/3 complex regulating protein \\
\hline 114966 & 6 & 1 & Arp2/3 complex member \\
\hline 57120 & 6 & 0 & Formin \\
\hline 415488 & 3 & 0 & Arp2/3 complex member \\
\hline 192562 & 2 & 0 & Arp2/3 complex member \\
\hline 493018 & 2 & 0 & Verprolin \\
\hline 421678 & 4 & 1 & Arp2/3 complex member \\
\hline 148867 & 2 & 0 & Formin \\
\hline 546441 & 1 & 0 & Arp2/3 complex member \\
\hline 465398 & 6 & 0 & WASP protein \\
\hline 544540 & 8 & 4 & WASP protein \\
\hline 176636 & 5 & 2 & Alpha tubulin \\
\hline 393528 & 6 & 1 & Beta-tubulin \\
\hline 359665 & 4 & 1 & Alpha tubulin \\
\hline 519006 & 3 & 1 & Beta tubulin \\
\hline 494087 & 3 & 1 & Kinesin \\
\hline 3150 & 6 & 0 & Kinesin \\
\hline 447928 & 3 & 0 & Kinesin \\
\hline 461075 & 12 & 9 & Kinesin \\
\hline 367694 & 9 & 5 & Kinesin \\
\hline 410146 & 12 & 7 & Kinesin \\
\hline 494863 & 10 & 4 & Kinesin \\
\hline 113001 & 6 & 0 & $\begin{array}{l}\text { Microtubule plus end tracking } \\
\text { protein }\end{array}$ \\
\hline 527694 & 4 & 1 & Tubulin specific chaperone. \\
\hline 441578 & 6 & 1 & Gamma-tubulin comlex member \\
\hline 361158 & 7 & 1 & Gamma tubulin \\
\hline 156038 & 5 & 2 & Gamma-tubulin comlex member \\
\hline 357668 & 5 & 0 & Gamma-tubulin comlex member \\
\hline 358462 & 6 & 1 & Gamma-tubulin comlex member \\
\hline 463163 & 4 & 1 & Gamma-tubulin comlex member \\
\hline 474549 & 4 & 0 & Dynactin complex member protein \\
\hline 447285 & 9 & 2 & Dynactin complex member protein \\
\hline 454161 & 4 & 0 & Dynactin complex member protein \\
\hline 488749 & 3 & 0 & Dynactin complex member protein \\
\hline 437053 & 3 & 1 & Dynactin complex member protein \\
\hline 415573 & 9 & 8 & Septin \\
\hline
\end{tabular}

\begin{tabular}{|c|c|c|c|c|c|c|c|c|c|c|c|c|}
\hline & & 2 & 4 & & & & 2 & $T$ & & & & \\
\hline PL242C & 2 & 1 & 2 & 2 & 1 & 2 & 1 & 1 & & 1 & 1 & 1 \\
\hline- & & 1 & 1 & 2 & 1 & 1 & 1 & 1 & & 1 & 2 & 1 \\
\hline- & 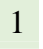 & 0 & 0 & 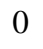 & 0 & 0 & & & & ( & & \\
\hline - & & & & & & 2 & & & & 1 & & \\
\hline BR234C & 4 & 1 & 2 & 1 & 1 & 1 & 1 & 1 & & 1 & 2 & 1 \\
\hline YDL029W & 2 & 1 & 2 & 1 & 0 & 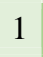 & 2 & 1 & & 1 & 1 & 1 \\
\hline DL146W & 4 & 1 & 1 & 1 & 1 & 1 & 2 & 1 & & 2 & 2 & 1 \\
\hline IL062C & 2 & 1 & 4 & 1 & 2 & 2 & 2 & 1 & & & 2 & \\
\hline IL159W & 2 & 1 & 2 & 2 & & 2 & & 1 & & & 2 & 1 \\
\hline JR065C & 1 & 2 & 2 & 0 & 1 & 1 & 1 & 1 & & 1 & 2 & 1 \\
\hline KL013C & 1 & 1 & 2 & 1 & 1 & 1 & 1 & 1 & & 1 & 2 & 1 \\
\hline LR337C & 2 & 1 & 0 & 1 & 0 & 1 & 1 & - & & 0 & 2 & 1 \\
\hline LR370C & 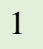 & 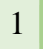 & 2 & 1 & 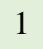 & 1 & 2 & & & & 4 & 1 \\
\hline NL271C & 2 & 1 & 1 & 1 & 1 & 1 & 2 & 1 & & 1 & 1 & 1 \\
\hline NR035C & 2 & 1 & 1 & 1 & 0 & 1 & 1 & 1 & & 1 & 1 & 1 \\
\hline OR181W & 2 & 1 & 1 & 2 & 0 & 2 & 1 & 1 & & 1 & 2 & 2 \\
\hline- & 4 & 1 & 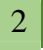 & 4 & 2 & 2 & 4 & 4 & & 1 & 0 & 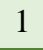 \\
\hline- & 4 & 1 & 2 & 0 & 1 & 1 & 1 & 2 & & 2 & 1 & 4 \\
\hline FL037W & 2 & 2 & 2 & 1 & 1 & 1 & 2 & 2 & & 4 & 1 & 1 \\
\hline ML085C & 4 & 2 & 0 & 0 & 1 & 1 & - & 1 & & & 2 & - \\
\hline - & 2 & 1 & 4 & 1 & 1 & 1 & 2 & 1 & & 1 & 1 & 1 \\
\hline- & 4 & 1 & 1 & 1 & 1 & 1 & 2 & 1 & & 1 & 1 & 1 \\
\hline- & 2 & 1 & 2 & 1 & 2 & 2 & 1 & 1 & & 1 & 2 & 1 \\
\hline 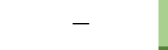 & 2 & 1 & 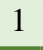 & 1 & 1 & 1 & 1 & 1 & & 1 & 2 & 1 \\
\hline - & 4 & 4 & 4 & 4 & 2 & 4 & 4 & . & & 2 & 2 & 4 \\
\hline YDR424C & 4 & 2 & 2 & 0 & 2 & 4 & 2 & 4 & 1 & 1 & 4 & 4 \\
\hline PL155C & 2 & 4 & 4 & 2 & 2 & 4 & 4 & 4 & 4 & 2 & 2 & 4 \\
\hline- & 4 & ( & 2 & 4 & 2 & 2 & 4 & 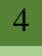 & & 1 & 2 & 1 \\
\hline ER016W & 2 & 1 & 1 & 2 & 1 & 1 & 2 & 2 & 2 & 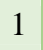 & 2 & 1 \\
\hline- & 2 & 1 & 2 & 2 & 1 & 1 & 1 & 1 & 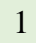 & 1 & 4 & 1 \\
\hline $\mathrm{HR} 172 \mathrm{~W}$ & 2 & 1 & 1 & 2 & 0 & 2 & 2 & 1 & & 1 & 4 & 1 \\
\hline YLR212C & 4 & 1 & 1 & 2 & 2 & 1 & 2 & 2 & 2 & 1 & 2 & 1 \\
\hline YNL126W & 4 & 1 & 1 & 2 & 1 & 1 & 2 & 2 & 1 & 1 & 4 & 1 \\
\hline - & 1 & 2 & 1 & 2 & 1 & 1 & 1 & 1 & & 2 & 2 & 1 \\
\hline 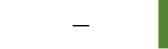 &. & - & 1 & 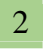 & 2 & 1 & 2 & 2 & & 1 & 1 & 1 \\
\hline - & 2 & 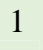 & 1 & 1 & 1 & 2 & 4 & 1 & & 1 & 2 & 1 \\
\hline- & 2 & 1 & 1 & 1 & 1 & 1 & 2 & 2 & 1 & 1 & 2 & 1 \\
\hline 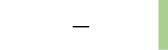 & 2 & 1 & 1 & 2 & 2 & 2 & 4 & 2 & 7 & 1 & 2 & 2 \\
\hline - & 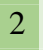 & 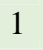 & 1 & 2 & 1 & 1 & 2 & 1 & 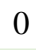 & 1 & 2 & 1 \\
\hline- & 2 & 1 & 1 & 1 & 1 & 1 & 1 & 1 & 1 & 1 & 2 & 2 \\
\hline OR269W & 2 & 1 & 1 & 1 & 1 & 1 & 1 & 1 & 4 & 1 & 2 & 1 \\
\hline & & $\boldsymbol{t}$ & 4 & 4 & 4 & 4 & 0 & 4 & & 1 & 0 & 2 \\
\hline
\end{tabular}




$\begin{array}{lcll}455217 & 10 & 7 & \text { Septin } \\ 367587 & 10 & 6 & \text { Septin } \\ 481878 & 6 & 3 & \text { Septin } \\ 416864 & 8 & 4 & \text { Septin } \\ 103773 & 5 & 1 & \text { Septin } \\ 103809 & 5 & 2 & \text { Septin } \\ 413977 & 4 & 2 & \text { Tubulin binding cofactor A } \\ 362068 & 4 & 0 & \text { Tubulin binding cofactor D }\end{array}$

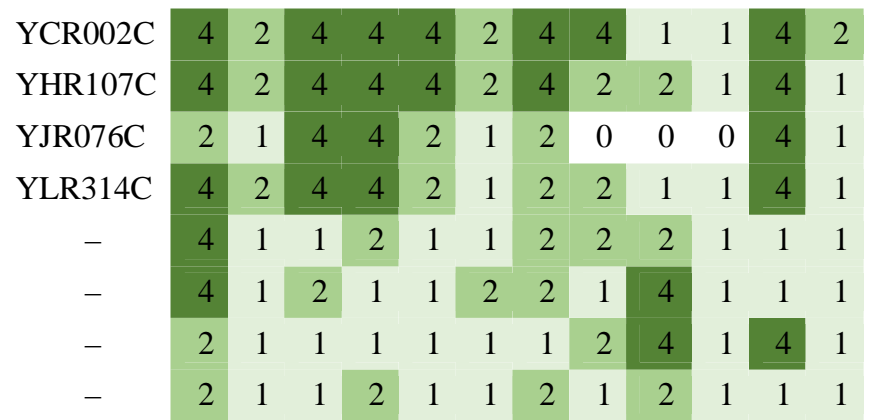

Table 36. Summary of developmental expression dynamics of CDE orthogroups of Cop9 signalosome genes across 12 species. Protein ID of a representative protein is given follows by the number of species in which the orthogroup is developmentally regulated at fold change 2 and 4 (FC>2 and FC>4, respectively). Putative function and ortholog in $S$. cerevisiae (if any) are also given. Abbreviations: 0-gene absent, 1-gene present but not developmentally regulated, 2 developmentally regulated at fold change $>2$, 4- developmentally regulated at fold change $>4$. Species names are abbreviated as: $\mathrm{Cc}-C$. cinerea, $\mathrm{Aa}-A$. ampla, Ab-A. bisporus, Ao - A. ostoyae, $\mathrm{Ca}-\mathrm{C}$. aegerita, $\mathrm{Lb}-\mathrm{L}$. bicolor, $\mathrm{Le}-\mathrm{L}$. edodes, $\mathrm{Lt}-\mathrm{L}$. tigrinus, $\mathrm{Mk}-\mathrm{M}$. kentingensis, $\mathrm{Pc}$ - Ph. chrysosporium, Po - P. ostreatus, Sc - S. commune.

Fold change

\begin{tabular}{|c|c|c|c|}
\hline ID & $>2$ & $>4$ & Putative function \\
\hline 357329 & 9 & 5 & Proteasome component \\
\hline 502637 & 6 & 0 & $\begin{array}{l}\text { Defective-in-cullin neddylation } \\
\text { protein }\end{array}$ \\
\hline 496080 & 4 & 2 & Nedd8-like ubiquitin \\
\hline 438017 & 7 & 1 & $\begin{array}{l}\text { COP9 signalosome complex subunit } \\
5\end{array}$ \\
\hline 497773 & 3 & 0 & $\begin{array}{l}\text { Ubiquitin carboxyl-terminal } \\
\text { hydrolase } 12\end{array}$ \\
\hline 494197 & 4 & 0 & $\begin{array}{l}\text { Cop9 signalosome complex subunit } \\
12\end{array}$ \\
\hline 450685 & 3 & 0 & $\begin{array}{l}\text { NEDD8-conjugating enzyme } \\
\text { UBC12 }\end{array}$ \\
\hline 503638 & 7 & 0 & $\begin{array}{l}\text { NEDD8-activating enzyme E1 } \\
\text { regulatory subunit }\end{array}$ \\
\hline 485679 & 8 & 3 & $\begin{array}{l}\text { NEDD8-activating enzyme E1 } \\
\text { catalytic subunit }\end{array}$ \\
\hline 237692 & 9 & 4 & $\begin{array}{l}\text { Ubiquitin carboxyl-terminal } \\
\text { hydrolase }\end{array}$ \\
\hline 439373 & 5 & 0 & $\begin{array}{l}\text { COP9 signalosome complex subunit } \\
1\end{array}$ \\
\hline 204652 & 3 & 0 & $\begin{array}{l}\text { COP9 signalosome complex subunit } \\
3\end{array}$ \\
\hline 444512 & 3 & 0 & $\begin{array}{l}\text { COP9 signalosome complex subunit } \\
4\end{array}$ \\
\hline 390754 & 4 & 0 & COP9 signalosome protein \\
\hline 35248 & 4 & 0 & $\begin{array}{l}\text { COP9 signalosome subunit } 6 \\
\text { protein }\end{array}$ \\
\hline 437024 & 4 & 1 & Csn8 subunit protein \\
\hline 442909 & 2 & 0 & Proteasome component \\
\hline 255029 & 2 & 0 & $\begin{array}{l}\text { COP9 signalosome complex subunit } \\
2\end{array}$ \\
\hline
\end{tabular}

S. cerevisiae $\quad$ Species ortholog Ce Aa Ab Ao Ca Lb Le Lt Mk Pc Po Sc

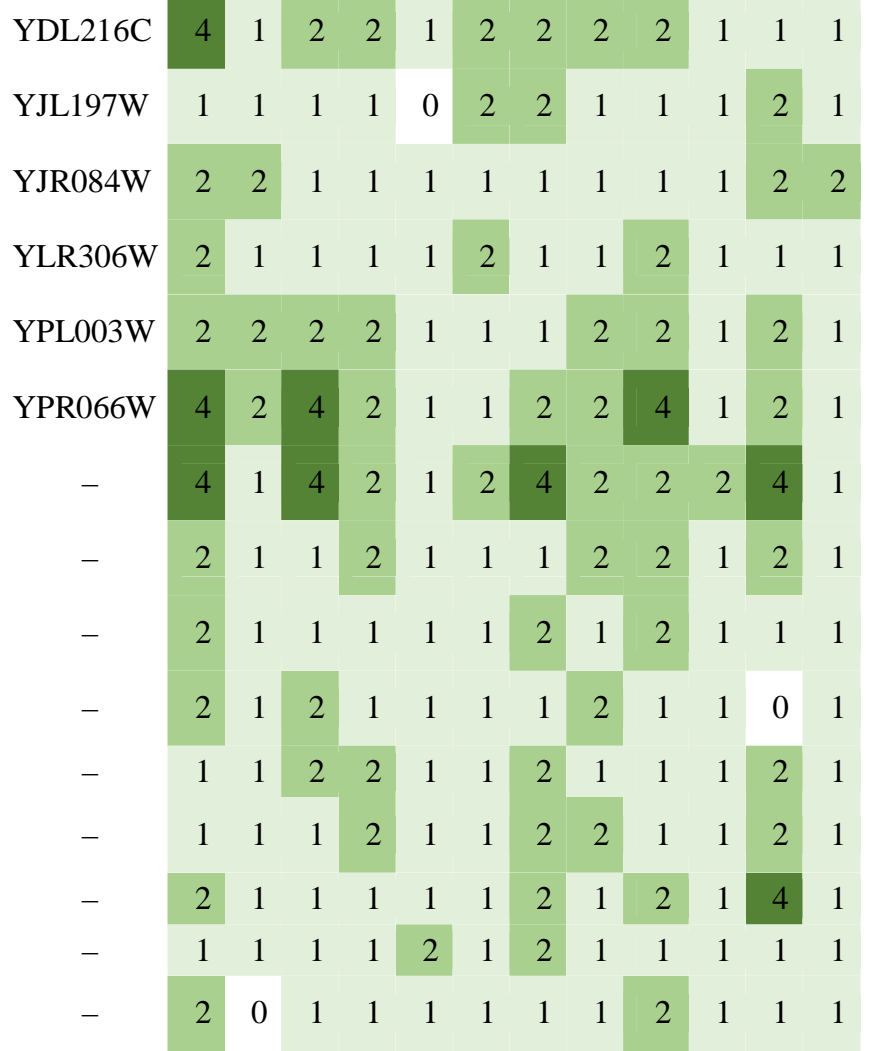


Table 37. Summary of developmental expression dynamics of CDE orthogroups of protein ubiquitination and the proteasome related genes across 12 species. Protein ID of a representative protein is given follows by the number of species in which the orthogroup is developmentally regulated at fold change 2 and 4 (FC>2 and $F C>4$, respectively). Putative function and ortholog in $S$. cerevisiae (if any) are also given. Abbreviations: 0-gene absent, 1-gene present but not developmentally regulated, 2 - developmentally regulated at fold change $>2$, 4- developmentally regulated at fold change $>4$. Species names are abbreviated as: $\mathrm{Cc}-C$. cinerea, $\mathrm{Aa}-A$. ampla, $\mathrm{Ab}$ - A. bisporus, Ao - A. ostoyae, $\mathrm{Ca}-C$. aegerita, Lb - L. bicolor, Le - L. edodes, Lt - L. tigrinus, Mk - M. kentingensis, $\mathrm{Pc}-\mathrm{Ph}$. chrysosporium, $\mathrm{Po}-\mathrm{P}$. ostreatus, $\mathrm{Sc}-\mathrm{S}$. commune.

\begin{tabular}{|c|c|c|c|c|c|c|c|c|c|c|c|c|c|c|c|c|}
\hline \multirow[b]{2}{*}{ ID } & \multicolumn{2}{|c|}{ Fold change } & \multirow[b]{2}{*}{ Putative function } & \multirow{2}{*}{$\begin{array}{c}S . \\
\text { cerevisiae } \\
\text { ortholog }\end{array}$} & \multicolumn{12}{|c|}{ Species } \\
\hline & $>2$ & $>4$ & & & $\mathrm{Cc}$ & Aa & $\mathbf{A b}$ & Ao & $\mathbf{C a}$ & $\mathbf{L b}$ & Le & $\mathbf{L t}$ & Mk & Pc & Po & Sc \\
\hline 354939 & 11 & 5 & $\begin{array}{l}\mathrm{BTB} / \mathrm{POZ} \text { domain containing } \\
\text { protein }\end{array}$ & - & 4 & 1 & 2 & 4 & 4 & 2 & 2 & 4 & 2 & 2 & 4 & 2 \\
\hline 379603 & 9 & 8 & $\begin{array}{l}\mathrm{BTB} / \mathrm{POZ} \text { domain containing } \\
\text { protein }\end{array}$ & - & 4 & 4 & 1 & 4 & 0 & 4 & 4 & 2 & 0 & 4 & 4 & 4 \\
\hline 383774 & 7 & 5 & $\begin{array}{l}\mathrm{BTB} / \mathrm{POZ} \text { domain containing } \\
\text { protein }\end{array}$ & - & 4 & 2 & 0 & 4 & 4 & 2 & 0 & 0 & 0 & 0 & 4 & 4 \\
\hline 405851 & 7 & 5 & $\begin{array}{l}\mathrm{BTB} / \mathrm{POZ} \text { domain containing } \\
\text { protein }\end{array}$ & - & 4 & 0 & 0 & 2 & 2 & 4 & 4 & 0 & 4 & 0 & 4 & 0 \\
\hline 446662 & 5 & 5 & $\begin{array}{l}\text { BTB/POZ domain containing } \\
\text { protein }\end{array}$ & - & 4 & 0 & 4 & 1 & 4 & 4 & 0 & 1 & 1 & 1 & 4 & 0 \\
\hline 449824 & 8 & 8 & $\begin{array}{l}\mathrm{BTB} / \mathrm{POZ} \text { domain containing } \\
\text { protein }\end{array}$ & - & 4 & 4 & 1 & 4 & 0 & 4 & 4 & 1 & 4 & 1 & 4 & 4 \\
\hline 451372 & 9 & 4 & $\begin{array}{l}\mathrm{BTB} / \mathrm{POZ} \text { domain containing } \\
\text { protein }\end{array}$ & - & 2 & 4 & 4 & 2 & 1 & 1 & 2 & 2 & 4 & 1 & 2 & 4 \\
\hline 459680 & 11 & 8 & $\begin{array}{l}\mathrm{BTB} / \mathrm{POZ} \text { domain containing } \\
\text { protein }\end{array}$ & - & 4 & 2 & 4 & 4 & 4 & 4 & 4 & 4 & 4 & 1 & 2 & 2 \\
\hline 374447 & 10 & 7 & $\begin{array}{l}\mathrm{BTB} / \mathrm{POZ} \text { domain containing } \\
\text { protein }\end{array}$ & - & 4 & 4 & 4 & 4 & 2 & 1 & 0 & 2 & 2 & 4 & 4 & 4 \\
\hline 545078 & 5 & 1 & $\begin{array}{l}\mathrm{BTB} / \mathrm{POZ} \text { domain containing } \\
\text { protein }\end{array}$ & - & 2 & 2 & 1 & 2 & 0 & 2 & 1 & 1 & 4 & 1 & 1 & 1 \\
\hline 358351 & 5 & 0 & Orthologs of A. nidulans GrrA & - & 2 & 1 & 1 & 2 & 2 & 1 & 1 & 1 & 2 & 1 & 2 & 1 \\
\hline 447658 & 10 & 5 & F-box protein & - & 4 & 4 & 1 & 4 & 1 & 2 & 4 & 2 & 4 & 2 & 2 & 2 \\
\hline 503496 & 9 & 5 & F-box protein & - & 4 & 1 & 4 & 4 & 2 & 2 & 4 & 4 & 2 & 1 & 2 & 1 \\
\hline 145676 & 10 & 6 & F-box protein & - & 4 & 2 & 4 & 4 & 2 & 4 & 4 & 2 & 4 & 1 & 2 & 1 \\
\hline 359613 & 10 & 6 & F-box protein & - & 2 & 2 & 4 & 4 & 4 & 2 & 4 & 4 & 4 & 1 & 2 & 1 \\
\hline 470790 & 10 & 6 & F-box protein & - & 4 & 2 & 4 & 4 & 2 & 4 & 4 & 2 & 4 & 1 & 1 & 2 \\
\hline 166096 & 9 & 7 & F-box protein & - & 4 & 0 & 0 & 4 & 2 & 4 & 4 & 4 & 4 & 2 & 4 & 0 \\
\hline 356986 & 9 & 6 & F-box protein & - & 4 & 1 & 4 & 4 & 2 & 4 & 2 & 2 & 4 & 1 & 4 & 1 \\
\hline 377171 & 7 & 2 & F-box protein & - & 2 & 2 & 2 & 4 & 1 & 1 & 1 & 2 & 4 & 1 & 1 & 2 \\
\hline 382134 & 7 & 4 & F-box protein & - & 4 & 1 & 1 & 4 & 2 & 1 & 4 & 2 & 0 & 1 & 4 & 2 \\
\hline 438595 & 7 & 7 & F-box protein & - & 4 & 1 & 0 & 4 & 1 & 4 & 4 & 0 & 4 & 1 & 4 & 4 \\
\hline 445665 & 8 & 7 & F-box protein & - & 4 & 1 & 4 & 4 & 4 & 4 & 4 & 2 & 4 & 1 & 0 & 1 \\
\hline 451935 & 7 & 6 & F-box protein & - & 1 & 4 & 1 & 4 & 0 & 4 & 0 & 4 & 0 & 4 & 4 & 2 \\
\hline 471789 & 6 & 6 & F-box protein & - & 4 & 1 & 4 & 4 & 4 & 4 & 4 & 0 & 1 & 0 & 0 & 1 \\
\hline 490594 & 7 & 3 & F-box protein & - & 4 & 1 & 1 & 2 & 2 & 1 & 4 & 1 & 2 & 1 & 4 & 2 \\
\hline 501200 & 12 & 9 & F-box protein & - & 4 & 4 & 4 & 4 & 4 & 4 & 4 & 2 & 2 & 2 & 4 & 4 \\
\hline 440772 & 11 & 7 & $\begin{array}{l}\text { RING-type zinc finger domain } \\
\text { containing protein }\end{array}$ & - & 4 & 4 & 2 & 4 & 2 & 2 & 2 & 0 & 4 & 4 & 4 & 4 \\
\hline
\end{tabular}




$\begin{array}{llll}364816 & 10 & 3 & \begin{array}{l}\text { RING-type zinc finger domain } \\ \text { containing protein }\end{array} \\ 378120 & 8 & 8 & \begin{array}{l}\text { RING-type zinc finger domain } \\ \text { containing protein }\end{array} \\ 455937 & 9 & 2 & \begin{array}{l}\text { RING-type zinc finger domain } \\ \text { containing protein }\end{array} \\ 466739 & 10 & 7 & \begin{array}{l}\text { RING-type zinc finger domain } \\ \text { containing protein }\end{array} \\ 467805 & 10 & 7 & \begin{array}{l}\text { RING-type zinc finger domain } \\ \text { containing protein }\end{array} \\ 543850 & 11 & 9 & \begin{array}{l}\text { RING-type zinc finger domain } \\ \text { containing protein }\end{array}\end{array}$

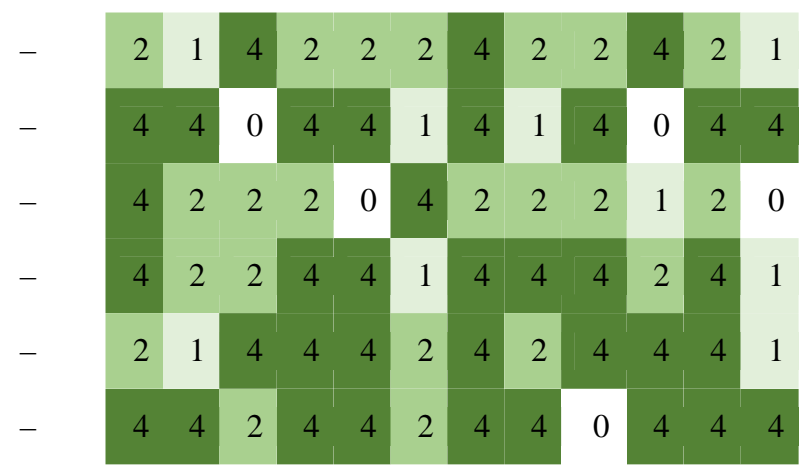

Table 38. Summary of developmental expression dynamics of CDE orthogroups of transporter encoding genes across 12 species. Protein ID of a representative protein is given follows by the number of species in which the orthogroup is developmentally regulated at fold change 2 and 4 (FC>2 and FC>4, respectively). Putative function and ortholog in $S$. cerevisiae (if any) are also given. Abbreviations: 0-gene absent, 1-gene present but not developmentally regulated, 2 developmentally regulated at fold change $>2$, 4- developmentally regulated at fold change $>4$. Species names are abbreviated as: $\mathrm{Cc}-C$. cinerea, $\mathrm{Aa}-A$. ampla, $\mathrm{Ab}-A$. bisporus, Ao $-A$. ostoyae, $\mathrm{Ca}-\mathrm{C}$. aegerita, $\mathrm{Lb}-$ L. bicolor, Le - L. edodes, $\mathrm{Lt}-$ L. tigrinus, Mk - M. kentingensis, $\mathrm{Pc}$ - Ph. chrysosporium, Po - P. ostreatus, Sc - S. commune.

\begin{tabular}{|c|c|c|c|}
\hline \multirow[b]{2}{*}{ ID } & \multicolumn{2}{|c|}{ Fold change } & \multirow[b]{2}{*}{ Putative function } \\
\hline & $>2$ & $>4$ & \\
\hline 538158 & 8 & 5 & Ammonium/urea transporter \\
\hline 404532 & 9 & 5 & $\begin{array}{l}\text { Major facilitator superfamily } \\
\text { protein }\end{array}$ \\
\hline 492885 & 9 & 5 & $\begin{array}{l}\text { Major facilitator superfamily } \\
\text { protein }\end{array}$ \\
\hline $\begin{array}{l}\text { S.c. } \\
253716 \\
3\end{array}$ & 9 & 5 & $\begin{array}{l}\text { Multi antimicrobial extrusion } \\
\text { protein }\end{array}$ \\
\hline 233439 & 9 & 6 & $\begin{array}{l}\text { Tetrapeptide transporter; OPT1/isp4 } \\
\text { family }\end{array}$ \\
\hline 30849 & 11 & 6 & 10TM phosphate transporter \\
\hline 371400 & 11 & 9 & $\begin{array}{l}\text { Major facilitator superfamily } \\
\text { protein }\end{array}$ \\
\hline 464027 & 7 & 6 & $\begin{array}{l}\text { Major facilitator superfamily } \\
\text { protein }\end{array}$ \\
\hline 468563 & 6 & 6 & $\begin{array}{l}\text { Major facilitator superfamily } \\
\text { protein }\end{array}$ \\
\hline 501434 & 9 & 9 & $\begin{array}{l}\text { Major facilitator superfamily } \\
\text { protein }\end{array}$ \\
\hline 436779 & 10 & 0 & Nuclear pore protein $84 / 107$ \\
\hline $\begin{array}{l}\text { S.c. } \\
228395 \\
7\end{array}$ & 8 & 6 & Sugar/inositol transporter \\
\hline 355160 & 10 & 9 & Threonine/Serine exporter; ThrE \\
\hline 432854 & 10 & 6 & Membrane transport protein family \\
\hline 449861 & 10 & 4 & GABA-specific permease \\
\hline 428934 & 10 & 6 & Sugar transporter STL1 \\
\hline 422892 & 10 & 2 & Purine-cytosine permease FCY2 \\
\hline 455616 & 5 & 5 & Zinc-regulated transporter 1 \\
\hline
\end{tabular}

$S$. cerevisiae
ortholog

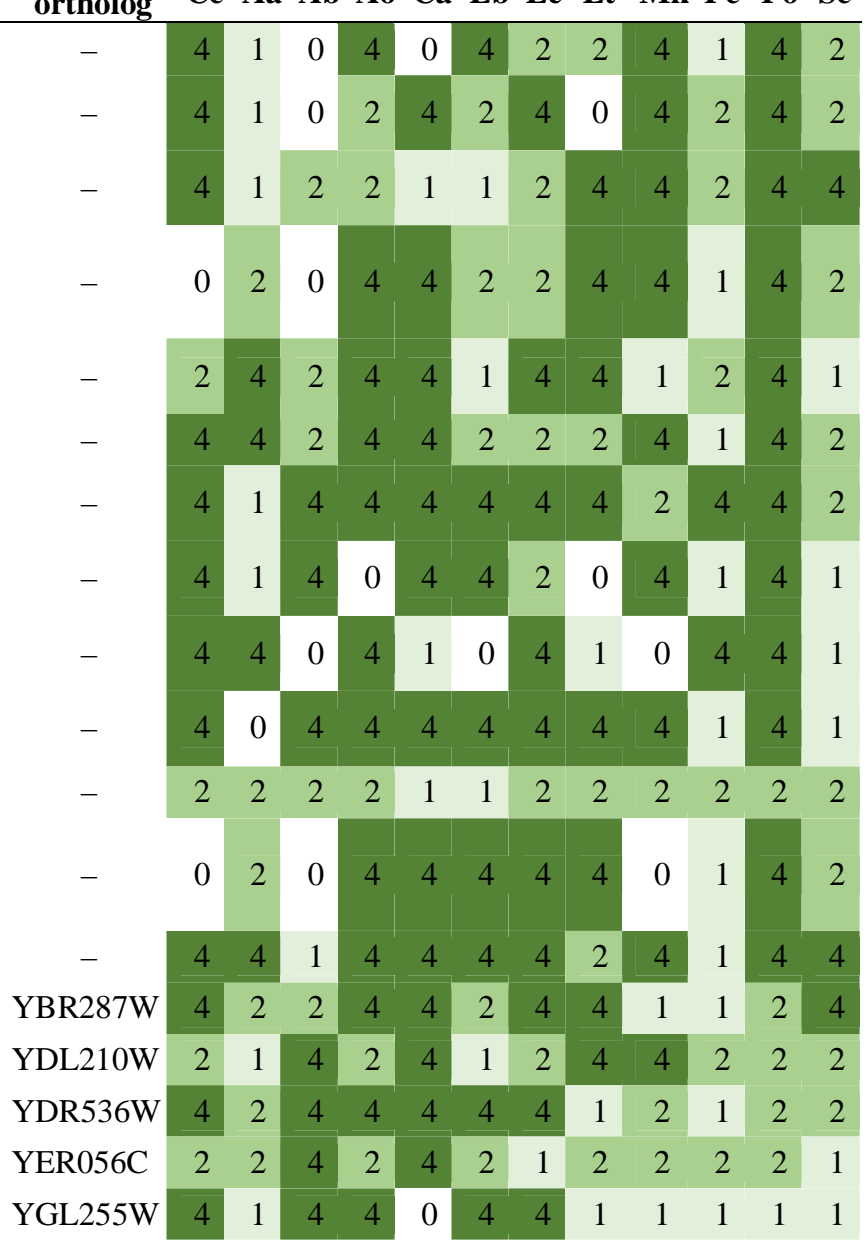




\begin{tabular}{|c|c|c|c|c|c|c|c|c|c|c|c|c|c|c|c|c|}
\hline 465413 & 9 & 6 & Urea transporter & YHL016C & 4 & 1 & 0 & 4 & 4 & 4 & 4 & 2 & 4 & 1 & 2 & 2 \\
\hline 496395 & 9 & 7 & Oligopeptide transporter 1 & YJL212C & 4 & 4 & 2 & 4 & 4 & 1 & 4 & 2 & 4 & 1 & 4 & 0 \\
\hline 502591 & 9 & 6 & Polyamine transporter 1 & YLL028W & 4 & 2 & 4 & 1 & 1 & 2 & 4 & 4 & 4 & 0 & 2 & 4 \\
\hline 360410 & 9 & 8 & $\begin{array}{l}\text { Thiamine pathway transporter } \\
\text { THI73 }\end{array}$ & YLR004C & 4 & 1 & 4 & 4 & 1 & 4 & 4 & 4 & 4 & 1 & 4 & 2 \\
\hline 495380 & 7 & 1 & $\begin{array}{l}\text { Transmembrane } 9 \text { superfamily } \\
\text { member } 1\end{array}$ & YLR083C & 4 & 2 & 2 & 2 & 1 & 1 & 2 & 1 & 0 & 1 & 2 & 2 \\
\hline 223186 & 11 & 3 & $\begin{array}{l}\text { Inorganic phosphate transporter } \\
\text { PHO84 }\end{array}$ & YML123C & 4 & 1 & 2 & 2 & 2 & 4 & 2 & 2 & 2 & 2 & 4 & 2 \\
\hline 393558 & 10 & 6 & $\begin{array}{l}\text { Vacuolar basic amino acid } \\
\text { transporter } 1\end{array}$ & YMR088C & 4 & 4 & 2 & 4 & 2 & 1 & 4 & 2 & 1 & 4 & 2 & 4 \\
\hline 502991 & 10 & 6 & $\begin{array}{l}\text { Major facilitator superfamily } \\
\text { protein }\end{array}$ & YMR279C & 4 & 4 & 4 & 4 & 2 & 2 & 4 & 2 & 0 & 1 & 4 & 2 \\
\hline 421680 & 7 & 3 & Calcium channel YVC1 & YOR087W & 2 & 2 & 2 & 4 & 1 & 1 & 4 & 1 & 1 & 1 & 2 & 4 \\
\hline 542873 & 10 & 5 & ATP-dependent permease PDR12 & YPL058C & 2 & 2 & 2 & 4 & 2 & 4 & 1 & 2 & 4 & 1 & 4 & 4 \\
\hline 462297 & 10 & 2 & Sulfate transporter YPR003C & YPR003C & 2 & 1 & 2 & 4 & 2 & 2 & 2 & 2 & 4 & 1 & 2 & 2 \\
\hline $\begin{array}{l}\text { S.c. } \\
250167 \\
7\end{array}$ & 9 & 6 & $\mathrm{Aqu}$ & - & 0 & 4 & 4 & 4 & 2 & 1 & 4 & 2 & 4 & 1 & 4 & 2 \\
\hline $\begin{array}{l}\text { A.o. } \\
253413\end{array}$ & 6 & 6 & $\begin{array}{l}\text { Major facilitator superfamily } \\
\text { protein }\end{array}$ & - & 0 & 0 & 4 & 4 & 0 & 4 & 4 & 4 & 0 & 0 & 4 & 0 \\
\hline 493842 & 9 & 6 & $\begin{array}{l}\text { Major facilitator superfamily } \\
\text { protein }\end{array}$ & - & 4 & 1 & 4 & 4 & 4 & 2 & 2 & 1 & 4 & 1 & 4 & 2 \\
\hline 495288 & 8 & 6 & $\begin{array}{l}\text { Major facilitator superfamily } \\
\text { protein }\end{array}$ & - & 4 & 4 & 2 & 4 & 4 & 4 & 0 & 4 & 1 & 1 & 2 & 1 \\
\hline 496145 & 9 & 7 & Sugar/inositol transporter & - & 4 & 4 & 1 & 4 & 2 & 1 & 4 & 4 & 4 & 2 & 4 & 1 \\
\hline 498282 & 10 & 6 & $\begin{array}{l}\text { Major facilitator superfamily } \\
\text { protein }\end{array}$ & - & 4 & 2 & 2 & 4 & 1 & 4 & 4 & 2 & 2 & 4 & 4 & 1 \\
\hline 474705 & 10 & 6 & $\mathrm{ABC}$ transporter & - & 4 & 2 & 0 & 4 & 2 & 4 & 4 & 1 & 2 & 2 & 4 & 4 \\
\hline 357414 & 6 & 5 & $\begin{array}{l}\text { Major facilitator superfamily } \\
\text { protein }\end{array}$ & - & 4 & 1 & 4 & 4 & 1 & 0 & 0 & 4 & 2 & 1 & 4 & 1 \\
\hline 420930 & 8 & 7 & $\begin{array}{l}\text { Major facilitator superfamily } \\
\text { protein }\end{array}$ & - & 4 & 4 & 4 & 4 & 2 & 4 & 4 & 1 & 0 & 1 & 4 & 1 \\
\hline 463045 & 9 & 6 & $\begin{array}{l}\text { Major facilitator superfamily } \\
\text { protein }\end{array}$ & - & 4 & 2 & 4 & 4 & 2 & 4 & 0 & 2 & 0 & 1 & 4 & 4 \\
\hline 465489 & 9 & 5 & $\begin{array}{l}\text { Major facilitator superfamily } \\
\text { protein }\end{array}$ & - & 4 & 1 & 4 & 4 & 0 & 1 & 2 & 2 & 4 & 2 & 4 & 2 \\
\hline 488859 & 9 & 5 & $\begin{array}{l}\text { Mitochondrial substrate/solute } \\
\text { carrier }\end{array}$ & - & 4 & 2 & 4 & 2 & 4 & 1 & 2 & 1 & 4 & 1 & 4 & 2 \\
\hline 451236 & 10 & 2 & $\begin{array}{l}\text { Secretory carrier membrane proteins } \\
\text { (SCAMP) }\end{array}$ & - & 2 & 2 & 1 & 4 & 2 & 2 & 4 & 2 & 2 & 1 & 2 & 2 \\
\hline 374240 & 10 & 8 & Sodium/calcium exchanger & - & 4 & 1 & 0 & 4 & 2 & 4 & 4 & 2 & 4 & 4 & 4 & 4 \\
\hline 450674 & 9 & 7 & Sugar phosphate transporter & - & 4 & 2 & 4 & 4 & 2 & 1 & 4 & 4 & 4 & 1 & 4 & 1 \\
\hline 537526 & 10 & 7 & Sugar phosphate transporter & - & 4 & 4 & 2 & 4 & 4 & 2 & 2 & 1 & 4 & 1 & 4 & 4 \\
\hline 358584 & 7 & 5 & Sugar/inositol transporter & - & 4 & 4 & 2 & 4 & 1 & 0 & 1 & 4 & 1 & 1 & 4 & 2 \\
\hline 487841 & 11 & 8 & Sugar/inositol transporter & - & 4 & 4 & 4 & 4 & 2 & 4 & 4 & 4 & 2 & 2 & 1 & 4 \\
\hline 502428 & 7 & 5 & Sugar/inositol transporter & - & 4 & 2 & 2 & 4 & 1 & 1 & 1 & 4 & 4 & 1 & 4 & 1 \\
\hline $\begin{array}{l}\text { S.c. } \\
262770 \\
3\end{array}$ & 6 & 5 & Sugar/inositol transporter & - & 0 & 2 & 4 & 4 & 0 & 0 & 4 & 0 & 4 & 0 & 0 & 4 \\
\hline 397645 & 9 & 6 & High glucose sensor RGT2 & YDL138W & 4 & 4 & 4 & 2 & 0 & 1 & 2 & 2 & 4 & 1 & 4 & 4 \\
\hline 463015 & 8 & 7 & Hexose transporter HXT10 & YFL011W & 4 & 4 & 0 & 4 & 4 & 0 & 4 & 4 & 0 & 1 & 4 & 2 \\
\hline
\end{tabular}


Table 39. Summary of developmental expression dynamics of CDE orthogroups of stress response genes across 12 species. Protein ID of a representative protein is given follows by the number of species in which the orthogroup is developmentally regulated at fold change 2 and 4 (FC>2 and $\mathrm{FC}>4$, respectively). Putative function and ortholog in $S$. cerevisiae (if any) are also given.

Abbreviations: 0-gene absent, 1-gene present but not developmentally regulated, 2 developmentally regulated at fold change $>2$, 4- developmentally regulated at fold change $>4$. Species names are abbreviated as: $\mathrm{Cc}-C$. cinerea, $\mathrm{Aa}-A$. ampla, Ab-A. bisporus, Ao - A. ostoyae, $\mathrm{Ca}-\mathrm{C}$. aegerita, $\mathrm{Lb}-$ L. bicolor, Le - L. edodes, $\mathrm{Lt}-$ L. tigrinus, $\mathrm{Mk}-M$. kentingensis, $\mathrm{Pc}$ - Ph. chrysosporium, Po - P. ostreatus, Sc - S. commune.

\begin{tabular}{|c|c|c|c|}
\hline \multirow[b]{2}{*}{ ID } & \multicolumn{2}{|c|}{ Fold change } & \multirow[b]{2}{*}{ Putative function } \\
\hline & $>2$ & $>4$ & \\
\hline 475934 & 7 & 7 & $12 \mathrm{kDa}$ heat shock protein \\
\hline 457314 & 8 & 5 & $\begin{array}{l}\text { ATP-dependent molecular } \\
\text { chaperone HSC } 82\end{array}$ \\
\hline 492695 & 6 & 5 & $\begin{array}{l}\text { Highly conserved plasma membrane } \\
\text { protein }\end{array}$ \\
\hline 496981 & 9 & 7 & Small heat shock protein HSP20 \\
\hline 23471 & 11 & 5 & $\begin{array}{l}\text { Endoplasmic reticulum chaperone } \\
\mathrm{BiP}\end{array}$ \\
\hline 367102 & 11 & 6 & Small heat shock protein HSP20 \\
\hline 420157 & 8 & 5 & Catalase \\
\hline 472224 & 11 & 7 & Peroxisomal catalase A \\
\hline 432313 & 11 & 11 & D-arabinono-1;4-lactone oxidase \\
\hline 365550 & 7 & 2 & D-arabinono-1;4-lactone oxidase \\
\hline 356399 & 11 & 8 & $\begin{array}{l}\text { Ergothioneine biosynthesis protein } \\
\text { Egt1 }\end{array}$ \\
\hline 355021 & 9 & 7 & Peroxiredoxin \\
\hline 258252 & 5 & 4 & $\begin{array}{l}\text { Ergothioneine biosynthesis protein } \\
\text { Egt2 }\end{array}$ \\
\hline 28769 & 8 & 2 & $\begin{array}{l}\text { Glutathione peroxidase-like } \\
\text { peroxiredoxin } 2\end{array}$ \\
\hline 415694 & 7 & 0 & Monothiol glutaredoxin-6 \\
\hline 441808 & 7 & 3 & Glutaredoxin \\
\hline 378207 & 5 & 1 & Monothiol glutaredoxin-4 \\
\hline 412620 & 5 & 2 & Glutamate--cysteine ligase \\
\hline 370027 & 5 & 1 & Glutathione synthetase \\
\hline 356216 & 7 & 1 & $\begin{array}{l}\text { Monothiol glutaredoxin-5, } \\
\text { mitochondrial }\end{array}$ \\
\hline 499716 & 5 & 3 & Gluthatione reductase \\
\hline 39451 & 7 & 3 & Glutaredoxin \\
\hline 378471 & 4 & 0 & Peroxiredoxin \\
\hline 379600 & 9 & 6 & Peroxiredoxin DOT5 \\
\hline 444300 & 10 & 4 & Peroxiredoxin AHP1 \\
\hline 468305 & 12 & 8 & Peroxiredoxin TSA1 \\
\hline 437202 & 8 & 6 & Peroxiredoxin \\
\hline 449056 & 9 & 7 & Peroxiredoxin \\
\hline $\begin{array}{l}\text { S.c. } \\
263240\end{array}$ & 5 & 3 & Peroxiredoxin \\
\hline
\end{tabular}

$S$. cerevisiae $\quad$ Species

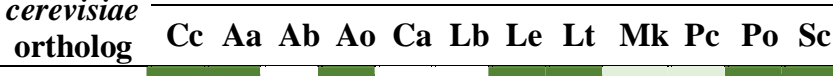

\begin{tabular}{llllll|llll|l|l|lllll} 
YFL014W & 4 & 4 & 0 & 4 & 0 & 0 & 4 & 4 & 1 & 1 & 4 & 4 \\
\hline
\end{tabular}
\begin{tabular}{lll|l|l|l|l|l|l|l|l|l|l} 
YMR186W & 4 & 1 & 4 & 4 & 2 & 1 & 4 & 1 & 2 & 1 & 4 & 2
\end{tabular} e

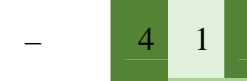

YJL034W
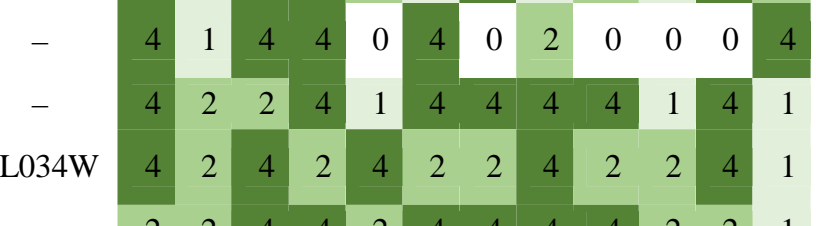

YDR256C

YML086C

YMR041C

YBL064C
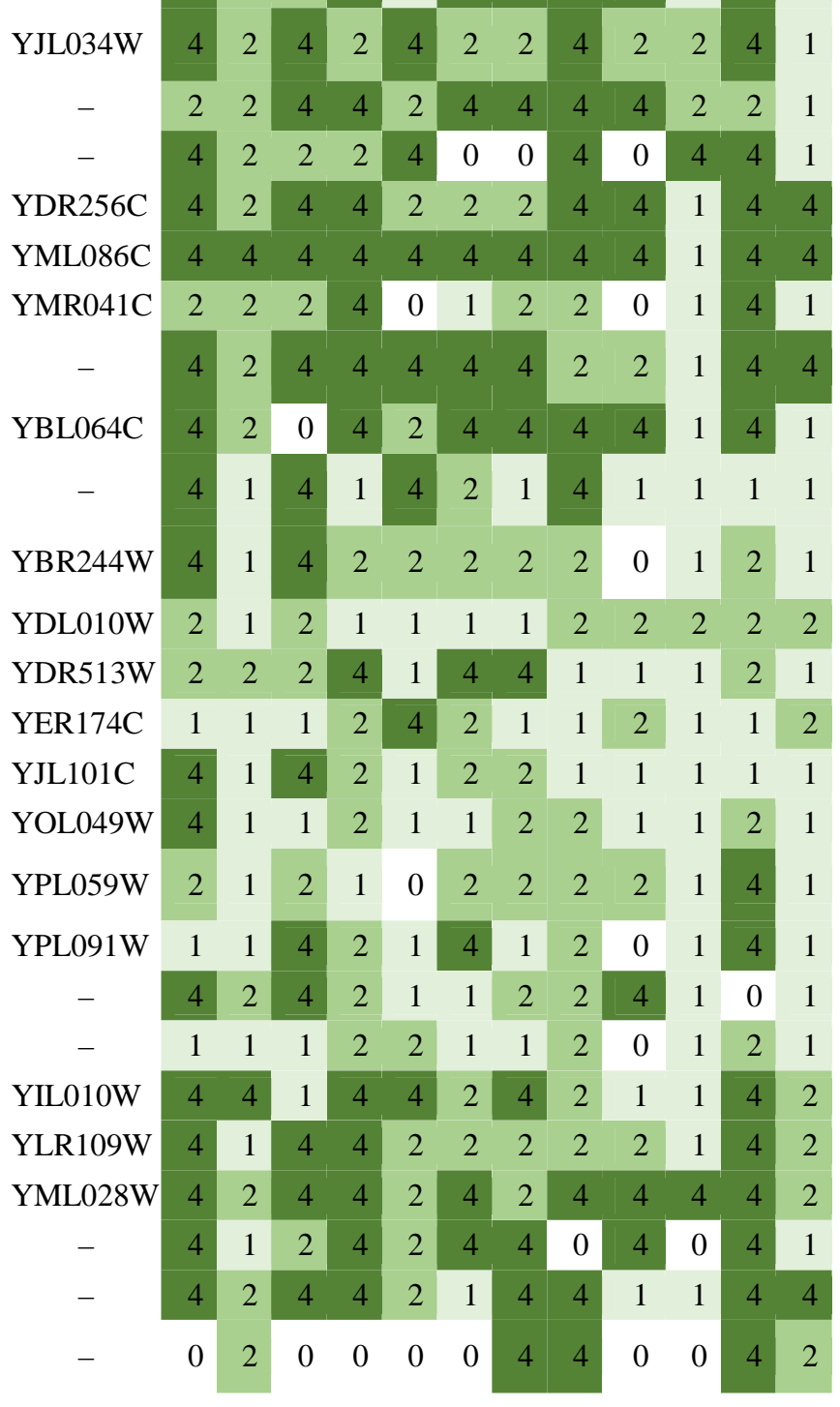
Table 40. Summary of developmental expression dynamics of CDE orthogroups of ferric reductase genes across 12 species. Protein ID of a representative protein is given follows by the number of species in which the orthogroup is developmentally regulated at fold change 2 and 4 (FC>2 and $\mathrm{FC}>4$, respectively). Putative function and ortholog in $S$. cerevisiae (if any) are also given.

Abbreviations: 0-gene absent, 1-gene present but not developmentally regulated, 2 developmentally regulated at fold change $>2$, 4- developmentally regulated at fold change $>4$. Species names are abbreviated as: $\mathrm{Cc}-C$. cinerea, $\mathrm{Aa}-A$. ampla, Ab-A. bisporus, Ao - A. ostoyae, $\mathrm{Ca}-C$. aegerita, $\mathrm{Lb}-$ L. bicolor, Le - L. edodes, Lt - L. tigrinus, Mk $-M$. kentingensis, $\mathrm{Pc}$ - Ph. chrysosporium, Po - P. ostreatus, Sc - S. commune.

\begin{tabular}{lccl} 
& \multicolumn{2}{c}{ Fold change } & \\
\cline { 2 - 3 } ID & $>\mathbf{2}$ & $\mathbf{> 4}$ & Putative function \\
\hline 354442 & 9 & 5 & Ferric reductase \\
152927 & 9 & 5 & Ferric reductase \\
260659 & 5 & 1 & Ferric reductase \\
448679 & 3 & 1 & Ferric reductase \\
461481 & 4 & 0 & Ferric reductase
\end{tabular}

S. cerevisiae
ortholog Ce Aa Ab Ao Ca Lb Le Lt Mk Pc Po Sc

\begin{tabular}{|c|c|c|c|c|c|c|c|c|c|c|c|c|}
\hline- & 4 & 0 & 4 & 4 & 0 & 4 & 4 & 2 & 2 & 2 & 2 & 1 \\
\hline YOR384W & 4 & 1 & 1 & 2 & 4 & 2 & 4 & 2 & 4 & 4 & 2 & 1 \\
\hline- & 1 & 1 & 4 & 2 & 2 & 1 & 1 & 1 & 2 & 1 & 2 & 1 \\
\hline - & 1 & 1 & 2 & 1 & 1 & 1 & 1 & 1 & 4 & 1 & 2 & 1 \\
\hline - & 2 & 1 & 2 & 2 & 2 & 1 & 1 & 1 & 0 & 1 & 1 & 1 \\
\hline
\end{tabular}

